\title{
Transformations of Energy-Related Small Molecules at Dinuclear Complexes
}

\author{
Dissertation \\ zur Erlangung des \\ mathematisch-naturwissenschaftlichen Doktorgrades \\ „Doctor rerum naturalium“ \\ der Georg-August-Universität Göttingen \\ im Promotionsprogramm Chemie \\ der Georg-August-University School of Science (GAUSS) \\ vorgelegt von \\ Jana Lücken \\ aus Oldenburg
}

Göttingen 2020 


\section{Betreuungsausschuss}

Prof. Dr. Franc Meyer

Institut für Anorganische Chemie, Georg-August-Universität Göttingen

Prof. Dr. Inke Siewert

Institut für Anorganische Chemie, Georg-August-Universität Göttingen

\section{Prüfungskomitee}

Referent: Prof. Dr. Franc Meyer

Institut für Anorganische Chemie, Georg-August-Universität Göttingen

Korreferentin: Prof. Dr. Inke Siewert

Institut für Anorganische Chemie, Georg-August-Universität Göttingen

Prof. Dr. Sven Schneider

Institut für Anorganische Chemie, Georg-August-Universität Göttingen

Prof. Dr. Ricardo Mata

Institut für Physikalische Chemie, Georg-August-Universität Göttingen

Jun.-Prof. Dr. Nathalie Kunkel

Institut für Anorganische Chemie, Georg-August-Universität Göttingen

Dr. Michael John

Institut für Organische und Biomolekulare Chemie, Georg-August-Universität Göttingen

Datum der mündlichen Prüfung: 04. November 2020 


\section{Abstract}

The urgent need for the development of carbon-neutral energy conversion schemes has inspired chemists worldwide to investigate catalytic water splitting using sunlight as the energy source. A key component for the preparation of devices employing this process is the water oxidation catalyst (WOC), and understanding the factors that govern its catalytic performance is among the major challenges. Beyond the well-established WOCs based on ruthenium, catalysts containing abundant first-row transition metals are a desirable target but require a deeper understanding before their large-scale application is viable. Along these lines, this work focusses on both the in-depth analysis of diruthenium WOCs, as well as the search for new catalysts based on copper and cobalt. A glance is also cast at dioxygen activation by dicopper complexes, which is not only of interest on its own but can additionally provide insight into intermediates that are relevant to water oxidation.

Two rationally designed diruthenium WOCs are presented bearing a compartmental ligand scaffold with a central pyrazolate moiety and peripheral carboxylate groups, beneficially preorganizing two metal ions in close proximity and lowering the overall charge of the complex, respectively. The two new complexes are completed with four axial sulfonated pyridine ligands that ensure high water solubility over a large $\mathrm{pH}$ range, enabling extensive water oxidation catalysis investigations and mechanistic studies. Distinction arises from the exogenous unit in the bimetallic cleft: an S,O-bridging DMSO in $3^{\text {dmso }}$ or two $\mathrm{H}_{2} \mathrm{O}$ ligands in $3^{\mathbf{H 2 0}}$. The complexes were thoroughly characterized electrochemically - using standard techniques such as cyclic voltammetry and square wave voltammetry, culminating in the construction of a Pourbaix diagram for $3^{\mathbf{H} 20}$ - and investigated in regard to their catalytic proficiency in chemical, electrochemical and photo-driven water oxidation. Using ceric ammonium nitrate (CAN) as chemical oxidant, $3^{\text {dmso }}$ and $3^{\mathbf{H} 20}$ exhibit similar turnover frequencies ( 0.188 and $0.201 \mathrm{~s}^{-1}$, respectively) but distinct overall catalytic profiles with retardation of catalysis by $\mathrm{Ce}^{\mathrm{III}}$ and nitrate from CAN. A foot-of-the-wave analysis demonstrates efficient electrocatalytic behavior for $3^{\mathbf{H 2 0}}$ with a $k_{\text {cat }}$ of $22.5 \pm 2.8 \mathrm{~s}^{-1}$ at $\mathrm{pH} 5.6$, and both complexes are shown to photochemically generate $\mathrm{O}_{2}$ using $\left[\mathrm{Ru}\left((\mathrm{COOEt})_{2} \mathrm{bpy}\right)_{2}(\mathrm{bpy})\right]^{2+}$ as photosensitizer and $\mathrm{Na}_{2} \mathrm{~S}_{2} \mathrm{O}_{8}$ as sacrificial electron acceptor. Although photocatalytic activity stops after around 130 turnovers in the applied experimental setup, control experiments suggest that this is not attributed to the degradation of the catalyst. Surprisingly, UV/vis spectroelectrochemical measurements indicate that the DMSO moiety in $3^{\text {dmso }}$ remains intact and within the bimetallic pocket during both chemical and electrochemical water oxidation at least for a few turnovers, implying different catalytically active species for the two WOCs. Three possible mechanistic scenarios are considered for $3^{\text {dmso }}$ based on the well-known hemilability of peripheral carboxylate groups and bridging exogenous ligands, as well as the potential expansion of the coordination environment of one ruthenium center from six to seven at high oxidation states. The unexpected stability of the DMSO moiety in the bimetallic pocket of $3^{\text {dmso }}$ during catalysis suggests that the role and fate of DMSO ligands in ruthenium-based WOCs should be carefully assessed.

A set of dinuclear copper and cobalt complexes based on a bis(tetradentate) platform with a central pyrazolate bridge and triazacyclononane (tacn) sidearms connected via methylene units are introduced, differing by the substituents of the tacn macrocycles $\left(\mathrm{Me}_{2}\right.$-tacn in $\left(\mathrm{L}^{2 \mathrm{a}}\right)$ - or the more bulky $i \operatorname{Pr}_{2}$-tacn in $\left.\left(\mathrm{L}^{2 \mathrm{~b}}\right)^{-}\right)$and the exogenous ligand in the in,in-position. Of the ( $\left.\mathrm{L}^{2 \mathrm{a}}\right)^{-}-$ligated complexes, the formate-bridged dicopper(II) compound $\mathbf{5}^{\mathrm{HCO} 2}$ and the MeCN-containing dicobalt(II) complex $\mathbf{7}^{\text {MecN }}$ were examined with respect to their ability to serve as electrocatalysts for water oxidation. Both complexes feature an irreversible wave in the cyclic voltammogram, which could be assigned to catalytic water oxidation by dioxygen detection experiments in combination with controlled potential electrolysis (CPE) for $\mathbf{5}^{\mathbf{H C O}}$ (52 \% faradaic efficiency). Rinse tests after cyclic voltammetry ( $7^{\mathrm{MeCN}}$ ) and CPE $\left(5^{\mathrm{HCO}}\right)$ clearly reveal a catalytically active deposit on the electrode surface, suggesting that the compounds merely act as precatalysts and quickly degrade under turnover conditions. In case of $\mathbf{5}^{\mathbf{H C O}}$, the deposited material could be identified via X-ray photoelectron spectroscopy as a copper oxide/hydroxide species. The decomposition of $5^{\mathrm{HCO}}$ and $7^{\mathrm{MecN}}$ under the harsh catalytic conditions 
emphasizes the necessity for oxidatively robust ligand systems. In addition to the analysis of the electrocatalytic water oxidation ability, magnetic susceptibility measurements were conducted for several of the dicopper and dicobalt complexes bearing the pyrazolate/tacn hybrid ligands, viz. $7^{\text {MeCN, }}$ $\mathbf{5}^{\mathrm{HCO}}$ and its acetate-bridged congener $\mathbf{5}^{\mathbf{O A c}}$ as well as the $\left(\mathrm{L}^{2 \mathrm{~b}}\right)^{-}$-ligated dicobalt analogs $\mathbf{8}^{\mathrm{HCO}}$ and $\mathbf{8}^{\mathbf{O A c}}$. Interestingly, $\mathbf{8}^{\mathbf{H C O 2}}$ and $\mathbf{8}^{\mathbf{0 A c}}$ feature slow relaxation of magnetization with relaxation times $\tau_{0}$ of $7.7 \cdot 10^{-6} \mathrm{~s}^{-1}$ and $3.7 \cdot 10^{-6} \mathrm{~s}^{-1}$, as well as energy barriers $U_{\text {eff }}$ of 8.9 and $6.0 \mathrm{~cm}^{-1}$, respectively, and thus belong to a very small circle of homodinuclear cobalt(II) complexes with SMM behavior.

$\mathbf{5}^{\mathbf{O A c}}$ and $\mathbf{5}^{\mathbf{H C O 2}}$ were further investigated in regard to dioxygen activation following the strategy: (i) reduction of the dicopper(II) complex for the in situ generation of a dicopper(I) species ( $5^{\mathrm{I} / \mathrm{I}}$ ) and (ii) addition of dry $\mathrm{O}_{2}$ to $\mathbf{5}^{\mathbf{I} / \mathbf{I}}$ for copper-oxygen adduct formation. Surprisingly, reduction of $\mathbf{5}^{\mathbf{A A c}}$ to $\mathbf{5}^{\mathbf{I} / \mathbf{I}}$

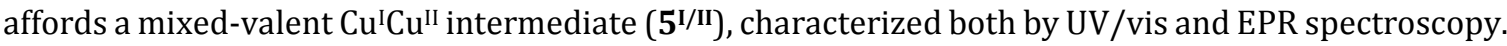
This observation is unprecedented for dinuclear copper complexes bearing pyrazolate/tacn hybrid ligands and is attributed to the acetate unit in the bimetallic pocket, which is proposed to change its coordination mode from bridging to chelating upon one-electron reduction in order to stabilize the $\mathrm{Cu}^{\mathrm{II}}$ site. Addition of molecular oxygen to $\mathbf{5}^{\mathbf{I} / \mathbf{I I}}$ monitored by time-resolved UV/vis spectroscopy in a stopped-flow setup at $-40{ }^{\circ} \mathrm{C}$ results in a superoxo species $\left(\mathbf{5}^{\mathbf{S}}\right)$ as primary $\mathrm{Cu}^{\mathrm{II}_{2}} \mathrm{O}_{2}$ adduct, in line with a $1 \mathrm{e}^{-}$reduction of the dioxygen. The generated $\mathbf{5}^{\mathbf{S}}$ rapidly converts to a $\mu-1,2$-peroxo compound $\left(\mathbf{5}^{\mathbf{P}}\right)$, distinguished by its characteristic UV/vis-spectroscopic features, by reduction with either an additional $\mathbf{5}^{\mathbf{I} / \mathbf{I I}}$ species or a second $\mathbf{5}^{\mathbf{S}}$ compound. On the other hand, $\mathbf{5}^{\mathbf{P}}$ is directly formed when exposing $5^{\mathrm{I} / \mathrm{I}}$ to $\mathrm{O}_{2}$, and can be electrochemically oxidized at $-0.55 \mathrm{~V} v$. $\mathrm{Fc}^{+/ 0}\left(\mathrm{O}_{2}{ }^{2-} / \mathrm{O}_{2}{ }^{--}\right.$redox couple $)$ at $-45^{\circ} \mathrm{C}$ in MeCN. The low stability observed for the copper-oxygen adducts of $\mathbf{5}$ is attributed to the highly exposed dioxygen derived ligands associated with the methylene linker between the pyrazolate moiety and tacn sidearms as well as the compact methyl residues at the macrocycles. As previously shown, $i \mathrm{Pr}_{2}$-tacn congeners of $\mathbf{5}$ bearing a methylene or ethylene linker yield significantly more stable $\mathrm{Cu}_{2} \mathrm{O}_{2}$ adducts with a low oxidation/oxygenation reactivity towards organic substrates, which is assumed to be higher for the herein presented complexes.

Intriguingly, the reduction of $5^{\mathrm{HCO}}$ monitored by NMR spectroscopy does not generate a dicopper(I) species but instead an oligonuclear copper(I) hydride cluster $\left(\mathbf{9}^{\mathbf{H}}\right)$ under carbon dioxide release. Isotopic labeling studies proceeding from the $\mathrm{DCO}_{2}^{-}\left(\mathbf{5}^{\mathrm{DCO}}\right)$ and $\mathrm{H}^{13} \mathrm{CO}_{2}{ }^{-}\left(\mathbf{5}^{\left.\mathbf{H}\{\mathbf{1 3 C}\} \mathbf{O}^{\mathbf{O}}\right)}\right.$ congeners of $5^{\mathrm{HCO}}$ reveal that both the $\mathrm{H}^{-}$ligands and $\mathrm{CO}_{2}$ originate from the $\mu_{1,3}$-bridging formate molecule. Formate decarboxylation and hydride abstraction can be imagined happening via a transient $\left[\left(\mathrm{L}^{2 \mathrm{a}}\right) \mathrm{Cu}_{2} \mathrm{I}_{2}\left(\mu-\mathrm{O}_{2} \mathrm{CH}\right)\right]$ species or a mixed-valent $\left[\left(\mathrm{L}^{2 \mathrm{a}}\right) \mathrm{Cu}^{\mathrm{I} C \mathrm{Cu}^{\mathrm{II}}}\left(\mu-\mathrm{O}_{2} \mathrm{CH}\right)\right]$ species followed by further reduction. The exact rearrangement of ligand strands and metal ions to the hexacopper(I) product $\left[\left(\mathrm{L}^{2 \mathrm{a}}\right)_{2} \mathrm{Cu}_{6}(\mathrm{H})_{2}\right]^{2+}$ containing two $\mathrm{Cu}_{3}\left(\mu_{3}-\mathrm{H}\right)$ subunits remains unclear, however the full reaction equation of this process could be determined, further comprising a free ligand molecule (identified by ESI mass spectrometry and NMR spectroscopy) and an unbound formate unit (detected by NMR spectroscopy) on the product side. Typical hydride reactivity of $\mathbf{9}^{\mathbf{H}}$ is demonstrated by the reaction with phenylacetylene retaining the $\left[\left(\mathrm{L}^{2 \mathrm{a}}\right)_{2} \mathrm{Cu}_{6}\right]$ core but replacing the hydrides with $\mathrm{PhC} \equiv \mathrm{C}^{-}$ligands bridging two $\mathrm{Cu}^{\mathrm{I}}$ ions in end-on fashion and serving as a side-on alkyne ligand ( $\pi$-bonding) to the more distant third $\mathrm{Cu}^{\mathrm{I}}$ center (968)5 $)$. By means of DFT calculations, four different isomers for both $\mathbf{9}^{\mathbf{H}}$ and 9C8H5 were identified featuring trans- and cis-arrangement of both the tacn sidearms of (L2a)- and the $\mathrm{H}^{-} / \mathrm{PhC} \equiv \mathrm{C}^{-}$moieties with respect to the pyrazolate plane in all possible combinations, which aid in explaining the dynamics observed in solution by variable temperature NMR spectroscopy. Control experiments performed with the $\left(\mathrm{L}^{2 \mathrm{~b}}\right)^{-}$analogs of $\mathbf{5}^{\mathbf{O A c}}$ and $\mathbf{5}^{\mathbf{H C O}}$, viz. $\mathbf{6}^{\mathbf{O A c}}$ and $\mathbf{6}^{\mathbf{H C O}}$, highlight that the formation of the mixed-valent $\mathrm{Cu}^{\mathrm{I} C \mathrm{Cu}^{\mathrm{II}}}$ species $\left(\mathbf{5}^{\mathrm{I} / \mathrm{II}}\right)$ as well as the hexacopper(I) hydride cluster $\left(\mathbf{9}^{\mathrm{H}}\right)$ are unique for the reduction of the $\mathrm{Me}_{2}$-tacn complexes, suggesting that steric hindrance is too high with isopropyl residues at the tacn moieties to accommodate the respective coordination motifs. 


\section{Contents}

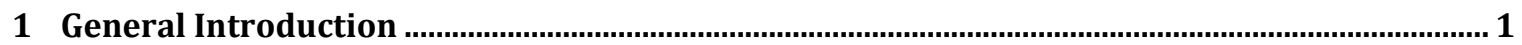

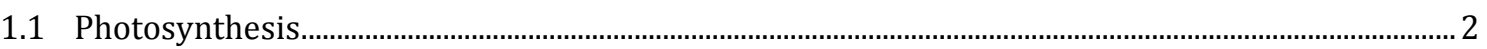

1.2 Artificial Photosynthesis ...................................................................................................................

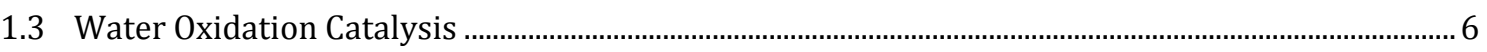

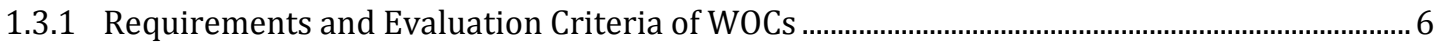

1.3.2 Molecular vs. Non-Molecular, Precious vs. Base Metal WOCs......................................................... 7

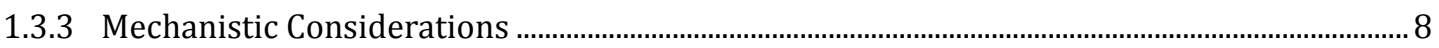

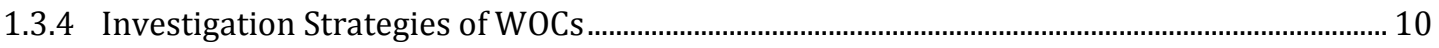

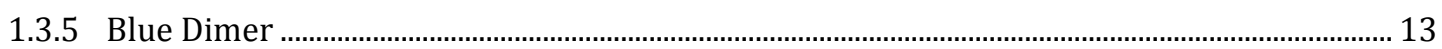

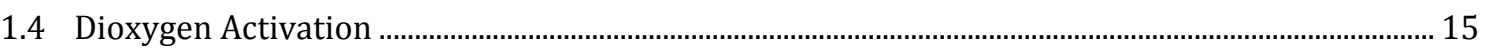

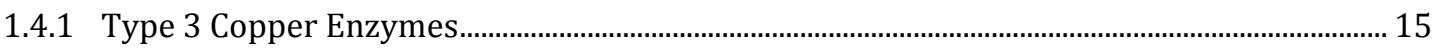

1.4.2 Synthetic Modeling...................................................................................................................... 16

2 Objectives

3 Water Oxidation Catalysis with Ruthenium Complexes …….......................................................23

3.1 Ligand Synthesis and Ruthenium Complexation ............................................................................... 27

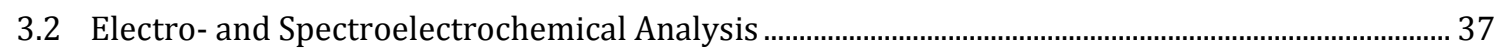

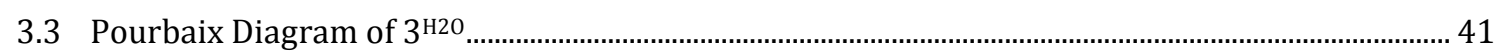

3.4 Electrochemical Water Oxidation Catalysis.................................................................................. 45

3.5 Chemical Water Oxidation Catalysis and Mechanistic Investigations ................................................ 46

3.5.1 Order of the Reaction and Comparison between the Catalytic Behavior of $3^{\mathrm{dmso}}$ and $3^{\mathrm{H} 2 \mathrm{O}} 46$

3.5.2 Inhibition of the Catalysis by Anation of Nitrate Ions and Interaction with Cerium ............ 49

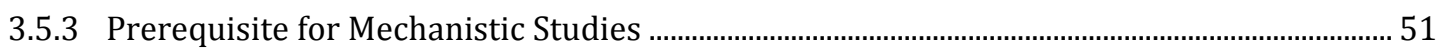

3.5.4 DMSO vs. Aquo Bridge: What is the Active Species? ……………………………...................... 54

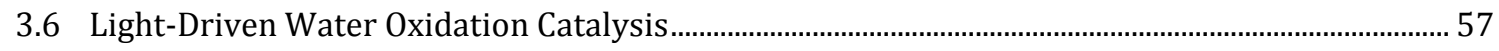

3.7 Summary and Conclusion ................................................................................................................... 61

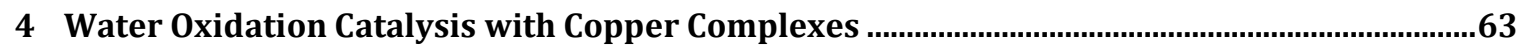

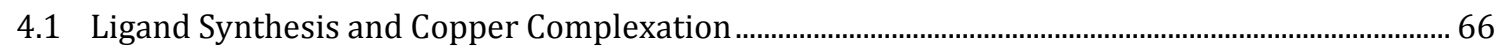

4.2 Magnetic Properties ................................................................................................................. 70

4.3 Electro- and Spectroelectrochemical Analysis ................................................................................. 71

4.3.1 Under Inert Conditions .................................................................................................... 71

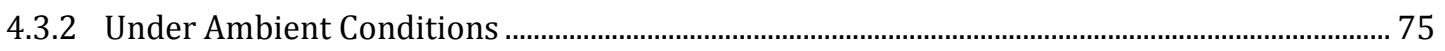

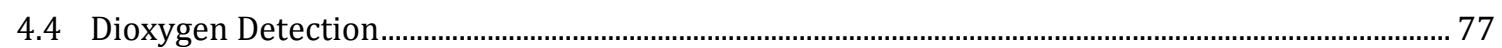

4.5 Characterization of the Surface Deposit...................................................................................... 78

4.6 Summary and Conclusion .................................................................................................................... 79

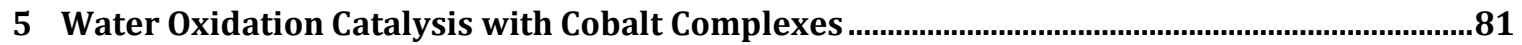

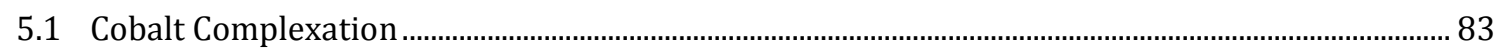

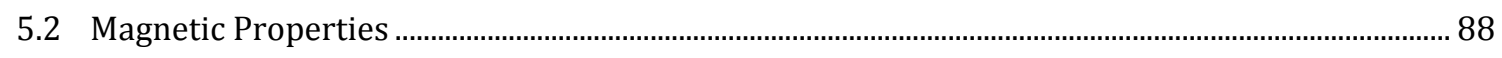

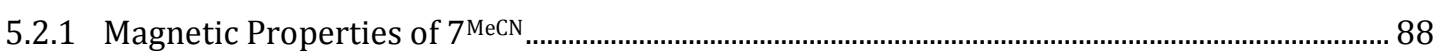


5.2.2 Magnetic Properties of $8^{\mathrm{HCO} 2}$ and $8^{\mathrm{OAc}}$ 89

5.3 Electro- and Spectroelectrochemical Analysis ....................................................................................92

5.3.1 Electro- and Spectroelectrochemical Analysis of $8^{\mathrm{HCO} 2}$ and $8^{\mathrm{OAc}}$............................................92

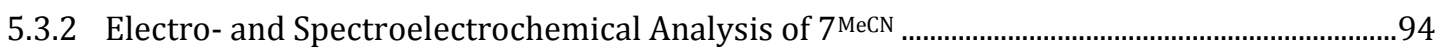

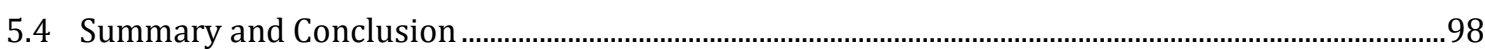

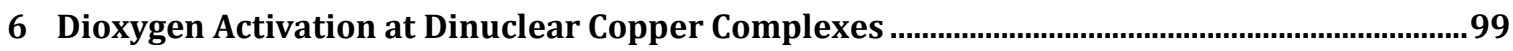

6.1 Reduction of 5 ${ }^{\mathrm{OAc}}$ via a Mixed-Valent $\mathrm{Cu}^{\mathrm{I}} \mathrm{Cu}^{\mathrm{II}}$ Intermediate ................................................................... 101

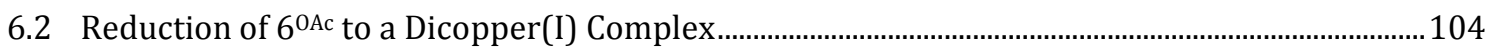

6.3 Dioxygen Activation at Dicopper Complexes ..................................................................................105

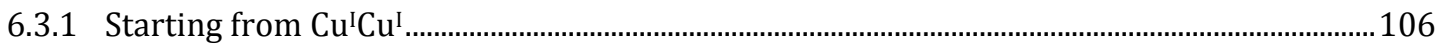

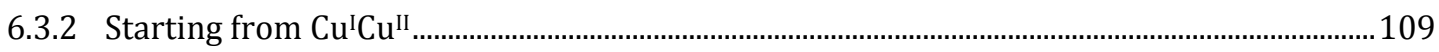

6.4 Reduction of $5^{\mathrm{HCO} 2}$ to an Oligonuclear Copper(I) Hydride Complex...............................................113

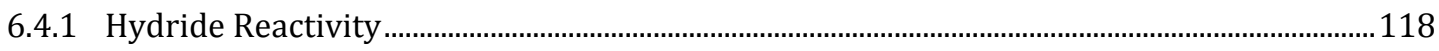

6.4.2 Origin of the Hydrides in $9^{\mathrm{H}}$ and Fate of the Formate .............................................................123

6.5 Reduction of $6 \mathrm{HCO}^{\mathrm{HCO}}$ to a Dicopper(I) Complex ...................................................................................125

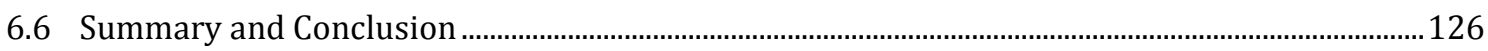

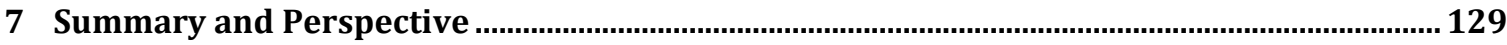

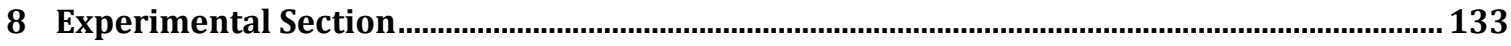

8.1 Materials and Methods.............................................................................................................. 133

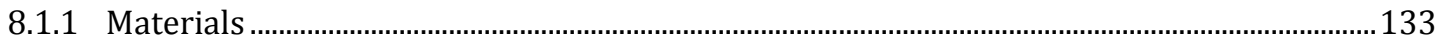

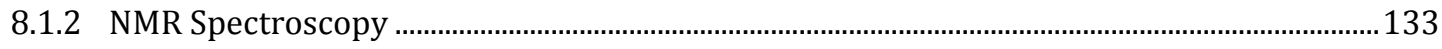

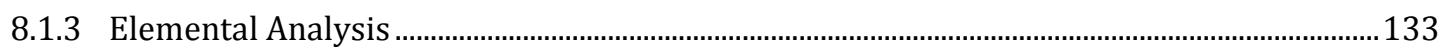

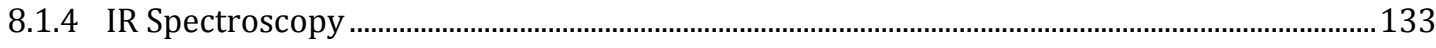

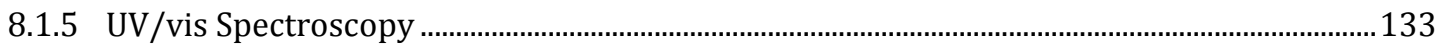

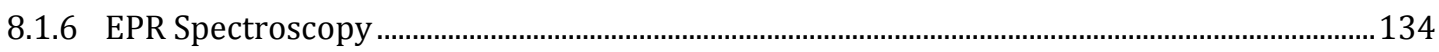

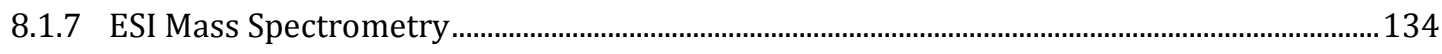

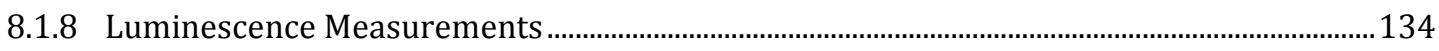

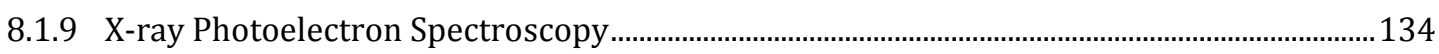

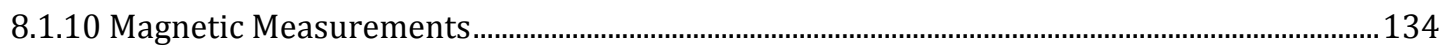

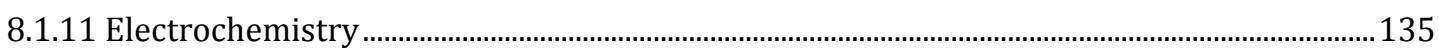

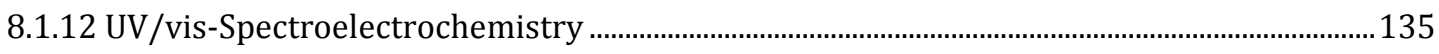

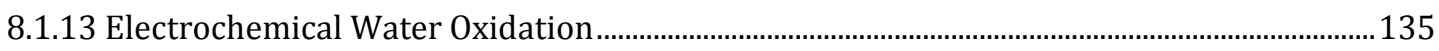

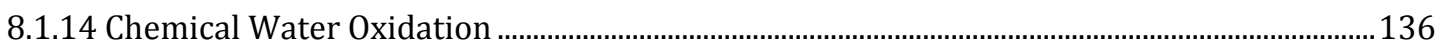

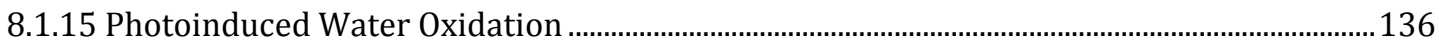

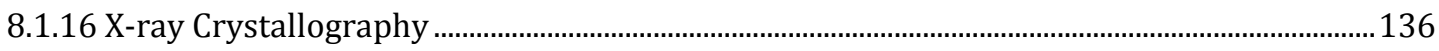

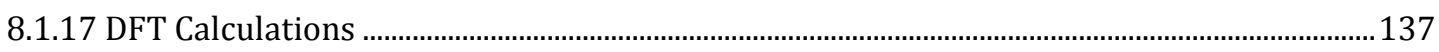

8.1.18 Formic Acid Dehydrogenation Catalysis Tests .......................................................................138

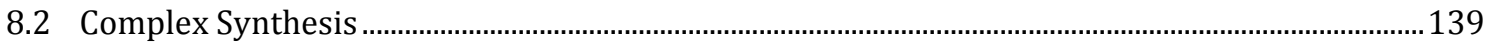

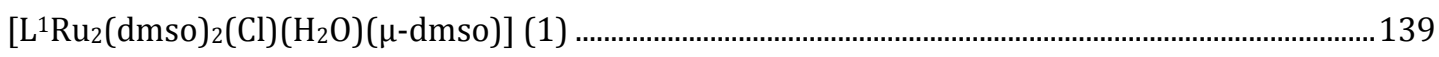

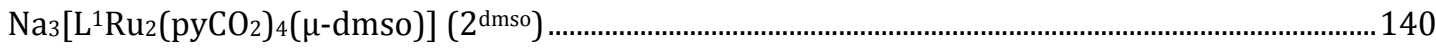

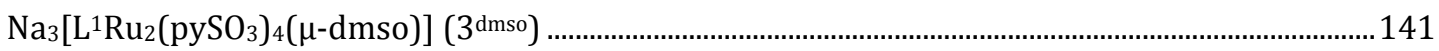




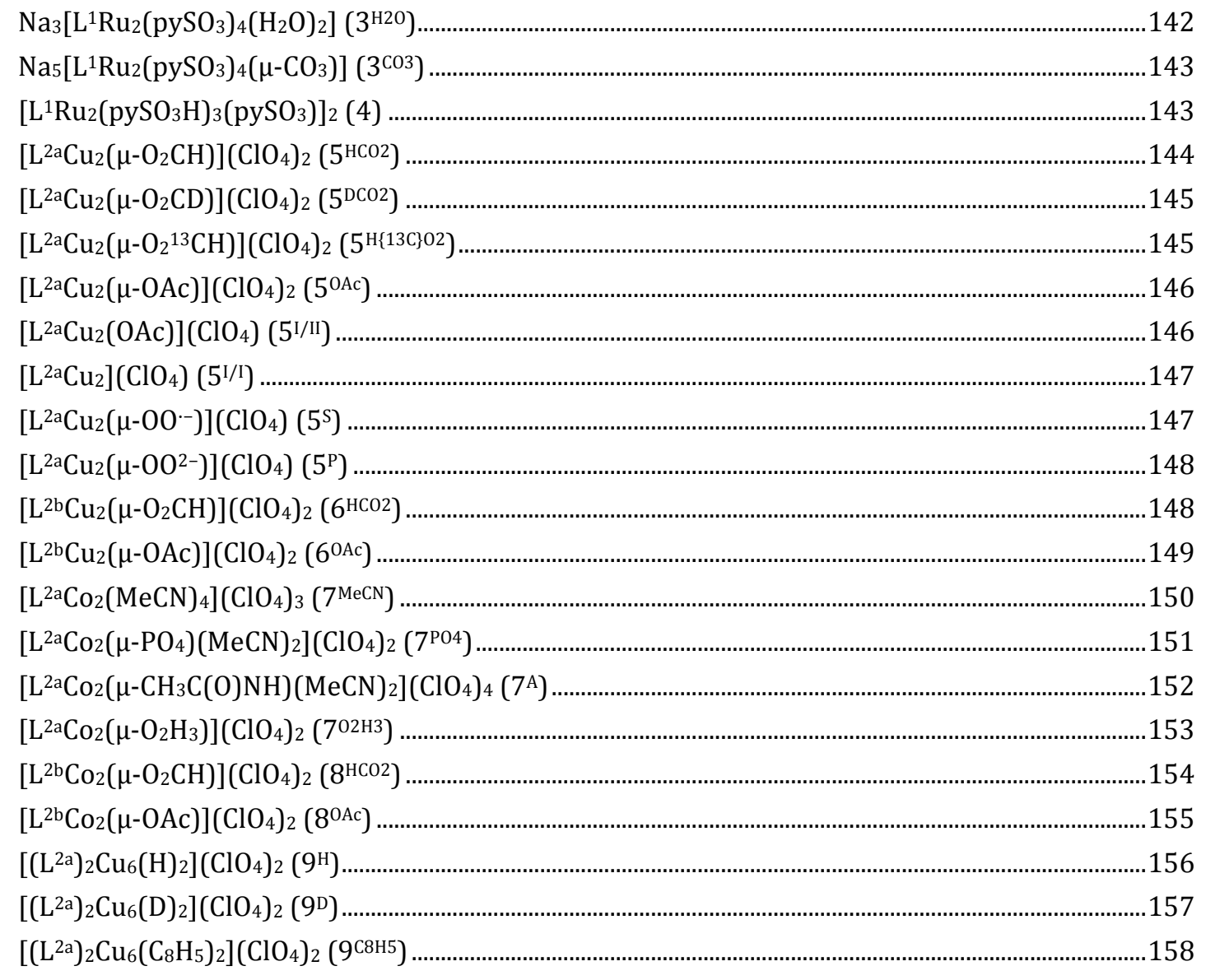

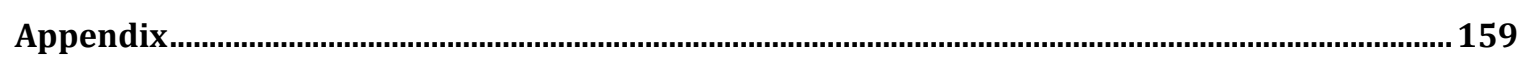

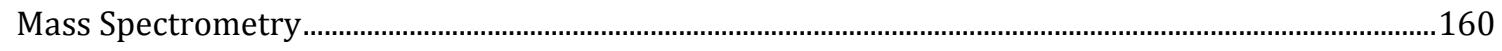

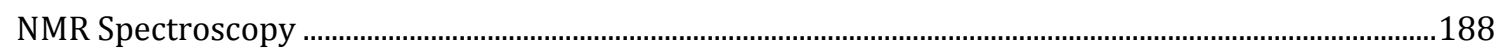

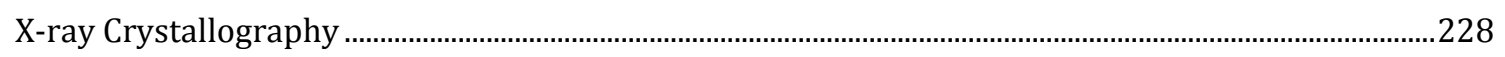

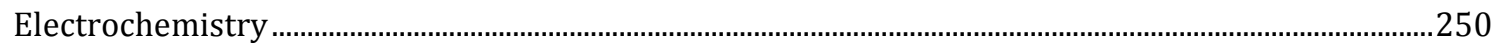

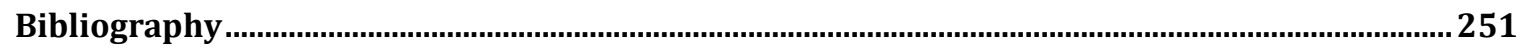

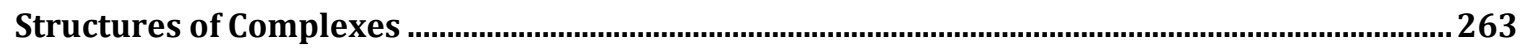

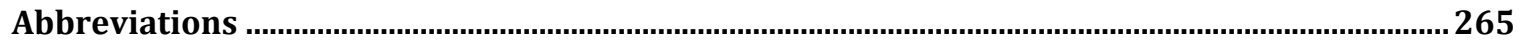

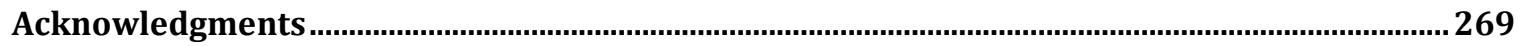

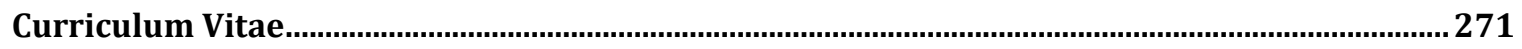





\section{1}

\section{General Introduction}

The continuously growing world population and expanding global economy, as well as higher standards of living are all factors which severely impact the global energy demand. In fact, according to the International Energy Agency, the world's energy consumption is expected to increase annually by $1.3 \%$ until 2040.[1] The dramatic energy thirst is currently, for the most part, met by fossil fuel combustion. However, with fossil resources slowly depleting, new technologies are necessary to comply with these future energy demands. The search for an energy solution is considered as one of the most important challenges of the 21st century. ${ }^{[2]}$

In order to ensure a secure energy supply, new methods of using existing carbon-based fuels are necessary. Nevertheless, this is not sufficient as it neglects the vast environmental damages that are connected to energy-related carbon dioxide emissions. Current government plans fail to reach emission reduction needs in order to limit the long-term global average temperature rise to well below $2{ }^{\circ} \mathrm{C}$ compared to pre-industrial levels as stated in the Paris Agreement of 2016. However, according to the Intergovernmental Panel on Climate Change, this is necessary to avert the most severe implications for our climate system associated with global warming. ${ }^{[3]}$ In order to reach this goal with $66 \%$ probability, anthropogenic $\mathrm{CO}_{2}$ emissions must be limited to a total of 790 gigatonnes between 2015 and 2100. On the prevailing path, this $\mathrm{CO}_{2}$ budget will have been exceeded by 2037.[4,5] Consequently, there is an urgent need for the development of new and amplification of existing schemes for carbonneutral energy production.

Among these are nuclear power and renewable energy sources such as photovoltaics (PV), wind and biomass. In 2019, 6.4 and $14.8 \%$ of the primary energy consumption in Germany came from nuclear and renewable energy, respectively.[6] Increasing the amount of nuclear power in the total energy mix would involve the rapid construction of multiple new power plants. Due to the exhaustibility of terrestrial uranium sources, it also merely postpones the approaching issue. ${ }^{[7]}$ Additionally, nuclear power is a constant topic of public debate, especially in regard to its safety and the storage of radioactive waste products. On the other hand, the current renewable energy technologies have significant drawbacks and limitations. Accordingly, PV and wind are sensitive to fluctuations in solar radiation and wind strength, and demand efficient transport and storage. Furthermore, biomass can be considered a competitor to food production as it requires the widespread use of agricultural land..$^{[8]}$ Finally, the current renewable technologies all provide electricity, which merely accounts for $20 \%$ of the energy consumption. ${ }^{[9]}$ Alternatives to fossil resources are required, especially in the fuel and heat sector.

By far the most abundant source of renewable energy is provided by the sun. In fact, within just over an hour of sunlight enough energy strikes the earth to cover the current global energy consumption of an entire year. ${ }^{[7]}$ However, the sun's energy is diffuse and thus difficult to harvest in large amounts. Additionally, the diurnal cycle of the sun limits its use as a primary energy source for 
society. Accordingly, schemes are necessary to store solar energy in order to use it whenever and for whatever desired.

Nature demonstrates the storage of solar-converted energy to generate a fuel by means of photosynthesis (see Section 1.1). In this process, the sun's energy is used to oxidize water to dioxygen, concomitantly providing reducing equivalents to convert $\mathrm{CO}_{2}$ into carbohydrates. Inspired by photosynthesis, its artificial replica (see Section 1.2) aims at storing solar-converted energy in the form of chemical bonds using water as the raw material. The oxidative half reaction (see Section 1.3), known as the oxygen evolution reaction (OER), is considered the bottleneck in this endeavor.[10,11] Consequently, the development of catalysts to facilitate this reaction is of great interest. As both the OER and its reducing counterpart, the oxygen reduction reaction (ORR), are suggested to involve metal-oxygen intermediates, ${ }^{[12,13]}$ an investigation of dioxygen activation at transition metal complexes can also bring forth fruitful insight (see Section 1.4).

\subsection{Photosynthesis}

Natural oxygenic photosynthesis is the biochemical process that paved the way for more complex life forms on earth. Initially emerging around two and a half billion years ago, this energy consuming process provided both reduced carbon-based compounds (including fossil resources) and vital dioxygen, which allowed for the formation of the ozone layer and the evolution of aerobic respiration. Overall, water and carbon dioxide are converted to carbohydrates (with $\mathrm{CH}_{2} \mathrm{O}$ stoichiometry) and molecular oxygen, using sunlight as energy (eq 1.1). As the energy content of the products exceeds that of the reactants, the photosynthetically formed carbohydrates represent chemically stored solar energy. ${ }^{[14]}$

$$
\mathrm{H}_{2} \mathrm{O}+\mathrm{CO}_{2} \stackrel{\text { light }}{\longrightarrow} \frac{1}{\mathrm{n}}\left(\mathrm{CH}_{2} \mathrm{O}\right)_{\mathrm{n}}+\mathrm{O}_{2}
$$

Photosynthesis is a membrane-spanning process that takes place in the thylakoid membrane in chloroplasts in cyanobacteria, green plants and algae. Its light reactions can generally be sub-divided into four steps: light harvesting, charge separation by electron transfer, water oxidation and the formation of energy carriers. The latter comprise adenosine triphosphate (ATP) and nicotinamide adenine dinucleotide phosphate (NADPH), which provide chemical and reducing power, respectively. These are subsequently inserted into a complex reaction cycle, known as the Calvin cycle, in which they are used for the conversion of atmospheric $\mathrm{CO}_{2}$ into carbohydrates in the so-called dark reactions of photosynthesis. ${ }^{[14,15]}$

The light-driven processes take place at the photosystems PSI and PSII (Scheme 1.1). Here, sunlight is harvested by antenna pigments, and the energy is transferred to reaction center chlorophylls (P700 and P680). At the PSII, the photoexcited P680* transfers an electron to pheophytin (Pheo), generating the cation radical P680+ (charge separation). In turn, Pheo reduces the protein-bound plastoquinone $Q_{A}$, which passes on the electron to $Q_{B}$. The plastoquinone $Q_{B}$ cycles between an oxidized $\left(Q_{B}\right)$ and doubly-reduced plastoquinol $\left(\mathrm{H}_{2} \mathrm{Q}_{B}\right)$ form, the latter of which is released into the hydrophobic region of the membrane. This allows $\mathrm{H}_{2} \mathrm{Q}_{B}$ to be in exchange with the so-called quinone pool of the membrane, enabling electron transfer outside of the protein.[16,17]

Subsequently, the pathway of the electrons crosses the cytochrome $b_{6} f$ complex (cyt $b_{6} f$ ), which mediates the electron flow from PSII to PSI. This transmembrane complex transfers electrons from $\mathrm{H}_{2} \mathrm{Q}_{B}$ to plastocyanin (PC), concomitantly pumping protons from the outside (stroma) to the inside (lumen) of the thylakoid membrane. The thus generated proton gradient drives the phosphorylation of adenosine diphosphate (ADP) to ATP by the enzyme ATP synthase. Thereupon, the water-soluble PC reduces the radical cation $\mathrm{P} 700{ }^{++}$at $\mathrm{PSI} .{ }^{[16]}$ 
Light triggered charge separation at PSI is responsible for electron transfer from PC to ferredoxin (FD) via a series of iron-sulfur clusters (FeS). The high-potential electrons stored in two FD molecules are ultimately used for the reduction of $\mathrm{NADP}^{+}$to $\mathrm{NADPH}$ catalyzed by ferredoxin-NADP ${ }^{+}$reductase (FNR). This proceeds in the stroma and, thus, contributes to the generation of the proton gradient along the thylakoid membrane, as it involves the uptake of an $\mathrm{H}^{+} .[16]$

On the other side of the electron transport chain (PSII), the electron deficiency at P680++ is compensated by the oxidation of water at the oxygen evolving complex (OEC) via a redox-active tyrosine $\left(\mathrm{Y}_{\mathrm{z}}\right)$. The oxidized $\mathrm{Y}_{\mathrm{z}}{ }^{+}$is stabilized by reversible proton transfer to an adjacent histidine moiety, thus generating a neutral tyrosyl radical. Concomitant to the reduction of $\mathrm{Y}_{\mathrm{z}} \cdot$ by the OEC, it is re-protonated by the neighboring histidine.[17-21]

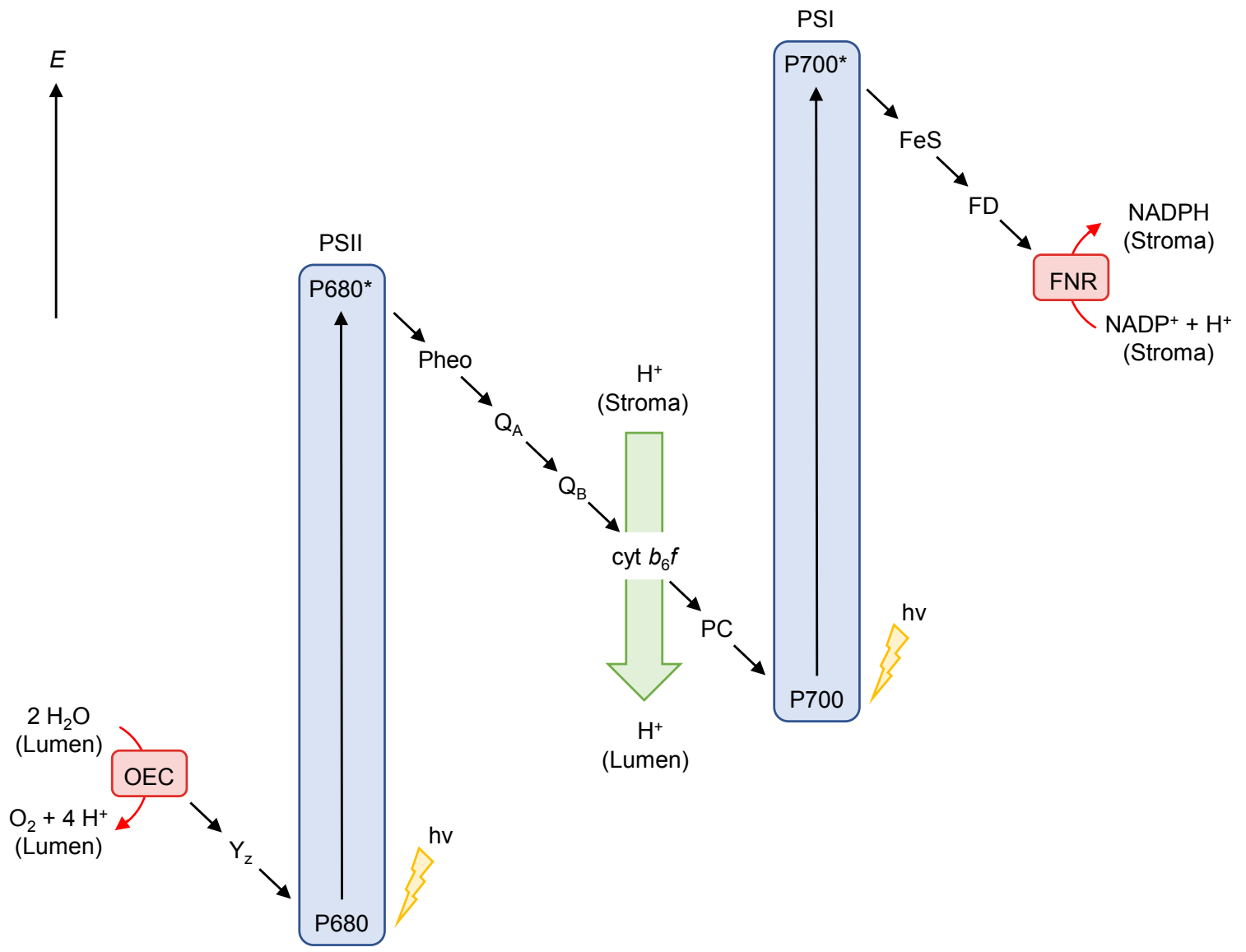

Scheme 1.1: Simplified Z-scheme of the light reactions of natural oxygenic photosynthesis (OEC: oxygen evolving complex, $\mathrm{Y}_{\mathrm{z}}$ : tyrosine z, PS: photosystem, Pheo: pheophytin, Q: plastoquinone, cyt $b_{6} f$ : cytochrome $b_{6} f$ complex, PC: plastocyanin, FeS: iron-sulfur cluster, FD: ferredoxin, FNR: ferredoxin-NADP+ reductase, NADPH: nicotinamide adenine dinucleotide phosphate). The short black arrows denote the direction of the electron flow.

The OEC acts as a charge accumulator, coupling light-induced one-electron transfer to the multielectron catalytic process of oxidizing water to dioxygen. Only through the combination of the two photosystems, a sufficient potential can be obtained to enable this extremely energy intensive reaction. An essential feature of the OEC is that it prevents the formation of detrimental free reactive oxygen species (ROS), such as the hydroxyl radical ${ }^{\circ} \mathrm{OH}$, hydrogen peroxide $\mathrm{H}_{2} \mathrm{O}_{2}$ and superoxide $\mathrm{O}_{2}{ }^{-}$. As the formation of molecular oxygen is accompanied by the release of four protons on the lumenal side of the thylakoid membrane, water oxidation by the OEC also contributes to the generation of the protonmotive force for ATP synthesis. ${ }^{[16,22]}$

The central unit of the OEC is a $\left[\mathrm{Mn}_{4} \mathrm{CaO}_{5}\right]$ cluster (Figure 1.1, left) with a chair-like structure, the base of which, a distorted cube, is formed by three manganese (Mn1-3) and one calcium ion linked via 
$\mu-\mathrm{OH}^{-}$and $\mu-\mathrm{O}^{2-}$ ligands $(01-3,05)$. Accordingly, $\mathrm{Mn} 4$ and 04 constitute the back of the chair. The metal ions are further coordinated by several carboxylate and a histidine residue from the peptide side chains of the protein. Several water molecules, representing the substrate of the OEC, are situated in close vicinity to the cluster core. ${ }^{[23]}$

In 1970, Kok et. al. reported a four-step mechanism for the oxidation of water to dioxygen at the OEC (Figure 1.1, right).[24] The respective five intermediates, known as the S-states $S_{0}$ to $S_{4}$, are formed by the successive storage of four oxidizing equivalents (as indicated by their subscript). Whereas the calcium ion is redox inactive, the manganese ions toggle between $\mathrm{Mn}^{\mathrm{III}}$ and $\mathrm{Mn}^{\mathrm{IV}}$ throughout the process. Concomitant to the oxidation of the metal ions, protons are removed from the cofactor in socalled proton-coupled electron transfer (PCET) steps in order to avoid charge accumulation. In the last transition of the so-called Kok cycle $\left(\mathrm{S}_{4} \rightarrow \mathrm{S}_{0}\right)$, dioxygen is released. The exact mechanism of this process has not been elucidated thus far; however, multiple suggestions are in debate (see Section 1.3.3).[21,25-28]

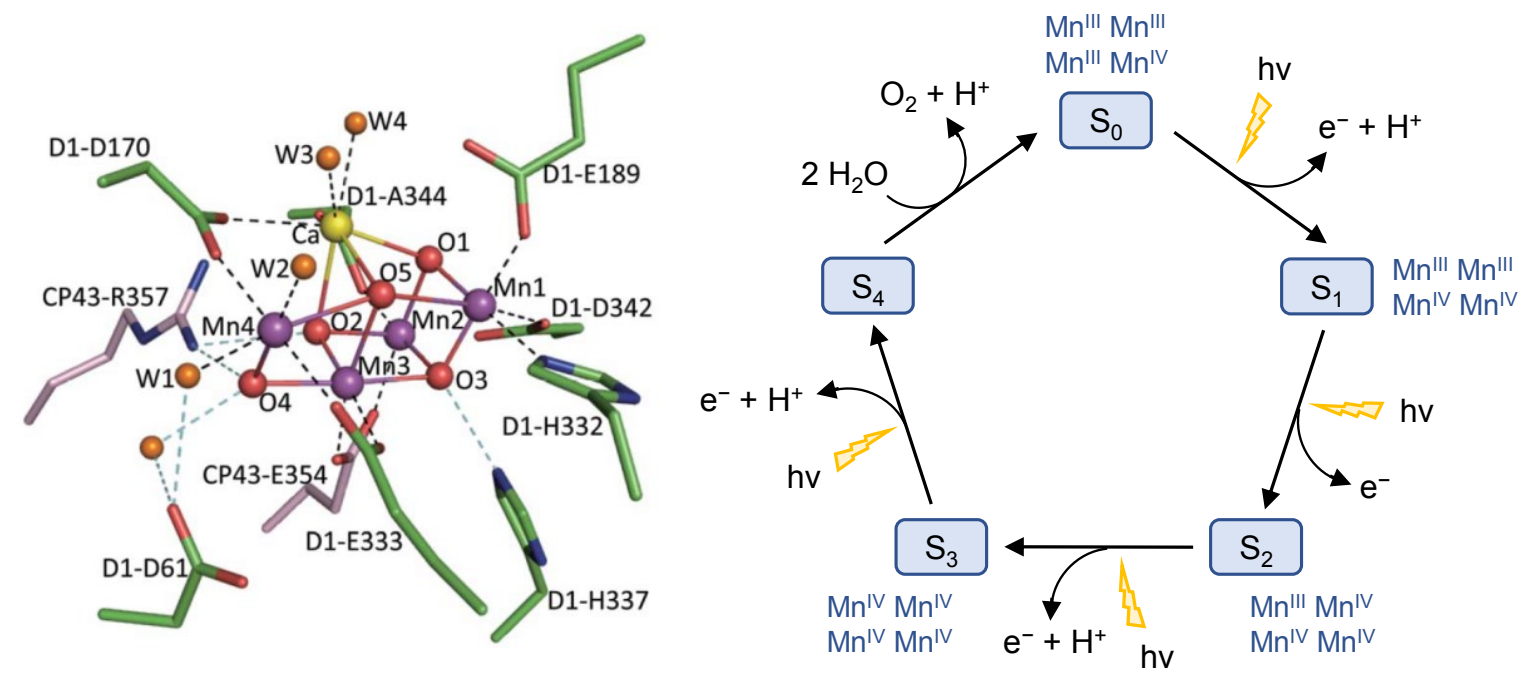

Figure 1.1: Water oxidation at the OEC. Left: Molecular structure of the OEC at the PSII extracted from Thermosynechococcus vulcanus at a resolution of $1.9 \AA$ (manganese: purple, calcium: yellow, oxygen: red, nitrogen: blue). Reprinted with permission from reference [23]. Right: Schematic diagram of the Kok cycle for water splitting at the OEC involving five intermediates, $\mathrm{S}_{0}$ to $\mathrm{S}_{4}$.

\subsection{Artificial Photosynthesis}

Owing to the diffuse $\left(\rho \sim 100 \mathrm{~mW} \cdot \mathrm{cm}^{-2}\right)$ and intermittent (sensitive to daytime, weather and geographical location) nature of solar energy, effective capture, conversion and storage methods are necessary to make it compatible with human energy requirements. Existing options include the conversion of sunlight to electricity (PV), and its storage in batteries or by transformation to mechanical energy (to drive turbines). However, the latter is not suitable to compensate the diurnal cycle of the sun and current batteries are far too expensive to compete with fossil resources. Thus, in order to make use of the sun as a primary energy source, the emulation of natural photosynthesis by conversion of solar energy into chemicals, i.e. to generate so-called solar fuels, is the most promising approach.[2,29]

Storing solar energy in chemical bonds can be achieved by both direct and indirect methods. The latter include biomass to biogas conversion and the use of electricity generated from solar energy to drive electrocatalytic reactions. In the direct pathway, referred to as artificial photosynthesis (AP), sunlight is converted to a solar fuel in an integrated system, without intermittent energy carriers. The successful imitation of the natural energy-storing procedure requires the orchestration of several 
intricate processes. These include light harvesting, charge separation and electron transfer, as well as coupling the one-photon/one-electron charge separation events with multi-electron catalysis.[29]

In principle, a device capable of AP, also referred to as a photoelectrochemical cell (PEC), consists of the same components as its natural counterpart: a photosensitizer responsible for light harvesting, as well as catalysts for water oxidation and the fuel-forming reaction. Hydrogen gas is considered an ideal fuel as it combines a high gravimetric energy density with a non-toxic byproduct upon combustion, viz. water. However, a transition to an $\mathrm{H}_{2}$ based economy bears significant challenges. As an alternative, the electrons resulting from water oxidation can be used for the formation of a carbonbased fuel by reductive fixation of $\mathrm{CO}_{2}$, e.g. in an alcohol. Although this might be more compatible with the current energy sector, the catalysis involved is significantly more challenging due the inherent structural stability of $\mathrm{CO}_{2}$. Furthermore, this pathway would maintain the carbon dioxide cycle.[29,30]

A schematic representation of a PEC for water splitting is illustrated in Scheme 1.2. The two halfreactions (anode: water oxidation, cathode: proton reduction) are located in different compartments, connected through an external circuit for electron flow but physically separated by a proton exchange membrane (PEM). The latter allows protons to pass from the oxidative to the reductive module, while keeping $\mathrm{H}_{2}$ and $\mathrm{O}_{2}$ well separated. This simplifies product collection and simultaneously prevents their potentially detrimental backreaction to $\mathrm{H}_{2} \mathrm{O}$. Each compartment comprises an electrode, of which at least one is coupled to light absorption (photoactive).[31]

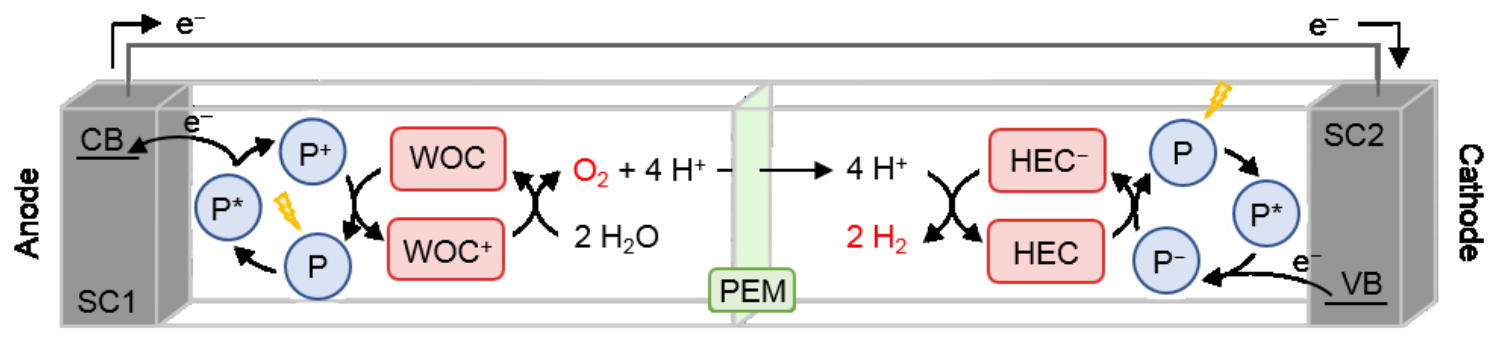

Scheme 1.2: Representation of a photoelectrochemical cell (PEC) for water splitting (SC: semiconductor, CB/VB: conduction/valence band, P: photosensitizer, WOC: water oxidation catalyst, PEM: proton exchange membrane, HEC: hydrogen evolving catalyst).[31]

Light harvesting can either be achieved by a semiconductor (SC) with an appropriate band gap ${ }^{[2,33]}$ or sensitized with a suitable chromophore (P), ${ }^{[34-36]}$ as known from dye-sensitized solar cells (DSSCs). ${ }^{[37,38]}$ High photochemical stability, extended absorption in the visible region, long-lived excited states, as well as a suitable redox potential to oxidize the water oxidation catalyst (WOC) or reduce the hydrogen evolving catalyst (HEC) constitute the demanding requirements for light absorbers. The SC is coupled to the catalyst, which would favorably be of heterogenous nature. This includes immobilized molecular catalysts or chromophore-catalyst dyads, as well as non-molecular systems.[39-41]

Given the high complexity of the artificial photosynthetic process - the thermodynamic and kinetic conditions of numerous chemical reactions have to be matched to prevent back electron transfer and unwanted side products - a reasonable approach for the construction of a PEC is to divide the overall process into its two half reactions. Along this strategy, the two catalyst systems for oxidative $\mathrm{O}_{2}$ and reductive $\mathrm{H}_{2}$ production can independently be developed. Initially, the catalytic proficiency is studied by means of a chemical redox agent or by applying a potential with a potentiostat. A suitable catalyst can then be coupled to light absorption, with a sacrificial electron acceptor (for $\mathrm{H}_{2} \mathrm{O}$ oxidation) or donor (for $\mathrm{H}^{+}$reduction) mimicking the complementary half reaction. Here, the typically irreversible nature of the sacrificial electron agents, as well as their large excess provide a strong driving force for forward electron transfer. Alternatively, one module can be replaced by a counter electrode and a suitable potential applied to probe the desired half reaction. Once separately optimized, the modules need to be combined in the final device.[29,31,42] 
Although these steps may seem straightforward, harmonizing all involved reactions to achieve efficient light harvesting, fast electron transfer, and ultimately high substrate $\left(\mathrm{H}_{2} \mathrm{O} / \mathrm{H}^{+}\right)$conversion is far from trivial. Accordingly, only few systems capable of artificial photosynthesis have been produced thus far. Due to the high complexity of assembling two photoactive modules, most current devices are single-photoelectrode cells. ${ }^{[39,43,44]}$

\subsection{Water Oxidation Catalysis}

As an essentially inexhaustible, environmentally benign, inexpensive and widely available material, water is the ideal feedstock for generating a solar fuel. However, in order to develop sustainable energy conversion schemes based on water splitting to $\mathrm{O}_{2}$ and $\mathrm{H}_{2}$ by sunlight, the underlying processes, viz. water oxidation and proton reduction, must be fully understood. Whereas the latter is already successfully conducted by catalysts based on both noble (e.g. rhodium, platinum) ${ }^{[45-48]}$ and earthabundant metals (e.g. nickel, cobalt, iron, molybdenum), ${ }^{[49-51]}$ the most efficient and stable molecular HECs being nickel-containing complexes bearing diphosphine ligands with pendant amines, ${ }^{[52-55]}$ the oxidation of water to dioxygen (eq 1.2) remains the major challenge. Given the complexity of this reaction, the development of efficient and robust WOCs is considered the bottleneck in this endeavor. ${ }^{[10,11]}$

$$
2 \mathrm{H}_{2} \mathrm{O} \rightarrow \mathrm{O}_{2}+4 \mathrm{H}^{+}+4 \mathrm{e}^{-}
$$

\subsubsection{Requirements and Evaluation Criteria of WOCs}

The oxidation of water to molecular oxygen is both thermodynamically and kinetically challenging. This expresses itself in a high standard redox potential of $1.23 \mathrm{~V}$ (all redox potentials herein are reported against the normal hydrogen electrode, NHE) at $\mathrm{pH}$, which corresponds to a Gibbs free energy of $237 \mathrm{~kJ} \cdot \mathrm{mol}^{-1}$, as well as the enormous complexity of the reaction from a mechanistic perspective. Accordingly, two protons and electrons must each be removed from two water molecules, and an $\mathrm{O}-\mathrm{O}$ double bond must be formed. As this multielectron catalysis must happen in one step in order to avoid the formation of highly reactive radical oxygen species, a suitable catalyst is required to accumulate four electron holes in close proximity. In other words, the metal centers in WOCs must have access to high oxidation states. Given the high thermodynamic energy associated with the water oxidation process, catalysts must further comply with a high oxidative stability, i.e. possess oxidatively robust and substitution resistant ligands. If this is not the case, the initial WOC could unknowingly be transformed to another species or all the way to the respective metal oxide, leaving the nature of the actual catalyst unknown. Finally, a potential WOC should feature an aquo ligand or an open coordination site at which a water molecule can bind in order to generate the crucial metal-oxo entity necessary for 0-0 bond formation (see Section 1.3.3).[31,56]

Various criteria can be applied for the evaluation of WOCs. These include the turnover number (TON) and frequency (TOF), the faradaic efficiency (FE), as well as the over- $(\eta)$ and catalytic potential $\left(E_{\text {cat }}\right)$ of water oxidation. Whereas the former two are used to evaluate the chemical, electrochemical and light-driven water oxidation proficiency of WOCs (see Section 1.3.4), the latter three are only relevant to electrocatalysts. ${ }^{[57]}$

The over- and catalytic potential are measures to assess the ability of a WOC to decrease the kinetic barrier for water oxidation and are more or less substitutable, in that $\eta$ refers to the deviation between $E_{\text {cat }}$ and the thermodynamic potential of water oxidation at a given $\mathrm{pH}$. However, the definition of $E_{\text {cat }}$ differs between publications, designating the potential at the onset of the catalytic wave, or at half maximum or maximum catalytic current and thus comparisons between WOCs regarding these parameters should be made with caution. Herein, the onset and catalytic potential are used as synonyms. ${ }^{[57]}$ 
The TON and TOF of a catalyst are defined as the number of dioxygen molecules produced by a catalyst molecule in total (before degradation or inactivation) and per unit time, respectively. They provide a measure of the stability/lifetime (TON) and catalytic proficiency (TOF) of a WOC. It is important to note that the methodology and experimental conditions strongly influence the TON and TOF parameters and thus should be taken into consideration when comparing WOCs. ${ }^{[57]}$

Finally, the FE describes the efficiency with which a catalyst utilizes charge to drive an electrocatalytic reaction. It corresponds to the ratio of the experimentally achieved and theoretically possible (assuming that $100 \%$ of the charge is transferred to water oxidation) number of catalytic cycles mediated by a WOC.

\subsubsection{Molecular vs. Non-Molecular, Precious vs. Base Metal WOCs}

The search for high-performance WOCs has led to the development of a large body of both homogenous ${ }^{[58-64]}$ and heterogenous ${ }^{[65-70]}$ catalysts. A big emphasis in this field has been put on molecular WOCs due to their distinct advantages over non-molecular systems. These include:[31,39]

(i) tunability - rational design of WOCs through deliberate synthetic modifications. This entails the choice of transition metal, oxidation state, geometry, and the introduction of functional groups to vary the solubility and electronic properties of the WOC, as well as enable anchoring on solid supports. Subtle variations in catalysts can provide insightful structure-activity relationships.

(ii) mechanistic elucidation - full comprehension of the catalytic process on a detailed level. Various spectroscopic techniques can be used to identify intermediates on the way to the desired reaction. This is especially useful in combination with the tunability of molecular WOCs as adjustments can be made based on the gained knowledge to generate more efficient catalysts.

(iii) metal-atom economy - efficient utilization of metal atoms (especially relevant for catalysts based on rare metals). In contrast to non-molecular systems, in which catalysis only takes place at a small number of metal atoms situated at surfaces, edges, corners or heterojunctions, metal utilization in homogenous molecular catalysts can reach $100 \%$. This is especially relevant in regard to the desired large-scale application of WOCs in devices for AP. Note that in homogenous electrocatalysis only the catalyst molecules at the electrode surface are active.

In contrast, non-molecular systems often possess a higher stability as they are less susceptible to degradation or inactivation. In order to combine the advantages of both the molecular and nonmolecular fields, the heterogenization of molecular WOCs by anchoring on solid supports is a useful technique. Various strategies exist to accomplish this, including physisorption, electrostatic interaction, encapsulation and chemisorption. The major challenge is to maintain the intrinsic high activity of the homogenous catalyst after immobilization. Next to a prolonged stability, the decreased translational mobility of the molecular component also bears the benefit of providing deeper insight into potential deactivation pathways. ${ }^{[39,71]}$

Ruthenium-based complexes provide the largest and best-characterized body of molecular WOCs. ${ }^{[57,71-74]}$ The extensive application of ruthenium in this regard results from its richness in terms of available oxidation states (-II to +VIII), coordination numbers and supporting ligand scaffolds. Additionally, ruthenium-based compounds are frequently sufficiently long-lived to enable detailed mechanistic investigations.

Although good catalytic activities (TON and TOF) have been achieved with WOCs based on noble metals, such as ruthenium and iridium, the limited supply and high cost of these metals restrict their widespread application. Consequently, enhanced research is going towards the incorporation of abundant and cheap first-row transition metals in water oxidation catalysts. Inspired by the OEC, manganese-based compounds were among the first group of WOCs developed in this regard. Although the structural imitations of the OEC exhibit a rather low proficiency for the oxidation of water to 
dioxygen, they give an insight into the dynamics of such structures. ${ }^{[75]}$ Nowadays, the field of WOCs based on base metals has expanded, including complexes with iron, cobalt, nickel and copper. ${ }^{[63,76-80]}$

A large drawback of first-row transition metal WOCs is their poor stability in comparison to those of precious and rare metals. Accordingly, a significant number of such catalysts have been shown to decompose under turnover conditions to the respective heterogenous metal oxides or nanoclusters, which are often still active towards water oxidation. ${ }^{[81-89]}$ This poor robustness is largely an effect of the substitutional lability of base metal complexes in comparison to their precious metal congeners. Correspondingly, $\mathrm{H}_{2} \mathrm{O}$ exchange in $\left[\mathrm{M}\left(\mathrm{H}_{2} \mathrm{O}\right)_{6}\right]^{\mathrm{n}+}$ is up to eight orders of magnitude higher for both $\mathrm{Fe}^{\mathrm{II}}$ and $\mathrm{Fe}^{\mathrm{III}}$ than their respective $\mathrm{Ru}^{\mathrm{II}}$ and $\mathrm{Ru}^{\mathrm{III}}$ counterparts. ${ }^{[90,91]}$ Whereas the $\mathrm{Ru}-\mathrm{OH}_{2}$ bond is the only reactive site in the typical polypyridyl-type catalysts, $\mathrm{H}_{2} \mathrm{O}$ competes with the ancillary ligands for coordination sites in base metal WOCs. The stability of the latter thus strongly depends on the coligands, and also the solution $\mathrm{pH}$ has a large effect. At too low $\mathrm{pH}$, donor groups of auxiliary ligands can be protonated leading to decoordination. ${ }^{92,93]}$ At too high $\mathrm{pH}$, base metal complexes are likely to degrade to the respective metal oxides or hydroxides, which can form thin films on the electrode surface or precipitate from the solution as colloids, thus providing an additional driving force for decomposition. ${ }^{[94]}$ Given that the $\mathrm{pH}$ can dramatically change during water oxidation catalysis (see eq 1.2), the use of a buffer is crucial. Furthermore, especially for first-row transition metal WOCs, the homogenous nature of the catalysis must be investigated.[56,64]

\subsubsection{Mechanistic Considerations}

The mechanism of water oxidation to dioxygen by transition metal complexes with a bound $\mathrm{H}_{2} \mathrm{O}$ molecule can be separated into three main phases: (i) generation of high-valent metal-oxo species, (ii) $\mathrm{O}-\mathrm{O}$ bond formation, and (iii) $\mathrm{O}_{2}$ evolution. In the first step, the WOC is activated via a series of PCET steps, a phenomenon first discovered by the Meyer group in the late 1970s, ${ }^{[95,96]}$ transforming the initial $\left[\mathrm{M}^{\mathrm{n}-2}-\mathrm{OH}_{2}\right]$ to an $\left[\mathrm{M}^{\mathrm{n}}=0\right]$ entity. The simultaneous removal of electrons and protons from the catalyst allows the formation of the critical high-valent metal-oxo species at comparably low potential in comparison to the exclusive transfer of electrons. ${ }^{[97]}$

The effect of PCET is nicely exemplified by the related mononuclear ruthenium complexes cis$\left[\mathrm{Ru}^{\mathrm{II}}(\mathrm{bpy})_{2} \mathrm{Cl}_{2}\right]$ and $c i s-\left[\mathrm{Ru}^{\mathrm{II}}(\mathrm{bpy})_{2}(\mathrm{py})\left(\mathrm{OH}_{2}\right)\right]^{2+}$ (bpy: 2,2'-bipyridine, py: pyridine). Whereas in the chlorido complex, the $\mathrm{Ru}^{\mathrm{III}} / \mathrm{Ru}^{\mathrm{II}}$ and $\mathrm{Ru}^{\mathrm{IV}} / \mathrm{Ru}^{\mathrm{III}}$ redox events are situated at 0.56 and $2.22 \mathrm{~V}$, ${ }^{[98]}$ respectively, the separation between these couples is reduced to $0.11 \mathrm{~V}$ upon oxidation and concomitant deprotonation in the aquo complex (0.66 and $0.77 \mathrm{~V}$, respectively, at $\mathrm{pH} 7)^{\left[{ }^{[95]}\right.}$

$$
\begin{gathered}
{\left[\mathrm{Ru}^{\mathrm{II}}(\text { bpy })_{2} \mathrm{Cl}_{2}\right] \stackrel{-\mathrm{e}^{-}}{\longrightarrow}\left[\mathrm{Ru}^{\mathrm{III}}(\text { bpy })_{2} \mathrm{Cl}_{2}\right]^{+} \stackrel{-\mathrm{e}^{-}}{\longrightarrow}\left[\mathrm{Ru}^{\mathrm{IV}}(\text { bpy })_{2} \mathrm{Cl}_{2}\right]^{2+}} \\
{\left[\mathrm{Ru}^{\mathrm{II}}(\mathrm{bpy})_{2}(\text { py })\left(\mathrm{OH}_{2}\right)\right]^{2+} \stackrel{-\mathrm{e}^{-} / \mathrm{H}^{+}}{\longrightarrow}\left[\mathrm{Ru}^{\mathrm{III}}(\text { bpy })_{2}(\text { py })(\mathrm{OH})\right]^{2+} \stackrel{-\mathrm{e}^{-} / \mathrm{H}^{+}}{\longrightarrow}\left[\mathrm{Ru}^{\mathrm{IV}}(\text { bpy })_{2}(\text { py })(\mathrm{O})\right]^{2+}}
\end{gathered}
$$

The beneficial effect of PCET steps, viz. reaching high oxidation states at the metal centers in WOCs at relatively low potential, results from two main contributions. ${ }^{[97]}$ On the one hand, simultaneous $\mathrm{e}^{-} / \mathrm{H}^{+}$removal prevents charge accumulation and the formation of high energy intermediates. On the other hand, the successive deprotonation of the aquo molecule gradually generates a ligand with higher electron-donating character $\left(\mathrm{H}_{2} \mathrm{O}<\mathrm{HO}^{-}<\mathrm{O}^{2-}\right)$ that is capable of stabilizing the high oxidation states of the metal ion. The oxidative activation of a WOC can thereby be achieved by using either a chemical oxidant, applying an external potential or by using sunlight as an energy source (see Section 1.3.4).

The thus generated metal-oxo units trigger oxygen-oxygen bond formation, the key and often ratedetermining step (RDS) of water oxidation catalysis. For this process, two major mechanistic pathways exist, involving either an external water molecule (water nucleophilic attack, WNA) or a second $\left[\mathrm{M}^{\mathrm{n}}=0\right.$ ] unit (interaction of two M-O entities, I2M). In the WNA pathway (Scheme 1.3), a substrate $\mathrm{H}_{2} \mathrm{O}$ 
molecule attacks the electron-deficient high-valent metal-oxo group, resulting in a formal two-electron reduction of the metal center and the formation of a hydroperoxo intermediate. Further oxidation and deprotonation of $\left[\mathrm{M}^{\mathrm{n}-2}-\mathrm{OOH}\right]$ leads to the liberation of $\mathrm{O}_{2}$ and reformation of the initial reduced complex on aquation. ${ }^{[61,99]}$

In the typical WNA mechanism, the formation of the hydroperoxo species proceeds via a concerted two-electron pathway in a single step. Recently, a slightly modified WNA mechanism was reported to be operative in a few copper-based WOCs, viz. single electron transfer (SET) WNA. In this case, the oxygen-oxygen bond formation occurs via two one-electron transfer steps involving an intermediate with an $(\mathrm{HO} \cdots \mathrm{OH})^{--}$radical anion fragment featuring a partial $\mathrm{O}-\mathrm{O}$ bond. The second SET then generates the hydroperoxide group $(\mathrm{HO}-\mathrm{OH}) \cdot[100,101]$

The WNA mechanism is favorable if the metal-oxo fragment is sufficiently electrophilic to be attacked by a nucleophilic substrate and the accessibility of the M-O moiety for a water molecule is given. As hydroxide is a far better nucleophile than an $\mathrm{H}_{2} \mathrm{O}$ molecule, the rate of water oxidation via WNA can significantly be enhanced by the strategic introduction of a proton acceptor/base, which simultaneously deprotonates the incoming substrate. This is often referred to as atom proton transfer (APT) WNA. The concentration of hydroxide nucleophiles in the aqueous solvent environment is also increased at high $\mathrm{pH}$, thus rendering WNA more likely under these conditions. ${ }^{[74]}$
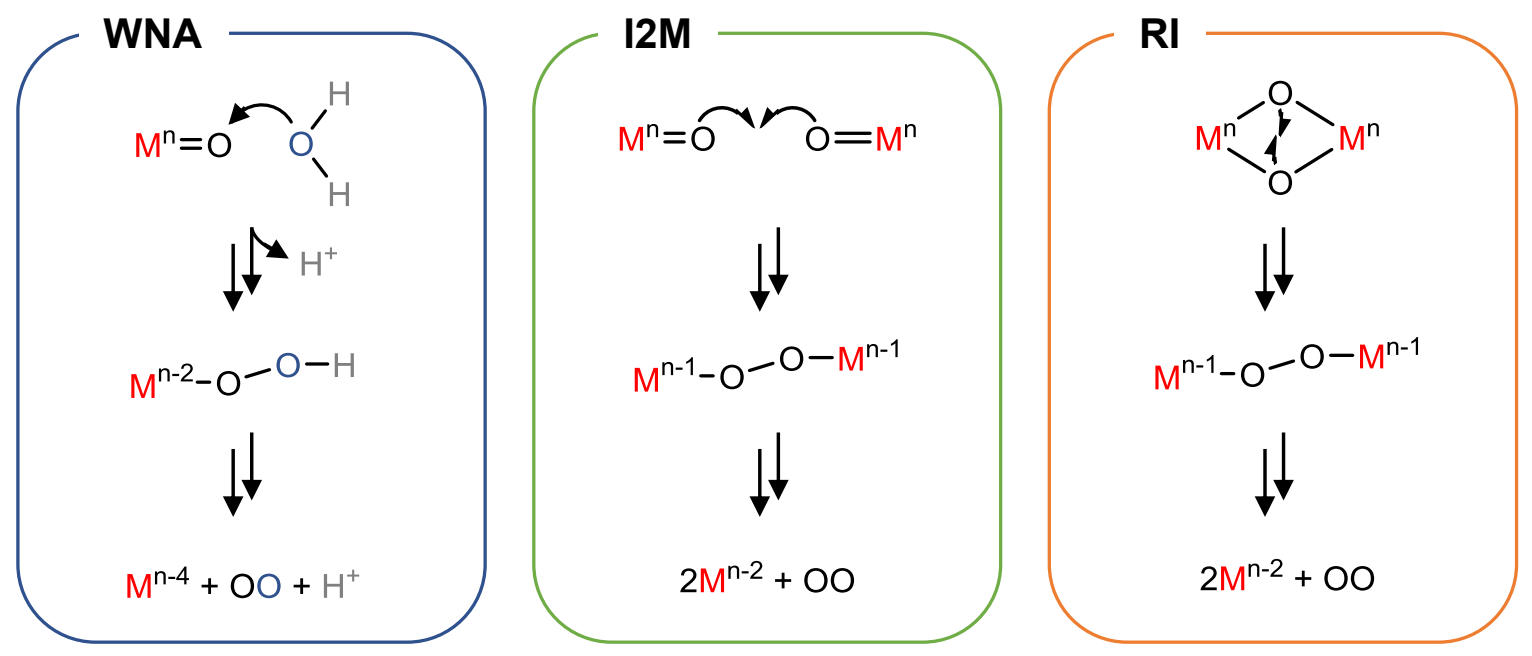

Scheme 1.3: Generalized 0-O bond formation mechanisms involving high-valent metal oxo species (WNA: water nucleophilic attack, I2M: interaction of two M-O entities, RI: redox isomerization). The oxygen atoms in blue and black are chosen to indicate a different degree of ${ }^{180}$-labeling in the transition metal complex and solvent water.

By contrast, the I2M pathway (Scheme 1.3) involves the radical coupling of two metal-oxo units and can occur in an intra- and intermolecular manner. In both scenarios, the $\mathrm{O}-\mathrm{O}$ bond is formed in an $\left[\mathrm{M}^{\mathrm{n}-1}-\mathrm{O}-\mathrm{O}-\mathrm{M}^{\mathrm{n}-1}\right]$ species, which undergoes further oxidation to produce $\mathrm{O}_{2}$. Requirements for this mechanistic pathway include that the $\left[\mathrm{M}^{\mathrm{n}}=0\right]$ fragment bears significant radical character, such that it might better be defined as [ $\left.\mathrm{M}^{\mathrm{n}-1}-\mathrm{O} \cdot\right]$, and the geometric state of the complex is compatible with such an interaction. Accordingly, the respective complexes generally contain flexible ligand scaffolds to accommodate the two metal-oxo units in a distance and angle suitable for $\mathrm{O}-\mathrm{O}$ bond formation.[61,99]

While WNA is the most widely proposed mechanism for both molecular and metal oxide based WOCs, few examples have been reported to operate via I2M.[102-105] Despite this mechanistic progress, a precise understanding regarding the parameters that favor one mechanism over the other, including ligand-dependent preferences (flexibility/rigidity, electron donating/withdrawing ability, possible $\mathrm{H}$ bonding interactions to substrate $\mathrm{H}_{2} \mathrm{O}$ ), remains one of the major challenges in the field of water oxidation catalysis. This endeavor is complicated by the fact that the energy difference between these 
two mechanisms can be minimal and only subtle changes regarding the structural or electronic properties of the catalysts can have a large impact on the reaction pathway.[56]

Quite recently, a third possible 0-0 bond formation pathway was introduced designated redox isomerization (RI, Scheme 1.3). This mechanism has thus far only been reported for copper-based WOCs and resembles the extensive biomimetic chemistry of copper complexes with dioxygen.[106] Accordingly, a dimeric bis( $\mu$-oxo) compound isomerizes to a $\mu-1,2$-peroxo species, featuring an $0-0$ bond and two one-electron reduced metal centers. Further oxidation of the peroxo unit leads to the release of $\mathrm{O}_{2}$ and regenerates the starting point of the catalytic cycle.[107]

Experimentally, the 0-O bond formation pathway can be elucidated by means of kinetic and spectroscopic techniques, as well as reactivity tests. Particularly useful are labeling experiments where the transition metal complex $\left(\mathrm{M}-\mathrm{OH}_{2}\right)$ and the aqueous solvent have a different degree of ${ }^{18} \mathrm{O}$ labeling. ${ }^{[103,108-111]}$ Here, the two major mechanistic pathways (WNA/I2M) can unambiguously be differentiated by isotopically analyzing the molecular oxygen generated in the first turnover using online mass spectrometry (see Scheme 1.3). For validation, these experiments should be performed at different degrees of catalyst and solvent labeling. In regard to this experimental procedure, mechanistic elucidations require transition metal complexes that are soluble in an aqueous medium. The experimental investigations are often complemented by theoretical calculations.

Given the lack of experimental data, the operating pathway for 0-O bond formation in the OEC is still unknown. Nevertheless, different mechanistic proposals exist depending on whether the oxidation from $\mathrm{S}_{3}$ to $\mathrm{S}_{4}$ (Kok cycle, see Figure 1.1) is ligand- or metal-centered (Scheme 1.4). In the former case, the generation of an $\mathrm{Mn}^{\mathrm{IV}}$-oxyl moiety is expected and $\mathrm{O}-\mathrm{O}$ bond formation is assumed to proceed via a radical coupling mechanism in either an open (A) or closed (B) cubane structure. In case of a metal oxidation event, the $\mathrm{O}-\mathrm{O}$ bond is presumed to be formed via nucleophilic attack of an $\mathrm{H}_{2} \mathrm{O}$ molecule, e.g. bound to the calcium ion, to an electrophilic $\mathrm{Mn}^{\mathrm{V}}$-oxo entity (C). Here, the [ $\mathrm{Mn}^{\mathrm{V}}=0$ ] unit could alternatively be attacked by a neighboring oxo group. ${ }^{[21]}$

A

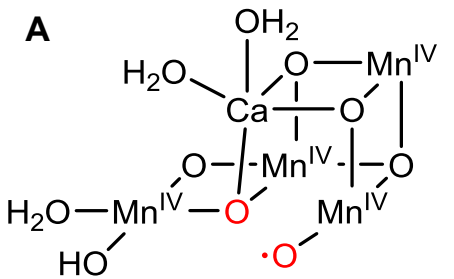

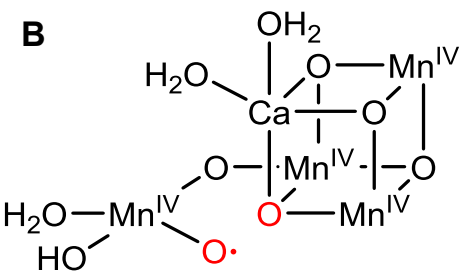

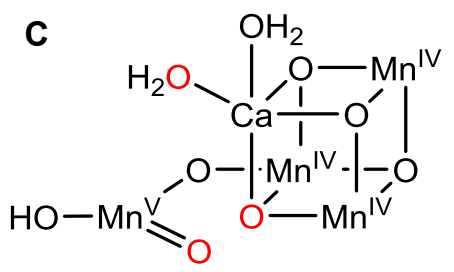

Scheme 1.4: Three potential structures of the $\mathrm{S}_{4}$ state in the Kok cycle preceding 0-O bond formation in the OEC. The oxygen atoms involved in $\mathrm{O}-\mathrm{O}$ bond formation are marked in red. Coordinating amino acids are omitted for clarity reasons.[21]

\subsubsection{Investigation Strategies of WOCs}

Essentially three different methods exist for the evaluation of the water oxidation capability of transition metal complexes in the homogenous phase. These involve the chemical, electrochemical and photochemical oxidation of the potential catalysts to generate the crucial metal-oxo fragments for $\mathrm{O}-\mathrm{O}$ bond formation. Rapid screening of WOCs is most easily performed using chemical oxidants in bulk solution. However, this technique is far from mimicking the conditions employed in artificial photosynthetic devices. By contrast, electrochemical studies most closely resemble the conditions found in a PEC. In light of the future goal of generating such a practical device, a promising WOC must be capable of the light-driven oxidation of water to dioxygen, which can be probed in combination with a photosensitizer. 


\section{Chemical Water Oxidation}

Typically, the sacrificial oxidant is added to an aqueous solution of the WOC or the other way around, and the generated dioxygen is measured in the solution or gas phase using a Clark electrode, mass spectrometry or gas chromatography. The most common oxidizing agent applied in this regard is ceric ammonium nitrate (CAN), a strong one-electron oxidant with a redox potential of $1.75 \mathrm{~V}$ at pH 0.9 in an aqueous perchlorate solution $(\sim 50 \mathrm{mM}) \cdot{ }^{[112]}$ The ability of CAN to act as a one-electron oxidant is advantageous, as it enables the successive oxidation of the catalyst as observed in the natural system (see Section 1.1). However, a large drawback in the application of CAN as sacrificial oxidant is its instability in aqueous solutions with $\mathrm{pH}>1$, thus limiting its use to screening acid resistant catalysts. ${ }^{[59]}$ Furthermore, studies have shown that $\mathrm{Ce}^{\mathrm{IV}}$ can interact with the catalyst and have an influence on the mechanistic scenario.[113-116]

Alternatively, a number of strong two-electron oxidants can be applied for the catalytic investigation of WOCs, including sodium hypochlorite ( $\mathrm{NaClO})$, sodium periodate $\left(\mathrm{NaIO}_{4}\right)$ and potassium peroxymonosulfate $\left(\mathrm{KHSO}_{5}\right)^{[112,117,118]}$ The main advantage of these sacrificial oxidants is their activity under neutral or alkaline conditions. However, a large concern exists regarding the use of two-electron oxidants to screen WOCs, due to their ability to serve as oxo-transfer reagents.[119,120] Consequently, the liberated $\mathrm{O}_{2}$ could contain an oxygen atom from the chemical oxidant, thus questioning their practicability to probe actual water oxidation. Studies have also shown that CAN can act as an oxygen atom transfer reagent, however examples are rare.[109,121]

An alternative and attractive one-electron oxidant is $\left[\mathrm{Ru}(\mathrm{bpy})_{3}\right]^{3+}$, which features a potential of $1.26 \mathrm{~V}$ and can be applied at near neutral $\mathrm{pH}$. However, its relatively high cost and low thermodynamic potential have rendered it quite uncommon for evaluating molecular WOCs. Nevertheless, as $\left[\mathrm{Ru}(\mathrm{bpy})_{3}\right]^{3+}$ can be photogenerated from the $\left[\mathrm{Ru}(\mathrm{bpy})_{3}\right]^{2+}$ complexes, it has found widespread application in probing light-driven water oxidation (see below). ${ }^{[59]}$ It should be noted that attention must be paid when using chemical oxidants as an external driving force for water oxidation, as the typically large excess of the oxidant relative to the WOC provide highly oxidizing conditions, which can promote catalyst decomposition processes.

\section{Electrochemical Water Oxidation}

Electrochemical methods provide a useful analysis technique for the investigation of both heterogenous and homogenous WOCs. In addition to the characterization of the catalyst, they provide a valuable tool to extract thermodynamic and kinetic information for electrocatalytic processes. Conventionally, the catalytic proficiency of electrocatalysts has been evaluated using the classical catalytic plateau current analysis, providing the observed rate constant $k_{\text {obs }}$ for the homogenous reaction according to equation 1.3 (ipl: catalytic plateau current, $i_{\mathrm{p}}$ : forward current in absence of substrate, $n$ : electrons transferred in catalytic process, $n^{\prime}$ : electrons transferred in respective noncatalytic process, $R$ : ideal gas constant, $T$ : temperature, $F$ : faraday constant, $v$ : scan rate).[122] However, the application of this method requires the forward and backward scan of the cyclic voltammogram (CV) to be fully superimposed (S-shaped voltammogram), which is rarely observed experimentally.

$$
\frac{i_{\mathrm{pl}}}{i_{\mathrm{p}}}=\frac{n}{0.4463 \cdot n^{\prime}} \cdot \sqrt{\frac{R T}{F v} \cdot \frac{k_{\mathrm{obs}}}{\mathrm{n}^{\prime}}}
$$

In 2012, Costentin et al. developed an improved methodology for the evaluation of electrocatalysts, the so-called foot-of-the-wave analysis (FOWA).[123,124] An advantage of this method is that it does not require an S-shaped response in the CV. Furthermore, FOWA is not affected by common side phenomena such as catalyst deactivation or substrate consumption at the electrode surface, which 
usually prevent the extraction of kinetic information, as it is based on the analysis of the first few points of the catalysis where the catalytic response is unperturbed by such processes.

The mathematical FOWA equation was adapted to the investigation of molecular WOCs by Matheu et al. for cases in which the RDS is first- (eq 1.4) or second-order (eq 1.5) in catalyst concentration, corresponding to a WNA or intermolecular I2M pathway of $0-0$ bond formation, respectively $\left(i_{\mathrm{p}}\right.$ : forward current of a reversible electrochemical process, $E^{0}$ : potential of water oxidation catalysis, which can be determined by differential pulse voltammetry, $C_{\text {cat }}^{0}$ : initial bulk concentration of catalyst). ${ }^{[125]}$ Accordingly, next to the extraction of kinetic information, this methodology, when applied to concentration dependent measurements, allows the elucidation of the operating reaction mechanism (WNA or intermolecular I2M). It should be noted that a distinction between WNA and intramolecular I2M cannot be made, as both pathways are first-order in catalyst concentration.

$$
\begin{gathered}
\frac{i}{i_{\mathrm{p}}}=\frac{4 \cdot 2.24 \sqrt{\frac{R T}{F v} \cdot k_{\mathrm{WNA}}}}{1+\mathrm{e}^{\frac{F\left(E^{0}-E\right)}{R T}}} \\
\frac{i}{i_{\mathrm{p}}}=\frac{4 \cdot 2.24 \sqrt{\frac{R T}{F v} \cdot k_{\mathrm{D}} \cdot C_{\mathrm{cat}}^{0}}}{\left[1+\mathrm{e}^{\frac{F\left(E^{0}-E\right)}{R T}}\right]^{3 / 2}}
\end{gathered}
$$

From equations 1.4 and 1.5, the apparent WNA pseudo-rate constant $k_{\mathrm{WNA}}$ or dimerization constant $k_{\mathrm{D}}$ can be extracted by plotting $\frac{i}{i_{\mathrm{p}}} v s . \frac{1}{1+\mathrm{e}^{\frac{F\left(E^{0}-E\right)}{R T}}}$ or $\frac{1}{\left[1+\mathrm{e}^{\frac{F\left(E^{0}-E\right)}{R T}}\right]^{3 / 2}}$, respectively. While $k$ WNA corresponds to the maximum achievable TOF for electrocatalytic water oxidation via WNA, TOF $\max$ for the intermolecular I2M pathway can be obtained by multiplication of $k_{\mathrm{D}}$ with $\frac{1}{3} C_{\mathrm{cat}}^{0}{ }^{[125]}$

\section{Light-Driven Water Oxidation}

A general scheme for the processes occurring during photo-driven water oxidation is depicted in Scheme 1.5. Apart from the WOC itself, a photosensitizer (P) and a sacrificial electron acceptor (SEA) are necessary to probe the photocatalytic performance. Water oxidation is thereby typically triggered by $\left[\mathrm{Ru}(\mathrm{bpy})_{3}\right]^{3+-t y p e}$ oxidants, which are generated in situ by light absorption of the respective $\left[\mathrm{Ru}(\mathrm{bpy})_{3}\right]^{2+}$-type complexes and subsequent oxidative quenching by the SEA. Depending on the substitution pattern of the bipyridine units, these oxidants feature redox potentials between 1.10 and 1.54 V.[59]

Sodium persulfate is commonly used as the SEA to provide oxidative equivalents. Upon reduction of $\mathrm{S}_{2} \mathrm{O}_{8}{ }^{2-}$ by a photoexcited [Ru(bpy) $\left.)_{3}\right]^{2+*}$ complex, it generates a sulfate molecule as well as a sulfate radical, which in turn can thermally oxidize another $\left[\mathrm{Ru}(\mathrm{bpy})_{3}\right]^{2+}$ to $\left[\mathrm{Ru}(\mathrm{bpy})_{3}\right]^{3+} \cdot[126,127]$ Consequently, two equivalents of photons and persulfate are necessary to generate four equivalents of the oxidant. Given the high thermodynamic potential of the generated sulfate radical $(E>2.40 \mathrm{~V}),{ }^{[128]}$ it is capable of oxidizing the WOC itself. Consequently, when conducting light-driven water oxidation experiments a significantly higher concentration of the photosensitizer relative to the WOC should be used. As an alternative SEA, Co ${ }^{\mathrm{III}}$ has been applied.[129] 


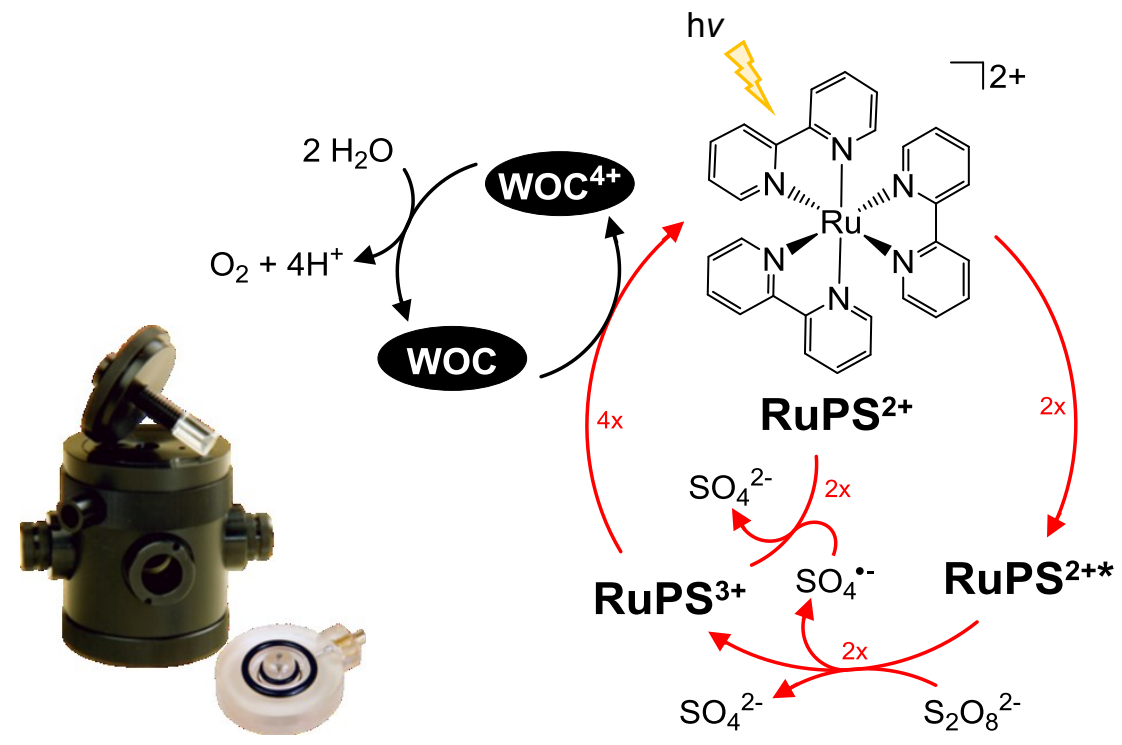

Scheme 1.5: General scheme for the processes occurring during photo-driven water oxidation catalysis. A dark chamber in which the experiments can be performed as well as the Clark-type electrode for $\mathrm{O}_{2}$ detection are shown on the left.

\subsubsection{Blue Dimer}

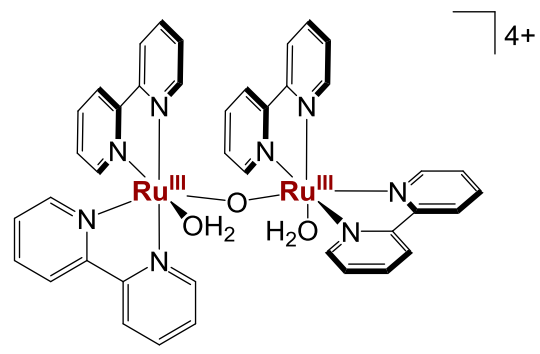

blue dimer

Scheme 1.6: Representation of the diruthenium WOC $\left[\left\{\mathrm{Ru}(\mathrm{bpy})_{2}\left(\mathrm{H}_{2} \mathrm{O}\right)\right\}_{2}(\mu-0)\right]^{4+}$, also known as the blue dimer.

In the early 1980s, Meyer and coworkers reported the first molecular compound capable of mediating the four-electron oxidation of water to dioxygen. ${ }^{[130]}$ The dinuclear complex $\left[\left\{\mathrm{Ru}(\mathrm{bpy})_{2}\left(\mathrm{H}_{2} \mathrm{O}\right)\right\}_{2}(\mu-\mathrm{O})\right]^{4+}$, referred to as the blue dimer due to its characteristic blue color, is comprised of two pseudo-octahedral cis- $\left[\mathrm{Ru}^{\mathrm{III}}(\mathrm{bpy})_{2}\left(\mathrm{H}_{2} \mathrm{O}\right)\right]$ units bridged by a dianionic oxo ligand (Scheme 1.6).[131] The presence of the oxo bridge was considered advantageous, as it promotes strong electronic coupling between the two ruthenium centers, thus facilitating the stabilization of high oxidation state species by electronic delocalization. In the presence of CAN as sacrificial oxidant, the

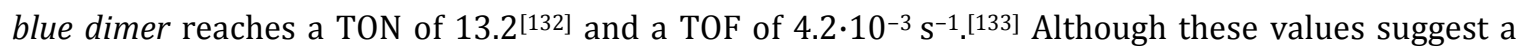
rather moderate catalytic proficiency, they provided a proof-of-concept that dinuclear ruthenium complexes are indeed capable of oxidizing $\mathrm{H}_{2} \mathrm{O}$ to $\mathrm{O}_{2}$.

Extensive mechanistic insight has been gained on the blue dimer by means of structural and kinetic studies in combination with theoretical calculations, however the results from ${ }^{18} 0$-labeling experiments are rather controversial (Scheme 1.7). In the latest mechanistic proposal by Meyer and coworkers (top), $\mathrm{O}-\mathrm{O}$ bond formation is suggested to proceed via WNA at a $\left[\mathrm{O}=\mathrm{Ru}^{\mathrm{V}}-\mathrm{O}-\mathrm{Ru}^{\mathrm{V}}=0\right]^{4+}$ species (bipyridine ligands are omitted for clarity reasons), which is generated by a series of PCET steps. ${ }^{[134,135]}$ The thus formed hydroperoxo intermediate releases $\mathrm{O}_{2}$ upon further oxidation by the 
second ruthenium center.[134] By contrast, labeling experiments by Hurst and coworkers propose ligand participation in the mechanistic pathway (bottom). Accordingly, a bipyridine ligand in the highvalent $\left[\mathrm{O}=\mathrm{Ru}^{\mathrm{V}}-\mathrm{O}-\mathrm{Ru}^{\mathrm{V}}=\mathrm{O}\right]^{4+}$ species is successively attacked at the $\alpha$ - and $\beta$-positions by two water molecules. Subsequent reduction of the ruthenium centers results in $\mathrm{O}-\mathrm{O}$ bond formation between the two hydroxide substituents. Upon liberation of dioxygen, the aromaticity in the pyridine moiety is recovered, thus providing the driving force for this step. ${ }^{[136]}$ Whereas $\mathrm{O}_{2}$ generation according to the latter mechanism seems improbable and additional support is lacking, this type of nucleophilic addition reaction has been considered a possible degradation pathway for polypyridyl complexes under oxidative conditions. ${ }^{[137,138]}$ Overall, the mechanistic pathway of water oxidation by the blue dimer is still a subject of intense debate.

The extensive investigations regarding the mechanism of water oxidation performed by the blue dimer have further provided detailed insight into degradation pathways responsible for the low TON. Next to the oxidation of the polypyridyl ligands as mentioned above, possible decomposition processes for the blue dimer and its derivatives include reductive cleavage of the bridging oxo ligand, particularly in the $\mathrm{Ru}^{\mathrm{II}} \mathrm{Ru}^{\mathrm{III}}$ state ${ }^{[131,139]}$ and oxidative cleavage of the oxo bridge via peroxidation of $\left[\mathrm{Ru}^{\mathrm{V}}-\mathrm{O}-\mathrm{Ru}^{\mathrm{V}}\right]$ to $\mathrm{Ru}^{\left.\mathrm{VI},{ }^{1} 140\right]}$ which both lead to a breakdown of the dimeric structure into non-active monomeric ruthenium complexes. Another factor that negatively influences the catalytic activity is a process called anation, i.e. the binding of allegedly innocent counter ions to the bimetallic complex thus displacing water from the coordination sphere of the ruthenium centers. ${ }^{[134,141]}$ It is assumed that the coordination of only one anion can prevent the formation of the catalytically active $\left[\mathrm{Ru}^{\mathrm{V}}-\mathrm{O}-\mathrm{Ru} \mathrm{v}^{\mathrm{V}}\right]$ species.
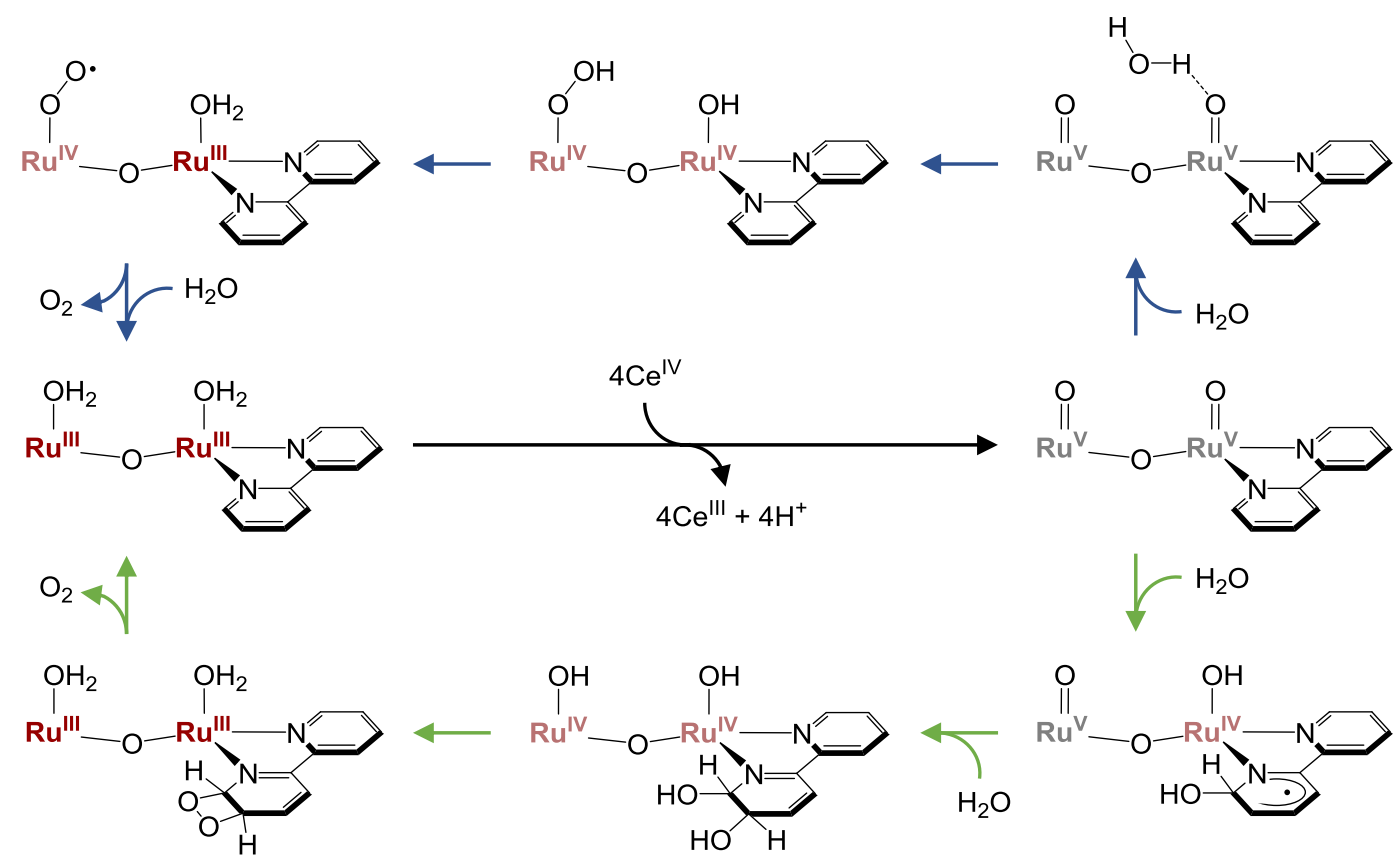

Scheme 1.7: Mechanistic scenarios discussed for the blue dimer based on ${ }^{18} 0$-labeling experiments (top: proposal by Meyer and coworkers, bottom: proposal by Hurst and coworkers). Non-participating bipyridine ligands have been omitted for clarity reasons.[137]

For over twenty years, the blue dimer and its $\mu$-oxo ligated derivatives were the only wellcharacterized molecular WOCs.[142,143] The eventual insight that an oxo bridge was not indispensable for water oxidation activity led to a significant enhancement in the field of ruthenium-based catalysts. Nowadays, a myriad of di- and mononuclear WOCs based on several first-, second- and third-row transition metals exist bearing a variety of polypyridyl ligands. ${ }^{[58-64]}$ Next to molecular catalysts, metal oxides have also received increased attention as WOCs. Whereas those of rare and precious metals have long been known to catalyze the oxidation of water to dioxygen, ${ }^{[144]}$ recent advances have been 
made in the development of highly active metal oxide catalysts based on non-toxic, cheap and earthabundant elements. [66,67,76]

\subsection{Dioxygen Activation}

The catalyzed evolution (OER) and reduction (ORR) of dioxygen are assumed to proceed via very similar intermediates involving various metal-oxygen species.[12,13] Consequently, it makes sense to take a deeper look at the reverse reactivity to water oxidation, which commences with dioxygen activation at transition metal complexes. Whereas binding and transport of $\mathrm{O}_{2}$ generally involves its two-fold reduction, breaking the $\mathrm{O}-\mathrm{O}$ bond requires four electrons and up to four protons, depending on the desired product.[145] Next to the generation of water, dioxygen is used for the oxidation and oxygenation of a large variety of organic substrates in nature. Responsible for these conversions are oxidases and oxygenases, which often possess copper ions in their active sites.[146,147]

Presently, copper enzymes are divided into seven classes denoted type 1 to 7 based on their spectroscopic features. ${ }^{[148,149]}$ These include mononuclear blue copper (T1) and "normal" copper (T2) proteins, dinuclear (T3) and trinuclear (T4, comprised of a T2 and T3 center) copper enzymes, the mixed-valent dinuclear $\mathrm{Cu}_{\mathrm{A}}$ site (T5), the $\mathrm{Cu}_{\mathrm{B}}$ center in close proximity to a mononuclear iron site (T6), as well as the tetranuclear $\mathrm{Cu}_{\mathrm{z}}$ center (T7). Out of these seven classes, types 2 to 4 are primarily involved in conversions using $\mathrm{O}_{2}$ as an electron acceptor. These enzymes all make use of the easily accessible $\mathrm{Cu}^{\mathrm{I}} / \mathrm{Cu}^{\mathrm{II}}$ redox couple, whereby initial $\mathrm{O}_{2}$ binding is facilitated by the $\mathrm{Cu}^{\mathrm{I}}$ state leading to the reduction of $\mathrm{O}_{2}$ and oxidation of the metal center. Herein, the focus is set on the respective active sites involving two copper centers.

\subsubsection{Type 3 Copper Enzymes}

Hemocyanin (Hc), tyrosinase (Tyr) and catechol oxidase (COx) are three members of the T3 class of copper enzymes. ${ }^{[149]}$ Whereas Hc is an oxygen transport protein found in anthropods and mollusks, the other two enzymes are catalytically active, responsible for the ortho-hydroxylation of monophenols (Tyr) and the oxidation of $o$-catechols to $o$-quinones (Tyr, COx).[150-154] All three enzymes possess almost identical active sites (Scheme 1.8), featuring two $\mathrm{Cu}^{\mathrm{I}}$ centers, each exclusively coordinated by three histidine residues of the protein backbone in the oxygen free (deoxy) form. Upon dioxygen binding (oxy form), the copper centers are oxidized to $\mathrm{Cu}^{\mathrm{II}}$, leading to a reduction of the dioxygen to the peroxide level. The $\mathrm{O}_{2}{ }^{2-}$ thereby adapts a bridging side-on $\left(\mu-\eta^{2}: \eta^{2}, \mathbf{s P}\right)$ coordination mode. In the oxy form, the two $\mathrm{Cu}^{\mathrm{II}}$ ions are strongly antiferromagnetically coupled, resulting in a singlet ground state and the concomitant absence of an EPR signal.[155,156]

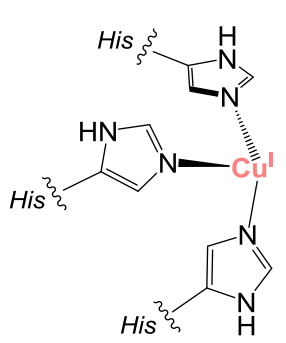
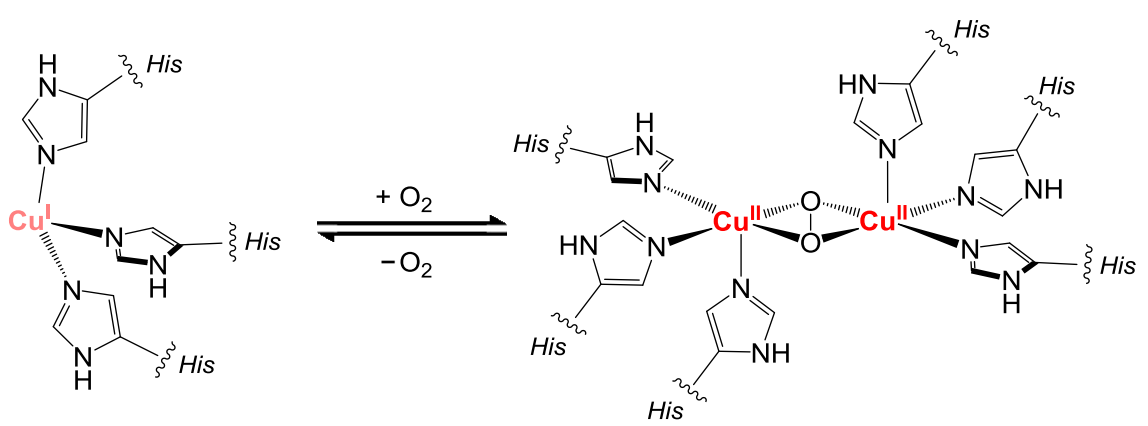

Scheme 1.8: Representation of the deoxy (left) and oxy (right) form of the active site of type 3 copper enzymes (His: histidine).

The exact pathway of dioxygen binding at the active site of T3 copper enzymes has been thoroughly investigated by means of DFT calculations based on structural and spectroscopic data of oxy Hc. [156-158] Especially interesting is how the spin-forbidden nature of this reaction is overcome, as triplet dioxygen 
must be converted to the singlet ground state in the oxy form. Initially, when $\mathrm{O}_{2}$ approaches the dicopper(I) site, both of its perpendicular $\pi^{*}$ orbitals simultaneously interact with each one $\mathrm{Cu}^{\mathrm{I}}$ ion (Scheme 1.9). This enables the synchronous transfer of two electrons from the copper ions to $\mathrm{O}_{2}$ and results in a butterfly distorted $\mathrm{Cu}_{2}\left(\mu-\eta^{2}: \eta^{2}-\mathrm{O}_{2}\right)$ core with ferromagnetically coupled $\mathrm{Cu}^{\mathrm{II}}$ ions $(S=1)$ due to nearly orthogonal magnetic orbitals. Relocation of the two unpaired charges onto the copper ions, which are further apart than the two oxygen atoms, thereby results in reduced electron-electron repulsion associated with a smaller energy difference between the triplet and singlet state. Planarization of the butterfly distortion thus induces intersystem crossing (ISC), as the change in the $\mathrm{Cu}_{2} \mathrm{O}_{2}$ core is accompanied with a reduced orthogonality of the magnetic orbitals and an increased superexchange coupling between the copper centers via the peroxide ligand. In the fully planar arrangement, the $\mathrm{Cu}^{\mathrm{II}}$ magnetic orbitals feature maximal overlap resulting in strong antiferromagnetic coupling $(S=0)$. The strong overlap between the two $\mathrm{Cu}^{\text {II }}$ magnetic orbitals and one of the peroxide $\pi^{*}$ HOMOs results in an intense $\mathrm{O}_{2}{ }^{2-} \rightarrow \mathrm{Cu}^{\mathrm{II}}$ charge transfer (CT) band in the UV/vis spectrum $(\sim 350 \mathrm{~nm}$, $\sim 20000 \mathrm{~L} \cdot \mathrm{mol}^{-1} \cdot \mathrm{cm}^{-1}$ ), giving the oxy form its deep blue color.[147] This illustrates how the spectroscopic features reflect the electronic structure of the copper oxygen core.

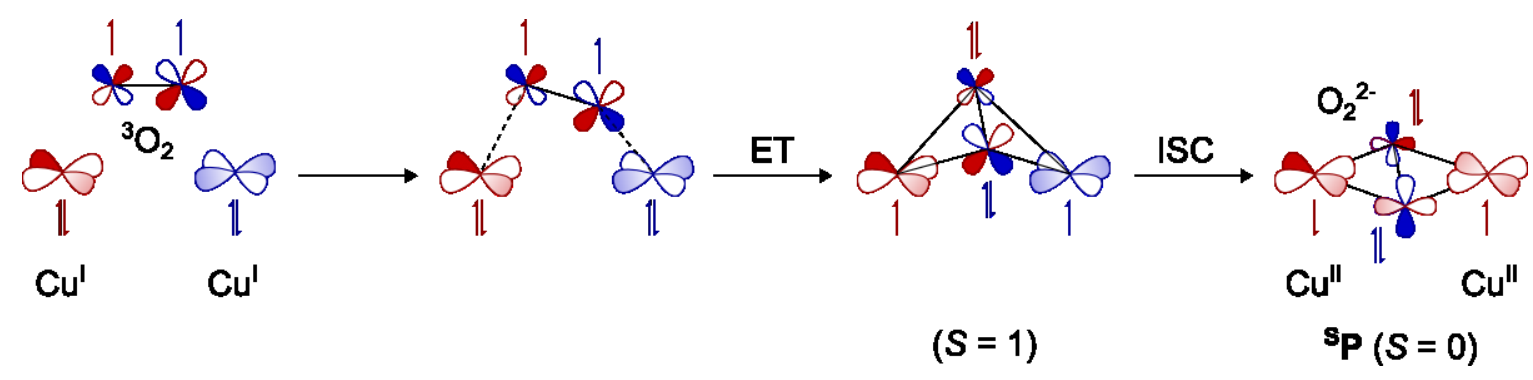

Scheme 1.9: Relevant steps in the dioxygen binding pathway in type 3 copper enzymes (ET: electron transfer, ISC: intersystem crossing, SP: denotation for $\mu-\eta^{2}: \eta^{2}$ coordination of the peroxo moiety).[158]

Despite the nearly identical active sites in $\mathrm{Hc}$ and Tyr/COx, only the latter two feature catalytic activity, which likely results from the limited accessibility of the $\mathrm{Cu}_{2} \mathrm{O}_{2}$ core in $\mathrm{Hc}$ for substrates. ${ }^{[159,160]}$ Tyr catalyzes the hydroxylation of L-Tyrosine to 3,4-dihydroxy-L-phenylalanine (L-DOPA), and the subsequent oxidation of L-DOPA to 3-(3,4-dioxocyclohexa-1,5-dien-1-yl)-L-alanine (L-DOPAquinone) is enabled by both Tyr and COx.[161] The latter is required for the formation of the brown polyphenolic pigment melanin. Early isotopic labeling studies showed that the oxygen atom incorporated into L-DOPA originates from dioxygen, ${ }^{[162]}$ however, despite spectroscopic and theoretical studies, it remains unclear whether the side-on peroxo complex or a high-valent dicopper(III) bis( $\mu$-oxo) species (so far only observed in synthetic model systems) is the hydroxylating intermediate.[147,161,163]

\subsubsection{Synthetic Modeling}

Synthetic chemistry has played a pivotal role in understanding how dioxygen interacts with copper metalloproteins. ${ }^{[147-149,157,164]}$ As initially crystal structures of parent enzymes, and especially their $\mathrm{O}_{2}$ adducts, were scarce, low molecular weight analogs were targeted to help resolve the copper-oxygen coordination modes in the proteins. One example is the type 3 copper enzyme hemocyanin: the ${ }^{\mathbf{S}} \mathbf{P}$ binding mode was first discovered in a synthetic model complex and, based on the spectroscopic agreement, concluded to correspond to the $\mathrm{Cu}_{2} \mathrm{O}_{2}$ adduct in $\mathrm{Hc} .{ }^{[165]}$ This was later confirmed by the crystallographic analysis of oxy Hc.[152]

Reaction of mono- and dinuclear copper(I) model complexes with $\mathrm{O}_{2}$ can result in a large variety of copper-oxygen species, some of which are depicted in Scheme 1.10.[106,166-168] The apparent diversity of these adducts is a product of the tunability of the formal oxidation states of both copper ( $\mathrm{Cu}^{\mathrm{II}} / \mathrm{Cu}^{\mathrm{III}}$ ) and the dioxygen derived ligands $\left(\mathrm{O}_{2}{ }^{-} / \mathrm{O}_{2}{ }^{2-} / \mathrm{O}^{2-}\right)$, but not all have been identified in the parent enzymes. Distinctive spectroscopic features, especially in UV/vis (CT bands) and resonance Raman 
spectroscopy ( $0-0$ stretching frequency), allow the differentiation of these copper-oxygen binding motifs. Inspired by the coordination environment of the natural systems, the majority of the model complexes bear nitrogen donating ligands, however $\mathrm{O}, \mathrm{S}$ and $\mathrm{P}$ donors are also represented. Adjustments in the donor atom type, denticity, charge, chelate ring size and steric demand of the ligand thereby have a large influence on which copper-oxygen adduct is formed.

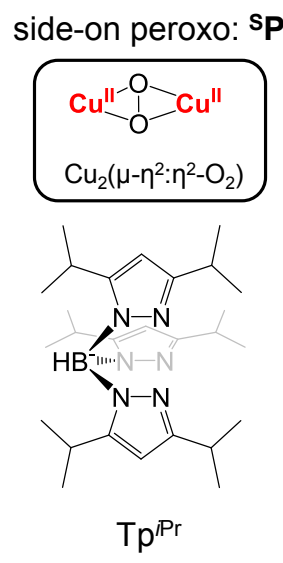

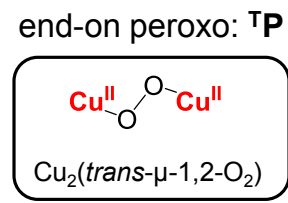

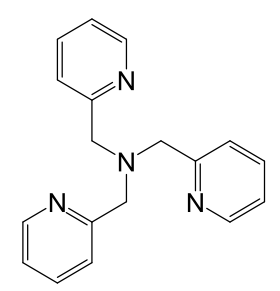

tpa end-on peroxo: ${ }^{\mathbf{C}} \mathbf{P}$

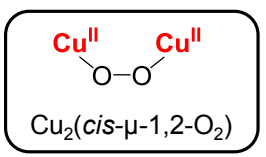<smiles>CC(C)N1CCN(Cc2cc(CN3CCN(C(C)C)CCN(C(C)C)CC3)[nH]n2)CCN(C(C)C)CC1</smiles>

$\mathrm{HL}^{2 b}$ bis $(\mu-0 \times 0): 0$

$$
\begin{gathered}
\mathrm{Cu}^{\mathrm{III}}{ }_{\mathrm{O}}^{\mathrm{O}}-\mathrm{Cu}^{\mathrm{III}} \\
\mathrm{Cu}_{2}(\mu-\mathrm{O})_{2}
\end{gathered}
$$

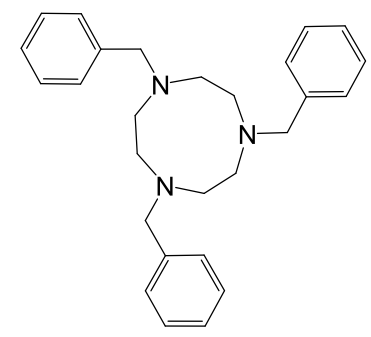

$\mathrm{Bz}_{3}$-tacn $\mu$-1,1-hydroperoxo

$$
\begin{gathered}
\mathrm{Cu}^{\prime \prime} \mathrm{Cu}^{\prime \prime} \\
\mathrm{O}^{-} \\
\mathrm{OH}_{2}(\mu-1,1-\mathrm{OOH}) \\
\mathrm{Cu}
\end{gathered}
$$<smiles>CC(C)N1CCN(CCc2cc(CCN3CCN(C(C)C)CCN(C(C)C)CC3)[nH]n2)CCN(C(C)C)CC1</smiles>

$H L^{2 b(E t)}$

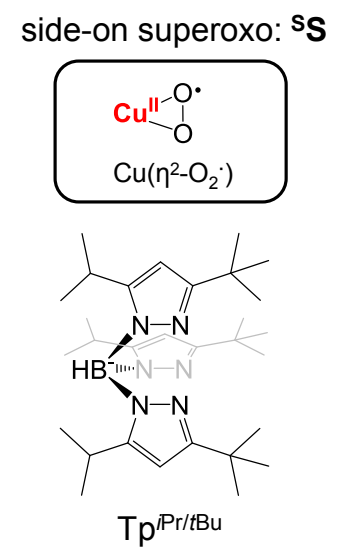

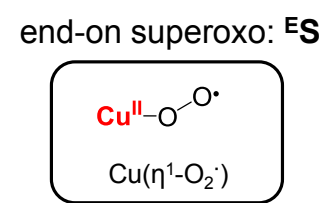<smiles>CN(C)CCN(CCN=C(N)N(C)O)CCNC(=O)N(C)C</smiles>

$\mathrm{TMG}_{3}$ tren $\eta^{1}$-hydroperoxo

$\mathrm{Cu}{ }^{11}-\mathrm{O}^{-} \mathrm{OH}$
$\mathrm{Cu}\left(\eta^{1}-\mathrm{OOH}\right)$

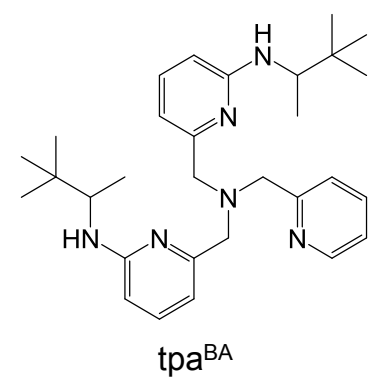

Scheme 1.10: Various mono- and dinuclear copper-oxygen adducts including their respective denotations, as well as the synthetic ligand system with which they were first structurally characterized. Please note, the $\eta^{1-}$ hydroperoxo complex was not generated with $\mathrm{O}_{2}$, but by use of $\mathrm{H}_{2} \mathrm{O}_{2}$. 
The first crystallographically characterized copper-oxygen complex was reported by Karlin, Zubieta and coworkers in 1988. ${ }^{[169]}$ Upon addition of $\mathrm{O}_{2}$ to the copper(I) precursor, bearing the tripodal tetradentate tris(2-pyridylmethyl)amine ligand (tpa; for schematic representations of the ligands see Scheme 1.10), a peroxo dicopper(II) species was formed, with a trans $\mu-1,2-\mathrm{O}_{2}{ }^{2-}$ group (end-on, ${ }^{\mathrm{T}} \mathbf{P}$ ) bridging the two $\mathrm{Cu}^{\mathrm{II}}$ ions. The corresponding cis $\mu-1,2-\mathrm{O}_{2}{ }^{2-}$ motif (end-on, ${ }^{\mathrm{CP}}$ ) was not characterized until 2014 with the help of the dinucleating pyrazolate/triazacyclononane (tacn) hybrid ligand scaffold $\mathrm{HL}^{2 \mathrm{~b}}$, which was also applied in the scope of this work. ${ }^{[170]}$ In 1989, the SP binding mode was identified in $\left[\left\{\mathrm{Cu}^{\mathrm{II}}\left(\mathrm{Tp}^{i \mathrm{Pr}}\right)\right\}_{2}\left(\mu-\mathrm{O}_{2}\right)\right]$ by Kitajima et al, in which the two copper centers are each ligated by a tridentate tris(pyrazolyl)borate ligand (Tp).[171] The last type of structurally characterized $\mathrm{Cu}_{2} \mathrm{O}_{2}$ species was first reported in 1996 upon application of a benzyl substituted tacn ligand. ${ }^{[172,173]}$ In this bis( $\mu$-oxo) dicopper(III) isomer (0), each $\mathrm{Cu}^{\mathrm{III}}$ ion has donated an additional electron to the dioxygen derived ligand, leading to $\mathrm{O}-\mathrm{O}$ bond scission. Although not yet discovered in natural systems, this $\mathrm{Cu}_{2} \mathrm{O}_{2}$ species is most frequently observed in synthetic model complexes, presumably due to the large variety of ligands that are capable of stabilizing $\mathbf{0}$ species.[167] Interestingly, it was shown that the $\mathbf{0}$ coordination mode can exist in equilibrium with both $\mathbf{S} \mathbf{P}$ and TP adducts (Scheme 1.11), interconversion being achieved e.g. by addition of a different solvent. ${ }^{[172,174-178]}$

The most prominent copper-oxygen binding modes are those with a $2 / 1 \mathrm{Cu}: \mathrm{O}_{2}$ stoichiometry, resulting mainly from the thermodynamically more favorable reduction of dioxygen to the peroxide $\left(2 \mathrm{e}^{-}\right)$than the superoxide (1e-) level.[167] Accordingly, mononuclear copper(I) precursors commonly dimerize to $\mathrm{Cu}_{2} \mathrm{O}_{2}$ complexes upon reaction with dioxygen (Scheme 1.11).[106,179,180] The respective transiently formed copper(II) superoxo species were initially observed in the early 1990s via low temperature stopped-flow UV/vis spectroscopy during investigations on TP systems. ${ }^{[181,182]}$ In order to trap the $\mathrm{Cu}^{\mathrm{II}}-\mathrm{O}_{2} \cdot-$ intermediates, sterically demanding ligands were introduced to prevent their reaction with a second equivalent of the mononuclear copper(I) complex. In line with this strategy, replacing one isopropyl residue at the pyrazole moieties in $\mathrm{Tp}$ with a tert-butyl group ( $\mathrm{Tp}{ }^{i \mathrm{Pr} / \mathrm{tBu}}$ ) allowed the isolation and crystallographic analysis of a side-on cupric superoxo complex $\left(\eta^{2}-\mathrm{O}_{2}-{ }^{-}, \mathbf{S}\right.$ S) instead of an $\mathbf{S P}$ copper-oxygen adduct.[183] Nevertheless, it could be shown that in solution both ${ }^{\text {SS }}$ and SP species are present, which could completely be shifted to the ${ }^{\mathbf{S}} \mathbf{S}$ side when replacing $t \mathrm{Bu}$ by adamantyl.[184] This example emphasizes how rational ligand design can bias the generated copperoxygen motif. In 2006, a mononuclear $\mathrm{Cu}^{\mathrm{II}}-\mathrm{O}_{2}{ }^{--}$compound was reported bearing a tetramethyl guanidino substituted tris(2-aminoethyl)amine ligand ( $\mathrm{TMG}_{3}$ tren) and featuring an end-on binding fashion of the superoxide moiety $\left(\eta^{1}-\mathrm{O}_{2}^{--},{ }^{E} \mathbf{S}\right) \cdot{ }^{[185,186]}$ The spectroscopic agreement between the ${ }^{\mathbf{E}} \mathbf{S}$ adduct and the features obtained from the stopped-flow analysis suggest that an end-on superoxo intermediate is transiently formed before the bimolecular process to form the corresponding $\mathbf{T} \mathbf{P}$ species.

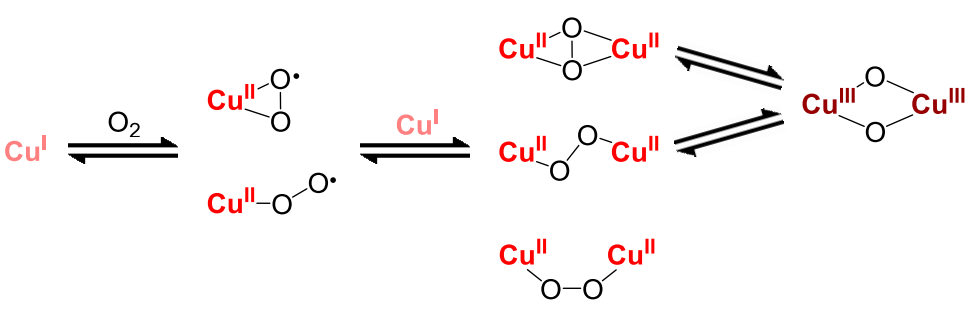

Scheme 1.11: Interconversion pathways between copper-oxygen adducts upon reaction of mononuclear copper(I) complexes with $\mathrm{O}_{2}$. 
Dinuclear S-type complexes can reversibly be generated by one-electron oxidation of the respective $\mathrm{Cu}_{2} \mathrm{O}_{2}$ peroxo intermediates. ${ }^{[187,188]}$ However, addition of dioxygen to a mixed-valent $\mathrm{Cu}^{\mathrm{I}} \mathrm{Cu}^{\mathrm{II}}$ precursor should allow the direct formation of a dicupric superoxo species. The latter was achieved with a dicopper complex based on the asymmetric phenol-containing dinucleating ligand UnOH (Scheme 1.12). Conversion of

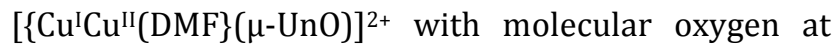
$-80^{\circ} \mathrm{C}$ afforded a dinuclear $\mathbf{S}$ species, in which the superoxide moiety is in equilibrium between a $\mu-1,2$ and $\mu-1,1$ binding fashion. ${ }^{[189,190]}$

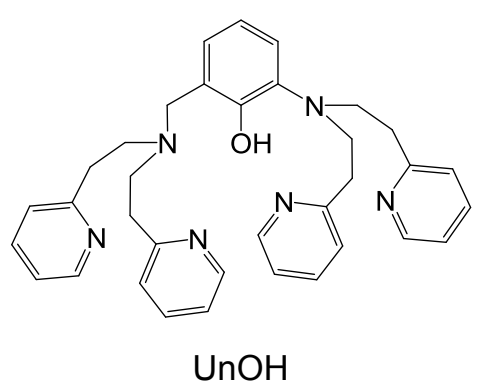

Scheme 1.12: Phenol-bridged dinucleating ligand scaffold $\mathrm{UnOH}$.

In regard to the four-electron reduction of $\mathrm{O}_{2}$ to $\mathrm{H}_{2} \mathrm{O}$, the dioxygen derived ligand has to be protonated at some point, likely leading to the formation of a hydroperoxo intermediate. Thus far, most well-characterized cupric hydroperoxo species are formed from the reaction of copper(II) complexes with $\mathrm{H}_{2} \mathrm{O}_{2},{ }^{1191-196]}$ such as the crystallographically characterized mononuclear $\eta^{1}$-hydroperoxo (endon) compound [(tpa $\left.\left.{ }^{\mathrm{BA}}\right) \mathrm{Cu}^{\mathrm{II}}(\mathrm{OOH})\right]^{+}$, bearing a butylamine substituted tpa ligand.[197,198] One of few dioxygen derived hydroperoxo complexes, formed by reversible protonation of the respective peroxo intermediate, was synthesized with the pyrazolate/tacn hybrid ligand $\mathrm{HL}^{2 \mathrm{~b}(\mathrm{Et})}$ in which the tacn sidearms are connected to the pyrazolate core by ethylene linkers. ${ }^{[199]}$ The $\left[\mathrm{Cu}^{\mathrm{II}} 2\left(\mu-\mathrm{L}^{2 \mathrm{~b}(\mathrm{Et})}\right)(\mu-\mathrm{OOH})\right]^{2+}$ complex, featuring a $\mu$-1,1-hydroperoxo motif, was not the first structurally investigated dinuclear $\mathrm{Cu}_{2}-\mathrm{OOH}$ species, ${ }^{[200]}$ but was unprecedented in that it allowed the reliable assignment of metric parameters due to the high quality of the X-ray diffraction (XRD) data. 


\section{Objectives}

Dinuclear ruthenium complexes for the catalytic oxidation of water to dioxygen have a history in the Meyer group. The first and most thoroughly characterized amongst them is a diruthenium catalyst based on the dinucleating bis(bipyridyl)pyrazolate ligand scaffold ${ }^{\mathrm{Me}} \mathrm{bbp}^{-}$, which was investigated both in the homogenous and heterogenous phase (see introduction of Chapter 3).[108,201-203] The versatility of this WOC is provided by its axial pyridine ligands, which can easily be varied during the synthetic procedure. Accordingly, substituted pyridines with anchoring groups were introduced for the immobilization on solid supports and the sulfonated analog was applied to render the catalyst water soluble, enabling detailed mechanistic studies.

The second generation of diruthenium complexes for water oxidation was developed by replacing the outmost pyridine moieties by anionic carboxylate groups in the equatorial ligand (Scheme 2.1, left; $\mathrm{R}^{1}=\mathrm{H}$ ), favorably stabilizing the high oxidation states required for oxygen-oxygen bond formation. ${ }^{[204]}$ This compound is the most efficient diruthenium WOC reported to date, however, its poor solubility in aqueous media hampered detailed mechanistic investigations. Consequently, the first goal of this work aims at the synthesis of a water-soluble congener equipped with sulfonated pyridine or isonicotinic acid as axial ligands. A subsequent comprehensive study on the water oxidation catalysis of the new WOC is expected to provide valuable information on how ligand modifications influence the mechanism of the $\mathrm{O}-\mathrm{O}$ bond forming step.
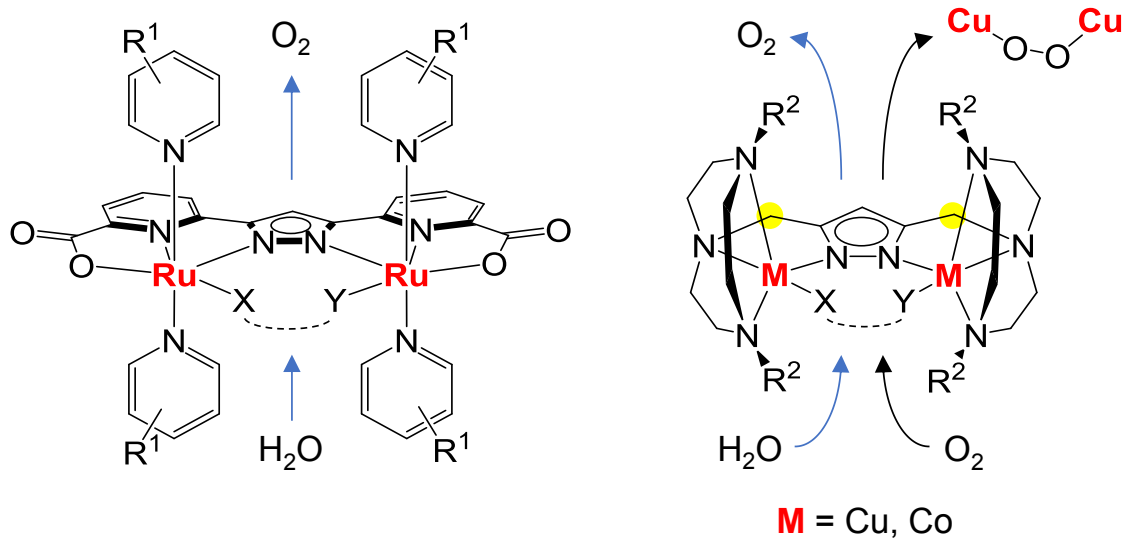

Scheme 2.1: Targeted dinuclear complexes based on pyrazolate/carboxylate (left) and pyrazolate/tacn (right) hybrid ligands, as well as the desired small molecule activation reactions.

With the future goal of a large-scale application of molecular WOCs in solar fuel devices, a remaining challenge is the design of efficient catalysts based on cheap and abundant base metals. Pyrazolatebridged ligand systems with amine sidearms have been shown to accommodate various first-row transition metal ions (including copper and cobalt) with suitable bimetallic distances for water 
binding.[205] Thus, the next goal is to develop new 3d transition metal based WOCs with a pyrazolate/tacn hybrid ligand (Scheme 2.1, right). Preliminary investigations have been conducted on a corresponding dicopper complex $\left(\mathrm{R}^{2}=i \mathrm{Pr}\right)$, which were complicated by the necessity of acetonitrile as a co-solvent (see introduction of Chapter 4).[188] This solubility issue is intended to be solved by introducing methyl residues at the tacn periphery $\left(\mathrm{R}^{2}=\mathrm{Me}\right)$.

Dicopper complexes bearing a pyrazolate/tacn hybrid ligand have been established as a suitable platform for dioxygen activation (see introduction of Chapter 6),[188,206,207] giving rise to various copper-oxygen species, which have also been considered as intermediates during water oxidation. So far, the influence of the linker $\left(\mathrm{CH}_{2} v s . \mathrm{CH}_{2} \mathrm{CH}_{2}, \mathrm{R}^{2}=i \mathrm{Pr}\right)$ between the pyrazolate and tacn moieties (Scheme 2.1, highlighted in yellow) on the structural and electronic properties of the obtained $\mathrm{Cu}_{2} \mathrm{O}_{2}$ species has been investigated. The impact of variations in the tacn periphery $\left(\mathrm{R}^{2}=i \mathrm{Pr} v s . \mathrm{Me}\right)$ is of interest to complete the picture and is hence the final goal of this work. In addition to further elucidating the effect of subtle ligand variations on the respective dioxygen activated species and their reactivity, this investigation could complement the water oxidation analysis.

Beyond the intended studies on small molecule activation and transformation, a large part of this work is aimed at the in-depth characterization of the involved transition metal complexes and putative intermediates with various techniques, including NMR, UV/vis, IR and EPR spectroscopy, as well as electrochemistry, X-ray diffraction and ESI mass spectrometry. 


\section{Water Oxidation Catalysis with Ruthenium Complexes}

Parts of this chapter have been adapted from Jana Lücken, Carolina Gimbert-Suriñach, Jordi BenetBuchholz, Antoni Llobet, Franc Meyer, "Diruthenium Complexes of a Pyrazolate/Carboxylate Hybrid Ligand for Chemically, Electro- and Photo-Driven Catalytic Water Oxidation", under revision. The corresponding sections are marked by footnotes.

Since the catalytic activity of the blue dimer was revealed in 1982 (see Section 1.3.5), dinuclear ruthenium-based WOCs have received increased attention. The availability of two metal centers was considered especially advantageous, enabling cooperativity to take effect. A significant enhancement in the field was achieved with the realization that a single metal site can be sufficient to promote the multi-electron oxidation of water to dioxygen. In the following, a glance will be taken at these two types of ruthenium-based catalysts for water oxidation. ${ }^{i}$

\section{Mononuclear Ruthenium-Based WOCs}

Owing to the catalytic proficiency of the blue dimer and the multimetallic core of the OEC, it was long assumed that an artificial WOC requires more than one metal center to accumulate the four oxidizing equivalents necessary for water oxidation. This hypothesis was proven wrong in 2005, when the first single-site catalyst capable of oxidizing water to dioxygen was presented by Thummel and coworkers. ${ }^{[208]}$ The mononuclear ruthenium complex $\left[\left(\mathrm{LI}^{\mathrm{I}}\right) \mathrm{Ru}(\mathrm{pic})_{2}\left(\mathrm{H}_{2} \mathrm{O}\right)\right]^{2+}(\mathrm{I}$, Scheme 3.1), composed of the neutral bis-naphthyridyl pyridine ligand $\mathrm{L}^{\mathrm{I}}$ and 4-picoline (pic), was shown to evolve $\mathrm{O}_{2}$ with a turnover number (TON) of 260 and a turnover frequency (TOF) of $0.014 \mathrm{~s}^{-1}$ in the presence of ceric ammonium nitrate (CAN) as sacrificial oxidant. ${ }^{[208,209]}$ By means of ${ }^{18}$ O-labeling studies, the $0-0$ bond formation mechanism was identified as WNA involving either a $\left[\mathrm{Ru}^{\mathrm{IV}}=0\right]^{2+}$ or $\left[\mathrm{Ru}^{\mathrm{V}}=0\right]^{3+}$ species (all scaffold ligands are omitted for clarity reasons).[210]

A significant improvement of the catalytic efficiency was achieved by incorporating anionic carboxylate groups into the ligand scaffold with the aim of accessing high-valent $\mathrm{Ru}=\mathrm{O}$ species at lower oxidation potential. The first example of a corresponding mononuclear complex is [(bda)Ru(pic) 2 ] (IIpic, Scheme 3.1) based on the dianionic ligand 2,2'-bipyridine-6,6'-dicarboxylate (bda ${ }^{2-}$ ).[211] Using CAN as electron acceptor, IIpic exhibits a high TOF of $32 \mathrm{~s}^{-1 .[104]}$ Investigations of the catalytic cycle of IIpic revealed $\mathrm{O}_{2}$ evolution to proceed via an intermolecular I2M pathway, in which two monomeric $\left[\mathrm{Ru}^{\mathrm{V}}=\mathrm{O} / \mathrm{Ru}^{\mathrm{IV}}-\mathrm{O}^{\circ}\right]^{+}$species rapidly dimerize to the peroxo bridged complex $\left[\mathrm{Ru}^{\mathrm{IV}}-\mathrm{O}-\mathrm{O}-\mathrm{Ru}^{\mathrm{IV}}\right]^{2+}$. From the latter, dioxygen is liberated after oxidation to the corresponding superoxo species in the presence of excess oxidant, regenerating the initial single-site catalyst.[104,105] The dimerization process is favored by $\pi-\pi$ stacking of the axial ligands, as clearly shown by the related complex IIisoq, in which the axial picoline ligands are exchanged by $\pi$-extended isoquinolines. Under identical conditions, II ${ }^{\text {isoq }}$ exhibits

i Please note, the catalytic proficiency of catalysts can only be compared if their analysis proceeds under identical conditions. Consequently, the values given herein should be considered with care. 
an order of magnitude higher TOF ( $303 \mathrm{~s}^{-1}$ ), which is comparable to that of the OEC at the PSII (100$\left.400 \mathrm{~s}^{-1}\right),{ }^{[212]}$ and is attributed to the lowering of the activation free energy barrier of the dimerization process (rate-determining step, RDS).[104] Concomitantly, the maximum TON is increased from 2000 (II ${ }^{\text {pic})}$ to 8000 (II $\left.{ }^{\text {isoq }}\right)$. Further improvements in TON (100000) and TOF $\left(1000 \mathrm{~s}^{-1}\right)$ were achieved by subtle variations of the axial ligands. ${ }^{[213-216]}$

Besides the carboxylate moieties in the ligand scaffold, there is another feature that made the Rubda catalysts (II) very unique at the time: the increase of the coordination number $(\mathrm{CN})$ of the ruthenium center from six to seven prior to catalysis. In its $\mathrm{Ru}^{\mathrm{II}}$ state, water binds to II at the expense of one of the hemilabile carboxylates, ${ }^{[217]}$ however, upon twofold oxidation, the electrophilicity of the $\mathrm{Ru}^{\mathrm{IV}}$ ion forces coordination of all $\mathrm{bda}^{2-}$ donor atoms along with the deprotonated hydroxide moiety (CN7).[104] This expansion of the coordination environment was first observed in the crystal structure of a $\mathrm{Ru}^{\mathrm{IV}}$ dimer of II ${ }^{\mathrm{pic}}$ with an $[\mathrm{HOHOH}]^{-}$bridge ${ }^{[211]}$ and is considered as one of the crucial elements for the extremely high catalytic proficiency of the Ru-bda catalysts. For a ruthenium complex to be able to reach CN7, it requires flexibility $(\mathrm{F})$, adaptability $(\mathrm{A})$, multidenticity $(\mathrm{M})$ and equatorial (E) coordination of the ligand scaffold, wherefore they are also referred to as FAME ligands. ${ }^{[64,218]}$

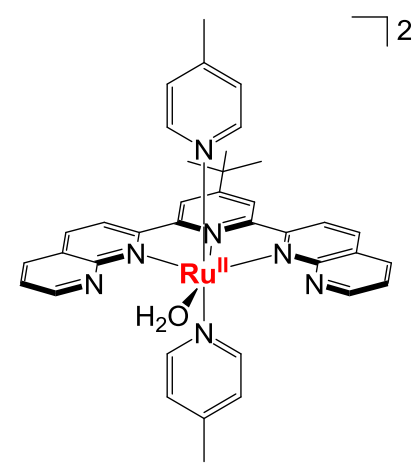

I

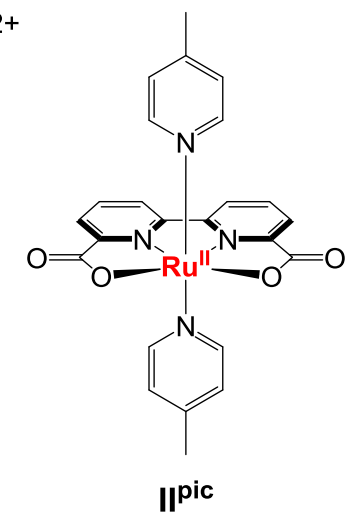

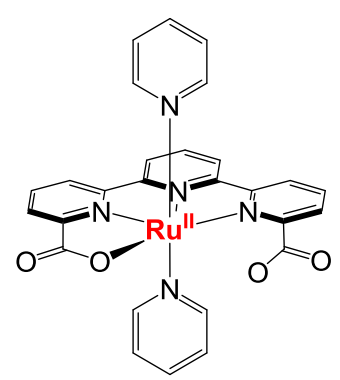

III

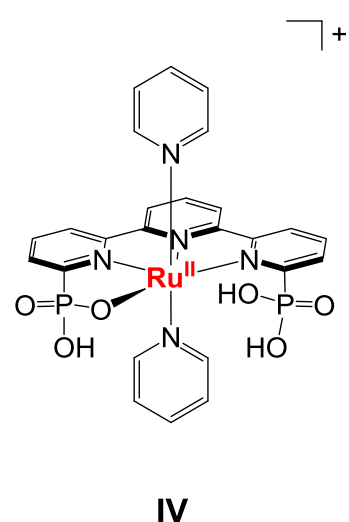

IV

Scheme 3.1: Representations of the mononuclear ruthenium-based WOCs I-IV.

Another system with a so-called FAME ligand is [( $\mathrm{N}^{3} \mathrm{O}$-tda) Ru(py) $\left.)_{2}\right]$, III (Scheme 3.1), based on $2,2^{\prime}: 6^{\prime}, 2^{\prime \prime}$-terpyridine-6,6"-dicarboxylate $\left(\mathrm{tda}^{2-}\right)$ and encompassing pyridine (py) as axial ligands. ${ }^{[219]}$ In the $\mathrm{Ru}^{\mathrm{II}}$ state, the tda ${ }^{2-}$ ligand binds in a tetradentate fashion $\left(N^{3} \mathrm{O}\right)$ with a dangling carboxylate. The latter is ligated upon $2 \mathrm{e}^{-}$oxidation to $\mathrm{Ru}^{\mathrm{IV}}\left(N^{3} \mathrm{O}^{2}, \mathrm{CN} 7\right)$ and is substituted by $\mathrm{OH}^{-}$at $\mathrm{pH} \geq 7$, generating the catalytically active $\left[\left(N^{3} \mathrm{O} \text {-tda) } \mathrm{Ru}^{\mathrm{lv}}(\mathrm{py})_{2}(\mathrm{OH})\right]^{+}\right.$species. Using FOWA analysis, an impressive maximum TOF (TOF $\max$ ) of $\sim 8000 \mathrm{~s}^{-1}$ at $\mathrm{pH} 7$, which is increased to $\sim 50000 \mathrm{~s}^{-1}$ at pH 10 for the electrocatalytic water oxidation of III was determined.[125,219] In contrast to II, $\mathbf{O}-\mathbf{O}$ bond formation in III takes place via a WNA mechanism. This pathway is favored over I2M due to the dangling carboxylate, which acts as a proton acceptor during the $\mathrm{O}-\mathrm{O}$ bond formation step and thus strongly reduces the activation free energy barrier of the WNA RDS. ${ }^{[219]}$ The WNA pathway of O-O bond formation also enables III to retain its solution-phase catalytic proficiency when anchored onto conductive solid oxide supports[220] or light-absorbing materials ${ }^{[221,222]}$ (attached via the apical ligands). In fact, immobilization on the former provides the most stable molecular anode for water oxidation reported to date, with around one million turnovers. By contrast, anchoring of II on graphitic surfaces results in degradation of the catalyst to $\mathrm{RuO}_{2}$, as the restricted mobility prevents dimerization (I2M), forcing it to access high energy pathways, which concomitantly facilitate ligand decomposition. ${ }^{[223]}$

Among the best molecular WOCs, in terms of TOFs, is the mononuclear ruthenium catalyst $\left[\left(N^{3} \mathrm{O}-\right.\right.$ $\mathrm{H}_{3}$ tpha)Ru(py) $]^{+}$(IV, Scheme 3.1), encompassing the deprotonated FAME ligand 2,2':6,2'terpyridine-6,6"-diphosphononic acid (H3tpha-).[224] By means of the FOWA technique, an unprecedented $\mathrm{TOF}_{\max }$ at $\mathrm{pH} 7.2$ of $\sim 16000 \mathrm{~s}^{-1}$ independent of the catalyst concentration $(0.5-3 \mathrm{mM})$ 
is obtained for IV. As in II and the related complex III, oxidation of IV to $\mathrm{Ru}^{\mathrm{IV}}$ leads to an increase of the coordination number of the ruthenium ion to seven. Interestingly, the catalytically active species of IV possesses an oxygenated ligand, which is formed by intramolecular $\mathrm{O}$ insertion into the para $\mathrm{C}-\mathrm{H}$ bond of one of the outer pyridine moieties by the highly reactive $\left[\mathrm{Ru}^{\mathrm{V}}=0\right]^{-}$species. ${ }^{[224]}$

\section{Dinuclear Ruthenium-Based WOCs ${ }^{\mathrm{ii}}$}

A new synthetic approach in the design of dinuclear WOCs was taken by Llobet and coworkers in 2004 by replacing the oxo bridge with a rigid polypyridyl-based backbone ligand equally bridging two ruthenium centers in close proximity. This was envisioned to avoid degradation processes based on the cleavage of the oxo bridge, common to all ruthenium-based WOCs until then, and thus enable more efficient water oxidation catalysis. Indeed, the combination of 2,2':6',2"'-terpyridine (trpy) and the compartmental dinucleating ligand 3,5-bis(2-pyridyl)pyrazolate $\left({ }^{-b p p p}{ }^{-}\right)$in $\left[\left\{\mathrm{Ru}(\operatorname{trpy})\left(\mathrm{H}_{2} \mathrm{O}\right)\right\}_{2}\right.$ $\left.\left(\mu-{ }^{H} \mathbf{b p p}\right)\right]^{3+}\left(\mathbf{V}^{\mathbf{H} 20}\right.$, Scheme 3.2) yielded a WOC with increased activity and stability. ${ }^{[225]}$ The active catalyst with two aquo ligands in the in,in-position, $\mathbf{V}^{\mathbf{H 2 0}}$, is obtained by dissolution of the acetatobridged precursor complex in an acidic aqueous solution at room temperature.

In the presence of 100 eq CAN as sacrificial oxidant (TON $\max =25), \mathbf{V}^{\mathbf{H} 20}$ reaches a TON of 17.5 $(70 \%)$ with an initial TOF $\left(\mathrm{TOF}_{\mathrm{i}}\right)$ of $0.014 \mathrm{~s}^{-1} .{ }^{[225,226]}$ The TON can be increased up to 512 by employing more oxidant, demonstrating the stability of $\mathbf{V}^{\mathbf{H 2 0}}$. These results compare favorably to those obtained for the blue dimer (see Section 1.3.5), and thus showed that a $\mu$-oxo ligand is not indispensable for the ability of ruthenium complexes to serve as WOCs, opening up the possibility of a rational synthetic ligand design. The main degradation pathway of $\mathbf{V}^{\mathbf{H} 20}$ was proven to be oxidative decomposition of the ligand at the pyrazolate moiety.

With the help of ${ }^{18} \mathrm{O}$-labeling experiments, I2M was determined as mechanism of the $\mathrm{O}-0$ bond formation step, encouraged by the strong through-space interaction of the two $\mathrm{Ru}-\mathrm{OH}_{\mathrm{x}}$ groups. Accordingly, the reactive $\mathrm{Ru}=\mathrm{O}$ groups, formed by multiple PCET steps, instantly couple to make an 0-0 bond. These results constitute the first experimental evidence of such a mechanistic pathway. ${ }^{[103]}$

Henceforth, preorganized complexes with rigid compartmental backbone ligands received increased attention. Another step forward was taken by introducing monodentate axial ligands instead of another polypyridyl ligand, as the exchangeable axial moieties offer tunability of the electronic properties and solubility of the catalyst by using pyridines with different substituents. The most thoroughly characterized amongst these is $\left[\left\{\mathrm{Ru}\left(\mathrm{pySO}_{3}\right)_{2}\left(\mathrm{H}_{2} \mathrm{O}\right)\right\}_{2}(\mu-\mathrm{Me} \text { bbp })\right]^{-}\left(\mathbf{V I}^{\mathbf{H} 20}\right.$, Scheme 3.2$)$ based on the anionic ligand system 3,5-bis\{6-(2,2'-bipyridyl)\}-4-methyl-pyrazolate (Mebbp-).[108] The monodentate pyridine-3-sulfonato $\left(\mathrm{pySO}_{3}{ }^{-}\right.$) is coordinated in the axial positions in order to overcome water solubility issues, thus allowing mechanistic investigations.

Using 100 eq CAN as sacrificial oxidant, VIH20 reaches a TON of 22.6 (90\%) with a TOF of $0.068 \mathrm{~s}^{-1}$.108] The improved catalytic efficiency in comparison to $\mathbf{V}^{\mathbf{H} 20}$ is associated with the methyl group at the pyrazolate backbone of $\mathrm{Mebbp}^{-}$, which prevents oxidative decomposition of the ligand, the main degradation pathway of $\mathbf{V}^{\mathbf{H} 20}$. By means of ${ }^{18} \mathrm{O}$-labeling experiments, the $\mathrm{O}-\mathrm{O}$ bond formation step in $\mathbf{V I}^{\mathbf{H 2 O}}$ was shown to take place via WNA at a $\mathrm{Ru}=0$ unit. The different mechanistic pathways observed in $\mathbf{V}^{\mathbf{H 2 0}}$ and $\mathbf{V I}^{\mathbf{H 2 0}}$, despite their very similar core structures and metal ion coordination environments, were explained by subtle geometric changes, which drastically reduce the degree of through-space interaction of the two $\mathrm{Ru}-\mathrm{OH}_{\mathrm{x}}$ groups in $\mathbf{V I}{ }^{\mathbf{H} 20}$, thus favoring WNA over I2M.[108] The mechanistic distinctions for related complexes illustrate that the activation barriers for these two pathways can be relatively close in energy. Thorough investigations on the correlation between the geometry of a catalyst and its $\mathrm{O}-\mathrm{O}$ bond formation pathway might help elucidate the details of the OEC reaction

\footnotetext{
ii Parts of this section were adapted from Jana Lücken, Carolina Gimbert-Suriñach, Jordi BenetBuchholz, Antoni Llobet, Franc Meyer, "Diruthenium Complexes of a Pyrazolate/Carboxylate Hybrid Ligand for Chemically, Electro- and Photo-Driven Catalytic Water Oxidation”, under revision.
} 
mechanism. Related diruthenium complexes based on the Mebbp- ligand platform[201] have also been shown to serve as efficient electrocatalysts after anchoring on solid oxide supports. [202,203]

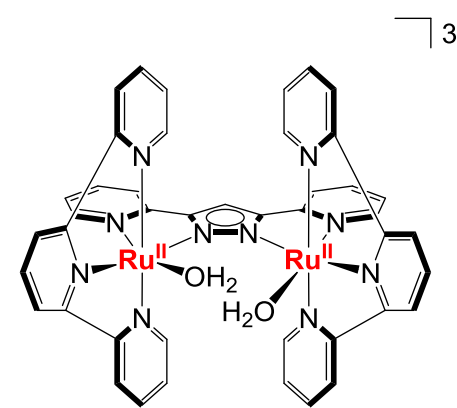

$\mathrm{V}^{\mathrm{H} 2 \mathrm{O}}$

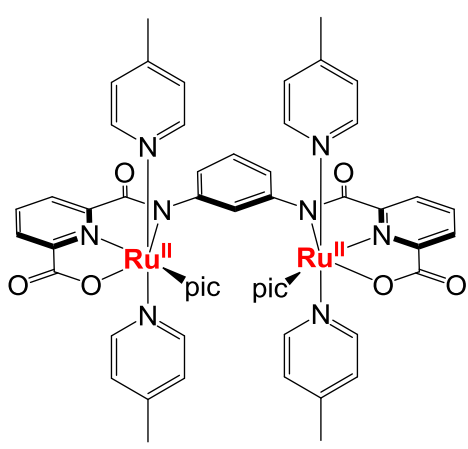

VIII

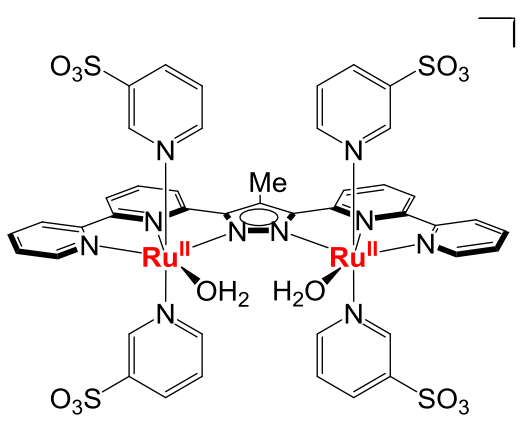

$\mathrm{VI}^{\mathrm{H} 2 \mathrm{O}}$

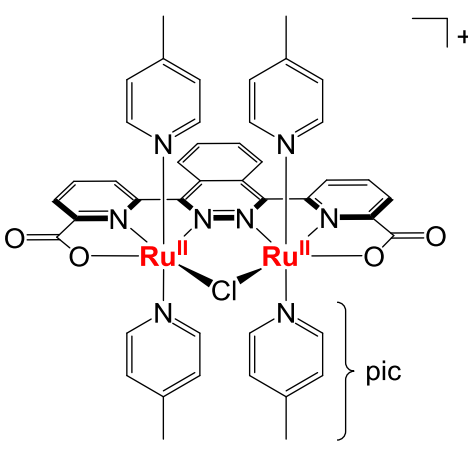

VII

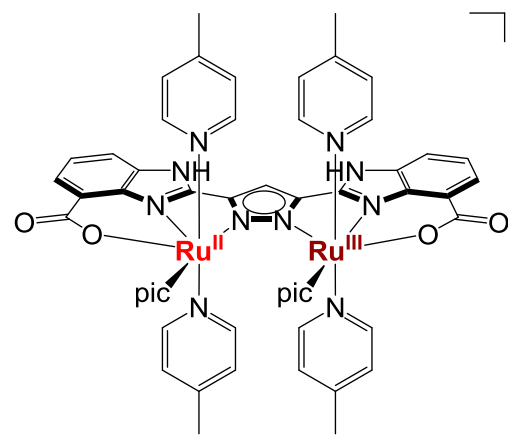

IX
$2+$

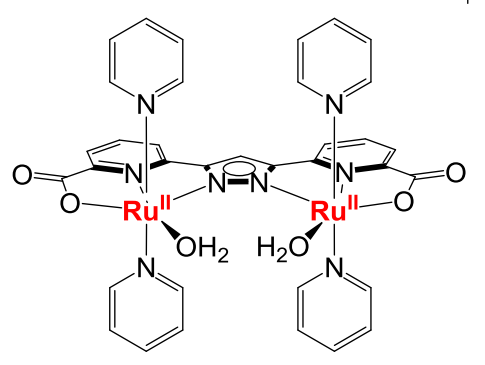

$\mathrm{X}^{\mathrm{H} 2 \mathrm{O}}$

Scheme 3.2: Representations of the diruthenium WOCs V-X.

The most significant improvement in water oxidation proficiency of dinuclear Ru-WOCs was achieved by the introduction of electron-rich carboxylate-containing ligand scaffolds. Among these is the complex $\left[\left\{\mathrm{Ru}(\mathrm{pic})_{2}\right\}_{2}(\mu-\mathrm{Cl})\left(\mu-\mathrm{L}^{\mathrm{VII}}\right)\right]^{+}$(VII, Scheme 3.2) based on the trianionic ligand scaffold 1,4bis $\{6$-(2-carboxypyridyl)\} phthalazine. Using CAN as sacrificial oxidant, VII reaches a TON of 10400 (42\%) with a TOFi of $1.2 \mathrm{~s}^{-1}$, far superior to other dinculear WOCs at the time..[27] Also in the presence of CAN, $\left[\left\{\mathrm{Ru}(\mathrm{pic})_{3}\right\}_{2}\left(\mu-\mathrm{L}^{\mathrm{VIII}}\right)\right]\left(\left(\mathrm{L}^{\mathrm{VIII}}\right)^{3-}=6,6^{\prime}-((1,3-\right.$ phenylenebis(azanediyl)$)$ bis-(carbonyl))dipicolinic acid), VIII (Scheme 3.2), exhibits a TON of 1400 (11\%) at a maximum rate of $1.17 \mathrm{~s}^{-1} .{ }^{[228]}$

In view of the future goal of incorporating such complexes into photoelectrochemical water splitting devices, attractive WOCs must not only be capable of chemically and electrochemically oxidizing water to dioxygen, but also efficiently mediate the light-driven reaction under neutral conditions. Next to the catalyst itself, the latter requires a sacrificial electron acceptor, typically sodium persulfate, and a photosensitizer. An attractive approach is thereby to drive water oxidation by mild one-electron $\left[\mathrm{Ru}(\mathrm{bpy})_{3}\right]^{3+}$-type oxidants, formed in situ by photo-irradiation of the $\left[\mathrm{Ru}(\mathrm{bpy})_{3}\right]^{3+}$-type complexes in the presence of $\mathrm{S}_{2} \mathrm{O}_{8}{ }^{2-}$.

In the photochemical system, the dinuclear ruthenium complex $\left[\left\{\mathrm{Ru}_{2}(\mathrm{pic})_{3}\right\}_{2}\left(\mu-\mathrm{H}_{2} \mathrm{~L}^{\mathrm{IX}}\right)\right]^{2+}, \mathbf{I X}$ (Scheme 3.2), based on the trianionic ligand scaffold 3,5-bis(4-carboxylate-1H-benzimidazol-2-yl)pyrazolate, proved to be an efficient photocatalyst achieving a TON of 830 in aqueous phosphate buffer $(\mathrm{pH} 7.2)$ in the presence of $\left[\mathrm{Ru}(\mathrm{bpy})_{2}\left((\mathrm{COOEt})_{2} \mathrm{bpy}\right)\right]^{2+}(\mathrm{RuP} 1)^{[229,230]}$ Under similar conditions, but using the weaker $\left[\mathrm{Ru}(\mathrm{bpy})_{2}\left((\mathrm{COOH})_{2} \mathrm{bpy}\right)\right]^{3+}(\mathrm{RuP} 2)$ as oxidant, VIII exhibits a TON of 146 with a $\mathrm{TOF}_{\max }$ of $0.03 \mathrm{~s}^{-1}$ under blue LED irradiation. ${ }^{[228]}$ Of the presented dinuclear carboxylate-containing WOCs, only VII is capable of mediating light-driven water oxidation using the parent complex $\left[\mathrm{Ru}(\mathrm{bpy})_{3}\right]^{2+}(\mathrm{RuP} 3)$ as the light-absorbing unit, however with a low TON of 60 and a TOF of $0.1 \mathrm{~s}^{-1}$. These numbers are significantly increased to 420 and $0.77 \mathrm{~s}^{-1}$ in the presence of RuP1.[227] Also catalyst 
$\mathbf{V I}^{\mathrm{H} 20}$ was shown to mediate photo-driven water oxidation using RuP1. In phosphate buffer (pH 7), $\mathbf{V I}^{\mathrm{H} 20}$ reaches a remarkable total TON of 5300 with a high TOF of $11.1 \mathrm{~s}^{-1 .[231]}$

The currently most efficient dinuclear ruthenium catalyst for chemical water oxidation was presented by Llobet, Meyer and coworkers in 2015.[204] Upon addition of 100 eq CAN as sacrificial oxidant, the rugged diruthenium complex $\left[\left\{\mathrm{Ru}(\mathrm{py})_{2}\left(\mathrm{H}_{2} \mathrm{O}\right)\right\}_{2}\left(\mu-\mathrm{L}^{1}\right)\right]^{+}\left(\mathbf{X}^{\mathrm{H} 20}\right.$, Scheme 3.2$)$ exhibits a TON of $24.0(96 \%)$ and a TOFi of $1.4 \mathrm{~s}^{-1}$. This is two orders of magnitude higher than those reported for the related pyrazolate-based complexes $\mathbf{V}^{\mathbf{H} 20}\left(0.014 \mathrm{~s}^{-1}\right)$ and $\mathbf{V I}^{\mathbf{H} 20}\left(0.068 \mathrm{~s}^{-1}\right)$, and comes close to the recorded TOFs of IIpic and IIisoq under identical conditions (TOF of 2.8 and $8.6 \mathrm{~s}^{-1}$, respectively).[104,204]

$\mathbf{X}^{\mathbf{H 2 0}}$ is a great example of how rational ligand design, founded on knowledge gained from previously studied WOCs, can lead to exceedingly improved catalysts. Accordingly, the trianionic scaffold 3,5-bis $\{6$-(2-carboxypyridyl) $\}$ pyrazolate, $\left(\mathrm{L}^{1}\right)^{3-}$, was designed as a hybrid of the ligands observed in the diruthenium complex $\mathbf{V I}^{\mathbf{H} 20}$, as well as the efficient mononuclear catalyst II. Given the dimerization process proposed in the catalytic cycle of the latter, it was assumed that preorganizing two ruthenium centers with otherwise similar coordination environments as II in a dinuclear complex, could lead to a catalyst with most favorable properties.

Due to the poor solubility of $\mathbf{X}^{\mathbf{H} 20}$ in aqueous media, however, more thorough investigations were not possible. This chapter discusses the attempt of improving the water solubility of $\mathbf{X}^{\mathbf{H} 20}$ by introducing substituted pyridine groups in the axial positions, a method that was previously proven successful for $\mathbf{V I}^{\mathbf{H} 20}{ }^{[108]}$ Next to a thorough spectroscopic and electrochemical characterization of the thus formed new complexes, their ability to chemically, electrochemically and photochemically oxidize water to dioxygen is investigated. In combination with these experiments, UV/visspectroelectrochemical (SEC) measurements reveal surprising results regarding the robustness of a bridging DMSO ligand and the catalytically active species of this diruthenium platform.

\subsection{Ligand Synthesis and Ruthenium Complexation ${ }^{i i i}$}

The proligand of interest, $\mathrm{H}_{3} \mathrm{~L}^{1}$, can be synthesized via a multi-step process adapted from literature procedures starting from dipicolinic acid (a) (Scheme 3.3). ${ }^{[204,232,233]}$ In an acidic medium, compound a is esterified using methanol to yield dimethyl 2,6-pyridinedicarboxylate (b). Subsequently, by means of sodium borohydride, one ester group is reduced to an alcohol to give rise to methyl 6(hydroxymethyl) picolinate (c). Ethyl acetate and sodium ethanolate convert c to a $\beta$-ketoester, which hydrolyzes to 2-ethanone-6-(hydroxymethyl)pyridine (d) in the presence of acid. A subsequent pseudo-Claisen condensation combines the pyridyl ester (c) and ketone (d) to the diketone (e), which can be converted to compound $f$ by ring closure with hydrazine. In this work, the ligand precursor $f$ was provided by Andreas Schwarz. Finally, oxidation with potassium permanganate in water yields the pyrazolate/carboxylate hybrid proligand $\mathrm{H}_{3} \mathrm{~L}^{1}$. The ligand was characterized by ${ }^{1} \mathrm{H}-\mathrm{NMR}$ spectroscopy, showing good agreement with reported data. ${ }^{[204]}$

For the preparation of the dinuclear ruthenium complexes, the proligand $\mathrm{H}_{3} \mathrm{~L}^{1}$ was initially converted to $\left[\mathrm{Ru}_{2}(\mathrm{dmso})_{2}\left(\mathrm{H}_{2} \mathrm{O}\right)(\mathrm{Cl})(\mu-\mathrm{dmso})\left(\mu-\mathrm{L}^{1}\right)\right](\mathbf{1}$, Scheme 3.4$)$ by reaction with the ruthenium precursor $\mathrm{RuCl}_{2}(\mathrm{dmso})_{4}$. In order to prevent the formation of mono- or multimetallic grid or grid-like complexes with a metal to ligand ratio smaller than $2: 1$, which have been shown to form with these types of ligands and various $3 \mathrm{~d}$ and $4 \mathrm{~d}$ metals, ${ }^{[234-241]}$ a dilute solution of $\mathrm{H}_{3} \mathrm{~L}^{1}$ and $\mathrm{NEt}_{3}$ was slowly added to $\mathrm{RuCl}_{2}(\mathrm{dmso})_{4}$. From this reaction mixture, intermediate $\mathbf{1}$ precipitated at low temperature. Crystals of $\mathbf{1}$ ', in which the axial water ligand is replaced by $\mathrm{MeOH}$, were obtained by diffusion of $\mathrm{Et}_{2} \mathrm{O}$ into a solution of $\mathbf{1}$ in $\mathrm{MeOH}$ and analyzed by X-ray diffraction (Scheme 3.4, top left).

iii This section was adapted from Jana Lücken, Carolina Gimbert-Suriñach, Jordi Benet-Buchholz, Antoni Llobet, Franc Meyer, "Diruthenium Complexes of a Pyrazolate/Carboxylate Hybrid Ligand for Chemically, Electro- and Photo-Driven Catalytic Water Oxidation", under revision. 
<smiles>COC(=O)c1cccc(C(=O)OC)n1</smiles>

b

C

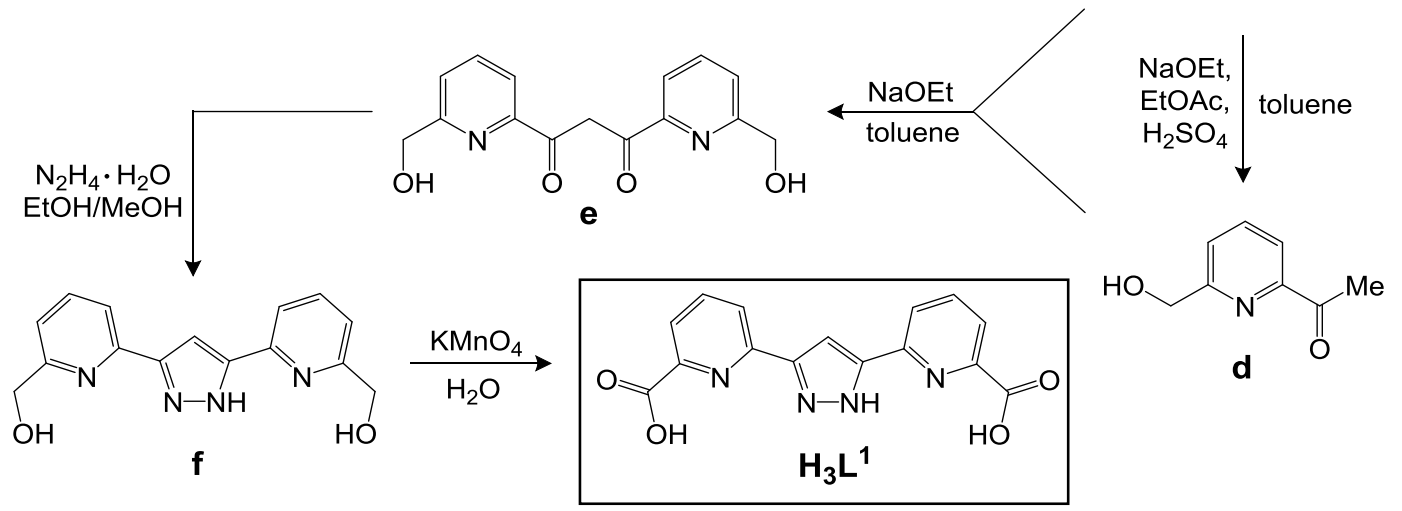

Scheme 3.3: Synthetic procedure for the preparation of the pyrazolate/carboxylate hybrid proligand $\mathrm{H}_{3} \mathrm{~L}^{1}$.

Compound 1' crystallizes in the monoclinic space group $C c$. One complex as well as four solvent molecules can be found in the asymmetric unit. The absence of counter ions, in combination with the trianionic ligand scaffold and a monoanionic chlorido moiety in one of the axial positions, suggests that the ruthenium atoms are in the oxidation state $+\mathrm{II}$. The $\mathrm{Ru}^{\mathrm{II}}$ ions are crystallographically independent and are coordinated by an $\left\{\mathrm{N}_{2} \mathrm{O}_{3} \mathrm{~S}\right\}$ - as well as a $\left\{\mathrm{ClN}_{2} \mathrm{OS}_{2}\right\}$ - donor set in an octahedral fashion. They are $4.15 \AA$ apart and bridged by the pyrazolate unit of the compartmental dinucleating ligand as well as a DMSO moiety - a rather unusual binding motif for DMSO. Besides the previously reported $\left[\mathrm{Ru}_{2}(\mathrm{dmso})_{2}\left(\mathrm{H}_{2} \mathrm{O}\right)(\mathrm{Cl})(\mu-\mathrm{dmso})(\mu-\mathrm{Me} b b p)\right]^{2+}(\mathbf{X I}),{ }^{[201]}$ this is only the second example of a complex with a highly preorganized $\mathrm{N}$-donating ligand system and DMSO combined in a six-membered ring. Both complexes exhibit very similar metal $\cdots$ metal distances and bond lengths regarding the DMSO moiety (Table 3.1). In case of $\mathbf{1}^{\prime}$, the equatorial ligand $\left(\mathrm{L}^{1}\right)^{3-}$, the two ruthenium atoms as well as the DMSO in the in,in-position are located almost perfectly in one plane. This is illustrated by the small torsion angles for $\mathrm{Ru}-\mathrm{N}-\mathrm{N}-\mathrm{Ru}$ and $\mathrm{Ru}-\mathrm{O}-\mathrm{S}-\mathrm{Ru}$ of $2.46^{\circ}$ and $-0.58^{\circ}$, respectively. In order to quantify the deviation of the coordination environment to an ideal polyhedron (not regarding the type of ligands, but merely the geometry), the method of Continuous Symmetry Measures (CSM) ${ }^{[242-245]}$ can be applied. Overall, a dimensionless value of $0-100$ is assigned to the ML6-system - the smaller the value, the closer the structure is to an ideal octahedron. The deviation of $\mathrm{Ru}(1)$ (1.32) is slightly lower than that of $\mathrm{Ru}(2)$ (1.55), which is coordinated by the sulfur atom of the bridging DMSO and the chlorido ligand (Table 3.1). Nevertheless, both values suggest a close to ideal octahedral geometry.

The integrity of 1 in solution was confirmed by ESI-mass spectrometry and NMR spectroscopy, enabled by the diamagnetic character of low-spin $\mathrm{d}^{6} \mathrm{Ru}^{\mathrm{II}}$ ions. The ESI(+)-MS in MeOH displays a signal at $m / z 802.9$ and the ESI(-)-MS at $m / z 732.9$ corresponding to $\left[\mathrm{M}-\mathrm{H}_{2} \mathrm{O}+\mathrm{Na}\right]^{+}$and $\left[\mathrm{M}-\mathrm{H}_{2} \mathrm{O}-\mathrm{dmso}+\mathrm{OMe}^{-}\right.$, respectively (appendix, Figures A.1-A.4). The ${ }^{1} \mathrm{H}-\mathrm{NMR}$ spectrum of $1 \mathrm{in} \mathrm{DMF-} d_{7}$ (Figure 3.1; for complete NMR characterization see appendix, Figures A.55-A.60) shows seven signals in the aromatic region for $\left(\mathrm{L}^{1}\right)^{3-}$, corresponding to a complex without a $C_{2}$ axis or mirror plane perpendicular to the $\left(\mathrm{L}^{1}\right)^{3-}$ ligand plane. Four singlet signals in the aliphatic region $(2.68,2.56,2.43$ and $2.01 \mathrm{ppm}$ ) can be assigned to the methyl groups of the $S$-coordinated terminal DMSO ligands, whereas those at $3.93 \mathrm{ppm}$ originate from the $S, O$-bridging DMSO. This is in good agreement with compound $\mathbf{X I}^{\mathrm{Cl}}$, in which the axial $\mathrm{H}_{2} \mathrm{O}$ from $\mathbf{X I}$ is replaced by a chlorido ligand, where the singlet signals of the DMSO in the in,in-position are situated at 3.93 and $3.82 \mathrm{ppm}$. 
Table 3.1: Selected distances, bond lengths, torsion angles and Continuous Symmetry Measures (CSM) of 1' in comparison to XI.[201]

\begin{tabular}{lll}
\hline distance / $\AA$ & $\mathbf{1}^{\prime}$ & $\mathbf{X I}^{[201]}$ \\
\hline \hline $\mathrm{Ru} \cdots \mathrm{Ru}$ & $4.1522(6)$ & $4.1545(5)$ \\
\hline bond lengths / $\AA$ & & \\
\hline \hline $\mathrm{Ru}-\mathrm{S}$ & $2.2827(8)$ & $2.321(1)$ \\
$\mathrm{Ru}-\mathrm{O}$ & $2.185(2)$ & $2.171(3)$ \\
$\mathrm{S}-\mathrm{O}$ & $1.521(2)$ & $1.516(3)$ \\
\hline torsion angle ${ }^{\circ}$ & & \\
\hline \hline $\mathrm{Ru}-\mathrm{N}-\mathrm{N}-\mathrm{Ru}$ & $2.462(498)$ & $19.157(615)$ \\
$\mathrm{Ru}-\mathrm{O}-\mathrm{S}-\mathrm{Ru}$ & $-0.583(243)$ & $9.582(300)$ \\
\hline${ }^{\mathrm{iv} C S M ~} S\left(O_{\mathrm{h}}\right)^{\mathrm{a}}$ & & \\
\hline \hline $\mathrm{Ru}(1)$ & 1.32 & $-\mathrm{b}$ \\
$\mathrm{Ru}(2)$ & 1.55 & $-\mathrm{b}$ \\
\hline
\end{tabular}

a Continuous Symmetry Measures for octahedral geometry

b not reported
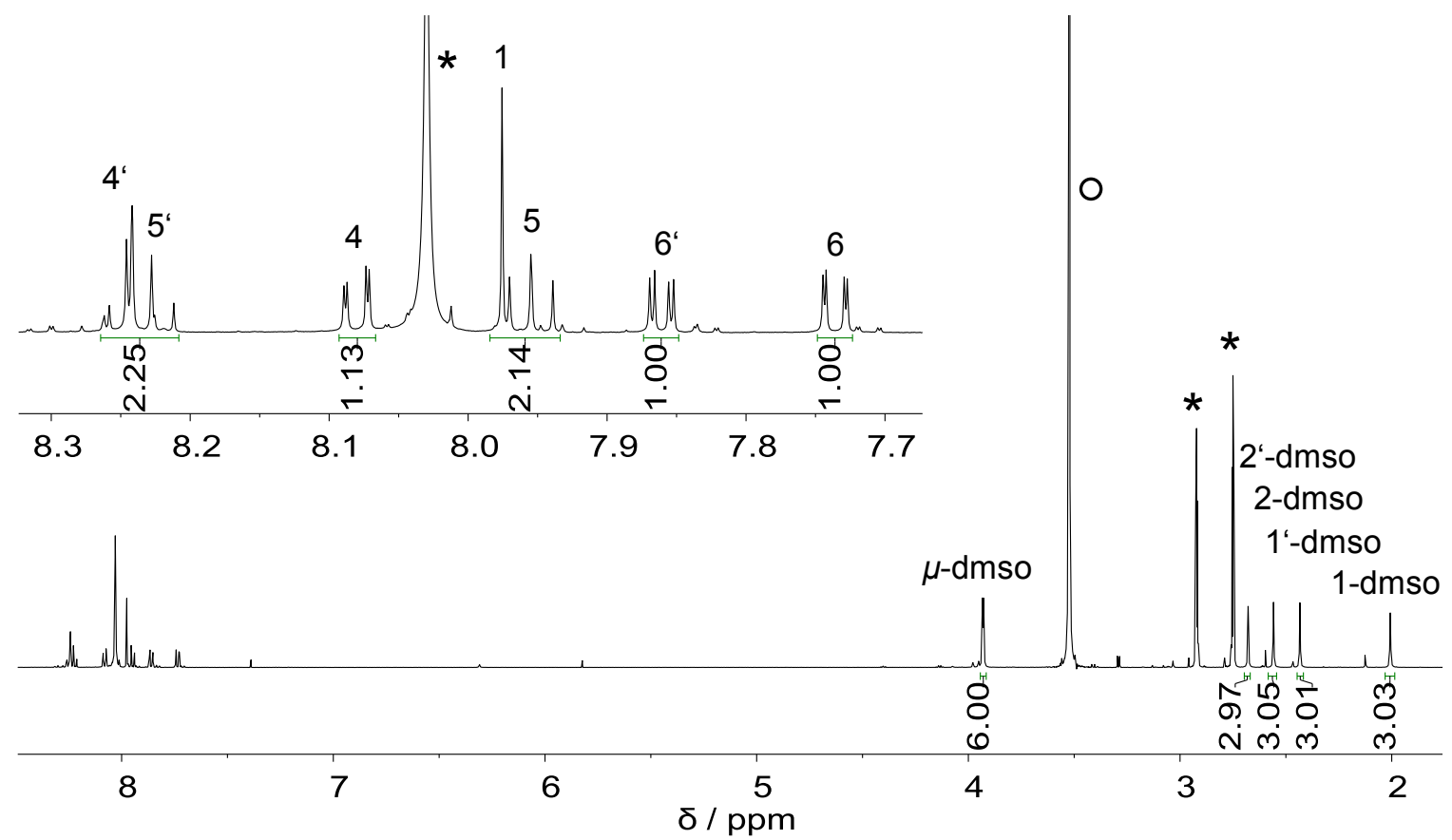

Figure 3.1: ${ }^{1} \mathrm{H}-\mathrm{NMR}$ spectrum $(500 \mathrm{MHz})$ of 1 in DMF- $d_{7}$. The inset depicts an expansion of the aromatic region (7.7-8.3 ppm). The peaks are assigned according to the labeling scheme in Scheme 3.4. The asterisks denote the residual solvent peaks and the circle points out $\mathrm{H}_{2} \mathrm{O}$. Please note, the distinction between prime and non-prime is not possible and is merely done for simplification.

iv The geometry of the coordination environment was compared to the reference shape "octahedron (with central atom)". 

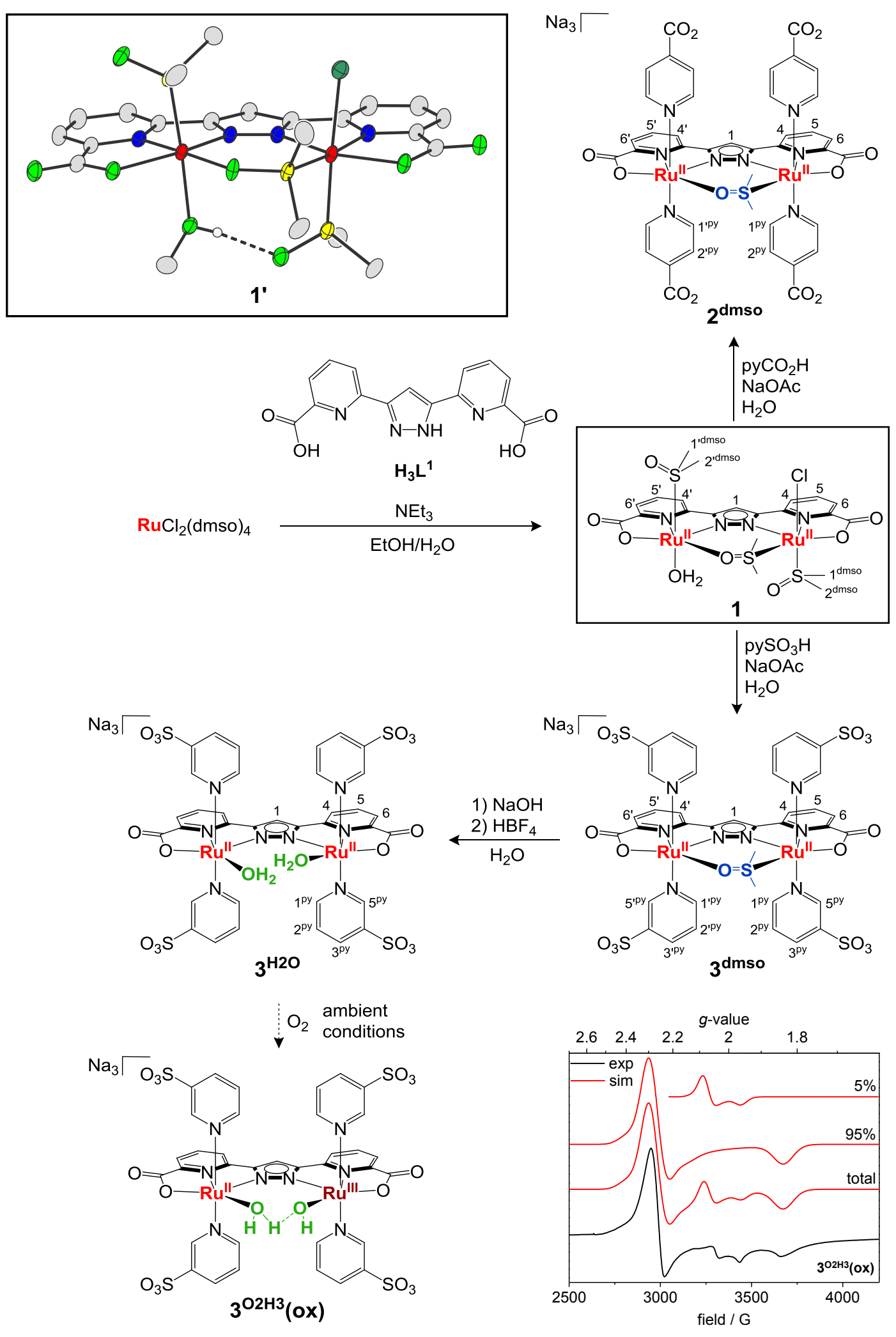

Scheme 3.4: Representation of the synthesis of the dinuclear ruthenium complexes $\mathbf{2}^{\text {dmso, }}$, as well as $\mathbf{3}^{\mathbf{d m s}}$ and $3^{\mathbf{H} 20}$ proceeding from the synthetic key intermediate $\mathbf{1}$. The inset at the top left corner depicts the molecular structure of 1' drawn as thermal ellipsoids at the $30 \%$ probability level (ruthenium: red, nitrogen: blue, oxygen: light green, sulfur: yellow, chloride: dark green, carbon: grey, hydrogen: white). All hydrogen atoms (except the one involved in intramolecular hydrogen bonding) and additional solvent molecules are omitted for clarity reasons. Please note, during crystallization the $\mathrm{H}_{2} \mathrm{O}$ molecule was exchanged for $\mathrm{MeOH}$. The inset at the bottom right corner depicts the X-band EPR spectrum of solid $\mathbf{3}^{\mathbf{3} 2 \mathrm{H} \mathbf{3}}(\mathbf{o x})$ at $145 \mathrm{~K}$. A signal can be observed, because $3^{\mathbf{H} 20}$ is gradually oxidized during synthesis under ambient conditions. 
The synthesis via an isolated intermediate - in contrast to the one-pot synthesis of the parent complex $\mathbf{X}^{\mathbf{H 2 O}[204]}$ - was chosen in order to have the possibility of introducing a variety of pyridine ligands with different substituents in the axial positions to optimize the electronic properties and tune the solubility of the complex. Introduction of pyridine carboxylate $\left(\mathrm{pyCO}_{2}{ }^{-}\right)$and pyridine sulfonate ( $\mathrm{pySO}_{3}{ }^{-}$) as axial ligands fulfilled the goal of generating highly water-soluble compounds based on the trianionic ligand scaffold $\left(\mathrm{L}^{1}\right)^{3-}$. For this, intermediate $\mathbf{1}$ was converted with either $\mathrm{pyCO}_{2} \mathrm{H}$ or $\mathrm{pySO}_{3} \mathrm{H}$ and sodium acetate in water (Scheme 3.4). The ligand exchange could visually be observed as the initial suspension of the water-insoluble compound $\mathbf{1}$ turned into a solution over time. Addition of acetone to the reaction mixture led to the precipitation of $\mathbf{2}^{\mathrm{dmso}}$ and $\mathbf{3}^{\mathrm{dmss}}$.

Complex $2^{\text {dmso }}$ was characterized in solution by ESI-mass spectrometry and NMR spectroscopy. The ESI(-)-MS displays a signal at $m / z 1122.8$ corresponding to [M+2Na]- (appendix, Figures A.5 and A.6). In the ${ }^{1} \mathrm{H}-\mathrm{NMR}$ spectrum in $\mathrm{D}_{2} \mathrm{O}$ (Figure 3.2, $\mathrm{A}$; for complete NMR characterization see appendix, Figures A.61-A.66) seven signals are observed for the $\left(\mathrm{L}^{1}\right)^{3-}$ ligand and four for the para-substituted pyCO $_{2}{ }^{-}$moieties. This is in agreement with a $C_{\mathrm{s}}$-symmetric complex with a mirror plane spanned by the $\left(\mathrm{L}^{1}\right)^{3-}$ ligand, the $\mathrm{Ru}^{\mathrm{II}}$ ions, as well as the $\mathrm{S}$ - and $\mathrm{O}$-atoms of the DMSO in the bimetallic pocket. By means of two-dimensional NMR spectroscopy $\left({ }^{1} \mathrm{H}-{ }^{1} \mathrm{H}-\mathrm{COSY},{ }^{1} \mathrm{H}-{ }^{1} \mathrm{H}-\mathrm{NOESY},{ }^{1} \mathrm{H}-{ }^{13} \mathrm{C}-\mathrm{HSQC},{ }^{1} \mathrm{H}-{ }^{13} \mathrm{C}-\mathrm{HMBC}\right)$ the carbon and hydrogen signals could largely be assigned - merely the distinction between the signals labeled prime and non-prime for the $\left(\mathrm{L}^{1}\right)^{3-}$ ligand is unclear. The ${ }^{1} \mathrm{H}-{ }^{-1} \mathrm{H}-\mathrm{NOESY}$ spectrum (appendix, Figure A.64) further confirmed the exact positioning of the DMSO moiety in the in,in-position by showing through-space interactions between the DMSO (singlet at $3.24 \mathrm{ppm}$ ) and the $1^{\mathrm{py}-\mathrm{H}}$ and $2^{\mathrm{py}-\mathrm{H}}$ signals of the $\mathrm{pyCO}_{2}{ }^{-}$ligands. The two doublets at 7.74 and $8.60 \mathrm{ppm}$ appear to belong to an additional pyCO $2_{2}^{-}$molecule (one equivalent considering each signal corresponds to two $\mathrm{H}$-atoms). According to the DOSY spectrum (appendix, Figure A.67) they do not belong to the complex, which is supported by the fact that they show no correlations in the 2D-NMR spectra except with each other. However, the doublets are shifted to higher field in comparison to free isonicotinic acid (doublets at 8.24 and $8.81 \mathrm{ppm})$.

An interesting observation made for $2^{\text {dmso }}$ was a somewhat self-cleaning procedure after dissolution. As apparent from the ${ }^{1} \mathrm{H}-\mathrm{NMR}$ spectra directly after sample preparation and five days later in the same solution (Figure 3.2, B), certain impurities (marked by an $\mathrm{x}$ ) seem to disappear over time without outer influence. At the same time, the two doublets of the additional $\mathrm{pyCO}_{2}{ }^{-}$molecule increase in intensity. Although multiple attempts were made to purify $2^{\text {dmso }}$, these impurities continued to appear when the complex was redissolved and, thus, hampered further analysis. Consequently, the related complex $\mathbf{3}^{\mathbf{d m s o}}$ was used for all further studies.

Complex $3^{\mathrm{dmso}}$ was also characterized in solution by ESI-mass spectrometry and NMR spectroscopy. As in the case of $\mathbf{2}^{\mathrm{dmso}}$, the ${ }^{1} \mathrm{H}-\mathrm{NMR}$ spectrum (Figure 3.3, bottom; for complete NMR characterization see appendix, Figures A.68-A.73) portrays a $C_{s}$-symmetric complex, now with 15 signals in the aromatic region of which eight stem from the meta-substituted $\mathrm{pySO}_{3}{ }^{-}$moieties. A singlet at $3.33 \mathrm{ppm}$ can be assigned to the methyl groups of the DMSO in the bimetallic pocket - once more the NOESY correlations confirm its exact orientation (appendix, Figure A.71). The ESI(-)-MS displays signals at $\mathrm{m} / \mathrm{z} 531.4$ and 1085.8 , corresponding to $\left[\mathrm{M}-\mathrm{pySO}_{3}\right]^{2-}$ and $[\mathrm{M}-\mathrm{pySO}+\mathrm{Na}]^{-}$, respectively (appendix, Figures A.7 and A.8). 

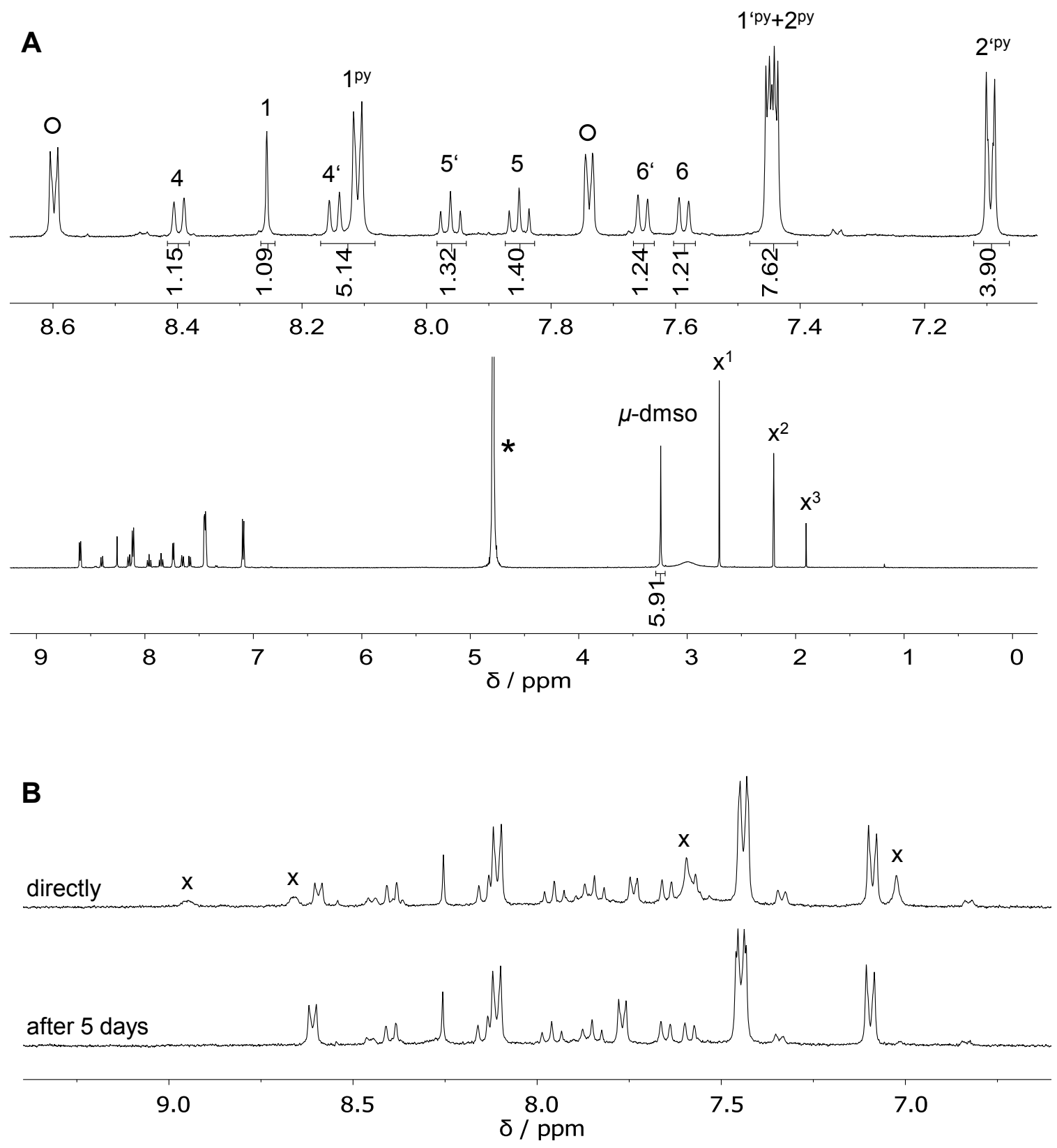

Figure 3.2: NMR spectroscopy of $\mathbf{2}^{\text {dmso }}$ in $\mathrm{D}_{2} \mathrm{O}$. A: ${ }^{1} \mathrm{H}-\mathrm{NMR}$ spectrum $(500 \mathrm{MHz}$ ). The top spectrum depicts an expansion of the aromatic region (7.1-8.6 ppm). The peaks are assigned according to the labeling scheme in Scheme 3.4. The asterisk denotes the residual solvent peak and the circles point out an additional pyCO $_{2}^{-}$molecule. $\mathrm{x}^{1}, \mathrm{x}^{2}$ and $\mathrm{x}^{3}$ mark free DMSO, acetone and acetate, respectively. Please note, the distinction between prime and non-prime for the $\left(\mathrm{L}^{1}\right)^{3-}$ ligand is not possible and is merely done for simplification. B: ${ }^{1} \mathrm{H}-\mathrm{NMR}$ spectrum $(300 \mathrm{MHz})$ of the aromatic region directly after dissolution and after remaining in the same solution for 5 days. The impurities marked with an $\mathrm{x}$ seem to disappear over time. 

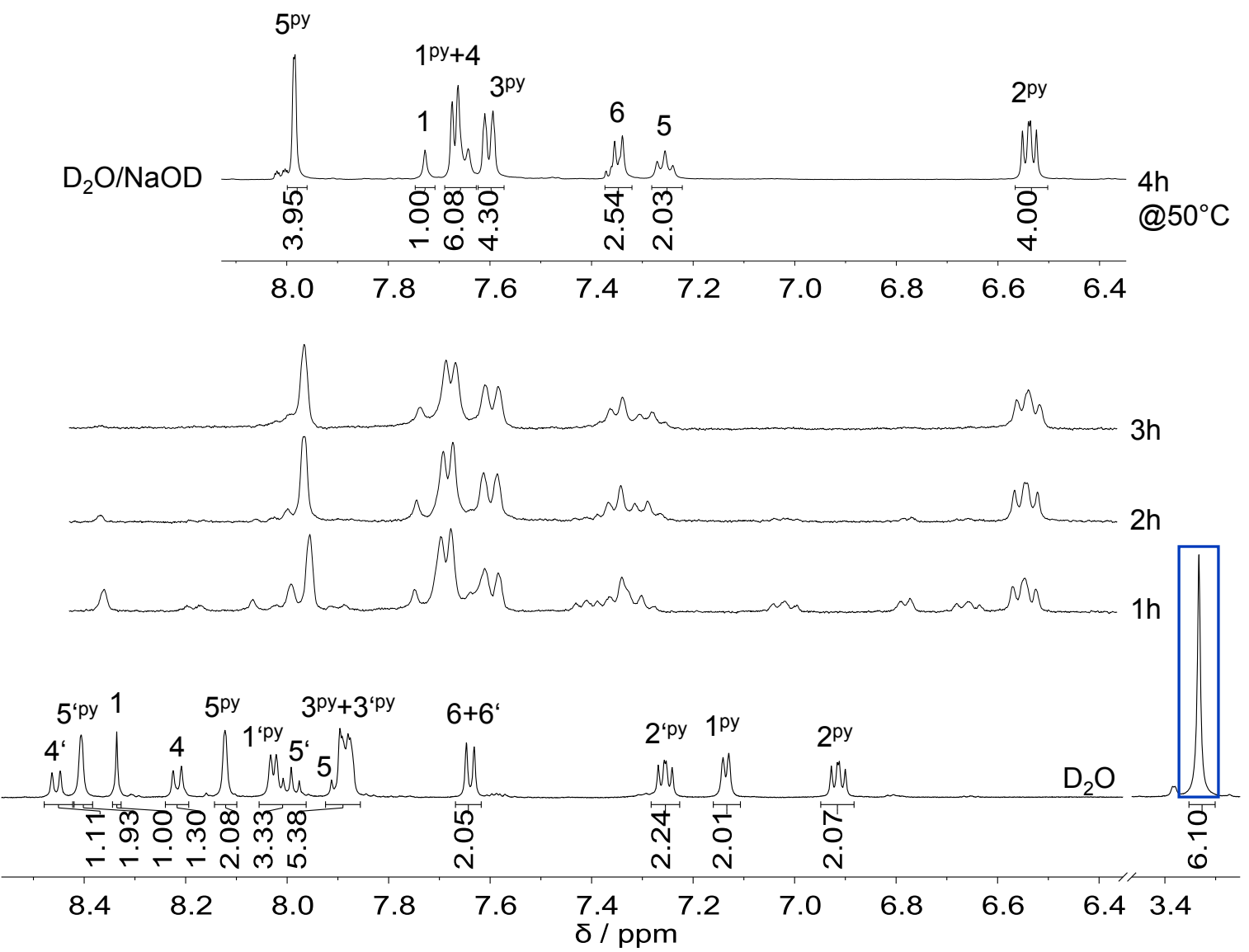

Figure 3.3: ${ }^{1} \mathrm{H}-\mathrm{NMR}$ spectra of the conversion of $\mathbf{3}^{\mathrm{dmso}}$ to $\mathbf{3}^{\mathbf{3} 2 \mathrm{H} 3}$ in $\mathrm{D}_{2} \mathrm{O} / \mathrm{NaOD}(1.5 \mathrm{M})$. Bottom: ${ }^{1} \mathrm{H}-\mathrm{NMR}$ spectrum

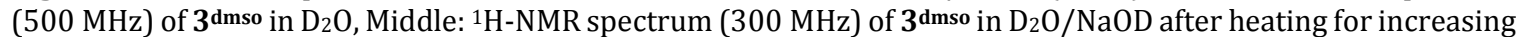
times at $50^{\circ} \mathrm{C}$, Top: ${ }^{1} \mathrm{H}-\mathrm{NMR}$ spectrum $(500 \mathrm{MHz})$ of $\mathbf{3}^{\mathbf{0 2} \mathrm{H}}$ in $\mathrm{D}_{2} \mathrm{O} / \mathrm{NaOD}$. The peaks are assigned according to the labeling scheme in Scheme 3.4. Please note, the distinction between prime and non-prime for the ( $\left.\mathrm{L}^{1}\right)^{3-}$ ligand is not possible and is merely done for simplification.

Exchange of the DMSO in the in,in-position with water was achieved within a few hours in a sodium hydroxide solution. This conversion can clearly be monitored by NMR spectroscopy, which shows the transition from a complex of lower to one of higher symmetry (Figure 3.3). After four hours at $50{ }^{\circ} \mathrm{C}$ the ${ }^{1} \mathrm{H}$-NMR spectrum displays only eight signals in the aromatic region in accordance with a complex having apparent $C_{2 \mathrm{v}}$-symmetry (for complete NMR characterization see appendix, Figures A.74-A.79). No signals are observed in the aliphatic region (there is no signal for free DMSO as it is deuterated under the basic conditions). A peak at $m / z 1183.8$ can be seen in the ESI(-)-MS corresponding to $\left[\mathrm{M}-\mathrm{OH}_{2}+\mathrm{Na}\right]^{-}$(appendix, Figures A.9 and A.10) - in this case the ruthenium ions are in the oxidation state II/III, which is a result of the gradual oxidation of $3^{\text {н20 }}$ during its synthesis under ambient conditions. This is confirmed by the observation of an EPR signal in the solid-state X-band spectrum of $\mathbf{3}^{\mathbf{0 2 H}}$ (ox) at $145 \mathrm{~K}$ (Scheme 3.4, bottom right), as only the oxidized species is paramagnetic, whereas $3^{\mathrm{H} 20}$ with two $\mathrm{Ru}^{\mathrm{II}}$ ions is diamagnetic and, thus, would show no signal. ${ }^{\mathrm{V}}$

\footnotetext{
${ }^{\mathrm{v}}$ Under ambient or slightly basic conditions, $3^{\mathrm{H} 2 \mathrm{O}}$ is expected to possess two aquo ligands and is given the superscript $\mathrm{H} 2 \mathrm{O}$. Upon oxidation to $\mathrm{Ru}^{\mathrm{II}} \mathrm{Ru}^{\mathrm{III}}$, one water molecule is likely deprotonated, thus its superscript is adapted to $02 \mathrm{H} 3$, referring to the aquo/hydroxo moiety in the bimetallic cleft. The latter is also used for the complex in its $\mathrm{Ru}^{\mathrm{II}} \mathrm{Ru}^{\mathrm{II}}$ state, when experiments were conducted in more basic solution, e.g. the NMR experiment in 1.5 M NaOD (Figure 3.3). These superscripts were chosen based on the information withdrawn from the Pourbaix diagram (see Section 3.3).
} 
The EPR spectrum was simulated with two species, each containing ruthenium(III). The major species $(95 \%)$ is assumed to be the $\mathrm{Ru}^{\mathrm{II}} \mathrm{Ru}^{\mathrm{III}}$ complex, the minor (5\%) possibly a mononuclear ruthenium(III) impurity. Both species were simulated with one $\mathrm{Ru}^{\mathrm{III}}$. They each show a pseudo-axial spectrum without hyperfine splitting of the ruthenium ion $(I=5 / 2)$. The corresponding EPR parameters are listed in Table 3.2.

Table 3.2 EPR parameters for $\mathbf{3}^{\mathbf{0 2}} \mathrm{H}^{3}(\mathrm{ox})$.

\begin{tabular}{lll} 
& major species & minor species \\
\hline \hline fraction / \% & 95 & 5 \\
$g_{1}$ & 1.84 & 1.96 \\
$g_{2}=g_{3}$ & 2.28 & 2.07 \\
gaverage $_{\mathrm{A}_{1} / \mathrm{MHz}}$ & 2.14 & 2.03 \\
$\mathrm{~A}_{2}=\mathrm{A}_{3} / \mathrm{MHz}$ & 80 & 50 \\
linewidth / G & 180 & 80 \\
\hline
\end{tabular}

As can be seen in Figure 3.3, compounds $3^{\text {dmso }}$ and $3^{\mathbf{H 2 0}}$ are spectroscopically pure according to NMR, but the combustion analysis shows that the powder samples do not exclusively contain the respective target complexes (see experimental section). Consequently, further analysis was conducted to investigate the possible impurities. According to XPS (Figure 3.4, left), the powder sample of $\mathbf{3}^{\mathrm{dmso}}$ contains no further elements other than those expected, whereas an unexpected large sodium peak as well as additional fluorine is observed for $3^{\mathbf{H 2 O}}$. This is further confirmed by the powder diffractogram of $3^{\mathrm{H} 20}$ (Figure 3.4, right) which coincides with that of $\mathrm{NaF}$ to $40 \%$. In conclusion, the results suggest that the powder sample of $\mathbf{3}^{\mathrm{dmso}}$ contains water molecules next to the target complex (the combustion analysis is quite accurate for $3^{\mathrm{dmso}} .9 \mathrm{H}_{2} \mathrm{O}$ ), and a significant amount of $\mathrm{NaF}$ next to water is additionally present in the powder sample of $\mathbf{3}^{\mathbf{H} 20}$. Generally, the presence of inorganic salts and water is a common phenomenon for complexes with sulfonate groups and multiple cations (three $\mathrm{Na}^{+}$in case of $3^{\mathrm{dmso}}$ and $3^{\mathbf{H 2 0}}$ ) that tend to engage in extended H-bonded networks. ${ }^{[246-249]}$ Nevertheless, the large amount of $\mathrm{NaF}$ in the powder sample of $3^{\mathbf{H} 20}$ can influence the catalytic performance and thus the respective results should be treated with care.
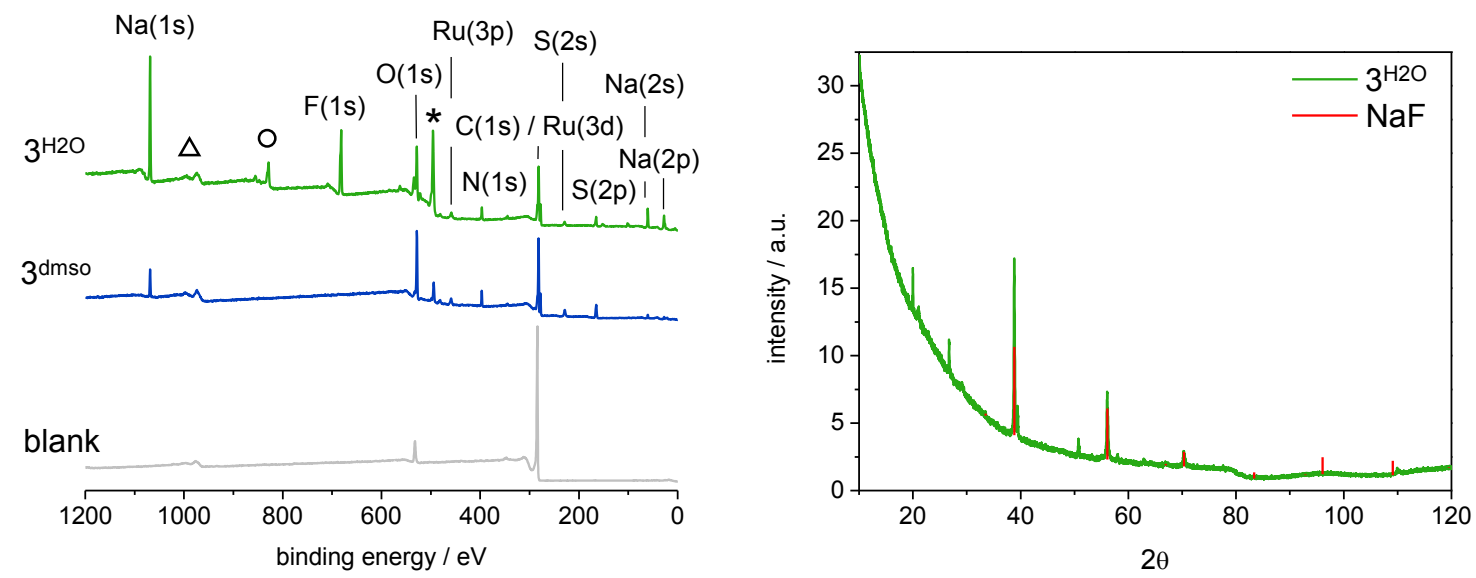

Figure 3.4: Analysis of the purity of $\mathbf{3}^{\mathbf{d m s o}}$ and $\mathbf{3}^{\mathbf{H 2 0}}$. Left: XPS spectra (wide) of $\mathbf{3}^{\mathrm{dmso}}$ (blue) and $\mathbf{3}^{\mathbf{H} 20}$ (green) dropcasted on a glassy carbon surface (bank: grey, pure glassy carbon surface). The asterisk, circle and triangle denote the Auger peaks of Na, F and O, respectively. Right: Powder diffractogram of the powder sample of $3^{\mathbf{H} 20}$ (green) at $298 \mathrm{~K}$ in comparison to that of $\mathrm{NaF}$ (red). The comparison shows that there is a significant amount of NaF in the powder sample of $\mathbf{3}^{\mathbf{H 2 0}}$. 
Unfortunately, no crystals suitable for X-ray diffraction could be obtained for complexes $\mathbf{3}^{\text {dmso }}$ and $3^{\mathrm{H2O}}$. However, in two cases crystalline material of slightly modified congeners could be received, which unambiguously confirm the $\left[\mathrm{L}^{1} \mathrm{Ru}_{2}\left(\mathrm{pySO}_{3}\right)_{4}\right]$ core of the complexes. Diffusion of acetone into an aqueous solution of $3^{\mathbf{0 2}} \mathbf{H}$ containing sodium hydroxide under aerobic conditions led to the incorporation of $\mathrm{CO}_{2}$ from air in the bimetallic cleft to form a carbonate bridge. The resulting complex

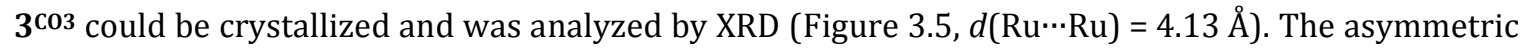
unit contains one molecule of the metal complex. Based on the symmetry $P-1$, the molecule forms a dimer through a sodium cation which bridges the $\mathrm{CO}_{3}{ }^{2-}$ moieties of two monomers (Figure 3.6, left). The $\mathrm{Na}^{+}$is further coordinated by the sulfonate groups of two axially coordinated $\mathrm{pySO}_{3}{ }^{-}$ligands (one of each metal complex), as well as two solvent water molecules (Figure 3.6, right). The ruthenium ions are coordinated by an $\left\{\mathrm{N}_{4} \mathrm{O}_{2}\right\}$ donor set in a close to ideal octahedral fashion as quantified by the very small $\operatorname{CSM} S\left(O_{\mathrm{h}}\right)$ values (Table 3.3). This result indirectly corroborates the presence of the $\left[\mathrm{L}^{1} \mathrm{Ru}_{2}\left(\mathrm{pySO}_{3}\right)_{4}\right]$ core in the aquo-bridged $\mathbf{3}^{\mathrm{H} 2 \mathrm{O}}$.

When acetone was diffused into a solution of $3^{\mathbf{4 2 0}}$ in $\mathrm{H}_{2} \mathrm{O}$ at neutral $\mathrm{pH}$, some crystals of a dimerized tetrametallic complex $\mathbf{4}$ were obtained. The molecular structure (Figure 3.7) shows that in $\mathbf{4}$ the sulfonate group of one of the axial pyridines is hosted in the diruthenium pocket of the second $\left[\mathrm{L}^{1} \mathrm{Ru}_{2}\left(\mathrm{pySO}_{3}\right)_{4}\right]$ unit, and vice versa. The asymmetric unit only contains a monomer of the structure $(d(\mathrm{Ru} \cdots \mathrm{Ru})=4.34 \AA)$. The dimer is generated through an inversion center and has $C_{\mathrm{i}}$-symmetry. Also here, the ruthenium ions are almost ideally octahedrally coordinated by an $\left\{\mathrm{N}_{4} \mathrm{O}_{2}\right\}$ donor set (Table 3.3). A careful check of the $\mathrm{SO}_{3}$-groups shows that the three which are not within the bimetallic cleft have two short S-O bonds (1.38-1.47 $\AA$ ) and one long S-O bond (1.47-1.56 $\mathrm{A}$ ). The long bond would correspond to $\mathrm{S}-\mathrm{OH}$; the two others to $\mathrm{S}=\mathrm{O}$. The final $\mathrm{SO}_{3}$ group is coordinated to the ruthenium ions via two $\mathrm{O}$-atoms ( $\mathrm{S}-\mathrm{O}$ distances of $1.47 \AA$ ) with one free $\mathrm{S}=\mathrm{O}$ (distance $1.43 \AA$ ). Thus, the structure could be refined as $\left[\mathrm{L}^{1} \mathrm{Ru}_{2}\left(\mathrm{pySO}_{3} \mathrm{H}\right)_{3}\left(\mathrm{pySO}_{3}\right)\right]_{2}$, which likely crystallized from water because it is charge neutral. This structure reflects the high tendency of the $\left[\mathrm{L}^{1} \mathrm{Ru}_{2}\left(\mathrm{pySO}_{3}\right)_{4}\right]$ core to accommodate exogenous entities in a bridging mode within the bimetallic cleft. It should be mentioned that no evidence for dimerization as observed for solid $\mathbf{4}$ is seen in the NMR spectra of $3^{\mathbf{0 2} \mathrm{H} 3}$ (note that $\mathbf{4}$ has reduced symmetry of the bimetallic core) or ESI mass spectra, suggesting that dimerization is not relevant in solution.

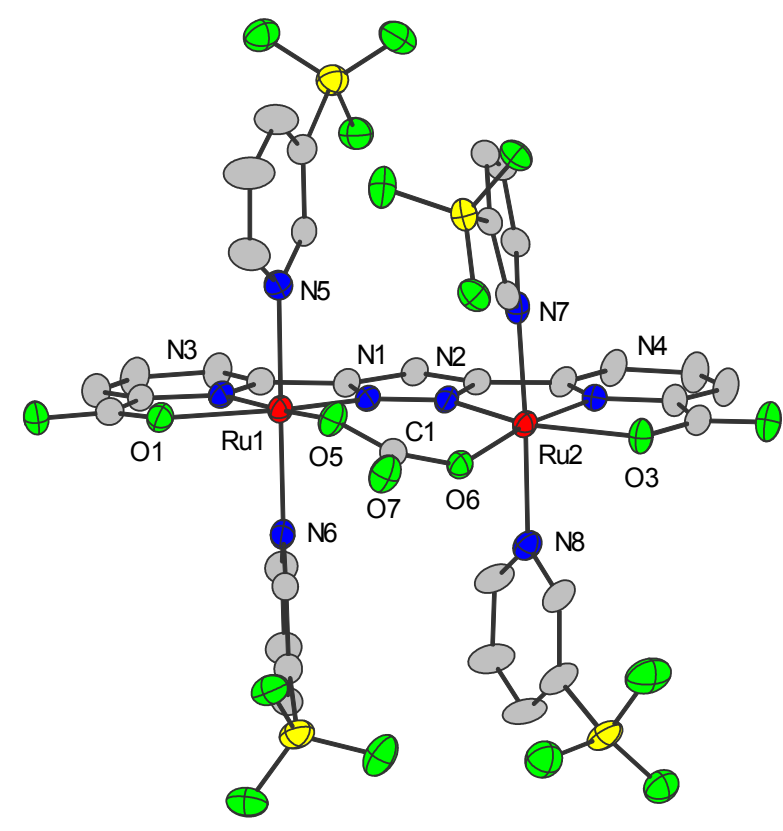

Figure 3.5: Molecular structure of the cationic core of $3^{\mathrm{co3}}$ drawn as thermal ellipsoids at the $30 \%$ probability level, respectively (ruthenium: red, nitrogen: blue, oxygen: green, sulfur: yellow, carbon: grey). All hydrogen atoms, solvent molecules and counter ions are omitted for clarity reasons. 

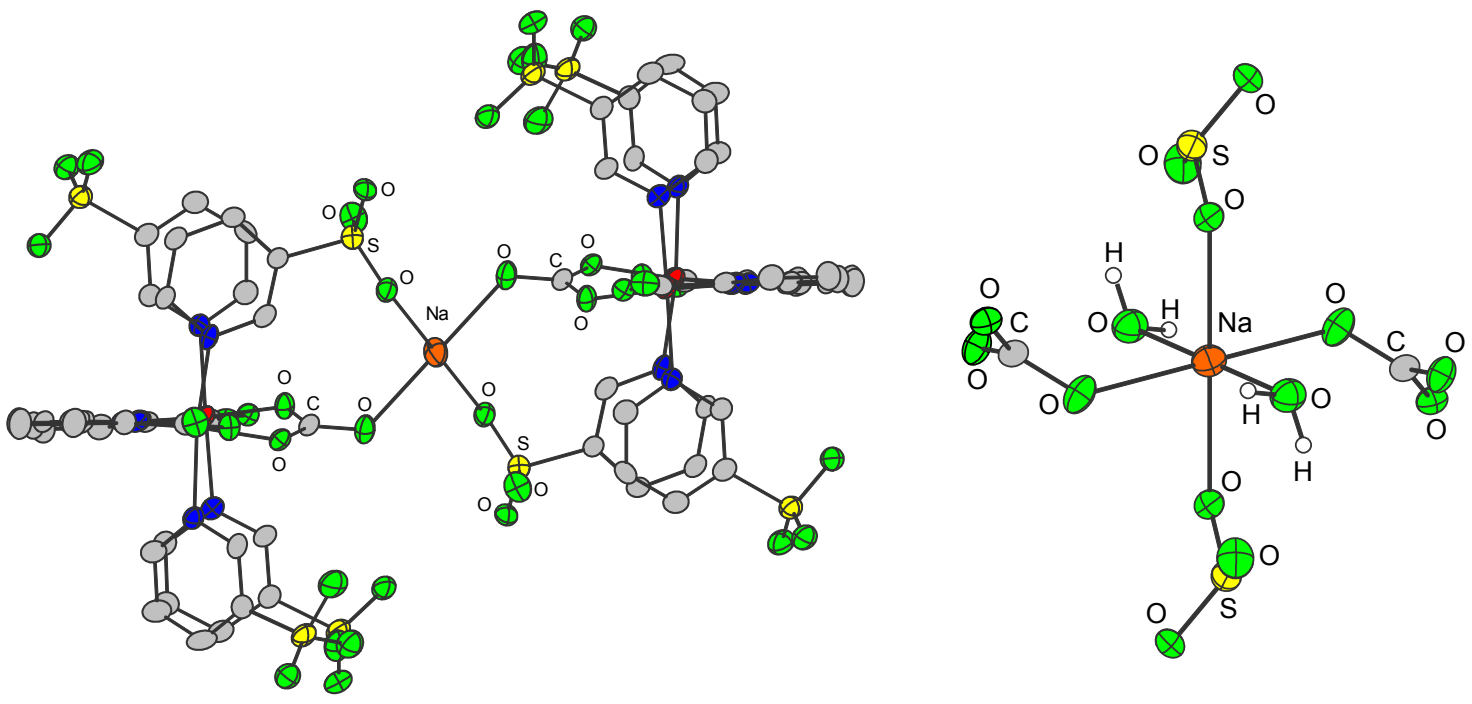

Figure 3.6: Additional views of the molecular structure of $3^{\mathrm{co3}}$ illustrating the dimerization via a sodium cation (left) and the full coordination environment of the $\mathrm{Na}^{+}$ion (right) made up of two $\mathrm{CO}_{3}{ }^{2-}$ moieties, two sulfonate groups from the axial pyridines, as well as two water molecules (ruthenium: red, nitrogen: blue, oxygen: green, sulfur: yellow, carbon: grey, hydrogen: white, sodium: orange).

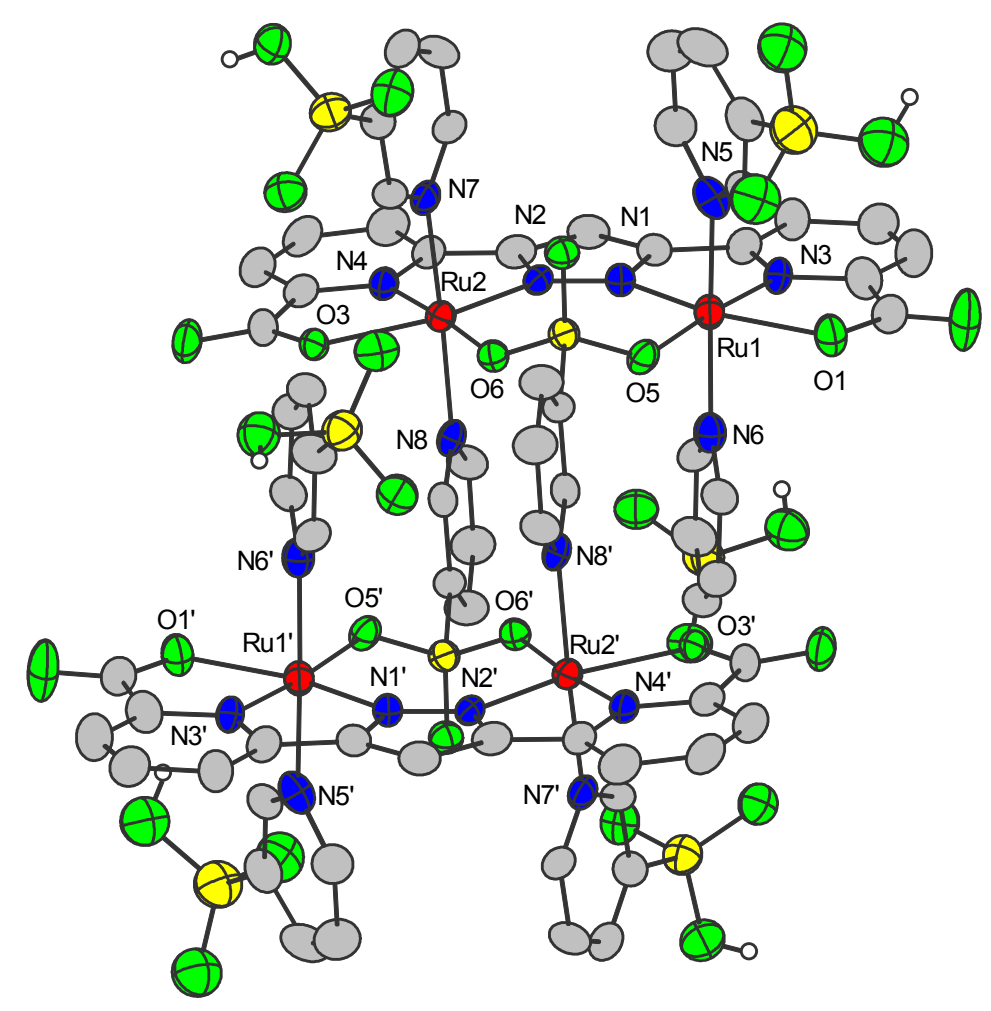

Figure 3.7: Molecular structure of 4 drawn as thermal ellipsoids at the $20 \%$ probability level, respectively (ruthenium: red, nitrogen: blue, oxygen: green, sulfur: yellow, carbon: grey, hydrogen: white). All hydrogen atoms (except those of the $\mathrm{SO}_{3} \mathrm{H}$ groups) and solvent molecules are omitted for clarity reasons. 
Table 3.3: Selected distances, torsion angles and Continuous Symmetry Measures (CSM) of 3co3 and 4.

\begin{tabular}{lll}
\hline distance $/ \AA$ & $\mathbf{3}^{\text {C03 }}$ & $\mathbf{4}$ \\
\hline \hline $\mathrm{Ru} \cdots \mathrm{Ru}$ & $4.1349(14)$ & $4.3364(14)$ \\
\hline${\text { torsion angle } /{ }^{\circ}}^{\mathrm{Ru}-\mathrm{N}-\mathrm{N}-\mathrm{Ru}}$ & & \\
$\mathrm{Ru}-\mathrm{O}-\mathrm{O}-\mathrm{Ru}$ & $7.7(9)$ & $-0.434(1458)$ \\
\hline $\mathrm{vi} \mathrm{CSM} S\left(O_{\mathrm{h}}\right)^{\mathrm{a}}$ & $35.004(301)$ & $2.539(437)$ \\
\hline $\mathrm{Ru}(1)$ & & \\
$\mathrm{Ru}(2)$ & 1.23 & 1.20 \\
\hline
\end{tabular}

a Continuous Symmetry Measures for octahedral geometry

\subsection{Electro- and Spectroelectrochemical Analysis ${ }^{\text {vii }}$}

The complexes $\mathbf{3}^{\text {dmso }}$ and $\mathbf{3}^{\mathbf{H 2 0}}$ were electrochemically characterized by means of cyclic voltammetry and square wave voltammetry in aqueous triflic acid at $\mathrm{pH} 1$. All potentials herein are given vs. the normal hydrogen electrode (NHE).

Complex $3^{\text {dmso }}$ exhibits one reversible redox process at $0.86 \mathrm{~V}$, which is $\mathrm{pH}$ independent, followed by a large irreversible wave associated with a catalytic process with $E_{\text {onset }}=1.38 \mathrm{~V}$ (Figure 3.8 , blue trace). The reversible redox wave is attributed to a two-electron process from $\mathrm{Ru}^{\mathrm{II}} \mathrm{Ru}^{\mathrm{II}}$ to $\mathrm{Ru}^{\mathrm{III}} \mathrm{Ru}^{\mathrm{III}}$ by comparing the UV/vis spectra of the one- and twofold chemically oxidized species with the species obtained by electrochemical oxidation at $\sim 1 \mathrm{~V}$, which will be discussed in more detail below. The observation of an electrocatalytic process in the cyclic voltammogram (CV) of $\mathbf{3}^{\text {dmso }}$ is somewhat surprising, as this complex is missing aquo ligands, which are required for the formation of high-valent $\mathrm{Ru}=\mathrm{O}$ moieties $-\mathrm{a}$ necessity for the $\mathrm{O}-\mathrm{O}$ bond formation step in water oxidation catalysis. If, however, the DMSO moiety in the in,in-position were to be substituted by aquo ligands as in $\mathbf{3}^{\mathbf{H} 20}$, thus establishing the foundation for water oxidation, a change in the CV would be expected.

In contrast to $\mathbf{3}^{\mathbf{d m s o}}$, the voltammogram of $\mathbf{3}^{\mathbf{H 2 0}}$ exhibits two $\mathrm{pH}$ dependent redox processes at 0.69 and $0.83 \mathrm{~V}$, corresponding to the oxidations from $\mathrm{Ru}^{I I} \mathrm{Ru}^{\mathrm{II}}$ to $\mathrm{Ru}^{\mathrm{II}} \mathrm{Ru}^{\mathrm{III}}$ and further to $\mathrm{Ru}^{\mathrm{III}} \mathrm{Ru}^{\mathrm{III}}$, respectively, followed by a catalytic process with $E_{\text {onset }}=1.36 \mathrm{~V}$ (Figure 3.8, green trace). Consequently, the lack of changes in the $\mathrm{CV}$ of $\mathbf{3}^{\mathbf{d m s o}}$ suggest that there is a more complicated situation regarding its catalytic species, which will be discussed thoroughly throughout this chapter.

The redox potentials of complexes $3^{\text {dmso }}$ and $3^{\mathbf{H} 20}$, as well as those of related dinuclear ruthenium complexes are compiled in Table 3.4. Interestingly, $\mathbf{3}^{\mathrm{dmso}}$ is the only complex that shows a two-electron oxidation, while similar complexes without water molecules as the bridging moiety (IOAc and IIOAc) exhibit two redox processes which are separated by $320-480 \mathrm{mV}\left(\Delta E_{1 / 2}\right)$. Furthermore, the redox potentials are significantly higher for these two complexes. A comparison between $3^{\mathbf{H} 20}$ and its parent complex $\mathbf{X}^{\mathbf{H 2 0}}$, measured under identical conditions, reveals a shift of 80 and $140 \mathrm{mV}$ to higher potential for $3^{\mathbf{H} 20}$ for the first and second oxidation, respectively, which could be associated with the electron withdrawing effect of the sulfonate moieties. The potential separation between the redox couples in $\mathbf{3}^{\mathbf{H 2 0}}\left(\Delta E_{1 / 2}=140 \mathrm{mV}\right)$ corresponds to a comproportionation constant $K_{\text {com }}$ of 233.

\footnotetext{
vi The geometry of the coordination environment was compared to the reference shape "octahedron (with central atom)".

vii This section was adapted from Jana Lücken, Carolina Gimbert-Suriñach, Jordi Benet-Buchholz, Antoni Llobet, Franc Meyer, "Diruthenium Complexes of a Pyrazolate/Carboxylate Hybrid Ligand for Chemically, Electro- and Photo-Driven Catalytic Water Oxidation”, under revision.
} 


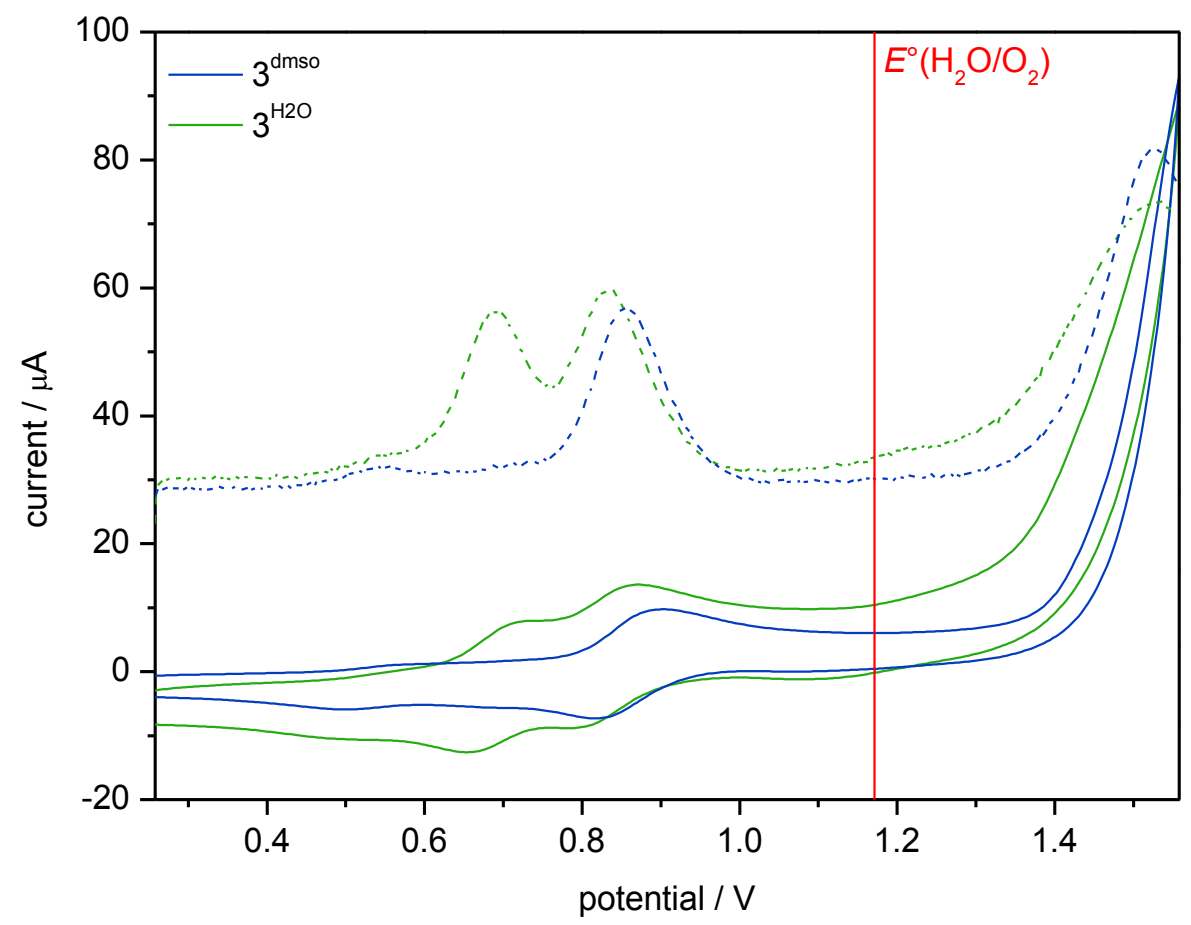

Figure 3.8: $\mathrm{CV}$ (solid, $100 \mathrm{mV} / \mathrm{s}$ ) and SWV (dashed) of a $1 \mathrm{mM}$ solution of $\mathbf{3}^{\text {dmso }}$ (blue) and $\mathbf{3}^{\mathrm{H} 20}$ (green) in $0.1 \mathrm{M}$ aqueous triflic acid ( $\mathrm{pH} 1$ ) using a glassy carbon disc working electrode, a Pt disc as auxiliary and an MSE as the reference electrode. The potentials were converted to the NHE scale by adding $0.658 \mathrm{~V}$. The red line marks the thermodynamic potential of water oxidation at the given $\mathrm{pH}$.

Table 3.4: Redox potentials of $3^{\mathrm{dmso}}$ and $\mathbf{3}^{\mathrm{H} 20}$ at $\mathrm{pH}$ 1, and comparison data for other relevant complexes.

\begin{tabular}{|c|c|c|c|c|c|}
\hline \multirow[t]{2}{*}{ complex } & \multirow[t]{2}{*}{ solvent $^{\mathrm{a}}$} & \multicolumn{2}{|c|}{$E_{1 / 2} / \mathrm{V} v s . \operatorname{NHE}\left(\Delta E_{\mathrm{p}} / \mathrm{mV}\right)^{\mathrm{b}}$} & \multirow[t]{2}{*}{$\Delta E_{1 / 2} / \mathrm{mV}^{\mathrm{c}}$} & \multirow[t]{2}{*}{ reference } \\
\hline & & II,III / II,II & III,III / II,III & & \\
\hline $3^{\text {dmso }}$ & pH 1 & \multicolumn{2}{|c|}{$0.86(73)^{d}$} & - & this work \\
\hline $3^{\mathrm{H} 2 \mathrm{O}}$ & pH 1 & $0.69(67)$ & $0.83(69)$ & 140 & this work \\
\hline $\mathrm{X}^{\mathrm{OAc}}$ & DCM & 0.45 & 0.87 & 420 & [204] \\
\hline $\mathrm{X}^{\text {Меон }}$ & DCM & 0.30 & 0.88 & 580 & [204] \\
\hline $\mathrm{X}^{\mathrm{H} 2 \mathrm{O}}$ & pH 1 & 0.61 & 0.72 & 110 & [204] \\
\hline VIOAc & pH 1 & 0.75 & 1.23 & 480 & [108] \\
\hline $\mathrm{VI}^{\mathrm{H} 2 \mathrm{O}}$ & pH 1 & 0.80 & 1.10 & 300 & [108] \\
\hline VoAc & DCM & 0.96 & 1.28 & 320 & [225] \\
\hline $\mathrm{V}^{\mathrm{H} 2 \mathrm{O}}$ & pH 1 & 0.82 & 0.88 & 60 & [225] \\
\hline
\end{tabular}

a DCM - dichloromethane, $\mathrm{pH} 1$ - aqueous triflic acid

b $\Delta E_{\mathrm{p}}$ - peak-to-peak separation of one redox process

c $\Delta E_{1 / 2}$ - separation between the two reversible redox processes

d two-electron process

On the basis of the electrochemical results, UV/vis-spectroelectrochemical (SEC) measurements were conducted. Next to the spectroscopic characterization of the electrochemically oxidized species of $3^{\mathrm{dmso}}$ and $3^{\mathbf{H} 20}$, the experiments provided information about the reversibility of the oxidations as well as helped distinguish the nature (one- or two-electron oxidation) of the redox process in the CV of $\mathbf{3}^{\text {dmso }}$. For the latter, $\mathbf{3}^{\text {dmso }}$ was both chemically and electrochemically oxidized whilst monitoring the $\mathrm{UV} /$ vis spectra. For the chemical oxidation, subsequently one and two equivalents of CAN were added, 
revealing the spectroscopic features of $3^{\text {dmso }}$ as a $\mathrm{Ru}^{\mathrm{II}} \mathrm{Ru}^{\mathrm{II}} / \mathrm{Ru}^{\mathrm{III}} \mathrm{Ru} \mathrm{u}^{\mathrm{III}}$ mixture (Figure 3.9, left, blue spectrum) and in its $\mathrm{Ru}^{\mathrm{III}} \mathrm{Ru}^{\mathrm{III}}$ (red spectrum) state, respectively. $3^{\text {dmso }}$ in its $\mathrm{Ru}^{\mathrm{II}} \mathrm{Ru}^{\mathrm{II}}$ state (black spectrum) possesses local absorption maxima at $275\left(\varepsilon=24100 \mathrm{~L} \cdot \mathrm{mol}^{-1} \cdot \mathrm{cm}^{-1}\right)$ and $346 \mathrm{~nm}(27600)$, as well as a shoulder at $423 \mathrm{~nm}$ (21000), the latter two of which are likely metal-ligand charge transfer (MLCT) bands. These MLCT transitions are partially $\left(\mathrm{Ru}^{\mathrm{II}} \mathrm{Ru}^{\mathrm{II}} / \mathrm{Ru}^{\mathrm{III}} \mathrm{Ru}^{\mathrm{III}}\right.$ mixture) or fully $\left(\mathrm{Ru}^{\mathrm{III}} \mathrm{Ru}^{\mathrm{III}}\right)$ vanished in the oxidized species. In addition to this, the absorbance at $275 \mathrm{~nm}$ gradually increases and a new band forms at $537 \mathrm{~nm}$ upon oxidation. Another significant difference between the spectrum of the $\mathrm{Ru}^{\mathrm{III}} \mathrm{Ru}^{\mathrm{III}}$ species of $\mathbf{3}^{\mathrm{dmso}}$ and the other two is the initial dip at $300 \mathrm{~nm}$, which turns into a new peak upon twofold oxidation. The final UV/vis spectrum obtained upon electrochemical oxidation at $\sim 1 \mathrm{~V}$ (Figure 3.9, right, red spectrum) clearly shows the same spectroscopic features as the chemically oxidized $\mathrm{Ru}^{\mathrm{III}} \mathrm{Ru}^{\mathrm{III}}$ species, thus demonstrating that the reversible redox process in the voltammogram of $3^{\text {dmso }}$ is the two-electron oxidation from $\mathrm{Ru}^{\mathrm{II}} \mathrm{Ru}^{\mathrm{II}}$ to $\mathrm{Ru}^{\mathrm{III}} \mathrm{Ru}^{\mathrm{III}}$.
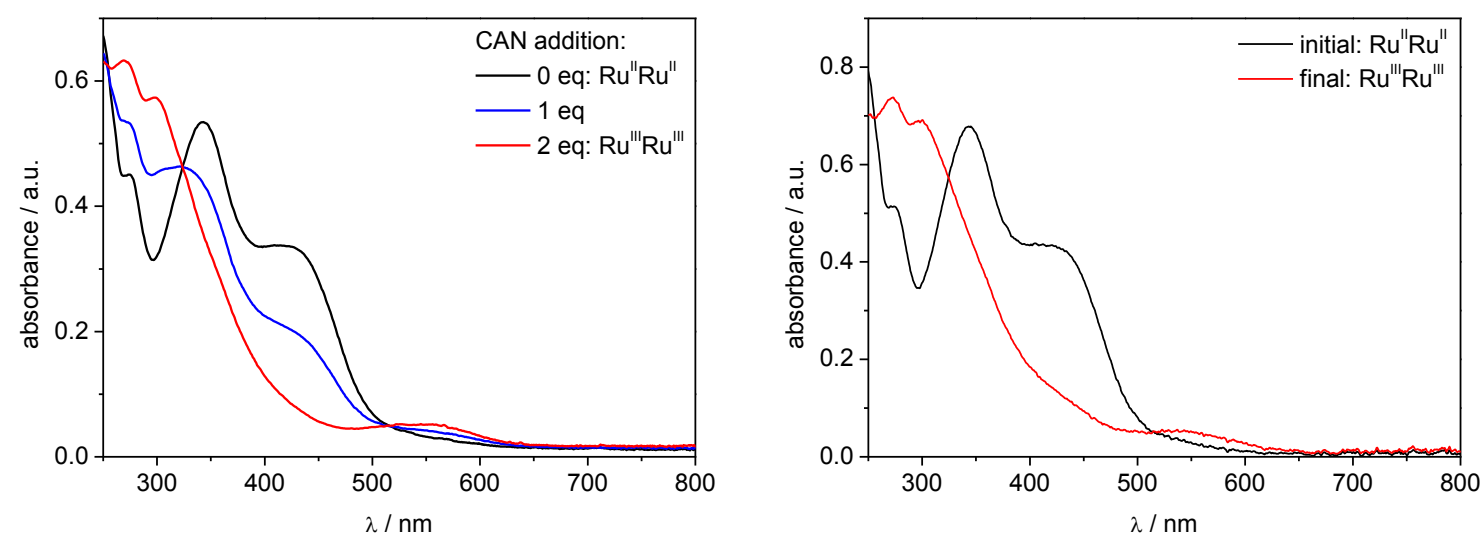

Figure 3.9: Comparison of the UV/vis spectra of $3^{\mathrm{dmso}}$ after chemical and electrochemical oxidation in $0.1 \mathrm{M}$ aqueous triflic acid (pH 1). Left: Chemical oxidation with 1 eq (blue) and 2 eq (red) of CAN leading from the $\mathrm{Ru}^{\mathrm{II}} \mathrm{Ru}^{\mathrm{II}}$ state (black) to a $\mathrm{Ru}^{\text {II }} \mathrm{Ru}^{\mathrm{II}} / \mathrm{Ru}^{\mathrm{III}} \mathrm{Ru}^{\mathrm{III}}$ mixture (blue) and $\mathrm{Ru}^{\mathrm{III}} \mathrm{Ru}{ }^{\mathrm{III}}$ (red), respectively. Right: UV/vis spectra before (black) and after (red) the electrochemical oxidation at $1.06 \mathrm{~V}$. The comparison of the two graphs shows that the redox wave in the $\mathrm{CV}$ is a two-electron process going directly from $\mathrm{Ru}^{\mathrm{II}} \mathrm{Ru}^{\mathrm{II}}$ to $\mathrm{Ru}^{\mathrm{III}} \mathrm{Ru}^{\mathrm{III}}$.

A measure of the reversibility of the electrochemical processes is given by the difference between the anodic and cathodic peak potentials $\Delta E_{\mathrm{p}}$. For an electrochemically reversible redox process, a theoretical $\Delta E_{\mathrm{p}}$ of $59 \mathrm{mV} / n$ ( $n$ is the number of transferred electrons) at $25^{\circ} \mathrm{C}$ is expected according to the Nernst equation. For a performed measurement, however, the $\Delta E_{\mathrm{p}}$ value of a reversible process is generally larger due to uncompensated solution resistance and non-linear diffusion. ${ }^{250]}$ With peak-topeak separations of $73 \mathrm{mV}$ for the two-electron oxidation of $3^{\mathrm{dmso}}$, as well as $67 \mathrm{and} 69 \mathrm{mV}$ for the first and second oxidation of $3^{\mathbf{H 2 0}}$, respectively, these electrochemical processes can be considered reversible (Table 3.4). Additional support for the reversibility of these oxidations is given by UV/visSEC measurements. In these experiments, the potential was increased from 0.36 to $1.06 \mathrm{~V}$, corresponding to the oxidation from $\mathrm{Ru}^{\mathrm{II}} \mathrm{Ru}^{\mathrm{II}}$ to $\mathrm{Ru}^{\mathrm{III}} \mathrm{Ru}^{\mathrm{III}}$, whilst monitoring the $\mathrm{UV} / \mathrm{vis}$ spectra, and then reversed. Specifically of interest is the comparison of the first and last UV/vis spectrum of this series, as a full recovery of the initial spectroscopic features shows that the electrochemical process is reversible. Upon re-reduction (Figure 3.10, right) of $3^{\mathbf{d m s o}}$ at $0.36 \mathrm{~V}$, the MLCT bands at 346 and $423 \mathrm{~nm}$ reappear, while the newly formed transitions at 300 and $537 \mathrm{~nm}$ disappear, thus reproducing the initial spectrum. This becomes obvious as the spectrum before oxidation (dashed black) nearly perfectly overlaps with the one after re-reduction (blue). The $\Delta$ absorbance traces with respect to the spectrum before oxidation (Figure 3.10, bottom) emphasize the $\lambda$-dependent intensity loss and gain upon oxidation, as well as the reversibility of the electrochemical process, as the trace after rereduction (blue) more or less coincides with the baseline. 

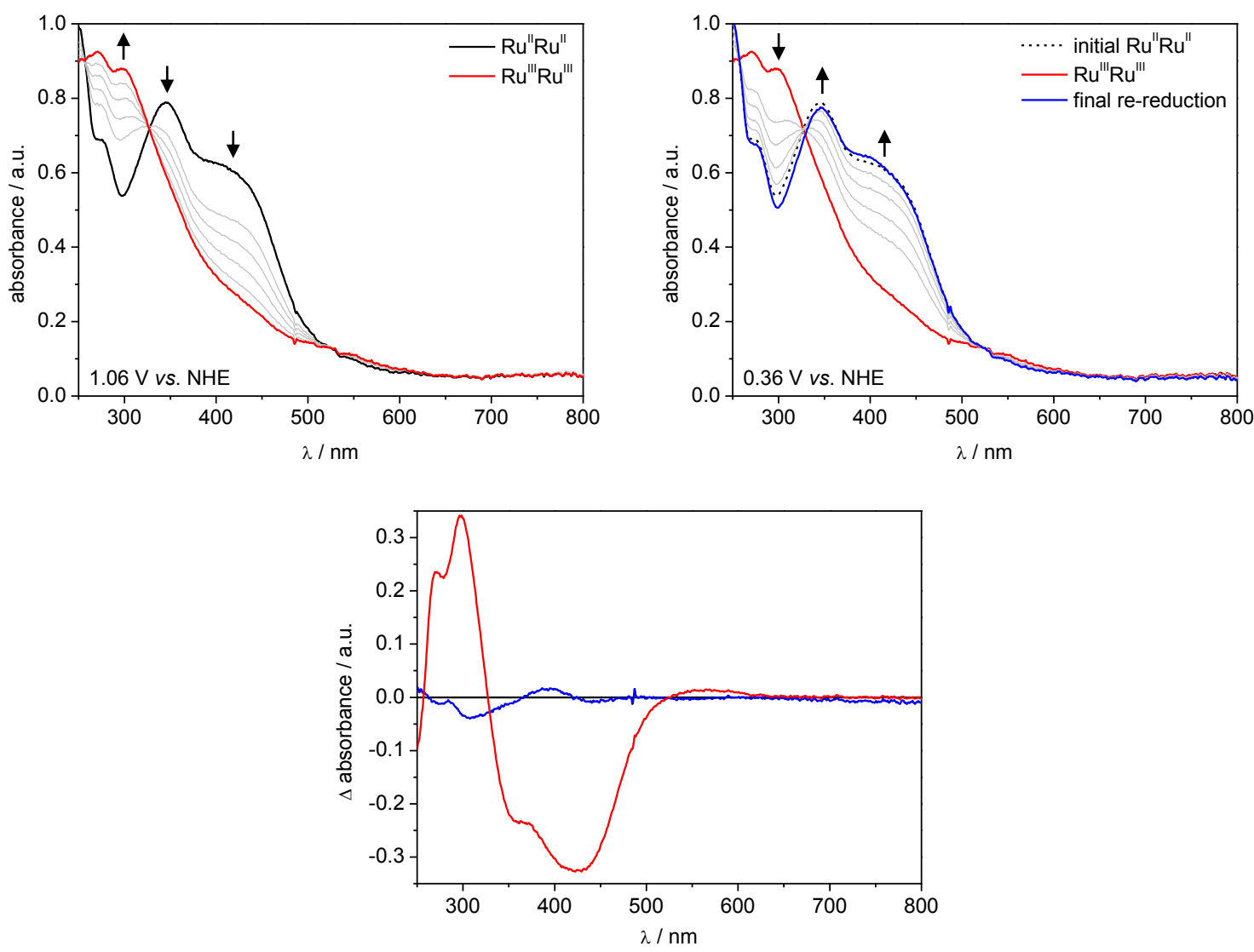

Figure 3.10: UV/vis-spectroelectrochemical analysis of $3^{\mathrm{dmso}}$ in $0.1 \mathrm{M}$ triflic acid (pH 1) (c $\left.=2.86 \cdot 10^{-4} \mathrm{M}\right)$. Top left: $\mathrm{UV} /$ vis spectra of the electrochemical oxidation from $\mathrm{Ru}^{\mathrm{II}} \mathrm{Ru}^{\mathrm{II}}$ to $\mathrm{Ru}^{\mathrm{III}} \mathrm{Ru}^{\mathrm{III}}$ at $1.06 \mathrm{~V}$ vs. NHE (black: beginning, red: end). Top right: UV/vis spectra of the re-reduction from $\mathrm{Ru}^{\mathrm{III}} \mathrm{Ru}^{\mathrm{III}}$ to $\mathrm{Ru}^{\mathrm{II}} \mathrm{Ru}^{\mathrm{II}}$ at $0.36 \mathrm{~V}$ vs. NHE (red: beginning, blue: end, black dashed: initial UV/vis spectrum before oxidation). Bottom: $\Delta$ absorbance of the spectra after oxidation (red) and re-reduction (blue) with respect to the spectrum before oxidation (baseline).

Complex $3^{\mathrm{H} 20}\left(\mathrm{Ru}^{\mathrm{II}} \mathrm{Ru} \mathrm{II}^{\mathrm{II}}\right)$ possesses local absorption maxima at $282\left(\varepsilon=23200 \mathrm{~L} \cdot \mathrm{mol}^{-1} \cdot \mathrm{cm}^{-1}\right)$ and $393 \mathrm{~nm}$ (22800), as well as a shoulder at $352 \mathrm{~nm}$ (19300) (Figure 3.11, left, black spectrum). Upon oxidation to $\mathrm{Ru}^{\mathrm{III}} \mathrm{Ru}^{\mathrm{III}}$ at $1.06 \mathrm{~V}$ (Figure 3.11, left), these transitions disappear, and new peaks arise at 274 and $294 \mathrm{~nm}$ with a shoulder at $417 \mathrm{~nm}$. Upon re-reduction to $\mathrm{Ru}^{\mathrm{II}} \mathrm{Ru}^{\mathrm{II}}$ at $0.36 \mathrm{~V}$ (Figure 3.11, right), the MLCT bands at 352 and $393 \mathrm{~nm}$ regain intensity and the newly formed peaks disappear. The final spectrum after re-reduction (blue) nicely overlaps with the spectrum before oxidation (dashed black). Once more, the reversibility of the electrochemical process is emphasized by the $\Delta$ absorbance trace (Figure 3.11, bottom). It is interesting to note that the UV/vis spectra of $3^{\mathrm{dmso}}$ and $\mathbf{3}^{\mathrm{H} 2 \mathrm{O}}$ in their $\mathrm{Ru}^{\mathrm{II}} \mathrm{Ru}^{\mathrm{II}}$ state significantly differ from one another. This circumstance was of great use for further studies as will be discussed in Section 3.5.4. 

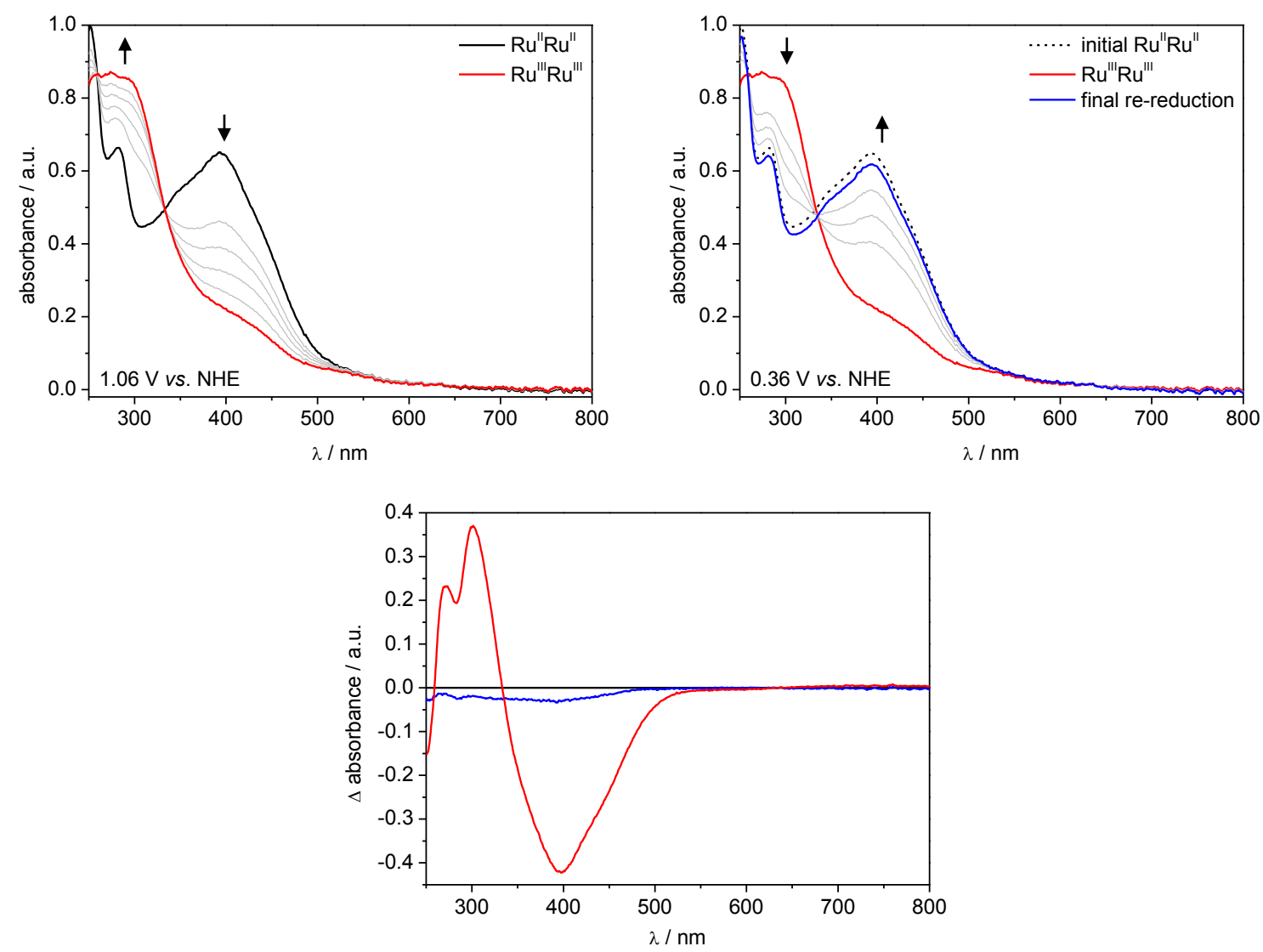

Figure 3.11: UV/vis-spectroelectrochemical analysis of $\mathbf{3}^{\mathbf{H} 20}$ in $0.1 \mathrm{M}$ triflic acid (pH 1) (c $\left.=2.86 \cdot 10^{-4} \mathrm{M}\right)$. Top left: $\mathrm{UV} /$ vis spectra of the electrochemical oxidation from $\mathrm{Ru}^{\mathrm{II}} \mathrm{Ru}^{\mathrm{II}}$ to $\mathrm{Ru}^{\mathrm{III}} \mathrm{Ru}^{\mathrm{III}}$ at $1.06 \mathrm{~V} v s$. NHE (black: beginning, red: end). Top right: UV/vis spectra of the re-reduction from $\mathrm{Ru}^{\mathrm{III}} \mathrm{Ru}^{\mathrm{III}}$ to $\mathrm{Ru}^{\mathrm{II}} \mathrm{Ru}^{\mathrm{II}}$ at $0.36 \mathrm{~V} v$ s. NHE (red: beginning, blue: end, black dashed: initial UV/vis spectrum before oxidation). Bottom: $\Delta$ absorbance of the spectra after oxidation (red) and re-reduction (blue) with respect to the spectrum before oxidation (baseline).

\subsection{Pourbaix Diagram of $3^{\text {H2Oviii }}$}

In water oxidation catalysis, multiple PCET processes by metal-aquo groups $\left(\mathrm{M}-\mathrm{OH}_{2}\right)$ are required before the $0-0$ bond is formed, and dioxygen is released (see Section 1.3.3). The potentials of these redox processes are dependent on the $\mathrm{pH}$ value. This $\mathrm{pH}$ dependency can be quantified based on the Nernst equation. For a PCET process in which $m$ protons and $n$ electrons are transferred (eq 3.1), the Nernst equation (at $298 \mathrm{~K}$ ) can be rewritten as shown in equation 3.2. Next to a pH independent part ( $E_{\text {const }}$ ), containing $E^{0}$ and the concentration of the reduced and oxidized species, the equation shows that the redox potentials $\left(E_{1 / 2}\right)$ decrease by $\frac{m}{n} 0.059 \mathrm{~V}$ per $\mathrm{pH}$ unit.

$$
\begin{gathered}
{[\mathrm{Ox}]+m \mathrm{H}^{+}+n \mathrm{e}^{-} \rightarrow[\text { Red }]} \\
E_{1 / 2}=\underbrace{E^{0}-\frac{0.059 \mathrm{~V}}{n} \lg \frac{[\text { Red }]}{[\mathrm{Ox}]}}_{E_{\text {const }}}-\frac{m}{n} \cdot 0.059 \mathrm{~V} \cdot \mathrm{pH}
\end{gathered}
$$

viii Parts of this section were adapted from Jana Lücken, Carolina Gimbert-Suriñach, Jordi BenetBuchholz, Antoni Llobet, Franc Meyer, "Diruthenium Complexes of a Pyrazolate/Carboxylate Hybrid Ligand for Chemically, Electro- and Photo-Driven Catalytic Water Oxidation", under revision. 
A Pourbaix diagram, also referred to as a potential/pH diagram, visualizes the $\mathrm{pH}$ dependency of redox processes. More precisely, a pure electron transfer $\left(n \mathrm{e}^{-} / 0 \mathrm{H}^{+}\right)$possesses a slope of 0 , whereas PCET processes of the type $n \mathrm{e}^{-} / n \mathrm{H}^{+}$or $n \mathrm{e}^{-} / 2 n \mathrm{H}^{+}$exhibit slopes of $-59 \mathrm{mV} / \mathrm{pH}$ or $-118 \mathrm{mV} / \mathrm{pH}$, respectively. Concomitantly, the Pourbaix diagram allows one to distinguish the proton content of each species and derive $\mathrm{p} K_{\mathrm{a}}$ values.

For the generation of a Pourbaix diagram for $3^{\mathbf{H} 20}$ (Figure 3.12), electrochemical measurements were conducted in the $\mathrm{pH}$ range of 1-8. To precisely determine the redox potentials, both cyclic voltammograms and differential pulse voltammograms (DPVs) were recorded. The experiments were performed in an aqueous $\mathrm{HBF}_{4}$ solution as it does not contain any coordinating anions in contrast to triflic acid and phosphate buffer. However, this implies that the solution is not buffered, which could be problematic as during water oxidation catalysis protons are released; and so, the $\mathrm{pH}$ value was monitored before and after each measurement. The $\mathrm{pH}$ value was adjusted by the addition of small amounts of an aqueous $\mathrm{NaOH}$ solution. To ensure that the complex concentration remained almost the same throughout all experiments, a highly concentrated $\mathrm{NaOH}$ solution was used.

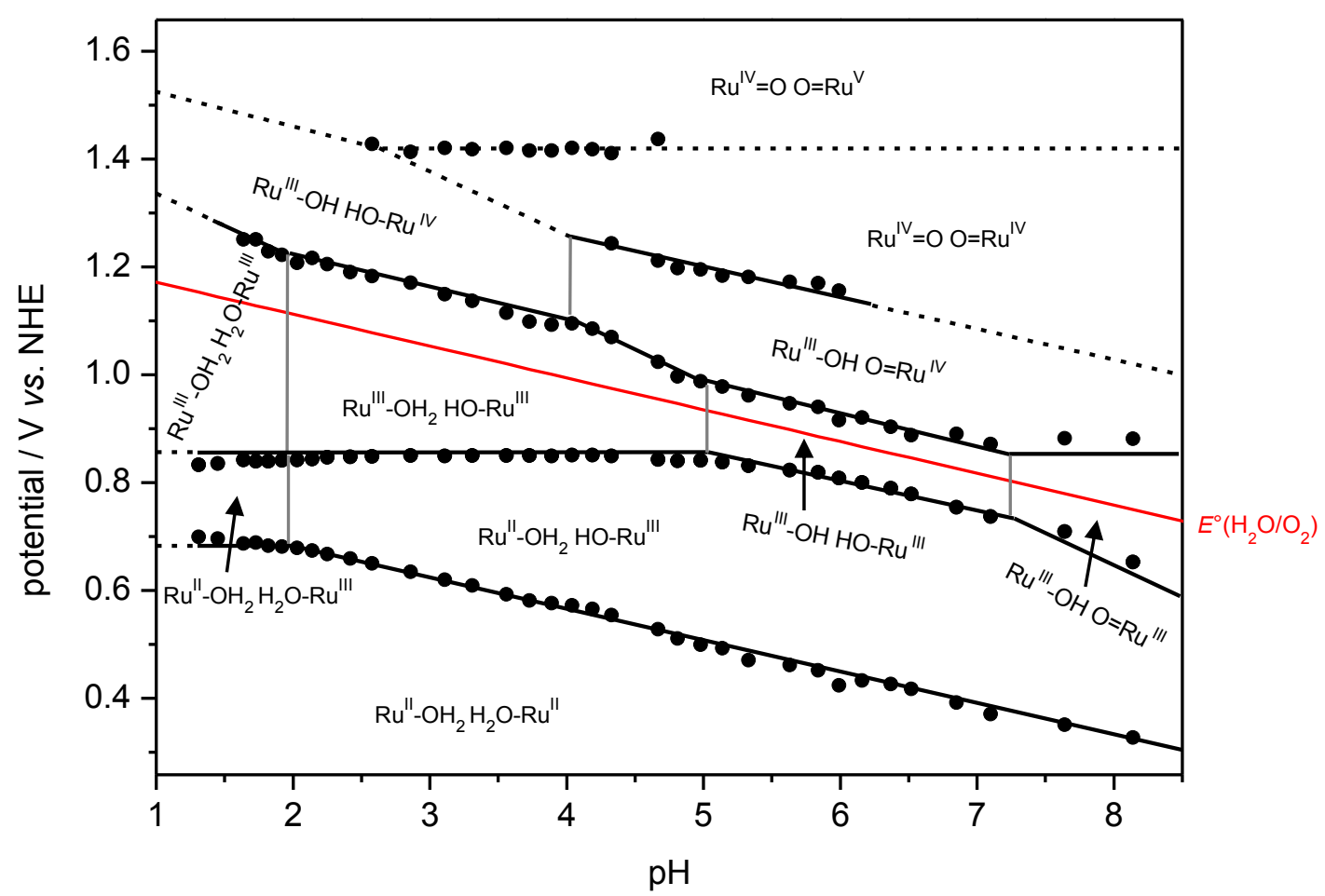

Figure 3.12. Pourbaix diagram of $\mathbf{3}^{\mathbf{H} 20}$ in a $\mathrm{pH}$ range of $1-8$ in aqueous $\mathrm{HBF}_{4}$. The red line indicates the thermodynamic potential of the water oxidation reaction. Dashed lines are drawn when the processes could not be observed in the electrochemical measurements or not unambiguously assigned. Vertical lines mark p Ka values. The $\mathrm{pH}$ was adjusted by the addition of aqueous $\mathrm{NaOH}$ to $3^{\mathrm{H} 20}$ in an aqueous $\mathrm{HBF}_{4}$ solution.

A series of CVs and DPVs of $\mathbf{3}^{\mathrm{H} 20}$ at various $\mathrm{pH}$ values is displayed in Figure 3.13. Whereas certain redox processes can easily be observed, others are barely visible as shoulders, hinting at the difficulty of generating a complete Pourbaix diagram. The redox potentials were determined both from the CV (half-wave potential $E_{1 / 2}$ ) and DPV (peak potential $E_{\mathrm{p}}$ ); at most, the first three redox processes were clearly visible in the CV. A list of the potentials of all redox processes used to compile the Pourbaix diagram is given in the appendix (Table A.23). All redox potentials that could be determined from both voltammograms were averaged. With this data, a potential/pH-diagram could be constructed. 

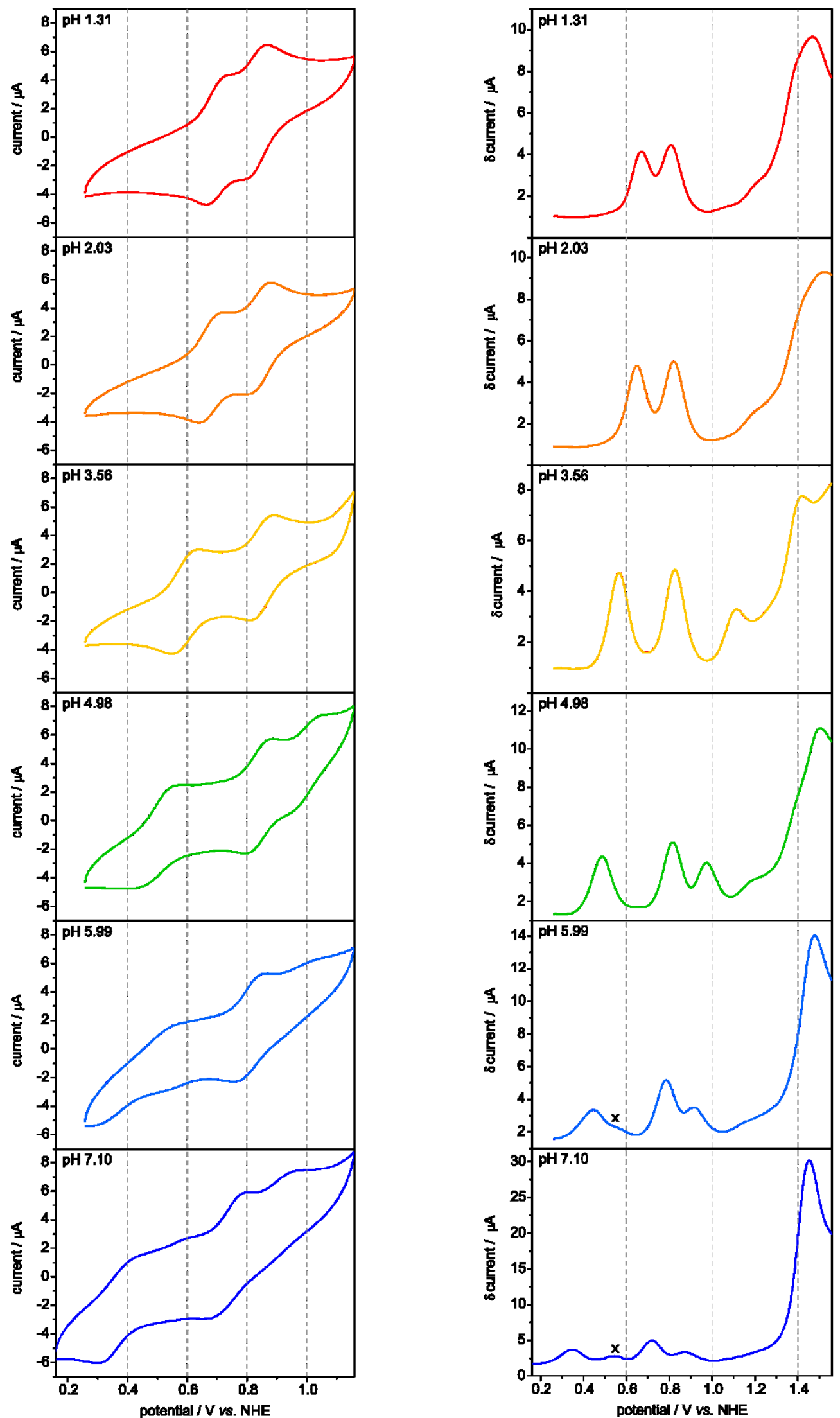

Figure 3.13: Electrochemical measurements of $3^{\mathrm{H} 20}$ in an aqueous $\mathrm{HBF}_{4}$ solution at different $\mathrm{pH}$ values. The $\mathrm{pH}$ was adjusted by addition of an aqueous $\mathrm{NaOH}$ solution. Left: $\mathrm{CVs}$ at $100 \mathrm{mV} / \mathrm{s}$ in a potential range of $0.16-1.16 \mathrm{~V}$. Right: DPVs in a potential range of $0.16-1.56 \mathrm{~V}$. The $\mathrm{x}$ marks an impurity that appeared at elevated pH. A glassy carbon disc was used as the working electrode, a Pt disc as auxiliary and an MSE as the reference electrode. The potentials were converted to the NHE scale by adding $0.658 \mathrm{~V}$. 
The thorough analysis of the PCET processes allowed the assignment of the proton content of each species in order to complete the Pourbaix diagram (Figure 3.12). Overall, the potentials of the first three redox processes (from $\mathrm{Ru}^{\mathrm{II}} \mathrm{Ru}^{\mathrm{II}}$ to $\mathrm{Ru}^{\mathrm{III}} \mathrm{Ru}^{\mathrm{IV}}$ ) could be determined in quite good accuracy, whereas at higher potentials the Pourbaix diagram becomes less precise (indicated by the dashed lines). Nevertheless, it supports the presence of two aquo-type ligands in $\mathbf{3}^{\mathbf{H 2 0}}$. Above this, it suggests that $\left[\mathrm{Ru}^{\mathrm{IV}}=\mathrm{O} O=\mathrm{Ru}^{\mathrm{V}}\right]^{2-}\left(\mathrm{L}^{3-}\right.$ and $\mathrm{pySO}_{3}{ }^{-}$ligands are omitted for clarity reasons) is the catalytically active species in which the $\mathrm{O}-\mathrm{O}$ bond is formed, and dioxygen is released.

In the Pourbaix diagram, each horizontal line represents an electron transfer and each vertical line a proton transfer, thus revealing the $\mathrm{p} K_{\mathrm{a}}$ values of certain species (Table 3.5). Surprisingly, the $\mathrm{p} K_{\mathrm{a}}$ values of $\left[\mathrm{Ru}^{\mathrm{II}}-\mathrm{OH}_{2} \mathrm{H}_{2} \mathrm{O}-\mathrm{Ru}^{\mathrm{IIII}}\right]^{2-}$ and $\left[\mathrm{Ru}^{\mathrm{III}}-\mathrm{OH}_{2} \mathrm{H}_{2} \mathrm{O}-\mathrm{Ru}^{\mathrm{III}}\right]-$ are the same, which is very unexpected and cannot be explained at this point. The redox potential of the first oxidation process from $\mathrm{Ru}^{\mathrm{II}} \mathrm{Ru}^{\mathrm{II}}$ to $\mathrm{Ru}^{\mathrm{II}} \mathrm{Ru}^{\mathrm{III}}$ remains unchanged until $\mathrm{pH} 2$ after which it decreases steadily with a slope of $\sim 60 \mathrm{mV}$, supporting a $1 \mathrm{e}^{-} / 1 \mathrm{H}^{+}$PCET process. Accordingly, the redox process can be phrased as (all scaffold ligands are omitted for clarity reasons):

$$
\begin{aligned}
& {\left[\mathrm{Ru}^{\mathrm{II}-}-\mathrm{OH}_{2} \mathrm{H}_{2} \mathrm{O}-\mathrm{Ru}^{\mathrm{IIII}}\right]^{2-}+\mathrm{e}^{-} \rightarrow\left[\mathrm{Ru}^{\mathrm{II}}-\mathrm{OH}_{2} \mathrm{H}_{2} \mathrm{O}-\mathrm{Ru}^{\mathrm{II}}\right]^{3-} \text { from } \mathrm{pH} 1-2} \\
& {\left[\mathrm{Ru}^{\mathrm{II}-\mathrm{OH}_{2}} \mathrm{HO}-\mathrm{Ru}^{\mathrm{III}}\right]^{3-}+\mathrm{e}^{-}+\mathrm{H}^{+} \rightarrow\left[\mathrm{Ru}^{\mathrm{II}}-\mathrm{OH}_{2} \mathrm{H}_{2} \mathrm{O}-\mathrm{Ru}^{\mathrm{II}}\right]^{3-} \text { from } \mathrm{pH} 2-8.1}
\end{aligned}
$$

The second oxidation ( $\mathrm{Ru}^{\text {II }} \mathrm{Ru}^{\text {III }}$ to $\left.\mathrm{Ru}^{\mathrm{III}} \mathrm{Ru}^{\mathrm{III}}\right)$ features three different slopes in the examined $\mathrm{pH}$ range, corresponding to a pure electron transfer, as well as $1 \mathrm{e}^{-} / 1 \mathrm{H}^{+}$and $1 \mathrm{e}^{-} / 2 \mathrm{H}^{+}$PCET process. As there are two different initial species, four processes are observed:

$$
\begin{aligned}
& {\left[\mathrm{Ru}^{\mathrm{III}}-\mathrm{OH}_{2} \mathrm{H}_{2} \mathrm{O}-\mathrm{Ru}^{\mathrm{III}}\right]^{-}+\mathrm{e}^{-} \rightarrow\left[\mathrm{Ru}^{\mathrm{II}}-\mathrm{OH}_{2} \mathrm{H}_{2} \mathrm{O}-\mathrm{Ru}^{\mathrm{III}}\right]^{2-} \text { from } \mathrm{pH} 1-2} \\
& {\left[\mathrm{Ru}^{\mathrm{III}}-\mathrm{OH}_{2} \mathrm{HO}-\mathrm{Ru}^{\mathrm{III}}\right]^{2-}+\mathrm{e}^{-} \rightarrow\left[\mathrm{Ru}^{\mathrm{II}}-\mathrm{OH}_{2} \mathrm{HO}-\mathrm{Ru}^{\mathrm{III}}\right]^{3-} \text { from } \mathrm{pH} 2-5} \\
& {\left[\mathrm{Ru}^{\mathrm{III}}-\mathrm{OH} \mathrm{HO}-\mathrm{Ru}^{\mathrm{III}}\right]^{3-}+\mathrm{e}^{-}+\mathrm{H}^{+} \rightarrow\left[\mathrm{Ru}^{\mathrm{II}}-\mathrm{OH}_{2} \mathrm{HO}-\mathrm{Ru}^{\mathrm{III}}\right]^{3-} \text { from } \mathrm{pH} \text { 5-7.2 }} \\
& {\left[\mathrm{Ru}^{\mathrm{III}}-\mathrm{OH} \mathrm{O}=\mathrm{Ru}^{\mathrm{III}}\right]^{4-}+\mathrm{e}^{-}+2 \mathrm{H}^{+} \rightarrow\left[\mathrm{Ru}^{\mathrm{II}}-\mathrm{OH}_{2} \mathrm{HO}-\mathrm{Ru}^{\mathrm{III}}\right]^{3-} \text { from } \mathrm{pH} 7.2-8.1}
\end{aligned}
$$

Finally, the third process can be split up into five separate sections in which the four initial species $\left(\mathrm{Ru}^{\mathrm{III}} \mathrm{Ru}^{\mathrm{III}}\right)$ with different proton content convert to two species in the oxidation state $\mathrm{Ru}^{\mathrm{III}} \mathrm{Ru}^{\mathrm{IV}}$ :

$$
\begin{aligned}
& {\left[\mathrm{Ru}^{\mathrm{III}}-\mathrm{OH} \mathrm{HO}-\mathrm{Ru}^{\mathrm{IV}}\right]^{2-}+\mathrm{e}^{-}+2 \mathrm{H}^{+} \rightarrow\left[\mathrm{Ru}^{\mathrm{III}}-\mathrm{OH}_{2} \mathrm{H}_{2} \mathrm{O}-\mathrm{Ru}^{\mathrm{III}}\right]^{-} \text {from } \mathrm{pH} \text { 1-2 }} \\
& {\left[\mathrm{Ru}^{\mathrm{III}}-\mathrm{OH} \mathrm{HO}-\mathrm{Ru}^{\mathrm{IV}}\right]^{2-}+\mathrm{e}^{-}+\mathrm{H}^{+} \rightarrow\left[\mathrm{Ru}^{\mathrm{III}}-\mathrm{OH}_{2} \mathrm{HO}-\mathrm{Ru}^{\mathrm{IIII}}\right]^{2-} \text { from } \mathrm{pH} 2-4} \\
& {\left[\mathrm{Ru}^{\mathrm{III}}-\mathrm{OH} \mathrm{O}=\mathrm{Ru}^{\mathrm{IV}}\right]^{3-}+\mathrm{e}^{-}+2 \mathrm{H}^{+} \rightarrow\left[\mathrm{Ru}^{\mathrm{III}}-\mathrm{OH}_{2} \mathrm{HO}-\mathrm{Ru}^{\mathrm{IIII}}\right]^{2-} \text { from } \mathrm{pH} 4-5} \\
& {\left[\mathrm{Ru}^{\mathrm{III}}-\mathrm{OH} \mathrm{O}=\mathrm{Ru}^{\mathrm{IV}}\right]^{3-}+\mathrm{e}^{-}+\mathrm{H}^{+} \rightarrow\left[\mathrm{Ru}^{\mathrm{III}}-\mathrm{OH} \text { HO- } \mathrm{Ru}^{\mathrm{III}}\right]^{3-} \text { from } \mathrm{pH} \text { 5-7.2 }} \\
& {\left[\mathrm{Ru}^{\mathrm{III}}-\mathrm{OH} \mathrm{O}=\mathrm{Ru}^{\mathrm{IV}}\right]^{3-}+\mathrm{e}^{-} \rightarrow\left[\mathrm{Ru}^{\mathrm{III}}-\mathrm{OH} \mathrm{O}=\mathrm{Ru}^{\mathrm{III}}\right]^{4-} \text { from } \mathrm{pH} \text { 7.2-8.1 }}
\end{aligned}
$$

Subsequent oxidation processes could not be easily distinguished from the electrochemical measurements and will thus not be discussed in detail. They were largely assigned through the overall picture of the Pourbaix diagram and cannot be considered completely accurate. Nevertheless, from pH 4-6 a $1 \mathrm{e}^{-} / 1 \mathrm{H}^{+}$PCET process could quite clearly be observed in the DPV (see Figure 3.13, right, $\mathrm{pH}$ 4.98) corresponding to the oxidation:

$$
\left[\mathrm{Ru}^{\mathrm{IV}}=\mathrm{O} \quad \mathrm{O}=\mathrm{Ru}^{\mathrm{IV}}\right]^{3-}+\mathrm{e}^{-}+\mathrm{H}^{+} \rightarrow\left[\mathrm{Ru}^{\mathrm{III}}-\mathrm{OH} \quad \mathrm{O}=\mathrm{Ru}^{\mathrm{IV}}\right]^{3-}
$$


After this there seems to be a further oxidation to $\left[\mathrm{Ru}^{\mathrm{IV}}=\mathrm{O} O=\mathrm{Ru}^{\mathrm{V}}\right]^{2-}$ in which the $0-0$ bond is formed, and dioxygen is released. The potential of this last process is $\mathrm{pH}$ independent; however, it fluctuates over the measured $\mathrm{pH}$ range making it difficult to determine the exact redox potential.

Table 3.5. $\mathrm{pK}_{\mathrm{a}}$ values of various species of $\mathbf{3}^{\mathbf{H} 20}$.

\begin{tabular}{|c|c|}
\hline conversion & $\mathrm{p} K \mathrm{a}$ \\
\hline 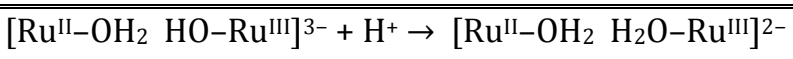 & 1.96 \\
\hline$\left[\mathrm{Ru}^{\mathrm{III}-O \mathrm{OH}_{2}} \quad \mathrm{HO}-\mathrm{Ru}^{\mathrm{III}}\right]^{2-}+\mathrm{H}^{+} \rightarrow\left[\begin{array}{lll}\mathrm{Ru}^{\mathrm{III}}-\mathrm{OH}_{2} & \mathrm{H}_{2} \mathrm{O}-\mathrm{Ru}^{\mathrm{IIII}}\end{array}\right]^{-}$ & 1.96 \\
\hline$\left[\mathrm{Ru}^{\mathrm{III}}-\mathrm{OH} \quad \mathrm{HO}-\mathrm{Ru}^{\mathrm{III}}\right]^{3-}+\mathrm{H}^{+} \rightarrow\left[\mathrm{Ru}^{\mathrm{III}}-\mathrm{OH}_{2} \mathrm{HO}-\mathrm{Ru}^{\mathrm{III}}\right]^{2-}$ & 5.02 \\
\hline$\left[\mathrm{Ru}^{\mathrm{III}}-\mathrm{OH} \quad \mathrm{O}=\mathrm{Ru}^{\mathrm{III}}\right]^{4-}+\mathrm{H}^{+} \rightarrow\left[\mathrm{Ru}^{\mathrm{III}}-\mathrm{OH} \quad \mathrm{HO}-\mathrm{Ru}^{\mathrm{III}}\right]^{3-}$ & 7.24 \\
\hline$\left[\mathrm{Ru}^{\mathrm{III}}-\mathrm{OH} \quad \mathrm{O}=\mathrm{Ru}^{\mathrm{IV}}\right]^{3-}+\mathrm{H}^{+} \rightarrow\left[\mathrm{Ru}^{\mathrm{III}}-\mathrm{OH} \mathrm{HO}-\mathrm{Ru}^{\mathrm{IV}}\right]^{2-}$ & 4.02 \\
\hline
\end{tabular}

A comparison of the Pourbaix diagram of $\mathbf{3}^{\mathbf{H 2 O}}$ with those of $\mathbf{V}^{\mathbf{H 2 O}}{ }^{226]}$ and $\mathbf{V I}^{\mathbf{H 2 O}[108,231]}$ is not trivial as different species are formed in these related complexes; however, the overall $\mathrm{p} K_{\mathrm{a}}$ values of $\mathbf{3}^{\mathbf{H 2 0}}$ seem to be higher. For example, the $\mathrm{p} K_{\mathrm{a}}$ value of the $\left[\mathrm{Ru}^{\mathrm{III}}-\mathrm{OH} \mathrm{HO}-\mathrm{Ru}^{\mathrm{IV}}\right]$ species (all scaffold ligands are omitted for clarity reasons, the charge is not given as it differs for the complexes $\mathbf{V}^{\mathbf{H} 20} / \mathbf{V I}^{\mathbf{H 2 0}}$ and $\mathbf{3}^{\mathbf{H 2 0}}$ ) is 0.7 and 4.0 for $\mathbf{V}^{\mathbf{H} 20}$ and $3^{\mathbf{H} 20}$, respectively. Furthermore, the $\mathrm{p} K_{\mathrm{a}}$ value of $\left[\mathrm{Ru}^{\mathrm{II}}-\mathrm{OH}_{2} \mathrm{H}_{2} \mathrm{O}-\mathrm{Ru}^{\mathrm{II}}\right]$, which lies at 6.5 and 6.8 for $\mathbf{V}^{\mathbf{H} 20}$ and $\mathbf{V I}^{\mathbf{H} 20}$, respectively, is not found within the measured pH range for complex $\mathbf{3}^{\mathbf{H 2 0}}$. Another significant difference to the Pourbaix diagrams of $\mathbf{V}^{\mathbf{H} 20}$ and $\mathbf{V I}^{\mathbf{H} 20}$ is that the fully protonated [ $\left.\mathrm{Ru}^{\mathrm{III}}-\mathrm{OH}_{2} \mathrm{H}_{2} \mathrm{O}-\mathrm{Ru}^{\mathrm{III}}\right]$ species can be observed for $3^{\mathrm{H} 20}$ but not for the previously reported systems. These observations are all in accordance with the lower overall charge of $3^{\mathbf{H} 20}$ compared to $\mathbf{V}^{\mathbf{H 2 0}} / \mathbf{V I}^{\mathbf{H} 20}$, because of the anionic carboxylate groups in the present complex.

\subsection{Electrochemical Water Oxidation Catalysis ${ }^{i x}$}

At pH 1, the extrapolation of the Pourbaix diagram of $3^{\mathbf{H} 20}$ suggests that there are two PCET processes after $1.36 \mathrm{~V} v$ s. NHE (onset of the catalytic wave in the CV; Figure 3.8), namely the oxidation from $\left[\mathrm{Ru}^{\mathrm{III}}-\mathrm{OH}_{2} \mathrm{H}_{2} \mathrm{O}-\mathrm{Ru}^{\mathrm{III}}\right]^{-}$to $\left[\mathrm{Ru}^{\mathrm{III}}-\mathrm{OH} \mathrm{HO}-\mathrm{Ru}^{\mathrm{IV}}\right]^{2-}(1.34 \mathrm{~V})$ and further to $\left[\mathrm{Ru}^{\mathrm{III}}=\mathrm{O} \mathrm{O}=\mathrm{Ru}^{2}\right]^{2-}(1.53 \mathrm{~V})$ before dioxygen is released. Well-separated redox processes can be observed between $\mathrm{pH} 4$ and 6, and thus allow one to distinguish the beginning of the catalytic wave in order to perform a FOWA analysis and obtain kinetic information on the catalysis (see Section 1.3.4). For an accurate and reproducible analysis, multiple CVs of $\mathbf{3}^{\mathbf{H 2 0}}$ in $\mathrm{HBF}_{4}$ (pH 5.6) were recorded at scan rates 20, 50, 100 and $200 \mathrm{mV} / \mathrm{s}$. Before data was extracted from the $\mathrm{CV}$, the background scan was subtracted. A corresponding background-subtracted CV exemplarily for $100 \mathrm{mV} / \mathrm{s}$ as well as its graphical analysis is shown in Figure 3.14. A $k_{\text {cat }}$ value was determined for every scan rate (Table 3.6) and averages to $22.5 \pm 2.8 \mathrm{~s}^{-1}$ for $3^{\mathbf{H 2 O}}$ at $\mathrm{pH}$ 5.6. It is important to note that kinetic parameters for catalytic reactions, which are derived from electrochemical measurements, are extremely sensitive to reaction conditions and analysis methods, and thus, should only be compared with great caution. ${ }^{[251]}$ Furthermore, please keep in mind that the powder sample of $3^{\mathbf{H} 20}$ contains significant amounts of NaF (see Section 3.1), which can influence the catalytic response.

\footnotetext{
ix This section was adapted from Jana Lücken, Carolina Gimbert-Suriñach, Jordi Benet-Buchholz, Antoni Llobet, Franc Meyer, "Diruthenium Complexes of a Pyrazolate/Carboxylate Hybrid Ligand for Chemically, Electro- and Photo-Driven Catalytic Water Oxidation", under revision.
} 

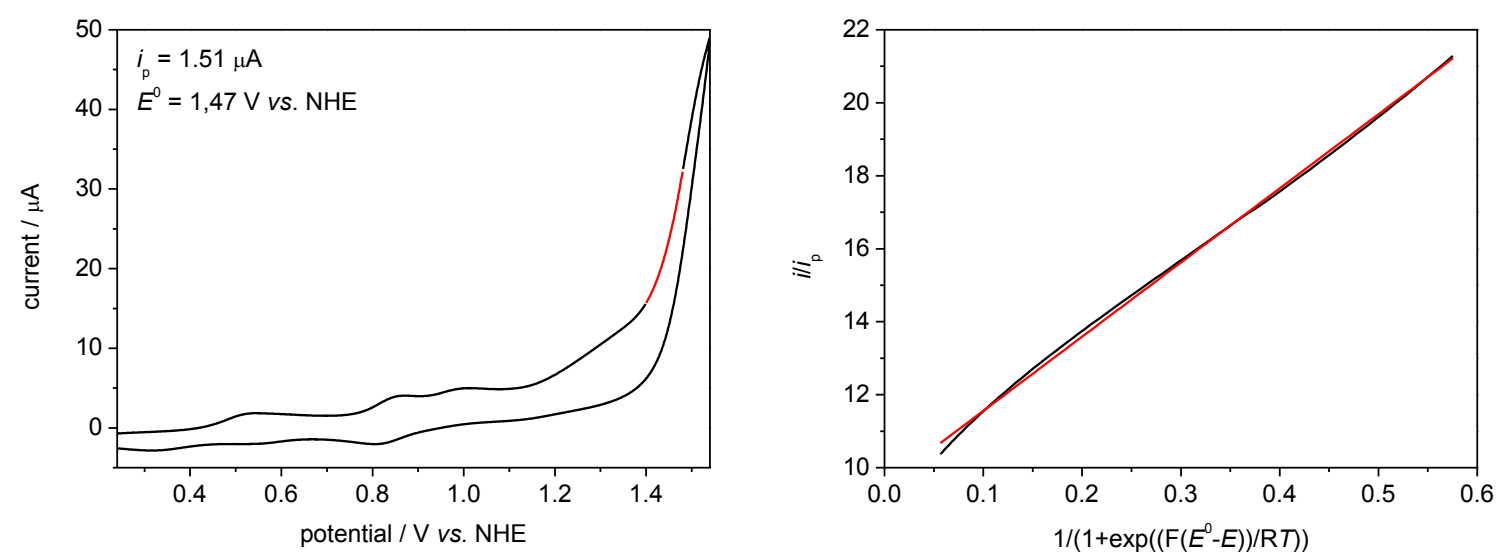

Figure 3.14: FOWA analysis of $3^{\mathbf{H} 20}$ in aqueous $\mathrm{HBF}_{4}\left(\mathrm{pH}\right.$ 5.6). Left: $\mathrm{CV}$ of $\mathbf{3}^{\mathbf{H} 20}$ from $0.24-1.54 \mathrm{~V}$ at $100 \mathrm{mV} / \mathrm{s}$. The red section marks the data used for the FOWA analysis. Right: Plot $\frac{i}{i_{\mathrm{p}}} v s . \frac{1}{1+\mathrm{e}^{\frac{F\left(E^{0}-E\right)}{R T}}}$ (for the red region in the CV; black: experimental, red: linear fit).

Table 3.6: $k_{\text {cat }}$ values from the FOWA analysis of complex $3^{\mathbf{H} 20}$ at various scan rates.

\begin{tabular}{cll} 
scan rate $/ \mathrm{mV} / \mathrm{s}$ & $k_{\text {cat }} / \mathrm{s}^{-1}$ & $\emptyset / \mathrm{s}^{-1}$ \\
\hline \hline 20 & 23.1 & \\
50 & 19.4 & \\
100 & 20.6 & $22.5 \pm 2.8$ \\
200 & 26.7 & \\
\hline
\end{tabular}

As for complex $\mathbf{3}^{\mathbf{H 2 0}}$, the $\mathrm{CV}$ of $\mathbf{3}^{\mathrm{dmso}}$ at $\mathrm{pH} 1$ exhibits a large irreversible wave associated with an electrocatalytic process beginning at very similar potential $\left(E_{\text {onset }}=1.38 \mathrm{~V}\right.$ for $3^{\text {dmso }} v s$. $E_{\text {onset }}=1.36 \mathrm{~V}$ for $3^{\mathbf{H 2 0}}$; Figure 3.8). Nevertheless, a FOWA analysis of $3^{\text {dmso }}$ at this $\mathrm{pH}$ is not feasible due to further oxidation processes before dioxygen formation that cannot be distinguished in the $\mathrm{CV}$, as stated for $3^{\mathbf{H} 20}$ above. In contrast to $\mathbf{3}^{\mathbf{H 2 0}}$, a FOWA analysis of $\mathbf{3}^{\mathbf{d m s o}}$ at higher $\mathrm{pH}$ was not possible, because under these conditions the DMSO in the bridging pocket is partially replaced by aquo ligands, which is in accordance with the synthetic procedure for the formation of $3^{\mathbf{H 2 O}}$. At $\mathrm{pH} 2$, however, in the presence of phosphate buffer, complex $\mathbf{3}^{\mathrm{dmso}}$ proved to be stable over the course of $20 \mathrm{CV}$ cycles. At this $\mathrm{pH}$, no exchange of the DMSO moiety in the bimetallic pocket was observed as confirmed by ESI-MS. Likewise, the stability of the bridging DMSO group at low pH was confirmed by NMR spectroscopy in DOTf (pH 1).

\subsection{Chemical Water Oxidation Catalysis and Mechanistic Investigations}

\subsubsection{Order of the Reaction and Comparison between the Catalytic Behavior of $3 \mathrm{dmso}$ and $3^{\text {H20x }}$}

The catalytic proficiency of complexes $\mathbf{3}^{\mathrm{dmso}}$ and $\mathbf{3}^{\mathrm{H} 20}$ for chemically driven water oxidation was tested using ceric ammonium nitrate as sacrificial oxidant. CAN is a one-electron oxidant with a redox potential of $1.75 \mathrm{~V} v s$. NHE $(\sim 50 \mathrm{mM})$ at $\mathrm{pH} 0.9$ in an aqueous perchlorate solution. ${ }^{[112]}$ While this allows for the study of WOCs with overpotentials of up to nearly $580 \mathrm{mV}\left(E^{0}\left(\mathrm{H}_{2} \mathrm{O} / \mathrm{O}_{2}\right)=1.17 \mathrm{~V} v\right.$ s. NHE

\footnotetext{
× This section was adapted from Jana Lücken, Carolina Gimbert-Suriñach, Jordi Benet-Buchholz, Antoni Llobet, Franc Meyer, "Diruthenium Complexes of a Pyrazolate/Carboxylate Hybrid Ligand for Chemically, Electro- and Photo-Driven Catalytic Water Oxidation", under revision.
} 
at $\mathrm{pH}$ 1), it may also open up new side reactions that could for example lead to the degradation of the catalyst.

In a typical experiment, 100 eq of CAN were added to a $1 \mathrm{mM}$ complex solution in triflic acid (pH 1) at $25{ }^{\circ} \mathrm{C}$ resulting in a maximum turnover number (TON) of 25 (100\% efficiency) for the four-electron oxidation of water to dioxygen. The produced dioxygen was simultaneously detected by a manometer and an optical oxygen sensor or a Clark electrode. For the manometric measurements, the pressure difference to a reference cell was recorded and subsequently converted to the amount of dioxygen produced by means of the ideal gas law. The determined oxygen partial pressure $p_{02}$ from the optical oxygen sensor was treated likewise. Alternatively, the measured potential at the Clark electrode was used to determine the concentration of dioxygen in the gas phase with the help of a previously recorded calibration curve. These two analytical methods (manometry and oxygen sensor) are employed simultaneously in order to confirm the absence of any gases other than dioxygen. The sole use of online manometry is not sufficient, as the manometric measurement cannot distinguish between produced dioxygen and for example carbon dioxide from the oxidative decomposition of the complex.

In order to determine the order of reaction of water oxidation on the complex concentration, chemical water oxidation experiments of $\mathbf{3}^{\text {dmso }}$ were conducted. For this, CAN was added to $\mathbf{3}^{\text {dmso }}$ in $0.1 \mathrm{M}$ triflic acid ( $\mathrm{pH}$ 1) to reach a final oxidant concentration of $100 \mathrm{mM}$ and the evolution of gas was monitored by on-line manometry (Figure 3.15, left). The initial rate of water oxidation for each concentration was determined from this data by a linear fit of the first 20 seconds after addition of CAN and revealed a linear dependency on the amount of $\mathbf{3}^{\mathbf{d m s o}}$ in solution (Figure 3.15 , right). Consequently, water oxidation catalysis is first-order in complex concentration.
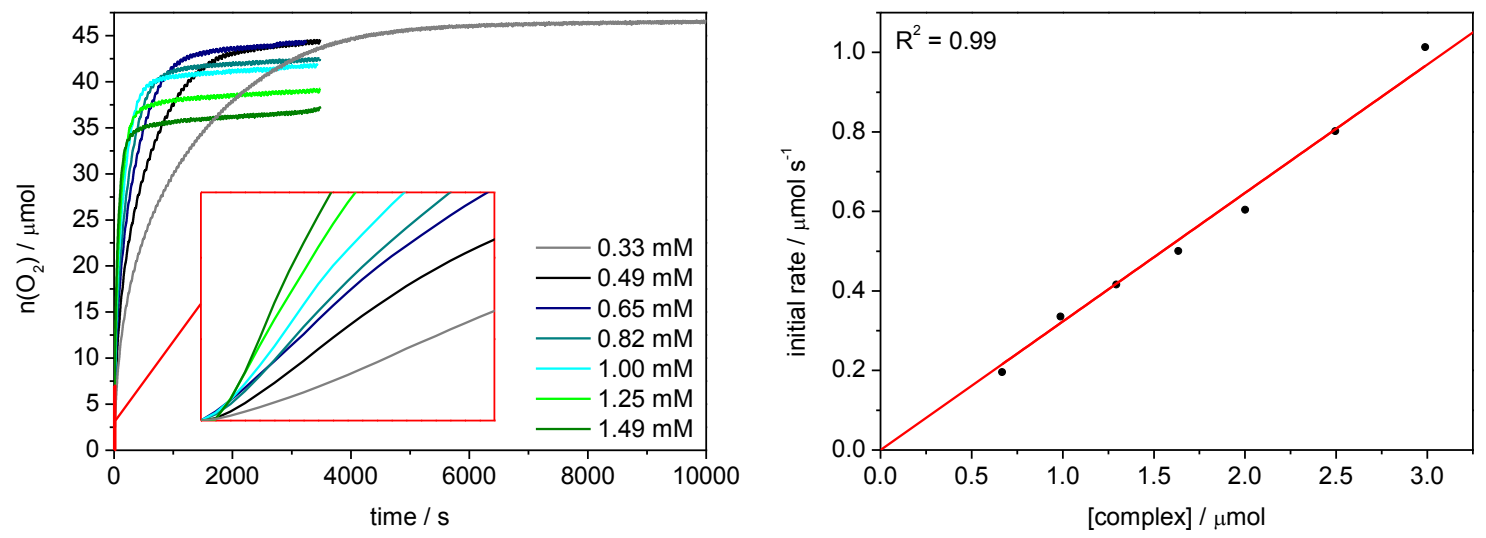

Figure 3.15: Concentration dependent chemical water oxidation of $3^{\text {dmso }}$ in $0.1 \mathrm{M}$ aqueous triflic acid (pH 1$)$ at $25^{\circ} \mathrm{C}$. Left: Formation of dioxygen at different complex concentrations detected by on-line manometry (the inset depicts the first 20 seconds of oxygen formation). The concentration of CAN was left constant (100 mM). Right: Initial rate of water oxidation determined in the first 30 seconds after addition of CAN. Linear fit with an $\mathrm{R}^{2}$ of 0.99 .

Typical traces for two consecutive additions of 100 eq CAN are shown in Figure 3.16 for complexes $\mathbf{3}^{\text {dmso }}$ (left) and $\mathbf{3}^{\text {H20 }}$ (right). ${ }^{\text {i }}$ The maxima recorded by the manometer (black) and the oxygen sensor (red) are in quite good agreement suggesting the absence of significant amounts of any gases other than $\mathrm{O}_{2}$. The often-encountered delayed response of the oxygen sensor can be attributed to the fact that the liquid phase is first enriched with molecular oxygen before being released to the gas phase in which it is then detected. The first water oxidation run (solid lines) is significantly faster than the second one (dashed lines), which is portrayed in the TOF $\mathrm{s}-0.188$ and $0.201 \mathrm{~s}^{-1}$ for complexes $3^{\mathrm{dmso}}$ and $3^{\mathbf{H} 20}$, respectively, for the first run and 0.014 and $0.017 \mathrm{~s}^{-1}$ for the second (Table 3.7). These results suggest that either the catalysis is inhibited, or that different species are responsible for the catalysis

xi Please keep in mind that the powder sample of $3^{\mathbf{H} 20}$ contains significant amounts of $\mathrm{NaF}$ (see Section 3.1), which can influence the catalytic response. 
in the first and second rounds. The $\mathrm{TOF}_{\mathrm{i}}$ values obtained for $\mathbf{3}^{\mathrm{dmso}}$ and $\mathbf{3}^{\mathbf{H} 2 \mathbf{O}}$ compare favorably to those reported for the related pyrazolate-based catalysts $\mathbf{V}^{\mathbf{H 2 O}}\left(0.014 \mathrm{~s}^{-1}\right)^{[225]}$ and $\mathbf{V I}^{\mathbf{H 2 O}}\left(0.068 \mathrm{~s}^{-1}\right)^{[108]}$ under the same conditions. However, they are nearly an order of magnitude lower than that of the parent complex $\mathbf{X}^{\mathbf{H 2 O}}\left(1.4 \mathrm{~s}^{-1}\right),{ }^{[204]}$ which lacks the peripheral sulfonate groups (Table 3.7). It has previously been shown that pendant sulfonate groups in the second coordination sphere of mononuclear ruthenium catalysts can significantly alter the rates of CAN-driven water oxidation.[115]
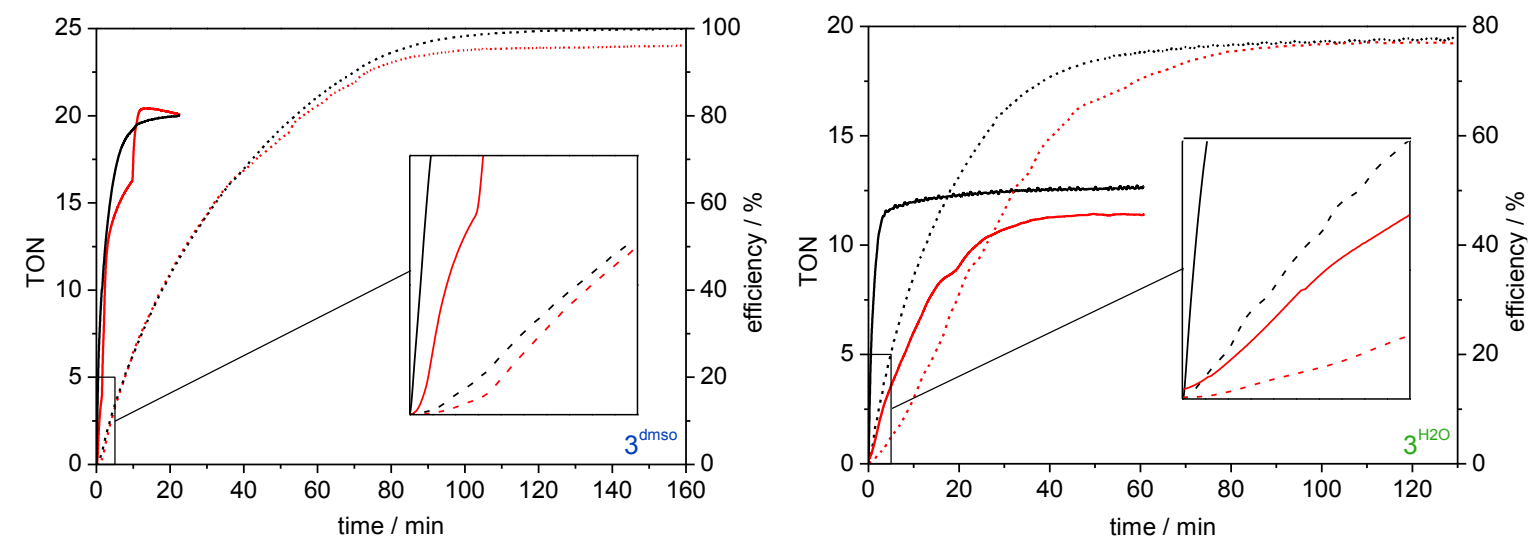

Figure 3.16. Catalytic activity monitored by on-line manometry (black) and an oxygen sensor (red) of $3^{\mathrm{dmso}}$ (left) and $3^{\mathrm{H} 20}$ (right) upon addition of 100 eq CAN (TON $\max =25$ (100\% efficiency)) in 0.1 M aqueous triflic acid (pH 1) at $25^{\circ} \mathrm{C}$ (catalyst concentration: $1 \mathrm{mM}$ ). The solid lines represent the first and the dashed lines the second addition of CAN. The inset depicts the first five minutes of dioxygen evolution.

Table 3.7. TON and TOF for the chemical water oxidation catalysis of $\mathbf{3}^{\mathbf{d m s o}}$ and $\mathbf{3}^{\mathbf{H} 20}$, as well as other relevant complexes $\left(\mathrm{TON}_{\max }=25(100 \%)\right)$ derived from manometric data. ${ }^{\mathrm{a}}$

\begin{tabular}{|c|c|c|c|c|c|c|c|}
\hline \multirow[t]{2}{*}{ complex } & \multicolumn{3}{|c|}{ TON (eff. / \%) } & \multicolumn{3}{|c|}{$\mathrm{TOF}_{\mathrm{i}}^{\mathrm{b}} / \mathrm{s}^{-1}$} & \multirow[t]{2}{*}{ ref. } \\
\hline & $1^{\mathrm{c}}$ & $2^{c}$ & $3^{c}$ & $1^{c}$ & $2^{c}$ & $3^{c}$ & \\
\hline $3^{\text {dmso }}$ & $20.0(80)$ & $24.9(>99)$ & $24.0(96)$ & 0.188 & ב0.014 & ב0.002 & this work \\
\hline $3^{\mathrm{H} 2 \mathrm{O}}$ & $12.6(50)$ & $19.4(78)$ & - & 0.201 & 0.017 & - & this work \\
\hline $\mathrm{X}^{\mathrm{H} 2 \mathrm{O}}$ & $24.0(96)$ & $24.0(96)$ & - & 1.4 & - & - & [204] \\
\hline VIH2O & $22.6(90)$ & - & - & 0.068 & - & - & [108] \\
\hline$V^{H 2 O}$ & $17.5(70)$ & - & - & 0.014 & - & - & {$[225,226]$} \\
\hline VIII $^{d}$ & $10400(42)$ & - & - & 1.2 & - & - & [227] \\
\hline VIII'e & $1400(11)$ & - & - & 1.17 & - & - & [228] \\
\hline
\end{tabular}

a conditions: cat $=1 \mathrm{mM}, \mathrm{CAN}=100 \mathrm{mM}$ (cat/CAN $=1 / 100$ ), in $0.1 \mathrm{M}$ aqueous triflic acid at $25^{\circ} \mathrm{C}$

$\mathrm{b}$ initial turnover frequency at the start of catalysis under identical conditions.

c refers to the first (1), second (2) and third (3) addition of CAN to the same complex solution

${ }^{\mathrm{d}}$ conditions: cat $=50 \mathrm{nM}, \mathrm{CAN}=5 \mathrm{mM}\left(\mathrm{cat} / \mathrm{CAN}=1 / 100000, \mathrm{TON}_{\max }=25000\right)$, in 0.1 M aqueous triflic acid $/ 10 \mu \mathrm{L}$ $\mathrm{MeCN}$

${ }^{\mathrm{e}}$ conditions: cat $=2 \mu \mathrm{M}, \mathrm{CAN}=100 \mathrm{mM}\left(\right.$ cat $\left./ \mathrm{CAN}=1 / 50000, \mathrm{TON}_{\max }=12500\right)$, in $0.1 \mathrm{M}$ aqueous triflic acid $/ 10 \%$ $\mathrm{MeCN}$

For the first addition of oxidant, complex $3^{\text {dmso }}$ reaches a TON of 20.0 (80 \% efficiency), whereas $3^{\mathbf{H 2 0}}$ has a maximum TON of only 12.6 (50 \%). The low TON of $\mathbf{3}^{\mathbf{H 2 0}}$ is a consequence of the formation of insoluble $\mathrm{CeF}_{4}$ from $\mathrm{CAN}$ and $\mathrm{F}^{-}$ions (present in the bulk material of $\mathbf{3}^{\mathbf{H} 20}$ from $\mathrm{HBF}_{4}$ used during synthesis, see experimental section), and therefore not all of the added oxidant is available for water oxidation (Figure 3.17, top). This could be confirmed by XPS measurements, in which the abovementioned solid (green trace) was compared to commercially available $\mathrm{CeF}_{4}$ (black) and a precipitate 
formed upon addition of CAN to a $0.1 \mathrm{M}$ aqueous $\mathrm{HBF}_{4}$ solution ( $\mathrm{pH} 1$, red). In all three cases, the XPS $\mathrm{F}(1 \mathrm{~s})$ and $\mathrm{Ce}(3 \mathrm{~d})$ spectra are very similar, suggesting that indeed $\mathrm{CeF}_{4}$ is formed (Figure 3.17, bottom). In both $3^{\text {dmso }}$ and $3^{\mathrm{H} 20}$, a significantly higher TON is reached for the second run of water oxidation, viz. 24.9 ( $>99 \%$ ) and 19.4 (78 \%), respectively. A third addition of 100 eq CAN to $3^{\text {dmso }}$ was also attempted leading to a TON and TOFi of 24.0 and $0.002 \mathrm{~s}^{-1}$, respectively, with good agreement between the manometry and oxygen sensor traces, demonstrating the robustness of the catalyst (Table 3.7).
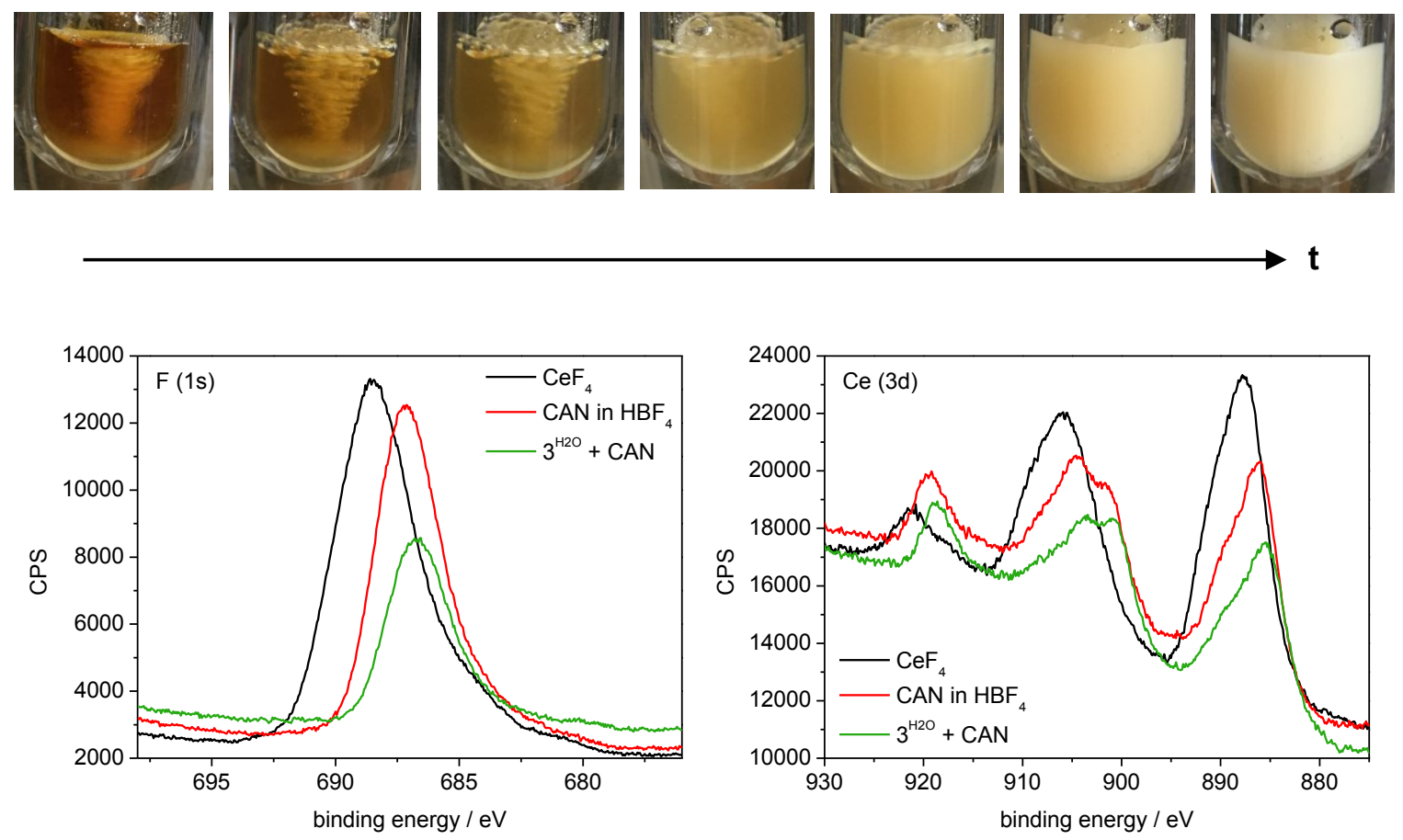

Figure 3.17: Observations during the chemical water oxidation of $3^{\mathbf{H} 20}$ in $0.1 \mathrm{M}$ triflic acid (pH 1) with CAN. Top: Pictures showing the gradual formation of a white solid upon addition of CAN to $3^{\mathbf{H} 20}$. Bottom: XPS spectra of the $\mathrm{F}(1 \mathrm{~s})$ (left) and $\mathrm{Ce}(3 \mathrm{~d})$ (right) areas of the above-mentioned powder (green) in comparison to commercially available $\mathrm{CeF}_{4}$ (black) and a powder formed upon the dissolution of $\mathrm{CAN}$ in $0.1 \mathrm{M}$ aqueous $\mathrm{HBF}_{4}$ (pH 1) (red). As can be seen, all traces look very similar, suggesting that the powder samples formed correspond to $\mathrm{CeF}_{4}$.

\subsubsection{Inhibition of the Catalysis by Anation of Nitrate Ions and Interaction with Cerium ${ }^{x i i}$}

The significant differences in $\mathrm{TOF}_{i}$ for the first and second catalytic runs for both complexes could be a result of a detrimental nitrate effect, as $\mathrm{NO}_{3}{ }^{-}$is added with every equivalent of CAN $(100 \mathrm{mM}$ $\mathrm{CAN}=600 \mathrm{mM} \mathrm{NO}_{3}^{-}$). Nitrate, next to other anions, is able to compete with the substrate (viz. water) for the active site of the diruthenium center, as has been previously shown for $\mathbf{X}^{\mathbf{H} 20}$ and other ruthenium-based WOCs, thus resulting in a lower TOF for the second run of water oxidation. ${ }^{[141,204,252-}$ 254] In order to demonstrate the effect of nitrate anation in the present case, water oxidation experiments with different amounts of $\mathrm{NaNO}_{3}$ added prior to catalysis were conducted for $3^{\text {dmso }}$. However, as can be seen in Figure 3.18 (left), the effect of additional 600 eq (corresponding to the same total concentration of nitrate in solution as after the second addition of CAN) or even 1200 eq $\mathrm{NaNO}_{3}$ during the first run of water oxidation (mid-blue and light-blue traces, respectively) is significantly smaller than the retardation observed during the second run without additional $\mathrm{NaNO}_{3}$ (dashed, dark blue trace). In case of 600 eq added $\mathrm{NaNO}_{3}$, the $\mathrm{TOF}_{\mathrm{i}}$ is decreased by approximately half to $0.095 \mathrm{~s}^{-1}$

xii This section was adapted from Jana Lücken, Carolina Gimbert-Suriñach, Jordi Benet-Buchholz, Antoni Llobet, Franc Meyer, "Diruthenium Complexes of a Pyrazolate/Carboxylate Hybrid Ligand for Chemically, Electro- and Photo-Driven Catalytic Water Oxidation", under revision. 
and the TON remains unchanged $(\sim 21)$ in the first run, in contrast to the values found for the second run, viz. $0.014 \mathrm{~s}^{-1}$ and a TON of $\sim 25$. The same trend is observed upon addition of $\mathrm{NaNO}_{3}$ (600 eq) prior to the second run. Here, the deceleration $\left(\mathrm{TOF}_{\mathrm{i}}=0.007 \mathrm{~s}^{-1}\right)$ does not reach the same extent as during a third run of water oxidation $\left(0.002 \mathrm{~s}^{-1}\right.$; Table 3.7), although in both cases a total amount of $1800 \mathrm{eq}$ $\mathrm{NO}_{3}{ }^{-}$is present in solution, thereby showing no difference if the $\mathrm{NaNO}_{3}$ is added prior to the first or second addition of CAN. Consequently, the disparities that are observed in the first and second runs (Figure 3.18, left) cannot exclusively be attributed to the effect of the nitrate in CAN. In contrast, for the non-sulfonated analog $\mathrm{X}^{\mathrm{H} 2 \mathrm{O}}$ it could be shown that the decrease in the $\mathrm{TOF}_{\mathrm{i}}$ for the second run is purely due to anation by nitrate, as nearly identical $\mathrm{TOF}_{\mathrm{i}}$ values are obtained for the second addition of CAN or when $600 \mathrm{eq} \mathrm{KNO}_{3}$ are added prior to the first run.[204]

Next to released nitrate from CAN, the cerium ions are also capable of interacting with the catalyst especially in the presence of sulfonate moieties, as has been previously demonstrated for a mononuclear terpyridine-based ruthenium WOC with pendant $\mathrm{SO}_{3}{ }^{-}$groups. ${ }^{[115]}$ In order to investigate the influence of cerium(III), a WO catalysis experiment in the presence of $100 \mathrm{eq} \mathrm{Ce}\left(\mathrm{NO}_{3}\right)_{3}$ and $300 \mathrm{eq}$ $\mathrm{NaNO}_{3}$ was conducted; these additives mimic the conditions after one run of water oxidation with 100 eq CAN (Figure 3.18, right). Indeed, the effect of added 100 eq Ce ${ }^{I I I}$ and $600 \mathrm{eq} \mathrm{NO}_{3}{ }^{-}$prior to the first run coincides with the retardation of catalysis in the second run without any additives, which clearly suggests that the observed deceleration of catalysis from the first to the second run is an effect of both nitrate and cerium(III).
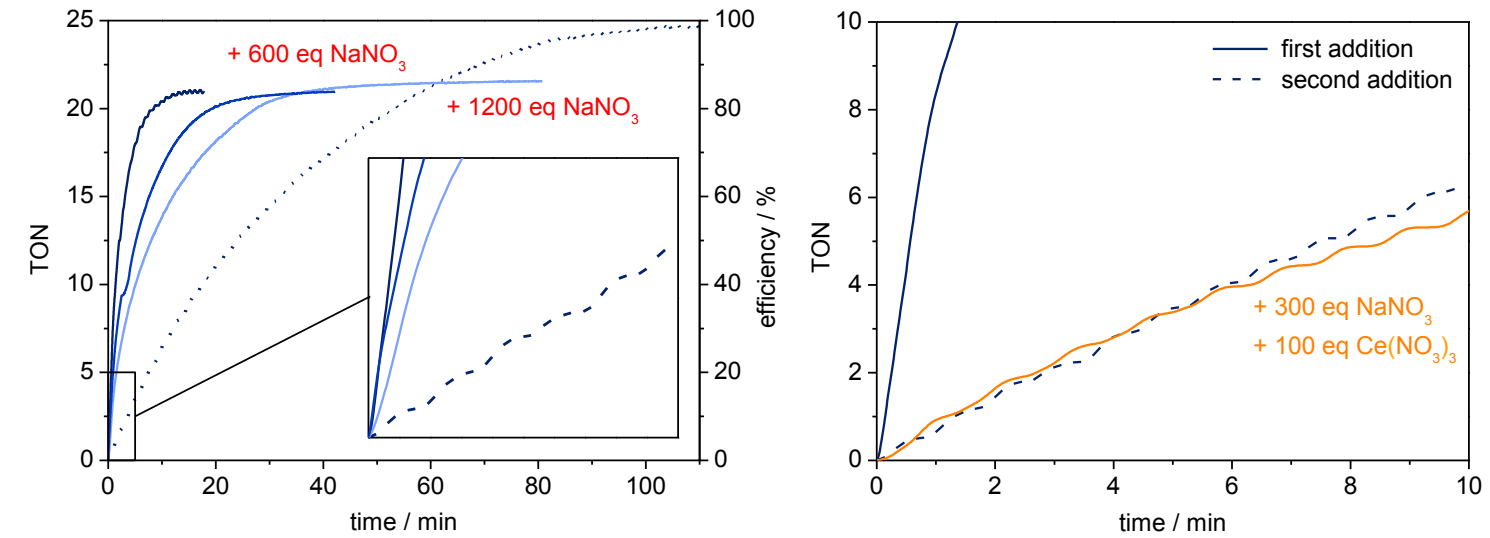

Figure 3.18: Influence of nitrate and Ce $\mathrm{III}^{\mathrm{III}}$ on the water oxidation catalysis of $\mathbf{3}^{\mathrm{dmso}}$ upon addition of 100 eq CAN $\left(\mathrm{TON}_{\max }=25\right)$ in $0.1 \mathrm{M}$ aqueous triflic acid $(\mathrm{pH} 1)$ at $25^{\circ} \mathrm{C}$ monitored by on-line manometry. Left: Catalytic runs with and without added $\mathrm{NaNO}_{3}$ prior to the first run (dark blue: no addition of $\mathrm{NaNO}_{3}$, blue: 600 eq, light blue: 1200 eq). Left: First 10 minutes of the catalytic runs with and without added $\mathrm{NaNO}_{3}$ and $\mathrm{Ce}\left(\mathrm{NO}_{3}\right)_{3}$ prior to the first run (dark blue: no additive, orange: 300 eq $\mathrm{NaNO}_{3}$ and 100 eq $\left.\mathrm{Ce}\left(\mathrm{NO}_{3}\right)_{3}\right)$. The solid lines represent the first and the dashed lines the second addition of CAN. Please note, 600 eq NO$_{3^{-}}$and 100 eq Ce ${ }^{\mathrm{III}}$ come from 100 eq CAN.

In order to further analyze the effect of nitrate, cyclic voltammograms of $\mathbf{3}^{\mathrm{dmso}}$ were measured in the potential range of $0.66-1.56 \mathrm{~V}$ at various $\mathrm{NaNO}_{3}$ concentrations with a dwell time of one minute in the catalytic regime (1.56 V). As can be seen in Figure 3.19 (left), next to the expected reductive peak at $0.85 \mathrm{~V}$ (original species), a new species with cathodic peak potential at $0.94 \mathrm{~V}$ can be observed in the reverse scan (dark blue $\mathrm{CV}$, no additional nitrate), suggesting that the original complex is transformed into an active new species during catalysis (possible catalytically active species are discussed in Section 3.5.4). Upon addition of nitrate, the new reductive wave shifts, which indicates that this catalytic species is subjected to anation (new anated species). However, anation appears to occur only in higher oxidation states as ${ }^{1} \mathrm{H}$ NMR spectroscopy of the $\mathrm{Ru}^{\mathrm{II}} \mathrm{Ru}^{\mathrm{II}}$ complex $3^{\mathrm{dmso}}$ in the presence of 100 eq $\mathrm{NaNO}_{3}$ does not show any significant changes. Furthermore, regardless of the amount of nitrate present in solution, the newly formed species disappears when the complex is reduced back to its $\mathrm{Ru}^{\mathrm{II}} \mathrm{Ru}^{\mathrm{II}}$ state, as can be seen when cycling the $\mathrm{Ru}^{\text {III }} \mathrm{Ru}^{\mathrm{III}} / \mathrm{Ru}^{\mathrm{II}} \mathrm{Ru}^{\mathrm{II}}$ wave after the dwell time in the catalytic regime (Figure 3.19, right; exemplarily for $0 \mathrm{mM} \mathrm{NaNO}$ ). Consequently, the active new species, which is 
formed during catalysis, only remains intact if the ruthenium ions are at least in the oxidation state +III. Furthermore, the reaction to form the new active species must be quite simple, without major structural changes, as it seems to be easy to reverse the process and regain original $3^{\text {dmso }}$.
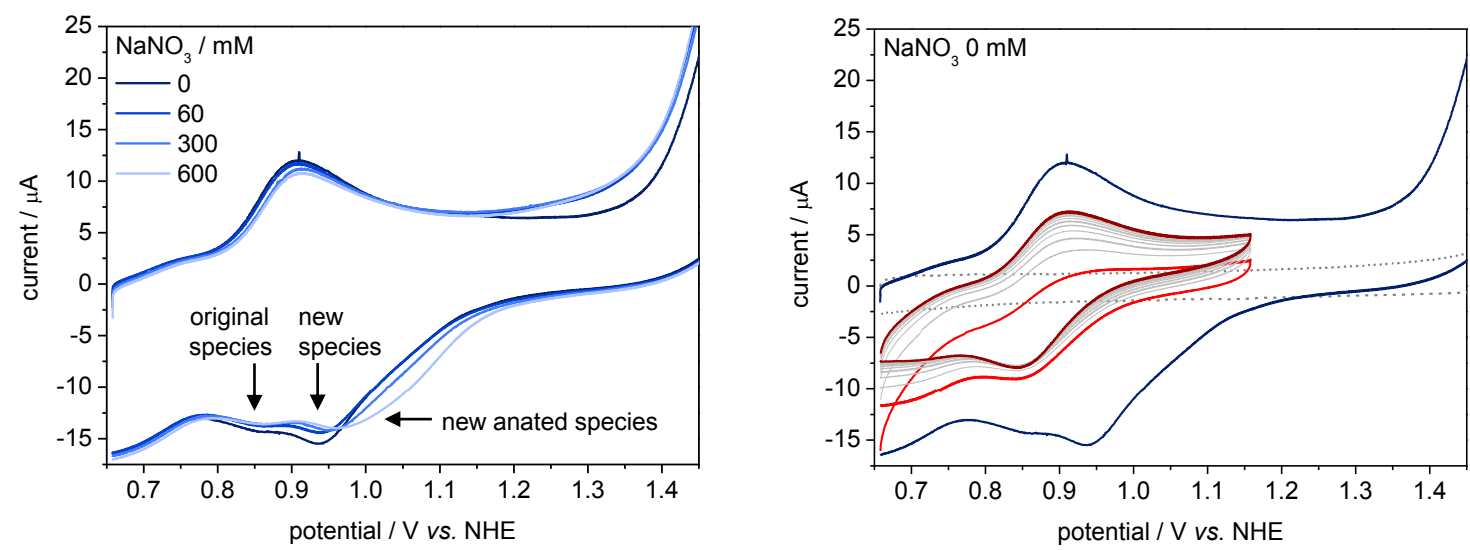

Figure 3.19: CVs of a $1 \mathrm{mM}$ solution of $3^{\text {dmso }}$ in $0.1 \mathrm{M}$ aqueous triflic acid (pH 1 ) in the potential range of $0.66-$ $1.56 \mathrm{~V}$ with a dwell time of one minute in the catalytic regime $(1.56 \mathrm{~V})$ using a glassy carbon disc working electrode, a Pt disc as auxiliary and an MSE as the reference electrode. The potentials were converted to the NHE scale by adding $0.658 \mathrm{~V}$. Left: CVs at various nitrate concentrations (0-600 $\mathrm{mM}$, dark to light blue, respectively). Right: $\mathrm{CV}$ at $0 \mathrm{mM} \mathrm{NaNO} 3$ with subsequent cycling from $0.66-1.16 \mathrm{~V}$ (10 times, red: first, dark red: last scan) showing that upon re-reduction to $\mathrm{Ru}^{\mathrm{II}} \mathrm{Ru}^{\mathrm{II}}$ the new species disappears.

In order to investigate the effect of cerium(III) on the electrochemistry of $\mathbf{3}^{\text {dmso, }}$ SWVs were measured in the presence of $\mathrm{Ce}\left(\mathrm{NO}_{3}\right)_{3}$. As can be seen in Figure 3.20, the addition of $\mathrm{Ce}\left(\mathrm{NO}_{3}\right)_{3}$ leads to an anodic shift of the oxidation potential of $3^{\text {dmso }}$. This could be explained by the interaction of Ce $\mathrm{e}^{\mathrm{III}}$ with the complex, thus removing electron density and making the oxidation more difficult. As the shift is only minimal (addition of 100 eq $\mathrm{Ce}\left(\mathrm{NO}_{3}\right)_{3}$ increases the oxidation potential by $19 \mathrm{mV}$ ), it could be assumed that the CeIII ion binds rather far from the ruthenium centers, e.g. at the sulfonate groups of the axial pyridine ligands, as has previously been observed for a mononuclear ruthenium WOC.[115]

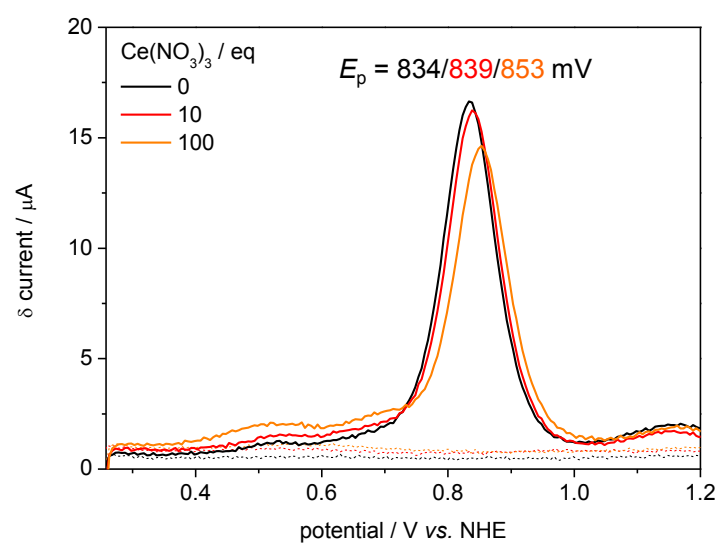

Figure 3.20: SWVs of a $1 \mathrm{mM}$ solution of $3^{\mathrm{dmso}}$ in $0.1 \mathrm{M}$ aqueous triflic acid ( $\mathrm{pH}$ 1) upon addition of $\mathrm{Ce}\left(\mathrm{NO}_{3}\right)_{3}$ using a glassy carbon disc working electrode, a Pt disc as auxiliary and an MSE as the reference electrode (black: no additional $\mathrm{Ce}\left(\mathrm{NO}_{3}\right)_{3}$, red: 10 eq, orange: $\left.100 \mathrm{eq}\right)$. The potentials were converted to the NHE scale by adding $0.658 \mathrm{~V}$.

\subsubsection{Prerequisite for Mechanistic Studies}

In order to distinguish the mechanism of water oxidation, more precisely the $\mathrm{O}-\mathrm{O}$ bond formation step (typically I2M or WNA; see Section 1.3.3), ${ }^{18} 0$ labeling experiments are a valuable tool. An essential requirement for such studies is a slow exchange of the bound water molecules with the solvent 
(compared to the timescale of the experiment), as a different degree of labeling of the catalyst and the substrate (viz. water) is necessary. Thus, the estimation of the exchange kinetics of the aquo ligands is indispensable, bearing in mind that in case of fast exchange no mechanistic information can be withdrawn.

An easily applicable method is to investigate the substitution kinetics with acetonitrile by UV/vis spectroscopy as has previously been reported for the related complexes $\mathbf{V}^{\mathbf{H 2 O}}$ and $\mathbf{V I}^{\mathbf{H} 20}{ }_{[108,226]}$ For this, $3^{\mathrm{H} 20}$ was dissolved in $0.1 \mathrm{M}$ aqueous $\mathrm{HClO}_{4}(\mathrm{pH} 1)$ and $\sim 1500$ eq MeCN were added, ensuring pseudofirst-order conditions. As $3^{\mathbf{H 2 0}}$ was already partially oxidized from synthesis (see Section 3.1), CAN was added prior to MeCN to reach the oxidation state $\mathrm{Ru}^{\mathrm{III}} \mathrm{Ru}^{\mathrm{III}}$ (also monitored by UV/vis). In any case, this is useful as it has been shown that the exchange rate significantly decreases upon oxidation of the ruthenium ions. ${ }^{[108,226]}$

As can be seen in Figure 3.21 (top), the spectral changes upon addition of $\mathrm{MeCN}$ to $3^{\mathrm{H} 20}\left(\mathrm{Ru}^{\mathrm{IIII}} \mathrm{Ru}^{\mathrm{III}}\right)$ are minimal. The absorbance slightly decreases at $284 \mathrm{~nm}$ and increases at $333 \mathrm{~nm}$ with isosbestic points at 304 and $416 \mathrm{~nm}$, suggesting a clean conversion from one species (A) to another (B) as shown below. The experimental data was evaluated with Specfit $/ 32^{\mathrm{TM}}$ using a global model for a pseudo firstorder process $(\mathrm{A} \rightarrow \mathrm{B})$. At room temperature, a rate constant of $5.3 \cdot 10^{-4} \mathrm{~s}^{-1}$ (half-life $t_{1 / 2}=22 \mathrm{~min}$ ) (Table 3.8, entry 8) was obtained, which is too fast for ${ }^{18} 0$ labeling experiments. The goodness of fit was confirmed by selected kinetic traces (Figure 3.21, bottom), which show that the experimental and simulated data are in good agreement. Additionally, calculated UV/vis spectra of the two species A and B (Figure 3.21, middle left), as well as a calculated species distribution (Figure 3.21, middle right) can be extracted from the theoretical treatment of the results.

$$
\underbrace{\left[\mathrm{Ru}^{\mathrm{III}}-\mathrm{OH}_{2} \mathrm{H}_{2} \mathrm{O}-\mathrm{Ru}^{\mathrm{III}}\right]^{-}}_{A}+\mathrm{MeCN} \rightarrow \underbrace{\left[\mathrm{Ru}^{\mathrm{III}}-\mathrm{OH}_{2} \mathrm{MeCN}-\mathrm{Ru}^{\mathrm{III}}\right]^{-}}_{B}+\mathrm{H}_{2} \mathrm{O}
$$

As the exchange rate turned out to be too high at $23^{\circ} \mathrm{C}$, the same experiment was conducted at lower temperatures $\left(5,10\right.$ and $15^{\circ} \mathrm{C}$, Table 3.8). From this data, an Eyring $\left(\ln \frac{k}{T} v s . \frac{1}{T}\right)$ and Arrhenius $\left(\ln k v s . \frac{1}{T}\right.$ ) plot were prepared (Figure 3.22), from which the activation energy $E_{\mathrm{A}}$ as well as the activation parameters $\Delta H^{\ddagger}$ and $\Delta S^{\ddagger}$ were extracted as shown in equations 3.3-3.7. Apart from the physical constants $\mathrm{R}, \mathrm{h}$ and $\mathrm{k}_{\mathrm{B}}$, the slope of the linear fit $(\mathrm{m})$ as well as the $y$-intercept were required for this. The analysis revealed that in the considered temperature range, the enthalpic contribution $\left(\Delta H^{\ddagger}=65 \mathrm{~kJ} \cdot \mathrm{mol}^{-1}\right)$ to the Gibbs energy barrier is approximately three times larger than the entropic one $\left(-T \Delta S^{\ddagger}=24-26 \mathrm{~kJ} \cdot \mathrm{mol}^{-1}\right)$. Furthermore, the negative $\Delta S^{\ddagger}$ value indicates an associative mechanism for the ligand exchange reaction. Reverting to the main reason for these studies: at the lowest temperature $\left(5^{\circ} \mathrm{C}\right)$, the exchange rate at the oxidation state $\mathrm{Ru}^{\mathrm{III}} \mathrm{Ru}^{\mathrm{III}}$ turned out to be $1.0 \cdot 10^{-4} \mathrm{~s}^{-1}$ $\left(t_{1 / 2}=115 \mathrm{~min}\right)$, eliminating labeling studies to elucidate the mechanism.

$$
\begin{gathered}
\ln \frac{k}{T}=-\frac{\Delta H^{\ddagger}}{R} \cdot \frac{1}{T}+\ln \frac{k_{\mathrm{B}}}{h}+\frac{\Delta S^{\ddagger}}{R} \\
\Delta S^{\ddagger}=R \cdot\left(y-\ln \left(\frac{k_{\mathrm{B}}}{h}\right)\right)=(-87 \pm 17) \frac{\mathrm{J}}{\mathrm{mol} \cdot \mathrm{K}} \\
\Delta H^{\ddagger}=-m \cdot R=(65 \pm 5) \frac{\mathrm{kJ}}{\mathrm{mol}} \\
\ln k=\ln A-\frac{E_{\mathrm{A}}}{R} \cdot \frac{1}{T} \\
E_{\mathrm{A}}=-m \cdot R=(67 \pm 5) \frac{\mathrm{kJ}}{\mathrm{mol}}
\end{gathered}
$$



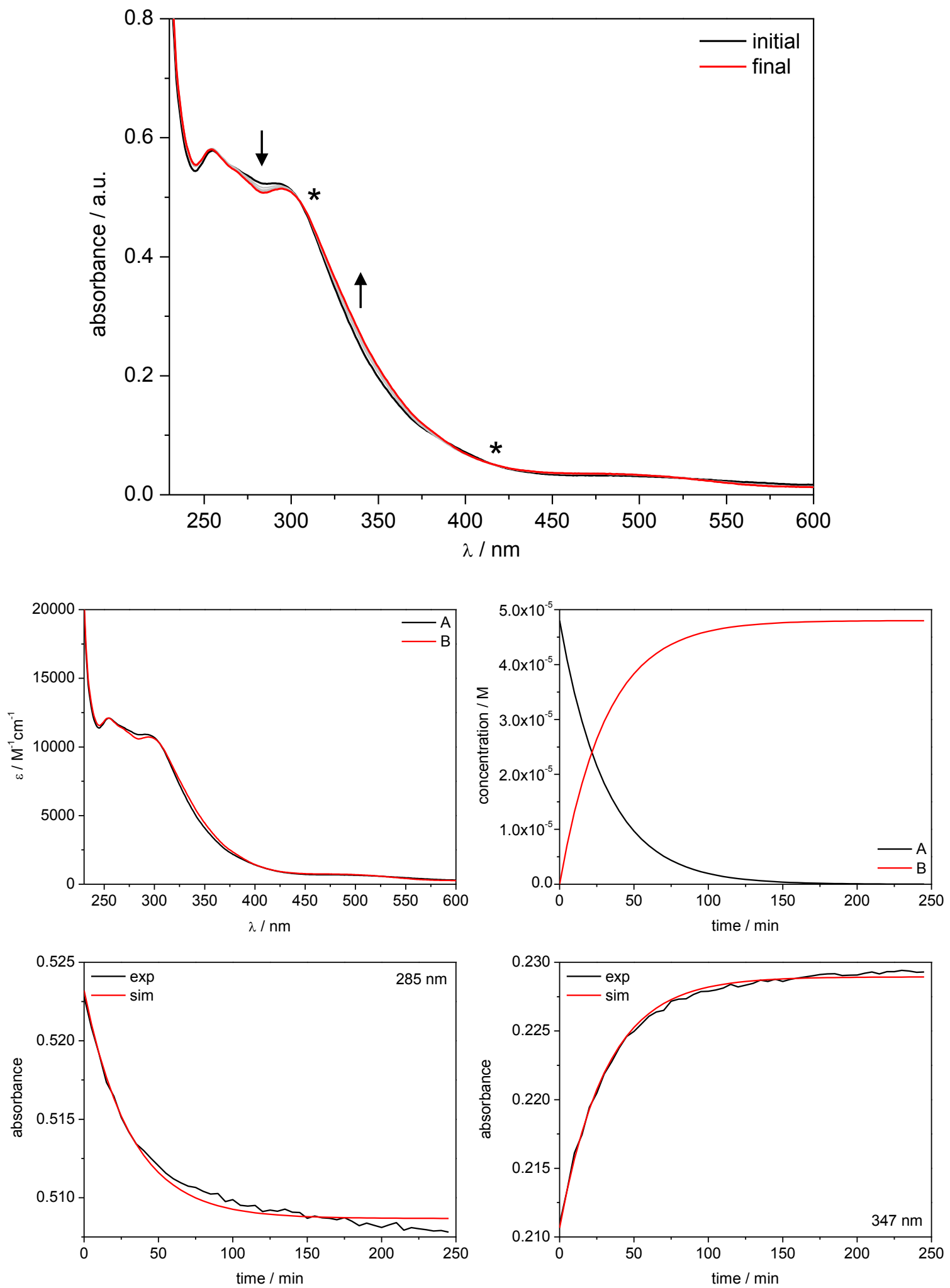

Figure 3.21: Exchange kinetics of $3^{\mathrm{H} 20}$ in the $\mathrm{Ru}^{\mathrm{III}} \mathrm{Ru}^{\mathrm{III}}$ state with $\mathrm{MeCN}$ in $0.1 \mathrm{M}$ triflic acid (pH 1 ) at $25^{\circ} \mathrm{C}$ (c $=5.0 \cdot 10^{-5} \mathrm{M}$ ). Large graph: UV/vis spectral changes upon addition of MeCN ( 1500 eq, initial spectrum: black, final: red). The asterisks denote isosbestic points. Small graphs: Specfit analysis consisting of the calculated UV/vis spectra (middle left, initial species: A, final species: B), the calculated species distribution (middle right), as well as the experimental (black) and simulated (red) data for selected wavelengths (bottom left: $285 \mathrm{~nm}$, bottom right: $347 \mathrm{~nm})$. 
Table 3.8: Rate constants for the substitution reaction of $3^{\mathrm{H} 2 \mathrm{O}}$ in $0.1 \mathrm{M}$ aqueous $\mathrm{HClO}_{4}$ (pH 1) with $\mathrm{MeCN}$. $^{\mathrm{a}}$ Prior to the addition of MeCN, the complex was oxidized to $\mathrm{Ru}^{\mathrm{III}} \mathrm{Ru}^{\mathrm{III}}$ through the addition of CAN.

\begin{tabular}{ccccc} 
entry & oxidation state & temperature $/{ }^{\circ} \mathrm{C}$ & rate constant $/ \mathrm{s}^{-1}$ & average $\emptyset / \mathrm{s}^{-1}$ \\
\hline \hline 1 & $\mathrm{Ru}^{\mathrm{III}} \mathrm{Ru}^{\mathrm{III}}$ & 5 & $(0.964 \pm 0.080) \cdot 10^{-4}$ & $(0.983 \pm 0.064) \cdot 10^{-4}$ \\
2 & $\mathrm{Ru}^{\mathrm{III}} \mathrm{Ru}^{\mathrm{III}}$ & 5 & $(1.001 \pm 0.048) \cdot 10^{-4}$ & \\
3 & $\mathrm{Ru}^{\mathrm{III}} \mathrm{Ru}^{\mathrm{III}}$ & 10 & $(2.197 \pm 0.191) \cdot 10^{-4}$ & $(2.340 \pm 0.198) \cdot 10^{-4}$ \\
4 & $\mathrm{Ru}^{\mathrm{III}} \mathrm{Ru}^{\mathrm{III}}$ & 10 & $(2.483 \pm 0.204) \cdot 10^{-4}$ & \\
5 & $\mathrm{Ru}^{\mathrm{III}} \mathrm{Ru}{ }^{\mathrm{III}}$ & 15 & $(2.978 \pm 0.227) \cdot 10^{-4}$ & \\
6 & $\mathrm{Ru}^{\mathrm{III}} \mathrm{Ru}^{\mathrm{III}}$ & 15 & $(3.620 \pm 0.492) \cdot 10^{-4}$ & \\
7 & $\mathrm{Ru}^{\mathrm{III}} \mathrm{Ru}^{\mathrm{III}}$ & 23 & $(6.735 \pm 0.122) \cdot 10^{-4}$ & \\
8 & $\mathrm{Ru}^{\mathrm{III}} \mathrm{Ru}^{\mathrm{III}}$ & 23 & $(5.338 \pm 0.244) \cdot 10^{-4}$ & $(5.854 \pm 0.195) \cdot 10^{-4}$ \\
9 & $\mathrm{Ru}^{\mathrm{III}} \mathrm{Ru}^{\mathrm{III}}$ & 23 & $(5.490 \pm 0.219) \cdot 10^{-4}$ & \\
\hline
\end{tabular}

a $\sim 1500$ eq MeCN were added
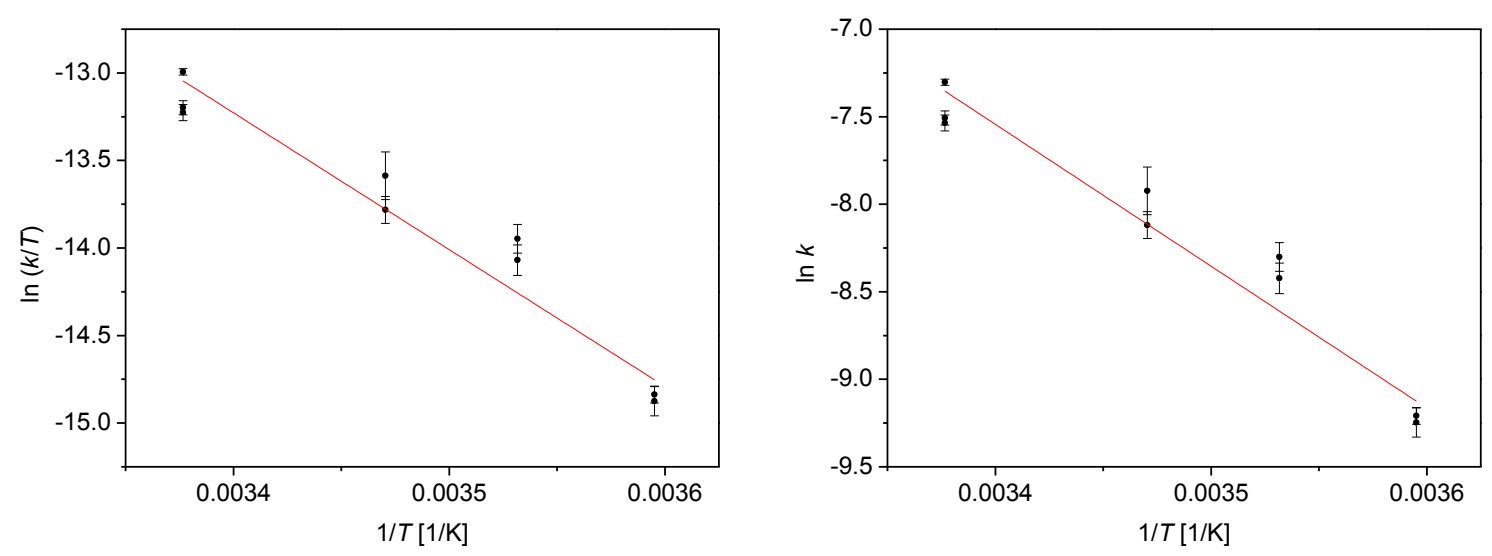

Figure 3.22: Eyring (left) and Arrhenius (right) plots for the substitution reaction of $3^{\mathbf{H} 20}$ in $0.1 \mathrm{M}$ aqueous $\mathrm{HClO}_{4}$ $(\mathrm{pH}$ 1) with MeCN at various temperatures.

\subsubsection{DMSO vs. Aquo Bridge: What is the Active Species?xiii}

Although complexes $3^{\text {dmso }}$ and $\mathbf{3}^{\mathbf{H} 20}$ show similar catalytic behavior under electrochemical (Figure 3.8) and chemical (Figure 3.16) conditions, their profiles are not identical, suggesting that distinct active species are responsible for catalysis. For dinuclear complexes of types $\mathbf{V}$, VI and $\mathbf{X}$ with exogenous bridging ligands such as acetate or carbonate (Scheme 3.2 and Table 3.4), substitution of the bridge by water is necessary before catalysis. The resulting aquo ligands enable PCET processes that lead to the required high oxidation state $\mathrm{Ru}$-oxo groups, which will then be involved in the $0-0$ bond formation step. Thus, we expected that compound $3^{\text {dmso }}$ would convert into $3^{\mathbf{H} 20}$ before the catalytic process started.

In order to gain insight into the active species derived from $\mathbf{3}^{\mathrm{dmss}}$, UV/vis-SEC measurements were carried out. In these experiments, the potential was successively increased from 0.36 to $1.06 \mathrm{~V}$ and further to $1.56 \mathrm{~V}$, corresponding to $\mathrm{Ru}^{\mathrm{II}} \mathrm{Ru}^{\mathrm{II}} \rightarrow \mathrm{Ru}^{\mathrm{III}} \mathrm{Ru}^{\mathrm{III}} \rightarrow$ catalysis, whilst monitoring the UV/vis spectra, and then reversed. Specifically of interest is a comparison of the first and last UV/vis spectrum of this series. Importantly, the UV/vis spectra of compounds $3^{\text {dmso }}$ and $3^{\mathbf{H} 20}$ differ significantly from

xiii This section was taken from Jana Lücken, Carolina Gimbert-Suriñach, Jordi Benet-Buchholz, Antoni Llobet, Franc Meyer, "Diruthenium Complexes of a Pyrazolate/Carboxylate Hybrid Ligand for Chemically, Electro- and Photo-Driven Catalytic Water Oxidation", under revision. 
one another in the $\mathrm{Ru}^{\text {IIRuII }}$ state (see Section 3.2 ) so that an exchange of the DMSO moiety with water after catalysis can easily be detected. Whereas complex $3^{\text {dmso }}$ possesses local absorption maxima at 275 and $346 \mathrm{~nm}$, as well as a shoulder at $423 \mathrm{~nm}$ (Figure 3.23, top left, black spectrum), complex $3^{\mathbf{H 2 0}}$ possesses local absorption maxima at 282 and $393 \mathrm{~nm}$, as well as a shoulder at $352 \mathrm{~nm}$ (Figure 3.23, bottom left, black). Upon oxidation of $\mathbf{3}^{\mathrm{dmso}}$ at $1.06 \mathrm{~V}$, a potential at which the complex reaches its $\mathrm{Ru}^{\text {III }} \mathrm{Ru}^{\text {III }}$ state, the MLCT transitions $(346 / 423 \mathrm{~nm})$ largely vanish, and new bands appear at $301 \mathrm{~nm}$ and $537 \mathrm{~nm}$ (Figure 3.23, top left, red spectrum). Upon catalysis, the latter peak intensifies and new bands emerge at $263 \mathrm{~nm}$ with a shoulder at $294 \mathrm{~nm}$ as well as $409 \mathrm{~nm}$ (Figure 3.23, top left, grey spectrum). These spectral changes are emphasized by the $\Delta$ absorbance traces with respect to the $\mathrm{Ru}^{\mathrm{II}} \mathrm{Ru}^{\mathrm{II}}$ spectrum (Figure 3.23, top right).
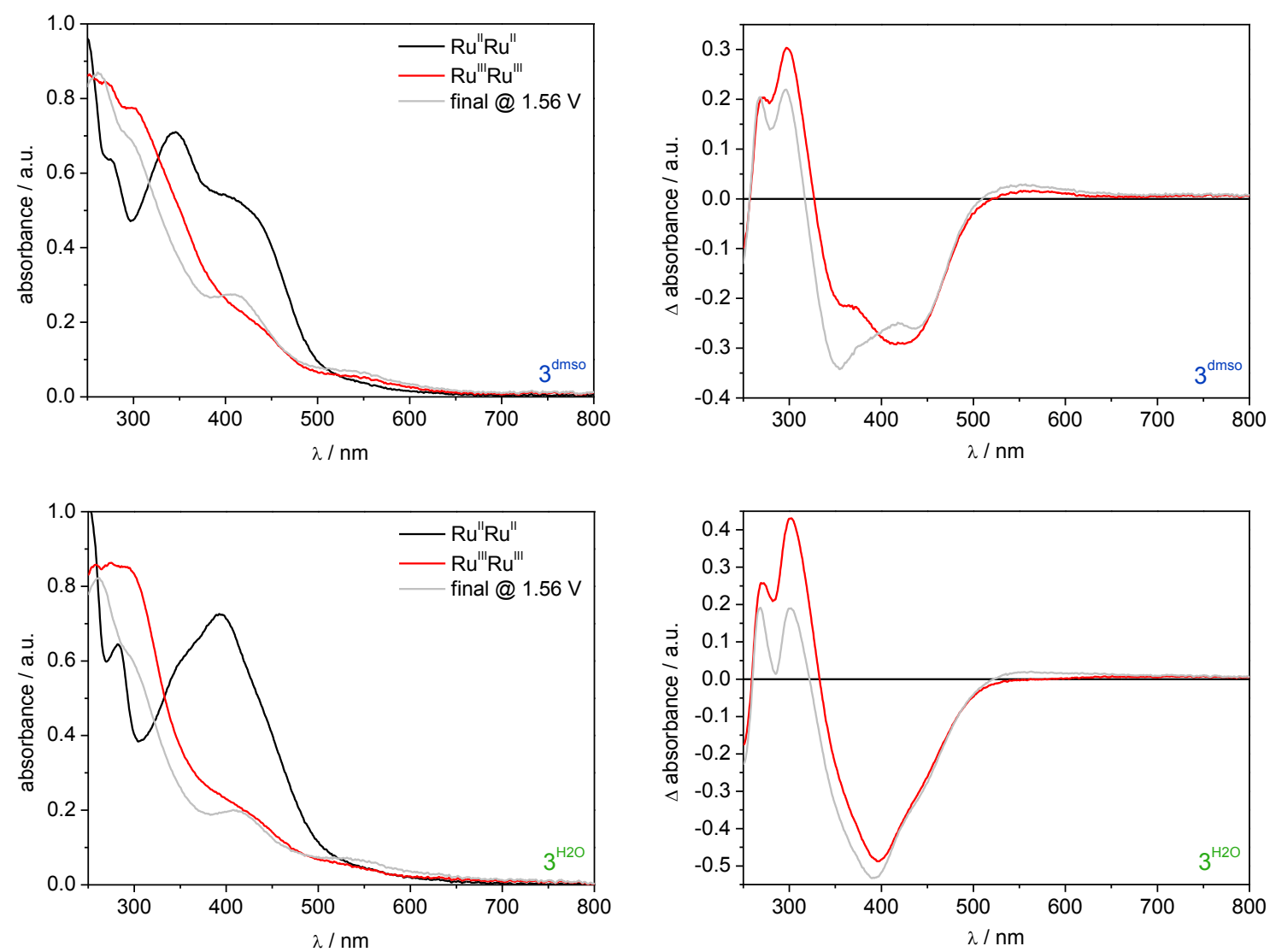

Figure 3.23: UV/vis-spectroscopic characterization of the oxidized species of $\mathbf{3}^{\text {dmso }}$ (top) and $\mathbf{3}^{\mathbf{H} 20}$ (bottom) in $0.1 \mathrm{M}$ triflic acid (pH 1). Left: UV/vis spectra in the $\mathrm{Ru}^{\mathrm{II}} \mathrm{Ru}^{\mathrm{II}}$ (black) and $\mathrm{Ru}^{\mathrm{III}} \mathrm{Ru}^{\mathrm{III}}$ (red) states as well as during catalysis (grey) at $0.36,1.06$ and $1.56 \mathrm{~V} v s$. NHE, respectively. Right: $\Delta$ absorbance of the spectra after oxidation at 1.06 (red) and $1.56 \mathrm{~V} v s$. NHE (grey) with respect to the spectrum before oxidation (baseline).

The potential was left in the catalytic regime for 10, 30 and 60 minutes, before reducing back at $0.36 \mathrm{~V}$. The resulting spectra in the $\mathrm{Ru}^{\mathrm{II}} \mathrm{Ru}^{\mathrm{II}}$ state are shown in black (after 10 minutes catalysis), dark grey (30) and light grey (60) in Figure 3.24 (left). Whereas after 10 and 30 minutes in catalysis and subsequent re-reduction the UV/vis spectra are nearly identical to the initial spectrum of $\mathbf{3}^{\mathrm{dmso}}$, there seems to be a mixture of both complexes $3^{\text {dmso }}$ and $3^{\text {H20 }}$ in solution after remaining at $1.56 \mathrm{~V}$ for 60 minutes. Overall, this suggests that the DMSO moiety either remains in the bridging pocket or reattaches after catalysis. In order to rule out the latter, the same experiment was conducted with $3^{\mathbf{H} 20}$ in the presence of 10 eq DMSO; however, the characteristic signatures of the DMSO complex $3^{\text {dmso }}$ were not observed in the final UV/vis spectrum (Figure 3.25). These results strongly suggest that the DMSO remains in the bimetallic pocket during water oxidation mediated by $3^{\text {dmso }}$ (at least for some time) and that $\mathbf{3}^{\mathrm{dmso}}$ does not convert to catalyst $\mathbf{3}^{\mathbf{H} 20}$, in contrast to what occurs for related complexes $\mathbf{V}, \mathbf{V I}$ and $\mathbf{X}$. 

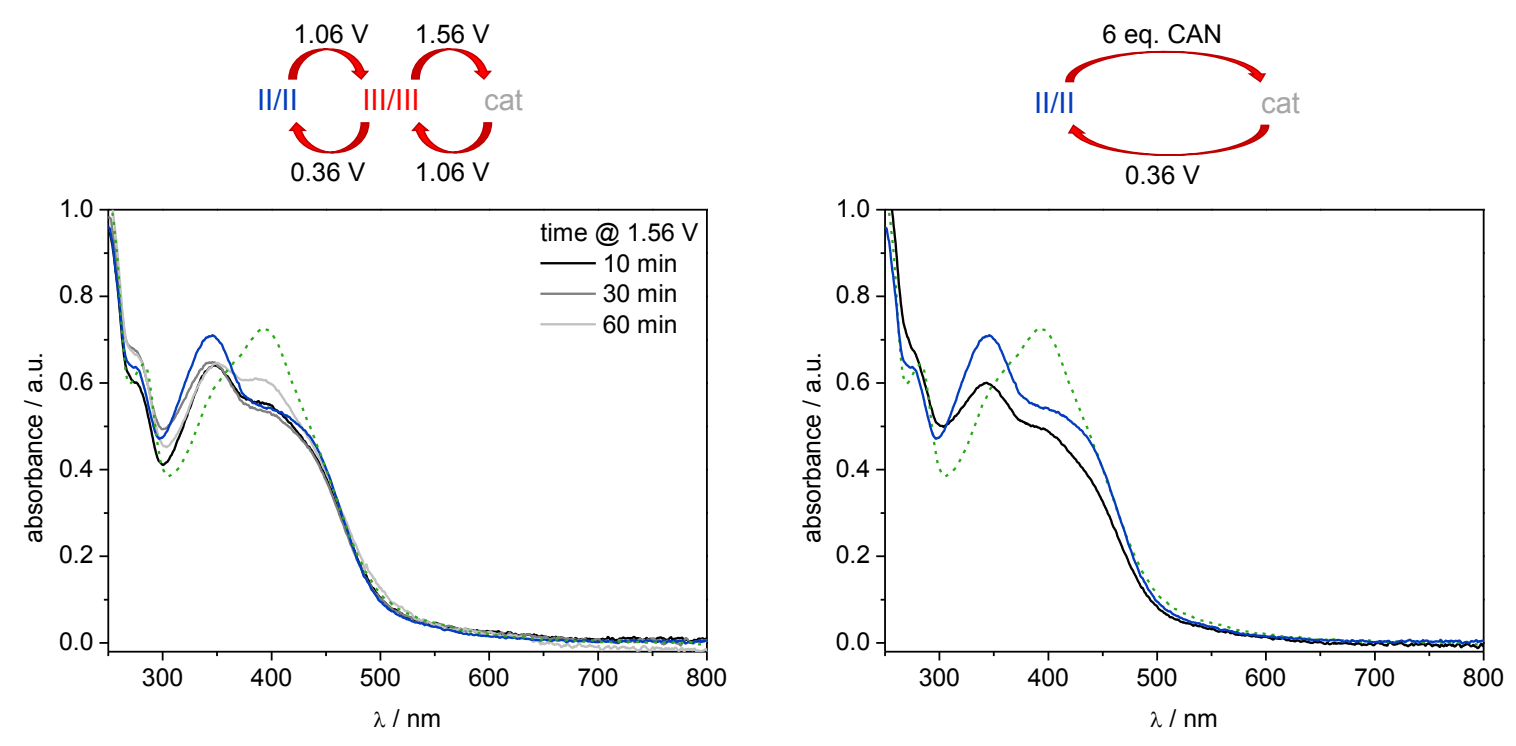

Figure 3.24. UV/vis-spectroelectrochemical analysis of $\mathbf{3}^{\mathrm{dmso}}$ in $0.1 \mathrm{M}$ aqueous triflic acid ( $\left.\mathrm{pH} 1\right)\left(\mathrm{c}=2.86 \cdot 10^{-4} \mathrm{M}\right)$. Left: Comparison of the final UV/vis-spectra at $0.36 \mathrm{~V}$ vs. NHE after catalysis for 10 (black), 30 (grey) and 60 (light grey) minutes with the spectroscopic signatures of complexes $\mathbf{3}^{\mathrm{dmso}}$ (solid blue) and $\mathbf{3}^{\mathbf{H} 2 \mathrm{O}}$ (dashed green) in their $\mathrm{Ru}^{\mathrm{II}} \mathrm{Ru}^{\mathrm{II}}$ state. Right: Comparison of the final UV/vis spectrum after chemical oxidation with 6 eq CAN (black) with complexes $\mathbf{3}^{\text {dmso }}$ (solid blue) and $\mathbf{3}^{\mathrm{H} 20}$ (dashed green).

The same conclusion is reached under chemical water oxidation catalysis conditions using a solution of $3^{\mathrm{dmso}}$ in the presence of 6 eq CAN, thus ensuring one turnover. After chemical oxidation, the solution was reduced electrochemically at $0.36 \mathrm{~V}$, corresponding to the potential regime for the $\mathrm{Ru}^{\mathrm{II}} \mathrm{Ru}^{\mathrm{II}}$ species. As can be seen in the right graph of Figure 3.24, the final UV/vis spectrum (black) shows the typical characteristics of the DMSO complex and, thus, suggests that also during chemical water oxidation the DMSO moiety remains within the bimetallic pocket. Interestingly, the mononuclear complex [Ru(bda)(dmso) $\left.\left(\mathrm{L}^{\mathrm{im}}\right)\right]$ ( $\mathrm{L}^{\mathrm{im}}$ : 5 -bromo- $N$-methylimidazole) with a terminally bound DMSO moiety in the axial position was also shown to efficiently catalyze the oxidation of water to dioxygen. A significant decrease in the catalytic activity was observed when the DMSO group was exchanged with

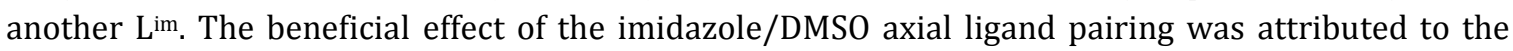
ability of the ligands to enable unhindered coupling between terminal oxygen atoms in an I2M mechanism, as opposed to the complex with two axial imidazoles.[255]
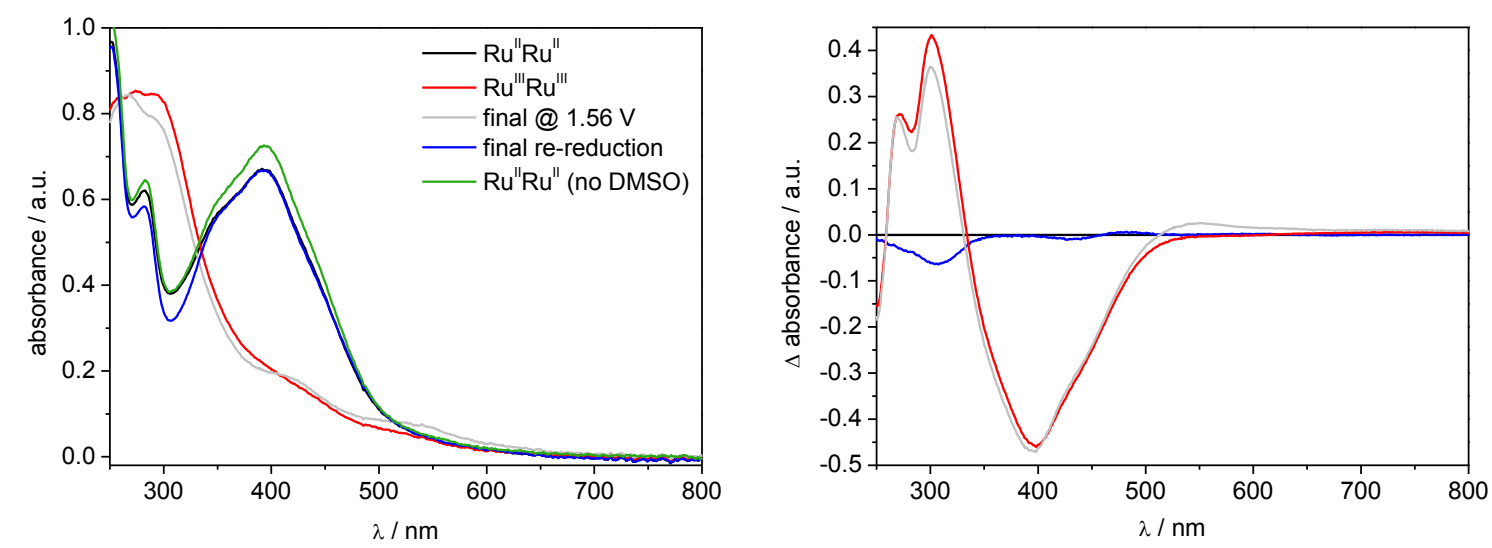

Figure 3.25: UV/vis-spectroelectrochemical characterization of $3^{\mathbf{H} 20}$ in $0.1 \mathrm{M}$ triflic acid (pH 1) in the presence of 10 eq DMSO. Left: UV/vis spectra in the $\mathrm{Ru}^{\mathrm{II}} \mathrm{Ru}^{\mathrm{II}}$ (black) and $\mathrm{Ru}^{\mathrm{III}} \mathrm{Ru}^{\mathrm{III}}$ (red) states as well as during catalysis (grey) at $0.36,1.06$ and $1.56 \mathrm{~V}$ vs. NHE, respectively, as well as the final UV/vis spectrum after re-reduction (blue) and the UV/vis spectrum of $\mathbf{3}^{\mathbf{H} 20}$ in the $\mathrm{Ru}^{\mathrm{II}} \mathrm{Ru}^{\mathrm{II}}$ state without dmso present (green). The spectra demonstrate that the DMSO does not attach in the in,in-position after catalysis. Right: $\Delta$ absorbance of the spectra after oxidation at 1.06 (red) and $1.56 \mathrm{~V} v$ s. NHE (grey), as well as after re-reduction (blue) with respect to the spectrum before oxidation (baseline). 
If the DMSO bridge in $3^{\text {dmso }}$ does not leave during catalytic turnover, there are only three possibilities to generate the active $\mathrm{Ru}$-aquo group: (i) substitution of a carboxylate group of the bridging $\left(\mathrm{L}^{1}\right)^{3-}$ ligand (A), (ii) direct coordination of an aquo ligand to one of the ruthenium ions, generating a seven coordinated (CN7) metal ion (B), or (iii) partial decoordination of the DMSO moiety in the bimetallic cleft (C) (Scheme 3.5). Interestingly, the first option leaves a dangling carboxylate that may assist a putative water nucleophilic attack in the $\mathrm{O}-\mathrm{O}$ bond formation step, as reported before for mononuclear complexes. ${ }^{[219]}$ The aquo substitution reaction must take place at high oxidation states (either $\mathrm{Ru}^{\mathrm{III}} \mathrm{Ru}^{\mathrm{III}}$ or $\mathrm{Ru}^{\mathrm{III}} \mathrm{Ru}^{\mathrm{IV}}$ ) because the starting complex $3^{\mathrm{dmso}}$ in the oxidation state $\mathrm{Ru}^{\mathrm{II}} \mathrm{Ru}^{\mathrm{II}}$ is stable under aqueous conditions as described in the synthetic protocol. The two former types of active species with CN6 and CN7 have previously been observed for WOCs containing carboxylate groups in the first and second coordination sphere, ${ }^{[104,211,218,256-258]}$ but the data presently available does not allow to unravel which of the scenarios is operative for $3^{\mathrm{dms}}$. In all of these scenarios, however, water oxidation mediated by $\mathbf{3}^{\mathrm{dms}}$ takes place at a single ruthenium center. Hence, in view of the first-order dependence of the initial rate on complex concentration (Figure 3.15), the mechanism for $3^{\text {dmso }}$ should be WNA.
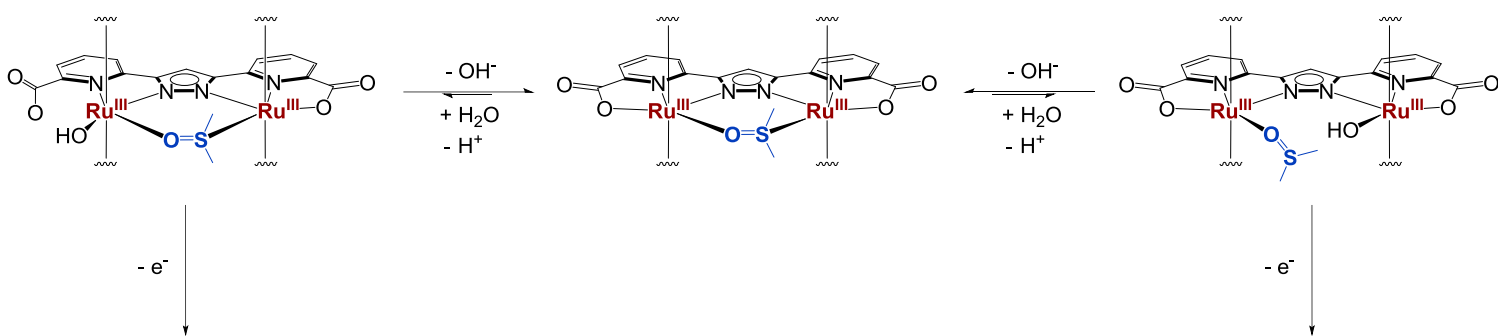

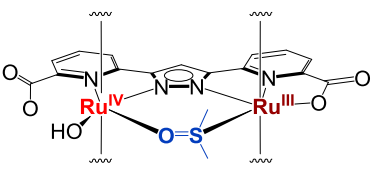

A
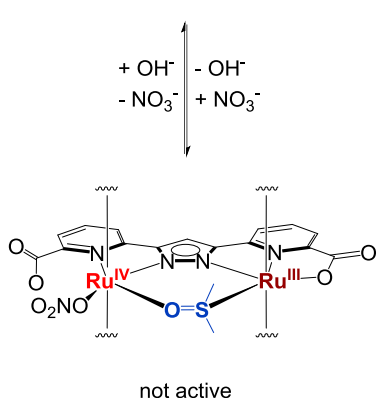

not active

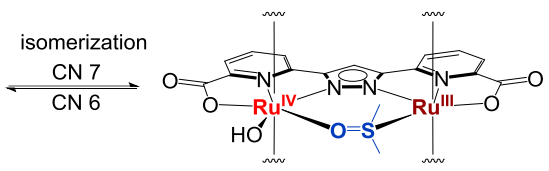

B
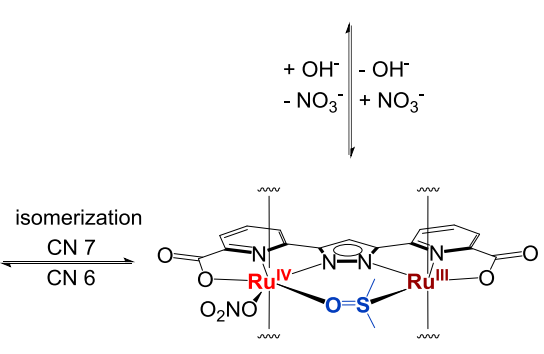

not active

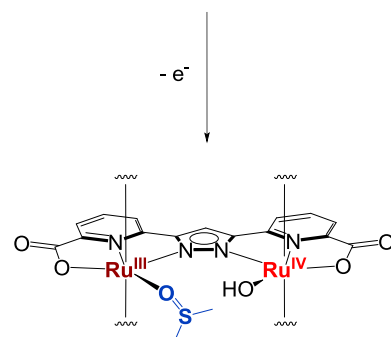

C
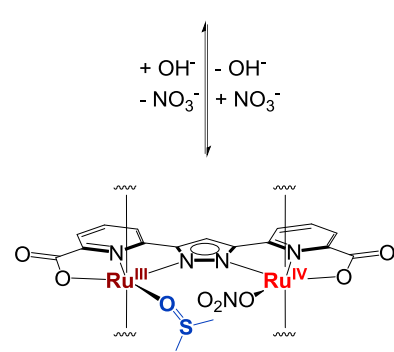

not active

Scheme 3.5: Illustration of the proposed formation of the three possible catalytic species derived from $3^{\text {dmso }}$ with CN6 (dangling carboxylate, A, or partial decoordination of the DMSO moiety, C) and CN7 (B) (tentatively shown for the $\mathrm{Ru}^{\mathrm{III}} \mathrm{Ru}$ III state), as well as their products after anation (bottom).

\subsection{Light-Driven Water Oxidation Catalysis}

The ability of complexes $3^{\mathrm{dmso}}$ and $\mathbf{3}^{\mathrm{H} 20}$ to photochemically oxidize water to dioxygen was analyzed using $\left[\mathrm{Ru}\left((\mathrm{COOEt})_{2} \text { bpy }\right)_{2} \text { (bpy) }\right]^{2+}(\mathrm{RuP} 1)$ as photosensitizer and sodium persulfate as SEA (see Section 1.3.4). It was not possible to use the homoleptic $\left[\mathrm{Ru}(\mathrm{bpy})_{3}\right]^{2+}(\mathrm{RuP} 3)$ photosensitizer, as it has an insufficient redox potential to induce water oxidation $\left(E^{0}=1.26 \mathrm{vs} .1 .62 \mathrm{~V}\right.$ in phosphate buffer at $\mathrm{pH} 7$

xiv This section was adapted from Jana Lücken, Carolina Gimbert-Suriñach, Jordi Benet-Buchholz, Antoni Llobet, Franc Meyer, "Diruthenium Complexes of a Pyrazolate/Carboxylate Hybrid Ligand for Chemically, Electro- and Photo-Driven Catalytic Water Oxidation", under revision. 
for RuP3 and RuP1, respectively).[231] In a typical experiment, $\mathrm{RuP} 1\left(2 \cdot 10^{-4} \mathrm{M}\right)$ and $\mathrm{Na}_{2} \mathrm{~S}_{2} \mathrm{O}_{8}$ (SEA, $10^{-2} \mathrm{M}$ ) were dissolved in $\mathrm{H}_{2} \mathrm{O}$ and added to a dark chamber reaction vessel (total volume $2 \mathrm{~mL}$, cooling jacket at $\left.25^{\circ} \mathrm{C}\right)$. After addition of the catalyst $\left(2 \cdot 10^{-6} \mathrm{M}\right)$, the solution was degassed and the chamber closed, thereby removing all headspace. Once a stable baseline was reached, the solution was irradiated to initiate water oxidation. The produced dioxygen was measured in the liquid phase by an integrated Clark-type electrode. A typical trace for consecutive runs is given in Figure 3.26 for complex $3^{\mathrm{dms}}$. Once a plateau was reached, the solution was degassed and irradiated once again. The second run mostly produced around one sixth of the amount of dioxygen measured in the first run. Resumed dioxygen production after deaeration suggests that the produced dioxygen quenched the excited state of the photosensitizer and, thus, hindered oxidant formation, which is inevitable in a setup with no headspace. However, the much lower dioxygen yield in the second run indicates that other deactivation mechanisms are also operative, and accordingly no significant amount of dioxygen could be detected in a third run.

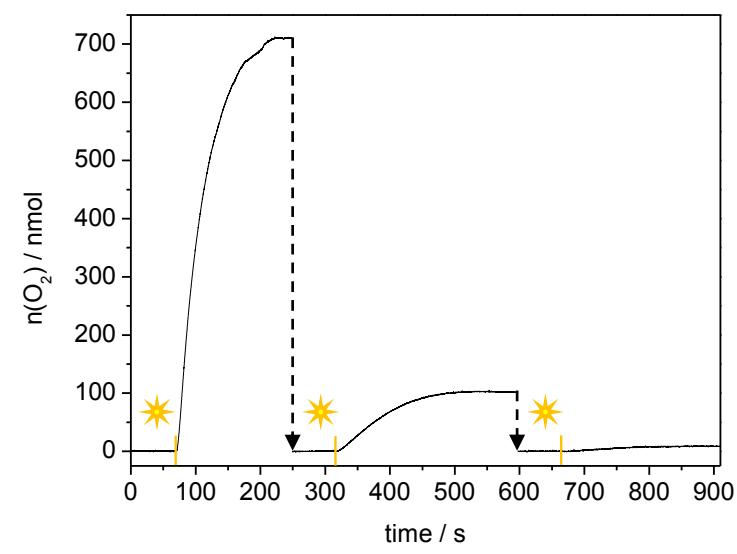

Figure 3.26. Dioxygen production monitored by a Clark-type electrode in the liquid phase during photo-driven water oxidation catalysis of $3^{\mathrm{dmso}}$ in $\mathrm{H}_{2} \mathrm{O}$ at $25^{\circ} \mathrm{C}$. The vertical yellow lines indicate the start of irradiation. The dashed arrows mark when the solution was degassed.

The photochemical reactions were performed in unbuffered aqueous conditions because poor activity was observed when working in either phosphate buffer $(\mathrm{pH} 7)$ or in aqueous $\mathrm{HBF}_{4}$ solution (pH 1 and $\mathrm{pH}$ 5.6). Thus, the $\mathrm{pH}$ of the catalytic mixture was determined after every measurement; values between $\mathrm{pH}$ 2.3-2.5 were received after the first run and $\mathrm{pH} 2.1-2.3$ after the second.

For both complexes $3^{\text {dmso }}$ and $3^{\text {H20 }}$ very similar traces of dioxygen production were observed reaching a plateau between $564-888 \mathrm{nmol}$ and $80-122 \mathrm{nmol}$ for the first and second run, respectively (values are not background-subtracted and do not consider the entries marked in red for $\mathbf{3}^{\mathbf{d m s o}}$; Table 3.10 and Table 3.11, and Figure 3.27, top). As shown in Table 3.9, irradiating a mixture of RuP1 and sodium persulfate without catalyst already leads to the formation of a significant amount of molecular oxygen ( $273 \mathrm{nmol}$ ). A possible reason for this has been described by Yoon and coworkers, who showed that not only $\left[\mathrm{Ru}(\mathrm{bpy})_{3}\right]^{2+}$ but also $\left[\mathrm{Ru}(\mathrm{bpy})_{3}\right]^{3+}$ is photoexcited under the given conditions. The photoexcited $\left[\mathrm{Ru}(\mathrm{bpy})_{3}\right]^{3+*}$ can equally react with $\mathrm{S}_{2} \mathrm{O}_{8}{ }^{2-}$ to produce an intermediate, which decomposes into catalytically active ruthenium $\mu$-oxo dimers under low $[\mathrm{RuP}]$ conditions $(<0.2 \mathrm{mM})$. In contrast, at higher $\left[\mathrm{Ru}(\mathrm{bpy})_{3}\right]^{2+}$ concentrations, catalytically inactive ruthenium $\mu$-oxo oligomers are formed. ${ }^{[259]}$ Overall, for the two consecutive catalytic runs, the total amount of produced dioxygen ranges between $380-683 \mathrm{nmol}$ (background-subtracted) for $3^{\text {dmso }}$ and $392-737 \mathrm{nmol}$ for $3^{\text {H20 }}$ leading to an average TON of 130 and 137, respectively. ${ }^{\mathrm{xv}}$

xv Please keep in mind that the powder sample of $3^{\mathbf{H 2 0}}$ contains significant amounts of $\mathrm{NaF}$ (see Section 3.1), which can influence the catalytic response. 
In order to elucidate what could eventually stop the evolution of $\mathrm{O}_{2}$, one or more variables were changed after the second run. Typically, the degradation of the dye due to nucleophilic attack at the bipyridine units ${ }^{[260]}$ or the decrease of the $\mathrm{pH}$ after water oxidation are the limiting factors. In fact, the UV/vis spectra before (solid) and after (dashed) photoinduced water oxidation clearly show that the significant features of the $\left[\mathrm{Ru}(\mathrm{bpy})_{3}\right]^{2+}$-type complex - the MLCT $\left(\mathrm{d} \rightarrow \pi^{*}\right)$ transitions at 440 and $483 \mathrm{~nm}$, as well as the metal-centered transitions at 364 and $397 \mathrm{~nm}$ and the ligand-centered $\left(\pi \rightarrow \pi^{*}\right)$ transition at $309 \mathrm{~nm}^{[261]}$ - have disappeared after the photocatalytic experiment, indicating decomposition of the dye (Figure 3.27, bottom). However, neither the addition of RuP1, SEA (half an equivalent) or even catalyst (one equivalent), as well as combinations of these, nor the increase of the $\mathrm{pH}$ with an aqueous $\mathrm{NaOH}$ solution successfully restored the catalytic activity of the reaction mixture (Table 3.10 and Table 3.11, $3^{\text {rd }}$ run). Even though in some cases renewed dioxygen formation was observed (Table 3.10, entries 6 and 7), the overall picture suggests that it does not result from catalytic turnover of the complex but that other mechanisms are on hand as described above (also the addition of RuP1 and SEA to the blank mixture re-induces dioxygen production; Table 3.9, entry 2). This suggests that it is not the degradation of the catalyst ( $3^{\text {dmso }}$ or $3^{\mathbf{H 2 0}}$ ) that stops $\mathrm{O}_{2}$ evolution, but rather some sort of inhibition of the photocatalytic system. This could result from the increase in the ionic strength during water oxidation (for every turnover, four molecules of sulfate are produced; see Section 1.3.4), which can affect the quenching of the excited state of the dye by persulfate, as well as promote anation of the WOC, as has been observed for other catalysts. ${ }^{[231,262]}$
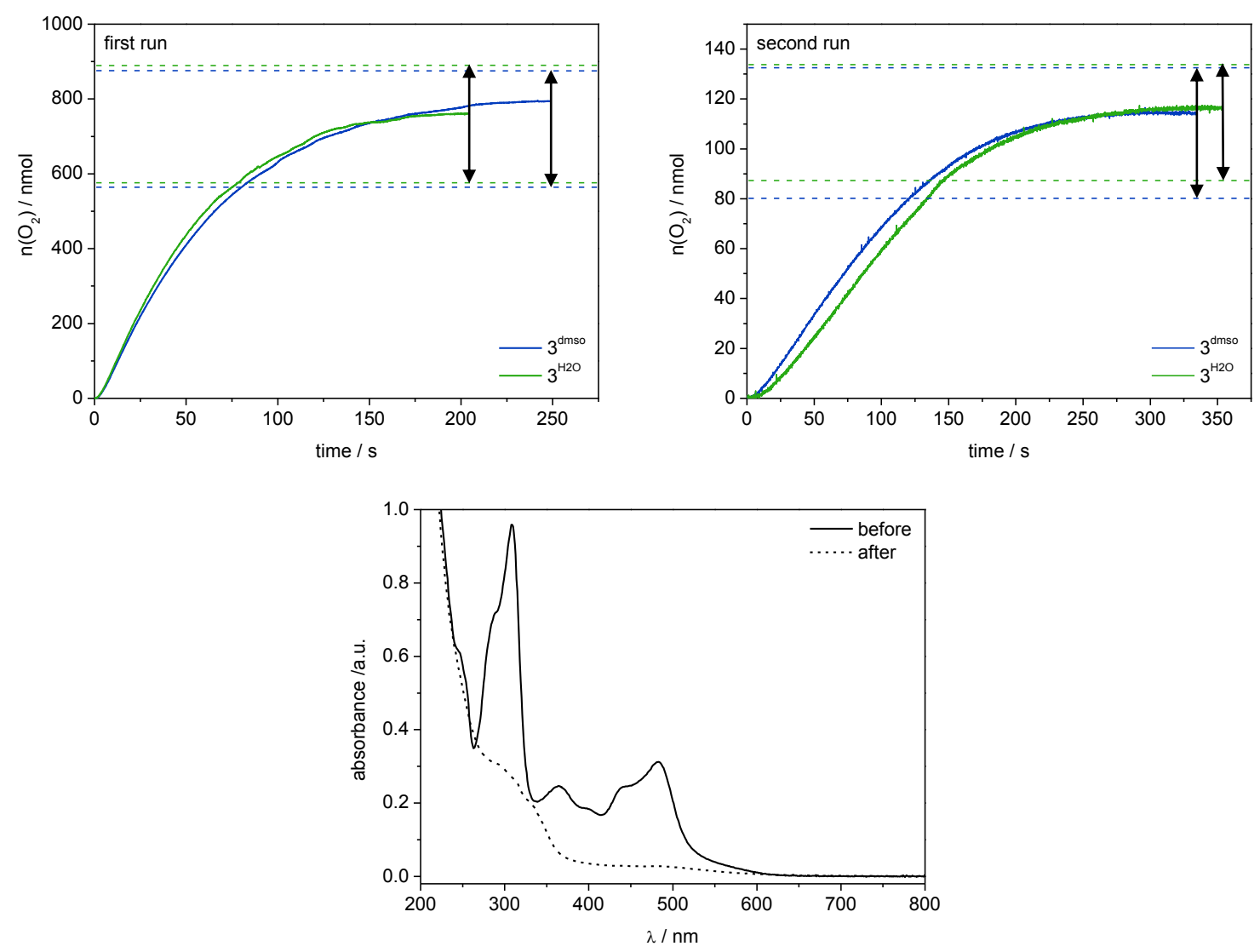

Figure 3.27: Photocatalytic water oxidation experiments. Top: Oxygen production by $3^{\text {dmso }}$ (blue) and $\mathbf{3}^{\mathbf{H} 20}$ (green) in the first (left) and second run (right) monitored by a Clark-type electrode. The dashed lines frame the range that was obtained over multiple experiments. Bottom: UV/vis spectra of the catalytic mixture before (solid) and after (dashed) photoinduced water oxidation. 
Table 3.9: Dioxygen evolution monitored upon irradiation of the RuP 1 and SEA mixture.a Two runs were performed undisturbed, degassing after the first. After the second run, one or more components were added prior to degassing ( $3^{\text {rd }}$ run).

\begin{tabular}{|c|c|c|c|c|}
\hline \multicolumn{5}{|c|}{ blanks } \\
\hline \multirow{2}{*}{ entry } & \multicolumn{3}{|c|}{$\mathrm{O}_{2} / \mathrm{nmol}$} & \multirow{2}{*}{$\emptyset / \mathrm{nmol}$} \\
\hline & $1^{\text {st }}$ run & $2^{\text {nd }}$ run & $3^{\text {rd }}$ run $^{\mathrm{b}}$ & \\
\hline \multirow{2}{*}{1} & 198 & 73 & RuP1 & \multirow{4}{*}{273} \\
\hline & & & 85 & \\
\hline \multirow{2}{*}{2} & 206 & 69 & \multirow{2}{*}{$\begin{array}{c}\text { RuP1 / SEA } \\
117\end{array}$} & \\
\hline & & & & \\
\hline
\end{tabular}

a conditions: [RuP1] $2 \cdot 10^{-4} \mathrm{M},\left[\mathrm{Na}_{2} \mathrm{~S}_{2} \mathrm{O}_{8}\right] 10^{-2} \mathrm{M}$

$\mathrm{b}$ one or more components added (see table); RuP1/SEA: half an equivalent

Table 3.10: Light-driven water oxidation catalysis experiments in the presence of $3^{\text {dmso }}$ a Two runs were performed undisturbed, degassing after the first. After the second run, one or more components were added prior to degassing in an attempt to restore the catalytic activity ( $3^{\text {rd }}$ run).

\begin{tabular}{|c|c|c|c|c|c|c|}
\hline \multicolumn{7}{|c|}{$3^{\text {dmso }}$} \\
\hline \multirow{2}{*}{ entry } & \multicolumn{3}{|c|}{$\mathrm{O}_{2} / \mathrm{nmol}$} & \multirow{2}{*}{$\begin{array}{l}\text { cat / } \\
\text { nmol }\end{array}$} & \multirow{2}{*}{ TONb } & \multirow{2}{*}{$\varnothing$} \\
\hline & $1^{\text {st }}$ run & $2^{\text {nd }}$ run & $3^{\text {rd }}$ run $^{\mathrm{c}}$ & & & \\
\hline \multirow{2}{*}{1} & 710 & 103 & \multirow{2}{*}{$\begin{array}{c}\text { degassed }^{d} \\
9\end{array}$} & \multirow{2}{*}{3.989} & \multirow{2}{*}{135} & \multirow{20}{*}{130} \\
\hline & \multicolumn{2}{|c|}{$813 / 540^{b}$} & & & & \\
\hline \multirow{2}{*}{2} & 849 & 80 & \multirow{2}{*}{$\begin{array}{c}\mathrm{pH}=6.18^{\mathrm{e}} \\
1\end{array}$} & \multirow{2}{*}{3.989} & \multirow{2}{*}{164} & \\
\hline & \multicolumn{2}{|c|}{$929 / 656^{b}$} & & & & \\
\hline \multirow{2}{*}{3} & 1209 & 78 & RuP1 & \multirow{2}{*}{3.989} & \multirow{2}{*}{254} & \\
\hline & \multicolumn{2}{|c|}{$1287 / 1014^{b}$} & 65 & & & \\
\hline \multirow{2}{*}{4} & 580 & 89 & SEA & \multirow{2}{*}{4.003} & \multirow{2}{*}{99} & \\
\hline & \multicolumn{2}{|c|}{$669 / 396^{b}$} & 13 & & & \\
\hline \multirow{2}{*}{5} & 568 & 88 & cat & \multirow{2}{*}{4.003} & \multirow{2}{*}{96} & \\
\hline & \multicolumn{2}{|c|}{$656 / 383^{b}$} & 22 & & & \\
\hline \multirow{2}{*}{6} & 822 & 134 & \multirow{2}{*}{$\begin{array}{c}\text { RuP1 / SEA / cat } \\
216\end{array}$} & \multirow{2}{*}{4.003} & \multirow{2}{*}{171} & \\
\hline & & & & & & \\
\hline \multirow{2}{*}{7} & 1603 & 188 & RuP1 / SEA & 4003 & 379 & \\
\hline & & $18^{\mathrm{b}}$ & & 4.003 & $3 / 9$ & \\
\hline 8 & 564 & 89 & - & 4004 & 95 & \\
\hline 8 & & & - & 4.004 & 95 & \\
\hline 9 & 684 & 115 & - & 4.004 & 131 & \\
\hline & & & - & & & \\
\hline 10 & 794 & 85 & - & 4004 & 151 & \\
\hline 10 & & & - & & 101 & \\
\hline
\end{tabular}

a conditions: [cat] $2 \cdot 10^{-9} \mathrm{M},[\mathrm{RuP} 1] 2 \cdot 10^{-4} \mathrm{M},\left[\mathrm{Na}_{2} \mathrm{~S}_{2} \mathrm{O}_{8}\right] 10^{-2} \mathrm{M}$; the entries marked in red showed extremely high activity in the $1^{\text {st }}$ run in comparison to the others and were not considered for the average

b background subtracted

c one or more components added (see table); cat: one equivalent, RuP1/SEA: half an equivalent

d no component added - just degassed

e $\mathrm{pH}$ adjusted to $6.18\left(3^{\mathrm{dmso}}\right) / 5.98\left(3^{\mathrm{H} 20}\right)$ with aqueous $\mathrm{NaOH}$ 
Table 3.11: Light-driven water oxidation catalysis experiments in the presence of $\mathbf{3}^{\mathbf{H} 20}$. $\mathbf{a}$ Two runs were performed undisturbed, degassing after the first. After the second run, one or more components were added prior to degassing in an attempt to restore the catalytic activity (3 $3^{\text {rd }}$ run).

\begin{tabular}{|c|c|c|c|c|c|c|}
\hline \multicolumn{7}{|c|}{$3^{\mathrm{H} 2 \mathrm{O}}$} \\
\hline entry & \multicolumn{3}{|c|}{$\mathrm{O}_{2} / \mathrm{nmol}$} & $\begin{array}{l}\text { cat / } \\
\text { nmol }\end{array}$ & $\mathrm{TON}^{\mathrm{b}}$ & $\varnothing$ \\
\hline \multirow{2}{*}{1} & 651 & 114 & \multirow{2}{*}{$\begin{array}{c}\text { degassed }^{\mathrm{d}} \\
36 \\
\end{array}$} & \multirow{2}{*}{3.985} & \multirow{2}{*}{123} & \multirow{10}{*}{137} \\
\hline & & & & & & \\
\hline \multirow{2}{*}{2} & 575 & 90 & \multirow{2}{*}{$\begin{array}{c}\mathrm{pH}=5.98^{\mathrm{e}} \\
0\end{array}$} & \multirow{2}{*}{3.985} & \multirow{2}{*}{98} & \\
\hline & & & & & & \\
\hline \multirow{2}{*}{3} & 888 & 122 & RuP1 & \multirow{2}{*}{3.985} & \multirow{2}{*}{185} & \\
\hline & & & 88 & & & \\
\hline \multirow{2}{*}{4} & 759 & 116 & SEA & \multirow{2}{*}{3.985} & \multirow{2}{*}{151} & \\
\hline & & & 16 & & & \\
\hline \multirow{2}{*}{5} & 697 & 87 & cat & \multirow{2}{*}{3.985} & \multirow{2}{*}{128} & \\
\hline & & & 17 & & & \\
\hline
\end{tabular}

a conditions: [cat] $2 \cdot 10^{-9} \mathrm{M},[\mathrm{RuP} 1] 2 \cdot 10^{-4} \mathrm{M},\left[\mathrm{Na}_{2} \mathrm{~S}_{2} \mathrm{O}_{8}\right] 10^{-2} \mathrm{M}$; the entries marked in red showed extremely high activity in the $1^{\text {st }}$ run in comparison to the others and were not considered for the average

b background subtracted

${ }^{\mathrm{c}}$ one or more components added (see table); cat: one equivalent, RuP1/SEA: half an equivalent

d no component added - just degassed

e $\mathrm{pH}$ adjusted to $6.18\left(3^{\mathrm{dmso}}\right) / 5.98\left(3^{\mathrm{H} 20}\right)$ with aqueous $\mathrm{NaOH}$

\subsection{Summary and Conclusion ${ }^{x v i}$}

The dinucleating compartmental ligand used in this study combines favorable components of ruthenium-based water oxidation catalysts, viz. (i) a rugged central pyrazolate bridge that preorganizes two metal ions in close proximity and (ii) peripheral carboxylate groups that lower the overall charge and may potentially engage in H-bonding interactions assisting water nucleophilic attack in the $\mathrm{O}-\mathrm{O}$ bond formation step. Furthermore, this study highlights the advantages of such complexes that are composed of a dinucleating equatorial scaffold and monodentate axial ligands, as the exchangeable axial ligands offer tunability of the electronic properties and solubility of the catalyst by using pyridines with different substituents. In this vein, the axial coordination of pyridine sulfonate instead of unsubstituted parent pyridine has rendered complex $\mathbf{X}$ highly soluble in aqueous media over a wide $\mathrm{pH}$ range. ${ }^{\text {xvi }}$ The thus formed new water-soluble complexes $3^{\text {dmso }}$ and $\mathbf{3}^{\mathbf{H} 20}$ were thoroughly characterized by ESI mass spectrometry, NMR spectroscopy and electrochemistry. A pH-dependent electrochemical analysis of $3^{\mathbf{H 2 O}}$ compiled in a Pourbaix diagram confirmed that the $\mathrm{p} K \mathrm{a}$ values of the aquo ligands are higher compared to related pyrazolate-based Ru2-WOCs that lack the peripheral carboxylates. Although $3^{\mathbf{H} 20}$ appears spectroscopically pure, XPS and powder diffractometry confirmed the presence of a significant amount of $\mathrm{NaF}$ in the powder sample, which can influence the catalytic response. Consequently, the respective results should be considered with care.

Complexes $3^{\text {dmso }}$ and $3^{\mathbf{H} 20}$ were analyzed in regard to their ability to electrochemically, chemically and photochemically oxidize water to dioxygen. Both complexes feature an irreversible wave in their cyclic voltammograms associated with catalytic water oxidation, and $3^{\mathrm{H} 20}$ was further shown to be an efficient electrocatalyst using the FOWA technique. In the presence of $\left[\mathrm{Ru}\left((\mathrm{COOE} t)_{2} \mathrm{bpy}\right)_{2}(\mathrm{bpy})\right]^{2+}$ as photosensitizer and sodium persulfate as sacrificial electron acceptor, $3^{\text {dmso }}$ and $3^{\text {H20 }}$ generate $\mathrm{O}_{2}$, with photocatalysis stopping after around 130 turnovers, likely due to the high ionic strength of the solution.

xvi This section was adapted from Jana Lücken, Carolina Gimbert-Suriñach, Jordi Benet-Buchholz, Antoni Llobet, Franc Meyer, "Diruthenium Complexes of a Pyrazolate/Carboxylate Hybrid Ligand for Chemically, Electro- and Photo-Driven Catalytic Water Oxidation", submitted.

xvii The pyridine carboxylate coordinated congener $\mathbf{2}^{\text {dmso }}$ was not further analyzed due to handling issues as discussed in Section 3.1. 
Efficient water oxidation is also observed using ceric ammonium nitrate as chemical oxidant, with retardation of catalysis due to large amounts of added nitrate and Ce ${ }^{\text {III. }}$. During CAN-driven catalysis, both complexes behave very similarly ( $\mathrm{TOF}_{\mathrm{i}}$ values), suggesting analogous mechanisms of water oxidation at first sight. However, UV/vis-spectroelectrochemical measurements have shown that the DMSO moiety in $3^{\text {dmso }}$ remains intact and bound to the diruthenium core during both electrochemical and chemical water oxidation at low $\mathrm{pH}$, at least for some time (few TON). The unexpected stability of the bridging DMSO in $\mathbf{3}^{\mathrm{dmso}}$ points out the interplay between coordination and decoordination of the carboxylate groups concomitant with the association and dissociation of an aquo (or $\left.\mathrm{OH}^{-}\right)$ligand; this is reminiscent of the catalytic scenario recently proposed for the mononuclear $\left[\left(N^{3} \mathrm{O}\right.\right.$-tda $\left.) \mathrm{Ru}(\mathrm{py})_{2}\right]$ catalyst (III, Scheme 3.1).[219]

For $3^{\text {dmso }}$, under the above-mentioned conditions, there are three possibilities for the formation of a catalytically active species with a Ru-aquo group formed at high oxidation states (viz. in the catalytic regime): (i) partial decoordination of the DMSO moiety in the bimetallic cleft, (ii) substitution of a carboxylate group of the bridging $\left(\mathrm{L}^{1}\right)^{3-}$ ligand (both $\mathrm{CN} 6$ ), or (iii) direct coordination of an aquo ligand to one of the ruthenium ions, generating a seven coordinated (CN7) metal ion (Scheme 3.5). The transformation of $\mathbf{3}^{\mathrm{dmso}}$ into an active new species during catalysis is supported by cyclic voltammetry, in which an additional peak is observed in the cathodic scan after remaining in the catalytic regime for one minute. In either case (CN6 or CN7), the bridging DMSO remains bound (at least partially), which demonstrates that the pyrazolate-based diruthenium core may support water oxidation catalysis activity not only within the bimetallic cleft but also at a single metal site, with the second metal serving as an adjacent redox reservoir. These findings offer new perspectives for the design of future powerful WOCs where the ruthenium-oxygen active species is generated in situ, which can greatly simplify the synthesis, isolation and purification of the complexes that will act as catalyst precursors. The stability of the bridging DMSO ligand in $\mathbf{3}^{\mathbf{d m s o}}$ during catalysis is surprising and motivates further studies addressing the role and fate of DMSO in those ruthenium-based WOCs that include DMSO ligands as part of the catalyst precursors. ${ }^{[255,263]}$ 


\section{4}

\section{Water Oxidation Catalysis with Copper Complexes}

At present, the most efficient water oxidation systems are based on second- and third-row transition metals. However, the high cost and toxicity, as well as limited supply of these rare elements make them inconvenient for large-scale applications. Consequently, the design of WOCs based on firstrow transition metals, such as copper, is exceedingly desirable. In addition to being cheap, low-toxic and earth abundant, the extensive biomimetic chemistry of copper complexes with dioxygen offers new avenues to explore water oxidation catalysis. ${ }^{[106]}$ Accordingly, multiple copper(I) complexes with nitrogen donors have been reported to form dimeric bis( $\mu$-oxo) compounds upon reaction with $\mathrm{O}_{2}$, which can undergo facile and reversible $0-0$ bond formation resulting in peroxo dicopper species (redox isomerization, see Section 1.3.3). Despite their high affinity to oxygen atoms, copper complexes have only recently received increased attention in the field of water oxidation catalysis. ${ }^{[264]}$

In 2012, Mayer and coworkers presented the first homogenous copper-based complex capable of the electrocatalytic oxidation of water. ${ }^{[265]}$ The simple monomeric [(bpy)Cu(OH) $)_{2}$ (bpy: 2,2'bipyridine), $\mathbf{X I I}^{\mathbf{H}}$ (Scheme 4.1), was shown to oxidize water under basic conditions ( $\mathrm{pH} 12.5$ ) at an overpotential of around $750 \mathrm{mV}$ and with a high catalytic TOF of $100 \mathrm{~s}^{-1}$. EPR studies and control experiments with copper oxide indicate that $\mathbf{X I I}^{\mathrm{H}}$ is homogenous, however, the catalyst only possesses a low stability with $35 \%$ degrading after $\sim 30$ turnovers. Initially, the dimer $\left[\{(\mathrm{bpy}) \mathrm{Cu}\}_{2}(\mu-\mathrm{OH})_{2}\right] \mathrm{X}_{2}$ $\left(\mathrm{X}_{2}=(\mathrm{OAc})_{2},(\mathrm{OTf})_{2}\right.$ or $\left.\mathrm{SO}_{4}\right)$ is formed by conversion of the corresponding copper salts with bipyridine in aqueous solution $(\mathrm{pH}>12)$, but under catalytic conditions, the monomer $\mathbf{X I I}^{\mathbf{H}}$ is the dominant species. Computational work later suggested that $\mathrm{O}-\mathrm{O}$ bond formation in $\mathrm{XII}^{\mathrm{H}}$ occurs according to a single electron transfer-water nucleophilic attack (SET-WNA) mechanism at a $\left[(\mathrm{bpy}) \mathrm{Cu}^{\mathrm{III}}(\mathrm{OH})\left(\mathrm{O}^{\circ}\right)\right]^{+}$ species.[101]

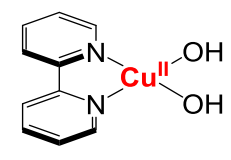

$X I^{H}$

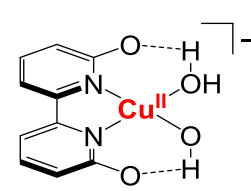

$\mathrm{XII}$ OH

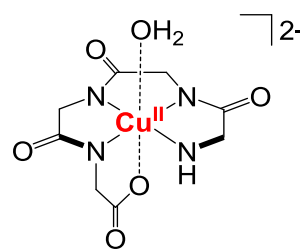

XIII

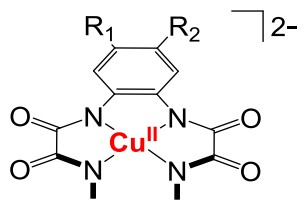

1: $R_{1}=R_{2}=H$

2: $R_{1}=R_{2}=M e$

3: $\mathrm{R}_{1}=\mathrm{H}, \mathrm{R}_{2}=\mathrm{OMe}$

4: $\mathrm{R}_{1}=\mathrm{R}_{2}=\mathrm{OMe}$

5: $R_{1}=H, R_{2}=$ pyrene

Scheme 4.1: Representations of the mononuclear copper-based WOCs XII-XIV.

Meyer and coworkers extended the field of copper-based WOCs with the self-assembled $\left[(\mathrm{TGG}) \mathrm{Cu}\left(\mathrm{H}_{2} \mathrm{O}\right)\right]^{2-}(\mathrm{XIII}$, Scheme 4.1$)$ bearing a triglycylglycine macrocyclic ligand (TGG $\left.{ }^{4-}\right){ }^{[266]} \mathbf{X I I I}$ reaches a TOF of $33 \mathrm{~s}^{-1}$, operating at an overpotential of $\sim 520 \mathrm{mV}$. The homogeneity of the catalyst is supported by optical spectroscopy (no spectroscopic changes after long-term electrolysis), electrochemistry (insignificant changes in peak currents and wave shapes over multiple CV cycles), rinse tests (no catalytic current with a "used" electrode in fresh electrolyte solution) and electrode 
surface analysis (SEM/XPS support absence of deposited material on the electrode surface). The 0-0 bond formation is assumed to proceed via a WNA mechanism involving a $\left[\mathrm{Cu}^{\mathrm{IV}}=0\right]^{2-}$ or $\left[\mathrm{Cu}^{\mathrm{III}}-\mathrm{O}\right]^{3+}$ species (all scaffold ligands are omitted due to clarity), which are stabilized by the tetraanionic TGG ${ }^{4-}$ ligand. Similar WOCs based on polypeptide ligands were studied by Pap and coworkers. ${ }^{[267,268]}$

The first copper-based WOC with a redox active ligand was introduced in 2014.[269] This was envisioned to be favorable as it provides an additional easily accessible redox equivalent, thus avoiding the formation of unstable high-valent copper(IV) species, concomitantly lowering the onset potential for water oxidation. Indeed, replacing the bipyridine unit in $\mathbf{X I I}^{\mathbf{H}}$ with the modified 6,6'-dihydroxy-2,2'bipyridine ligand (XIIOH, Scheme 4.1), results in a decrease of the overpotential by $\sim 200 \mathrm{mV}$ and an increase of the TON to $\sim 400$. Using square-wave voltammetry, Lin and coworkers showed that the ligand and metal oxidations are only $20 \mathrm{mV}$ apart, affording a two-electron oxidation of $\mathbf{X I I}^{\mathbf{O H}}$ at relatively low potentials. ${ }^{[269]}$ The thus formed $\left[\left(\mathrm{bpyO}^{++}\right) \mathrm{Cu}^{\mathrm{III}}(\mathrm{OH})_{2}\right]$ species ${ }^{\text {xviii }}$ is involved in $\mathrm{O}-\mathrm{O}$ bond formation by nucleophilic attack of a water molecule. Next to its redox active nature, the bpyO2- ligand also serves as an internal base for proton transfer. A large drawback of the decreased overpotential is, however, the concomitantly reduced driving force for water oxidation. Consequently, the catalytic rate of $\mathbf{X I I}^{\mathbf{O H}}$ is significantly lowered to $0.4 \mathrm{~s}^{-1 .[269]}$ The effect of the bpyO-2- ligand was also investigated by Gerlach et al.[270]

In 2015, Llobet and coworkers reported a new family of robust mononuclear dianionic copperbased WOCs containing a redox non-innocent ligand (XIV ${ }^{1-4}$, Scheme 4.1).[100] According to cyclic voltammetry, the $\left[\left(\mathrm{L}^{\mathrm{XIVn}}\right) \mathrm{Cu}\right]^{2-}$ complexes undergo two $1 \mathrm{e}^{-}$oxidation processes, of which the second is ligand-centered (aryl oxidation) and is associated with a large irreversible anodic current due to the electrocatalytic oxidation of water to dioxygen. Using FOWA analysis, XIV ${ }^{1}$ was shown to oxidize water with a TOF $\max$ of $3.6 \mathrm{~s}^{-1}$ and $12 \mathrm{~s}^{-1}$ at $\mathrm{pH} 11.5$ and 12.5 , respectively, operating at an overpotential of $700 \mathrm{mV}$. Upon increasing the electron-donating capacity at the aromatic ring $\left(\mathrm{H}_{4} \mathrm{~L}^{\mathrm{XIV} 1}\right.$ to $\left.\mathrm{H}_{4} \mathrm{~L}^{\mathrm{XIV} 4}\right)$, the latter is strongly reduced down to a record low of $170 \mathrm{mV}\left(\mathbf{X I V}^{4}\right)$ for first-row transition metal complexes. ${ }^{[100,271]}$ Nevertheless, this goes hand in hand with a decrease of the rate constant of the catalytic process $\left(0.16 \mathrm{~s}^{-1}\right.$ for $\mathbf{X I V}^{4}$ at $\left.\mathrm{pH} 11.5\right)$ as well as the oxidative stability of the complex. According to DFT calculations, $0-0$ bond formation in $\mathbf{X I V}^{\mathbf{1}}$ was shown to proceed via an SET-WNA mechanism at a $\left[\left(\mathrm{L}^{\mathrm{XIV} 1+}\right) \mathrm{Cu}^{\mathrm{III}}(\mathrm{OH})\right]^{-}$species. The thus formed peroxo intermediate $\left[\left(\mathrm{L}^{\mathrm{XIV} 1}\right) \mathrm{Cu}^{\mathrm{II}}(\mathrm{HO}-\right.$ $\mathrm{OH})]^{2-}$ exhibits no direct $\mathrm{Cu}-\mathrm{O}$ bond, but features strong hydrogen bonding between the (HO-OH) moiety and the [( $\left.\left.\mathrm{L}^{\mathrm{XIV} 1}\right) \mathrm{Cu}^{\mathrm{II}}\right]$ core. The $\mathrm{H}_{2} \mathrm{O}_{2}$ complex then undergoes a series of intramolecular electron transfer reactions before releasing $\mathrm{O}_{2}$ and regenerating the initial complex.[100,101]

Both XIV ${ }^{1}$ as well as the $\pi$-extended homologue $\mathbf{X I V}^{5}$, with a pyrene moiety in the backbone, were immobilized on solid support. ${ }^{[272]}$ In the homogenous phase at $\mathrm{pH} 12$, the $\pi$-delocalization in $\mathbf{X I V}^{5}$ leads to a significant increase in the TOF $\max$ from $6.2 \mathrm{~s}^{-1}$ (XIV) to $128 \mathrm{~s}^{-1}$ (XIV) and a cathodic shift of the catalytic wave by $\sim 150 \mathrm{mV}$. Heterogenization by anchoring on graphene electrodes ( $\pi-\pi$ stacking), results in the highest TOF $_{\max }$ values reported to date for water oxidation with molecular first-row transition metal complexes, viz. 320 and $540 \mathrm{~s}^{-1}$ for $\mathbf{X I V}^{1}{ }_{\text {het }}$ and $\mathbf{X I V}^{5}{ }_{\text {het, }}$, respectively (same surface coverage). Furthermore, the $\mathbf{X I V}^{5}{ }_{\text {het }}$ hybrid material exhibits a high stability, reaching a TON $>5300$. The relatively low faradaic efficiency of $24.5 \%$ was attributed to graphene oxidation, which occurs in parallel to water oxidation in basic solutions. Nevertheless, the catalyst was shown to remain intact after catalysis.

The complexes described thus far require strong alkaline conditions to drive catalytic water oxidation. In 2015, Zhang and coworkers reported the first copper-based WOC that functions at neutral $\mathrm{pH}^{\left[{ }^{273]}\right.}\left[\mathrm{Cu}_{2}(\mu-\mathrm{OH})(\mu-\mathrm{BPMAN})\right]^{3+}(\mathbf{X V}$, Scheme 4.2$)$ based on the ligand scaffold 2,7-[bis(2byridylmethyl)aminomethyl]-1,8-naphthyridine (BPMAN) was concomitantly the first reported dinuclear WOC with copper centers - and to date remains one of only very few. At $\mathrm{pH} 7, \mathbf{X V}$ is capable

xviii The expression of a radical cation $(\cdot+)$ is only used to illustrate that the ligand has been oxidized but does not refer to the overall charge of the ligand. 
of electrochemically oxidizing water to dioxygen with a modest TOF of $0.6 \mathrm{~s}^{-1}$ at an overpotential of $\sim 800 \mathrm{mV}$. According to DFT calculations, one pyridyl group is replaced by an aquo ligand in the $\mathrm{Cu}^{\mathrm{II}} \mathrm{Cu}^{\mathrm{II}}$ state. Upon two consecutive PCET steps to form a $\left[\left(\mathrm{Cu}^{\mathrm{III}}\right)_{2}(\mathrm{OH})(\mu-0)(\mu \text {-BPMAN })\right]^{3+}$ species, proton transfer from the hydroxide moiety to the dangling pyridyl triggers intramolecular $0-0$ bond formation between the terminal hydroxide and the $\mu-\mathrm{O}^{2-}$ unit. Subsequent oxidation yields $\mathrm{O}_{2}$ via a superoxo intermediate. ${ }^{[273,274]}$ Due to the presence of two copper centers, unstable high valent copper(IV) species are prevented.

On the basis of the bimetallic cooperative 0 -insertion pathway proposed for the $0-0$ bond formation in $\mathbf{X V}$, the hemilable methylpyridyl moiety was replaced by a non-coordinating methyl group in $\left[\mathrm{Cu}_{2}\left(\mathrm{H}_{2} \mathrm{O}\right)(\mu-\mathrm{OH})(\mu-\mathrm{TPMAN})\right]^{3+}(\mathrm{XVI}$, Scheme 4.2$)$, providing an empty site for water coordination. At pH 7, XVI reaches a TOF of $0.8 \mathrm{~s}^{-1}$, operating at an overpotential of $\sim 800 \mathrm{mV}$. The catalytic current is enhanced with increasing phosphate concentration (buffer), which seems to act as a proton acceptor in the absence of an intramolecular base as in XV. Apart from this, the 0-O bond formation is assumed to proceed according to the mechanism proposed for $\mathbf{X V}$.

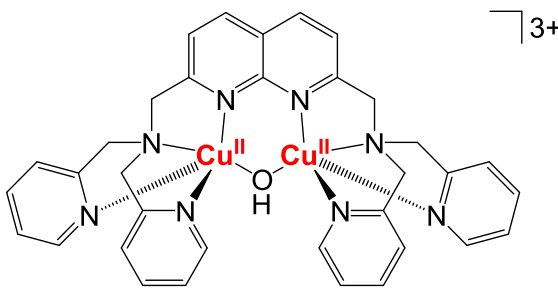

XV

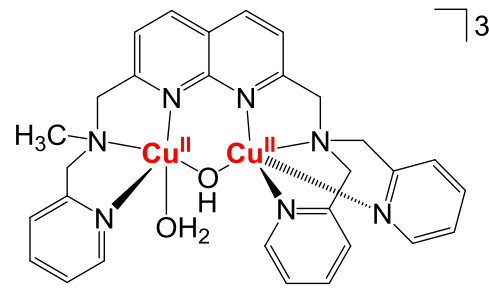

XVI

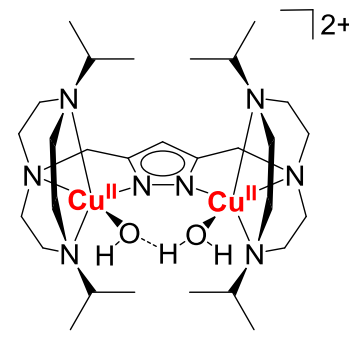

XVII

Scheme 4.2: Representations of the dicopper complexes XV-XVII.

Recently, Dr. Alexander Brinkmeier investigated the water oxidation proficiency of a dinuclear copper complex bearing a pyrazolate/tacn hybrid ligand.[188] The aquo/hydroxo compound $\left[\mathrm{Cu}_{2}\left(\mathrm{H}_{2} \mathrm{O}\right)(\mathrm{OH})\left(\mu-\mathrm{L}^{2 \mathrm{~b}}\right)\right]^{2+}(\mathbf{X V I I}$, Scheme 4.2$)$ is the decomposition product of the dioxygen activated peroxo, superoxo and hydroperoxo species, which have all been thoroughly characterized. As these three moieties constitute potential intermediates in the water oxidation cycle of dinuclear copper complexes (e.g. a superoxide species is suggested for $\mathbf{X V}$ and $\mathbf{X V I}$ ), XVII was considered a valuable candidate for water oxidation. Electrochemical investigations showed a pH-dependent irreversible wave between pH 11 and 13 in the CV of XVII associated with a catalytic process. Preliminary dioxygen experiments at $\mathrm{pH} 13$ exhibited $\mathrm{O}_{2}$ evolution to occur with faradaic efficiencies of 15-24\% during controlled potential electrolysis (CPE) at $1.45 \mathrm{~V}$ vs. NHE (20 minutes). Under these conditions, a rinse test suggested the absence of catalytically active electrodeposited material on the electrode surface. Furthermore, the conformity of the UV/vis spectra and CVs before and after CPE indicate the integrity of XVII after catalysis without apparent decay. However, long term electrolysis (four hours) at $1.25 \mathrm{~V}$ $v s$. NHE did reveal a surface deposit. No further investigations regarding the homogenous nature of water oxidation catalysis with XVII were conducted.

According to preliminary DFT studies and experimental evidence for the conversion of XVII to Int1-3 (see introduction of Chapter 6), a mechanistic scenario as shown in Scheme 4.3 is suggested for XVII. Two consecutive PCET steps and $\mathrm{O}-\mathrm{O}$ bond formation give rise to the hydroperoxo complex Int1, which is rapidly deprotonated under basic conditions to the peroxo species Int2. Subsequent oxidation results in the superoxo compound Int3, from which $\mathrm{O}_{2}$ is released as previously suggested for $\mathbf{X V}$ and XVI. Intramolecular $\mathrm{O}-\mathrm{O}$ bond formation is attributed to the coupling of a hydroxyl radical and the hydroxy moiety at the adjacent copper centers in a $\left[\mathrm{Cu}_{2}(\cdot \mathrm{OH})(\mathrm{OH})\left(\mu-\mathrm{L}^{2 \mathrm{~b}}\right)\right]^{3+}$ species $\left(\mathrm{Cu}^{\mathrm{II}} \mathrm{Cu}^{\mathrm{III}}\right)$ followed by the release of a proton. As for $\mathbf{X V}$ and $\mathbf{X V I}$, the formation of high-valent copper(IV) species is prevented. 


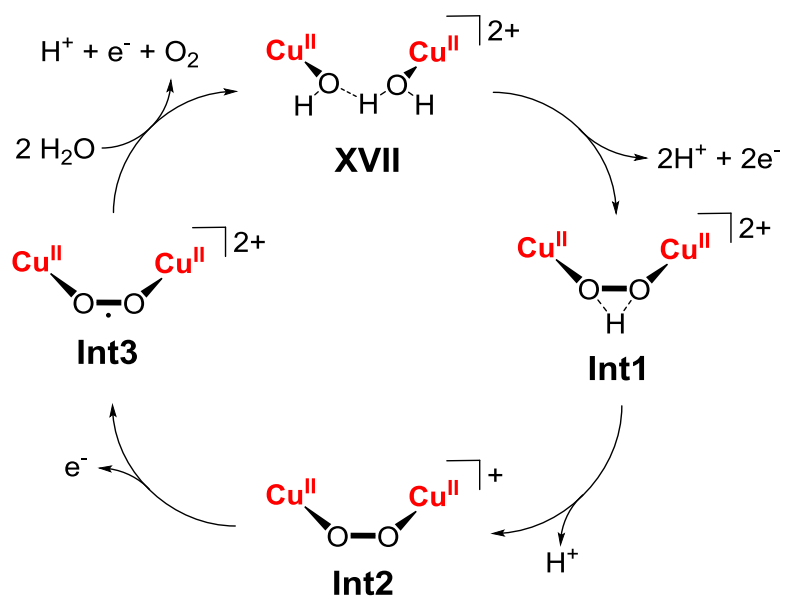

Scheme 4.3: Catalytic water oxidation cycle suggested for the dinculear copper complex XVII.

A large drawback for water oxidation studies of XVII is that it requires a minimal amount of acetonitrile as co-solvent to fully dissolve in aqueous phosphate buffer. Increasing the concentration of MeCN in the complex solution, however, resulted in an enhancement of the electrocatalytic wave. Reasons for this might be the oxidation of acetonitrile or an involvement of MeCN in the catalytic cycle, thus complicating mechanistic studies. ${ }^{[188]}$ To overcome this drawback, water soluble analogs of XVII based on the dinucleating ligand scaffold $\mathrm{HL}^{2 \mathrm{a}}$ with methyl periphery at the tacn moieties are specifically targeted. The following chapter discusses the synthesis of these water soluble dinuclear copper complexes with formate or acetate as bridging ligands, and their comprehensive spectroscopic and magnetometric characterizations, as well as electrochemical investigations under both inert and ambient conditions. Finally, the ability of the formate complex to electrocatalytically oxidize water to dioxygen is tested.

\subsection{Ligand Synthesis and Copper Complexation}

The proligands of interest, $\mathrm{HL}^{2 \mathrm{a}}$ and $\mathrm{HL}^{2 \mathrm{~b}}$, can be synthesized via a multi-step process according to literature procedures (Scheme 4.4). In an initial step, commercially available diethylenetriamine (g) and ethylene glycol (i) are tosylated to give $\mathrm{N}, \mathrm{N}^{\prime}, \mathrm{N}^{\prime \prime}$-tritosyl diethylenetriamine (h) and ditosyl ethylene glycol (j), respectively. ${ }^{[275]}$ These are combined in a ring-closure reaction to yield $\mathrm{N}, \mathrm{N}^{\prime}, \mathrm{N}^{\prime \prime}$-tritosyl-1,4,7triazacyclononane (Ts3-tacn, k).[276] By means of phenol and hydrobromic acid in acetic acid, two of the nitrogen atoms can selectively be deprotected to afford N-tosyl-1,4,7-triazacyclononane (H2Ts-tacn, l), which is the precursor for both tacn sidearms. ${ }^{[276,277]}$ Introduction of the methyl groups is achieved by an Eschweiler-Clarke reaction,[276] whereas the isopropyl residues are added using 2-bromopropane in a nucleophilic substitution. ${ }^{277]} \mathrm{Me}_{2} \mathrm{Ts}-\left(\mathrm{m}^{\mathrm{a}}\right)$ and $i \mathrm{Pr}_{2} \mathrm{Ts}$-tacn $\left(\mathrm{m}^{\mathrm{b}}\right)$ are subsequently deprotected with sulfuric acid to yield the free side arms $\mathrm{n}^{\mathrm{a}}$ and $\mathrm{n}^{\mathrm{b}}$ after distillation under reduced pressure $\left(60{ }^{\circ} \mathrm{C}\right) .[276,277]$

The pyrazole building block $1 H$-pyrazole-3,5-dicarbonyl dichloride (p) can be synthesized by chlorination of $1 H$-pyrazole-3,5-dicarboxylic acid (o) with thionyl chloride.[278] Combining one equivalent o with two equivalents $\mathrm{n}^{\mathrm{a} / \mathrm{b}}$ in dry THF leads to the formation of the ligand precursors $\mathrm{HL}^{2 \mathrm{a} / 2 \mathrm{~b}-\mathrm{ox}}$. Subsequent reduction of the amides with $\mathrm{LiAlH}_{4}$ yields the pyrazolate/tacn hybrid proligands $\mathrm{HL}^{2 \mathrm{a}}$ and HL ${ }^{2 \mathrm{~b}}[279,280]$ The ligands were characterized by ${ }^{1} \mathrm{H}-\mathrm{NMR}$ spectroscopy. Further characterization was not necessary, as the ligands are known and were reproduced in good spectroscopic agreement.

Both ligand scaffolds $\mathrm{HL}^{2 \mathrm{a}}$ and $\mathrm{HL}^{2 \mathrm{~b}}$ have previously been applied in the synthesis of dicopper,[170,188,207,281] diiron, ${ }^{[206,282,283]}$ dinickel[279] and dizinc ${ }^{[284]}$ complexes. In the Meyer group, they 
have found particular attention in the biomimetic formation of dicopper(I) complexes for dioxygen activation, which will be discussed in more detail in Chapter 6 .
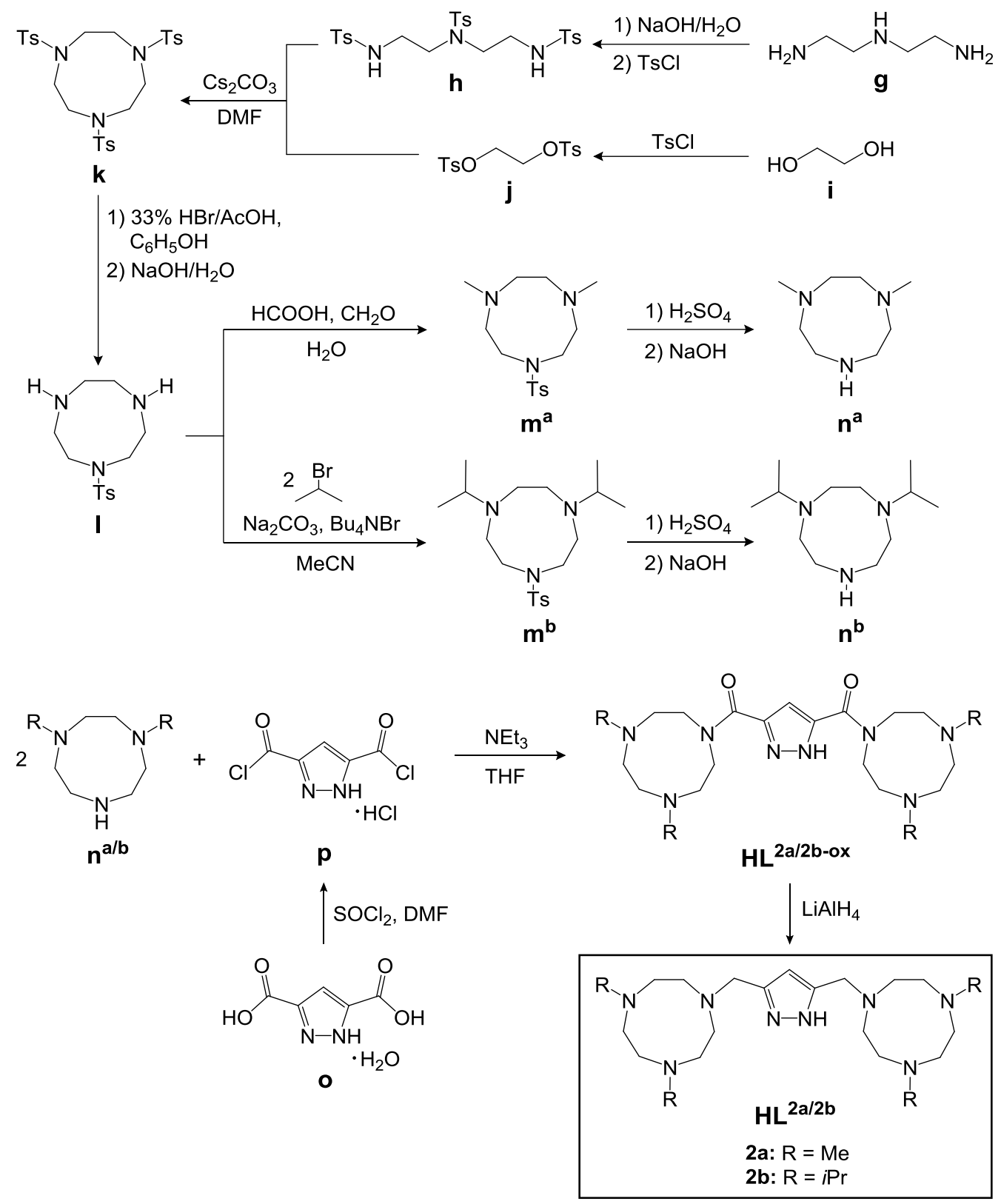

Scheme 4.4: Synthetic procedure for the preparation of the pyrazolate/tacn hybrid proligands HL $2 \mathrm{a}$ and HL2b.

The original aquo/hydroxo complex XVII (Scheme 4.2) based on HL $\mathrm{HL}^{2 \mathrm{~b}}$ was synthesized with copper(II) perchlorate in $\mathrm{MeOH} / \mathrm{MeCN}$ using $\mathrm{NaO}^{t} \mathrm{Bu}$ as base and adding an aqueous $\mathrm{NaOH}$ solution (5 M) during the workup. ${ }^{[188]}$ All attempts to synthesize its congener with $\mathrm{HL}^{2 \mathrm{a}}$ failed. When using $\mathrm{MeCN}$ as co-solvent, cyanide species were observed, and the formate-bridged complex $\mathbf{5}^{\mathrm{HCO}}$ was crystallized in pure $\mathrm{MeOH}$. To find out if the observed formate was an impurity from the solvent or formed through oxidation of $\mathrm{MeOH}$, experiments were attempted in dried solvent (all formate impurities should be removed during the drying procedure) both in an $\mathrm{N}_{2}$-atmosphere and under ${ }^{18} \mathrm{O}_{2}$ conditions. Unfortunately, these experiments were inconclusive, as the unlabeled formate complex was observed in both cases (not quantitative). Nevertheless, the syntheses showed that the $\left[\mathrm{Cu}_{2}\left(\mu-\mathrm{L}^{2 a}\right)\right]$ core has a high tendency to bind an anionic co-ligand, which was considered in all further syntheses. 
The dinuclear copper complexes $\mathbf{5}^{\mathrm{HCO}}$ and $\mathbf{5}^{\mathbf{O A c}}$ could be synthesized quantitatively starting from the proligand $\mathrm{HL}^{2 \mathrm{a}}$, which was deprotonated with $\mathrm{NEt}_{3}$ in $\mathrm{MeOH}$ (Scheme 4.5). Subsequent addition of copper(II) perchlorate and $\mathrm{NaHCO}_{2}$ or NaOAc, respectively, as well as a few drops of water to ensure dissolution, yielded the desired complexes as perchlorate salts. Crystals were obtained by diffusion of $\mathrm{Et}_{2} \mathrm{O}$ into the reaction mixture and analyzed by XRD (Figure 4.1). Multiple recrystallizations were necessary to remove excess $\mathrm{NaHCO}_{2}$ and $\mathrm{NaOAc}$. The syntheses of the related complexes $6^{\mathrm{HCO}}$ and $6^{\mathbf{O A c}}$ were performed in a solvent mixture of $\mathrm{MeOH} / \mathrm{MeCN}(1 / 1)$ without addition of water. Apart from their characterization, these two complexes will not be further discussed herein, but will reappear in Chapter 6. All analyses and follow-up experiments were conducted with crystalline material.

In contrast to XVII, $\mathbf{6}^{\mathrm{HCO}}$ and $\mathbf{6}^{\mathbf{O A c}}$, the complexes $\mathbf{5}^{\mathrm{HCO}}$ and $\mathbf{5}^{\mathbf{O A c}}$ are completely water soluble and do not require the addition of a co-solvent for water oxidation (WO) catalysis experiments. Single crystals of $\mathbf{5}^{\mathrm{HCO}}$ were previously obtained by diffusion of $\mathrm{Et}_{2} \mathrm{O}$ into a solution of $\left[\left(\mathrm{Cu}^{\mathrm{I}}\right)_{2}\left(\mu-\mathrm{L}^{2 \mathrm{a}}\right)\right]^{+}\left(\mathbf{5}^{\mathrm{I} / \mathrm{I}}\right)$ and $\mathrm{O}_{2}$ in dried $\mathrm{MeOH}$, stored at $-26^{\circ} \mathrm{C}$ overnight.[188] This complex has been observed as the decomposition product after dioxygen activation at $\mathbf{5}^{\mathbf{I} / \mathbf{I}}$.

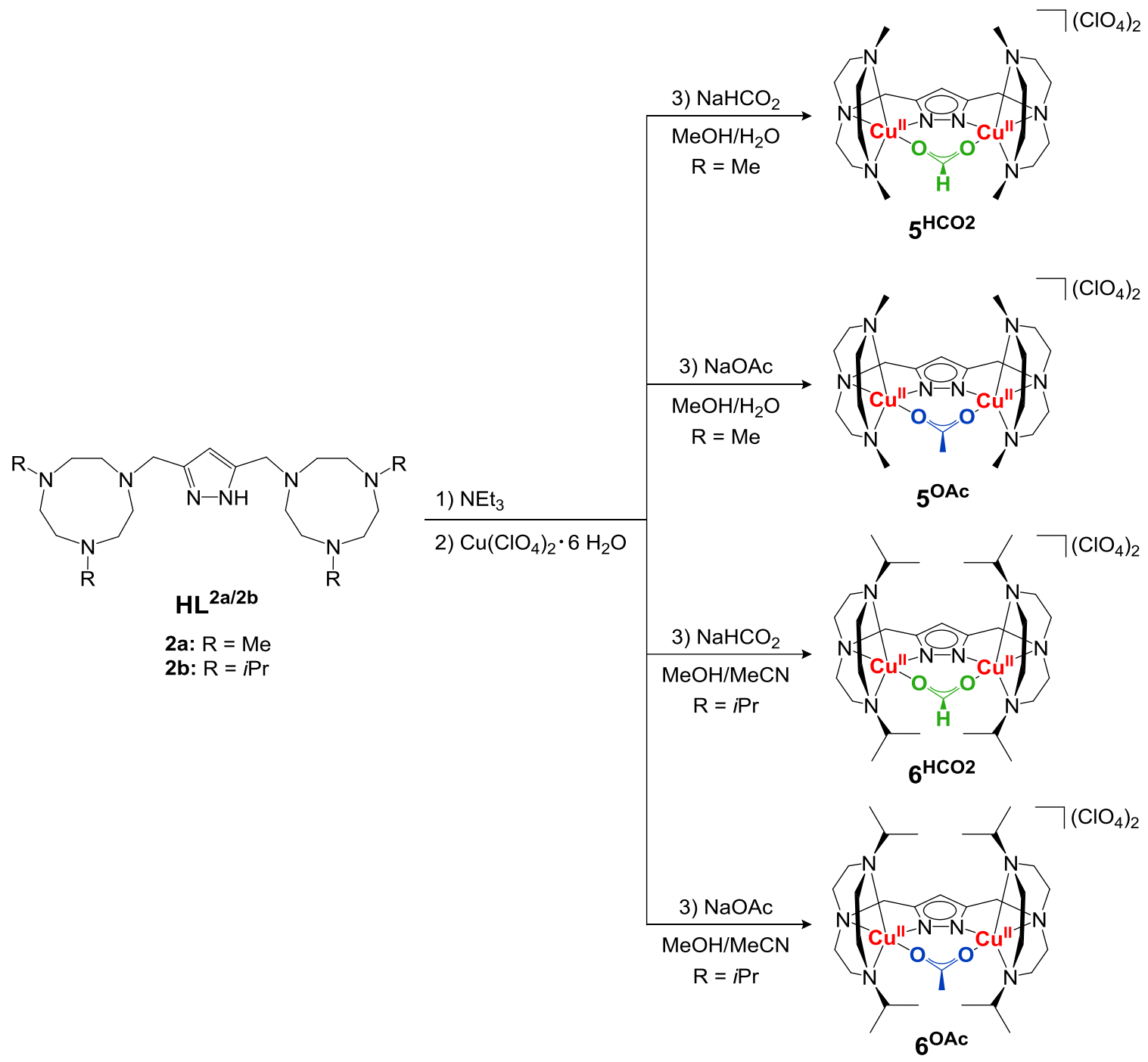

Scheme 4.5: Representation of the synthesis of the dinuclear copper complexes $\mathbf{5}^{\mathrm{HCO}}$ and $\mathbf{5}^{\mathbf{O A c}}$, as well as $\mathbf{6}^{\mathrm{HCO}}$ and $6^{0 A c}$. 
All four compounds, $5^{\mathrm{HCO} 2}$ (tetragonal, $I_{1} 1 c d$ ), $\mathbf{5}^{\mathrm{OAc}}$ (orthorhombic, $P m n 2_{1}$ ), $\mathbf{6}^{\mathrm{HCO}}$ (triclinic, $P 1$ ) and $\mathbf{6}^{\mathbf{A A c}}$ (monoclinic, P21), crystallize in different space groups (Table 4.1). In the case of $\mathbf{5}^{\mathbf{H C O}}$ and $\mathbf{5}^{\mathbf{0 A c}}$, one half of the dication as well as two perchlorate anions, and for the latter an MeCN molecule are found in the asymmetric unit. The full dication, two perchlorate moieties, as well as an $\mathrm{MeOH}$ or $\mathrm{MeCN}$ molecule make up the asymmetric units of $\mathbf{6}^{\mathbf{H C O}}$ and $\mathbf{6}^{\mathbf{0 A c}}$, respectively. Each $\mathrm{Cu}^{\mathrm{II}}$ ion is coordinated by an $\left\{\mathrm{N}_{4} \mathrm{O}\right\}$-donor set (Figure 4.1). Whereas for $\mathbf{5}^{\mathbf{H C O}}$ the ligands are coordinated in a close to ideal square pyramidal fashion, the geometry of the donor atoms is in between a square pyramid and a trigonal bipyramid in $\mathbf{5}^{\mathbf{O A c}}, \mathbf{6}^{\mathrm{HCO}}$ and $\mathbf{6}^{\mathbf{O A c}}$. This is nicely quantified by the CSM values (value from 0-100; the smaller, the closer the geometry is to the ideal polyhedron): For $\mathbf{5}^{\mathrm{HCO}}, \operatorname{CSM} S\left(C_{4 \mathrm{v}}\right)$ for square pyramidal geometry is very small (0.88) in comparison to $\operatorname{CSM} S\left(D_{3 \mathrm{~h}}\right)$ (trigonal bipyramid, 6.42). On the contrary, both values are very similar for $\mathbf{5}^{\mathbf{0 A c}}$, suggesting an in-between coordination environment, and the geometry of the ligated copper ions in $\mathbf{6}^{\mathrm{HCO}}$ and $\mathbf{6}^{\mathrm{OAc}}$ is slightly closer to a trigonal bipyramid (Table 4.1).

In all four complexes, the donor atoms are ligated to the metal centers with distances between 1.91 and $2.23 \AA$ A. Except in $\mathbf{5}^{\mathbf{H C O}}$, the shortest $\mathrm{M}-\mathrm{L}$ distance is found to the carboxylate moiety. The $\mathrm{Cu} \cdots \mathrm{Cu}$ separations are very similar in all cases, ranging from $4.15\left(5^{\mathbf{H C O}}\right)$ to $4.23 \AA\left(\mathbf{6}^{\mathbf{H C O}}\right)$. A direct comparison of the metric parameters can be found in Table 4.1.
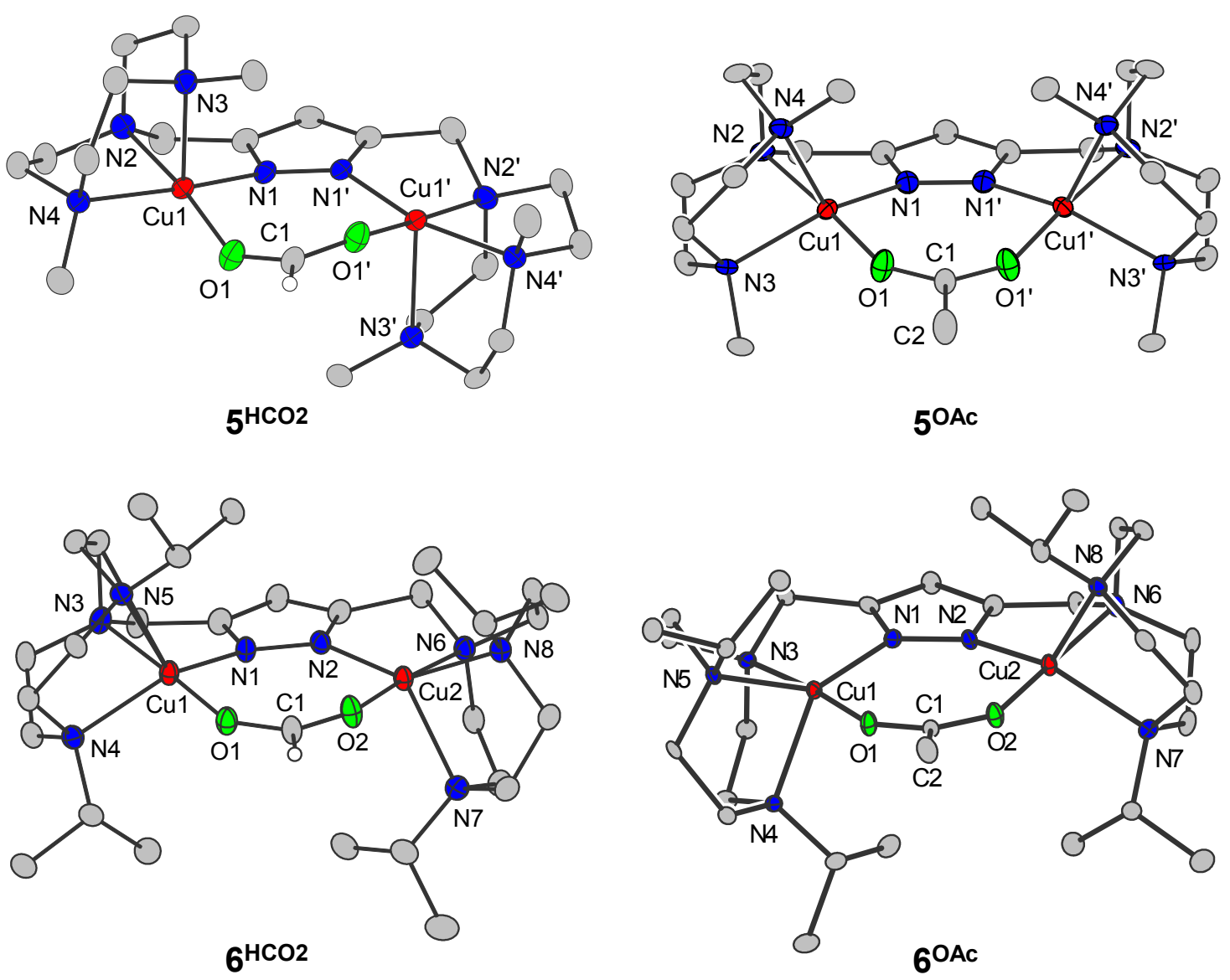

Figure 4.1: Molecular structures of the cationic cores of $\mathbf{5}^{\mathrm{HCO}}$ (top left), $\mathbf{5}^{\mathbf{O A c}}$ (top right), $\mathbf{6}^{\mathrm{HCO}}$ (bottom left) and $\mathbf{6}^{\mathbf{O A c}}$ (bottom right) drawn as thermal ellipsoids at the $30 \%$ probability level (copper: red, nitrogen: blue, oxygen: green, carbon: grey, hydrogen: white). All hydrogen atoms (except those of $\mathrm{HCO}_{2}{ }^{-}$), counter ions and additional solvent molecules are omitted for clarity reasons. 
Table 4.1: Selected crystal data, distances, bond lengths, torsion angles and Continuous Symmetry Measures $(\mathrm{CSM})$ of $5^{\mathrm{HCO}}, 5^{\mathrm{OAc}}, \mathbf{6}^{\mathrm{HCO}}$ and $\mathbf{6}^{\mathrm{OAc}}$.

\begin{tabular}{|c|c|c|c|c|}
\hline crystal data & $5^{\mathrm{HCO} 2}$ & $5^{0 A c}$ & $6^{\mathrm{HCO} 2}$ & $6^{\mathrm{OAc}}$ \\
\hline crystal system & tetragonal & orthorhombic & triclinic & monoclinic \\
\hline space group & $I 4_{1} c d$ & $P m n 2_{1}$ & $P 1$ & $P 2_{1}$ \\
\hline \multicolumn{5}{|l|}{ distance / $\AA$} \\
\hline$\overline{\mathrm{Cu} \cdots \mathrm{Cu}}$ & $4.1498(9)$ & $4.1712(14)$ & $4.2257(15)$ & $4.1850(14)$ \\
\hline \multicolumn{5}{|c|}{ bond lengths / } \\
\hline $\mathrm{Cu}(1)-\mathrm{O}(1)$ & $1.991(6)$ & $1.908(8)$ & $1.953(9)$ & $1.950(4)$ \\
\hline $\mathrm{Cu}(2)-\mathrm{O}(2)$ & - & - & $1.947(9)$ & $1.945(4)$ \\
\hline $\mathrm{Cu}-\mathrm{N}$ & $1.95-2.06$ & $1.98-2.20$ & $1.95-2.21$ & $1.95-2.23$ \\
\hline \multicolumn{5}{|c|}{ torsion angles $/^{\circ}$} \\
\hline $\mathrm{Cu}-\mathrm{N}-\mathrm{N}-\mathrm{Cu}$ & $33.129(994)$ & $0.000(1647)$ & $22.779(1963)$ & $-23.920(795)$ \\
\hline $\mathrm{Cu}-\mathrm{O} \cdots \mathrm{O}-\mathrm{Cu}$ & $-41.801(446)$ & $0.000(755)$ & $-14.255(913)$ & $5.710(368)$ \\
\hline \multicolumn{5}{|l|}{${ }^{\mathrm{xix}} \operatorname{CSM} S\left(C_{4 \mathrm{v}}\right)^{\mathrm{a}}$} \\
\hline $\mathrm{Cu}(1)$ & 0.88 & 3.41 & 4.10 & 3.76 \\
\hline $\mathrm{Cu}(2)$ & - & - & 3.82 & 4.47 \\
\hline \multicolumn{5}{|l|}{${ }_{\mathrm{xix}} \operatorname{CSM} S\left(D_{3 \mathrm{~h}}\right)^{\mathrm{b}}$} \\
\hline $\mathrm{Cu}(1)$ & 6.42 & 3.77 & 2.93 & 2.74 \\
\hline $\mathrm{Cu}(2)$ & - & - & 3.03 & 2.82 \\
\hline
\end{tabular}

a Continuous Symmetry Measures for square pyramidal geometry

b Continuous Symmetry Measures for trigonal bipyramidal geometry

The integrity of the complexes in solution was confirmed by ESI-mass spectrometry. The ESI(+)-MS in $\mathrm{MeOH}$ and $\mathrm{MeCN}$ for the $\left(\mathrm{L}^{2 \mathrm{a}}\right)^{-}-$and $\left(\mathrm{L}^{2 \mathrm{~b}}\right)^{-}$-complexes, respectively, display signals corresponding to the molecular ion peak $[\mathrm{M}]^{2+}$ and $\left[\mathrm{M}+\mathrm{ClO}_{4}\right]^{+}$for $\mathbf{5}^{\mathbf{H C O} 2}(\mathrm{~m} / \mathrm{z} 287.9 / 677.1), \mathbf{5}^{\mathbf{O A c}}(294.9 / 691.1), \mathbf{6}^{\mathbf{H C O}}$ (344.2/789.3) and $\mathbf{6}^{\mathbf{O A c}}(351.3 / 803.3)$. Additionally, compounds $\mathbf{6}^{\mathrm{HCO}}$ (987.1) and $\mathbf{6}^{\mathbf{O A c}}$ (1001.1) exhibit a peak in the ESI(-)-MS whose isotopic pattern matches the $\left[\mathrm{M}+3 \mathrm{ClO}_{4}\right]^{-}$species (appendix, Figures A.11, A.12, A.17, A.18 and A.20-A.27).

\subsection{Magnetic Properties}

Magnetic susceptibility measurements at $0.5 \mathrm{~T}$ in a temperature range from 2 to $295 \mathrm{~K}$ were performed on powdered samples of crystalline material of $\mathbf{5}^{\mathbf{H C O}}$ (Figure 4.2, left) and $\mathbf{5}^{\mathbf{O A c}}$ (right). The $\chi_{\mathrm{m}} T$ values at room temperature $\left(0.86 \mathrm{~cm}^{3} \mathrm{~K} \mathrm{~mol}^{-1}\right.$ at $295 \mathrm{~K}$ for both complexes $)$ correspond quite well with the expected spin-only value for two uncoupled $\mathrm{Cu}^{\mathrm{II}}$ ions $\left(0.75 \mathrm{~cm}^{3} \mathrm{~K} \mathrm{~mol}^{-1}\right.$ assuming $\left.g=2\right)$. An increase of $\chi_{\mathrm{m}} T$ towards lower temperatures with a maximum between $6-8 \mathrm{~K}$ indicates a moderate intramolecular ferromagnetic exchange interaction with coupling constants $J$ $\left(\widehat{H}=-2 J \hat{s}_{1} \hat{s}_{2}\right)$ of 9.1 and $6.4 \mathrm{~cm}^{-1}$ for $\mathbf{5}^{\mathbf{H C O 2}}$ and $\mathbf{5}^{\mathbf{O A c}}$, respectively. Simulation of the experimental susceptibility data further showed very similar isotropic $g$ values of $2.13\left(5^{\mathbf{H C O}}\right)$ and $2.12\left(5^{\mathbf{0 A c}}\right)$ for both complexes (see Table 4.2). These are higher than the $g$ value of a free electron $\left(g_{\mathrm{e}} \approx 2.0\right)$ and thus indicate significant spin-orbit coupling, which is typical in most transition metal complexes ( $g$ values of around 2.12 are quite common for copper(II) complexes). The best simulation results were obtained including temperature-independent paramagnetism (TIP) constants of $200 \cdot 10^{-6} \mathrm{~cm}^{3} \mathrm{~mol}^{-1}$ and

xix The geometry of the coordination environment was compared to self-made custom shapes of an ideal square pyramid and trigonal bipyramid with equal distances of all ligands to the central atom. 
$170 \cdot 10^{-6} \mathrm{~cm}^{3} \mathrm{~mol}^{-1}$ for $\mathbf{5}^{\mathbf{H C O}}$ and $\mathbf{5}^{\mathbf{O A c}}$, respectively. Additionally, Weiss temperatures $\Theta_{\mathrm{w}}$ were included to describe the decrease of $\chi_{\mathrm{m}} T$ at very low temperature. They were quantified as -0.33 and $-0.24 \mathrm{~K}$ for $\mathbf{5}^{\mathrm{HCO}}$ and $\mathbf{5}^{\mathbf{O A c}}$, respectively, thus indicating very small intermolecular interactions.
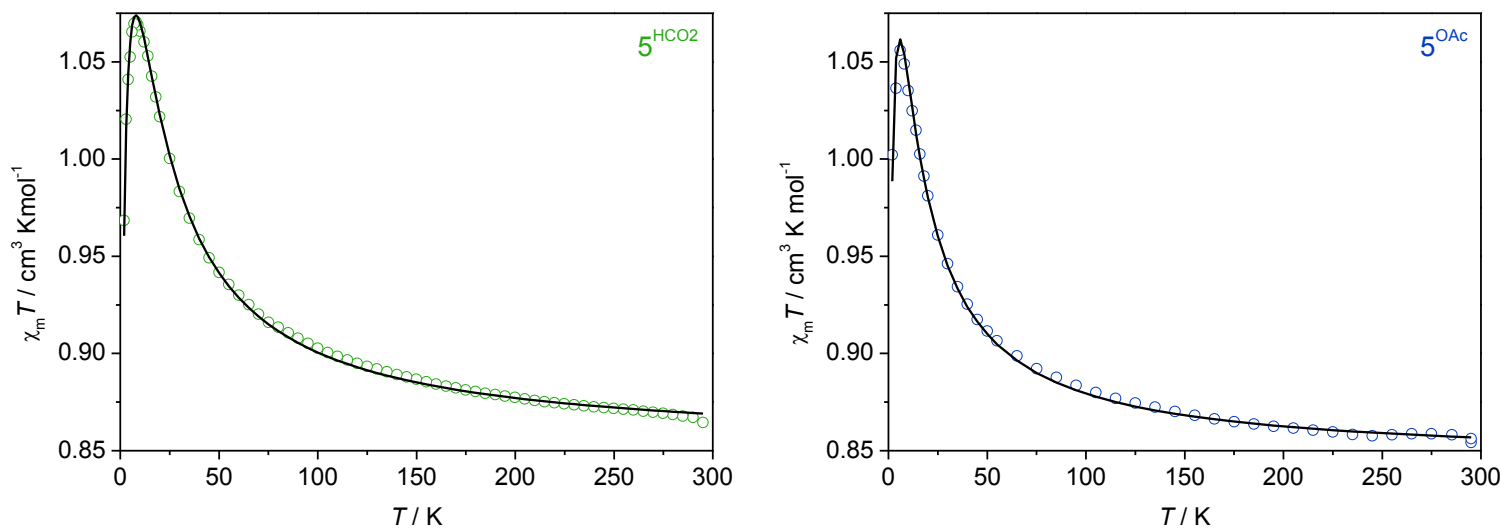

Figure 4.2: $\chi_{\mathrm{m}} T$ vs. T measurements of crystalline samples of $5^{\text {HCO2 }}$ (left) and $\mathbf{5}^{\mathbf{0 A c}}$ (right) in the temperature range $2-295 \mathrm{~K}$ at a field of $0.5 \mathrm{~T}$. The solid black lines represent the fits using the parameters presented in Table 4.2.

Table 4.2: Parameters extracted from the simulation of the $\chi_{\mathrm{m}} T v s . T$ measurements of $\mathbf{5}^{\mathrm{HCO2}}$ and $\mathbf{5}^{\mathbf{0 A c}}$.

\begin{tabular}{lll} 
parameters & $\mathbf{5}^{\text {HCO2 }}$ & $\mathbf{5}^{\mathbf{O A c}}$ \\
\hline \hline$g$-value $\left(g_{1}=g_{2}\right)$ & 2.13 & 2.12 \\
coupling constant $J / \mathrm{cm}^{-1}$ & 9.1 & 6.4 \\
$\mathrm{TIP} / 10^{-6} \mathrm{~cm}^{3} \cdot \mathrm{mol}^{-1}$ & 200 & 170 \\
$\Theta_{\mathrm{W}} / \mathrm{K}$ & -0.33 & -0.24 \\
\hline
\end{tabular}

\subsection{Electro- and Spectroelectrochemical Analysis}

The complexes $\mathbf{5}^{\mathrm{HCO}}$ and $\mathbf{5}^{\mathbf{H A c}}$ were electrochemically characterized by means of cyclic voltammetry and square wave voltammetry in $\mathrm{MeCN}\left(0.1 \mathrm{M} \mathrm{NBu}_{4} \mathrm{PF}_{6}\right)$ under inert conditions (all potentials are given $v s$. the ferrocenium/ferrocene $\left(\mathrm{Fc}^{+/ 0}\right)$ redox couple), as well as in phosphate buffer (ionic strength $I=0.1 \mathrm{M}$ ) under ambient conditions (all potentials are given $v s$. the normal hydrogen electrode (NHE)).

\subsubsection{Under Inert Conditions}

The cyclic voltammograms (CVs) of $\mathbf{5}^{\mathbf{H C O 2}}$ and $\mathbf{5}^{\mathbf{O A c}}$ at a scan rate of $100 \mathrm{mV} / \mathrm{s}$ (Figure 4.3 , left) in $\mathrm{MeCN}\left(0.1 \mathrm{M} \mathrm{NBu}_{4} \mathrm{PF}_{6}\right)$ display three irreversible peaks: two reductions in the cathodic scan and one oxidation in the anodic scan. From the square wave voltammograms (SWVs) (Figure 4.3, right), it becomes clear that these three peaks correspond to two redox couples. These are positioned at $-0.92 \mathrm{~V}$ $\left(E_{1}\right)$ and $-1.23 \mathrm{~V}\left(E_{2}\right)$, as well as $-1.07 \mathrm{~V}\left(E_{1}\right)$ and $-1.28 \mathrm{~V}\left(E_{2}\right)$ for $\mathbf{5}^{\mathrm{HCO}}$ and $\mathbf{5}^{\mathbf{A c}}$, respectively (Table 4.3). Consequently, reducing $\mathbf{5}^{\mathbf{H C O}}$ is slightly easier than $\mathbf{5}^{\mathbf{A A c}}$, which might be due to the electrondonating effect of the methyl group of the acetate molecule in the bimetallic pocket of $\mathbf{5}^{\mathbf{O A c}}$ in comparison to the formate moiety in $5^{\mathbf{H C O}}$. 

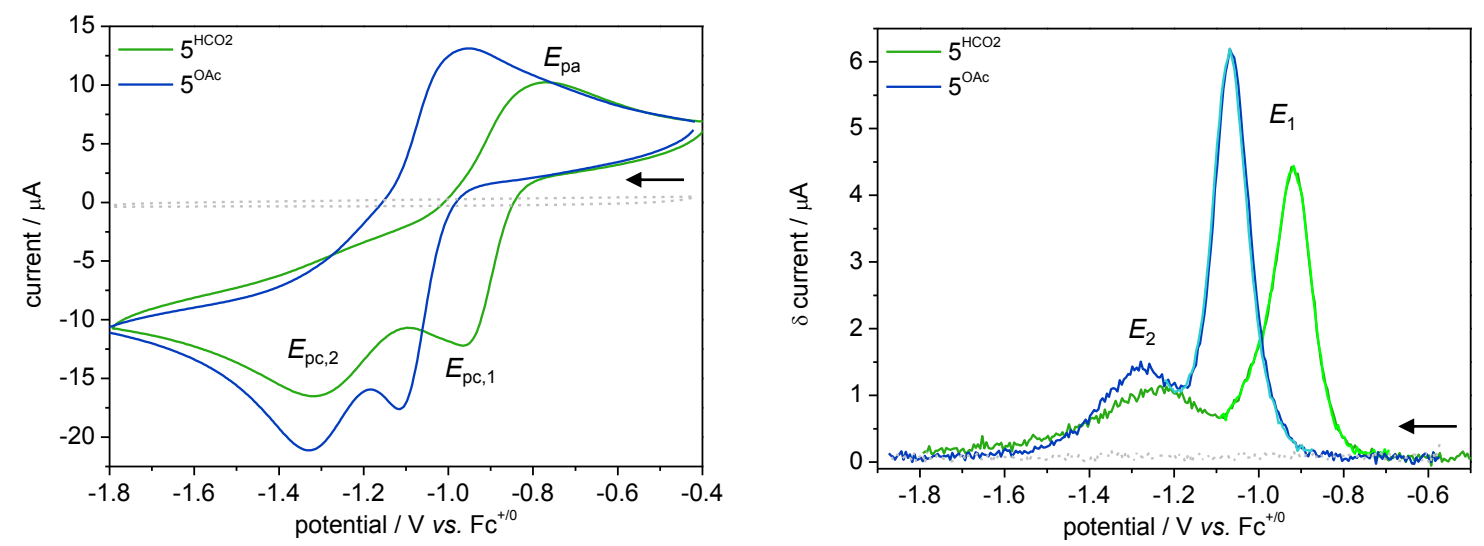

Figure 4.3: CV (left, $100 \mathrm{mV} / \mathrm{s}$ ) and SWV (right) of a $1 \mathrm{mM}$ solution of $\mathbf{5}^{\mathbf{H C O}}$ (green) and $\mathbf{5}^{\text {OAc }}$ (blue) in MeCN $(0.1 \mathrm{M}$ $\left.\mathrm{NBu}_{4} \mathrm{PF}_{6}\right)$ under inert conditions $(c=1 \mathrm{mM})$. The lighter traces (dashed) in the SWV represent a measurement of only the first electrochemical process $E_{1}$. The scanning direction is indicated by an arrow. A glassy carbon disc was used as the working electrode, a Pt disc as auxiliary and an Ag wire as the reference electrode. Ferrocene was used as an internal standard.

Table 4.3: Peak potentials extracted from the CVs and SWVs of $\mathbf{5}^{\mathrm{HCO}}$ and $\mathbf{5}^{\mathrm{OAc}}$ in $\mathrm{MeCN}\left(0.1 \mathrm{M} \mathrm{NBu}_{4} \mathrm{PF}_{6}\right)$ under inert conditions. All potentials are given $v s . \mathrm{Fc}^{+} / 0$.

\begin{tabular}{lccr}
$\mathrm{CV}$ & $\mathbf{5}^{\text {HCO2 }}$ & $\mathbf{5}^{\mathbf{O A c}}$ & \multicolumn{1}{c}{$\Delta E^{\mathrm{a}}$} \\
\hline \hline$E_{\mathrm{pc}, 1}$ & $-0.96 \mathrm{~V}$ & $-1.11 \mathrm{~V}$ & $150 \mathrm{mV}$ \\
$E_{\mathrm{pc}, 2}$ & $-1.32 \mathrm{~V}$ & $-1.33 \mathrm{~V}$ & $10 \mathrm{mV}$ \\
$E_{\mathrm{pa}}$ & $-0.76 \mathrm{~V}$ & $-0.95 \mathrm{~V}$ & $190 \mathrm{mV}$ \\
\hline $\mathrm{SWV}$ & & & \\
\hline \hline$E_{1}$ & $-0.92 \mathrm{~V}$ & $-1.07 \mathrm{~V}$ & $150 \mathrm{mV}$ \\
$E_{2}$ & $-1.23 \mathrm{~V}$ & $-1.28 \mathrm{~V}$ & $50 \mathrm{mV}$ \\
\hline
\end{tabular}

a $\Delta E$ between the peak potentials of $5^{\mathrm{HCO}}$ and $\mathbf{5}^{\mathrm{OAc}}$

When adjusting the potential range so that only $E_{1}$ is measured, a quasi-reversible redox process can be observed (Figure 4.4, top, exemplarily for $\mathbf{5}^{\mathbf{H C O 2}}$ ) with a peak-to-peak separation between $104 \mathrm{mV}(50 \mathrm{mV} / \mathrm{s})$ and $160 \mathrm{mV}(1000 \mathrm{mV} / \mathrm{s})$ depending on the scan rate (Table 4.4). The ratio of the currents of the cathodic and anodic peaks is rather large (for a reversible redox process: $i_{\mathrm{pc}} / i_{\mathrm{pa}}=1$ ), which is also a consequence of the inaccurate baselines that have to be extrapolated from the $\mathrm{CV}$ and to which the current is measured. Especially for the anodic peak $\left(E_{\mathrm{pa}, 1}\right)$, the determined baseline is too steep, as after reduction the potential has to be reversed quickly to prevent from measuring $E_{2}$.

The second redox event $E_{2}$ can only be observed when the potential is scanned cathodically to $-2.5 \mathrm{~V}$, as evidenced by an additional reduction peak $\left(E_{\mathrm{pc}, 2},-1.3 \mathrm{~V}\right)$ and a broadening of the anodic wave (Figure 4.4, bottom right). The latter splits into two at higher scan rates (500 and $1000 \mathrm{mV} / \mathrm{s}$ ), of which the second anodic peak at around $-0.2 \mathrm{~V}$ is then likely the counterpart to $E_{\mathrm{pc}, 2}$. 

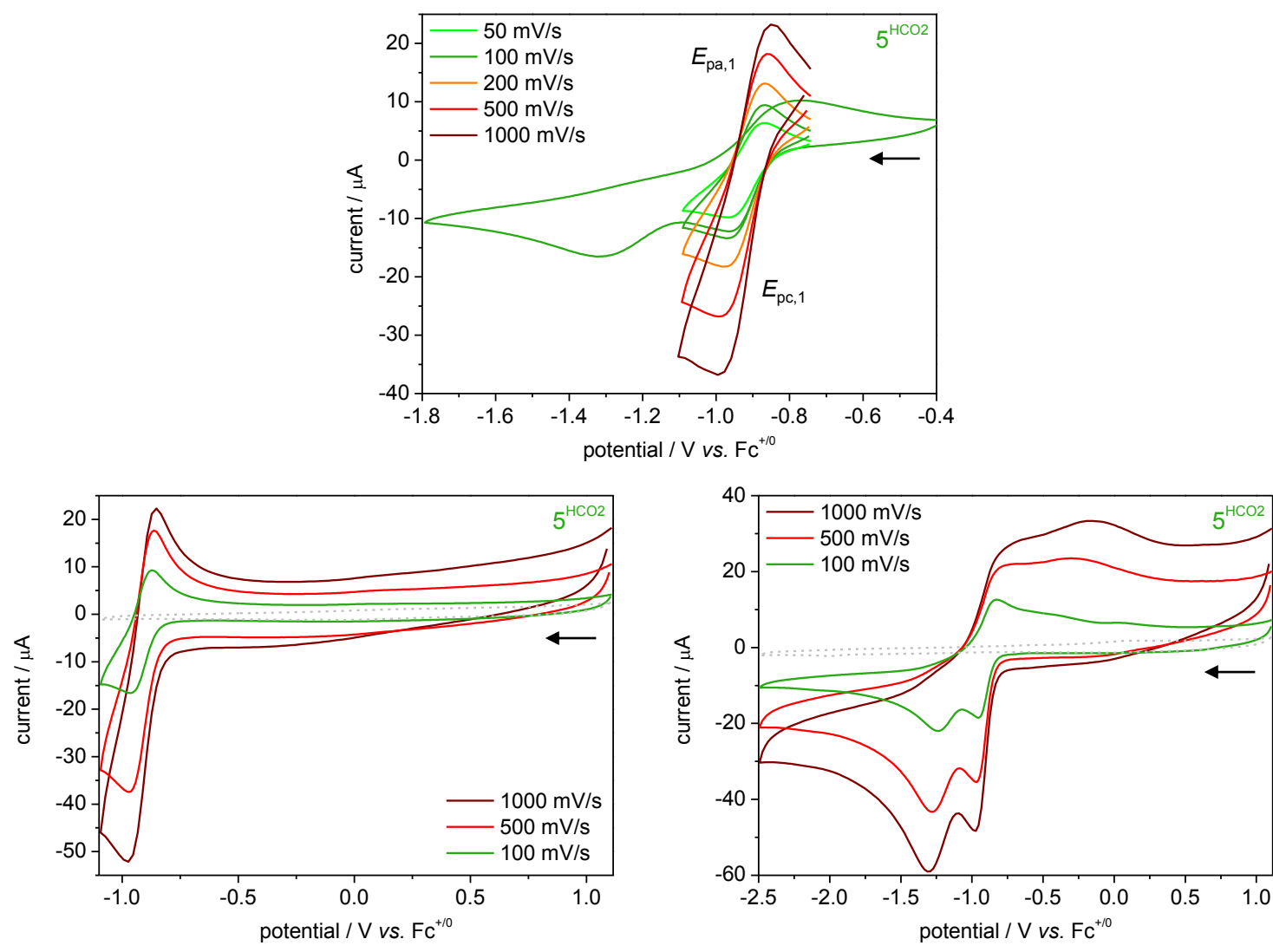

Figure 4.4: Electrochemical analysis of $\mathbf{5}^{\mathrm{HCO} 2}$ in $\mathrm{MeCN}\left(0.1 \mathrm{M} \mathrm{NBu}_{4} \mathrm{PF}_{6}\right)$ under inert conditions $(c=1 \mathrm{mM})$. Top: Scan rate dependence of the redox process $E_{1}$. Bottom: CVs at 100, 500 and $1000 \mathrm{mV} / \mathrm{s}$ from (-2.5)-1.1 V (left) and (-1.1)-1.1 V (right). The scanning direction is indicated by an arrow. A glassy carbon disc was used as the working electrode, a Pt disc as auxiliary and an Ag wire as the reference electrode. Ferrocene was used as an internal standard.

Table 4.4: Electrochemical data extracted from the scan rate dependent measurements of $\mathbf{5}^{\mathrm{HCO}}$ in $\mathrm{MeCN}(0.1 \mathrm{M}$ $\mathrm{NBu}_{4} \mathrm{PF}_{6}$ ) under inert conditions. The data refers to the first redox process $E_{1}$. All potentials are given $v s$. $\mathrm{Fc}^{+} / 0$.

\begin{tabular}{clllllll} 
scan rate $/ \mathrm{mV}$ & $E_{\mathrm{pc}} / \mathrm{mV}^{\mathrm{a}}$ & $i_{\mathrm{pc}} / \mu \mathrm{A}^{\mathrm{a}}$ & $E_{\mathrm{pa}} / \mathrm{mV}^{\mathrm{a}}$ & $i_{\mathrm{pa}} / \mu \mathrm{A}^{\mathrm{a}}$ & $E_{1 / 2} / \mathrm{V}^{\mathrm{b}}$ & $\Delta E_{\mathrm{p}} / \mathrm{mV}^{\mathrm{c}}$ & $i_{\mathrm{pc}} / i_{\mathrm{pa}} \mathrm{d}$ \\
\hline \hline 50 & -970 & 7.477 & -866 & 2.919 & -0.918 & 104 & 2.56 \\
100 & -970 & 9.848 & -866 & 4.434 & -0.918 & 104 & 2.22 \\
200 & -974 & 11.48 & -866 & 6.555 & -0.920 & 108 & 1.75 \\
500 & -992 & 17.97 & -862 & 7.587 & -0.927 & 130 & 2.37 \\
1000 & -1002 & 22.67 & -842 & 7.880 & -0.922 & 160 & 2.88 \\
\hline
\end{tabular}

a peak potentials and currents from the anodic (a) and cathodic (c) scan

b half-wave potential $E_{1 / 2}=\frac{E_{\mathrm{pf}}+E_{\mathrm{pr}}}{2}$

${ }^{\mathrm{c}}$ peak-to-peak separation $\Delta E_{\mathrm{p}}=E_{\mathrm{pf}}-E_{\mathrm{pr}}$

d ratio $i_{\mathrm{pc}} / i_{\mathrm{pa}}$ is so large as it is difficult to draw an accurate baseline for the reverse scan (large deviation of $i_{\mathrm{pc}} / i_{\mathrm{pa}}$ is attributed to the inaccurate baseline definition for the reverse scan)

In order to elucidate the nature of the redox processes, UV/vis-spectroelectrochemical (SEC) measurements were conducted in $\mathrm{MeCN}\left(0.1 \mathrm{M} \mathrm{NBu}_{4} \mathrm{PF}_{6}\right)$. The UV/vis spectrum of $\mathbf{5}^{\mathbf{H C O}}$ in the $\mathrm{Cu}^{\mathrm{II}} \mathrm{Cu}^{\mathrm{II}}$ state possesses local absorption maxima at 292 and $678 \mathrm{~nm}$, the latter of which corresponds to a d-d transition (Figure 4.5, top left, black spectrum). Upon reduction at $-0.92 \mathrm{~V}\left(E_{1}\right)$, the intensity of both transitions significantly decreases. A closer look at the $\mathrm{d}$-d transition regime reveals that the reduction 
process occurs in two steps. Initially, the d-d band decreases with an increased absorption at $480 \mathrm{~nm}$ associated with two isosbestic points at 424 and $588 \mathrm{~nm}$ (Figure 4.5, bottom left). These are lost upon further reduction, while the band at $678 \mathrm{~nm}$ continues to vanish (Figure 4.5, bottom right).

The observed spectroscopic changes suggest two conclusions: (i) $E_{1}$ corresponds to a metalcentered reduction, and (ii) the two-step process likely corresponds to two consecutive one-electron

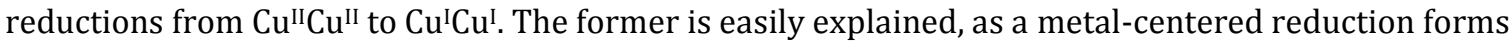
$\mathrm{d}^{10} \mathrm{Cu}^{\mathrm{I}}$ ions, whose $\mathrm{d}$-orbitals are completely filled. Hence, a decrease in the $\mathrm{d}$ - $\mathrm{d}$ transition peak supports the formation of $\mathrm{Cu}^{\mathrm{I}}$. Regarding the latter, the initial reduction process should lead to the formation of the mixed-valent $\mathrm{Cu}^{\mathrm{I} C \mathrm{Cu}^{\mathrm{II}}}$ species. This conversion seems to be clean as two isosbestic points can be observed. The subsequent reduction to $\mathrm{Cu}^{\mathrm{I} C \mathrm{Cu}^{\mathrm{I}}}$ shows no isosbestic points. A reason for this might be the formation of elemental copper, which was observed on the surface of the working electrode (Figure 4.5, top right). As a direct reduction to $\mathrm{Cu}^{0}$ at $-0.92 \mathrm{~V}$ cannot be expected, this observation suggests that the possible $\mathrm{Cu}^{\mathrm{I}} \mathrm{Cu}^{\mathrm{I}}$ complex disproportionates to elemental copper and $\mathrm{Cu}^{\mathrm{II}} \mathrm{Cu}^{\mathrm{II}}$ or $\mathrm{Cu}^{\mathrm{I}} \mathrm{Cu}^{\mathrm{II}}$, which might also explain why the d-d transition peak does not completely vanish. Furthermore, chemical reduction of $5^{\mathrm{HCO}}$ leads to the formation of an oligonuclear copper(I) hydride complex (see Section 6.4). However, the formation of such a complex under electrochemical reduction cannot be deduced from the UV/vis spectroscopic study.

Upon reoxidation at $-0.49 \mathrm{~V}$, the measurement is unsuccessful due to a current overload from the oxidation of the elemental copper on the electrode surface. Nevertheless, the observed formation of $\mathrm{Cu}^{0}$, which presumably succeeds the decomposition of the complex, anyhow excludes reversibility.
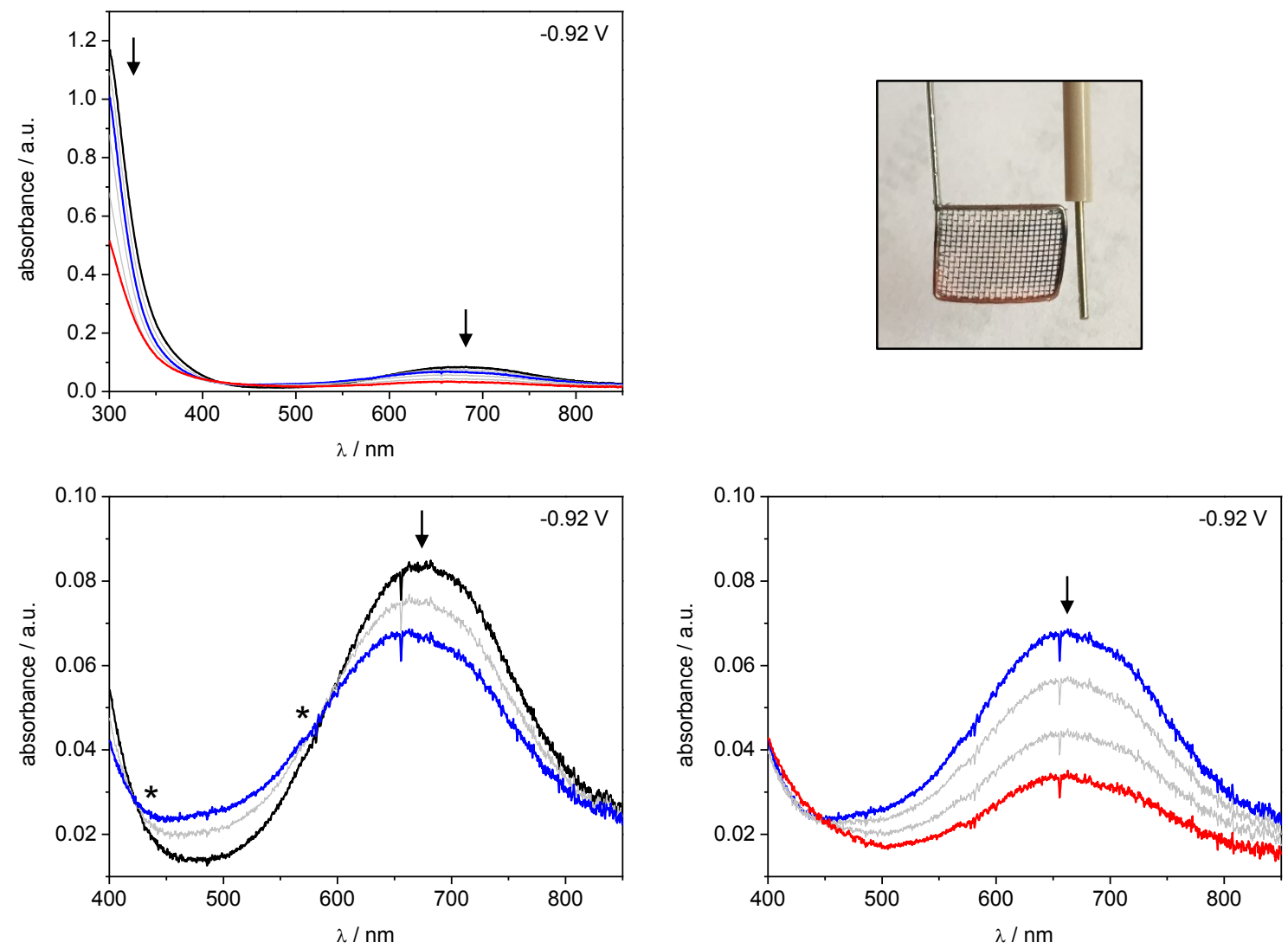

Figure 4.5: UV/vis-spectroelectrochemical analysis of $\mathbf{5}^{\mathrm{HCO}}$ in $\mathrm{MeCN}\left(0.1 \mathrm{M} \mathrm{NBu}_{4} \mathrm{PF}_{6}\right)$ under inert conditions. Top left: UV/vis spectra (full region, $250-850 \mathrm{~nm}$ ) of the electrochemical reduction at $-0.92 \mathrm{~V}$ (black: beginning, red: end). Bottom: Enlargement of the d-d transition peak showing a two-step reduction. In the first few minutes an intermediate species (blue) is formed (left, isosbestic points: $424 / 588 \mathrm{~nm}$ ), which then converts to the final species (right, no isosbestic point). The top right corner depicts the electrodes after electrochemical reduction; the Pt mesh working electrode exhibits deposited elemental copper. 
The suggested formation of a mixed-valent $\mathrm{Cu}^{\mathrm{I}} \mathrm{Cu}^{\mathrm{II}}$ species is a unique observation for dinuclear copper complexes based on pyrazolate/tacn hybrid ligands, which have extensively been studied in the Meyer group. ${ }^{[188,206,207]}$ As attended to in Section 6.1, the mixed-valent species $\mathrm{Cu}^{\mathrm{I}} \mathrm{Cu}^{\mathrm{II}}$ is also observed during chemical reduction of $\mathbf{5}^{\mathbf{O A c}}$.

In conclusion, the first redox event $\left(E_{1}\right)$ can be tentatively assigned to the reduction from $\mathrm{Cu}^{\mathrm{II}} \mathrm{Cu}^{\mathrm{II}}$ initially to $\mathrm{Cu}^{\mathrm{I}} \mathrm{Cu}^{\mathrm{II}}$ and possibly further to $\mathrm{Cu}^{\mathrm{I}} \mathrm{Cu}^{\mathrm{I}}$, which is quasi-reversible on the $\mathrm{CV}$ timescale. However, follow-up reactivity is observed and can be more complicated than expected, as will be discussed in Section 6.4. The nature of the electrochemical process $E_{2}$ could not be investigated using $\mathrm{UV} / \mathrm{vis}$-SEC. If $E_{2}$ corresponds to a metal-centered reduction to $\mathrm{Cu}^{0}$, a characteristic peak could be expected in the reverse scan of the $\mathrm{CV}$ due to anodic stripping of the elemental copper,[250] which is not observed. Electrochemical reduction of the pyrazolate/tacn hybrid ligand is not expected.

\subsubsection{Under Ambient Conditions}

In order to test whether $\mathbf{5}^{\mathbf{H C O}}$ and $\mathbf{5}^{\mathbf{0 A c}}$ can serve as WOCs, further electrochemical studies were performed in aqueous solution under ambient conditions. The CVs of $\mathbf{5}^{\mathrm{HCO}}$ and $\mathbf{5}^{\mathbf{O A c}}$ in phosphate buffer (pH 12.4, $I=0.1 \mathrm{M})$ exhibit an irreversible wave associated with a catalytic process with $E_{\text {onset }}=1.1 \mathrm{~V}$, which could originate from water oxidation (Figure 4.6, left). This coincides with an overpotential of $600 \mathrm{mV}$, as the thermodynamic potential of water oxidation at $\mathrm{pH} 12.4$ is $0.5 \mathrm{~V}$. Note that the stability of the complex under these conditions is difficult to evaluate due to the absence of a precatalytic wave, which is often used as an indication in a CV study, e.g. an unchanged precatalytic wave during multiple $\mathrm{CV}$ cycles suggests that the complex remains intact under the given conditions.
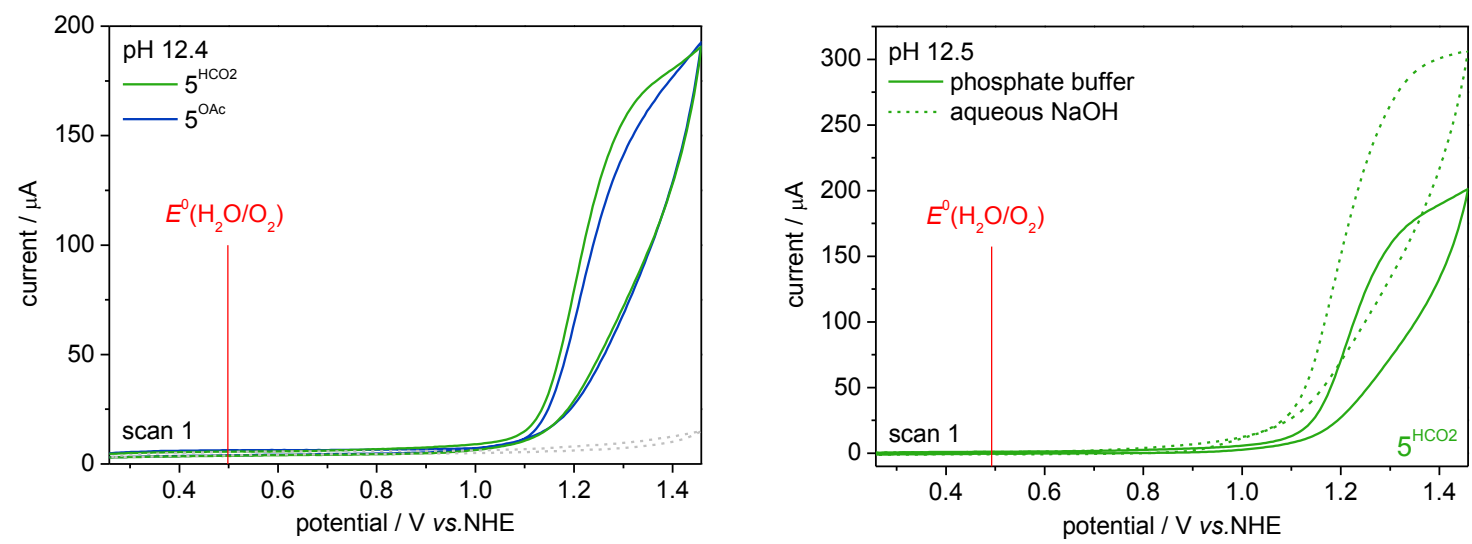

Figure 4.6: Electrochemistry of $\mathbf{5}^{\mathrm{HCO}}$ (green) and $\mathbf{5}^{\mathbf{O A c}}$ (blue) under ambient conditions $(c=1 \mathrm{mM})$. Left; Comparison of both complexes in phosphate buffer $(I=0.1 \mathrm{M}, \mathrm{pH} 12.4)$. Right: Comparison of $\mathbf{5}^{\mathrm{HCO}}$ in phosphate buffer (solid, $I=0.1 \mathrm{M}, \mathrm{pH} 12.5$ ) and aqueous $\mathrm{NaOH}$ solution ( $\mathrm{pH} 12.5$ ). A glassy carbon disc was used as working electrode, a Pt disc as auxiliary and an MSE as the reference electrode. The potentials were converted to the NHE scale by adding $0.658 \mathrm{~V}$. The red line marks the thermodynamic potential of water oxidation at the given $\mathrm{pH}$.

In order to identify the effect of the buffer solution on the catalytic wave, a $\mathrm{CV}$ of $\mathbf{5}^{\mathrm{HCO}}$ was conducted in an aqueous $\mathrm{NaOH}$ solution ( $\mathrm{pH}$ 12.5). The comparison to the $\mathrm{CV}$ in phosphate buffer under identical conditions, shows a significantly higher catalytic activity in a nonbuffered solution (Figure 4.6, right), suggesting that phosphate might interfere with the catalysis. Nevertheless, the use of a buffer is highly recommended, as during WO catalysis protons are formed, which can significantly decrease the $\mathrm{pH}$ of the solution. For the mononuclear complex $\left[\left(\mathrm{L}^{\mathrm{XVIII}}\right) \mathrm{Cu}^{\mathrm{II}}\right]^{2+}$ (Scheme 4.6, $\mathrm{L}^{\mathrm{XVIII}}=1,3-\operatorname{bis}(((1-$ methyl-1 $H$ imidazol-2-yl)methyl)amino)propan-2-ol)), XVIII, it could be shown

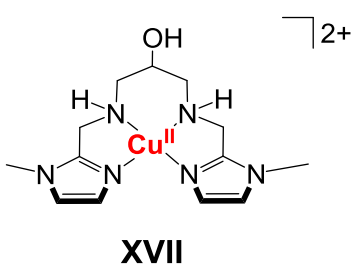

Scheme 4.6: Mononuclear complex XVIII. that the employed electrolyte has a strong influence on the catalytic current.[285] 
As complexes $\mathbf{5}^{\mathrm{HCO}}$ and $\mathbf{5}^{\mathbf{O A c}}$ appear to behave very similarly regarding their possible electrocatalytic WO proficiency, all further experiments were exclusively conducted with $\mathbf{5}^{\mathbf{H C O 2}}$.

To further analyze the observed irreversible process in the $\mathrm{CV}, \mathrm{pH}$ dependent cyclic voltammetry measurements were conducted. For this, $\mathbf{5}^{\mathbf{H C O} 2}$ was dissolved in phosphate buffer solutions $(I=0.1 \mathrm{M})$ between $\mathrm{pH} 10$ and 13. As shown in Figure 4.7, the catalytic wave increases with rising $\mathrm{pH}$ starting from 11.5. Over the course of $20 \mathrm{CV}$ cycles, the catalytic current gradually decreases (comparison scan 1 and scan 20). By contrast, between pH 12 and 13, the current of the background (pure electrolyte) slowly increases over twenty cycles, suggestive of a modification of the electrode surface under highly oxidative conditions and at elevated pH.[265,286]

The effect of the working electrode (WE) material was investigated by switching to a boron doped diamond (BDD) electrode. As with the glassy carbon electrode, a current loss was also observed with BDD over the course of several CV scans with a slightly more stable background measurement.
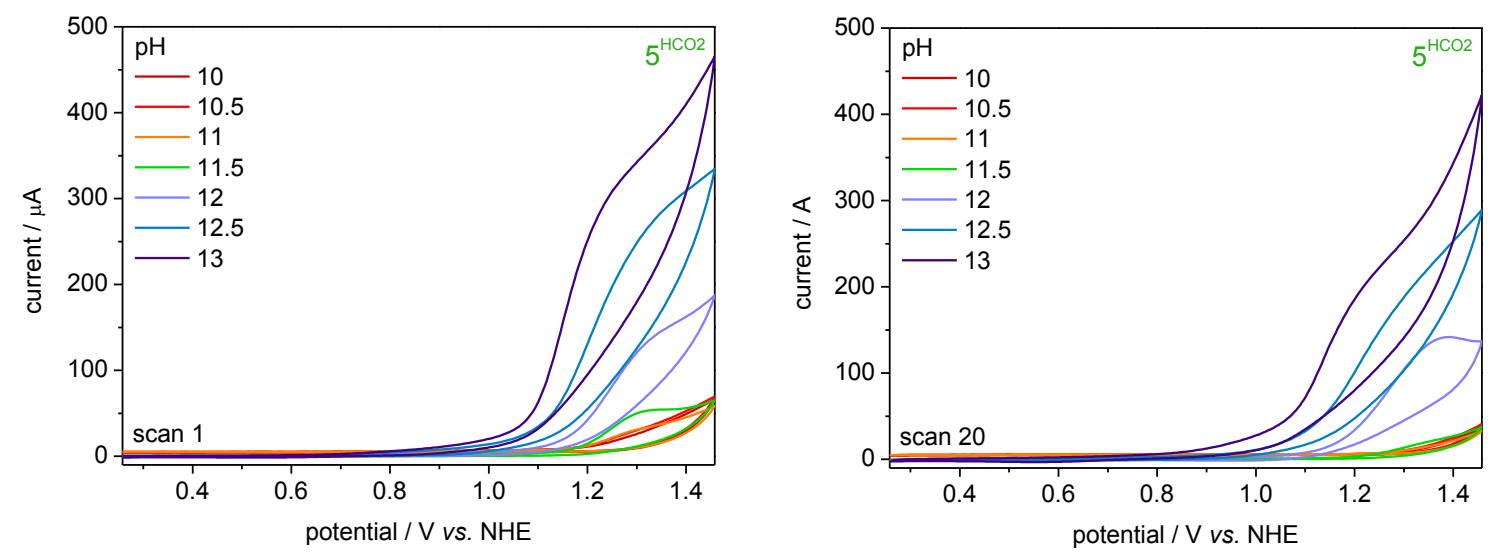

Figure 4.7: $\mathrm{pH}$ dependent CVs (left: scan 1, right: scan 20) of a $1 \mathrm{mM}$ solution of $\mathbf{5}^{\mathrm{HCO}}$ in phosphate buffer $(I=0.1 \mathrm{M})$ using a glassy carbon disc working electrode, a Pt disc as auxiliary and an MSE as the reference electrode. The potentials were converted to the NHE scale by adding $0.658 \mathrm{~V}$.

As WOCs based on earth-abundant metals are likely to undergo irreversible structural modifications under harsh conditions (basic $\mathrm{pH}$ and high potentials), ultimately leading to the transformation of the initially homogenous catalyst (then only the precatalyst) to a heterogenous metal oxide species, analyzing the stability of the WOCs is an essential element. ${ }^{[86]}$ This is especially important as the heterogenous species have been shown to be catalytically active in water oxidation. [287] Therefore, it must be elucidated if the pristine complex is in fact responsible for the catalytic activity.

Accordingly, the WE was first examined under CV experimental conditions. After cycling between 0.26 and $1.46 \mathrm{~V}$ for 20 scans in complex solution, no deposits were visible on the surface of the WE. The WE was then carefully rinsed with electrolyte and subsequently used for a measurement in a fresh blank solution (so-called rinse test). Surprisingly, the resulting CVs were not consistent. In some cases, a significant current similar to that in complex solution could be observed, while in other measurements the current was identical to the background scan. No trend was observed with increasing $\mathrm{pH}$ and even repeated measurements could not unambiguously confirm the presence or absence of a deposit on the electrode surface.

As discussed in the following section, the water oxidation capability and stability of $\mathbf{5}^{\mathbf{H C O}}$ was further investigated by means of controlled potential electrolysis (CPE) in combination with an oxygen sensor for $\mathrm{O}_{2}$ detection. Here, a constant oxidative potential is held for a longer time compared to the CV measurement. Consequently, a rinse test after this experiment is more meaningful, as it shows if the WOC is stable under turnover conditions. Furthermore, surface deposits can be detected by scanning or transmission electron microscopy (SEM/TEM), energy-dispersive X-ray spectroscopy (EDX) or $\mathrm{X}$-ray photoelectron spectroscopy (XPS). The formation of metal oxide nanoparticles in solution could be detected by dynamic light scattering experiments (DLS).[288,289] 


\subsection{Dioxygen Detection}

In order to confirm that the irreversible event observed in the CV (Figure 4.6) results from catalytic water oxidation, CPE experiments were conducted in combination with molecular oxygen detection. These measurements were performed by Nataliia Vereshchuk at the Institute of Chemical Research of Tarragona, Spain. Based on the results of the $\mathrm{pH}$ dependent cyclic voltammograms (Figure 4.7), the $\mathrm{O}_{2}$ detection experiments were performed at $\mathrm{pH}$ 11.65. This should ensure significant electrocatalytic behavior while reducing the chance of complex decomposition in comparison to experiments at higher $\mathrm{pH}$. The measurements were carried out in a two-compartment electrochemical cell separated by a frit and sealed by septa; one side holding the working electrode, and the other the counter and reference electrodes. For these experiments, a BDD plate was applied as working electrode, a Pt mesh as auxiliary and an MSE as reference electrode. Both compartments were filled with complex solution to prevent dilution in case solution passes through the frit.
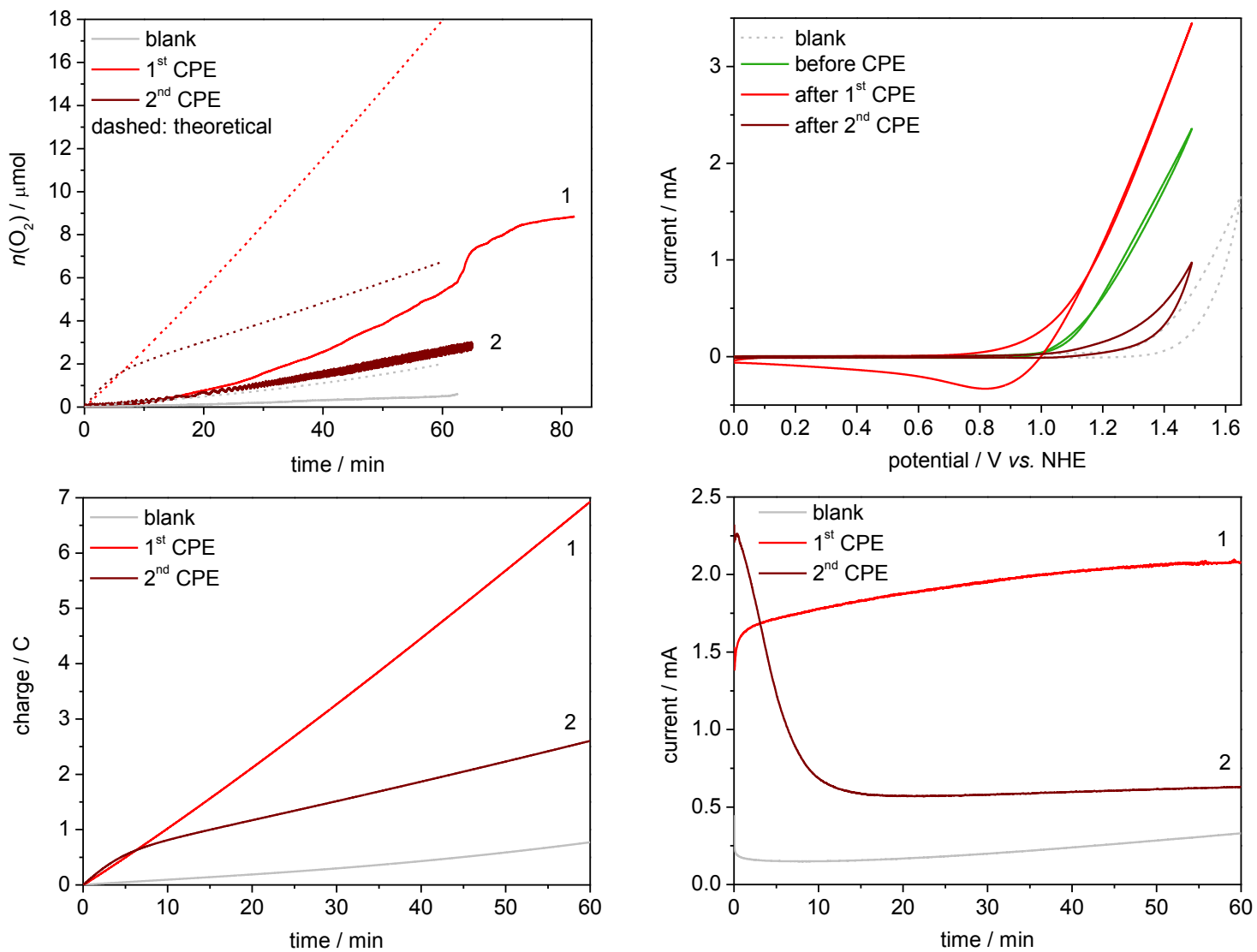

Figure 4.8: Electrochemical water oxidation catalysis of $\mathbf{5}^{\mathbf{H C O}}$ in phosphate buffer $(I=0.1 \mathrm{M}, \mathrm{pH} 11.65)$ using a BDD plate as a working electrode, a Pt mesh as an auxiliary and an MSE as reference electrode. The first controlled potential electrolysis (CPE, 1) was done in a complex solution $(0.5 \mathrm{mM})$, the second (2) in pure phosphate buffer without cleaning the working electrode (rinse test). Top left: $\mathrm{O}_{2}$ traces monitored by a Clark electrode during $\mathrm{CPE}$ for 60 minutes at $1.35 \mathrm{~V} v$ s. NHE (solid: experimentally observed, dashed: theoretical trace if all charge was used for $\mathrm{O}_{2}$ formation). For $1, \mathrm{O}_{2}$ detection was continued post-CPE until all formed bubbles were burst $\left(\mathrm{O}_{2}\right.$ detection only in gas phase). The bottom graphs depict the charge (left) and current (right) traces of the respective measurements. Top right: CVs before CPE (green) in a complex solution ( $0.5 \mathrm{mM})$ and after the first (light red) and second (dark red) CPE in a pure electrolyte. Accordingly, the current observed in the red CVs can only result from deposited substance. The potentials were converted to the NHE scale by adding $0.658 \mathrm{~V}$.

In a typical experiment, $\mathbf{5}^{\mathbf{H C O 2}}(0.5 \mathrm{mM})$ is dissolved in phosphate buffer $(\mathrm{pH} 11.65, I=0.1 \mathrm{M})$ and the solution is degassed by purging with $\mathrm{N}_{2}$. The dioxygen level is detected in the gas phase by a Clark electrode with a needle sensor pierced through the septum of the WE compartment. Once a stable baseline is reached, a constant potential of $1.35 \mathrm{~V} v$ s. NHE is applied. The CPE is run for 60 minutes, during which the produced molecular oxygen is detected by the Clark electrode. Within this hour, some 
$\mathrm{O}_{2}$ bubbles remain intact in the electrochemical cell and so the oxygen detection is continued even after the CPE ends. By adjusting the stirring speed, these bubbles are burst to ensure that all the produced dioxygen is measured.

Traces of the dioxygen detection are shown in Figure 4.8 (top left) in light red (1) and grey for the complex and blank measurements, respectively. The dashed lines correspond to the theoretical $\mathrm{O}_{2}$ values if all the transferred charge is responsible for water oxidation. The corresponding current and charge traces are displayed in Figure 4.8 (bottom) on the right and left, respectively. After subtraction of the blank measurement, a total amount of $8.24 \mu \mathrm{mol} \mathrm{O}_{2}$ are formed during the CPE (theoretically expected $n\left(\mathrm{O}_{2}\right)=15.95 \mu \mathrm{mol}$ ). This corresponds to a faradaic efficiency (experimentally detected $n\left(\mathrm{O}_{2}\right)$ divided by theoretically expected $n\left(\mathrm{O}_{2}\right)$ ) of $52 \%$, which is significantly higher than that observed for the parent complex XVII (15-24 \%, Scheme 4.2).[188]

After the first electrolysis, the BDD plate was carefully rinsed with phosphate buffer ( $\mathrm{pH} 11.65$ ) and a second CPE was run under identical conditions (1.35 V vs. NHE, 60 minutes), but in pure electrolyte without catalyst. During this CPE, a total amount of $2.23 \mu \mathrm{mol} \mathrm{O}_{2}$ are formed (Figure 4.8, top left, 2). The current is initially high when the potential is applied, but gradually decreases over the course of ten minutes after which it remains stable (Figure 4.8, bottom left, 2). This suggests that there is an active species on the electrode surface, which redissolves in the electrolyte solution; a situation that has previously been observed for surface deposits. ${ }^{287]}$ Additionally, cyclic voltammograms were measured before CPE (green) in complex solution, as well as after the first (light red) and second CPE (dark red) in pure phosphate buffer solution (Figure 4.8, top right). The CVs clearly show that the rinse test after the first CPE is positive, exhibiting a significantly higher catalytic current than the complex measurement before CPE with a less positive onset potential. This is further supported by the visual impression of the BDD plate after the first CPE, which exhibited a yellowish metallic gloss. After the second CPE, the voltammogram is quite similar to the blank measurement (grey), confirming that the surface deposit formed after the first CPE redissolves during the second run.

\subsection{Characterization of the Surface Deposit}

To distinguish the nature of the surface deposit, XPS measurements were conducted. For this, three samples were prepared: (i) a blank sample obtained by CPE in pure electrolyte solution (B), (ii) a drop cast samplex of $\mathbf{5}^{\mathrm{HCO}}$ (D), which should show the XPS features of the pristine complex, and (iii) a sample after CPE in a $0.5 \mathrm{mM}$ solution of $\mathbf{5}^{\mathrm{HCO}}$ in phosphate buffer (C). The conditions chosen were identical to those of the dioxygen detection experiments: a BDD plate as working electrode, $\mathrm{pH} 11.65$ phosphate buffer $(I=0.1 \mathrm{M})$ and electrolysis for 60 minutes at $1.35 \mathrm{~V} v s$. NHE. The applied BDD plates were pretreated by CPE for 5 minutes at $2.26 \mathrm{~V}$ vs. NHE in $\mathrm{pH} 2$ phosphate buffer $(I=0.1 \mathrm{M})$. The electrolysis was repeated twice, and a CV $((-0.14)-2.26 \mathrm{~V}, 100 \mathrm{mV} / \mathrm{s})$ was measured before and after each CPE to monitor any changes. In case of unstable CV measurements, the pretreatment proceeded again with another CPE. This was the same procedure performed before the dioxygen detection experiments.

The XPS spectra of the full range (top left), as well as the $\mathrm{Cu}(2 \mathrm{p}$ ) (top right), N(1s) (bottom left) and $\mathrm{O}(1 \mathrm{~s})$ (bottom right) areas are depicted in Figure 4.9 for all three samples. The $\mathrm{Cu}(2 \mathrm{p})$ traces of $\mathbf{C}$ and D exhibit peaks at around 935 and $955 \mathrm{eV}$ originating from $\mathrm{Cu}\left(2 \mathrm{p}^{3 / 2}\right)$ and $\mathrm{Cu}\left(2 \mathrm{p}^{1 / 2}\right)$, respectively, with satellite features at around 945 and $965 \mathrm{eV}$. The latter are indicative of $\mathrm{Cu}^{\mathrm{II},[290-293]}$ which is in line with the expected composition of $\mathbf{D}$. The N(1s) spectra are very significant, only displaying a peak for the drop cast sample ( $402 \mathrm{eV}$ ). This clearly shows that the pristine complex is not deposited on the surface during CPE (C). Finally, the O(1s) trace of $\mathbf{C}$ exhibits peaks at 532 and $536 \mathrm{eV}$, different to the spectra of $\mathbf{B}$ and $\mathbf{D}$, which implies that the surface deposit contains oxygen. Overall, this suggests that the electrodeposited material is a copper(II) oxide or hydroxide species. ${ }^{[290-293]}$ This concludes that the

xx For the drop cast sample, a highly concentrated solution of $\mathbf{5}^{\mathrm{HCO} 2}$ in phosphate buffer (pH 11.65, $I=0.1 \mathrm{M}$ ) was slowly dropped on the electrode surface and left to dry. 
observed electrocatalysis is heterogenous and $\mathbf{5}^{\mathrm{HCO}}$ merely acts as the precatalyst, which degrades to a catalytically active species under the given conditions.
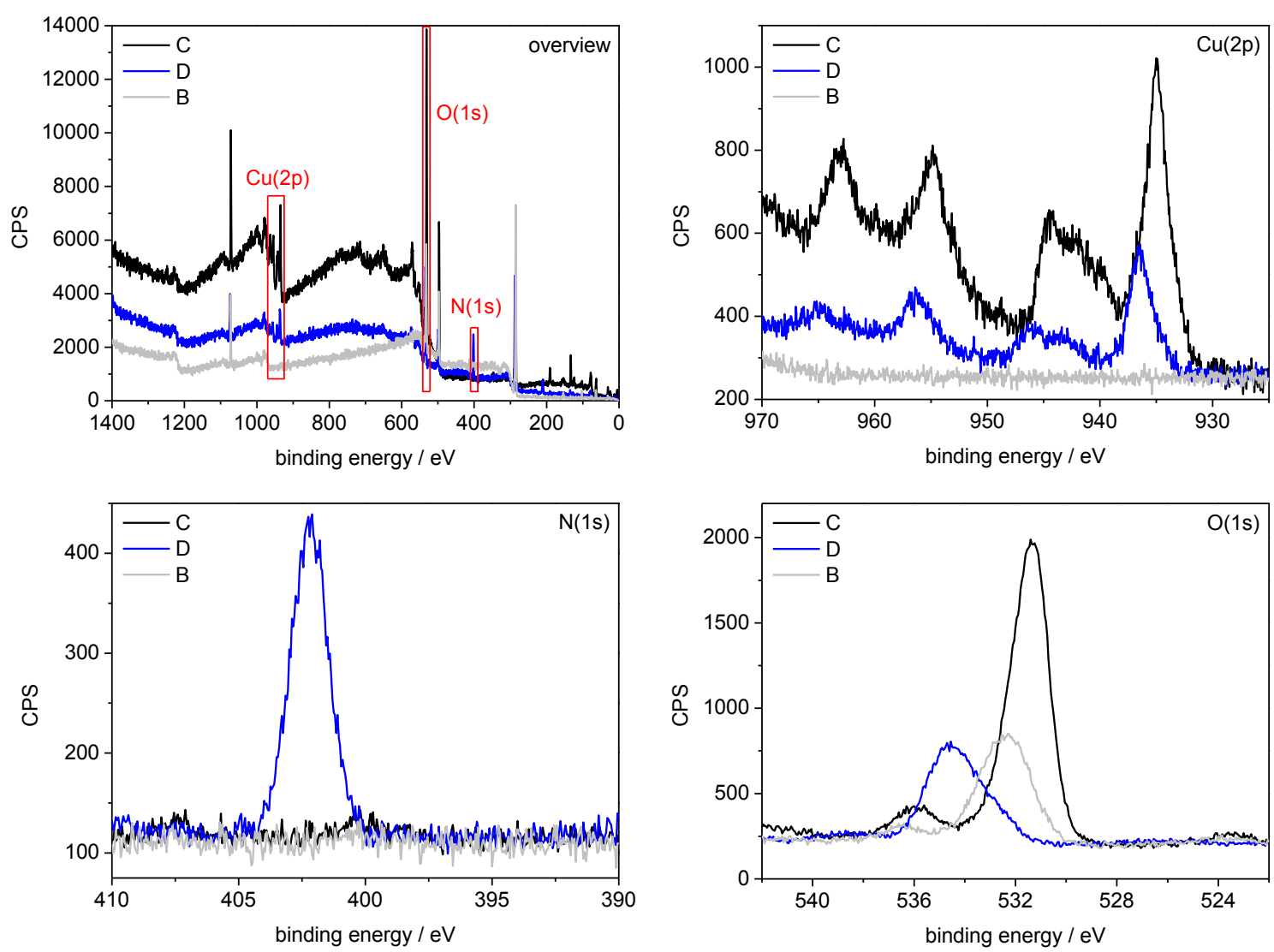

Figure 4.9: XPS spectra of the full range (top left), as well as the $\mathrm{Cu}(2 \mathrm{p})$ (top right), $\mathrm{N}(1 \mathrm{~s})$ (bottom left) and $\mathrm{O}(1 \mathrm{~s})$ (bottom right) areas of the BDD plate after CPE (60 minutes, $1.35 \mathrm{~V} v s$. NHE) of $5^{\mathrm{HCO}}$ in phosphate buffer $(I=0.1 \mathrm{M}$, $\mathrm{pH} 11.65$ ) (C, black) in comparison to a drop cast (D, blue) and a blank sample (B, grey). The Cu(2p), N(1s) and $\mathrm{O}(1 \mathrm{~s})$ spectra were adjusted so that the baseline is more or less the same for all three samples.

\subsection{Summary and Conclusion}

Previous investigations of the dinuclear copper(II) complex XVII with a pyrazolate/tacn hybrid ligand showed a large irreversible wave in the CV upon electrochemical oxidation, suggesting its potential to serve as a first-row transition metal-based WOC.[188] Inspired by this result, the dinuclear copper(II) complexes $\mathbf{5}^{\mathbf{H C O 2}}$ and $\mathbf{5}^{\mathbf{O A c}}$ were synthesized based on the proligand HL ${ }^{2 \mathrm{a}}$ with formate and acetate as co-ligand in the bimetallic cleft, respectively. Replacing the isopropyl periphery at the tacn moieties (as in XVII) with methyl groups rendered the complexes fully water soluble, benefiting the study of water oxidation. Additionally, the related compounds $6^{\mathrm{HCO}}$ and $\mathbf{6}^{\mathbf{0 A c}}$ were prepared based on $\mathrm{HL}^{2 \mathrm{~b}}$.

All four compounds were characterized by means of XRD, ESI-MS and combustion analysis. $\mathbf{5}^{\mathrm{HCO2}}$ and $5^{\mathbf{O A c}}$ were additionally investigated regarding their electrochemical behavior under inert (MeCN, $0.1 \mathrm{M} \mathrm{NBu}_{4} \mathrm{PF}_{6}$ ) and ambient conditions (phosphate buffer, $I=0.1 \mathrm{M}$ ). In MeCN, both complexes exhibit two redox processes ( $E_{1}$ and $E_{2}$ ). By means of UV/vis-SEC measurements, $E_{1}$ (quasi-reversible) could

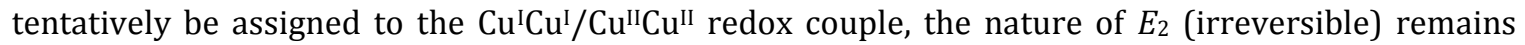
unclear. The reduction of $\mathbf{5}^{\mathbf{H C O}}$ is slightly easier than that of $\mathbf{5}^{\mathbf{O A c}}$, which might be due to the electrondonating effect of the methyl group of the acetate molecule in the bimetallic pocket of $\mathbf{5}^{\mathbf{0 A c}}$ in comparison to the formate moiety in $\mathbf{5}^{\mathbf{H C O}}$. In phosphate buffer ( $\left.\mathrm{pH} 12.4\right)$, both complexes exhibit an irreversible wave associated with a catalytic process with $E_{\text {onset }}=1.1 \mathrm{~V} v s$. NHE. This catalytic wave is 
$\mathrm{pH}$ dependent, supported by the observation of current increment and cathodical potential shift with rising $\mathrm{pH}$.

In order to confirm the WO catalysis activity of $5^{\mathrm{HCO} 2}$, dioxygen detection experiments with CPE were conducted in $\mathrm{pH} 11.65$ phosphate buffer solution. Within these experiments, $\mathrm{O}_{2}$ was detected with a faradaic efficiency of $52 \%$, confirming the WO nature of the irreversible oxidative process in the CV. However, by means of a rinse test, it was shown that a surface deposit is responsible for this activity. With the help of XPS measurements, the electrodeposited material could be identified as a copper(II) oxide or hydroxide species. This concludes that the observed electrocatalysis is heterogenous and $\mathbf{5}^{\mathrm{HCO}}$ merely acts as the precatalyst, which degrades to a catalytically active species under the given conditions. A glance at ( $\left.\mathrm{L}^{2 \mathrm{a}}\right)^{-}$quickly reveals weak spots in the multiple methylene groups, which are prone to oxidation, as previously observed in other complexes. ${ }^{[82,294-296]}$ These results once more demonstrate the indispensable necessity of oxidatively rugged ligand scaffolds for water oxidation catalysts. 


\section{Water Oxidation Catalysis with Cobalt Complexes}

Since the late 1960s, simple inorganic cobalt salts have been studied as potential catalysts for the oxidation of water to dioxygen.[297-301] Despite this knowledge, the interest in cobalt-based WOCs remained negligible, until in 2008 Nocera and coworkers showed that a heterogenous oxide material based on cobalt phosphate $\left(\mathrm{CoP}_{\mathrm{i}}\right)$ was highly active for water oxidation at neutral $\mathrm{pH}(\sim 7)$ and low overpotential. ${ }^{[302]}$ Further studies on $\mathrm{CoP}_{\mathrm{i}}$ revealed that $\mathrm{Co}^{\mathrm{IV}}$ is generated at potentials suitable for water oxidation ${ }^{[303]}$ and $\mathrm{O}-\mathrm{O}$ bond formation occurs at the edges of $\mathrm{CoO}_{6}$ octahedra, common in all

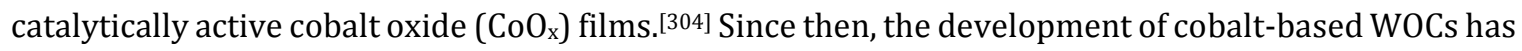
progressed steadily and has been focused on $\mathrm{Co}_{4} \mathrm{O}_{4}$ cubanes, ${ }^{[305-316]}$ inspired by the $\mathrm{Mn}_{4} \mathrm{CaO}_{5}$ core of the OEC, and polyoxometallates (POMs).[317-327] Beyond these, the research field of molecular cobaltbased WOCs remains underdeveloped.

In 2011, the Berlinguette group presented the first mononuclear $\mathrm{Co}-\mathrm{WOC}\left[\left(\mathrm{L}^{\mathrm{XIX}}\right) \mathrm{Co}\left(\mathrm{H}_{2} \mathrm{O}\right)\right]^{2+}, \mathbf{X I X}$ (Scheme 5.1), based on the oxidatively stable pentadentate ligand 2,6-(bis(bis-2-pyridyl) methoxymethane)pyridine ( $\mathrm{L}^{\mathrm{XIX}}{ }^{\left[{ }^{[328]}\right.}$ According to cyclic voltammetry, $\mathbf{X I X}$ is capable of electrocatalytically oxidizing water to dioxygen between $\mathrm{pH} 7.6$ and 10.3, exhibiting an overpotential of $\sim 510 \mathrm{mV}$ and a rate of $79 \mathrm{~s}^{-1}$ at $\mathrm{pH} 9.2$ (a fluorescence optical probe was used to confirm $\mathrm{O}_{2}$ evolution). Multiple experiments were conducted to confirm the homogeneity of XIX and rule out the formation of active $\mathrm{CoO}_{\mathrm{x}}$ films or nanoparticles, however, a contribution of the latter to the catalytic current could not unambiguously be excluded.[328,329] Based on DFT calculations, 0-0 bond formation is assumed to proceed via nucleophilic attack of a hydroxide on a $\mathrm{Co}^{\mathrm{IV}}{ }_{-o x y l}$ species.[330]

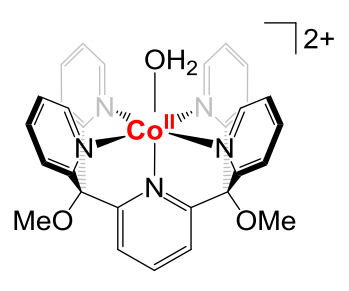

$\mathbf{X I X}$

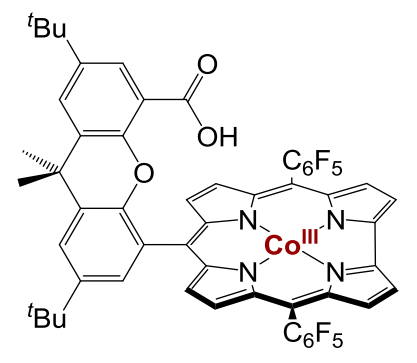

$\mathbf{X X}$

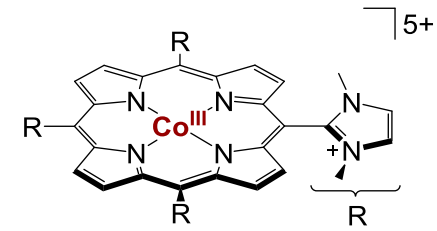

$\mathbf{X X I}$

Scheme 5.1: Representations of the mononuclear cobalt-based WOCs XIX-XXI.

In the same year, Nocera and coworkers reported the use of cobalt xanthene hangman corroles as WOCs.[331] Interestingly, the analogous porphyrin complexes were previously shown to catalyze the reverse reaction, viz. oxygen reduction. ${ }^{[32]}$ The mononuclear complex $\mathbf{X X}$ (Scheme 5.1) and its $\beta$-octafluorinated congener are capable of driving electrocatalytic water oxidation at $\mathrm{pH} 7$, the latter reaching a TOF of $0.81 \mathrm{~s}^{-1}$ and performing at an overpotential of $\sim 630 \mathrm{mV}$. Quantum chemical calculations revealed a non-innocent behavior of the corrole backbone and suggested that $0-0$ bond 
formation proceeds via WNA involving an $\left[\left(\mathrm{L}^{\mathrm{Xx} \cdot+}\right) \mathrm{Co}^{\mathrm{IV}=0}\right]^{-}$species. ${ }^{\mathrm{xx}[333,334]} \mathrm{A}$ beneficial effect for $\mathrm{O}-\mathrm{O}$ bond formation was thereby attributed to the xanthene hangman motif, which is capable of preorganizing and activating substrate water within the hangman cleft via hydrogen bonding. ${ }^{[335]}$

Cobalt porphyrin complexes have also been shown to serve as WOCs. Among these, [( $\left.\mathrm{LXI}^{\mathrm{XXI}} \mathrm{Co}^{\mathrm{IIII}}\right]^{5+}$ (XXI, Scheme 5.1), based on the highly electron-deficient meso-dimethylimidazolium porphyrin ( $\mathrm{L}^{\mathrm{XXI}}$ ), is the most efficient catalyst.[336] At pH 7, XXI reaches a high TOF of $1400 \mathrm{~s}^{-1}$, operating at an overpotential of $\sim 600 \mathrm{mV}$ for the electrocatalytic oxidation of water to dioxygen. By means of spectroscopic techniques and catalyst reisolation experiments, the homogenous nature of the observed catalysis was confirmed. The $\mathrm{O}-\mathrm{O}$ bond formation in XXI is assumed to proceed via WNA at a $\left[\left(\mathrm{LXI}^{\mathrm{XX}+}\right) \mathrm{Co}^{\mathrm{IV}}=\mathrm{O}\right]^{4+}$ species, in which the ligand has been oxidized to a radical cation. Cobalt porphyrin complexes were also demonstrated to serve as catalysts for photo-driven water oxidation. ${ }^{[337]}$

As for most first-row transition metals, the development of dinuclear WOCs based on cobalt is a widely unexplored field. In a joint effort, the groups of Llobet and Stahl reported the first dicobalt

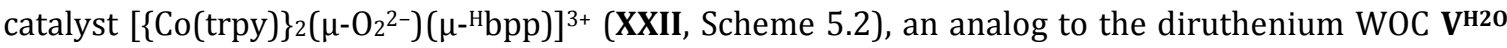
(see introduction of Chapter 3).[338] Interestingly, the same complex was shown to be capable of performing the $4 \mathrm{e}^{-}$reduction of dioxygen to water in the presence of octamethylferrocene as sacrificial reductant.[339] The peroxo complex XXII is readily formed under ambient conditions from cobalt(II) chloride, $\mathrm{Na}^{\mathrm{H}} \mathrm{bbp}$ and terpyridine in methanol. At $\mathrm{pH}$ 2.1, XXII exhibits an electrocatalytic wave at an overpotential of $\sim 800 \mathrm{mV}$. Replacing the ancillary terpyridine molecules by more electron-donating bis(N-methyl-imidazolyl)pyridine ligands (XXIII, Scheme 5.2) results in a cathodic shift of the catalytic wave by $70 \mathrm{mV}$ and an enhancement of the steady-state catalytic current. This systematic modulation of the catalytic activity provides evidence for the homogenous nature of water oxidation conducted by XXII and XXIII. In 2016, a thorough characterization of the reaction intermediates of XXII was accomplished, revealing $\mathrm{O}-\mathrm{O}$ bond formation to proceed via WNA involving the end-on superoxo complex $\left[\mathrm{H}_{2} \mathrm{O}-\mathrm{Co}^{\mathrm{III}} \mathrm{Co}^{\mathrm{III}}-\mathrm{O}_{2}{ }^{--}\right]^{4+}$ (all scaffold ligands are omitted due to clarity), which was characterized by resonance Raman, EPR and X-ray absorption spectroscopy. ${ }^{[13]}$ The proposed catalytic cycle was confirmed by DFT calculations.
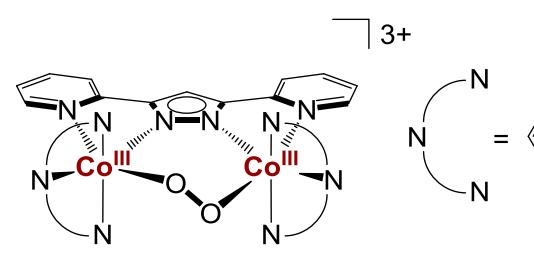

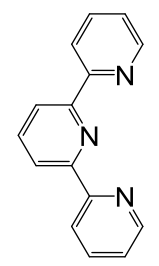

XXII

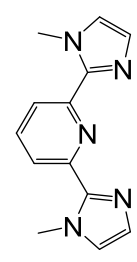

XXIII

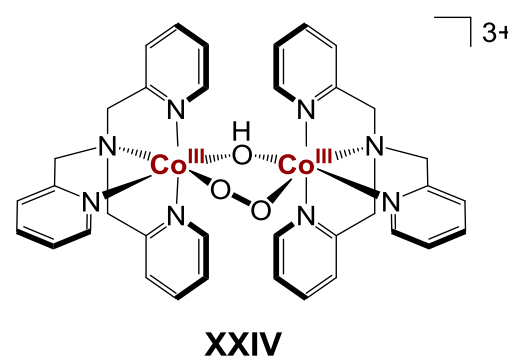

XXIV

Scheme 5.2: Representations of the dinuclear cobalt complexes XXII-XXIV for catalytic water oxidation.

A large drawback of Co-WOCs is their intrinsic instability, which often leaves warranted doubt concerning the molecular nature of the catalysts. ${ }^{[83,340]}$ Accordingly, multiple cobalt-based WOCs have been shown to decompose under the harsh catalytic conditions (high potentials and/or $\mathrm{pH}$ ) to active $\mathrm{CoO}_{\mathrm{x}}$ films or nanoparticles. ${ }^{[82,341,342]}$ In other cases, the catalytically active heterogenous material results from small impurities of cobalt(II) salts. ${ }^{[343]}$ One example of the former is the dicobalt complex $\left[\left\{(\right.\right.$ tpa $\left.) \mathrm{Co}_{2}(\mu-\mathrm{OH})\left(\mu-\mathrm{O}_{2}{ }^{2-}\right)\right], \mathbf{X X I V}$ (Scheme 5.2), based on tris(2-pyridylmethyl)amine (tpa). The original publication proposes XXIV to act as a molecular catalyst for the photo-driven oxidation of water to dioxygen. ${ }^{[344]}$ The homogeneity of XXIV was scrutinized by dynamic light scattering (DLS) experiments, revealing no $\mathrm{CoO}_{\mathrm{x}}$ particles. Two years later, a reinvestigation of the water oxidation

xxi The expression of a radical cation $(\cdot+)$ is only used to illustrate that the ligand has been oxidized but does not refer to the overall charge of the ligand. In the $\left[\left(\mathrm{LXX}^{\mathrm{X} \cdot+}\right) \mathrm{Co}^{\mathrm{IV}}=0\right]^{-}$species, the carboxylate moiety at the xanthene group is deprotonated. 
catalyzed by XXIV identified heterogenous $\mathrm{CoO}_{\mathrm{x}}$ as the actual catalyst. ${ }^{[82]}$ This emphasizes the caution that must be taken with respect to DLS, as the size of low molecular weight oxide clusters, including $\mathrm{CoP}_{\mathrm{i}},[345,346]$ can be smaller than the detection limit $(1-2 \mu \mathrm{m})$. Interestingly, oxidation induced $\mathrm{CoO}_{\mathrm{x}}$ formation from mononuclear cobalt complexes with organic ligands has also been exploited to prepare efficient heterogenous catalysts.[347-349]

The following chapter discusses the synthesis of five dicobalt complexes based on the dinucleating ligand scaffolds $\mathrm{HL}^{2 \mathrm{a}}$ and $\mathrm{HL}^{2 \mathrm{~b}}$, previously introduced in Chapter 4. In addition to a thorough spectroscopic and magnetometric characterization of the complexes, their electrochemical behavior under an $\mathrm{N}_{2}$ atmosphere was analyzed. Finally, one dicobalt compound was electrochemically studied under ambient conditions concerning its ability to electrocatalytically oxidize water to dioxygen.

\subsection{Cobalt Complexation}

The overall synthetic routes for the preparation of five dinuclear cobalt complexes from $\mathrm{HL}^{2 \mathrm{a}}$ and $\mathrm{HL}^{2 \mathrm{~b}}$ are summarized in Scheme 5.1. In each case, $\mathrm{NEt}_{3}$ was used as base to deprotonate the ligand and cobalt(II) perchlorate was selected as metal precursor. Varying the reaction conditions such as solvent, atmosphere (ambient or $\mathrm{N}_{2}$ ), as well as the addition of co-ligands gave rise to different complexes. Accordingly, (i) the aquo/hydroxo bridged complex $\mathbf{7}^{\mathbf{0 2}} \mathbf{H} \mathbf{3}$ was obtained in $\mathrm{MeOH}$, while addition of MeCN resulted in $7^{\text {MecN }}$ with $\mathrm{HL}^{2 a}$ under an $\mathrm{N}_{2}$ atmosphere, (ii) synthesis under inert or ambient conditions yielded either $\mathbf{7}^{\mathrm{MecN}}$ or the acetamide bridged complex $\mathbf{7}^{\mathrm{A}}$, respectively, based on $\left(\mathrm{L}^{2 \mathrm{a}}\right)^{-}$, and (iii) addition of either $\mathrm{HCO}_{2}-$ or $\mathrm{OAc}^{-}$as co-ligand was the key to the formation of $\mathbf{8}^{\mathrm{HCO}}$ or $\mathbf{8}^{\mathbf{0 A c}}$, respectively, from $\mathrm{HL}^{2 \mathrm{~b}}$ under ambient conditions.

Although the preparation and crystallization of $7^{\mathrm{A}}$ was originally conducted with deaerated solvents under an $\mathrm{N}_{2}$ atmosphere, the ${ }^{1} \mathrm{H}-\mathrm{NMR}$ spectrum (discussed below) shows a diamagnetic feature, suggesting that the cobalt ions were oxidized to $\mathrm{Co}^{\mathrm{III}}$ due to $\mathrm{O}_{2}$ contamination. Considering that the preferred geometry of $\mathrm{Co}^{\mathrm{III}}$ ions is octahedral due to the high crystal field stabilization energy of the $\left(\mathrm{t}_{2 \mathrm{~g}}\right)^{6}$ configuration, ${ }^{[350]}$ co-coordination of MeCN seems very likely in $\mathbf{7}^{\mathrm{A}}$. By contrast, the cobalt ions in $\mathbf{8}^{\mathrm{HCO}}$ and $\mathbf{8}^{\mathbf{0 A c}}$ were not oxidized to Co ${ }^{\mathrm{III}}$ during synthesis under ambient conditions. A reason for this could be the introduction of more bulky residues at the tacn moieties in $\left(\mathrm{L}^{2 \mathrm{~b}}\right)$ - in comparison to $\left(\mathrm{L}^{2 \mathrm{a}}\right)^{-}$, which prevents additional solvent coordination and thus, the $\mathrm{Co}^{\mathrm{II}}$ ions do not favor oxidation.

The diamagnetic character of the low-spin $\mathrm{d}^{6} \mathrm{Co}^{\mathrm{III}}$ ions in $7^{\mathrm{A}}$ enable its NMR spectroscopic analysis. The ${ }^{1} \mathrm{H}-\mathrm{NMR}$ spectrum in $\mathrm{CD}_{3} \mathrm{CN}$ under inert conditions (Figure 5.1, $\mathrm{A}$; for complete NMR characterization see appendix, Figures A.80-A.87) shows a large number of superimposed multiplets in the aliphatic region (1.75-4.75 ppm). The presence of only one pyrazole $1-\mathrm{H}$ resonance suggests a clean NMR sample. In order to simplify the ${ }^{1} \mathrm{H}-\mathrm{NMR}$ spectrum, a homonuclear decoupled ${ }^{1} \mathrm{H}-\mathrm{NMR}$ spectrum was recorded. The so-called pure shift ${ }^{1} \mathrm{H}$-NMR spectrum (Figure 5.1, B) shows 33 signals in the aliphatic region, which is in agreement with a $C_{1}$-symmetric complex with diastereotopic $\mathrm{H}$ atoms at each $\mathrm{CH}_{2}$ group. With the help of the pure shift ${ }^{1} \mathrm{H}-\mathrm{NMR}$ spectrum and two-dimensional NMR spectroscopy $\left({ }^{1} \mathrm{H}-{ }^{-1} \mathrm{H}-\mathrm{COSY},{ }^{1} \mathrm{H}-{ }^{1} \mathrm{H}-\mathrm{NOESY},{ }^{1} \mathrm{H}-{ }^{13} \mathrm{C}-\mathrm{HSQC},{ }^{1} \mathrm{H}-{ }^{-13} \mathrm{C}-\mathrm{HMBC}\right)$, all carbon and hydrogen signals could eventually be assigned.xxii A singlet at $1.74 \mathrm{ppm}$ is attributed to the $\mathrm{CH}_{3}$ group of the acetamide moiety in the bimetallic cleft. The ${ }^{1} \mathrm{H}-1 \mathrm{D}-\mathrm{NOESY}$ spectrum (Figure 5.1, C) after selective excitation of this resonance, shows correlations to the methyl groups 11 and 21, as well as to each one $\mathrm{H}$ atom of the methylene linkers of the ( $\mathrm{L}^{2 \mathrm{a}}$ - ligand scaffold. Furthermore, a NOESY correlation is observed to a resonance at $3.23 \mathrm{ppm}$, which could not be assigned to a $\mathrm{CH}_{2}$ or $\mathrm{CH}_{3}$ group and is thus assumed to originate from the NH proton of the acetamide moiety. An NMR analysis of the paramagnetic cobalt(II) complexes was also attempted, however, without success.

xxii Please note, the distinction between the diastereotopic $\mathrm{H}$ atoms of $\left(\mathrm{L}^{2 \mathrm{a}}\right)^{-}$and the two tacn sidearms is not possible and is merely done for simplification. 


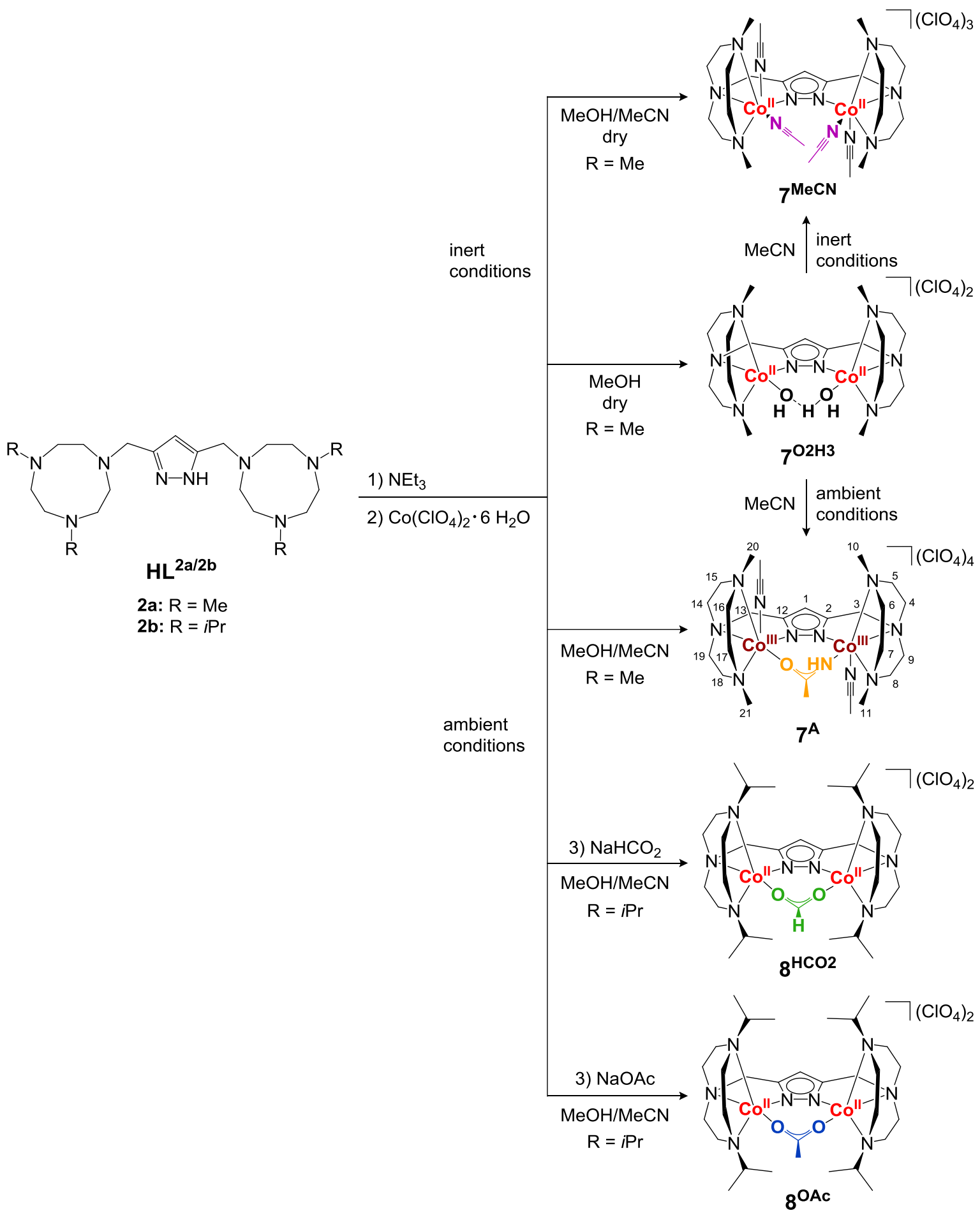

Scheme 5.3: Representation of the synthesis of the dinuclear cobalt complexes $7^{\mathrm{MecN}}, \mathbf{7 0 2}^{\mathbf{0} \mathbf{H} 3}$ and $\mathbf{7}^{\mathrm{A}}$, as well as $8^{\mathrm{HCO}}$ and $\mathbf{8}^{\mathbf{O A c}}$. The preparation and crystallization of $7^{\mathrm{A}}$ was originally attempted with deaerated solvents under an $\mathrm{N}_{2}$ atmosphere, however, the cobalt ions were oxidized to Co ${ }^{\text {III }}$ in the process, suggesting that there was $\mathrm{O}_{2}$ contamination. For simplification, it is thus classified as "synthesis under ambient conditions" in this scheme. The exact structures of $\mathbf{7}^{\mathbf{0 2 H}}$ and $\mathbf{7}^{\mathrm{A}}$ could not unambiguously be elucidated but are assumed as depicted due to their characterization (discussed in the text). 


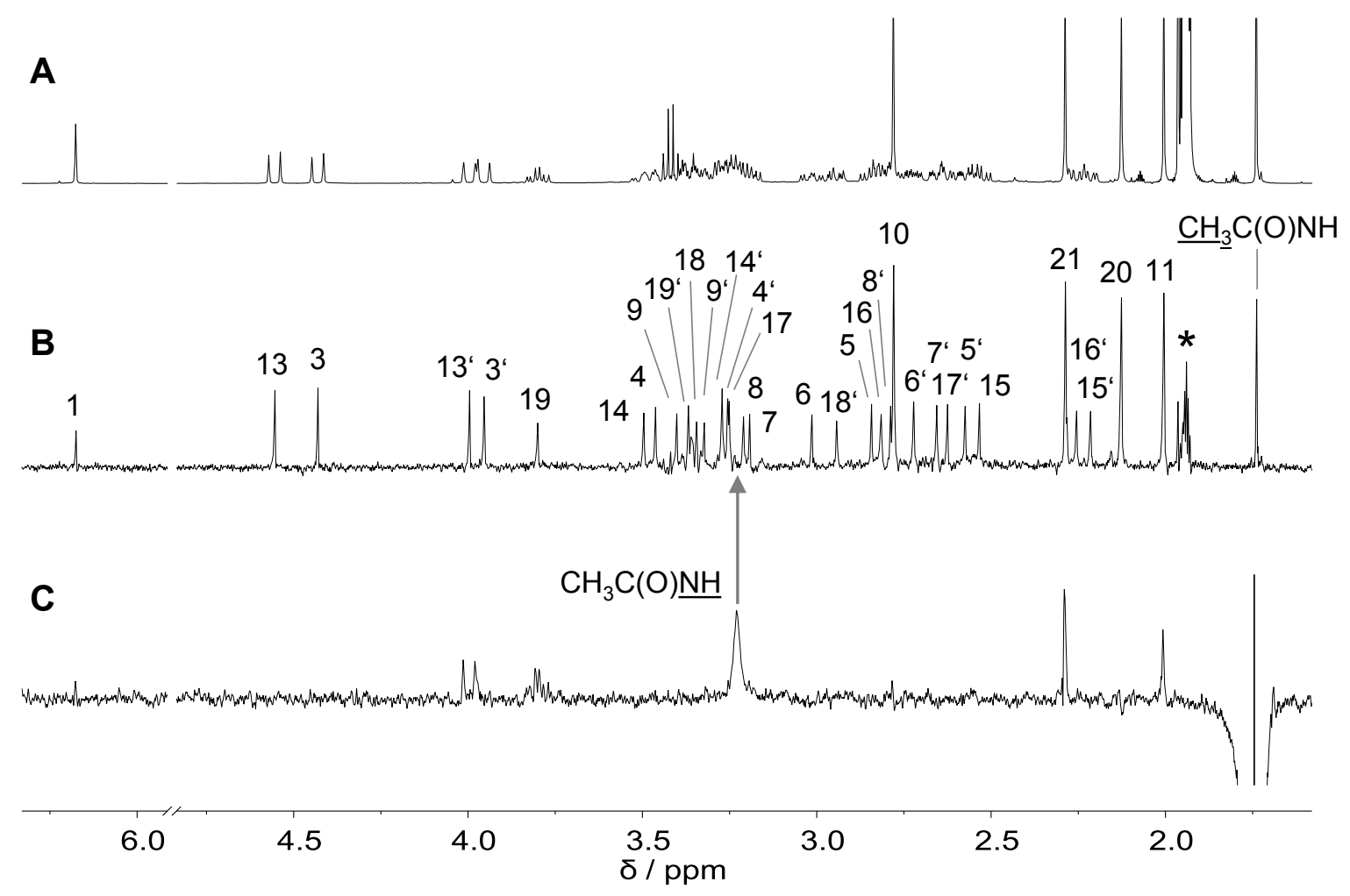

Figure 5.1: ${ }^{1} \mathrm{H}-\mathrm{NMR}$ spectroscopy $(500 \mathrm{MHz})$ of $7^{\mathrm{A}}$ in $\mathrm{CD}_{3} \mathrm{CN}$ at room temperature under inert conditions. A: ${ }^{1} \mathrm{H}-$ NMR spectrum. B: Pure shift ${ }^{1} \mathrm{H}-\mathrm{NMR}$ spectrum. C: ${ }^{1} \mathrm{H}-1 \mathrm{D}-\mathrm{NOESY}$ spectrum after selective excitation of the resonance at $1.74 \mathrm{ppm}$ corresponding to the methyl group of the acetamide moiety. The peaks are assigned according to the labeling scheme in Scheme 5.3. The asterisk denotes the residual solvent peak. Please note, the distinction between prime and non-prime (diastereotopic $\mathrm{H}$ atoms) for the ( $\mathrm{L}^{2 \mathrm{a}}$ )- ligand and the two tacn sidearms is not possible and is merely done for simplification.

An alternative synthetic route for the formation of compound $\mathbf{7}^{\mathrm{A}}$ is given by oxidation of $\mathbf{7}^{\mathbf{0 2} \mathrm{H} 3}$ in MeCN as could be nicely monitored by UV/vis spectroscopy (Figure 5.2). The UV/vis spectrum of $\mathbf{7}^{\mathbf{0 2 H}} \mathbf{3}$ in the $\mathrm{Co}^{\mathrm{II}} \mathrm{Co}^{\mathrm{II}}$ state possesses local absorption maxima at $452\left(\varepsilon=116 \mathrm{~L} \cdot \mathrm{mol}^{-1} \cdot \mathrm{cm}^{-1}\right)$ and $550 \mathrm{~nm}(238)$, which correspond to d-d transitions (Figure 5.2, left). Upon oxidation with dry $\mathrm{O}_{2}$, the overall intensity in the UV/vis spectrum increases and new bands arise at 297 and $359 \mathrm{~nm}$ with a shoulder at $479 \mathrm{~nm}$ (Figure 5.2, right; grey spectrum). After several days, an entirely new spectrum is observed with local absorption maxima at 303 and $480 \mathrm{~nm}$ (dashed grey), which is in very good agreement with the UV/vis spectrum of $7^{\mathrm{A}}$ (orange). The acetamide moiety is presumably formed by nucleophilic attack of the $\mathrm{OH}^{-}$ on an acetonitrile ligand, which replaces the aquo group in the bimetallic cleft upon dissolution of $7^{\mathbf{0 2}} \mathbf{H}$ in MeCN (Figure 5.2).

In contrast to the UV/vis spectrum of $7^{\mathrm{MeCN}}$ ( $\left.\mathrm{Co}^{\mathrm{II}} \mathrm{Co}{ }^{I I}\right)$, which exhibits a main absorption at $464 \mathrm{~nm}$ (152) with a shoulder at $543 \mathrm{~nm}$ (51) (see experimental section), $7^{\mathbf{0 2}} \mathbf{H 3}\left(\mathrm{Co}^{\mathrm{II}} \mathrm{Co}^{\mathrm{II}}\right)$ shows no typical absorptions for octahedrally coordinated high-spin cobalt(II) complexes.[350] Instead, its UV/vis spectrum is similar to those of $8^{\mathrm{HCO}}$ and $\mathbf{8}^{\mathbf{O A c}}$ (discussed in Section 5.3.1). This finding suggests that a coordination number (CN) of five best describes the environment of the cobalt ions in $\mathbf{7 0 2 H 3}^{\mathbf{2}}$.

The integrity of all five complexes in solution was confirmed by ESI-mass spectrometry. The ESI(+)MS in $\mathrm{MeOH}\left(7^{02 \mathrm{H}}\right)$ and $\mathrm{MeCN}\left(7^{\mathrm{MeCN}} / 7^{\mathrm{A}}\right)$ for the $\mathrm{Me}_{2}$-tacn complexes exhibit signals of both monoand divalent cationic species. In comparison to the simulated spectra, the dications could be assigned to $\left[\mathrm{M}-\mathrm{H}_{2} \mathrm{O}\right]^{2+}\left(\mathrm{m} / \mathrm{z} 269.9, \mathbf{7}^{\mathbf{0}} \mathrm{H}^{3}\right),\left[\mathrm{M}-4 \mathrm{MeCN}+\mathrm{ClO}_{4}\right]^{2+}\left(310.8,7^{\mathrm{MeCN}}\right)$ and $[\mathrm{M}-2 \mathrm{MeCN}]^{2+}\left(290.3,7^{\mathrm{A}}\right)$. Peaks at $m / z$ 639.1, 721.1 and 680.1 for $\mathbf{7}^{\mathbf{0 2}} \mathbf{H} 3,7^{\mathrm{MecN}}$ and $\mathbf{7}^{\mathrm{A}}$, respectively, were found to correspond to these particular species with an additional perchlorate anion. Beyond this, the ESI(-)-MS of 702 3 shows a signal at $m / z 838.9$ consistent with an $\left[\mathrm{M}-\mathrm{H}_{2} \mathrm{O}+3 \mathrm{ClO}_{4}\right]^{-}$species (appendix, Figures A.28-A.35). 
The ESI(+)-MS in MeCN for the $i \mathrm{Pr}_{2}$-tacn complexes display signals corresponding to the molecular ion peak $[\mathrm{M}]^{2+}$ and $\left[\mathrm{M}+\mathrm{ClO}_{4}\right]^{+}$for $\mathbf{8}^{\mathrm{HCO}}(\mathrm{m} / \mathrm{z} 339.9 / 779.2)$ and $\mathbf{8}^{\mathbf{O A c}}(346.9 / 793.2)$. Additionally, a peak is observed in the ESI(-)-MS at $m / z \quad 979.0$ and 993.1 for $\mathbf{8}^{\mathbf{H C O 2}}$ and $\mathbf{8}^{\mathbf{0 A c}}$, respectively, whose isotopic pattern matches the $\left[\mathrm{M}+3 \mathrm{ClO}_{4}\right]^{-}$species (appendix, Figures A.38-A.45).
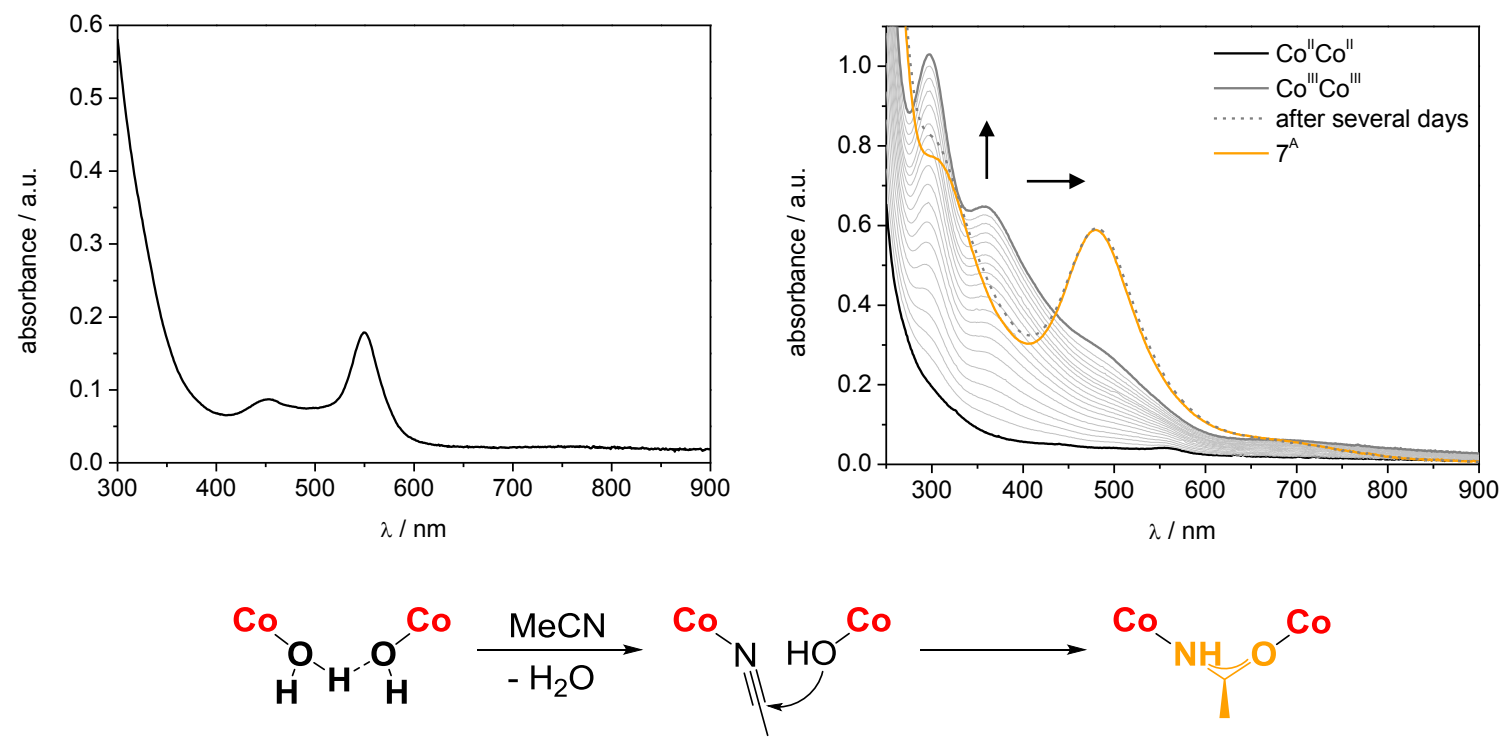

Figure 5.2: UV/vis-spectroscopic analysis of $\mathbf{7}^{\mathbf{0} 2 \mathbf{H} 3}$ in $\mathrm{MeCN}$ at room temperature. Left: UV/vis spectrum $(c=0.75 \mathrm{mM})$. Right: Spectroscopic changes upon addition of $\mathrm{O}_{2}$ (black: initial, grey: final, grey dashed: after several days) in comparison to the UV/vis spectrum of $7^{\mathbf{A}}$ (orange). The formation of an acetamide bridged species from the aquo/hydroxo complex in MeCN is depicted at the bottom.

Crystals of $\mathbf{7}^{\mathrm{MeCN}}, \mathbf{8}^{\mathrm{HCO2}}$ and $\mathbf{8}^{\mathbf{O A c}}$ were obtained by diffusion of $\mathrm{Et}_{2} \mathrm{O}$ into the reaction mixture and analyzed by XRD (Figure 5.3). In case of $\mathbf{8}^{\mathrm{HCO}}$ and $\mathbf{8}^{\mathbf{O A c}}$, multiple recrystallizations were necessary to remove excess $\mathrm{NaHCO}_{2}$ and $\mathrm{NaOAc}$, respectively. All analyses and follow-up experiments were conducted with crystalline material.

Compound $7^{\mathrm{MeCN}}$ crystallizes in the orthorhombic space group $P 2{ }_{1} 2{ }_{1} 2$, whereas $\mathbf{8}^{\mathbf{H C O}}$ and $\mathbf{8}^{\mathbf{O A c}}$ crystallize in the monoclinic space groups $C 2 / c$ and $P 2_{1}$, respectively. In case of 7 MecN, one half of the trication as well as three perchlorate anions, $1.2 \mathrm{MeCN}$ and $0.4 \mathrm{Et}_{2} \mathrm{O}$ molecules are found in the asymmetric unit. The full dication, two perchlorate moieties, as well as four $\mathrm{MeCN}$ or one $\mathrm{MeOH}$ molecule make up the asymmetric units of $\mathbf{8}^{\mathbf{H C O}}$ and $\mathbf{8}^{\mathbf{O A c}}$, respectively. The $\mathrm{Co}^{\mathrm{II}}$ ion in $7^{\mathrm{MeCN}}$ is coordinated by six $\mathrm{N}$-donors in a close to ideal octahedral fashion portrayed in the small CSM value for octahedral geometry of 0.88. Each Co ${ }^{\mathrm{II}}$ ion in $\mathbf{8}^{\mathrm{HCO}}$ and $\mathbf{8}^{\mathbf{O A c}}$ is ligated by an $\left\{\mathrm{N}_{4} \mathrm{O}\right\}$ donor set. According to the CSM $S\left(C_{4 \mathrm{v}}\right)$ and $S\left(D_{3 \mathrm{~h}}\right)$ values, the coordination environment is closer to a trigonal bipyramid than a square pyramid for $\mathbf{8}^{\mathrm{HCO}}$ and $\mathbf{8}^{\mathrm{OAc}}$ (see Table 5.1).

In comparison to $8^{\mathrm{HCO}}(d(\mathrm{Co} \cdots \mathrm{Co})=4.13 \AA)$ and $\mathbf{8}^{\mathrm{OAc}}(d(\mathrm{Co} \cdots \mathrm{Co})=4.14 \AA)$, the Co $\cdots$ Co separation is larger in 7 MecN $(d(\mathrm{Co} \cdots \mathrm{Co})=4.66 \AA)$. The same holds true for the average Co-X bond lengths. A direct comparison of the metric parameters can be found in Table 5.1. 

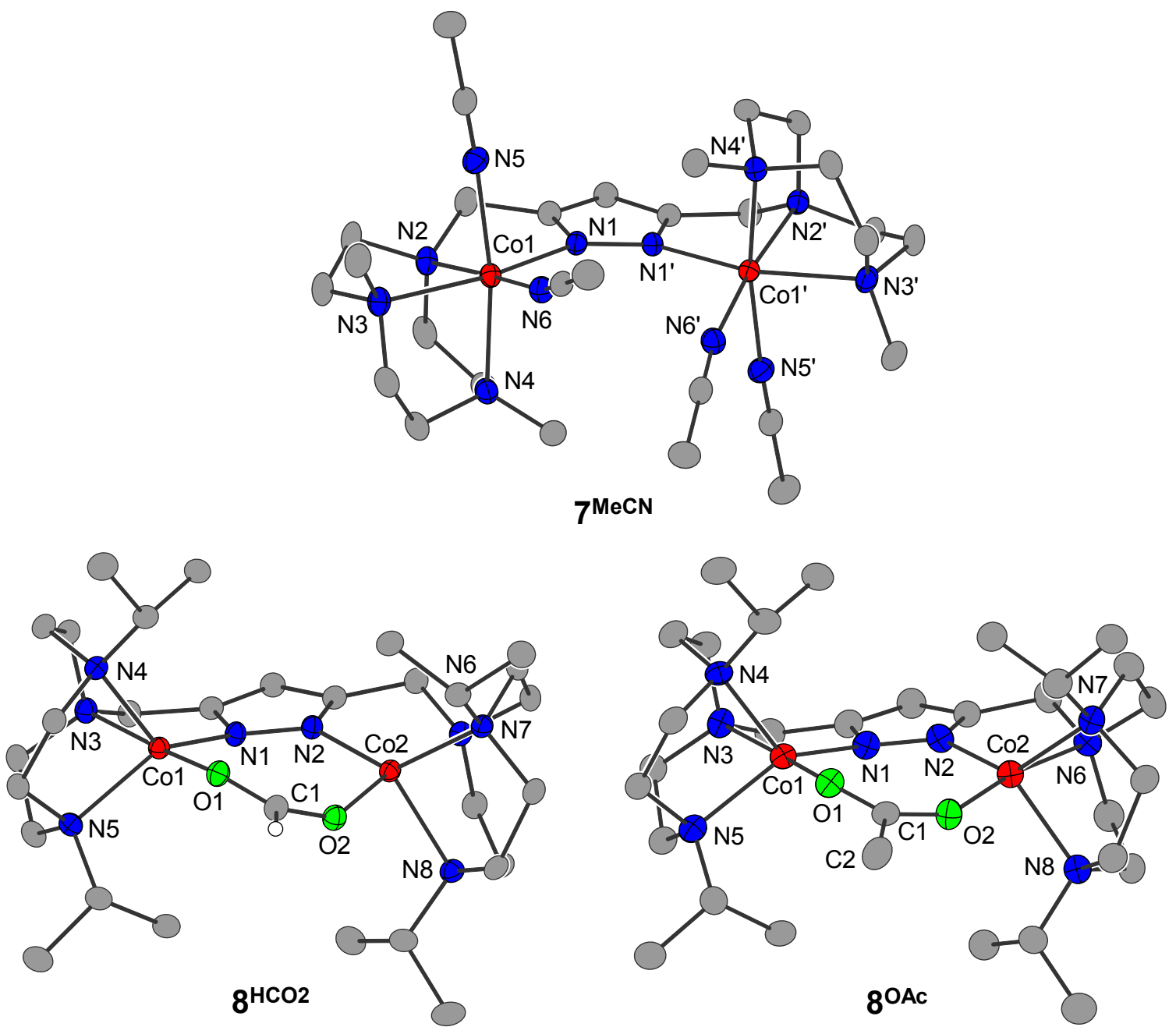

Figure 5.3: Molecular structures of the cationic cores of $7^{\mathrm{MeCN}}$ (top), $\mathbf{8}^{\mathrm{HCO}}$ (bottom left) and $\mathbf{8}^{\mathrm{OAc}}$ (bottom right) drawn as thermal ellipsoids at the $30 \%$ probability level (cobalt: red, nitrogen: blue, oxygen: green, carbon: grey, hydrogen: white). All hydrogen atoms (except that of $\mathrm{HCO}_{2}^{-}$), counter ions and additional solvent molecules are omitted for clarity reasons. 
Table 5.1: Selected crystal data, distances, bond lengths, torsion angles and Continuous Symmetry Measures (CSM) of $7^{\mathrm{MeCN}}, \mathbf{8}^{\mathrm{HCO}}$ and $\mathbf{8}^{\mathrm{OAc}}$.

\begin{tabular}{|c|c|c|c|}
\hline crystal data & $7^{\mathrm{MeCN}}$ & $8^{\mathrm{HCO2}}$ & $8^{\text {OAc }}$ \\
\hline crystal system & orthorhombic & monoclinic & monoclinic \\
\hline space group & $P 2{ }_{1}{ }_{1} 2$ & $C 2 / c$ & $P 2{ }_{1}$ \\
\hline \multicolumn{4}{|l|}{ distance / $\AA$} \\
\hline 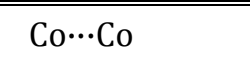 & $4.6553(6)$ & $4.1324(10)$ & $4.1437(14)$ \\
\hline \multicolumn{4}{|c|}{ bond lengths / $\AA$} \\
\hline $\mathrm{Co}(1)-\mathrm{O}(1)$ & - & $2.034(2)$ & $2.006(6)$ \\
\hline $\mathrm{Co}(2)-\mathrm{O}(2)$ & - & $2.021(2)$ & $2.004(6)$ \\
\hline Co-N & $2.09-2.20$ & $1.99-2.22$ & $2.01-2.23$ \\
\hline \multicolumn{4}{|c|}{ torsion angles $/^{\circ}$} \\
\hline Co-N-N-Co & $-7.233(577)$ & $32.777(350)$ & $28.660(1208)$ \\
\hline Сo-O…-Co & - & $36.165(157)$ & $25.152(525)$ \\
\hline \multicolumn{4}{|l|}{ xxiii $\operatorname{CSM} S\left(O_{\mathrm{h}}\right)^{\mathrm{a}}$} \\
\hline $\operatorname{Co}(1)$ & 0.88 & - & - \\
\hline \multicolumn{4}{|l|}{${ }^{\mathrm{xxiv}} \operatorname{CSM} S\left(C_{4 \mathrm{v}}\right)^{\mathrm{b}}$} \\
\hline $\operatorname{Co}(1)$ & - & 5.97 & 5.28 \\
\hline $\operatorname{Co}(2)$ & - & 5.70 & 6.10 \\
\hline \multicolumn{4}{|l|}{ 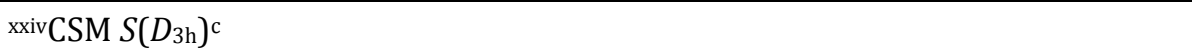 } \\
\hline Co(1) & - & 2.45 & 2.41 \\
\hline $\operatorname{Co}(2)$ & - & 2.50 & 2.61 \\
\hline
\end{tabular}

\subsection{Magnetic Properties}

\subsubsection{Magnetic Properties of $7 \mathrm{MeCN}$}

Magnetic susceptibility data of crystalline material of $7^{\mathrm{MecN}}$ was recorded at $0.5 \mathrm{~T}$ in a temperature range from 2 to $210 \mathrm{~K}$ (Figure 5.4, left). The high temperature $\chi_{\mathrm{m}} T$ value $\left(5.52 \mathrm{~cm}^{3} \mathrm{~K} \mathrm{~mol}^{-1}\right.$ at $\left.210 \mathrm{~K}\right)$ surpasses the expected spin-only value for two uncoupled high-spin $\mathrm{d}^{7} \mathrm{Co}^{\mathrm{II}}$ ions with $S=3 / 2$ $\left(3.75 \mathrm{~cm}^{3} \mathrm{~K} \mathrm{~mol}^{-1}\right.$ assuming $\left.g=2\right)$, which implies a significant orbital contribution to the magnetic moment. The initially gradual and later prompt decrease of $\chi_{\mathrm{m}} T$ towards lower temperatures can indicate two phenomena: (i) intramolecular antiferromagnetic exchange interaction and (ii) zero-field splitting of $\mathrm{Co}^{\mathrm{II}}$. In fact, the coupling constant $J\left(\widehat{H}=-2 J \hat{S}_{1} \hat{S}_{2}\right)$ was quantified as $-0.3 \mathrm{~cm}^{-1}$ and can be considered as very weak, and the axial zero-field splitting value was determined as $D=88 \mathrm{~cm}^{-1}$ with the help of variable temperature/variable field (VTVH) measurements (Figure 5.4, right). Simulation of the susceptibility data furthermore afforded large anisotropic $g$ values of $g_{1}=g_{2}=2.54$ and $g_{3}=2.25$ (see Table 5.2). The average $g$ value of 2.45 is higher than the $g$ value of a free electron $\left(g_{\mathrm{e}} \approx 2.0\right)$ and thus indicates significant spin-orbit coupling, which is typical in most transition metal complexes. The

xxii The geometry of the coordination environment was compared to the reference shape "octahedron (with central atom)".

xxiv The geometry of the coordination environment was compared to self-made custom shapes of an ideal square pyramid and trigonal bipyramid with equal distances of all ligands to the central atom. 
best simulation result was obtained including a temperature-independent paramagnetism (TIP) constant of $220 \cdot 10^{-6} \mathrm{~cm}^{3} \mathrm{~mol}^{-1}$.
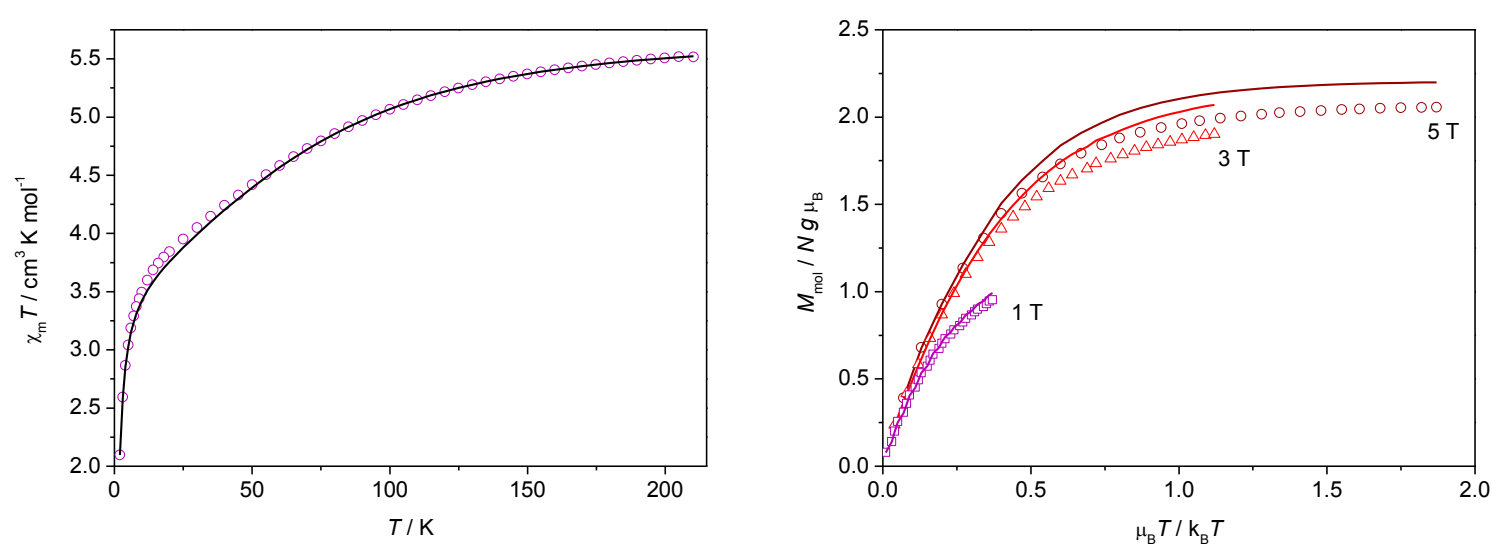

Figure 5.4: Magnetic measurements of a crystalline samples of 7 MecN. Left: $\chi_{\mathrm{m}} T v s . T$ measurement in the temperature range $2-210 \mathrm{~K}$ at a field of $0.5 \mathrm{~T}$. Right: Variable temperature/variable field (VTVH) measurement at fields of 1, 3 and 5 T. The solid lines represent the respective fits.

Table 5.2: Parameters extracted from the simulation of the magnetic measurements of $\mathbf{7}^{\mathrm{MeCN}}$.

\begin{tabular}{lc} 
parameters & 7 $^{\text {MecN }}$ \\
\hline \hline$g$-value $\left(g_{1}=g_{2}\right)$ & 2.54 \\
$g$-value $\left(g_{3}\right)$ & 2.25 \\
average $g$-value $g_{\mathrm{av}}$ & 2.45 \\
coupling constant $J / \mathrm{cm}^{-1}$ & -0.3 \\
TIP $/ 10^{-6} \mathrm{~cm}^{3} \cdot \mathrm{mol}^{-1}$ & 220 \\
zero-field splitting $D / \mathrm{cm}^{-1}$ & 88 \\
\hline
\end{tabular}

\subsubsection{Magnetic Properties of $8^{\mathrm{HCO}}$ and $8^{\mathrm{OAc}}$}

Magnetic susceptibility measurements at $0.5 \mathrm{~T}$ in a temperature range from 2 to $210 \mathrm{~K}$ were also performed on crystalline material of $\mathbf{8}^{\mathrm{HCO}}$ (Figure 5.5, left) and $\mathbf{8}^{\mathbf{0 A c}}$ (right). As for $\mathbf{7}^{\mathrm{MecN}}$, the $\chi_{\mathrm{m}} T$ values at high temperature (5.14 and $5.23 \mathrm{~cm}^{3} \mathrm{~K} \mathrm{~mol}^{-1}$ at $210 \mathrm{~K}$ for $\mathbf{8}^{\mathrm{HCO2}}$ and $\mathbf{8}^{\mathbf{0 A c}}$, respectively) surpass the expected spin-only value for two uncoupled high-spin $\mathrm{d}^{7} \mathrm{Co}^{\mathrm{II}}$ ions with $S=3 / 2\left(3.75 \mathrm{~cm}^{3} \mathrm{~K} \mathrm{~mol}^{-1}\right.$ assuming $g=2$ ), which implies a significant orbital contribution to the magnetic moment. Relatively constant $\chi_{\mathrm{m}} T$ values, which start to increase at around $30 \mathrm{~K}$ and exhibit a maximum between 5-6 K indicate weak intramolecular ferromagnetic exchange interaction, which was quantified in coupling constants $J\left(\widehat{H}=-2 J \hat{S}_{1} \hat{S}_{2}\right)$ of 0.8 and $1.5 \mathrm{~cm}^{-1}$ for $\mathbf{8}^{\mathrm{HCO}}$ and $\mathbf{8}^{\mathrm{OAc}}$, respectively. The prompt decline of $\chi_{\mathrm{m}} T$ below $5 \mathrm{~K}$ is attributed to zero-field splitting of $\mathrm{Co}^{\mathrm{II}}$, determined as $D=-4.4 \mathrm{~cm}^{-1}\left(8^{\mathrm{HCO}}\right)$ and $-27 \mathrm{~cm}^{-1}\left(\mathbf{8}^{\mathrm{OAc}}\right)$. Simulation of the susceptibility data was possible assuming very similar isotropic $g$ values of $2.32\left(\mathbf{8}^{\mathbf{H C O}^{\mathrm{CO}}}\right)$ and $2.33\left(\mathbf{8}^{\mathbf{O A c}}\right)$ for both complexes (see Table 5.3), which are higher than the $g$ value of a free electron $\left(g_{\mathrm{e}} \approx 2.0\right)$, thus suggesting significant spin-orbit coupling. For $\mathbf{8}^{\mathrm{HCO} 2}$, the best simulation result was obtained including a TIP constant of $80 \cdot 10^{-6} \mathrm{~cm}^{3} \mathrm{~mol}^{-1}$. 

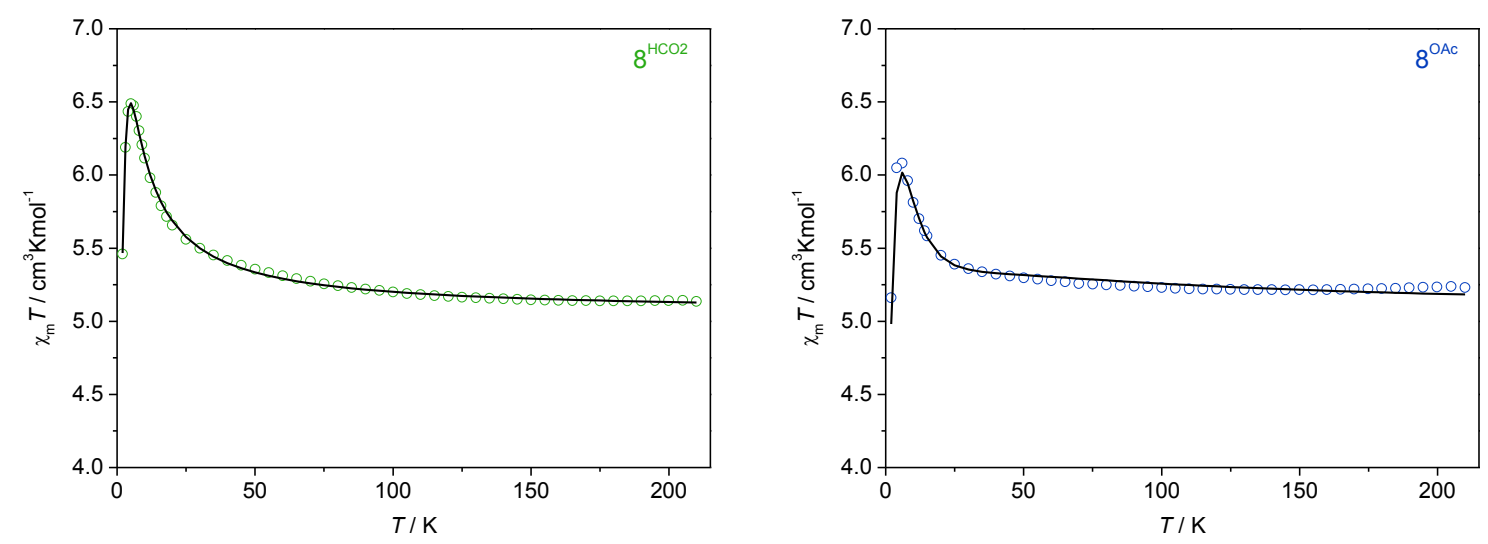

Figure 5.5: DC susceptibility measurements of crystalline samples of $\mathbf{8}^{\mathrm{HCO}}$ (left) and $\mathbf{8}^{\mathrm{OAc}}$ (right) in the temperature range $2-210 \mathrm{~K}$ at a field of $0.5 \mathrm{~T}$. The solid black lines represent the fits using the parameters presented in Table 5.3.

The presence of a high-spin ground state and a negative axial zero-field splitting parameter $D$ in $\mathbf{8}^{\mathrm{HCO} 2}$ and $\mathbf{8}^{\mathrm{OAc}}$, demonstrates their potential application as single molecule magnets (SMMs). SMMs are molecules that exhibit slow relaxation of the magnetization and thus retain the latter in the absence of an external field. They have received increased attention in the past two decades as potential applications are numerous, e.g. in high density data storage, quantum computing and spintronics. ${ }^{[351,352]}$ Cobalt(II) is a viable candidate for use in SMMs due to its non-quenched orbital angular momentum with a non-integer spin ground state $(S=3 / 2) \cdot{ }^{[353]}$

In order to determine slow magnetic relaxation, ac susceptibility measurements are conducted. Thereby, the dynamic susceptibility of a sample is investigated depending on an applied oscillating magnetic field. The dynamic susceptibility has an in-phase $\left(\chi^{\prime}\right)$ and out-of-phase ( $\left.\chi^{\prime \prime}\right)$ component, which vary with the frequency of the ac field as well as the temperature. ${ }^{[354]}$ Plotting $\chi^{\prime \prime} v s . \chi^{\prime}$ in a so-called Cole-Cole plot gives a semi-circle shaped trace, from which the frequency $v$ at which $\chi^{\prime \prime}$ reaches its maximum can be determined (data is fitted according to the Debye model). ${ }^{[355]}$ By means of equation 5.1, this gives rise to the relaxation time $\tau$, which follows the Arrhenius law for thermal Orbach relaxation according to equation 5.2.[356]

$$
\begin{gathered}
\tau=\frac{1}{2 \pi v} \\
\ln \tau=\ln \tau_{0}+\frac{U_{\text {eff }}}{\mathrm{k}_{\mathrm{B}} T}
\end{gathered}
$$

The effective barrier to spin reversal $U_{\text {eff }}$ can be derived from the slope of the $\ln \tau v s . \frac{1}{T}$ plot and is the most important property of an SMM: the larger, the better. 

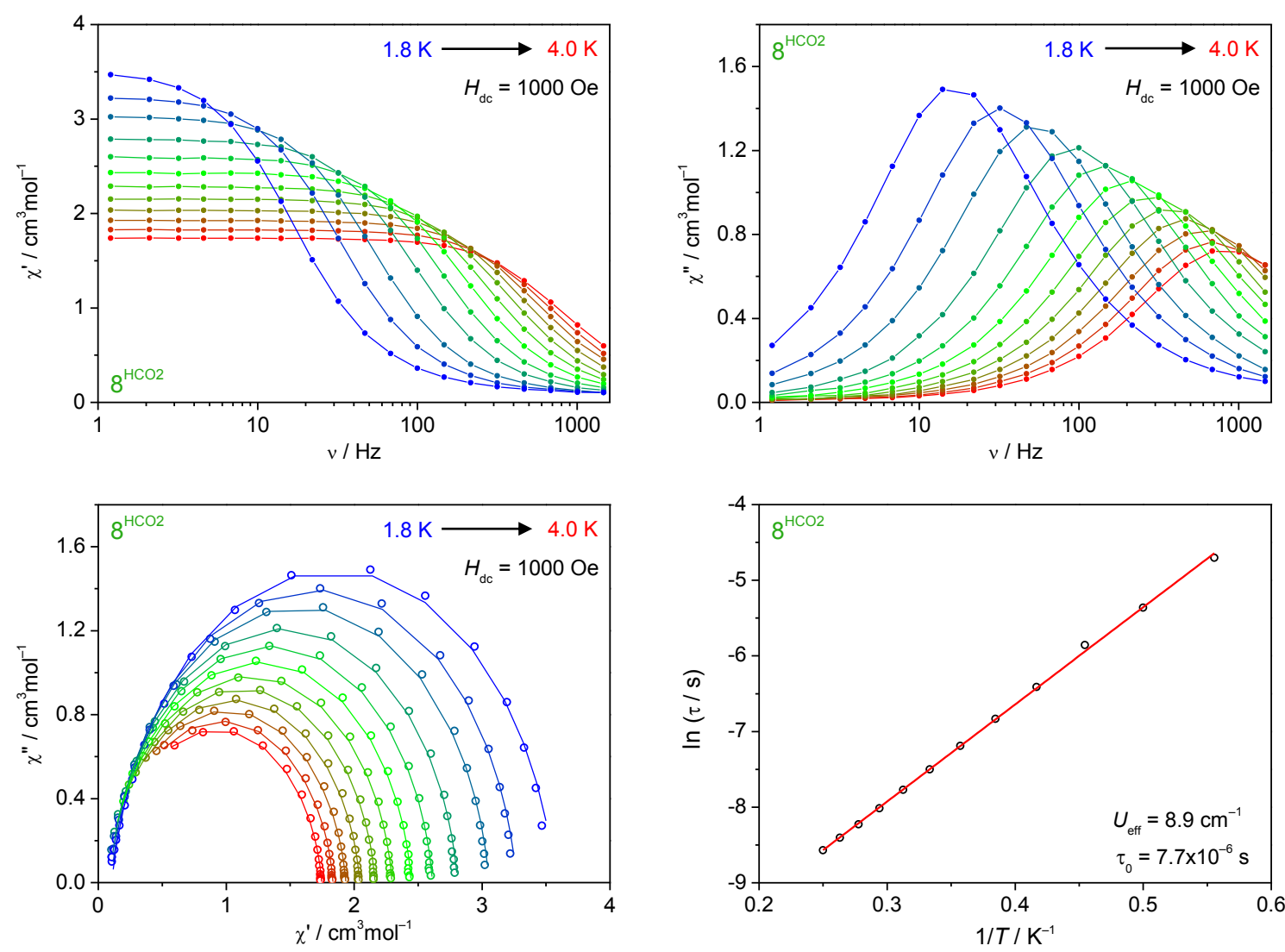

Figure 5.6: AC susceptibility measurements of $\mathbf{8}^{\mathbf{H C O 2}}$. Top: Frequency dependence of the in-phase ( $\chi^{\prime}$, left) and outof-phase ( $\chi$ ", right) components of the ac magnetic susceptibility for different temperatures $(0.2 \mathrm{~K}$ steps) under a 1000 Oe dc field. Bottom left: Cole-Cole plot for different temperatures ( $0.2 \mathrm{~K}$ steps) under a 1000 Oe dc field. The solid lines given for the ac susceptibility measurements are intended as optical guidance. Bottom right: Arrhenius plot of the relaxation time $\tau$ for the ac susceptibility measurements at different temperatures. The ac susceptibility data is very similar for $\mathbf{8}^{\mathbf{O A c}}$.

The in-phase (top left) and out-of-phase (top right) components of the ac magnetic susceptibility, as well as the corresponding Cole-Cole (bottom left) and Arrhenius (bottom right) plots are depicted in Figure 5.6 exemplarily for $\mathbf{8}^{\mathrm{HCO}}$ under a 1000 Oe dc field. From the latter, a relaxation time $\tau_{0}$ of $7.7 \cdot 10^{-6} \mathrm{~s}^{-1}$ and $3.7 \cdot 10^{-6} \mathrm{~s}^{-1}$, as well as an energy barrier $U_{\text {eff }}$ of 8.9 and $6.0 \mathrm{~cm}^{-1}$ were determined for $\mathbf{8}^{\mathrm{HCO2}}$ and $\mathbf{8}^{\mathbf{0 A c}}$, respectively (Table 5.3). These values are similar to other cobalt(II) SMMs. ${ }^{[239,241,357]}$ Although the barrier to spin reversal cannot be considered very high, $\mathbf{8}^{\mathrm{HCO}}$ and $\mathbf{8}^{\mathrm{OAc}}$ represent a very rare example of SMM behavior in homodinuclear cobalt(II) compounds. In fact, to the best of our knowledge, only one paper has so far been published in this exact field. ${ }^{[358]}$ In other dinuclear cobalt complexes, slow magnetic relaxation is achieved when one cobalt ion is silenced by oxidation to $\mathrm{Co}^{\mathrm{III}}$ or the cobalt ions are far apart so that there is no coupling. ${ }^{[359-361]}$ Such behavior then results from one $\mathrm{Co}^{\mathrm{II}}$ ion and these complexes are known as single ion magnets (SIMs). ${ }^{[362]}$

The absence of SMM behavior in $7^{\text {MeCN }}$ is likely due to the different coordination environment of the cobalt ions therein: whereas $\mathbf{8}^{\mathrm{HCO}}$ and $\mathbf{8}^{\mathrm{OAc}}$ exhibit trigonal bipyramidal $\mathrm{Co}^{\mathrm{II}}$ centers, the additional $\mathrm{MeCN}$ ligand in $7 \mathrm{MeCN}$ induces an octahedral geometry. Previous examples have shown that the local geometry at the $\mathrm{Co}^{\text {II }}$ center plays an important role for the single ion magnetic behavior. ${ }^{[363]}$ Furthermore, the zero-field splitting parameter $D$ was also shown to depend on the type and coordination environment of the metal ion. ${ }^{[364]}$ A strict octahedral environment, as is displayed in $\mathbf{7}^{\mathrm{MecN}}$ $\left(\operatorname{CSM} S\left(O_{\mathrm{h}}\right)=0.88\right.$, Table 5.1), is particularly unfavorable for SMM properties. 
Table 5.3: Parameters extracted from the dc (top) and ac (bottom) magnetic susceptibility measurements of $\mathbf{8}^{\mathrm{HCO} 2}$ and 80Ac

\begin{tabular}{lll} 
dc susceptibility & $\mathbf{8}^{\text {HCO2 }}$ & $\mathbf{8}^{\text {OAc }}$ \\
\hline \hline$g$-value $\left(g_{1}=g_{2}=g_{3}\right)$ & 2.32 & 2.33 \\
coupling constant $J / \mathrm{cm}^{-1}$ & 0.8 & 1.5 \\
TIP $/ 10^{-6} \mathrm{~cm}^{3} \cdot \mathrm{mol}^{-1}$ & 80 & 0 \\
zero-field splitting $D / \mathrm{cm}^{-1}$ & -4.4 & -27 \\
\hline ac susceptibility & & \\
\hline \hline$U_{\text {eff }} / \mathrm{cm}^{-1}$ & 8.9 & 6.0 \\
relaxation time $\tau_{0} / 10^{-6} \mathrm{~s}$ & 7.7 & 3.7 \\
\hline
\end{tabular}

\subsection{Electro- and Spectroelectrochemical Analysis}

The complexes $\mathbf{7}^{\mathrm{MeCN}}, \mathbf{8}^{\mathrm{HCO2}}$ and $\mathbf{8}^{\mathrm{AAc}}$ were electrochemically characterized by means of cyclic voltammetry in $\mathrm{MeCN}\left(0.1 \mathrm{M} \mathrm{NBu}_{4} \mathrm{PF}_{6}\right)$ under inert conditions (all potentials are given vs. the ferrocenium/ferrocene $\left(\mathrm{FC}^{+} / 0\right)$ redox couple). Compound $7^{\mathrm{MeCN}}$ was further investigated in phosphate buffer (ionic strength $I=0.1 \mathrm{M}$ ) under ambient conditions (all potentials are given vs. the normal hydrogen electrode (NHE)).

\subsubsection{Electro- and Spectroelectrochemical Analysis of $8^{\mathrm{HCO}}$ and $8^{\mathrm{OAc}}$}

\subsubsection{Under Inert Conditions}

The cyclic voltammograms (CVs) of $\mathbf{8}^{\mathbf{H C O 2}}$ and $\mathbf{8}^{\mathbf{O A c}}$ at a scan rate of $100 \mathrm{mV} / \mathrm{s}$ (Figure 5.7, top left) in $\mathrm{MeCN}\left(0.1 \mathrm{M} \mathrm{NBu}_{4} \mathrm{PF}_{6}\right)$ display four electrochemically irreversible peaks: two oxidations in the anodic scan and two reductions in the cathodic scan. When starting at the open circuit potential (OCP) and scanning in the negative direction, no reduction waves are observed, indicating that the reduction processes are the counter parts to the observed oxidations (Figure 5.7, top right). By changing the potential range of the $\mathrm{CV}$, the corresponding oxidation-reduction couples were distinguished (Figure 5.7, bottom). Consequently, the electrochemical events can be assigned accordingly:

$$
\mathrm{Co}^{\mathrm{II}} \mathrm{Co}^{\mathrm{II}} \stackrel{E_{\mathrm{pa}, 1}}{\longrightarrow} \mathrm{Co}^{\mathrm{II}} \mathrm{Co}^{\mathrm{III}} \stackrel{E_{\mathrm{pa}, 2}}{\longrightarrow} \mathrm{Co}^{\mathrm{III}} \mathrm{Co}^{\mathrm{III}} \stackrel{E_{\mathrm{pc}, 2}}{\longrightarrow} \mathrm{Co}^{\mathrm{II}} \mathrm{Co}^{\mathrm{III}} \stackrel{E_{\mathrm{pc}, 1}}{\longrightarrow} \mathrm{Co}^{\mathrm{II}} \mathrm{Co}^{\mathrm{II}}
$$

The large separation between the oxidation and corresponding reduction events indicates that a follow-up process might be associated with the electron transfer. This could include bond elongation or shortening, possibly due to spin crossover, as well as a subsequent chemical reaction. As Co ${ }^{\mathrm{III}}$ ions favor octahedral geometry, oxidation of $\mathrm{Co}^{\mathrm{II}}$ may induce ligand association to reach $\mathrm{CN} 6$, accompanied by a geometrical change. The resulting $\mathrm{Co}^{\mathrm{III}}$ ions are thus better stabilized, making their reduction more difficult, and hence cathodically shifting the corresponding potential. Upon reduction, the initial $\mathrm{Co}^{\mathrm{II}} \mathrm{Co}^{\mathrm{II}}$ species is fully recovered, as evidenced by the fact that no changes are observed in the CV over multiple scans.

Overall, the oxidation of $\mathbf{8}^{\mathbf{O A c}}$ is easier than that of $\mathbf{8}^{\mathbf{H C O 2}}$ (see Table 5.4). This might be due to the electron-donating effect of the methyl group of the acetate molecule in the bimetallic pocket of $\mathbf{8}^{\mathbf{O A c}}$ in comparison to the formate moiety in $\mathbf{8}^{\mathrm{HCO}}$, which leads to a slightly better stabilization of the $\mathrm{Co}^{\mathrm{III}}$ oxidation state. The scan rate ( $v$ ) dependent measurements of $\mathbf{8}^{\mathbf{H C O}}$ show a slight shift of the oxidation and reduction events with $v$, which is typical for irreversible electron transfer processes (Figure 5.7, bottom). 

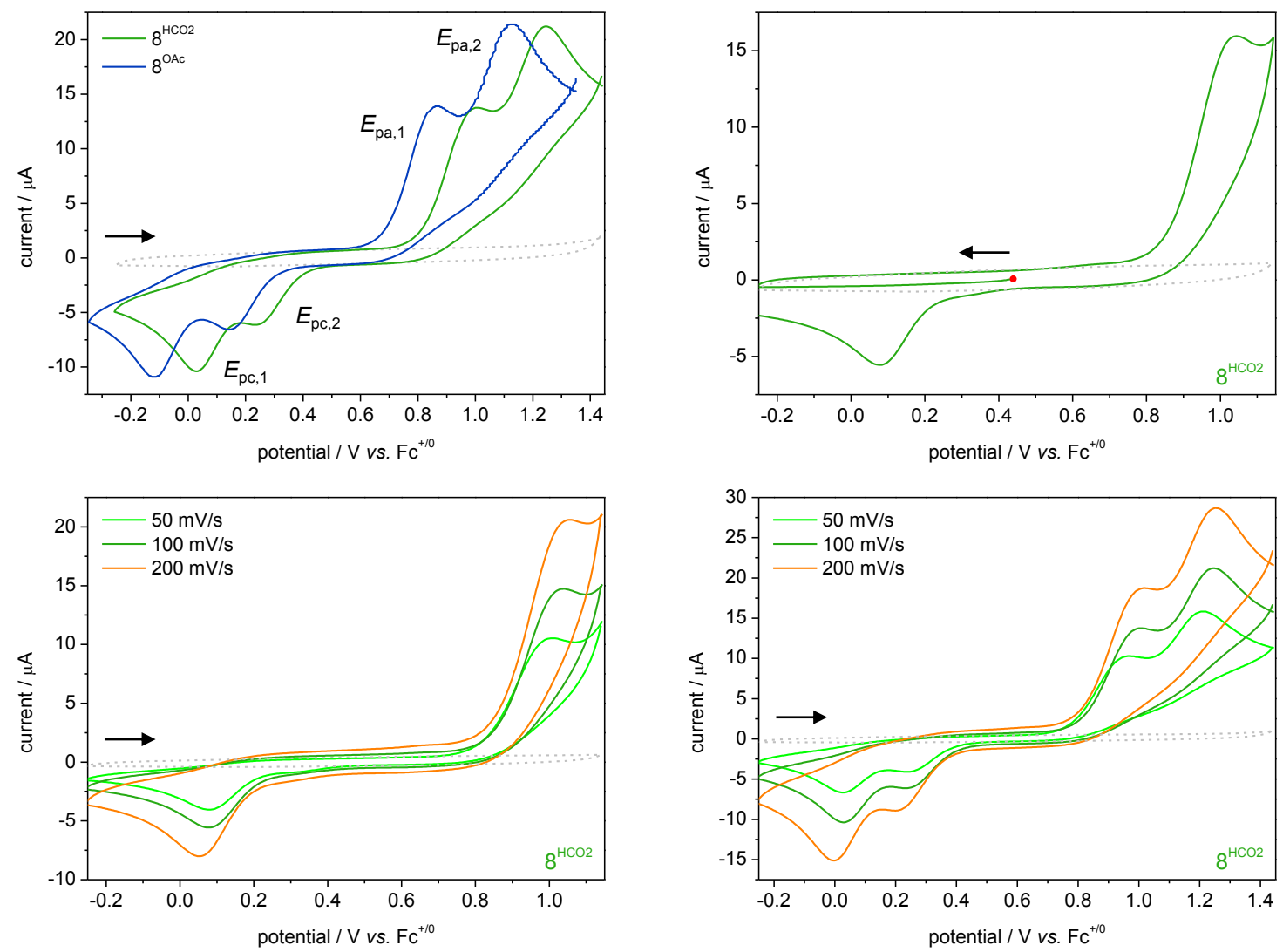

Figure 5.7: Electrochemical analysis of $\mathbf{8}^{\mathrm{HCO} 2}$ and $\mathbf{8}^{\mathrm{OAc}}$ in $\mathrm{MeCN}\left(0.1 \mathrm{M} \mathrm{NBu}_{4} \mathrm{PF}_{6}\right)$ under inert conditions $(c=1 \mathrm{mM})$.

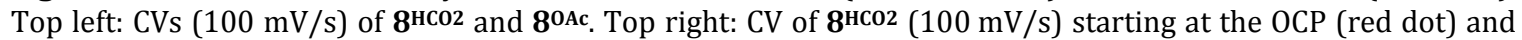
scanning cathodically. Bottom: Scan rate dependence of the first (left) and of both oxidation-reduction couples (right) of $\mathbf{8}^{\mathrm{HCO}}$. The scanning direction is indicated by an arrow. A glassy carbon disc was used as the working electrode, a Pt disc as auxiliary and an Ag wire as the reference electrode. Ferrocene was used as an internal standard.

Table 5.4: Peak potentials extracted from the CVs of $\mathbf{8}^{\mathbf{H C O}}$ and $\mathbf{8}^{\mathbf{O A c}}$ at $100 \mathrm{mV} / \mathrm{s}$ in MeCN (0.1 M NBu $\left.4 \mathrm{PF}_{6}\right) \mathrm{under}$ inert conditions. All potentials are given $v s . \mathrm{Fc}^{+/ 0}$.

\begin{tabular}{llll}
$\mathrm{CV}$ & $\mathbf{8}^{\mathrm{HCO2}}$ & $\mathbf{8}^{\text {OAc }}$ & assignment \\
\hline \hline$E_{\mathrm{pa}, 1} / \mathrm{V}^{\mathrm{a}}$ & 1.00 & 0.87 & $\mathrm{Co}^{\mathrm{II}} \mathrm{Co}^{\mathrm{II}} \rightarrow \mathrm{Co}^{\mathrm{II}} \mathrm{Co}^{\mathrm{III}}$ \\
$E_{\mathrm{pa}, 2} / \mathrm{V}^{\mathrm{a}}$ & 1.25 & 1.13 & $\mathrm{Co}^{\mathrm{II}} \mathrm{Co}^{\mathrm{III}} \rightarrow \mathrm{Co}^{\mathrm{III}} \mathrm{Co}^{\mathrm{III}}$ \\
$E_{\mathrm{pc}, 2} / \mathrm{V}^{\mathrm{a}}$ & 0.24 & 0.15 & $\mathrm{Co}^{\mathrm{III}} \mathrm{Co}^{\mathrm{III}} \rightarrow \mathrm{Co}^{\mathrm{II}} \mathrm{Co}^{\mathrm{III}}$ \\
$E_{\mathrm{pc}, 1} / \mathrm{V}^{\mathrm{a}}$ & 0.03 & -0.12 & $\mathrm{Co}^{\mathrm{II}} \mathrm{Co}^{\mathrm{III}} \rightarrow \mathrm{Co}^{\mathrm{II}} \mathrm{Co}^{\mathrm{II}}$ \\
\hline
\end{tabular}

a peak potentials from the anodic (a) and cathodic (c) scan

On the basis of the electrochemical results, UV/vis-spectroelectrochemical (SEC) measurements were conducted in $\mathrm{MeCN}\left(0.1 \mathrm{M} \mathrm{NBu}_{4} \mathrm{PF}_{6}\right)$ and will be discussed for $\mathbf{8}^{\mathrm{HCO}}$ as a representative example. The UV/vis spectrum of $\mathbf{8}^{\mathrm{HCO2}}$ in the $\mathrm{Co}^{\mathrm{II}} \mathrm{Co}^{\mathrm{II}}$ state possesses local absorption maxima at 444 $\left(\varepsilon=82 \mathrm{~L} \cdot \mathrm{mol}^{-1} \cdot \mathrm{cm}^{-1}\right.$ ), $504(75), 524(68), 552(298)$ and $720 \mathrm{~nm}(23)$, which correspond to d-d transitions. In order to distinguish these bands, a peak deconvolution (determination of the minimum amount of Gauss type peaks that reproduce the experimental spectrum with good accuracy) was conducted in Origin Pro 8.5 from OriginLab (Figure 5.8, bottom right). ${ }^{x x v}$ The large number of $d-d$

xxv Please note, the deconvolution was conducted in the energy scale and is only shown in the wavelength scale for simplicity. 
transitions observed for both $\mathbf{8}^{\mathbf{H C O}}$ and $\mathbf{8}^{\mathbf{O A c}}$ (see experimental section) is typical for CN5 cobalt complexes ${ }^{[365-369]}$ and results from the lower symmetry of the cobalt ions in comparison to the octahedral coordination in $\mathbf{7}^{\mathrm{MecN}}$.

Upon oxidation of $\mathbf{8}^{\mathrm{HCO}}$ at $1.64 \mathrm{~V}$, the overall intensity significantly increases with bands emerging at 413 and $558 \mathrm{~nm}$ (Figure 5.8, top left). Upon re-reduction (Figure 5.8, top right) at $-0.36 \mathrm{~V}$, these bands slowly vanish and the $\mathrm{d}-\mathrm{d}$ transitions between 400 and $600 \mathrm{~nm}$ reappear. The initial $\mathrm{Co}^{\mathrm{II}} \mathrm{Co}^{\mathrm{II}}$ species is fully re-established, supported by a nice overlap of the spectra before oxidation (dashed black) and after re-reduction (blue). The $\Delta$ absorbance traces with respect to the spectrum before oxidation (Figure 5.8 , bottom left) emphasize the $\lambda$-dependent intensity gain upon oxidation, as well as the apparent reversibility of the electrochemical process, as the trace after re-reduction (blue) more or less coincides with the baseline. This concludes that the follow-up process that causes the irreversibility in the $\mathrm{CV}$ is chemically reversible based on the UV/vis-SEC measurement.
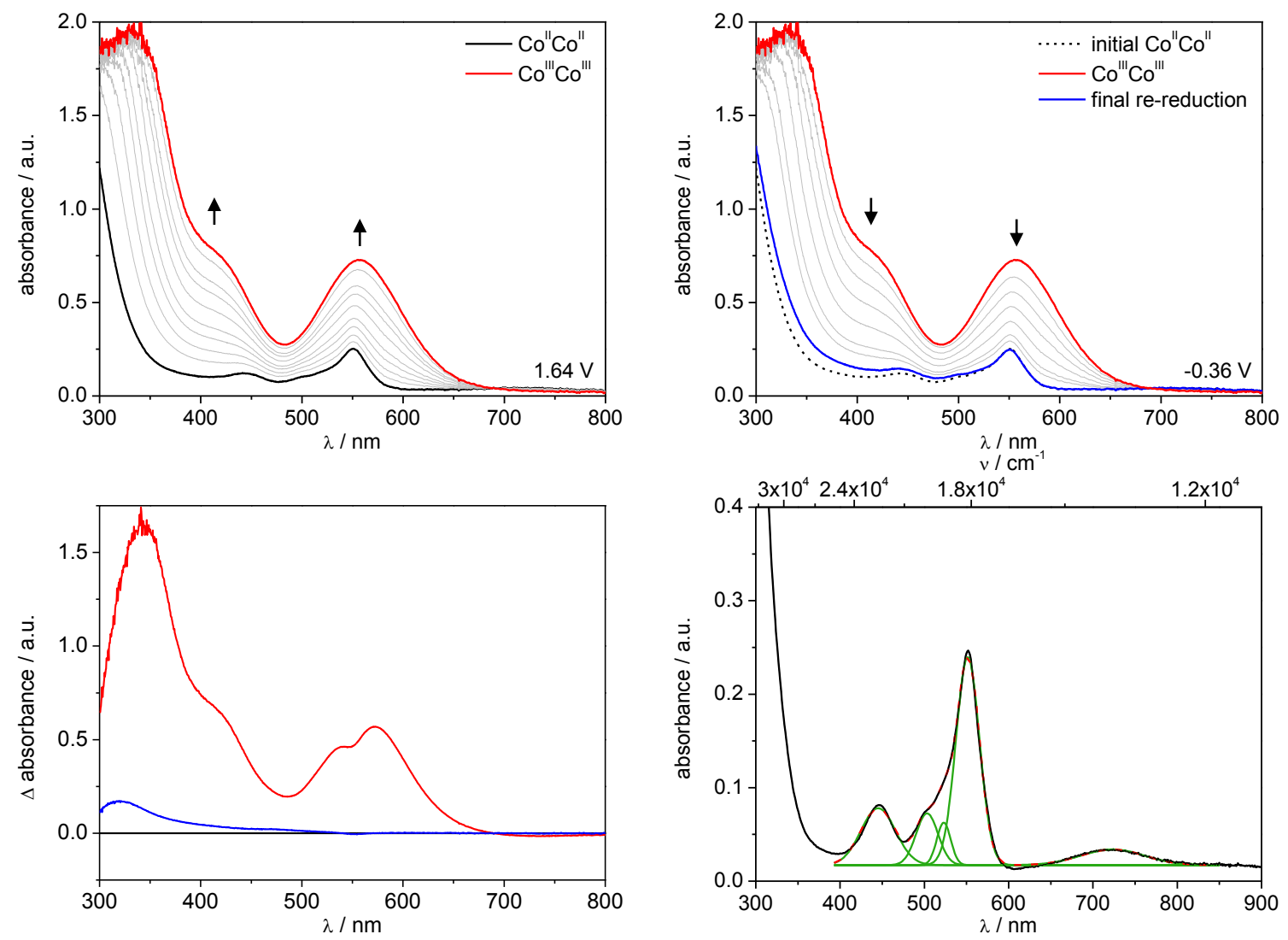

Figure 5.8: UV/vis-spectroelectrochemical analysis of $\mathbf{8}^{\mathrm{HCO} 2}$ in $\mathrm{MeCN}\left(0.1 \mathrm{M} \mathrm{NBu}_{4} \mathrm{PF}_{6}\right)$ under inert conditions. Top left: UV/vis spectra of the electrochemical oxidation from $\mathrm{Co}^{\mathrm{II}} \mathrm{Co}^{\mathrm{II}}$ to $\mathrm{Co}^{\mathrm{III}} \mathrm{Co}^{\mathrm{III}}$ at $1.64 \mathrm{~V}$ (black: beginning, red: end). Top right: UV/vis spectra of the re-reduction from $\mathrm{Co}^{\mathrm{III}} \mathrm{Co}^{\mathrm{III}}$ to $\mathrm{Co}^{\mathrm{II}} \mathrm{Co}^{\mathrm{II}}$ at $-0.36 \mathrm{~V}$ (red: beginning, blue: end, black dashed: initial UV/vis spectrum before oxidation). Bottom left: $\Delta$ absorbance of the spectra after oxidation (red) and re-reduction (blue) with respect to the spectrum before oxidation (baseline). Bottom right: Peak deconvolution of the $\mathrm{Co}^{\mathrm{II}} \mathrm{Co}^{\mathrm{II}}$ species' spectrum $(c=0.75 \mathrm{mM}) \times{ }^{\mathrm{xxv}}$ The red line corresponds to the cumulative peak fit.

\subsubsection{Electro- and Spectroelectrochemical Analysis of $7^{\mathrm{MecN}}$}

\subsubsection{Under Inert Conditions}

As for $\mathbf{8}^{\mathrm{HCO2}}$ and $\mathbf{8}^{\mathrm{OAc}}$, the $\mathrm{CV}$ of $\mathbf{7}^{\mathrm{MeCN}}$ (Figure 5.9) in $\mathrm{MeCN}\left(0.1 \mathrm{M} \mathrm{NBu} 4 \mathrm{PF}_{6}\right.$ ) displays four electrochemically irreversible peaks, which can be assigned to the oxidations from $\mathrm{Co}^{\mathrm{III}} \mathrm{Co}^{\mathrm{II}}$ to $\mathrm{Co}^{\mathrm{II}} \mathrm{Co}^{\mathrm{III}}$ and further to $\mathrm{Co}^{\mathrm{III}} \mathrm{Co}^{\mathrm{III}}$, with their corresponding reductions. The peak potentials shift with the scan rate $v$, especially at high $v$ (500 and $1000 \mathrm{mV} / \mathrm{s}$ ), supporting their irreversibility (Table 5.5). 

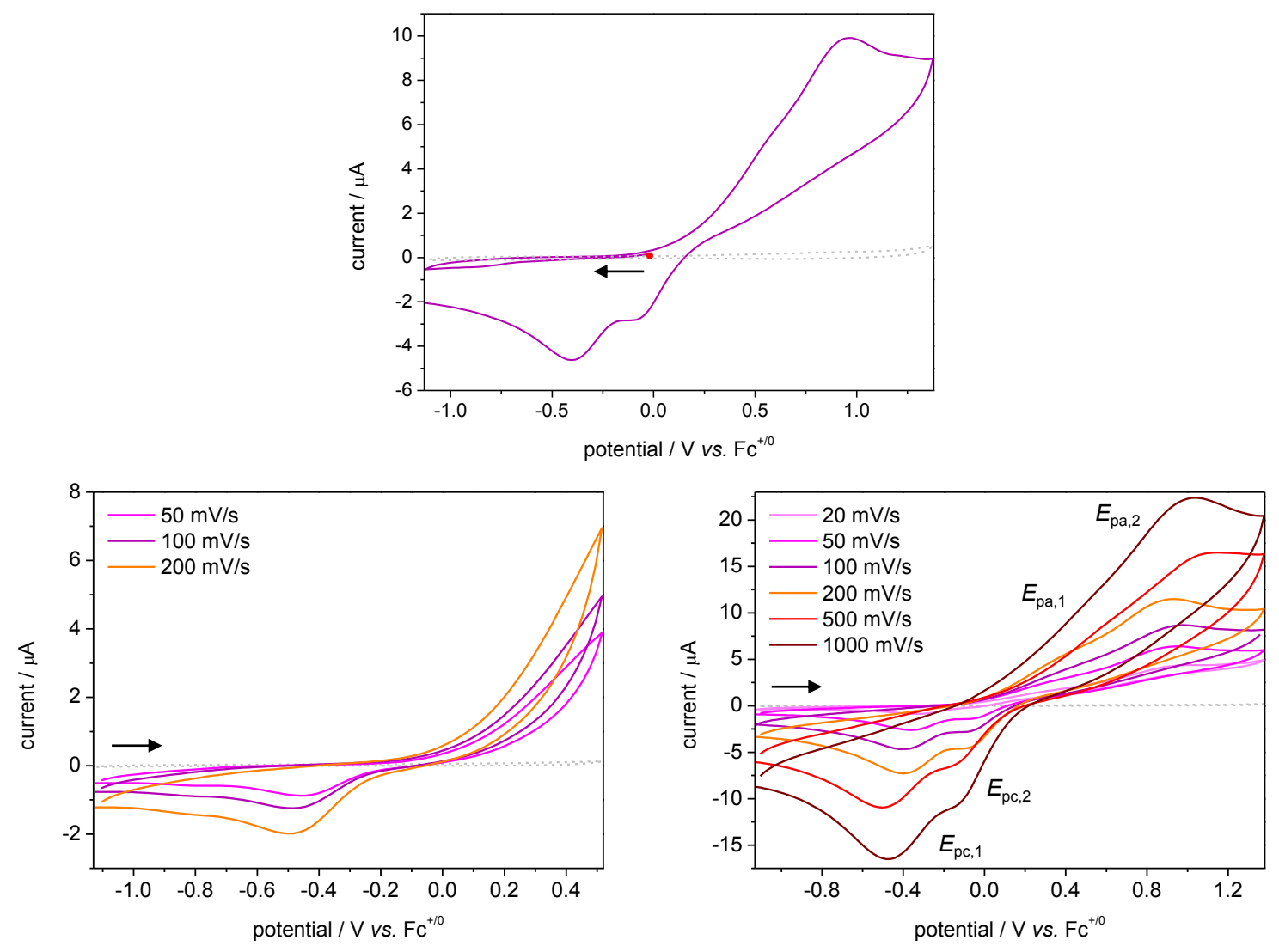

Figure 5.9: Electrochemical analysis of $7 \mathrm{MeCN}$ in $\mathrm{MeCN}\left(0.1 \mathrm{M} \mathrm{NBu}_{4} \mathrm{PF}_{6}\right)$ under inert conditions. Top: CV $(100 \mathrm{mV} / \mathrm{s})$ starting at the OCP (red dot) and scanning cathodically. Bottom: Scan rate dependence of the first (left) and of both oxidation-reduction couples (right). The scanning direction is indicated by an arrow. A glassy carbon disc was used as the working electrode, a Pt disc as auxiliary and an Ag wire as the reference electrode. Ferrocene was used as an internal standard.

Table 5.5: Peak potentials extracted from the scan rate dependent measurements of 7 MeCN in MeCN $(0.1 \mathrm{M}$ $\mathrm{NBu}_{4} \mathrm{PF}_{6}$ ) under inert conditions (Figure 5.11, right). All potentials are given $v s . \mathrm{Fc}^{+/ 0}$.

\begin{tabular}{cllll} 
scan rate $/ \mathrm{mV}$ & $E_{\mathrm{pa}, 1} / \mathrm{V}^{\mathrm{a}, \mathrm{b}}$ & $E_{\mathrm{pa}, 2} / \mathrm{V}^{\mathrm{a}}$ & $E_{\mathrm{pc}, 2} / \mathrm{V}^{\mathrm{a}}$ & $E_{\mathrm{pc}, 1} / \mathrm{V}^{\mathrm{a}}$ \\
\hline \hline 20 & 0.38 & 0.98 & -0.02 & -0.33 \\
50 & 0.38 & 0.95 & -0.05 & -0.36 \\
100 & 0.42 & 0.98 & -0.07 & -0.39 \\
200 & 0.42 & 0.93 & -0.08 & -0.39 \\
500 & 0.60 & 1.13 & -0.15 & -0.50 \\
1000 & 0.52 & 1.03 & -0.13 & -0.47 \\
\hline \multirow{2}{*}{ assignment } & $\mathrm{Co}^{\mathrm{II}} \mathrm{Co}^{\mathrm{II}}$ & $\mathrm{Co}^{\mathrm{III}} \mathrm{Co}^{\mathrm{III}}$ & $\mathrm{Co}^{\mathrm{III}} \mathrm{Co}^{\mathrm{III}}$ & $\mathrm{Co}^{\mathrm{II}} \mathrm{Co}^{\mathrm{III}}$ \\
& $\rightarrow \mathrm{Co}^{\mathrm{II}} \mathrm{Co}^{\mathrm{III}}$ & $\rightarrow \mathrm{Co}^{\mathrm{III}} \mathrm{Co}^{\mathrm{III}}$ & $\rightarrow \mathrm{Co}^{\mathrm{III}} \mathrm{Co}^{\mathrm{III}}$ & $\rightarrow \mathrm{Co}^{\mathrm{II}} \mathrm{Co}^{\mathrm{II}}$ \\
\hline
\end{tabular}

a peak potentials from the anodic (a) and cathodic (c) scan

${ }^{b}$ very rough approximation of the potentials as they are hardly visible in the $\mathrm{CV}$

In comparison to $\mathbf{8}^{\mathrm{HCO}}$ and $\mathbf{8}^{\mathbf{0 A c}}$, the oxidation of $7^{\mathrm{MeCN}}$ is significantly easier, occurring at around $0.42 \mathrm{~V}\left(\mathrm{Co}^{\mathrm{II}} \mathrm{Co}^{\mathrm{III}}\right)$ and $0.98 \mathrm{~V}\left(\mathrm{Co}^{\mathrm{III}} \mathrm{Co}^{\mathrm{III}}\right)$ at a scan rate of $100 \mathrm{mV} / \mathrm{s}$. ${ }^{\mathrm{xxvi}}$ This is presumably due to the fact that the coordination sites in $7^{\mathrm{MeCN}}$ feature octahedral geometry that is ideal for Co ${ }^{\text {III }}$, facilitating their

xxvi The first oxidation potential $\left(E_{\mathrm{pf}, 1}\right)$ is a very rough approximation as it is hardly visible in the CV 
oxidation. It further matches the observation that $7^{\mathrm{MecN}}$ is oxidized in the presence of oxygen. The large separation of the oxidation and corresponding reduction events presumably arises from a spin crossover and the concomitant large reorganization energy associated with the high-spin CoII $/$ low-spin $\mathrm{Co}^{\mathrm{III}}$ electron transfer, which is typical in octahedrally coordinated cobalt complexes. ${ }^{[350,370,371]}$

Based on the electrochemical results, UV/vis-SEC measurements were conducted in MeCN $(0.1 \mathrm{M}$ $\mathrm{NBu}_{4} \mathrm{PF}_{6}$ ). The UV/vis spectrum of $7 \mathrm{MeCN}^{\mathrm{M}}$ in the $\mathrm{Co}^{\mathrm{II}} \mathrm{Co}{ }^{\text {II }}$ state possesses local absorption maxima at $310 \mathrm{~nm}\left(\varepsilon=2.8 \cdot 10^{3} \mathrm{~L} \cdot \mathrm{mol}^{-1} \cdot \mathrm{cm}^{-1}\right)$ and $464 \mathrm{~nm} \mathrm{(152)}$ with a shoulder at $543 \mathrm{~nm}(51)$, the latter two of which correspond to $\mathrm{d}$-d transitions. Upon oxidation at $1.33 \mathrm{~V}$, the overall intensity significantly increases without any particular bands emerging (Figure 5.10, top left). Upon re-reduction (Figure 5.10 , top right) at $-0.97 \mathrm{~V}$, the absorption intensity once again decreases, however, the initial spectroscopic features of $\mathrm{Co}^{\mathrm{II}} \mathrm{Co}^{\mathrm{II}} 7^{\mathrm{MeCN}}$ are not reproduced, as shown in the dashed black and solid blue spectra. The $\Delta$ absorbance traces with respect to the spectrum before oxidation (Figure 5.10, bottom) emphasize the $\lambda$-dependent intensity gain and loss upon oxidation and re-reduction, as well as the irreversibility of the electrochemical process, as the trace after re-reduction (blue) significantly differs from the baseline. A possible reason for the irreversibility of the process might be that upon reduction one $\mathrm{MeCN}$ molecule at each $\mathrm{Co}^{\mathrm{II}}$ ion decoordinates; however, re-coordination of a solvent molecule would be expected upon reoxidation. Accordingly, the exact reason for the irreversibility is not clear.
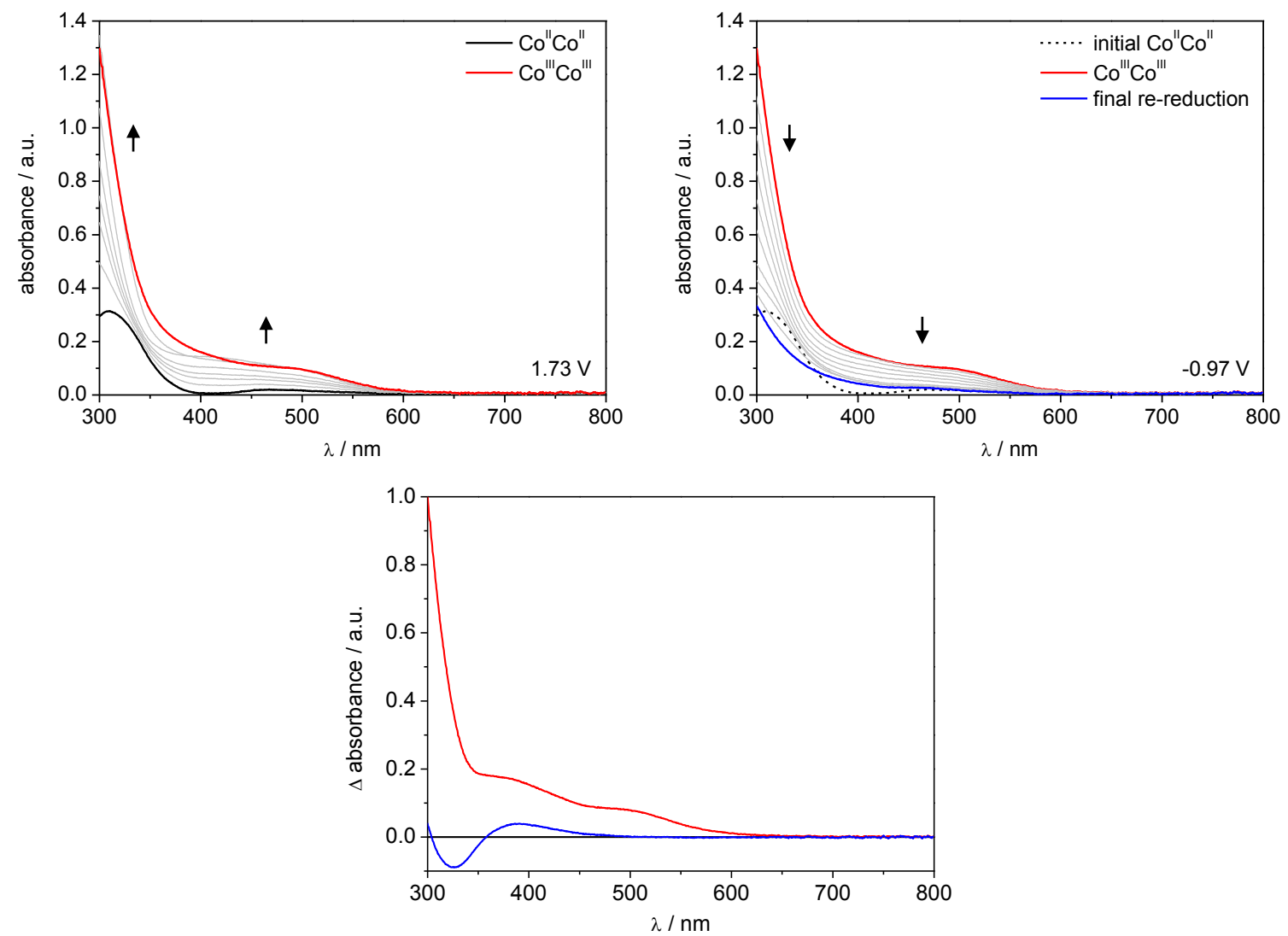

Figure 5.10: $\mathrm{UV} /$ vis-spectroelectrochemical analysis of $7^{\mathrm{MeCN}}$ in $\mathrm{MeCN}\left(0.1 \mathrm{M} \mathrm{NBu} 4 \mathrm{PF}_{6}\right)$ under inert conditions. Top left: UV/vis spectra of the electrochemical oxidation from $\mathrm{Co}^{\mathrm{II}} \mathrm{Co}^{\mathrm{II}}$ to $\mathrm{Co}^{\mathrm{III}} \mathrm{Co}^{\mathrm{III}}$ at $1.33 \mathrm{~V}$ (black: beginning, red: end). Top right: UV/vis spectra of the re-reduction from $\mathrm{Co}^{\mathrm{III}} \mathrm{Co}^{\mathrm{III}}$ to $\mathrm{Co}^{\mathrm{II}} \mathrm{Co}^{\mathrm{II}}$ at $-0.97 \mathrm{~V}$ (red: beginning, blue: end, black dashed: initial UV/vis spectrum before oxidation). Bottom: $\Delta$ absorbance of the spectra after oxidation (red) and re-reduction (blue) with respect to the spectrum before oxidation (baseline).

\subsubsection{Under Ambient Conditions}

In order to test whether $7^{\mathrm{MecN}}$ can serve as a WOC, further electrochemistry studies were performed in aqueous solution under ambient conditions. The $\mathrm{CV}$ of $7^{\mathrm{MeCN}}$ in phosphate buffer $(I=0.1 \mathrm{M})$ exhibits 
a pH dependent irreversible wave associated with a catalytic process, which could originate from water oxidation (Figure 5.11, top). The peak current of this wave initially arises at $\mathrm{pH} 5$ and increases with rising $\mathrm{pH}$. Over the course of $20 \mathrm{CV}$ cycles, the catalytic current stays relatively stable, however the peak maximum shifts anodically (compare scan 1 and scan 20). Furthermore, the rise in pH is associated with a color change of $7^{\mathrm{MecN}}$ in phosphate buffer, going from a very pale pink ( $\mathrm{pH} 2$ ) to a dark brown solution ( $\mathrm{pH}$ 8). This could be monitored by UV/vis spectroscopy and was accompanied by the emergence of a strong absorption at $462 \mathrm{~nm}$ (Figure 5.11, bottom right), which is reminiscent of the $\mathrm{Co}^{\mathrm{III}} \mathrm{Co}^{\mathrm{III}}$ spectra of $7^{\mathrm{A}}$ (Figure 5.2) and $\mathbf{8}^{\mathrm{HCO}}$ (Figure 5.8).
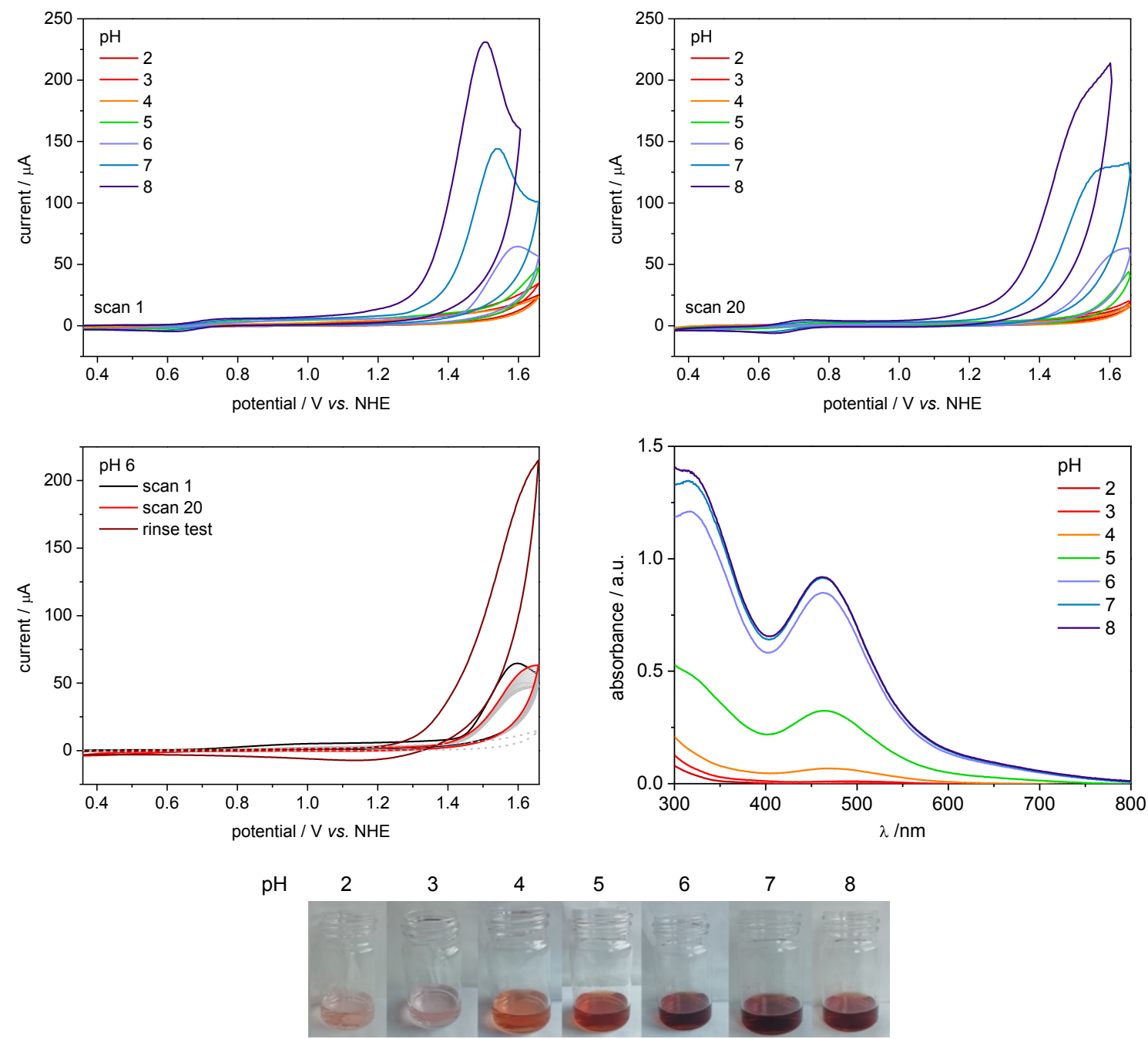

Figure 5.11: $\mathrm{pH}$ dependent analysis of a $1 \mathrm{mM}$ solution of $7 \mathrm{MeCN}$ in phosphate buffer $(I=0.1 \mathrm{M})$. Top: $\mathrm{pH}$ dependent CVs (left: scan 1, right: scan 20). Bottom left: CVs (20 cycles, black: scan 1, red: scan 20) at pH 6 in comparison to the rinse test after CPE at $1.54 \mathrm{~V}$ for 30 minutes (dark red). A glassy carbon disc was used as working electrode, a Pt disc as auxiliary and an MSE as the reference electrode. The potentials were converted to the NHE scale by adding 0.658 V. Bottom right: $\mathrm{pH}$ dependent UV/vis spectra. Pictures of the solutions at each pH are shown below.

In order to evaluate the stability of $\mathbf{7}^{\mathrm{MecN}}$ under the catalytic conditions, a rinse test was conducted in $\mathrm{pH} 6$ phosphate buffer $(I=0.1 \mathrm{M})$. For this, a controlled potential electrolysis (CPE) was performed for 30 minutes at $1.54 \mathrm{~V}$. Subsequently, the working electrode was carefully rinsed with electrolyte and a CV was measured in fresh blank solution without polishing. The resulting CV (dark red, Figure 5.11, bottom left) shows a significantly increased catalytic current with a less positive onset potential in comparison to the catalytic wave in a complex solution, indicating a positive rinse test after CPE. This concludes that the observed electrocatalysis is heterogenous and $7^{\mathrm{MeCN}}$ merely acts as the precatalyst, which degrades to a catalytically active species under the given conditions, as previously observed for 
the copper complex $\mathbf{5}^{\mathrm{HCO}}$ equally bearing ( $\mathrm{L}^{2 \mathrm{a}}$ )- (see Chapter 4). Not surprisingly, many examples exist of originally presumed homogenous cobalt WOCs, in which a heterogenous species turned out to be the actual active catalyst. ${ }^{[341,342,82,343]}$ Next to the degradation of the applied cobalt complexes, the presence of cobalt(II) salt impurities was the main source of eventual heterogeneity. The catalytic activity of cobalt oxide has been known for a long time. ${ }^{[372-375,144,376,377]}$

In the herein studied case, the availability of phosphate from the electrolyte might also be an issue, as it has previously been reported that highly active cobalt oxide particles $\left(\mathrm{CoP}_{\mathrm{i}}\right)$ are formed in a selfassembly process from cobalt and phosphate upon oxidation of $\mathrm{Co}^{\mathrm{II}}$ to $\mathrm{Co}^{\mathrm{III}}$ in an aqueous solution. ${ }^{[302,378]}$ In fact, this is the exact WOC that is applied in Daniel Nocera's artificial leaf - an amorphous silicon photovoltaic with $\mathrm{H}_{2}$ - and $\mathrm{O}_{2}$-evolving catalysts that functions under illumination in near-neutral conditions. ${ }^{[379]}$ ESI-MS experiments of $7^{\mathrm{MeCN}}$ in the presence of $\mathrm{Na}_{2} \mathrm{HPO}_{4}$ already showed coordination of phosphate in the bimetallic cleft, illustrating the high affinity of phosphate to cobalt ions (appendix, Figures A.36 and A.37).

\subsection{Summary and Conclusion}

Five dinuclear cobalt complexes were synthesized based on the pyrazolate/tacn hybrid proligands $\mathrm{HL}^{2 \mathrm{a}}\left(\mathbf{7}^{\mathrm{MeCN}} / \mathbf{7}^{\mathbf{0 2} \mathrm{H}} / \mathbf{7}^{\mathrm{A}}\right)$ and $\mathrm{HL}^{2 \mathrm{~b}}\left(\mathbf{8}^{\mathrm{HCO2}} / \mathbf{8}^{\mathrm{OAc}}\right)$. The complexes with $\left(\mathrm{L}^{2 \mathrm{a}}\right)^{-}$thereby proved to be sensitive to oxidation under ambient conditions, providing the ideal geometry for low-spin $\mathrm{d}^{6} \mathrm{Co}^{\mathrm{III}}$ ions by coordination of an additional axial ligand. The use of $\mathrm{HL}^{2 \mathrm{~b}}$ with sterically hindered $i \mathrm{Pr}$ peripherals, on the other hand, seems to prevent additional ligand coordination and the concomitant oxidation of the cobalt ions. Consequently, the cobalt(II) complexes $\mathbf{8}^{\mathrm{HCO}}$ and $\mathbf{8}^{\mathbf{0 A c}}$ featuring formate and acetate as bridging ligands in the bimetallic cleft, respectively, can be synthesized under ambient conditions.

All five compounds were characterized in solution by means of ESI mass spectrometry. The diamagnetic character of octahedrally coordinated low-spin $\mathrm{d}^{6} \mathrm{Co}^{\mathrm{III}}$ ions in $7^{\mathrm{A}}$ additionally enabled an NMR spectroscopic analysis. Furthermore, the solid state molecular structures of $7^{\mathrm{MeCN}}, \mathbf{8}^{\mathrm{HCO}}$ and $8^{\mathrm{OAc}}$ were authenticated by XRD. All follow-up analyses were conducted with these three complexes.

The magnetic susceptibility measurement of $7^{\mathrm{MecN}}$ revealed weak intramolecular antiferromagnetic exchange interaction and a positive axial zero-field splitting. By contrast, the $\chi_{\mathrm{m}} T v$ vs. $T$ data of $\mathbf{8}^{\mathrm{HCO}}$ and $8^{\text {OAc }}$ displayed weak ferromagnetic coupling and a negative $D$ parameter, rendering them possible candidates for SMMs. Indeed, ac susceptibility measurements demonstrated slow relaxation of the magnetization in $\mathbf{8}^{\mathrm{HCO}}$ and $\mathbf{8}^{\mathbf{0 A c}}$ with a relaxation time $\tau_{0}$ of $7.7 \cdot 10^{-6} \mathrm{~s}^{-1}$ and $3.7 \cdot 10^{-6} \mathrm{~s}^{-1}$, as well as an energy barrier $U_{\text {eff }}$ of 8.9 and $6.0 \mathrm{~cm}^{-1}$, respectively. These values are similar to other cobalt(II) SMMs. ${ }^{[239,241,357]}$

The electrochemical behavior of $7^{\mathrm{MecN}}, \mathbf{8}^{\mathrm{HCO2}}$ and $\mathbf{8}^{\mathbf{O A c}}$ was investigated under inert conditions. In MeCN (0.1 M NBu $\left.4 \mathrm{PF}_{6}\right)$, all three complexes exhibit four electrochemically irreversible peaks, which can be assigned to the consecutive oxidations from $\mathrm{Co}^{\mathrm{II}} \mathrm{Co}^{\mathrm{II}}$ to $\mathrm{Co}^{\mathrm{II}} \mathrm{Co}^{\mathrm{III}}$ and further to $\mathrm{Co}^{\mathrm{III}} \mathrm{Co}^{\mathrm{III}}$, and vice versa for the reductions. In comparison to $\mathbf{8}^{\mathbf{H C O}}$ and $\mathbf{8}^{\mathbf{O A c}}$, the oxidation of $\mathbf{7}^{\mathrm{MecN}}$ is significantly easier, presumably because the coordination sites in $7^{{ }^{M e C N}}$ feature the ideal geometry for $\mathrm{Co}^{\mathrm{III}}$, viz. octahedron, facilitating their oxidation. This is furthermore consistent with the synthetic observation that $7 \mathrm{MeCN}^{\mathrm{M}}$ oxidized in the presence of oxygen.

Complex $7^{\text {MecN }}$ was further electrochemically characterized under ambient conditions. In phosphate buffer, $7^{\mathrm{MecN}}$ exhibits a $\mathrm{pH}$ dependent irreversible wave associated with a catalytic process that initially arises at $\mathrm{pH} 5$ and increases with rising $\mathrm{pH}$. A positive rinse test conducted after CPE for 30 minutes at $1.54 \mathrm{~V} v$ s. NHE in $\mathrm{pH} 6$ phosphate buffer, showed that a surface deposit is responsible for the observed catalytic process. Consequently, $7^{\mathrm{MecN}}$ merely acts as the precatalyst, which degrades to a catalytically active species under the given conditions, as previously observed for the copper complex $\mathbf{5}^{\mathrm{HCO2}}$ equally based on ( $\left.\mathrm{L}^{2 \mathrm{a}}\right)^{-}$(see Chapter 4 ). 


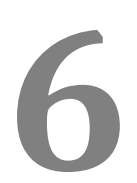

\section{Dioxygen Activation at Dinuclear Copper Complexes}

Parts of this chapter have been adapted from Jana Lücken, Thomas Auth, Sara Ida Mozzi, Franc Meyer, "Hexanuclear Copper(I) Hydride from the Reduction-Induced Decarboxylation of a Dicopper(II) Formate", Inorg. Chem. 2020, 59, 14347-14354. The corresponding sections are marked by footnotes.

Dinuclear copper complexes based on pyrazolate/tacn hybrid ligands have long been used for dioxygen activation in the Meyer group. $[170,187,188,199,206,207,380]$ The starting point of these conversions are dicopper(I) compounds, which facilitate the binding, activation and reduction of $\mathrm{O}_{2}$. So far, two related dinucleating pyrazolate/tacn ligand scaffolds have been applied for the synthesis of the latter (Scheme 6.1) - one bearing two methylene spacers $\left(6^{1 / 1}\right)$ and the other connecting the pyrazole unit and macrocyclic sidearm via ethylene groups (XXV). Ongoing studies are currently focused on the use of a non-symmetric ligand comprising both a methylene and an ethylene linker. These subtle ligand modifications result in significant structural differences, viz. the $\mathrm{Cu} \cdots \mathrm{Cu}$ distance is reduced from 4.153 (6'/I) to $3.968 \AA$ (XXV),[206,207] which also has a great impact on the follow-up chemistry.

Addition of dry molecular oxygen to the dicopper(I) complexes $\mathbf{6}^{\mathbf{I} / \mathbf{I}}$ and $\mathbf{X X V}$ leads to the formation of $\mu$-1,2-peroxo dicopper(II) species, in which the $\mathrm{O}_{2}$ has been reduced twice by the metal centers (Scheme 6.1). In fact, the peroxo complex $\mathbf{6}^{\mathbf{P}}$ constitutes the first ever structurally characterized ${ }^{\mathbf{C}} \mathbf{P}$ model complex. ${ }^{[170]}$ On the other hand, under evaluation of the $\mathrm{Cu}-\mathrm{O}-\mathrm{O}-\mathrm{Cu}$ torsion angle in $\mathbf{X X V}^{\mathbf{P}}$ $\left(104.2^{\circ}\right)$, it can be considered as an intermediate between a ${ }^{\mathbf{C P}}\left(65.2^{\circ}\right.$ in $\left.\mathbf{6}^{\mathbf{P}}\right)$ and $\mathbf{T} \mathbf{P}\left(\sim 180^{\circ}\right)$ binding mode. Together with this rather unique feature arises a ferromagnetic coupling between the copper centers, making $\mathbf{X X V}^{\mathbf{P}}$ the first example of a peroxo dicopper(II) complex with a triplet ground state. ${ }^{[380]}$

In addition to the different electronic structures of the peroxo complexes, the ligand scaffold also alters the accessibility of substrates to the peroxide moiety. Accordingly, the peroxo moiety in $\mathbf{X X V}^{\mathbf{P}}$ is strongly shielded by the isopropyl periphery at the tacn moieties, while the cavity in $\mathbf{6}^{\mathbf{P}}$ is more accessible. ${ }^{[188,206]}$ As a consequence, $\mathbf{X X V}^{\mathbf{P}}$ shows no interaction with redox inert alkali metal ions, but $6^{\text {P }}$ forms adducts with the latter, leading to a modification of its electronic structure. Both complexes display no oxygenation reactivity towards organic substrates. However, replacing the residues at the tacn moieties with less bulky groups can reduce the steric hindrance at the bridging peroxo moiety, and thus might enhance its reactivity.

The peroxo dicopper(II) complexes $\mathbf{6}^{\mathbf{P}}$ and $\mathbf{X X V}^{\mathbf{P}}$ can be converted to superoxo and hydroperoxo species by oxidation and protonation, respectively. The latter is achieved for both compounds with lutidinium triflate (HLutOTf), resulting in significant spectroscopic and structural changes. ${ }^{188,206]}$ In case of $\mathbf{X X V}$, the hydroperoxo dicopper(II) complex ( $\mathbf{X X V}^{\mathbf{0 0 H}}$ ) was structurally characterized by X-ray diffraction, exhibiting a monoatomic $\mu-1,1$ bridging mode of the hydroperoxo moiety (this type of coordination mode is not expected for $\mathbf{6}^{\mathbf{0 O H}}$ due to the significantly larger $\mathrm{Cu} \cdots \mathrm{Cu}$ distance, which usually does not accommodate monoatomic bridging ligands).[199] In addition, with an estimated halflife of 9 hours at room temperature, $\mathbf{X X V}^{\circ 0 \mathrm{oH}}$ is by far the most stable $\mathrm{Cu}_{2}-\mathrm{OOH}$ compound to date. Despite this stability, $\mathbf{X X V}{ }^{\mathbf{0 O H}}$ is capable of mediating the oxygenation of $\mathrm{PPh}_{3}$ to $\mathrm{OPPh}_{3}$ with a 
conversion of $(94 \pm 5) \%$ based on ${ }^{1} \mathrm{H}$ NMR experiments. For both complexes, the transformation to the hydroperoxo species is cleanly reversed by addition of diazabicycloundecene (DBU).

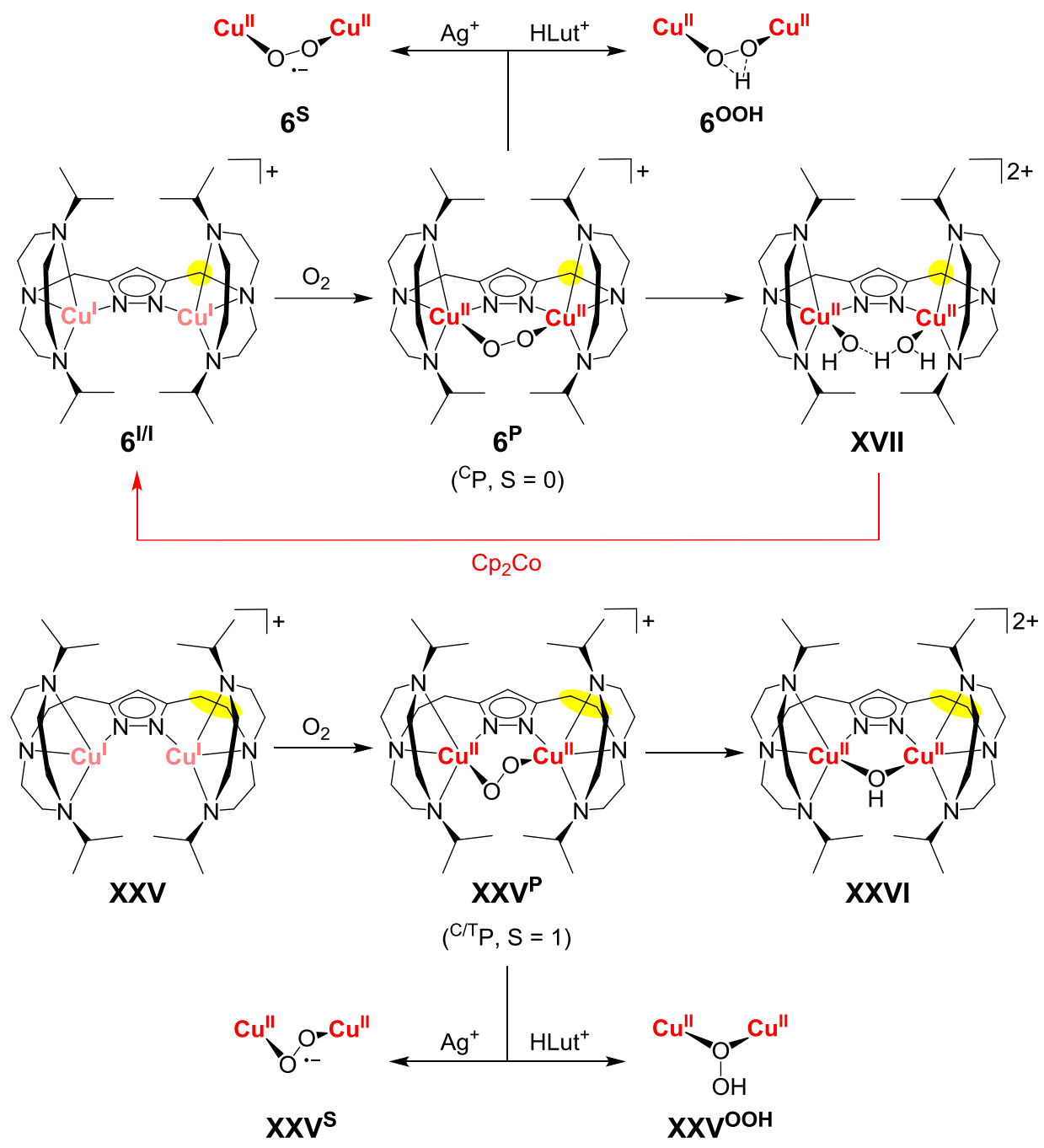

Scheme 6.1: Dicopper(I) complexes $\mathbf{6}^{\mathbf{1} / \mathbf{I}}$ and XXV with pyrazolate/tacn hybrid ligands, as well as the corresponding peroxo, superoxo and hydroperoxo dicopper(II) species and decomposition products. The targeted recycling of the aquo/hydroxo copper(II) decomposition product XVII is marked in red. The structural differences are highlighted in yellow.

Oxidation of $\mathbf{6}^{\mathbf{P}}$ and $\mathbf{X X V}^{\mathbf{P}}$ was achieved both electrochemically and chemically by means of $\mathrm{Ag}^{+}$. A $\mu-1,2$ binding motif of the superoxo moiety is assumed for both complexes, however a solid-state structure could only be obtained for $\mathbf{6}^{\mathbf{S}}$, making it the first ever structurally characterized superoxo dicopper(II) complex.[188] The superoxo binding mode of $\mathbf{X X V}^{\mathbf{S}}$ was deduced from the EPR spectrum and supported by DFT calculations. ${ }^{[187]}$ From the thermodynamic parameters $E^{0}$ for the $\mathrm{O}_{2}{ }^{2-} / \mathrm{O}_{2}-{ }^{--}$redox couple and the $\mathrm{p} K_{\mathrm{a}}$ of the hydroperoxo species, bond dissociation free energies (BDFEs) of $(71.4 \pm 0.3)$ and $(71.7 \pm 1.1) \mathrm{kcal} \cdot \mathrm{mol}^{-1}$ were determined for the $\mathrm{OO}-\mathrm{H}$ bonds in $\mathbf{6}^{\mathbf{6 0 H}}$ and $\mathbf{X X V}^{\mathbf{0 O H}}$, respectively. Accordingly, both superoxo complexes showed no reactivity toward $\mathrm{C}-\mathrm{H}$ bonds (BDFE $>72.9 \mathrm{kcal} \cdot \mathrm{mol}^{-1}$ ) but were capable of $\mathrm{H}$ atom abstraction (HAT) of the weaker $\mathrm{O}-\mathrm{H}$ bond in TEMPOH (66.5). Likewise, the hydroperoxo species is formed upon addition of phenylhydrazine $(\mathrm{N}-\mathrm{H}$,

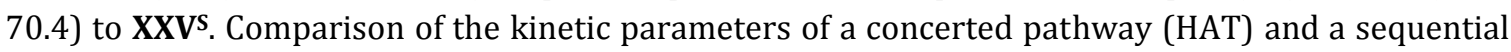
mechanism starting with electron or proton transfer, suggest the former for these conversions.

A challenge that must be met when handling oxygen activated compounds is their relative instability, especially at temperatures above $-25^{\circ} \mathrm{C}$ and in some cases also in the presence of traces of 
water. Common decomposition products are the aquo/hydroxo or hydroxo dicopper(II) complexes XVII and XXVI (Scheme 6.1), which are typically discarded, being the thermodynamic end product and unreactive to organic substrates. Recently, the recycling of XVII was achieved by reduction with cobaltocene. This led to the in situ formation of the dicopper(I) compound $6^{1 / 1}$ without an exogenous ligand in the bimetallic cleft, which was capable of re-binding and activating dioxygen. ${ }^{[188]}$

As a dicopper(I) complex could not be synthesized directly from the $\mathrm{Me}_{2}$-tacn ligand $\mathrm{HL}^{2 \mathrm{a}}$, the above-mentioned method was chosen in order to form a reduced copper species starting from $\mathbf{5}^{\mathbf{0 A c}}$ and $5^{\mathrm{HCO2}}$ with surprising results. Next to the reduction with cobaltocene, the subsequent activation of dioxygen will be discussed. Preliminary investigations of the $\mathrm{O}_{2}$ activation by the dicopper(I) compound $\mathbf{5}^{\mathbf{I} / \mathbf{I}}$ were previously attempted.[188]

\subsection{Reduction of $5^{\mathrm{OAc}}$ via a Mixed-Valent $\mathrm{Cu}^{\mathrm{I}} \mathrm{Cu}^{\mathrm{II}}$ Intermediate}

The reduction of $\mathbf{5}^{\mathbf{0 A c}}$ with cobaltocene in MeCN was monitored by UV/vis spectroscopy. As shown in Figure 6.1 (top), the addition of $\mathrm{Cp}_{2} \mathrm{Co}$ to $5^{\mathrm{OAc}}$ leads to the decrease of the $\mathrm{d}$-d transition at $684 \mathrm{~nm}$. Concomitantly, new bands rise at 400 and $508 \mathrm{~nm}$ corresponding to a cobaltocenium species and cobaltocene, respectively. ${ }^{[381]}$ After addition of 2.3 eq $\mathrm{Cp}_{2} \mathrm{Co}$, the $\mathrm{d}$ - $\mathrm{d}$ transition has completely vanished in line with the presence of a fully reduced dicopper(I) species. ${ }^{x x v i i}$ A closer look at the UV/vis spectra reveals two distinct processes denoted by different isosbestic points (Figure 6.1, bottom). Up to the addition of 1.2 eq cobaltocene (left), isosbestic points are observed at 355 and $585 \mathrm{~nm}$. The subsequent addition of a further 1.1 eq $\mathrm{Cp}_{2} \mathrm{Co}$ (right) is characterized by an isosbestic point at $457 \mathrm{~nm}$. This strongly suggests the presence of an intermediate species.

The occurrence of two consecutive reduction processes is emphasized by the absorbance traces of the isosbestic points (Figure 6.2). Up to 1.2 eq $\mathrm{Cp}_{2} \mathrm{Co}$, the traces at 355 (top right) and $585 \mathrm{~nm}$ (bottom left) remain stable, after which the former increases and the latter decreases. By contrast, the absorbance at $457 \mathrm{~nm}$ (bottom right) gradually increases until $1.2 \mathrm{eq}$ and then remains quite unchanged until 2.3 eq $\mathrm{Cp}_{2} \mathrm{Co}$ are reached. The trace of the $\mathrm{d}$-d transition (Figure 6.2, top left) also shows a slight change in slope between 0-1.2 eq and 1.2-2.3 eq $\mathrm{Cp}_{2} \mathrm{Co}$, however, the difference is not so pronounced as the band continues to decrease over the entire process. In any case, further addition of cobaltocene beyond 2.3 eq leads to distinct changes in the absorbance of all four wavelengths. Whereas for the isosbestic points a significant increase is observed, the absorbance of the d-d transition becomes stable. This indicates that the reduction process is finalized after 2.3 eq and further addition of cobaltocene leads to an overtitration. The necessity of 1.2 and 2.3 eq instead of 1.0 and 2.0 eq for the single and twofold reduction is likely a result of uncertainties regarding the experimental procedure.

In view of these results, it seems likely that a mixed-valent $\mathrm{Cu}^{\mathrm{I} C \mathrm{Cu}^{\mathrm{II}}}$ species $\left(\mathbf{5}^{\mathrm{I} / \mathrm{II}}\right)$ is formed upon single reduction of the original complex $\mathbf{5}^{\mathbf{0 A c}}$. Thus far, this is a unique observation for dinuclear copper complexes based on pyrazolate/tacn hybrid ligands, but has been observed for the related dicopper

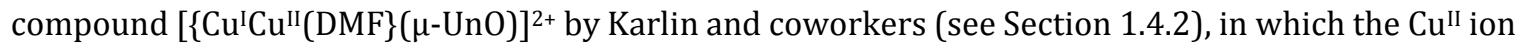
is additionally coordinated by a DMF molecule.[189,190] One significant difference between $\mathbf{5}^{\mathbf{0 A c}}$ and the $i \mathrm{Pr}_{2}$-tacn containing XVII, is the molecule in the bimetallic cleft, viz. acetate vs. aquo/hydroxo, respectively. Consequently, the acetate moiety might rearrange and stabilize the $\mathrm{Cu}^{\mathrm{II}}$ site in the mixedvalent species 5/III, allowing its formation (Scheme 6.2). In line with this, the ESI(+)-MS of a solution of $\mathbf{5}^{\mathbf{0 A c}}$ and cobaltocene (1.2 eq) in MeCN (Figure 6.3) shows a major peak at $m / z$ 590.3, corresponding to the molecular ion peak of $5^{\mathrm{I} / \mathrm{II}}$, viz. [ $\left.\mathrm{L}^{2 \mathrm{a}} \mathrm{Cu}_{2}(\mathrm{OAc})\right]^{+}\left(\mathrm{Cu}^{\mathrm{I}} \mathrm{Cu}^{\mathrm{II}}\right)$. Further peaks, however, can be attributed to both the fully reduced $\mathrm{Cu}^{\mathrm{I}} \mathrm{Cu}^{\mathrm{I}}$ complex $\left(\mathrm{m} / z\right.$ 531.2) and the starting material $\mathbf{5}^{\mathrm{OAc}}(\mathrm{m} / z 295.2$ and 689.2).

xxvii Upon addition of more than 2.3 eq $\mathrm{Cp}_{2} \mathrm{Co}$, the increase in absorbance from 350-400 nm results from the cobaltocene itself, which features a very intense band at $325 \mathrm{~nm}$, next to the one at $508 \mathrm{~nm}$, whose flanks reach past $400 \mathrm{~nm}$. 

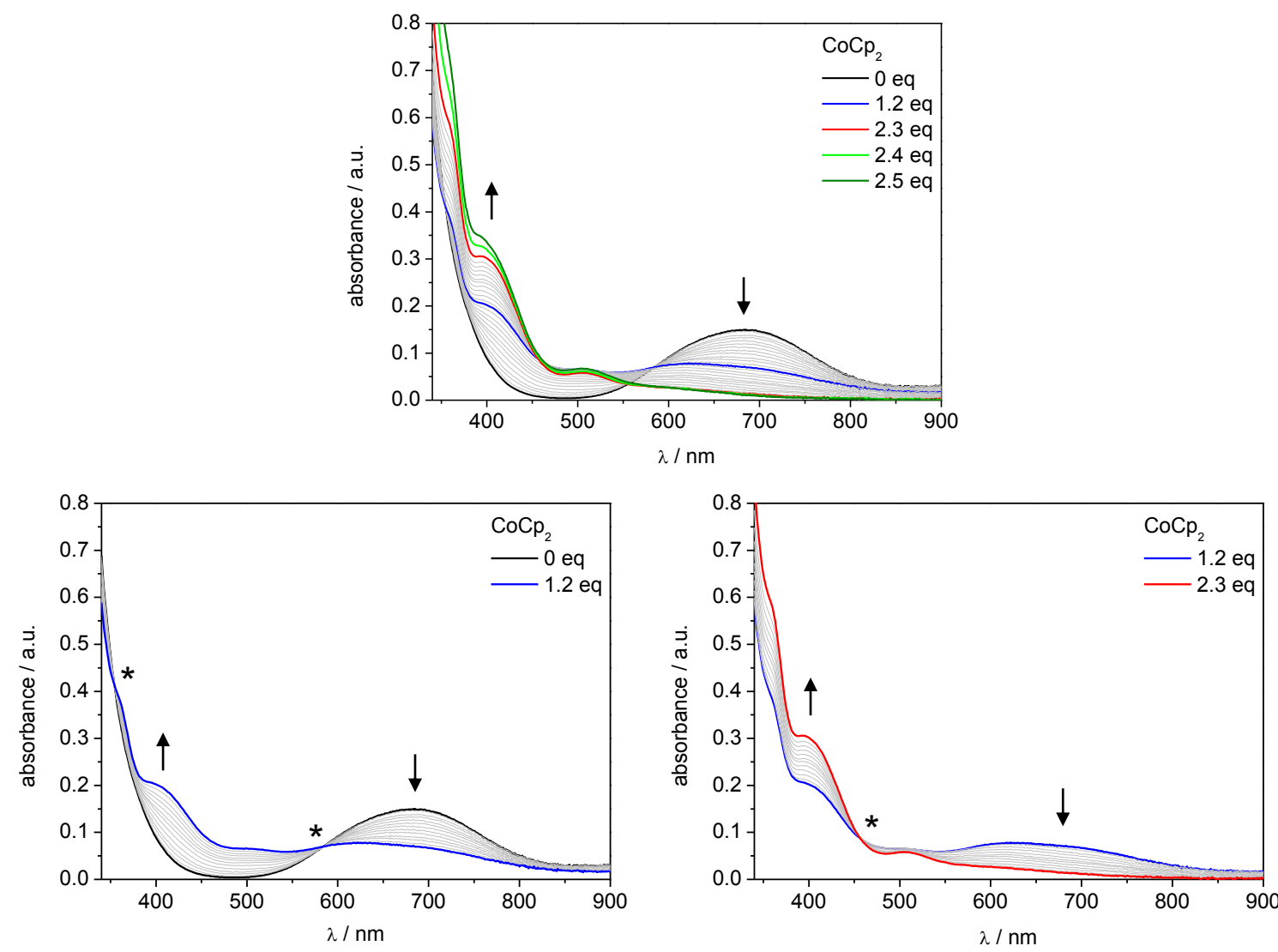

Figure 6.1: UV/vis titration of $\mathbf{5}^{\mathbf{O A c}}$ with $\mathrm{Cp}_{2} \mathrm{Co}$ in $\mathrm{MeCN}$ at room temperature under inert conditions. Top: UV/vis spectra of the complete addition of 2.5 eq $\mathrm{Cp}_{2} \mathrm{Co}$ in 0.1 eq steps. The reduction is complete after addition of $2.3 \mathrm{eq}$ $\mathrm{Cp}_{2} \mathrm{Co}$. There are no isosbestic points for the twofold reduction of $\mathbf{5}^{\mathbf{O A c}}$. Bottom: UV/vis spectra of the first (left, isosbestic points: 355 and $585 \mathrm{~nm}$ ) and second (right, isosbestic point: $457 \mathrm{~nm}$ ) reduction steps.
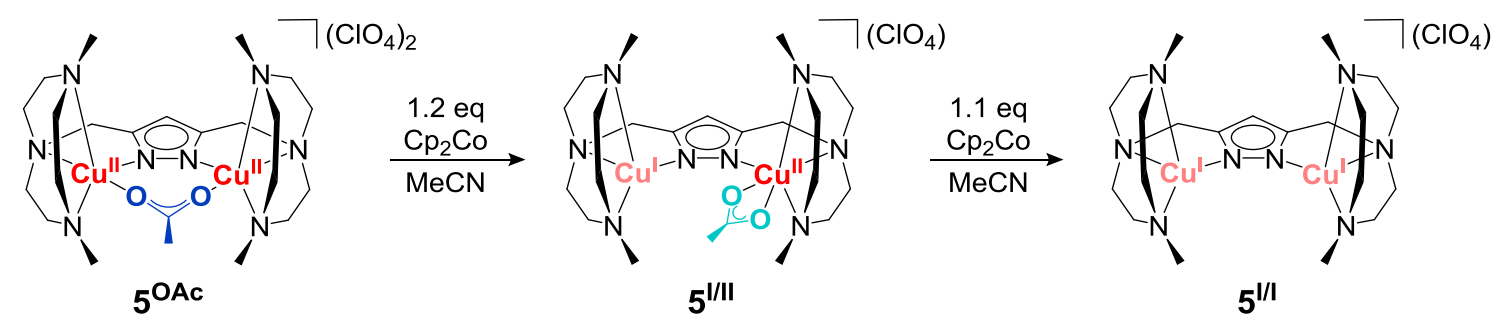

Scheme 6.2: Twofold reduction of $5^{\mathrm{OAc}}$ to the $\mathrm{Cu}^{\mathrm{I} C \mathrm{Cu}^{\mathrm{I}}}$ species $\mathbf{5}^{\mathbf{I} / \mathrm{I}}$ via the mixed-valent $\mathbf{5}^{\mathrm{I} / \mathrm{II}}$. The acetate molecule in $\mathbf{5}^{\mathbf{I} / \mathrm{II}}$ is assumed to stabilize the $\mathrm{Cu}^{\mathrm{II}}$ site. 

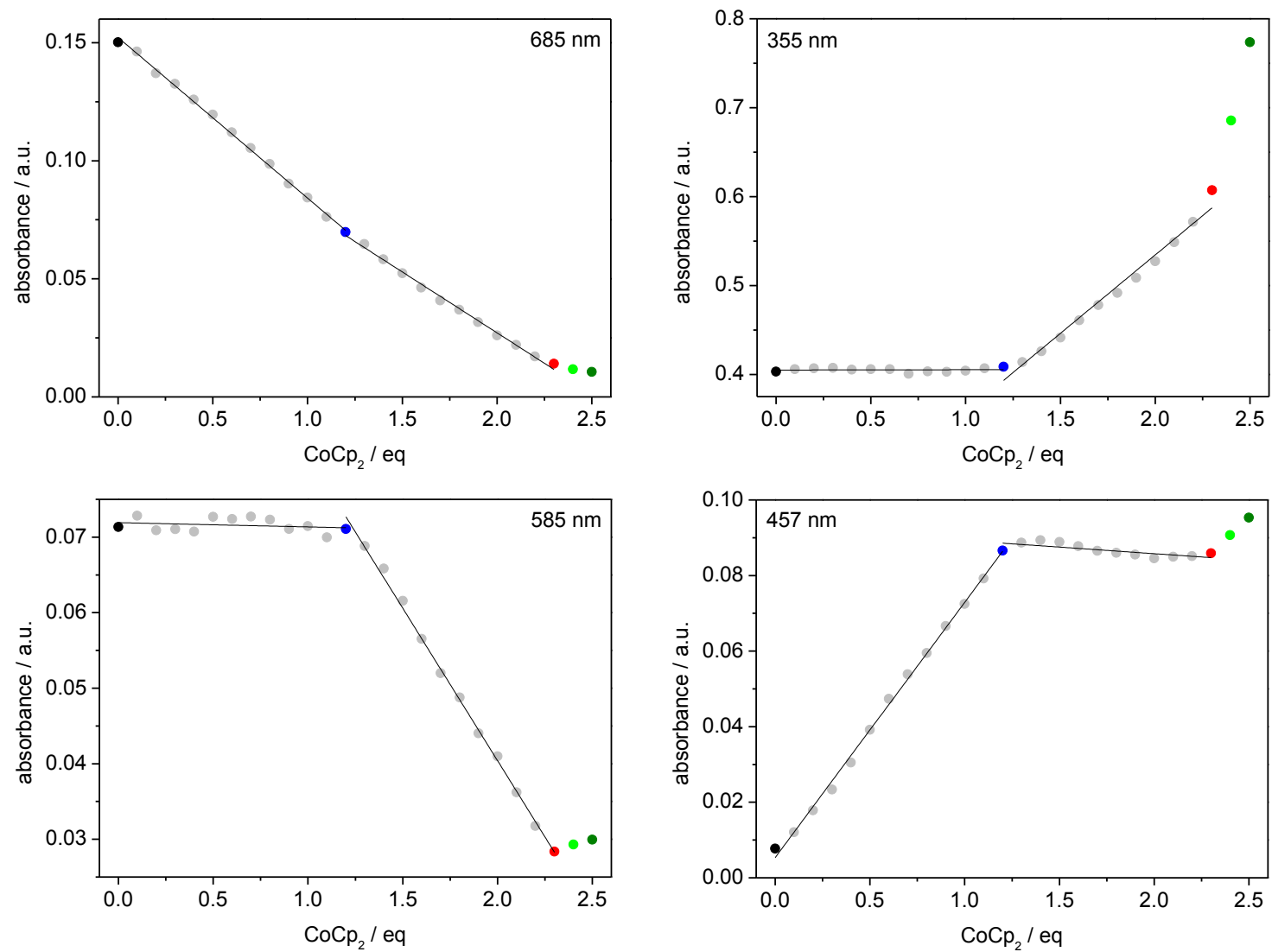

Figure 6.2: Traces of the UV/vis titration of $\mathbf{5}^{\mathbf{0 A c}}$ with $\mathrm{Cp}_{2} \mathrm{Co}$ in $\mathrm{MeCN}$ at room temperature under inert conditions. The traces of the $\mathrm{d}$-d transition $(685 \mathrm{~nm})$, as well as the three isosbestic points $(355,585$ and $457 \mathrm{~nm})$ are depicted.

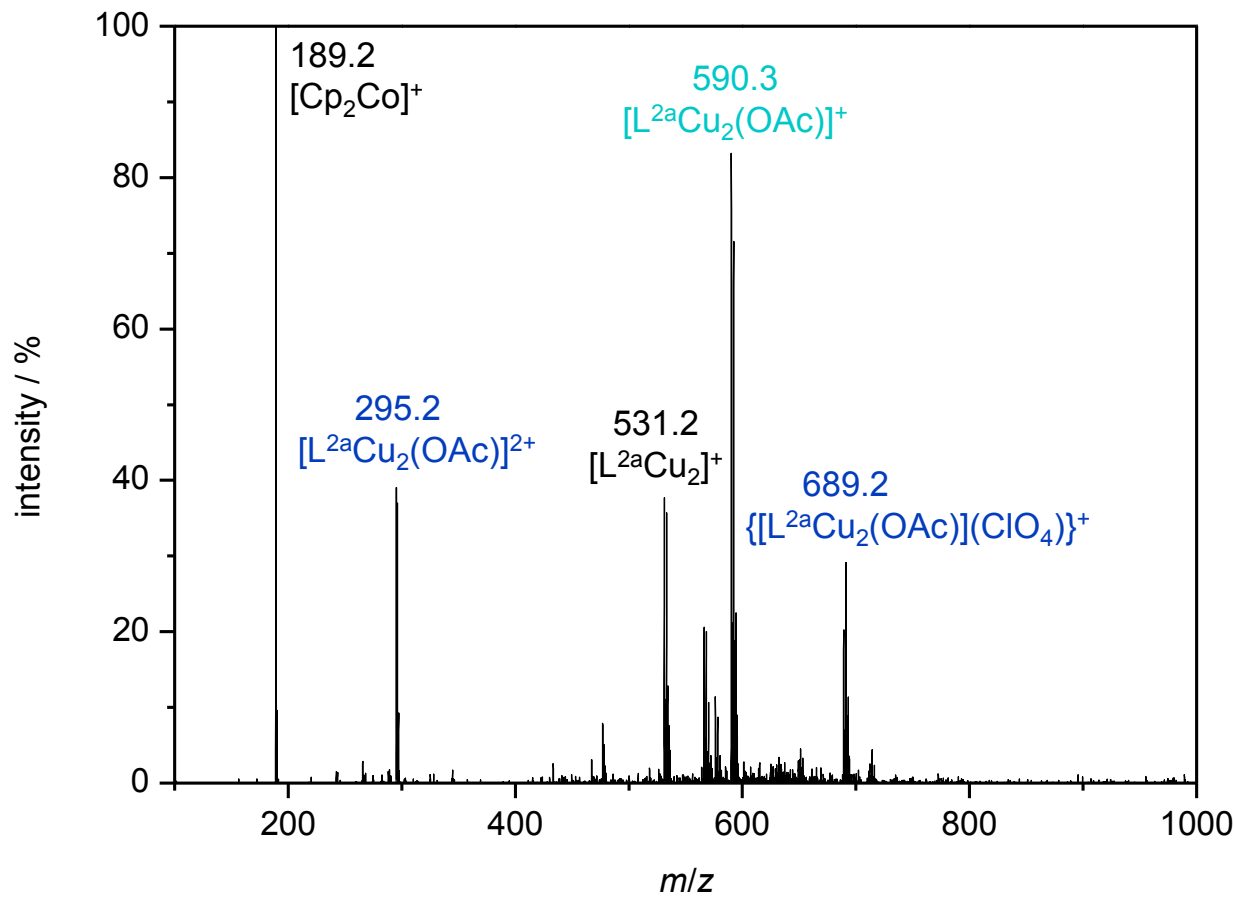

Figure 6.3: $\mathrm{ESI}(+)-\mathrm{MS}$ of $\mathbf{5}^{\mathbf{O A c}}+\mathrm{Cp}_{2} \mathrm{Co}(1.2 \mathrm{eq})$ in $\mathrm{MeCN}$ under inert conditions. Next to cobaltocenium $(\mathrm{m} / \mathrm{z}$ 189.2), species of the $\mathrm{Cu}^{\mathrm{II}} \mathrm{Cu}^{\mathrm{II}}$ (295.2/689.2), $\mathrm{Cu}^{\mathrm{I}} \mathrm{Cu}^{\mathrm{II}}$ (590.3) and $\mathrm{Cu}^{\mathrm{I} C u^{\mathrm{I}}}$ (531.2) complex are observed. 
To further confirm the suggested formation of the mixed-valent complex $\mathbf{5}^{\mathbf{I} / \mathrm{II}}$ after single reduction of $\mathbf{5}^{\mathbf{O A c}}$ with cobaltocene, EPR measurements were conducted. The spectrum of $\mathbf{5}^{\mathbf{O A c}}$ (Figure 6.4, left) is in accord with an $S=1$ system (ferromagnetic coupling of the two $\mathrm{Cu}^{\mathrm{II}}$ ions, as determined from the magnetic measurements, see Section 4.2), exhibiting a broad EPR signal at $3170 \mathrm{G}$, as well as a halffield peak at around $1500 \mathrm{G}$, corresponding to the forbidden $\Delta M_{\mathrm{s}}= \pm 2$ transition typical of triplet state EPR spectra. ${ }^{[382-385]}$ No hyperfine splitting is observed and the zero-field splitting, which was obtained from the simulation, arises from dipole-dipole interaction. After addition of 1.2 eq $\mathrm{Cp}_{2} \mathrm{Co}$ (Figure 6.4, right), the half-field signal disappears and a typical pseudo-axial $\mathrm{Cu}^{\mathrm{II}}$ spectrum is observed. The EPR spectrum was simulated with one $\mathrm{Cu}^{\mathrm{II}}$ ion $(S=1 / 2, I=3 / 2)$ revealing anisotropic $g$ values of $2.05,2.10$ and 2.24. Hyperfine splitting constants were distinguished as 94,50 and $480 \mathrm{MHz}$, however, the former two are overcompensated by the linewidth $(100 \mathrm{G})$. The corresponding parameters for $\mathbf{5}^{\mathbf{0 A c}}$ before and after addition of $\mathrm{Cp}_{2} \mathrm{Co}$ are compiled in Table 6.1. Overall, this coincides well with the expected EPR spectrum of the mixed-valent complex $5^{1 / I I}$, as the reduction of one $\mathrm{d}^{9} \mathrm{Cu}^{\mathrm{II}}$ to a $\mathrm{d}^{10} \mathrm{Cu}^{\mathrm{I}}$ makes it diamagnetic and thus EPR silent, leaving just one active $\mathrm{Cu}^{\mathrm{II}}$ center.
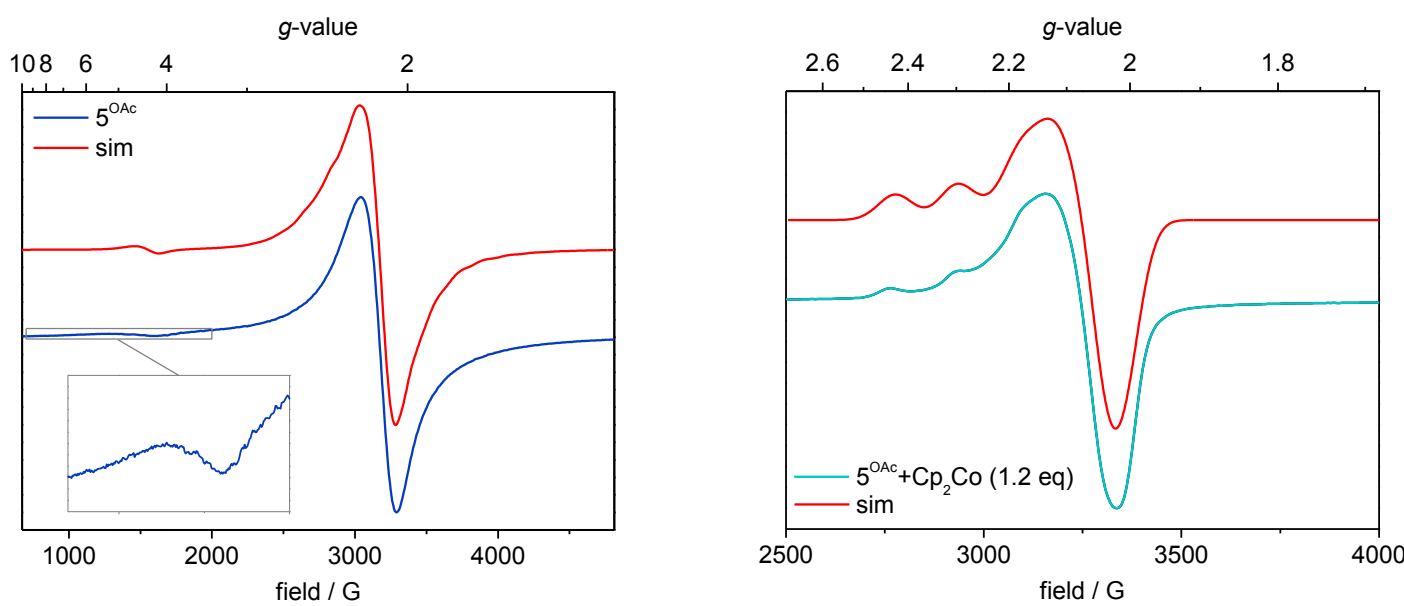

Figure 6.4: Frozen solution X-band EPR spectra of $5^{\text {OAc }}$ before (blue, left) and after (light blue, right) addition of $\mathrm{Cp}_{2} \mathrm{Co}(1.2 \mathrm{eq})$ in $\mathrm{MeCN}$ at $150 \mathrm{~K}$ under inert conditions, including the respective simulations.

Table 6.1: EPR parameters for $\mathbf{5}^{\mathrm{AAc}}$ before and after addition of $\mathrm{Cp}_{2} \mathrm{Co}(1.2 \mathrm{eq})$.

\begin{tabular}{lll} 
& $\mathbf{5}^{\mathbf{O A c}}$ & $\mathbf{5}^{\mathbf{O A c}}+\mathrm{Cp}_{2} \mathrm{Co}(1.2 \mathrm{eq})$ \\
\hline \hline$g_{1}$ & 2.03 & 2.05 \\
$g_{2}$ & 2.11 & 2.10 \\
$g_{3}$ & 2.25 & 2.24 \\
$g_{\text {average }}$ & 2.13 & 2.13 \\
$A_{1} / \mathrm{MHz}$ & - & 94 \\
$A_{2} / \mathrm{MHz}$ & - & 50 \\
$A_{3} / \mathrm{MHz}$ & - & 480 \\
linewidth $l w_{1} / \mathrm{G}$ & 94 & 100 \\
$l w_{2} / \mathrm{G}$ & 82 & 100 \\
$l w_{3} / \mathrm{G}$ & 117 & 100 \\
zero-field splitting $D / \mathrm{cm}^{-1}$ & -0.03 & - \\
\hline
\end{tabular}

\subsection{Reduction of $6^{\text {OAc }}$ to a Dicopper(I) Complex}

In order to determine if the occurrence of a mixed-valent species is solely an effect of the acetate in the bimetallic pocket, the same reduction was performed with the related complex $\mathbf{6}^{\mathbf{O A c}}$ with isopropyl 
instead of methyl residues at the tacn macrocycles. As shown in Figure 6.5 (left), titration of $\mathbf{6}^{\mathbf{0 A c}}$ with $\mathrm{Cp}_{2}$ Co leads to a decrease of the $\mathrm{d}-\mathrm{d}$ transition $(731 \mathrm{~nm})$ and the formation of new bands at 400 and $508 \mathrm{~nm}$ in the UV/vis spectrum. In contrast to the titration starting from $\mathbf{5}^{\mathbf{0 A c}}$, however, an isosbestic point is observed at $378 \mathrm{~nm}$ for the complete addition of $2.3 \mathrm{eq} \mathrm{Cp}_{2} \mathrm{Co}$, corresponding to the twofold reduction of $6^{\mathbf{O A c}}$ to $\mathbf{6}^{\mathbf{1} / \mathbf{I}}$ (Scheme 6.3). This is emphasized by the absorbance trace of the isosbestic point, which remains stable up until overtitration (Figure 6.5, right). The direct formation of a dicopper(I) complex was also confirmed by addition of $\mathrm{O}_{2}$, leading to a peroxo species (see Section 6.3.2).

In line with this, it seems that both the acetate molecule in the bimetallic cleft, as well as the methyl

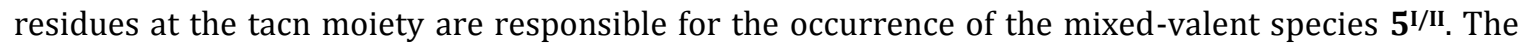
bulkier isopropyl residues in $\mathbf{6}^{\mathbf{0 A c}}$ might prevent the acetate molecule from changing its coordination mode from bridging to chelating, thus impeding the formation of a mixed-valent species. To summarize, the reduction process of $6^{\mathbf{0 A c}}$ can be stated as follows:

$$
\left[\mathrm{Cu}^{\mathrm{II}} \mathrm{Cu}^{\mathrm{II}}\right] \stackrel{\mathrm{e}^{-}}{\rightarrow} 0.5\left[\mathrm{Cu}^{\mathrm{I}} \mathrm{Cu}^{\mathrm{I}}\right]+0.5\left[\mathrm{Cu}^{\mathrm{II}} \mathrm{Cu}^{\mathrm{II}}\right] \stackrel{\mathrm{e}^{-}}{\rightarrow}\left[\mathrm{Cu}^{\mathrm{I}} \mathrm{Cu}^{\mathrm{I}}\right]
$$

By contrast, the reduction of $\mathbf{5}^{\mathbf{0 A c}}$ occurs as two consecutive processes:

$$
\left[\mathrm{Cu}^{\mathrm{II}} \mathrm{Cu}^{\mathrm{II}}\right] \stackrel{\mathrm{e}^{-}}{\rightarrow}\left[\mathrm{Cu}^{\mathrm{I}} \mathrm{Cu}^{\mathrm{II}}\right] \stackrel{\mathrm{e}^{-}}{\rightarrow}\left[\mathrm{Cu}^{\mathrm{I}} \mathrm{Cu}^{\mathrm{I}}\right]
$$
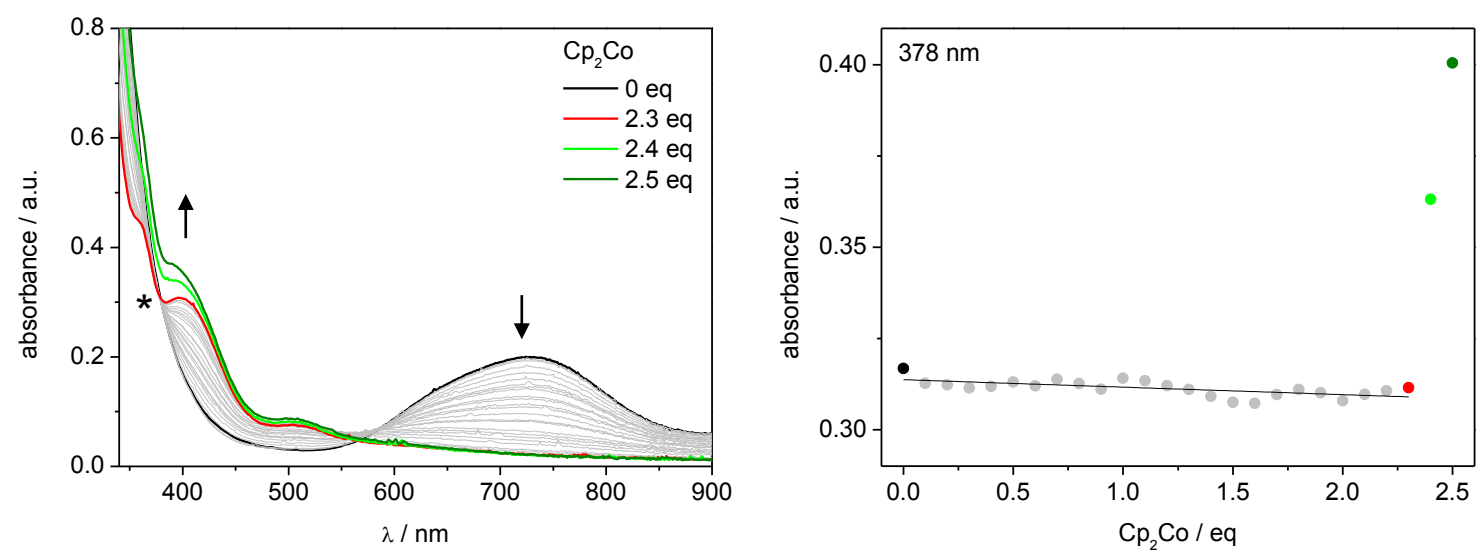

Figure 6.5: UV/vis titration of $6^{\mathbf{O A c}}$ with $\mathrm{Cp}_{2} \mathrm{Co}$ in $\mathrm{MeCN}$ at room temperature under inert conditions. Left: UV/vis spectra of the addition of 2.5 eq $\mathrm{Cp}_{2} \mathrm{Co}$ in 0.1 eq steps. The reduction is complete after addition of 2.3 eq $\mathrm{Cp}_{2} \mathrm{Co}$. Right: Trace of the isosbestic point for the twofold reduction at $378 \mathrm{~nm}$.
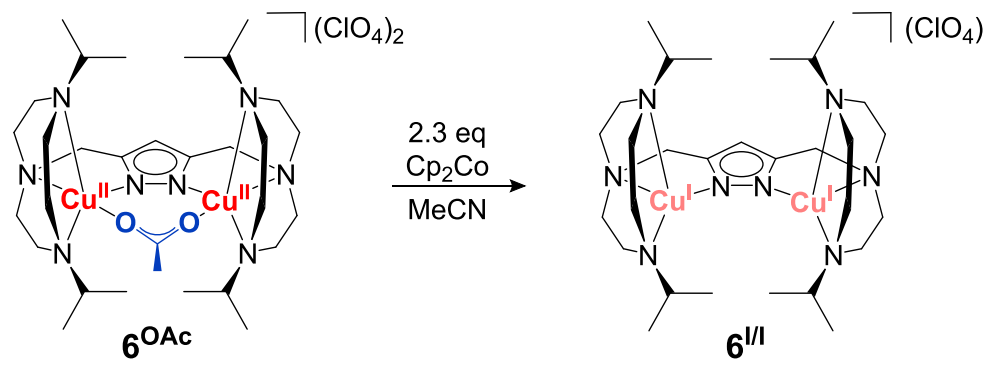

Scheme 6.3: Twofold reduction of $6^{\mathrm{OAc}}$ to the $\mathrm{Cu}^{\mathrm{I}} \mathrm{Cu}^{\mathrm{I}}$ species $6^{\mathrm{I} / \mathrm{I}}$.

\subsection{Dioxygen Activation at Dicopper Complexes}

In the typical dioxygen activation experiments, dry molecular oxygen is added to a dicopper(I) compound, leading to the formation of a peroxo complex, which can be converted to a superoxo or hydroperoxo moiety by oxidation or protonation, respectively. The occurrence of the $\mathrm{Cu}^{\mathrm{I} C \mathrm{Cu}^{\mathrm{II}}}$ species 
$\mathbf{5}^{\mathbf{I} / \mathrm{II}}$ should, however, allow the direct formation of the superoxo species $\mathbf{5}^{\mathbf{s}}$, as the $\mathrm{O}_{2}$ molecule can only be reduced once by the dicopper core. Consequently, depending on the starting material, $\mathbf{5}^{\mathbf{I} / \mathbf{I}}$ or $\mathbf{5}^{\mathbf{I} / \mathbf{I I}}$, different primary products of dioxygen activation can be expected (Scheme 6.4).

Preliminary investigations of the $\mathrm{O}_{2}$ activation by the dicopper(I) compound $\mathbf{5}^{\mathbf{I} / \mathbf{I}}$ were attempted by Dr. Alexander Brinkmeier and monitored via UV/vis spectroscopy. ${ }^{[188]}$ The motive for investigating the $\mathrm{Me}_{2}$-tacn complexes is their expected higher activity for substrate oxygenation than the $i \mathrm{Pr}_{2}$-tacn compounds, as the dioxygen derived ligand in the bimetallic cleft is assumed to be more accessible with less bulky residues. ${ }^{\text {xxviii }}$ Although highly active species are favorable for reactivity studies, tracking and isolating the possible reaction intermediates is significantly more challenging. In the following, the dioxygen activation at both the dicopper(I) and mixed-valent complexes will be discussed. For all experiments, $\mathbf{5}^{\mathbf{I} / \mathbf{I}}$ and $\mathbf{5}^{\mathbf{I} / \mathrm{II}}$ are formed in situ either chemically (addition of 2.3 eq or $1.2 \mathrm{eq} \mathrm{Cp}_{2} \mathrm{Co}$, respectively) or electrochemically.

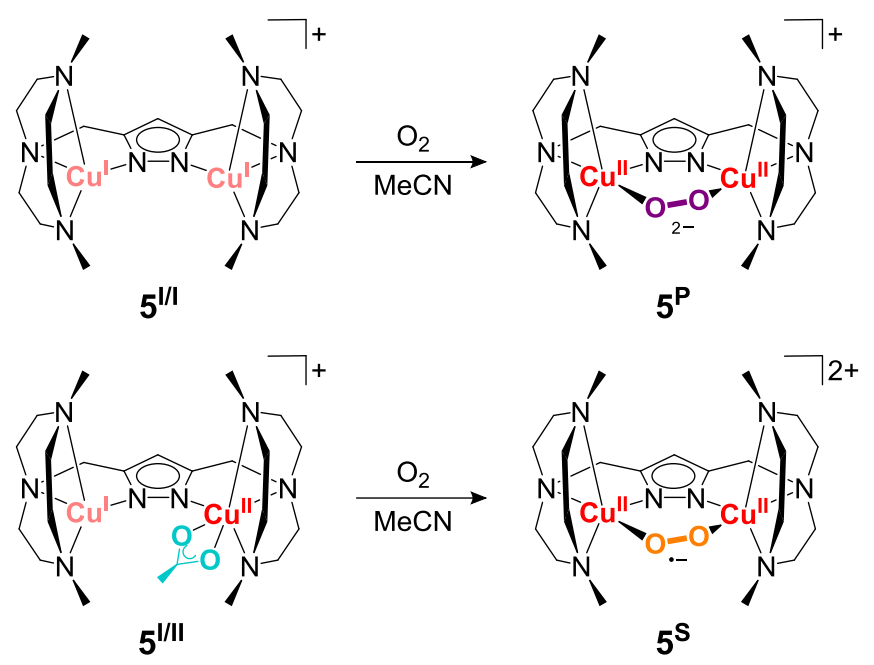

Scheme 6.4: Dioxygen activation at $\mathbf{5}^{\mathbf{I} / \mathbf{I}}$ (top) and $\mathbf{5}^{\mathbf{I} / \mathrm{II}}$ (bottom) to the expected peroxo $\left(\mathbf{5}^{\mathrm{P}}\right)$ and superoxo $\left(\mathbf{5}^{\mathbf{S}}\right)$ complexes, respectively.

\subsubsection{Starting from $\mathrm{Cu}^{\mathrm{I}} \mathrm{Cu}^{\mathrm{I}}$}

Exposing an acetonitrile solution of $\mathbf{5}^{\mathbf{I} / \mathbf{I}}$, formed in situ by addition of cobaltocene (2.3 eq) to $\mathbf{5}^{\mathbf{0 A c}}$, to dry dioxygen at $-20^{\circ} \mathrm{C}$ instantly led to a color change from yellow (cobaltocenium) to purple. This was accompanied by the formation of two new bands at 510 and $630 \mathrm{~nm}$ in the UV/vis spectrum (Figure 6.6, top left), which can be assigned as LMCT transitions and suggest a $\mu-1,2$ binding fashion of the peroxo moiety in the bimetallic cleft. ${ }^{[147,167,386,387]}$ The peroxo complex $\mathbf{5}^{\mathbf{P}}$ gradually decays, indicated by the disappearance of its typical spectroscopic features, and a compound is formed with a d-d transition at $616 \mathrm{~nm}$ (Figure 6.6, top right). Unfortunately, an exact decay rate could not be distinguished as multiple repetitions gave different rates, with half-life times between 30 and 80 minutes at $0{ }^{\circ} \mathrm{C}$. The ESI $(+)$-MS of the decomposition product showed the typical features of $5^{\mathbf{O A c} \text { : }}$ the molecular ion peak $[\mathrm{M}]^{2+}$ and $\left[\mathrm{M}+\mathrm{ClO}_{4}\right]^{+}$at $\mathrm{m} / \mathrm{z} 295.2$ and 691.2, respectively. An additional peak was observed at $m / z 649.3$, which was assigned to $\left[\mathrm{L}^{2 \mathrm{aa}} \mathrm{Cu}_{2}(\mathrm{OAc})_{2}\right]^{+}$(appendix, Figure A.19). Consequently, the decomposition product seems to be a dicopper(II) complex with at least one acetate ligand.

In order to investigate if any short-lived species are formed upon dioxygen activation at $\mathbf{5}^{\mathbf{1} / \mathbf{I}}$, timeresolved UV/vis spectra were collected with a stopped-flow instrument. As expected, upon rapid

xxviii $\mathrm{A}$ large difference in the accessibility of the bridging moiety is already achieved by changing from an ethylene to a methylene linker between the pyrazolate and the tacn moiety. 
mixing of an acetonitrile solution of $\mathbf{5}^{\mathbf{0 A c}}$ and $\mathrm{Cp}{ }_{2} \mathrm{Co}(2.3 \mathrm{eq})$ with $\mathrm{O}_{2}$ saturated $\mathrm{MeCN}$ at $-40{ }^{\circ} \mathrm{C}$, only the typical bands of the peroxo complex $\mathbf{5}^{\mathbf{P}}$ appear (Figure 6.6 , bottom left). The formation of $\mathbf{5}^{\mathbf{P}}$ is complete after around ten seconds, as can be seen from the temporal traces at 505 and $605 \mathrm{~nm}$ (Figure 6.6, bottom right). The low intensity of the UV/vis bands, equivalent to a low concentration of $5^{P}$ in solution, could be explained by: (i) ineffective reduction of $\mathbf{5}^{\mathbf{O A c}}$ to $\mathbf{5}^{\mathbf{I} / \mathbf{I}}$ or (ii) incomplete conversion of $\mathbf{5}^{\mathbf{I} / \mathbf{I}}$ to $\mathbf{5}^{\mathbf{P}}$ in the presence of $\mathrm{O}_{2}$. As will be seen in the following Section, dioxygen activation at $\mathbf{5}^{\mathbf{I} / \mathrm{II}}$ proved more effective. Consequently, it is assumed that the larger amount of cobaltocene/cobaltocenium in solution negatively effects the $\mathrm{O}_{2}$ activation.

Multiple attempts were made to convert $\mathbf{5}^{\mathbf{P}}$ to the superoxo or hydroperoxo species by addition of ferrocenium as oxidant or lutidinium as proton donor, respectively. Unfortunately, at no point spectroscopic features of either of the two other dioxygen activated complexes could be observed. Instead, a rapid decay of $\mathbf{5}^{\mathbf{P}}$ took place.
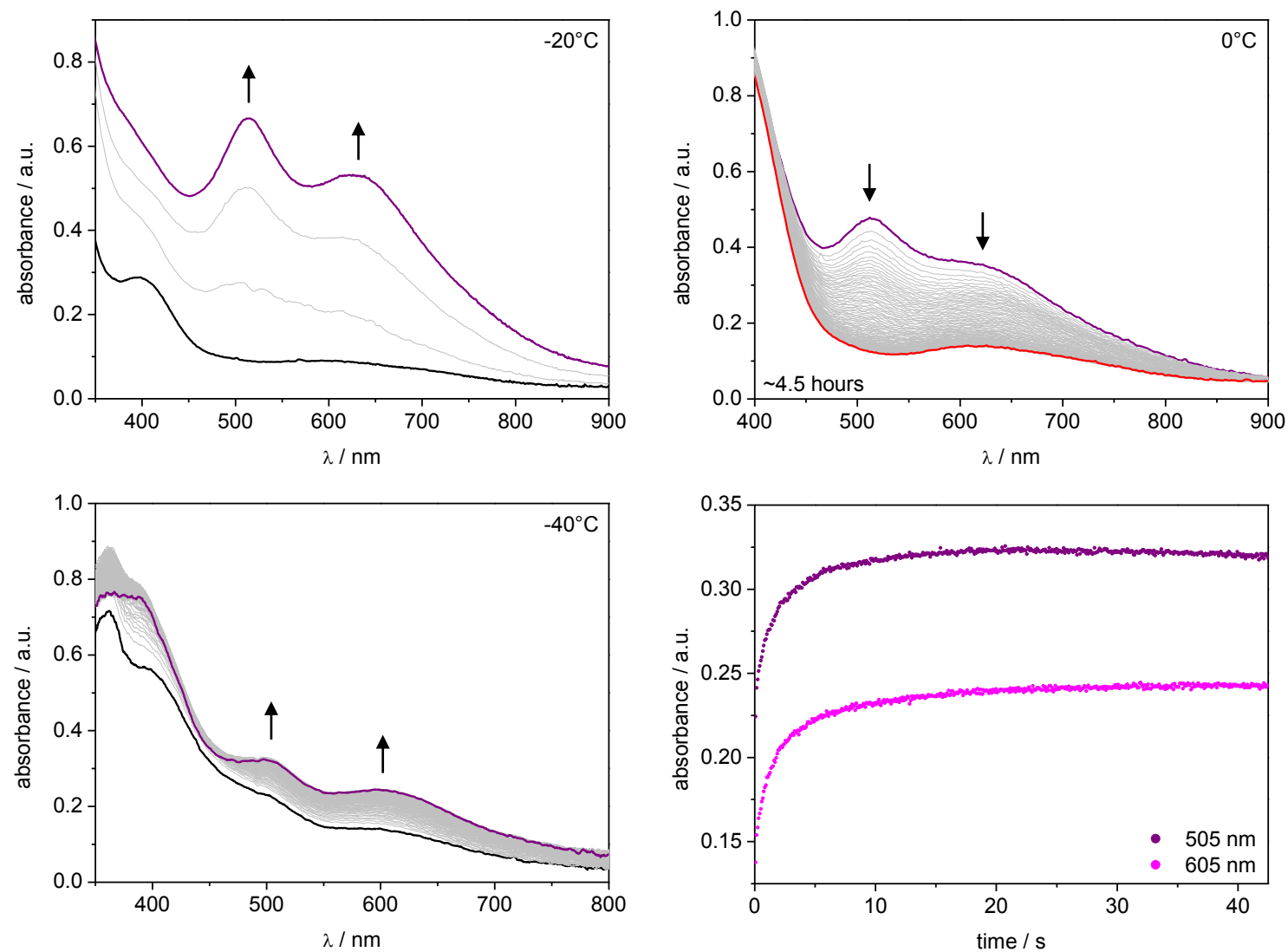

Figure 6.6: Monitoring dioxygen activation at 5i/l via UV/vis spectroscopy in MeCN under inert conditions. Top left: UV/vis spectra of the addition of dry $\mathrm{O}_{2}$ to $5^{\mathbf{I} / \mathbf{I}}$ at $-20^{\circ} \mathrm{C}$ (initial: black, final: purple). Top right: Decay of the peroxo species $5^{\mathbf{P}}$ at $0^{\circ} \mathrm{C}$ over $\sim 4.5$ hours (initial; purple, final: red). Bottom: Stopped-flow UV/vis spectra of the addition of $\mathrm{O}_{2}$ to $5^{\mathrm{I} / \mathrm{I}}$ at $-40^{\circ} \mathrm{C}$ (initial: black, final: purple) and the corresponding time traces at 505 and $605 \mathrm{~nm}$. In all experiments, the initial concentration of $\mathbf{5}^{\mathbf{0 A c}}$ was $0.5 \mathrm{mM}$.

Next to the UV/vis spectroscopic analysis, dioxygen activation by $\mathbf{5}^{\mathbf{I} / \mathbf{I}}$ was studied with electrochemical techniques. All potentials below are given $v s$. the ferrocenium $/$ ferrocene $\left(\mathrm{Fc}^{+} / 0\right)$ redox couple.

As discussed in Chapter 4 (Section 4.3.1), the cyclic voltammogram of $\mathbf{5}^{\mathrm{OAc}}$ in $\mathrm{MeCN}\left(0.1 \mathrm{M} \mathrm{NBu}_{4} \mathrm{PF}_{6}\right)$ under inert conditions exhibits a quasi-reversible redox process at $-1.07 \mathrm{~V}$ (here $-0.93 \mathrm{~V}$ ), , xix which was tentatively assigned to the twofold reduction from $\mathrm{Cu}^{\mathrm{II}} \mathrm{Cu}^{\mathrm{II}}$ to $\mathrm{Cu}^{\mathrm{I} C \mathrm{Cu}^{\mathrm{I}}}$ based on UV/vis-SEC

xxix The redox potential is shifted to more positive potentials in the herein described electrochemical experiment, presumably due to the different experimental conditions. 
investigations. Bearing this in mind, in the presence of $\mathrm{O}_{2}$, the electrochemically formed $\mathbf{5}^{\mathbf{1} / \mathbf{I}}$ should convert to the peroxo complex $\mathbf{5}^{\mathbf{P}}$. The latter is expected to exhibit an electrochemical feature corresponding to the $\mathrm{O}_{2}{ }^{2-} / \mathrm{O}_{2}{ }^{--}$redox couple. Indeed, when a $\mathrm{CV}$ is measured under an $\mathrm{O}_{2}$ atmosphere at $-45^{\circ} \mathrm{C}$ starting at significantly low potentials, a new quasi-reversible redox process appears at $-0.55 \mathrm{~V}$ (Figure 6.7, top left). The occurrence and current of this process strongly depends on the potential at which the measurement is started: with an initial potential of $-0.83 \mathrm{~V}$, no electrochemical feature is observed, starting at $-0.93 \mathrm{~V}$ reveals the peroxo/superoxo redox couple, and its intensity is significantly increased when measuring from $-1.03 \mathrm{~V}$ (Figure 6.7, top right). Over the course of multiple CV cycles, the current of the redox waves significantly decreases (Figure 6.7, bottom left). This is likely due to the severe instability of the electrochemically generated superoxo complex $\mathbf{5}$ s, even at $-45^{\circ} \mathrm{C}$. The processes occurring during the electrochemical analysis are summarized in Scheme 6.5.
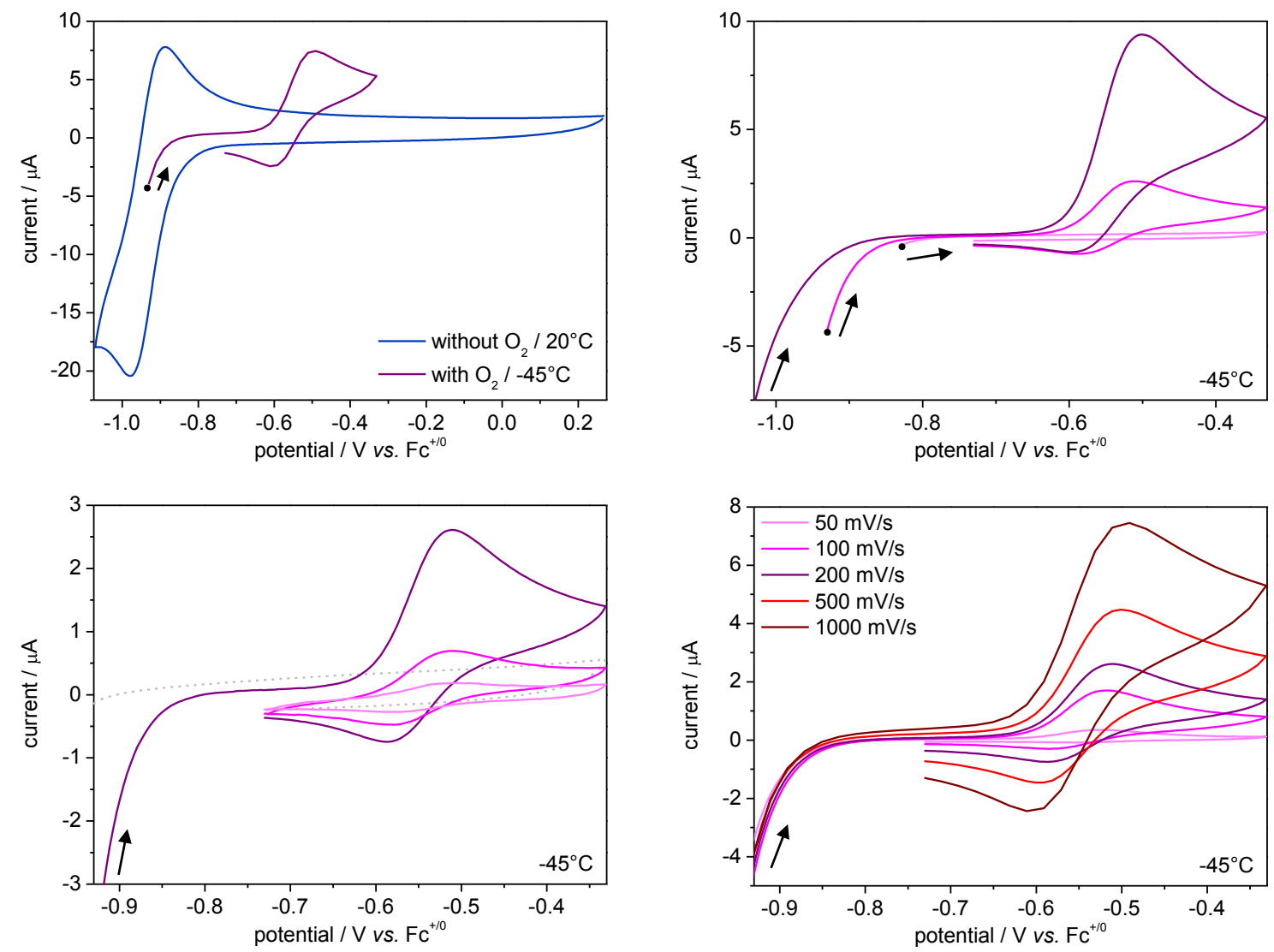

Figure 6.7: Electrochemical analysis of $\mathbf{5}^{\mathrm{OAc}}(3 \mathrm{mM})$ in the presence of $\mathrm{O}_{2}$ in dry $\mathrm{MeCN}\left(0.1 \mathrm{M} \mathrm{NBu}_{4} \mathrm{PF}_{6}\right)$ at $-45^{\circ} \mathrm{C}$. Top left: $\mathrm{CV}$ with (blue) and without (purple) $\mathrm{O}_{2}$ at different temperatures and scan rates. Top right: $\mathrm{CV}(200 \mathrm{mV} / \mathrm{s})$ starting at various reductive potentials $\left(-1.03 /-0.93 /-0.83 \mathrm{~V} v s . \mathrm{Fc}^{+/ 0}\right.$ : from dark to light purple). Bottom left: Multiple CV cycles at $200 \mathrm{mV} / \mathrm{s}$ (scan 1-3: dark to light purple). Bottom right: Scan rate dependence. The scanning direction is indicated by an arrow. A glassy carbon disc was used as the working electrode, a Pt disc as auxiliary and an $\mathrm{Ag}$ wire as the reference electrode. Ferrocene was used as an internal standard.

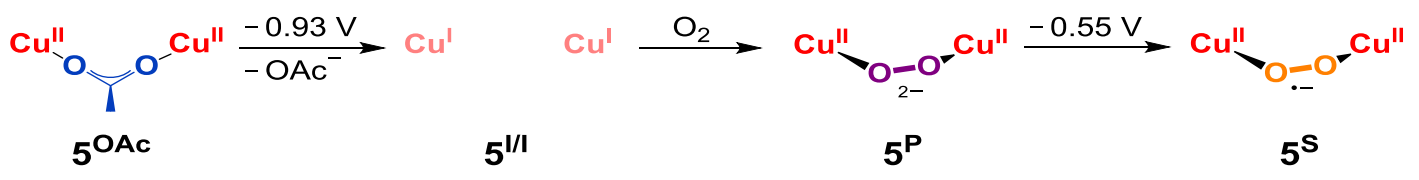

Scheme 6.5: Representation of the processes occurring during the electrochemical reduction and subsequent oxidation of $5^{\text {OAc }}$ under $\mathrm{O}_{2}$ atmosphere. 


\subsubsection{Starting from $\mathrm{Cu}^{\mathrm{I}} \mathrm{Cu}^{\mathrm{II}}$}

Addition of dry dioxygen at $-40^{\circ} \mathrm{C}$ to an acetonitrile solution of $\mathbf{5}^{\mathbf{I} / I I}$, formed in situ by addition of cobaltocene (1.2 eq) to $\mathbf{5}^{\mathbf{O A c}}$, did not, as expected, reveal the spectroscopic features of a superoxo complex. Instead, the formation of bands at 510 and $630 \mathrm{~nm}$ were observed in the UV/vis spectrum corresponding to the peroxo species $\mathbf{5}^{\mathbf{P}}$ (Figure 6.8, top; time range: 78 seconds). To further investigate this, time-resolved UV/vis spectra were collected with a stopped-flow instrument. Upon rapid mixing of an acetonitrile solution of $\mathbf{5}^{\mathrm{OAc}}$ and $\mathrm{Cp}_{2} \mathrm{Co}(1.2 \mathrm{eq})$ with $\mathrm{O}_{2}$ saturated $\mathrm{MeCN}$ at $-40{ }^{\circ} \mathrm{C}$, a short-lived species was observed with an intense band at $430 \mathrm{~nm}$ (first scan after $1.4 \mathrm{~ms}$ ), which rapidly converted to the peroxo complex $\mathbf{5}^{\mathrm{P}}$ (Figure 6.8, bottom left). The band at $430 \mathrm{~nm}$ can be attributed to an LMCT transition and is typical of dicopper(II) superoxo complexes, ${ }^{[187-190]}$ indicating that the short-lived species is $\mathbf{5}^{\mathbf{S}}$. Within 0.1 seconds (Figure 6.8 , bottom right), $\mathbf{5}^{\mathbf{S}}$ is transformed into $\mathbf{5}^{\mathbf{P}}$ suggesting one of two possibilities: (i) two $\mathbf{5}^{\mathbf{s}}$ molecules react to one peroxo species, a dicopper(II) complex and $\mathrm{O}_{2}$ or (ii) a second $\mathbf{5}^{\mathbf{I} / \mathrm{II}}$ complex provides the second reducing equivalent for the superoxo moiety (Scheme 6.6). In both cases, the products (simply speaking, $\mathbf{5}^{\mathbf{P}}$ and $\mathbf{5}^{\mathbf{O A c}}$ ) are the same, however, the former seems more likely as the first spectrum from the stopped-flow measurement exhibits $\mathbf{5}^{\mathbf{s}}$ in full intensity, suggesting that dioxygen activation at $\mathbf{5}^{\mathbf{I} / \mathrm{II}}$ to form $\mathbf{5}^{\mathbf{S}}$ is instantaneous.
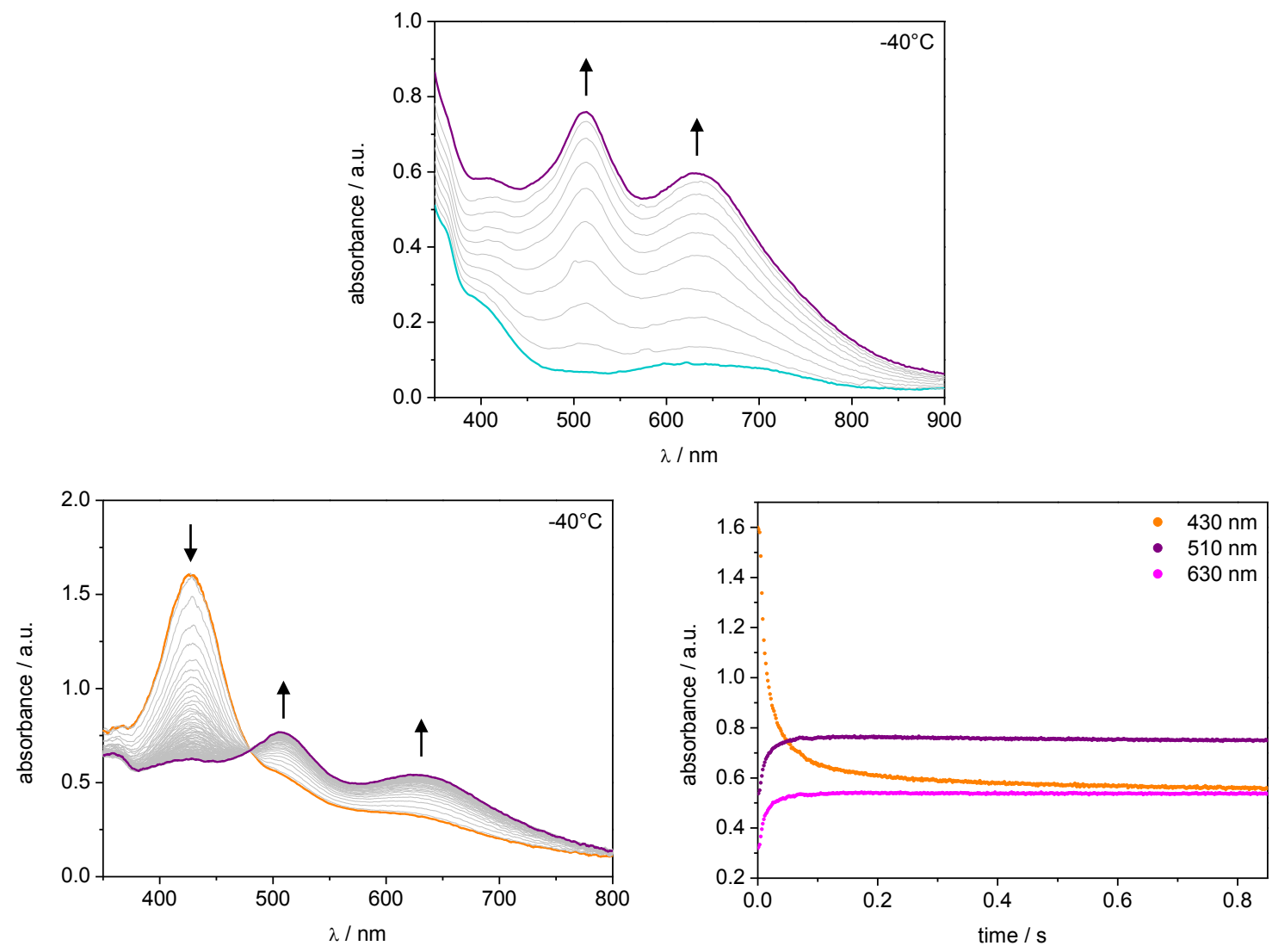

Figure 6.8: Monitoring dioxygen activation at 5//II via UV/vis spectroscopy in $\mathrm{MeCN}$ at $-40{ }^{\circ} \mathrm{C}$ under inert conditions. Top: UV/vis spectra of the addition of dry $\mathrm{O}_{2}$ to $\mathbf{5}^{\mathbf{I} / \mathbf{I I}}$ (initial: light blue, final: purple, time range: 78 seconds). Bottom: Stopped-flow UV/vis spectra of the addition of $\mathrm{O}_{2}$ to $\mathbf{5}^{\mathbf{1} / \mathrm{II}}$ (initial: orange, final: purple) and the corresponding traces at 430, 510 and $630 \mathrm{~nm}$. The orange UV/vis spectrum exhibits the typical features of a superoxo complex. In both experiments, the initial concentration of $\mathbf{5}^{\mathbf{0 A c}}$ was $0.5 \mathrm{mM}$. 


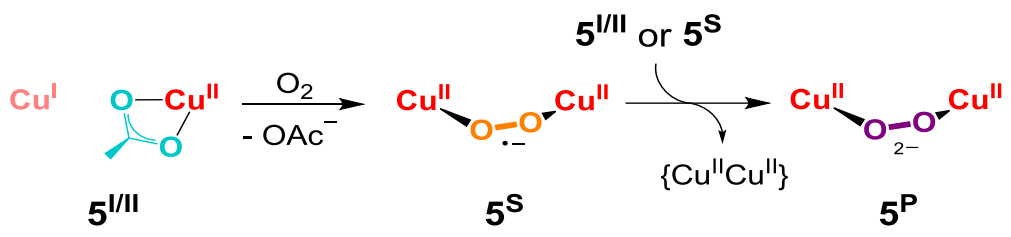

Scheme 6.6: Representation of the processes occurring during dioxygen activation at $\mathbf{5}^{\mathbf{I} / \mathrm{II}}$.

As proposed in Scheme 6.6, the formation of $\mathbf{5}^{\mathbf{P}}$ should be second-order in complex concentration. Consequently, concentration dependent stopped-flow UV/vis experiments were conducted and the initial rate of $\mathbf{5}^{\mathbf{P}}$ generation was distinguished from the temporal progress of the band at $510 \mathrm{~nm}$. Up to a complex concentration of $0.5 \mathrm{mM}$, a constant increase in the initial rate is observed, after which it seems to remain stable or even slightly decrease (Figure 6.9). A reason for this might be the amount of dissolved $\mathrm{O}_{2}$ in $\mathrm{MeCN}\left((8.1 \pm 0.6) \mathrm{mM}\right.$ at $\left.25^{\circ} \mathrm{C}\right),{ }^{[388]}$ which likely becomes a limiting factor at higher complex concentrations. Plotting the rates versus the simple (Figure 6.9, left) and squared (right) complex concentration and fitting the data up to $0.5 \mathrm{mM}$ reveals $\mathrm{R}^{2}$ values of 92 and $95 \%$, respectively. The good agreement for both plots is likely due to the large distribution of rates obtained during multiple repetitions at each concentration. As a result, the pathway for the formation of $\mathbf{5}^{\mathbf{P}}$ remains unclear. The decay of the superoxo complex $\mathbf{5}^{\mathbf{s}}(430 \mathrm{~nm})$ was analyzed in a similar manner, yielding equally inconclusive results.
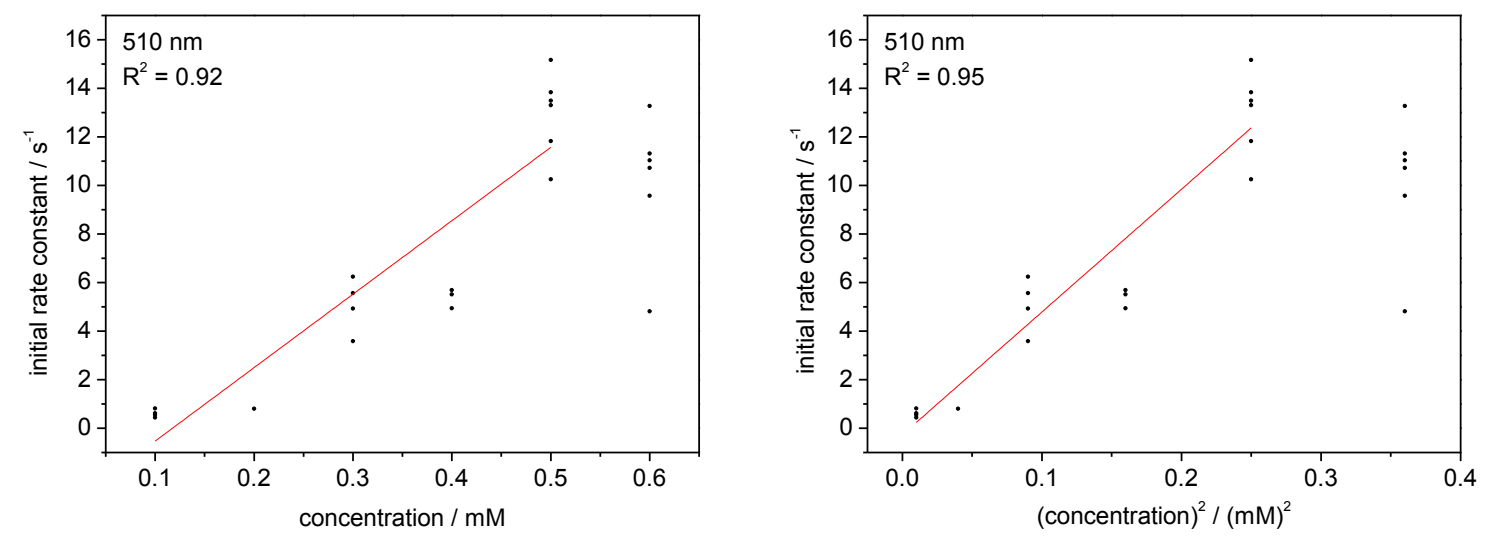

Figure 6.9: Concentration dependence of the formation of the peroxo complex $\mathbf{5}^{\mathrm{P}}$ by addition of dioxygen to $\mathbf{5}^{\mathrm{I} / \mathrm{II}}$ in $\mathrm{MeCN}$ at $-40{ }^{\circ} \mathrm{C}$. The initial rate constant is plotted $v$ s. the simple (left, $\mathrm{R}^{2}=92 \%$ ) and squared (right, $\mathrm{R}^{2}=95 \%$ ) concentration of $\mathbf{5}^{\mathbf{I} / \mathrm{II}}$. The absorbance trace at $510 \mathrm{~nm}$ was used for this analysis. No clear information can be withdrawn if the peroxo formation is first- or second-order in complex concentration.

The proposition that two complex molecules eventually generate one peroxo compound, further entails that the maximum concentration of $\mathbf{5}^{\mathbf{P}}$ is $\mathbf{5 0} \%$ of the initial $\mathbf{5}^{\mathbf{A A c}}$. In line with this, the full amount of $\mathbf{5}^{\mathbf{P}}$ in solution should be achieved upon addition of 0.5 eq dioxygen. Indeed, addition of $0.5,1$ eq or an excess of $\mathrm{O}_{2}$ to $5^{1 / I I}$ at $-40^{\circ} \mathrm{C}$ yielded the peroxo complex in very similar concentrations (Figure 6.10, left). On the other hand, reaction of $\mathbf{5}^{\mathbf{I} / \mathbf{I I}}$ with superoxide should provide $\mathbf{5}^{\mathbf{P}}$ in larger amount, as it is already reduced and, thus, can react $1: 1$ with $\mathbf{5}^{\mathbf{1} / \mathrm{II}}$ to form $\mathbf{5}^{\mathbf{P}}$. As expected, addition of an excess $\mathrm{KO}_{2}$ to $5^{\mathrm{I} / \mathrm{II}}$ at $-40^{\circ} \mathrm{C}$ yielded a significantly higher concentration of $\mathbf{5}^{\mathrm{P}}$ in solution, evidenced by the higher intensity in the UV/vis spectrum (Figure 6.10, right). These results strongly support the proposition that two molecules of the starting material $\mathbf{5}^{\mathbf{O A c}}$ are required to generate one molecule $\mathbf{5}^{\mathbf{P}}$ with $\mathrm{O}_{2}$. Furthermore, they indicate that under conditions of low $\mathrm{O}_{2}$ concentration, the second reduction equivalent to generate $5^{P}$ from $5^{S}$ is provided by a $5^{\mathbf{I} / I I}$ complex. 

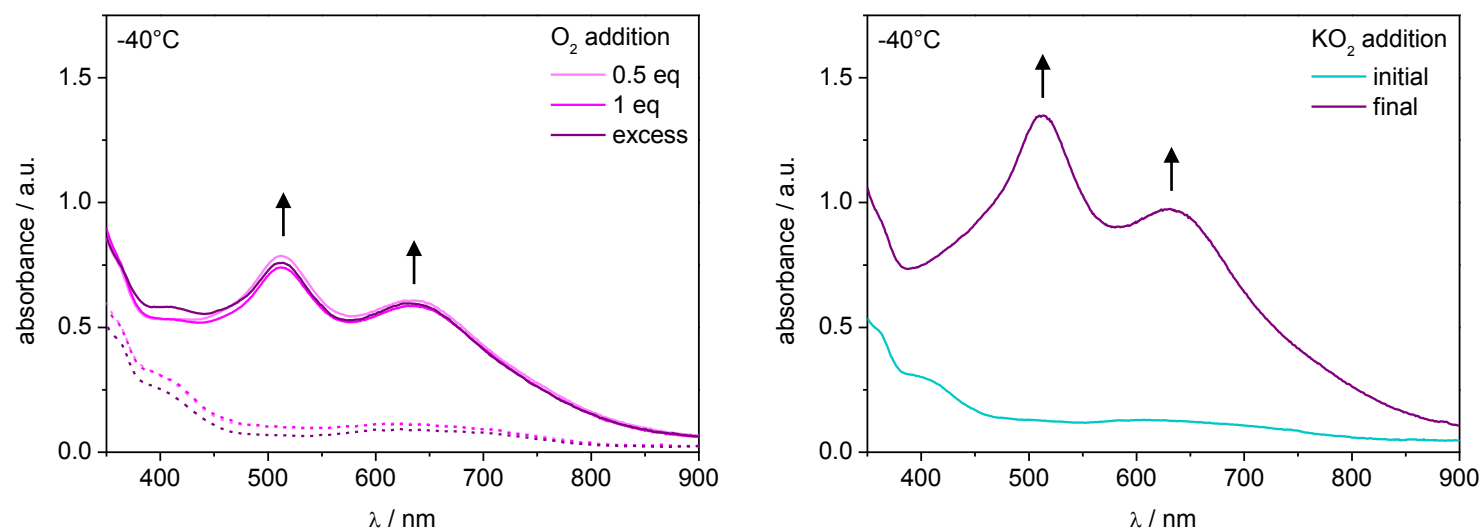

Figure 6.10: UV/vis spectra for the addition of $\mathrm{O}_{2}$ (left; from light to dark: $0.5,1$ eq, excess; initial: dashed, final: solid) and $\mathrm{KO}_{2}$ (right; excess; initial: light blue, final: purple) to $5^{\mathrm{I} / \mathrm{II}}$ in $\mathrm{MeCN}$ at $-40^{\circ} \mathrm{C}$ under inert conditions. In both experiments, the initial concentration of $5^{\mathbf{O A c}}$ was $0.5 \mathrm{mM}$.

In line with the above-mentioned observations, the following scenario is suggested for the formation of the peroxo complex via the mixed-valent species (Scheme 6.7): Addition of $1.2 \mathrm{eq}^{\mathrm{Cp}_{2} \mathrm{Co}}$ to $5^{\mathbf{O A c}}$ leads to the formation of the $\mathrm{Cu}^{\mathrm{I}} \mathrm{Cu}^{\mathrm{II}}$ complex $\mathbf{5}^{\mathrm{I} / \mathrm{II}}$. Subsequent exposure to an atmosphere of dry dioxygen initially yields the short-lived superoxo complex, which rapidly converts to $\mathbf{5}^{\mathbf{P}}$. This conversion can take place via two possible routes: either a second $\mathbf{5}^{\mathbf{s}}$ compound or an unreacted mixedvalent complex act as reductant. Based on the discussed experiments, it seems likely that the former takes place in the presence of large amounts of dioxygen and the latter occurs at low $\mathrm{O}_{2}$ concentrations. In both cases, a dicopper(II) complex is formed as side-product (5 $5^{\text {Ac }}$ balances the equation).

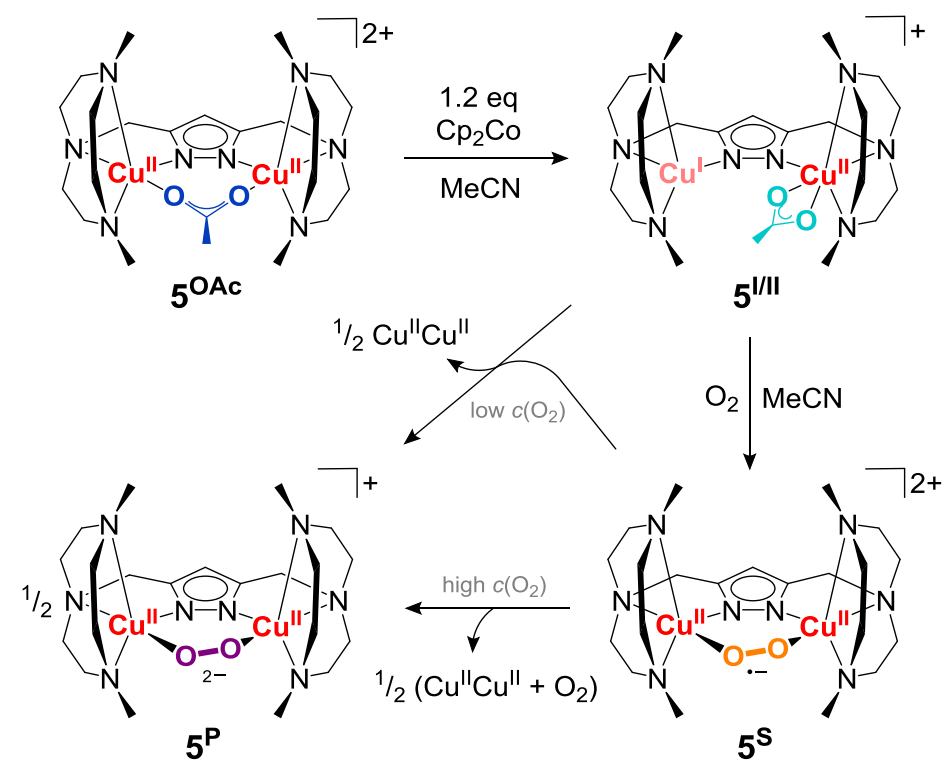

Scheme 6.7: Formation of the peroxo species $5^{\mathbf{P}}$ from $5^{\mathbf{O A c}}$ by reduction and $\mathrm{O}_{2}$ activation via two possible routes.

Several attempts were made to convert $\mathbf{5}^{\mathbf{s}}$ to the hydroperoxo complex by addition of TEMPOH; but remained unsuccessful. Furthermore, preliminary investigations on the effect of co-solvents, such as $\mathrm{MeOH}$ and TFE, on the generation of $\mathbf{5}^{\mathbf{P}}$ were conducted. For this, the co-solvent was added to the $\mathrm{O}_{2}$

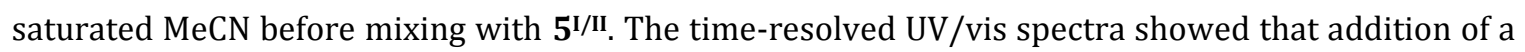
co-solvent traps $\mathbf{5}^{\mathbf{S}}$ and prevents the formation of $\mathbf{5}^{\mathbf{P}}$. Thereby, $\mathbf{5}^{\mathbf{S}}$ proved significantly more stable in the presence of TFE than $\mathrm{MeOH}$. Preliminary experiments were also conducted to investigate the

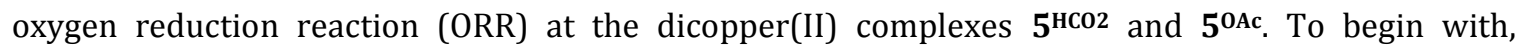


electrochemical techniques were applied to examine their behavior upon cathodic scanning in the presence of $\mathrm{O}_{2}$ both with and without addition of an acid. So far, these studies have been without success, however, before this is put aside, the conditions should be varied.

Lastly, the same dioxygen activation experiments were carried out for the $i \mathrm{Pr}_{2}$-tacn complex $6^{\mathbf{O A c} \text {. }}$ As shown in Figure 6.11 (top), upon addition of $\mathrm{O}_{2}$ to an acetonitrile solution of $\mathbf{6}^{\mathbf{0 A c}}$ and cobaltocene (1.2 eq), new bands arise in the UV/vis spectrum at 527 and $648 \mathrm{~nm}$ with a shoulder at $456 \mathrm{~nm}$ that could be assigned to the known peroxo complex $\mathbf{6}^{\mathrm{P} .}{ }^{[188]}$ Moreover, time-resolved UV/vis spectra negate the presence of any short-lived species (Figure 6.11, bottom). These results validate the direct reduction of $6^{\text {OAc }}$ from $\mathrm{Cu}^{\mathrm{II}} \mathrm{Cu}^{\mathrm{II}}$ to $\mathrm{Cu}^{\mathrm{I}} \mathrm{Cu}^{\mathrm{I}}$ without a mixed-valent species as intermediate. The spectroscopic features of the peroxo and superoxo species of all three dicopper complexes based on pyrazolate/tacn hybrid ligands are compiled in Table 6.2.
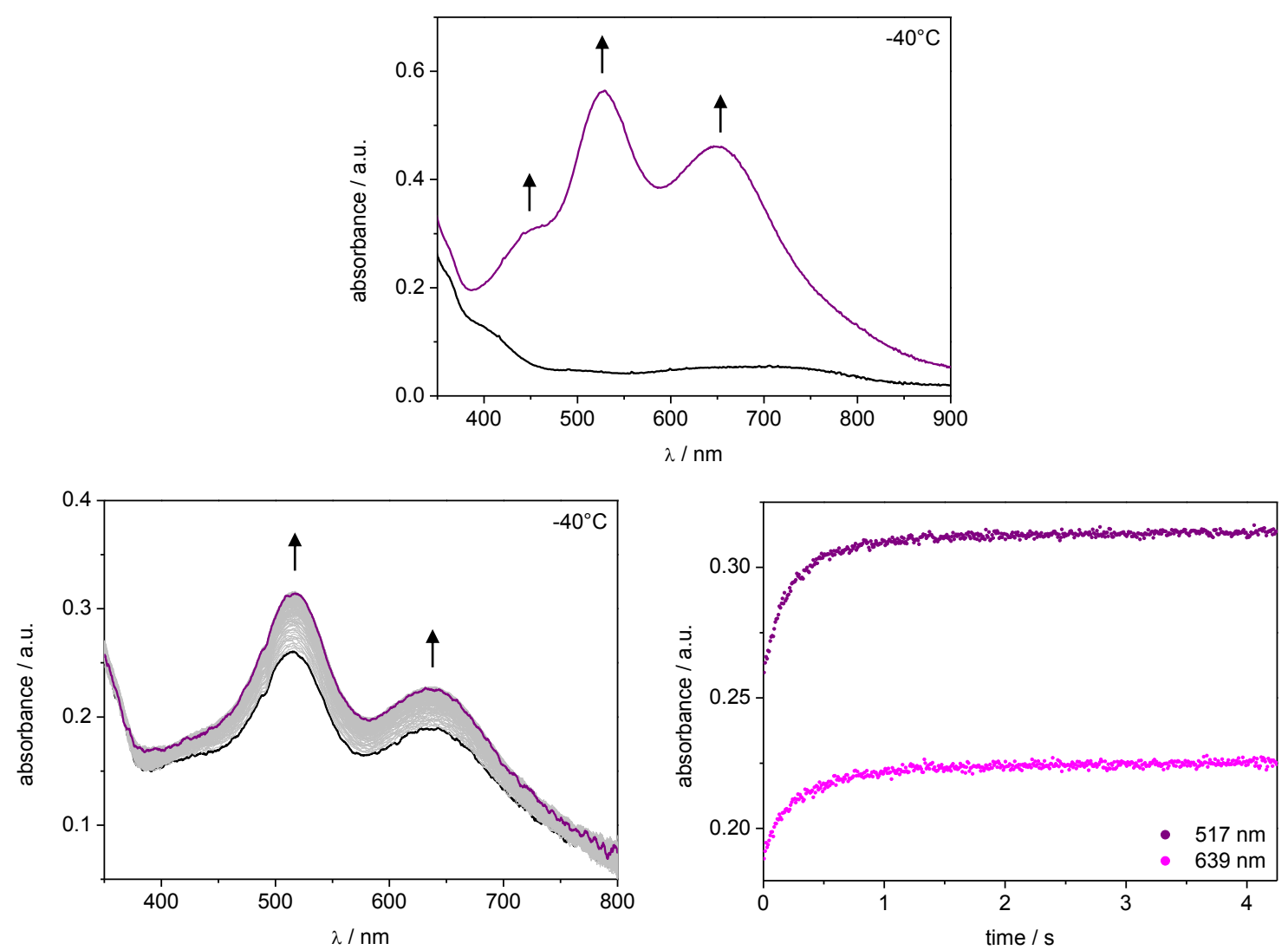

Figure 6.11: Monitoring dioxygen activation of $6^{0 \mathrm{Ac}}+\mathrm{Cp}_{2} \mathrm{Co}(1.2 \mathrm{eq})$ via $\mathrm{UV} / \mathrm{vis}$ spectroscopy in $\mathrm{MeCN}$ at $-40{ }^{\circ} \mathrm{C}$ under inert conditions. Top: UV/vis spectra of the addition of dry $\mathrm{O}_{2}$ to $\mathbf{6}^{\mathbf{O A c}}+\mathrm{Cp}_{2} \mathrm{Co}(1.2 \mathrm{eq})$ (initial: black, final: purple). Bottom: Stopped-flow UV/vis spectra of the addition of $\mathrm{O}_{2}$ to $\mathbf{6}^{\mathbf{O A c}}+\mathrm{Cp}_{2} \mathrm{Co}$ (1.2 eq) (initial: black, final: purple) and the corresponding traces at 517 and $639 \mathrm{~nm}$. In contrast to the Me-tacn complex, no superoxo species is observed. In both experiments, the initial concentration of $\mathbf{5}^{\mathbf{0 A c}}$ was $0.5 \mathrm{mM}$.

Table 6.2: Spectroscopic features of the peroxo and superoxo species of dinuclear copper complexes based on hybrid pyrazolate/tacn ligands.

\begin{tabular}{llll}
$\lambda / \mathrm{nm}$ & $\mathbf{5}$ & $\mathbf{6}^{[188]}$ & $\mathbf{X X V}^{[187,206]}$ \\
\hline \hline peroxo & $510 / 630$ & $456(\mathrm{sh}) / 527 / 648$ & $506 / 600(\mathrm{sh}) / 807$ \\
superoxo & 430 & $444 / 600$ & $440 / 545(\mathrm{sh})$ \\
\hline
\end{tabular}




\subsection{Reduction of $5^{\mathrm{HCO} 2}$ to an Oligonuclear Copper(I) Hydride Complex ${ }^{\mathrm{xxx}}$}

In comparison to $5^{\mathrm{OAc}}$ and $\mathbf{6}^{\mathrm{OAc}}$, the reduction of $\mathbf{5}^{\mathrm{HCO}}$ bore more of a surprise. Addition of decamethylcobaltocene (2.0 eq) or cobaltocene $(2.3 \mathrm{eq})$ to an acetonitrile solution of $\mathbf{5}^{\mathbf{H C O}}$ under inert

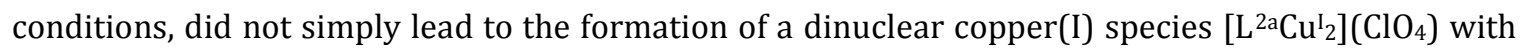
the release of formate, but to a hexanuclear complex with an $\left[\left(\mathrm{L}^{2 \mathrm{a}}\right)_{2} \mathrm{Cu}_{6} \mathrm{I}_{6}\right]$ core (Scheme 6.8). This was not directly visible from UV/vis spectroscopy, but crystals of $9^{\mathbf{H}}$ were obtained by diffusion of Et ${ }_{2} \mathrm{O}$ into the reaction mixture. X-ray diffraction (XRD) described the coordination sphere, however, the quality of the structure analysis was only moderate yielding an unequal charge balance (Figure 6.12, top). By means of ESI-mass spectrometry and NMR spectroscopy, enabled by the diamagnetic character of the $\mathrm{d}^{10} \mathrm{Cu}^{\mathrm{I}}$ ions, the complete constitution of $9^{\mathbf{H}}$ with two additional hydride ligands was determined. Next to a signal corresponding to $\left[\mathrm{Cp}_{2} \mathrm{Co}\right]^{+}(\mathrm{m} / z$ 189.0), a side product from synthesis which proved difficult to separate from $\mathbf{9 H}^{\mathbf{H}}$, the $\mathrm{ESI}(+)-\mathrm{MS}$ in $\mathrm{MeOH}$ exhibits a peak at $m / z 597.0$ whose isotopic pattern matches the molecular ion peak $[\mathrm{M}]^{2+}(\mathrm{M}$ corresponds to the cationic core of the complex including the hydride moieties) (appendix, Figures A.46 and A.47). The ${ }^{1} \mathrm{H}-\mathrm{NMR}$ spectrum of $\mathbf{9 H}^{\mathrm{H}}$ in $\mathrm{CD}_{3} \mathrm{CN}$ at $238 \mathrm{~K}$ (Figure 6.13; for complete NMR characterization see appendix, Figures A.88-A.94) shows 16 signals in the aliphatic region, which is in agreement with an $\left[\left(\mathrm{L}^{2 \mathrm{a}}\right)_{2} \mathrm{Cu}_{6}(\mathrm{H})_{2}\right]^{2+}$ complex of $D_{2}$ or $C_{2 \mathrm{~h}}$ symmetry (four equal quadrants) with diastereotopic $\mathrm{H}$ atoms at each $\mathrm{CH}_{2}$ group. With the help of the pure shift ${ }^{1} \mathrm{H}-\mathrm{NMR}$ spectrum and two-dimensional NMR spectroscopy $\left({ }^{1} \mathrm{H}-{ }^{1} \mathrm{H}-\mathrm{COSY},{ }^{1} \mathrm{H}-{ }^{1} \mathrm{H}-\mathrm{NOESY},{ }^{1} \mathrm{H}-{ }^{13} \mathrm{C}-\mathrm{HSQC}\right.$, ${ }^{1} \mathrm{H}-{ }^{13} \mathrm{C}-\mathrm{HMBC}$ ), all carbon and hydrogen signals could be assigned.xxi The singlet at -3.11 ppm arises from the hydride ligands; this chemical shift is similar to a recently reported trinuclear copper hydride complex based on a naphthyridine ligand reported by Tilley et al. $(\delta=-3.36 \mathrm{ppm}) .{ }^{[389]}$
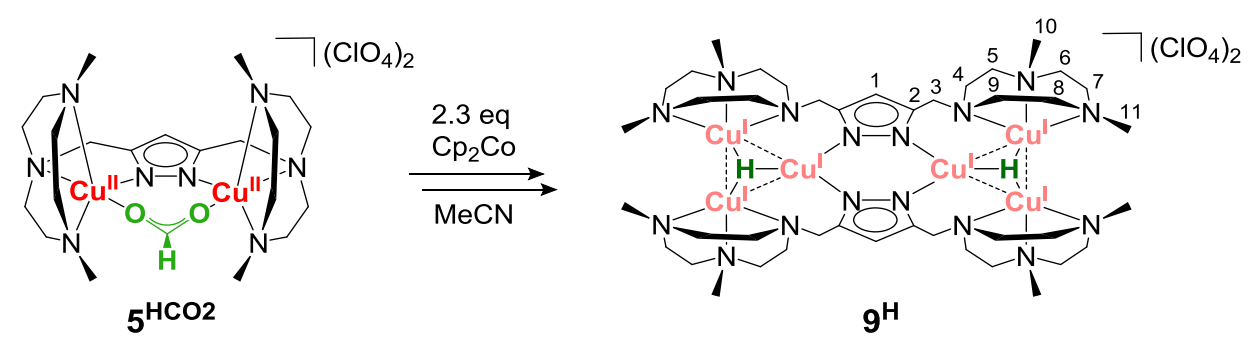

Scheme 6.8: Twofold reduction of $5^{\mathbf{H C O}}$ to the oligonuclear copper(I) hydride $\mathbf{9 H}^{\mathbf{H}}$

In order to validate the structural information extracted from XRD, NMR spectroscopy and ESI mass spectrometry, DFT geometry optimizations were performed by Thomas Auth (University of Göttingen) on the basis of the experimental data (for details see experimental section). Overall, the DFT calculations provided four structural isomers for the cationic core of $\mathbf{9}^{\mathrm{H}}$ that feature two $\mathrm{Cu}^{\mathrm{I}}$ triangles bridged by the pyrazolate groups from two ligand strands (Scheme 6.8) in accord with the XRD data. Each hydride is positioned either above or below the plane of the pyrazolates and is $\mu_{3}$-capping a $\mathrm{Cu}_{3}$ triangle (slightly closer to the outer two $\mathrm{Cu}^{\mathrm{I}}$ ions), a common structural motif in copper hydride chemistry, ${ }^{[390,391]}$ which has also been evidenced in the well-known Stryker/Osborn reagent. ${ }^{[392,393]}$ The four isomers of $9^{\mathbf{H}}$ encompass cis- and trans arrangement of both tacn moieties of (L $\left.{ }^{2 a}\right)^{-}$(prefix) and the hydrides (superscript) relative to the pyrazolate plane in all possible combinations, viz. cis-9 $\mathbf{9}^{\text {cis-H, }}$, cis-9trans-H, trans-9 $\mathbf{9}$ cis-H and trans-9trans-H (Figure 6.12 and Figure 6.14). According to the DFT calculations, cis-9 $\mathbf{9}^{\text {cis-H }}$ has the lowest $G_{298}$ value in MeCN but is not significantly more stable than the cis ${ }^{\text {trans }}$ isomer $\left(0.7 \mathrm{~kJ} \cdot \mathrm{mol}^{-1}\right.$, Table 6.3; this small difference is within the error range of the DFT method), while those with trans arrangement of the tacn $\left(\mathrm{L}^{2 \mathrm{a}}\right)^{-}$coordination are energetically less favorable by 4.7 and

xxx This section was adapted from Jana Lücken, Thomas Auth, Sara Ida Mozzi, Franc Meyer, "Hexanuclear Copper(I) Hydride from the Reduction-Induced Decarboxylation of a Dicopper(II) Formate", Inorg. Chem. 2020, 59, 14347-14354.

xxxi Please note, the distinction between the diastereotopic $H$ atoms of $\left(\mathrm{L}^{2 \mathrm{a}}\right)^{-}$is not possible and is merely done for simplification. 
$6.6 \mathrm{~kJ} \cdot \mathrm{mol}^{-1}$ for trans $\mathbf{- 9}$ cis- $\mathbf{H}$ and trans $\mathbf{- 9}$ trans- $\mathbf{H}$, respectively. A comparison of the point group symmetries of the cis- $\left(\mathrm{L}^{2 \mathrm{a}}\right)^{-}$structures obtained experimentally by XRD $\left(C_{\mathrm{i}}\right)$ and computationally from DFT $\left(C_{\mathrm{s}}\right.$ and $C_{\mathrm{i}}$ for cis-9 $\mathbf{9}^{\text {cis-H }}$ and cis-9trans-H, respectively, also when neglecting the $\mathrm{H}^{-}$ligands) suggests that the obtained crystals likely correspond to the cis $^{\text {trans }}$ isomer (Figure 6.12).

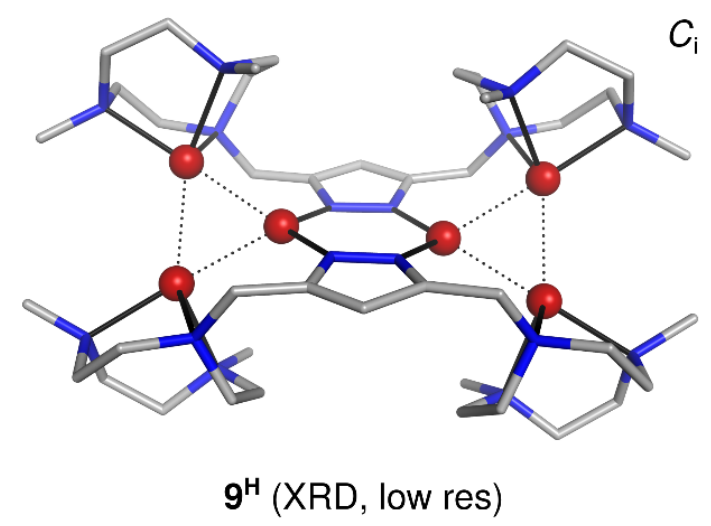

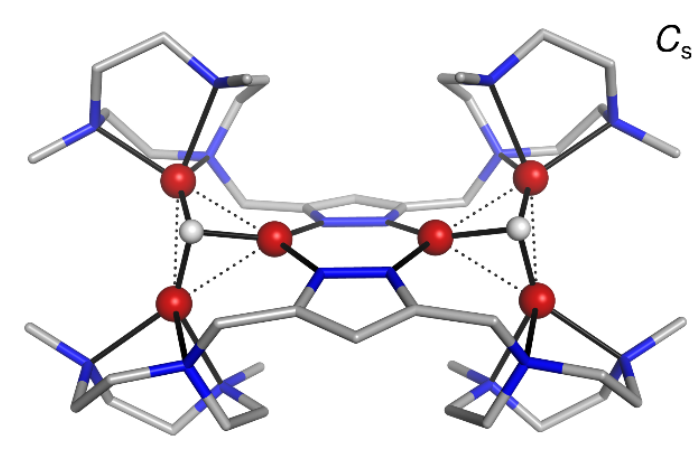

cis- $9^{\text {cis-H }}(\mathrm{DFT})$

$\Delta G_{298}=0.0 \mathrm{~kJ} \mathrm{~mol}^{-1}$

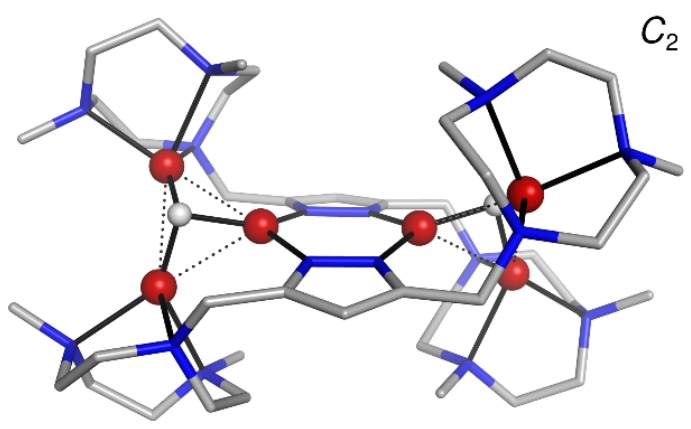

trans-9 $\mathbf{9}^{\text {cis-H }}(\mathrm{DFT})$

$\Delta G_{298}=4.7 \mathrm{~kJ} \mathrm{~mol}^{-1}$

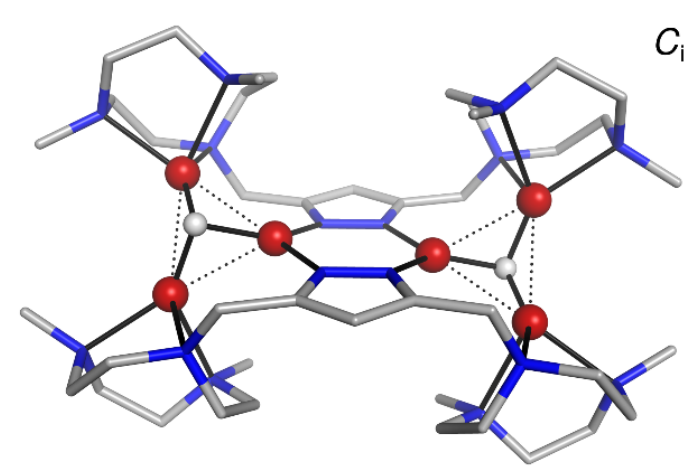

cis $-\mathbf{9}^{\text {trans-H }}$ (DFT)

$\Delta G_{298}=0.7 \mathrm{~kJ} \mathrm{~mol}^{-1}$

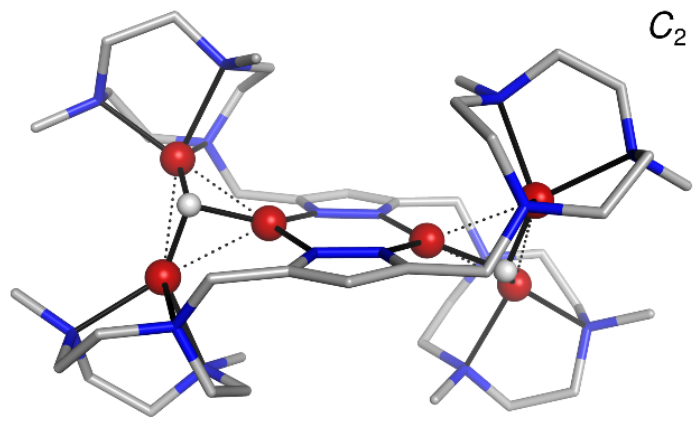

trans $-\mathbf{9}^{\text {trans }-\mathrm{H}}$ (DFT)

$\Delta G_{298}=6.6 \mathrm{~kJ} \mathrm{~mol}^{-1}$

Figure 6.12: Comparison of the molecular structures of the cationic core of $\mathbf{9 H}$ obtained from XRD and DFT. Top: Low-resolution XRD structure. Bottom: Molecular structures and relative Gibbs energies at $298 \mathrm{~K}$ in acetonitrile $\left(\Delta G_{298}\right)$ for the isomers obtained from PBE0-D3BJ/def2-SVP/ECP10MWB calculations. The $\Delta G_{298}$ values include SMD solvation energies. Next to each structure the respective point group is given. Hydrogen atoms of the ligand backbone are omitted for clarity; the latter is only represented by sticks (copper: red, nitrogen: blue, carbon: grey, hydride: white). The structures are shown from a different perspective in Figure 6.14. This figure was made by Thomas Auth. 


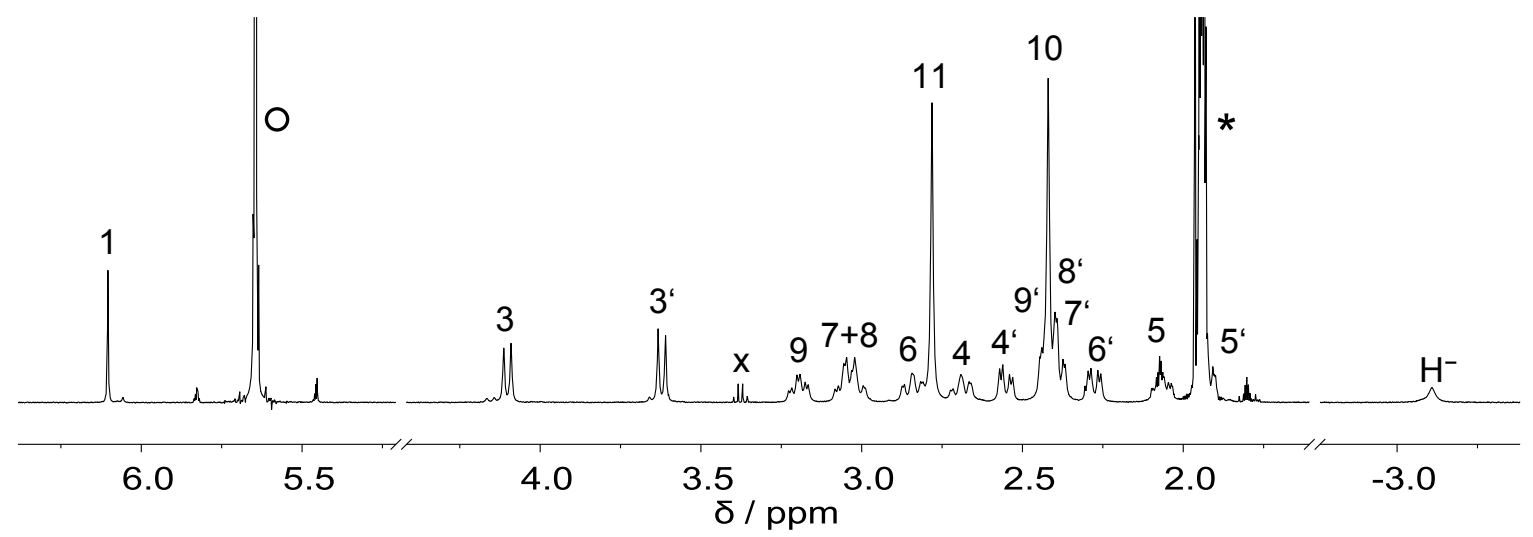

Figure 6.13: ${ }^{1} \mathrm{H}-\mathrm{NMR}$ spectrum $(500 \mathrm{MHz})$ of $9 \mathrm{H}$ in $\mathrm{CD}{ }_{3} \mathrm{CN}$ at $-35{ }^{\circ} \mathrm{C}$ under inert conditions. All signals are assigned according to the labeling scheme in Scheme 6.8. The asterisk denotes the residual solvent peak, the $\mathrm{x}$ points out $\mathrm{Et}_{2} \mathrm{O}$ and the circle marks $\left[\mathrm{Cp}_{2} \mathrm{Co}\right]^{+}$. Please note, the distinction between prime and non-prime (diastereotopic $\mathrm{H}$ atoms) is not possible and is merely done for simplification.

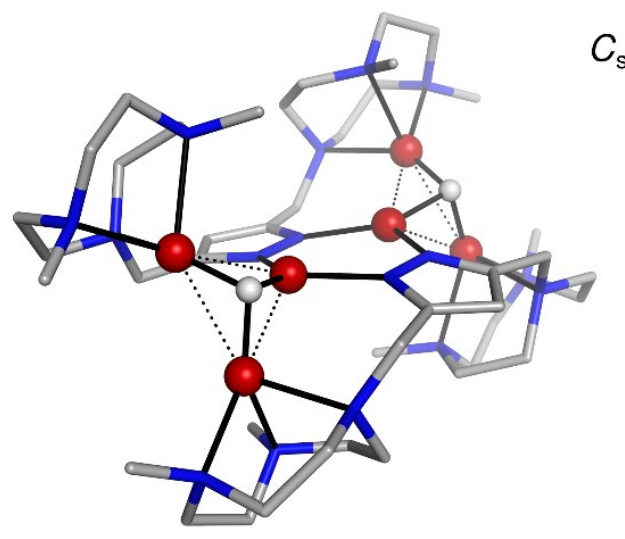

$$
\begin{gathered}
\text { cis-9 } 9^{\text {cis- } \mathrm{H}} \\
\Delta G_{298}=0.0 \mathrm{~kJ} \mathrm{~mol}^{-1}
\end{gathered}
$$

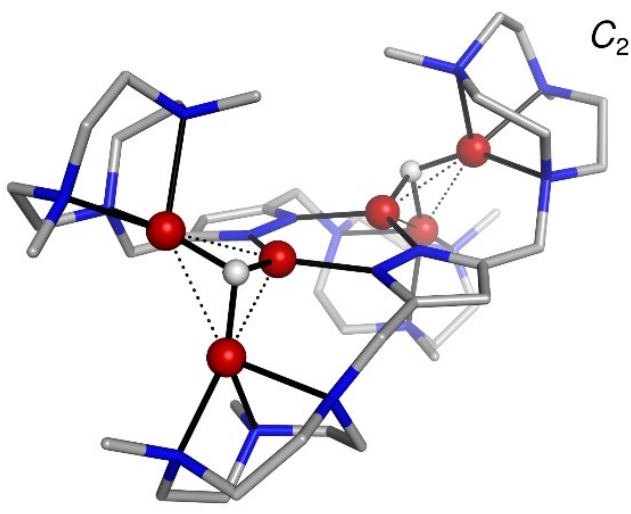

trans-9 ${ }^{\text {cis- } \mathrm{H}}$

$\Delta G_{298}=4.7 \mathrm{~kJ} \mathrm{~mol}^{-1}$

$C_{\mathrm{s}}$

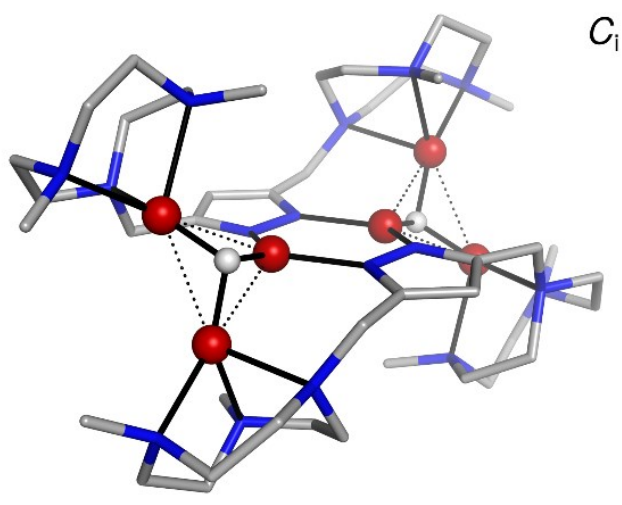

cis-9 $\mathbf{9}^{\text {trans- } \mathrm{H}}$

$\Delta G_{298}=0.7 \mathrm{~kJ} \mathrm{~mol}^{-1}$

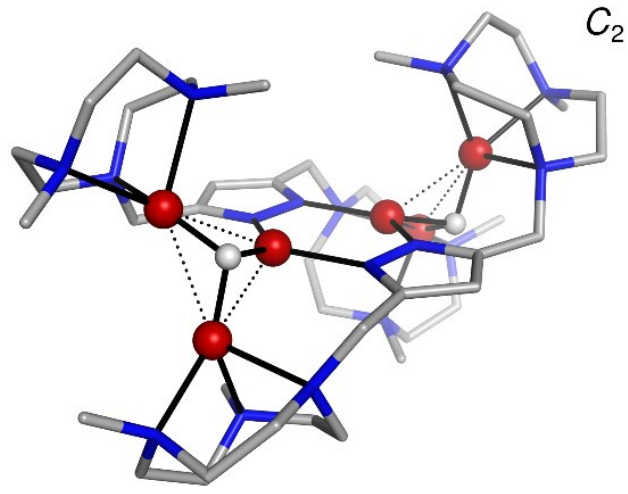

trans-9 $\mathbf{9}^{\text {trans- } \mathrm{H}}$

$\Delta G_{298}=6.6 \mathrm{~kJ} \mathrm{~mol}^{-1}$

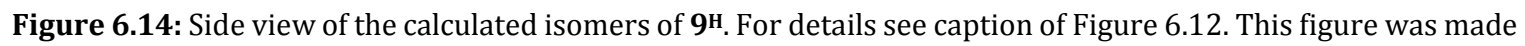
by Thomas Auth. 
Table 6.3: Relative electronic energies $\left(\Delta E_{\text {elec }}\right)$, relative enthalpies at $0 \mathrm{~K}\left(\Delta H_{0}\right)$ and relative Gibbs energies at $298 \mathrm{~K}$ in the gas phase $\left(\Delta G_{298}{ }^{\prime}\right)$ and in acetonitrile $\left(\Delta G_{298}\right)$ for the isomers of $\mathbf{9 H}^{\mathbf{H}}$ obtained from PBE0-D3BJ/def2SVP/ECP10MWB calculations. The $\Delta G_{298}$ values include SMD solvation energies. All values are given relative to the most stable isomer based on $G_{298}$.

\begin{tabular}{|c|c|c|c|c|}
\hline isomer & $\Delta E_{\text {elec }} / \mathrm{kJ} \cdot \mathrm{mol}^{-1}$ & $\Delta H_{0} / \mathrm{kJ} \cdot \mathrm{mol}^{-1}$ & $\Delta G_{298^{\prime}} / \mathrm{kJ} \cdot \mathrm{mol}^{-1}$ & $\Delta G_{298} / \mathrm{kJ} \cdot \mathrm{mol}^{-1}$ \\
\hline cis- $\mathbf{9}$ cis-H & 0.0 & 0.0 & 0.0 & 0.0 \\
\hline cis-9trans-H & 1.2 & -0.6 & -2.3 & 0.7 \\
\hline trans $\mathbf{- 9}{ }^{c i s-\mathbf{H}}$ & -4.0 & -3.5 & -1.6 & 4.7 \\
\hline trans $-\mathbf{9}$ trans $-\mathbf{H}$ & 1.1 & -0.2 & 0.7 & 6.6 \\
\hline
\end{tabular}

As DFT predicts significant population of at least two isomers, viz. cis-9 $\mathbf{9}^{\text {cis-H }}$ and cis-9 $\mathbf{9}^{\text {trans-H, }}$, the presence of one set of signals in the ${ }^{1} \mathrm{H}-\mathrm{NMR}$ spectrum suggests that both conformers have indistinguishable chemical shifts or undergo rapid interconversion (Figure 6.13). Fluxionality could also explain why the ${ }^{1} \mathrm{H}-\mathrm{NMR}$ spectrum implies four equal quadrants for $\mathbf{9}^{\mathrm{H}}$, although cis-9is-H and cis$\mathbf{9}^{\text {trans-H }} \mathbf{\mathrm { H }}$ feature only $C_{\mathrm{s}}$ and $C_{\mathrm{i}}$ symmetry, respectively (alternatively, this could result from indistinguishable chemical shifts of all tacn moieties).

Variable temperature (VT) NMR experiments were conducted to investigate possible temperature dependent dynamics of $9^{\mathrm{H}}$. Indeed, upon heating, the signals of the diastereotopic $\mathrm{H}$ atoms of the methylene linker (3/3') significantly broaden and eventually merge into one singlet (Figure 6.15; for full spectra see appendix, Figure A.95). On the basis of the calculated structures of $9^{\mathbf{H}}$, this finding can be rationalized by rapid switching between the cis- and trans-( $\left.\mathrm{L}^{2 \mathrm{a}}\right)^{-}$isomers at higher temperatures. By means of the full width half maximum (FWHM), the line broadening due to this dynamic with

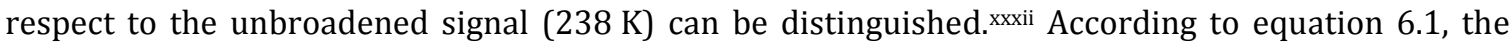
$\triangle \mathrm{FWHM}$ can then be converted to a rate constant for each temperature.

$$
\begin{gathered}
k=\Delta \mathrm{FWHM} \cdot \pi \\
\ln \frac{k}{T}=-\frac{\Delta H^{\ddagger}}{R} \cdot \frac{1}{T}+\ln \frac{k_{\mathrm{B}}}{h}+\frac{\Delta S^{\ddagger}}{R} \\
\ln k=\ln A-\frac{E_{\mathrm{A}}}{R} \cdot \frac{1}{T} \\
\Delta G^{\ddagger}=\Delta H^{\ddagger}-T \Delta S^{\ddagger}
\end{gathered}
$$

From this data, an Eyring $\left(\ln \frac{k}{T} v s \cdot \frac{1}{T}\right)$ and Arrhenius $\left(\ln k v s \cdot \frac{1}{T}\right)$ plot were prepared (Figure 6.16), from which the activation energy $E_{\mathrm{A}}$, as well as the activation parameters $\Delta H^{\ddagger}, \Delta S^{\ddagger}$ and $\Delta G^{\ddagger}$ were extracted as shown in equations 6.2-6.4. Apart from the physical constants $R, h$ and $k_{\mathrm{B}}$, the slope of the linear fit $(m)$ as well as the $y$-intercept were required for this. Alternatively, the rate constant $k_{c}$ and the standard Gibbs energy of activation $\Delta G_{c}^{\ddagger}$ can be determined at the coalescence temperature $T_{\mathrm{c}}$ (308 K) according to equations 6.5 and 6.6 , where $\Delta v$ is the separation of the two signals at low temperature and $J(\mathrm{AB})$ their coupling constant in $\mathrm{s}^{-1}$. All distinguished parameters are compiled in Table 6.4. From the VT-NMR spectra, a standard Gibbs energy of activation $\Delta G_{298}^{\ddagger}$ of $59 \pm 1 \mathrm{~kJ} \cdot \mathrm{mol}^{-1}$ for

xxxii This is possible as long as the two peaks can be distinguished from one another - here, up until $298 \mathrm{~K}$. 
the interconversion process was derived. This coincides well with the $\Delta G_{\mathrm{c}}^{\ddagger}$ of $59 \mathrm{~kJ} \cdot \mathrm{mol}^{-1}$ calculated from the coalescence temperature $T_{\mathrm{c}}(308 \mathrm{~K})$.

$$
\begin{gathered}
k_{\mathrm{c}}=\frac{\pi}{2} \sqrt{(\Delta v)^{2}+6 J(\mathrm{AB})^{2}} \\
\Delta G_{\mathrm{c}}^{\ddagger}=-R T_{\mathrm{c}} \ln \left(\frac{k_{\mathrm{c}} \cdot h}{k_{\mathrm{B}} \cdot T_{\mathrm{c}}}\right)
\end{gathered}
$$
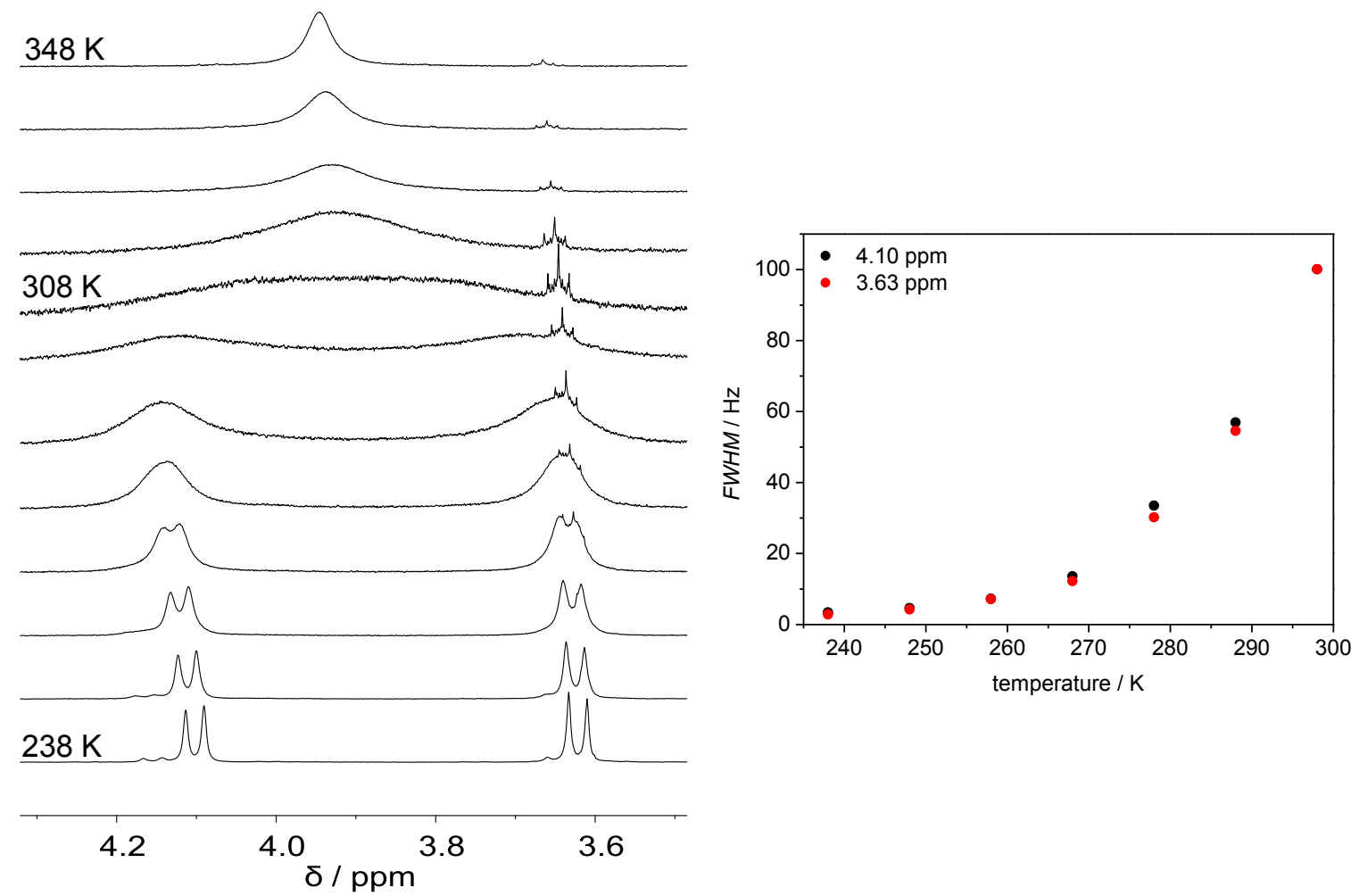

Figure 6.15: Variable temperature ${ }^{1} \mathrm{H}-\mathrm{NMR}$ spectra $(500 \mathrm{MHz})$ of $9^{\mathrm{H}}$ in $\mathrm{CD}_{3} \mathrm{CN}$ under inert conditions (238-348 K, $10 \mathrm{~K}$ steps) showing the 3/3'-H signals. For the full spectra see the appendix (Figure A.95).
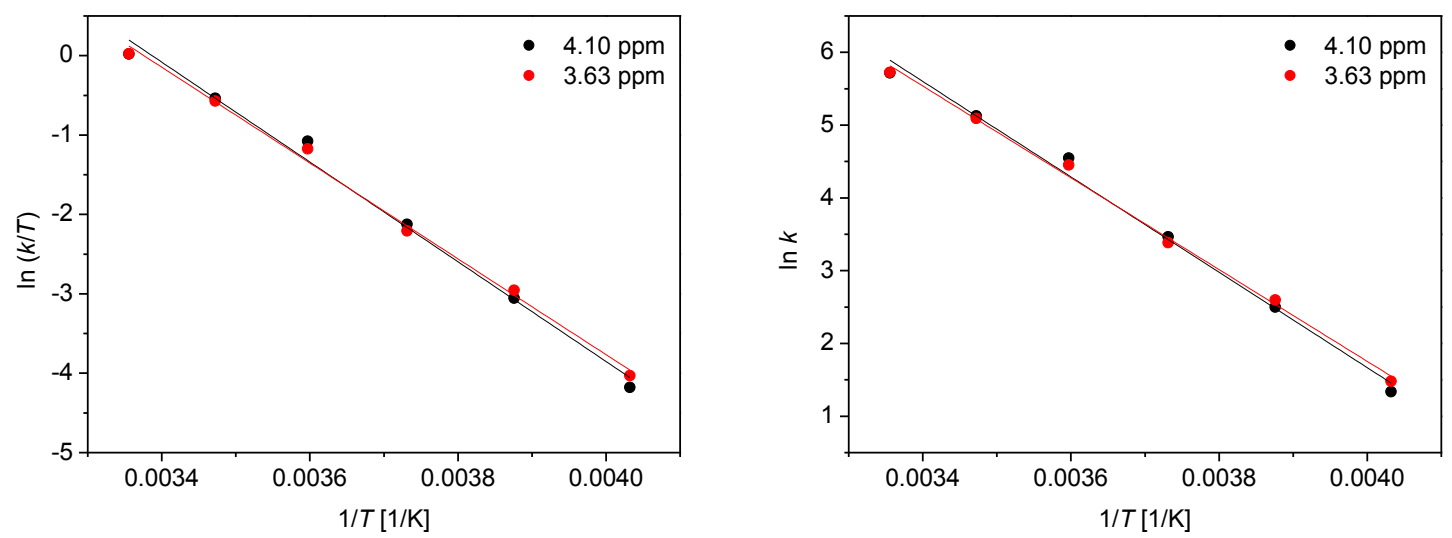

Figure 6.16: Eyring (left) and Arrhenius (right) plots for the dynamics of $9^{\mathrm{H}}$ in MeCN. 
Table 6.4: Activation parameters for the dynamics of $9^{\mathrm{H}}$ with temperature determined from VT-NMR studies by means of the FWHM and the coalescence temperature.

\begin{tabular}{lrrr} 
FWHM & $3.63 \mathrm{ppm}$ & $4.10 \mathrm{ppm}$ & weighted average \\
\hline \hline$E_{\mathrm{A}} / \mathrm{kJ} \cdot \mathrm{mol}^{-1}$ & $52.4 \pm 1.6$ & $54.5 \pm 2.4$ & $53.1 \pm 1.3$ \\
$\Delta H^{\ddagger} / \mathrm{kJ} \cdot \mathrm{mol}^{-1}$ & $50.2 \pm 1.6$ & $52.2 \pm 2.4$ & $50.8 \pm 1.3$ \\
$\Delta S^{\ddagger} / \mathrm{J} \cdot \mathrm{mol}^{-1} \cdot \mathrm{K}^{-1}$ & $-28.2 \pm 5.9$ & $-20.6 \pm 8.8$ & $-25.8 \pm 4.9$ \\
$\Delta G_{298^{\ddagger}} / \mathrm{kJ}^{-\mathrm{mol}^{-1}}$ & $58.6 \pm 0.7$ & $58.4 \pm 1.1$ & $58.5 \pm 0.6$ \\
\hline coalescence temperature & & & \\
\hline \hline$T_{\mathrm{c}} / \mathrm{K}$ & & 508 & \\
$k_{\mathrm{c}} / \mathrm{s}^{-1}$ & 537 & \\
$\Delta G_{\mathrm{c}} / \mathrm{kJ} \cdot \mathrm{mol}^{-1}$ & 59 & \\
\hline
\end{tabular}

a the errors were determined by Gaussian error propagation based on the standard errors from the linear regressions

Preliminary measurements were conducted to investigate the luminescence properties of $9^{\mathrm{H}}$. Taking into account the emission spectrum of $\left[\mathrm{Cp}_{2} \mathrm{Co}\right]\left(\mathrm{PF}_{6}\right)$, one rather broad emission peak remains at $586 \mathrm{~nm}$ after excitation at $342 \mathrm{~nm}$ (Figure 6.17, left), corresponding to a Stokes' shift of $244 \mathrm{~nm}$. The lifetime of this emission was determined to be $6.24 \mathrm{~ns}$ at room temperature (Figure 6.17, right). In fact, the observation of luminescence is quite typical for multinuclear coinage metal $\mathrm{d}^{10}$ complexes with $\mathrm{M}_{3}$ units.[394-409]
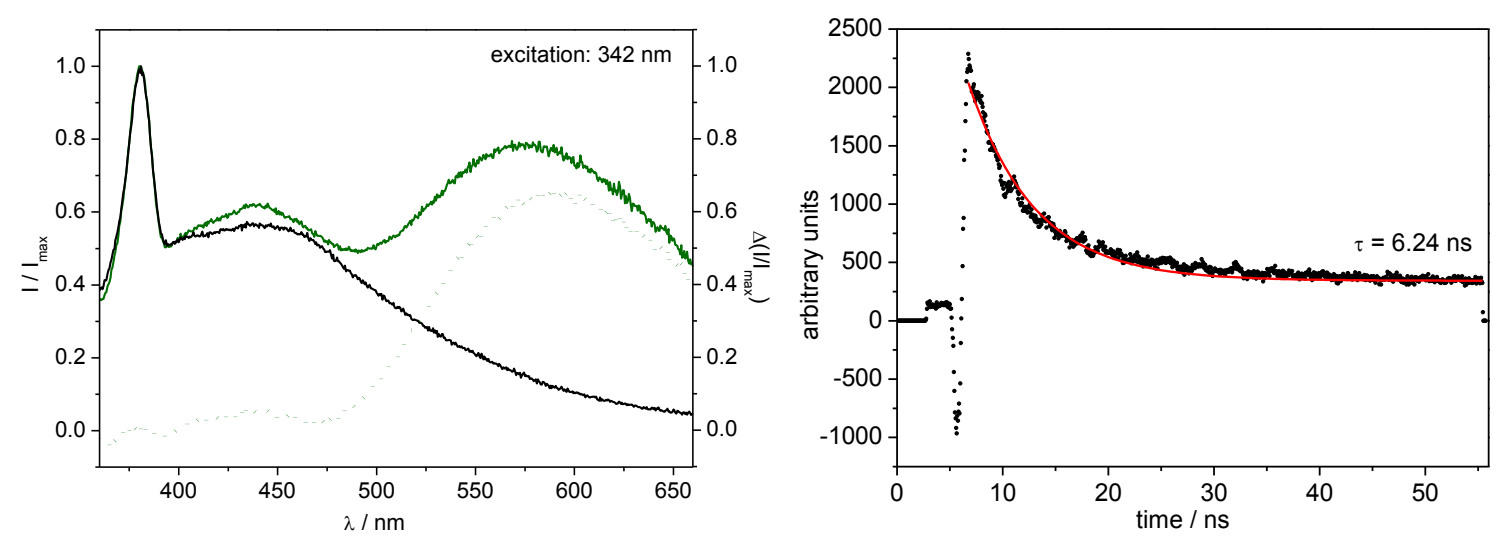

Figure 6.17: Luminescence properties of $9 \mathrm{H}$ in $\mathrm{MeCN}$ at room temperature under inert conditions. Left: Emission spectra of ${ }^{\mathbf{H}}\left(0.125 \mathrm{mM}\right.$, solid green) and $\left[\mathrm{Cp}_{2} \mathrm{Co}\right]\left(\mathrm{PF}_{6}\right)(0.884 \mathrm{mM}$, solid black) upon excitation at $342 \mathrm{~nm}$ normalized to $I_{\max }$ as well as $\Delta\left(\mathrm{I} / \mathrm{I}_{\max }\right)$ of $\mathbf{9 H}^{\mathbf{H}}$ (dotted green) with respect to the spectrum of [ $\mathrm{Cp}_{2} \mathrm{Co}_{0}\left(\mathrm{PF}_{6}\right)$. Right: Time-resolved luminescence intensity measurement of $\mathbf{9 H}^{\mathrm{H}}(0.125 \mathrm{mM})$ (excitation: $342 \mathrm{~nm}$, emission: $\left.568 \mathrm{~nm}\right)$.

\subsubsection{Hydride Reactivity}

To further support the presence of $\mu_{3}$-hydrides in $9^{\mathrm{H}}$ and to probe their reactivity, $9^{\mathrm{H}}$ was treated with phenylacetylene. This gave rise to $\mathrm{H}_{2}$ release $\left(4.55 \mathrm{ppm}\right.$ ) ${ }^{\mathrm{xxxiii}}$ and the formation of a new hexacopper complex $\left[\left(\mathrm{L}^{2 \mathrm{a}}\right)_{2} \mathrm{Cu}_{6}(\mathrm{CCPh})_{2}\right]\left(\mathrm{ClO}_{4}\right)_{2}\left(\mathbf{9}^{\mathrm{C} 8 \mathrm{H}}\right)$ that has two phenylacetylide ligands capping the $\mathrm{Cu}_{3}$ subunits (Figure 6.18). Crystals of $\mathbf{9}^{\mathbf{C} \mathbf{H} \mathbf{5}}$ were obtained by diffusion of $\mathrm{Et}_{2} \mathrm{O}$ into an acetonitrile solution of $\mathbf{9}^{\mathrm{H}}$ and phenylacetylene, and analyzed by XRD. $\mathbf{9}^{\mathrm{c}} \mathrm{H}^{\mathbf{5}}$ crystallizes in the triclinic space group $P-1$. The complex molecule, two perchlorate anions and an acetonitrile molecule are found in the asymmetric unit. The molecular structure of $9^{\text {C8H5 }}$ (Figure 6.19) shows that the overall hexacopper core

xxxiii The signal for $\mathrm{H}_{2}$ is so small, because the NMR tube was opened before the measurement. 
of $9^{\mathrm{H}}$ is retained. However, the $\mathrm{Cu}_{3}$ triangles are not isosceles because the $\mathrm{PhC} \equiv \mathrm{C}^{-}$ligands span a short $\mathrm{Cu} \cdots \mathrm{Cu}$ edge in end-on fashion $(d(\mathrm{Cu} 1 \cdots \mathrm{Cu} 3)=2.51 \AA ̊$; $d(\mathrm{Cu} 4 \cdots \mathrm{Cu} 6)=2.55 \AA ̊$ ), and via their $\pi$-system they additionally serve as side-on alkyne ligands towards the more distant third $\mathrm{Cu}^{1}$ ion (with $d(\mathrm{Cu} 2 \cdots \mathrm{Cu} 1 / 3)$ and $d(\mathrm{Cu} 5 \cdots \mathrm{Cu} 4 / 6)$ in the range 2.88-3.61 $\AA$ ). The combination of end-on $\left(\eta^{1}\right)$ and sideon $\left(\eta^{2}\right)$ binding modes is not uncommon for copper(I) alkynide complexes. ${ }^{[410]}$ The two $\mathrm{PhC} \equiv \mathrm{C}^{-}$ligands in $\mathbf{9}^{\mathbf{c}} \mathbf{H 5}$ are located cis to one another (viz. on the same side of the plane defined by the two pyrazolates). Moreover, in the solid state, the $\left[\left(\mathrm{L}^{2 \mathrm{a}}\right)_{2} \mathrm{Cu}_{6}\right]$ core of $\mathbf{9}^{\mathbf{c} 8 \mathrm{H} 5}$ features cis arrangement of the two tacn units of each ligand strand $\left(\mathrm{L}^{2 \mathrm{a}}\right)^{-}$, as was also observed for $\mathbf{9}^{\mathrm{H}}$. The $\mathrm{C} \equiv \mathrm{C}$ stretch of the bound phenylacetylides in $\mathbf{9}^{\mathbf{C}} \mathbf{H 5}$ is found at $1852 \mathrm{~cm}^{-1}$, i.e. significantly lower than in free $\mathrm{HC} \equiv \mathrm{CPh}$ $\left(2119 \mathrm{~cm}^{-1}\right)^{[411-413]}$ in accordance with side-on coordination to a $\mathrm{Cu}^{1}$ ion and substantial $\pi$ backbonding. ${ }^{[414-418]}$

Solutions of $\mathbf{9}^{\mathbf{c 8 H} 5}$ were characterized by ESI mass spectrometry and NMR spectroscopy. Next to the omnipresent $\left[\mathrm{Cp}_{2} \mathrm{Co}\right]^{+}$, the ESI(+)-MS exhibits a peak at $m / z$ 697.1 corresponding to the molecular ion peak $[\mathrm{M}]^{2+}$ (appendix, Figures A.53 and A.54). Noteworthy is the absence of the hydride signal at $-3.11 \mathrm{ppm}$ in the ${ }^{1} \mathrm{H}-\mathrm{NMR}$ spectrum and the occurrence of peaks in the aromatic region associated with the phenylacetylide moieties (Figure 6.18; for complete NMR characterization see appendix, Figures A.99-A.105). With the help of the pure shift ${ }^{1} \mathrm{H}$-NMR spectrum and two-dimensional NMR spectroscopy $\left({ }^{1} \mathrm{H}-{ }^{-1} \mathrm{H}-\mathrm{COSY},{ }^{1} \mathrm{H}-{ }^{1} \mathrm{H}-\mathrm{NOESY},{ }^{1} \mathrm{H}-{ }^{-13} \mathrm{C}-\mathrm{HSQC},{ }^{1} \mathrm{H}-{ }^{13} \mathrm{C}-\mathrm{HMBC}\right)$, all carbon and hydrogen signals except those of the alkyne ligands could be assigned.xxxiv. In contrast to $\mathbf{9 H}^{\mathrm{H}}$, two peaks for the pyrazole $1-\mathrm{H}$ as well as a doubling of several signals, e.g. the diastereotopic $\mathrm{H}$ atom $5^{\prime}$ and the $\mathrm{N}-\mathrm{CH}_{3}$ groups 10 and 11 , can be identified in the ${ }^{1} \mathrm{H}-\mathrm{NMR}$ spectrum. The two sets of signals have an approximate ratio of 3:1, indicating that they originate from two different isomers. These are shown by VT-NMR spectroscopy to interconvert at higher temperatures (Figure 6.18; for full spectra see appendix, Figure A.106). Although the diastereotopic $\mathrm{H}$ atoms of the methylene linker appear to be retained under these conditions, VTNOESY experiments show that they also exchange at higher temperatures (appendix, Figure A.107), however, due to the large chemical shift difference of the signals, the coalescence temperature is not reached. Overall, the VT-NMR experiments thus indicate that at high temperatures all isomers are in fast equilibrium (there is both a dynamic of the phenylacetylide ligands and the tacn moieties of the equatorial scaffold). At low temperatures, however, it is not fully understood which dynamic is preserved and which is frozen, especially as a correlation of both dynamic processes cannot be excluded. The observation of apparent $D_{2}$ or $C_{2 \mathrm{~h}}$ symmetry of each isomer according to ${ }^{1} \mathrm{H}$-NMR spectroscopy at $238 \mathrm{~K}$ is quite unexpected in view of the symmetry of the XRD structure (noncrystallographic $C_{\mathrm{s}}$ ) and suggests that the chemical shifts of the four sidearm tacn moieties in $\mathbf{9}^{\text {c8r5 }}$ are indistinguishable, as was also proposed for $\mathbf{9}^{\mathrm{H}}$ (see above).

As for $9^{\mathrm{H}}$, four isomers of $\mathbf{9}^{\mathbf{c} 8 \mathrm{H} 5}$ were identified by DFT geometry optimizations, viz. cis-9 $\mathbf{9}^{\text {cis-C8H5 }}$ (analogous to the XRD structure), cis-9trans-C8H5, trans-9 $\mathbf{9}^{\text {cis-C8H5 }}$ and trans-9trans-C8H5 (Figure 6.20 and Figure 6.21). The trans-9 $\mathbf{9}^{\text {cis-C8H5 }}$ structure features the lowest $G_{298}$ value in acetonitrile but is only slightly more stable than cis-9 ${ }^{\text {cis-c8H5 }}\left(1.2 \mathrm{~kJ} \cdot \mathrm{mol}^{-1}\right.$, Table 6.5$)$. The two isomers with trans-standing phenylacetylide moieties, cis-9 9 trans-C8H5 and trans-9 $\mathbf{9}$ trans-C8H5, are energetically less favorable by 7.8 and $14.9 \mathrm{~kJ} \cdot \mathrm{mol}^{-1}$, respectively. Changing the orientation of one of the tacn moieties of each $\left(\mathrm{L}^{2 \mathrm{a}}\right)^{-}$strand relative to the respective pyrazolate plane, i.e. converting cis-9 into trans-9, is correlated with a change in $\eta^{1}$ and $\eta^{2}$ binding sites in the $\mathrm{Cu}_{3}$ subunits. Although the trans ${ }^{\text {cis }}$ and ciscis isomers of $9^{\text {c8H5 }}$ have the lowest calculated $G_{298}$ values, the results of the VT-NMR experiments indicate that all isomers are in equilibrium at higher temperatures.

xxxiv Please note, the distinction between the diastereotopic $\mathrm{H}$ atoms of $\left(\mathrm{L}^{2 \mathrm{a}}\right)$ - is not possible and is merely done for simplification. 

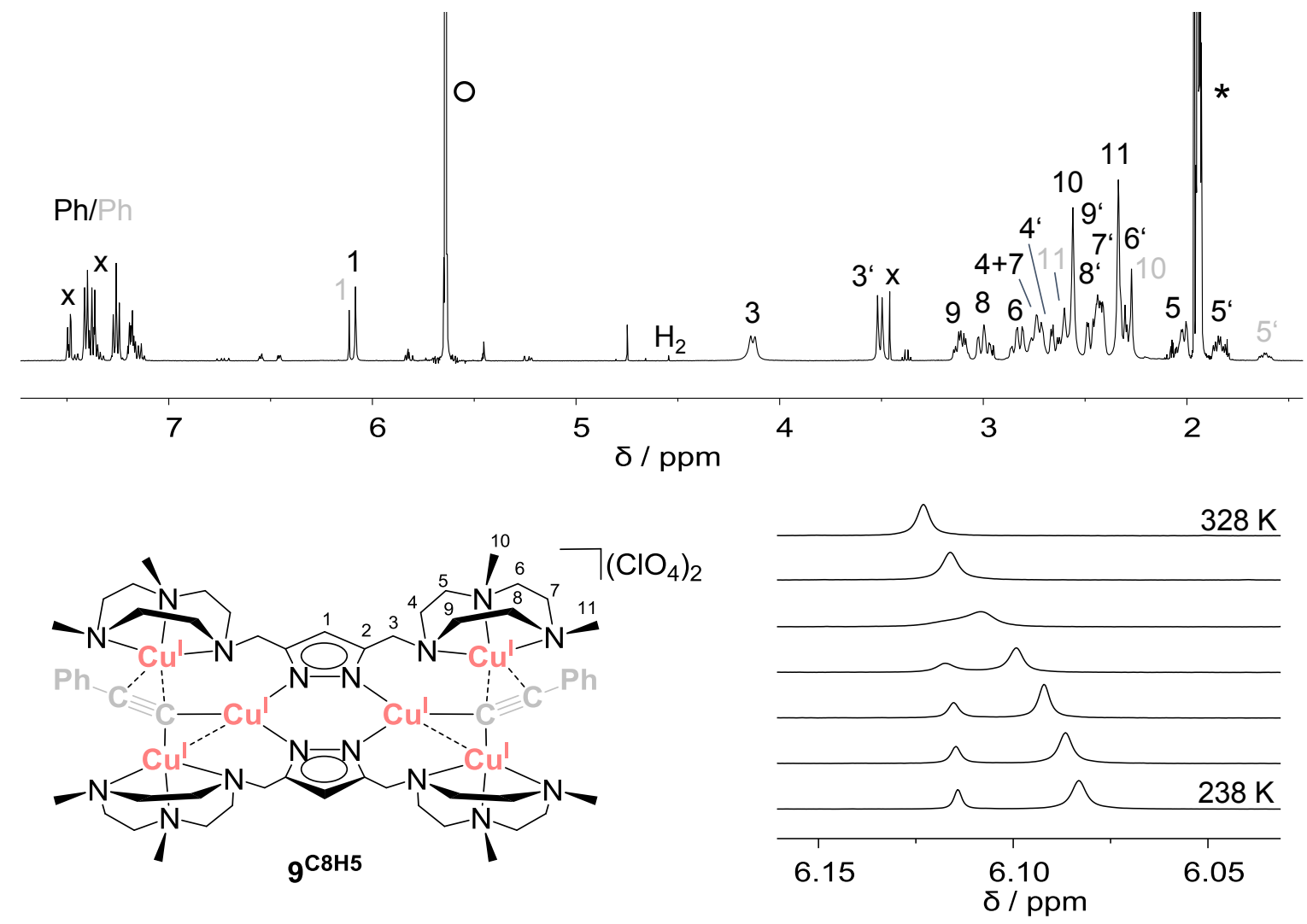

Figure 6.18: NMR spectroscopic characterization of $\mathbf{9}^{\mathrm{C} 8 \mathrm{H} 5}$ in $\mathrm{CD}_{3} \mathrm{CN}$ under inert conditions. Top: ${ }^{1} \mathrm{H}-\mathrm{NMR}$ spectrum $(500 \mathrm{MHz})$ at $238 \mathrm{~K}$. The signals labeled in grey are those of a second isomer (ratio 3:1). The asterisk denotes the residual solvent peak, the circle points out $\left[\mathrm{Cp}_{2} \mathrm{Co}\right]^{+}$and the $\mathrm{x}$ mark free phenyl acetylene. Bottom right: Excerpt of the variable temperature ${ }^{1} \mathrm{H}-\mathrm{NMR}$ spectra showing the 1 - $\mathrm{H}$ signals $(238-328 \mathrm{~K}, 15 \mathrm{~K}$ steps). For the full spectra see the appendix (Figure A.106).
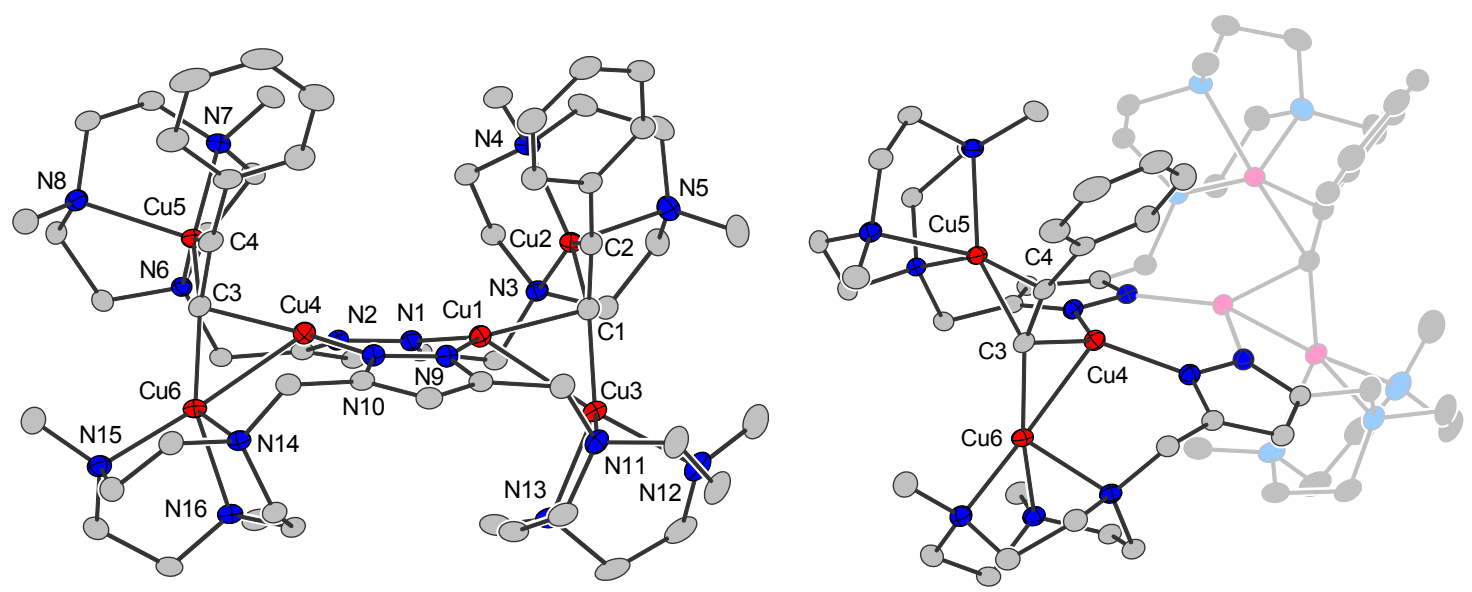

Figure 6.19: Molecular structure of the cationic core of $\mathbf{9}$ (8)5 drawn as thermal ellipsoids at the 30\% probability level (copper: red, nitrogen: blue, carbon: grey). All hydrogen atoms, solvent molecules and counter ions are omitted for clarity reasons. The view shown on the right highlights the binding mode of the $\mathrm{PhC} \equiv \mathrm{C}^{-}$ligands. 


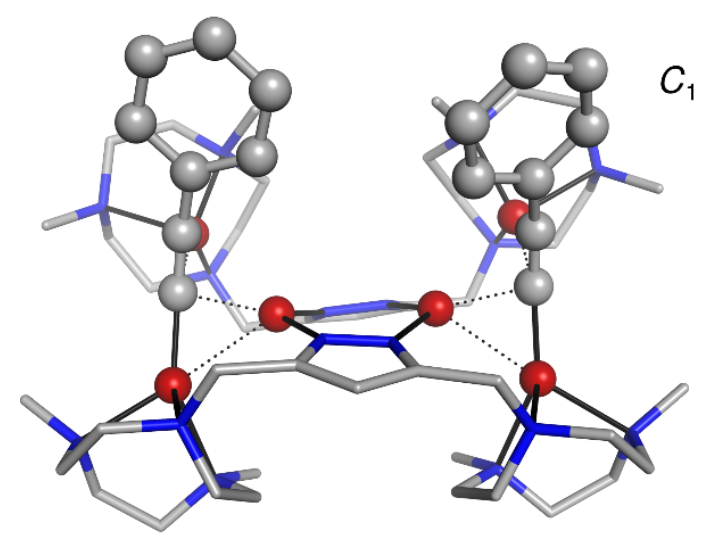

cis-9 $\mathbf{9}^{\text {cis-C8H5 }}$

$\Delta G_{298}=1.2 \mathrm{~kJ} \mathrm{~mol}^{-1}$

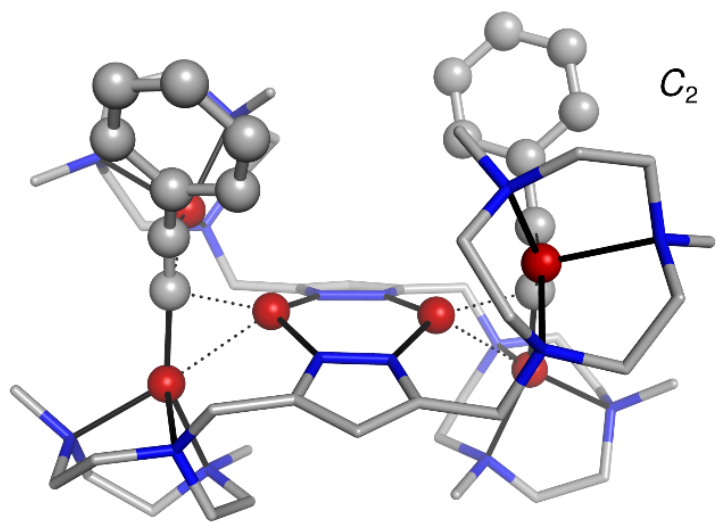

trans-9 $\mathbf{9}^{\text {cis-C8H5 }}$

$\Delta G_{298}=0.0 \mathrm{~kJ} \mathrm{~mol}^{-1}$

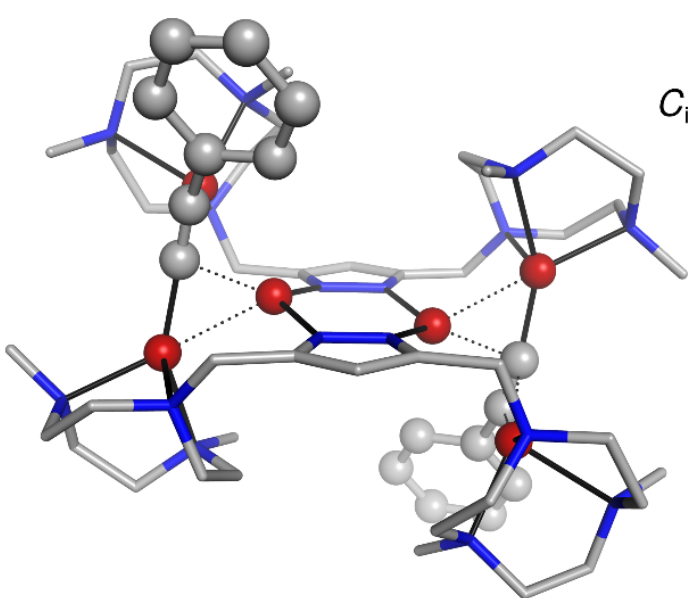

cis-9 $9^{\text {trans-C8H5 }}$

$\Delta G_{298}=7.8 \mathrm{~kJ} \mathrm{~mol}^{-1}$

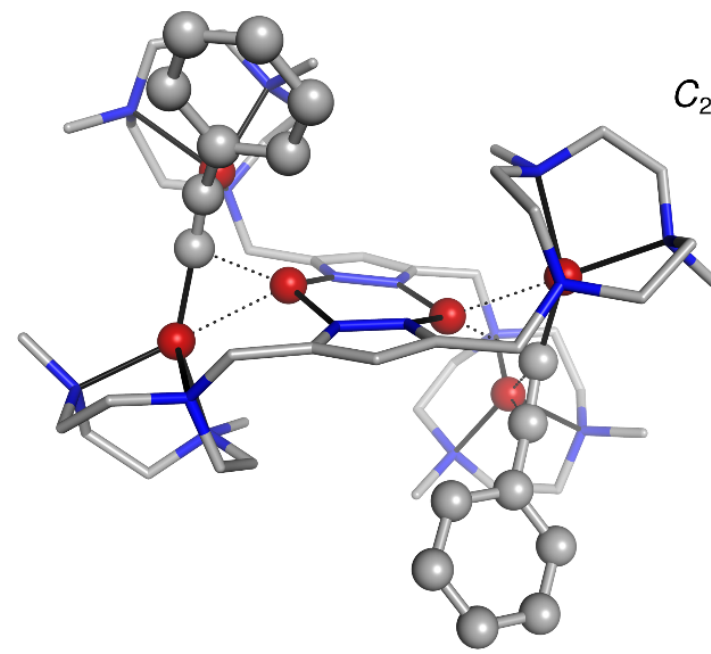

trans-9 $\mathbf{9}^{\text {trans-C8H5 }}$

$\Delta G_{298}=14.9 \mathrm{~kJ} \mathrm{~mol}^{-1}$

Figure 6.20: Molecular structures and relative Gibbs energies at $298 \mathrm{~K}$ in acetonitrile $\left(\Delta G_{298}\right)$ for the isomers of 9c8H5 obtained from PBE0-D3BJ/def2-SVP/ECP10MWB calculations. The $\Delta G_{298}$ values include SMD solvation energies. Next to each structure the respective point group is given. Hydrogen atoms of the ligand backbone are omitted for clarity; the latter is only represented by sticks (copper: red, nitrogen: blue, carbon: grey, hydride: white). The structures are shown from a different perspective in Figure 6.21. This figure was made by Thomas Auth. 


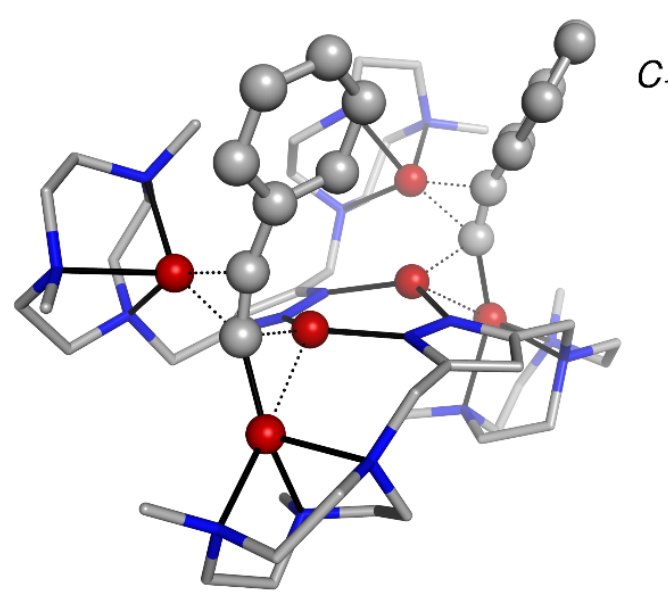

cis- $\mathbf{9}^{\text {cis- } \mathrm{C} 8 \mathrm{H} 5}$

$$
\Delta G_{298}=1.2 \mathrm{~kJ} \mathrm{~mol}^{-1}
$$

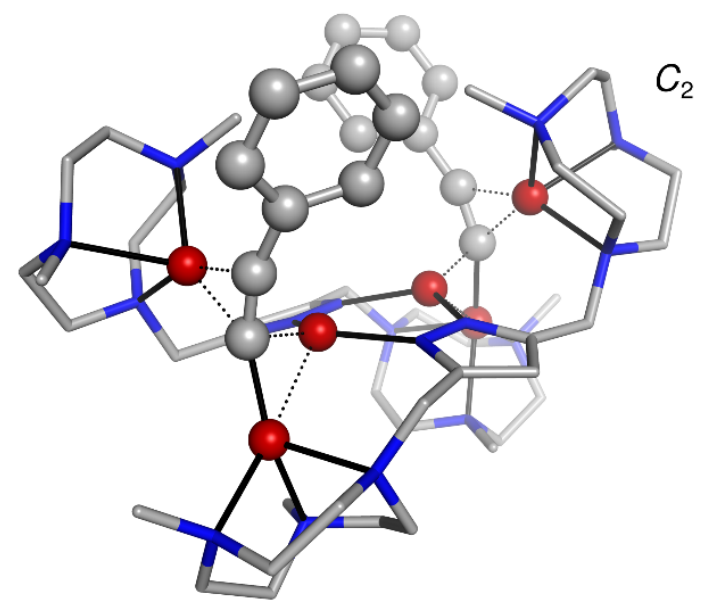

$$
\text { trans-9 } \mathbf{9}^{\text {cis-C8H5 }}
$$

$$
\Delta G_{298}=0.0 \mathrm{~kJ} \mathrm{~mol}^{-1}
$$

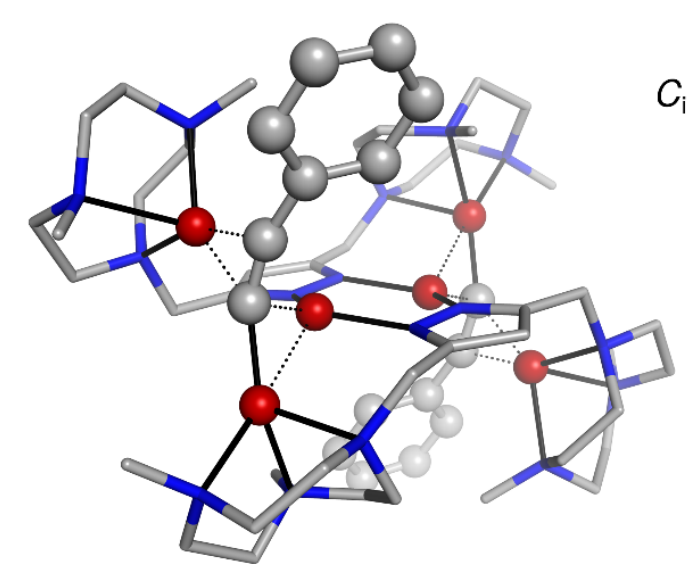

$$
\text { cis-9 } \mathbf{9}^{\text {trans-C8H5 }}
$$

$$
\Delta G_{298}=7.8 \mathrm{~kJ} \mathrm{~mol}^{-1}
$$

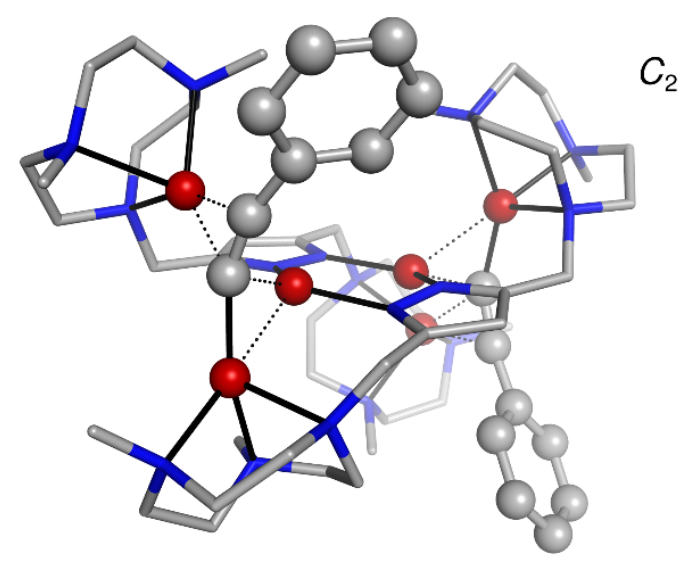

trans-9 $\mathbf{9}^{\text {trans-C8H5 }}$

$\Delta G_{298}=14.9 \mathrm{~kJ} \mathrm{~mol}^{-1}$

Figure 6.21: Side view of the calculated isomers of $\mathbf{9 c 8}^{\mathbf{c}} \mathbf{H 5}$. For details see caption of Figure 6.20. This figure was made by Thomas Auth

\begin{tabular}{|c|c|c|c|c|}
\hline isomer & $\Delta E_{\text {elec }} / \mathrm{kJ} \cdot \mathrm{mol}^{-1}$ & $\Delta H_{0} / \mathrm{kJ} \cdot \mathrm{mol}^{-1}$ & $\Delta G_{298^{\prime}} / \mathrm{kJ} \cdot \mathrm{mol}^{-1}$ & $\Delta G_{298} / \mathrm{kJ} \cdot \mathrm{mol}^{-1}$ \\
\hline cis-9 ${ }^{\text {cis- }-\mathbf{C 8 H 5}}$ & 8.4 & 9.0 & 5.8 & 1.2 \\
\hline cis-9trans-C8H5 & 14.2 & 12.9 & 8.7 & 7.8 \\
\hline trans-9cis-C8H5 & 0.0 & 0.0 & 0.0 & 0.0 \\
\hline trans-9trans- $\mathbf{C 8 H 5}$ & 14.6 & 15.5 & 16.0 & 14.9 \\
\hline
\end{tabular}

Table 6.5: Relative electronic energies $\left(\Delta E_{\text {elec }}\right)$, relative enthalpies at $0 \mathrm{~K}\left(\Delta H_{0}\right)$ and relative Gibbs energies at $298 \mathrm{~K}$ in the gas phase $\left(\Delta G_{298}{ }^{\prime}\right)$ and in acetonitrile $\left(\Delta G_{298}\right)$ for the isomers of $\mathbf{9}$ c8H5 obtained from PBE0-D3BJ/def2SVP/ECP10MWB calculations. The $\Delta G_{298}$ values include SMD solvation energies. All values are given relative to the most stable isomer based on $G_{298}$. 


\subsubsection{Origin of the Hydrides in $9^{\mathrm{H}}$ and Fate of the Formate}

To investigate the origin of the hydrides in $9^{\mathbf{H}}$ and the fate of the formate after reduction of $\mathbf{5}^{\mathrm{HCO}}$, isotopic labeling studies were performed. Therefore, the deuterated complex $\mathbf{5}^{\mathbf{D C O} 2}$ was synthesized using $\mathrm{NaDCO}_{2}$ instead of $\mathrm{NaHCO}_{2}$ (ESI-MS in the appendix, Figures A.13 and A.14). The reduction of $5^{\text {DCO2 }}$ was monitored by NMR spectroscopy, revealing no hydride signal in the high-field region of the ${ }^{1} \mathrm{H}$-NMR spectrum (Figure 6.22, middle). Instead, two peaks were observed in the ${ }^{2} \mathrm{H}$-NMR spectrum at -3.00 and $8.69 \mathrm{ppm}$, which could be assigned to deuteride moieties and free $\mathrm{DCO}_{2}^{-}$, respectively, and confirm the formation of $9^{\mathbf{D}}$ from $5^{\mathbf{D C O} 2}$ (Figure 6.22, top). Additionally, the molecular ion peak $[\mathrm{M}]^{2+}$ in the ESI(+)-MS is shifted by $m / z 1$ in comparison to the one of unlabeled $9^{\mathrm{H}}$, in accordance with the presence of two deuteride ligands in $9^{\mathrm{D}}$ (appendix, Figures A.51 and A.52). IR spectra of $9^{\mathrm{H}}$ and $\mathbf{9 D}^{\mathrm{D}}$ show no obvious differences, in compliance with DFT harmonic frequency calculations, which predict low intensity peaks at around $900 / 600 \mathrm{~cm}^{-1}$ for the $\mathrm{Cu}_{3}-\mathrm{H} / \mathrm{D}$ vibrations, where also other bands occur.

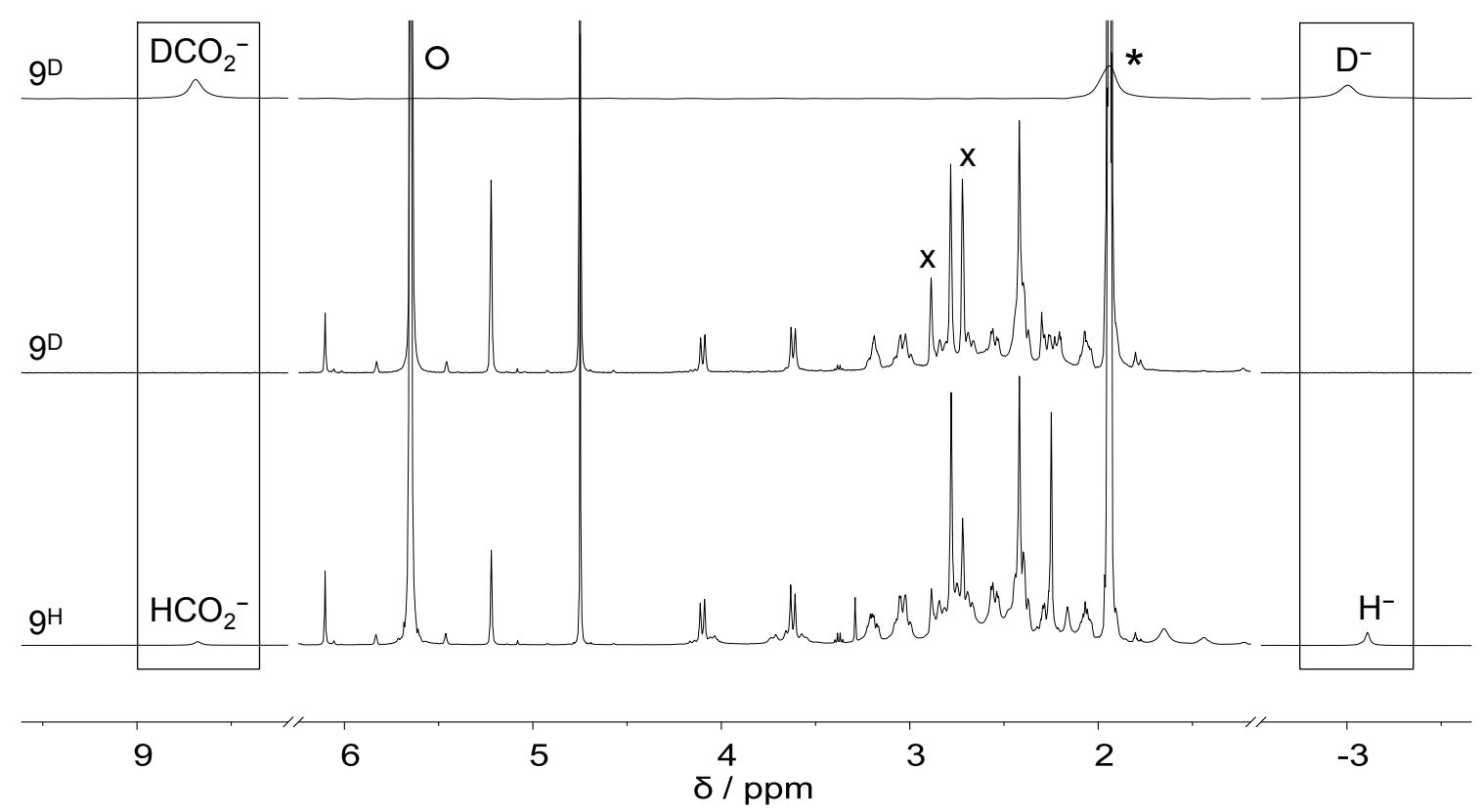

Figure 6.22: ${ }^{1} \mathrm{H}-\mathrm{NMR}$ spectrum $(500 \mathrm{MHz})$ of $\mathbf{9 H}^{\mathrm{H}}$ (bottom) and 9D (middle) in $\mathrm{CD}_{3} \mathrm{CN}$ and ${ }^{2} \mathrm{H}-\mathrm{NMR}$ spectrum ( $77 \mathrm{MHz}$ ) of 9D (top) in $\mathrm{CH}_{3} \mathrm{CN}$ at $238 \mathrm{~K}$ under inert conditions. The black rectangles highlight the areas in which the formate and hydride as well as their deuterated equivalents are located. The asterisk denotes the residual solvent peak, the circle points out $\left[\mathrm{Cp}_{2} \mathrm{Co}\right]^{+}$and the $\mathrm{x}$ marks an impurity.

When $\mathrm{NaH}^{13} \mathrm{CO}_{2}$ was used for the synthesis of the starting dicopper(II) complex and the resulting $5^{\mathrm{H}\{13 \mathrm{C}\} \mathbf{0 2}}$ (ESI-MS in the appendix, Figures A.15 and A.16) that has a ${ }^{13} \mathrm{C}$-labeled formate bridge was reduced with $\mathrm{Cp}_{2} \mathrm{Co}$, the ${ }^{13} \mathrm{C}-\mathrm{NMR}$ spectrum of the reaction mixture showed an intense signal for ${ }^{13} \mathrm{CO}_{2}$ at $125.3 \mathrm{ppm}$ (Figure 6.23, bottom). The combined findings indicate that the bound formate in $\mathbf{5}^{\mathrm{HCO}}$ splits into $\mathrm{H}^{-}$and $\mathrm{CO}_{2}$ upon reduction. Accordingly, no evidence for the formation of $\mathbf{9 H}^{\mathbf{H}}$ was obtained when the acetate-bridged dicopper(II) complex $\mathbf{5}^{\mathbf{O A c}}$ was reduced with $\mathrm{Cp}_{2} \mathrm{Co}$ (see Section 6.1).

Obviously, the transformation of dinuclear $\mathbf{5}^{\mathrm{HCO}}$ into hexanuclear $\mathbf{9}^{\mathrm{H}}$ is a complicated multi-step process with reshuffling of metal ions. Considering the different metal-to-ligand ratios in $\mathbf{5}^{\mathbf{H C O 2}}$ (2:1) and $9^{\mathrm{H}}(6: 2)$, this means that one ligand molecule ( $\left.\mathrm{L}^{2 \mathrm{a}}\right)^{-}$must be released during the reaction. Indeed, free pyrazolate-based ligand could be detected in the ${ }^{13} \mathrm{C}$-NMR spectrum of the residue after crystallization of $\mathbf{9 H}^{\mathrm{H}}$, as well as the reaction mixture of $\mathbf{5}^{\mathrm{HCO}}$ and $\mathrm{Cp}_{2} \mathrm{Co}$ (2.3 eq) (Figure 6.24, A and C, respectively). Which signals correspond to the free ligand could be distinguished by addition of $\mathrm{HL}^{2 a}$ (Figure 6.24, B and D). Furthermore, the ESI(+)-MS of the residual solvent after crystallization of $\mathbf{9}^{\mathbf{H}}$ displays dominant signals at $m / z 189.1$ and 407.3 corresponding to $\left[\mathrm{Cp}_{2} \mathrm{Co}\right]^{+}$and $\left[\mathrm{HL}^{2 \mathrm{a}}+\mathrm{H}\right]^{+}$, 
respectively (appendix, Figure A.48). With all this information at hand, the full reaction equation for the reduction of formate-bridged dicopper(II) complex $5^{\mathrm{HCO}}$ can be formulated as:

$$
3\left[\mathrm{~L}^{2 \mathrm{a}} \mathrm{Cu}_{2}\left(\mathrm{HCO}_{2}\right)\right]^{2+}+6 \mathrm{Cp}_{2} \mathrm{Co} \rightarrow \underbrace{\left[\left(\mathrm{L}^{2 \mathrm{a}}\right)_{2} \mathrm{Cu}_{6}(\mathrm{H})_{2}\right]^{2+}}_{\mathrm{NMR}}+\underbrace{6\left[\mathrm{Cp}_{2} \mathrm{Co}\right]^{+}+\left(\mathrm{L}^{2 \mathrm{a}}\right)^{-}}_{\text {ESI-MS+NMR }}+\underbrace{\mathrm{HCO}_{2}^{-}}_{{ }^{1} \mathrm{H}-\mathrm{NMR}}+\underbrace{2 \mathrm{CO}_{2}}_{{ }_{13} \mathrm{C}-\mathrm{NMR}}
$$

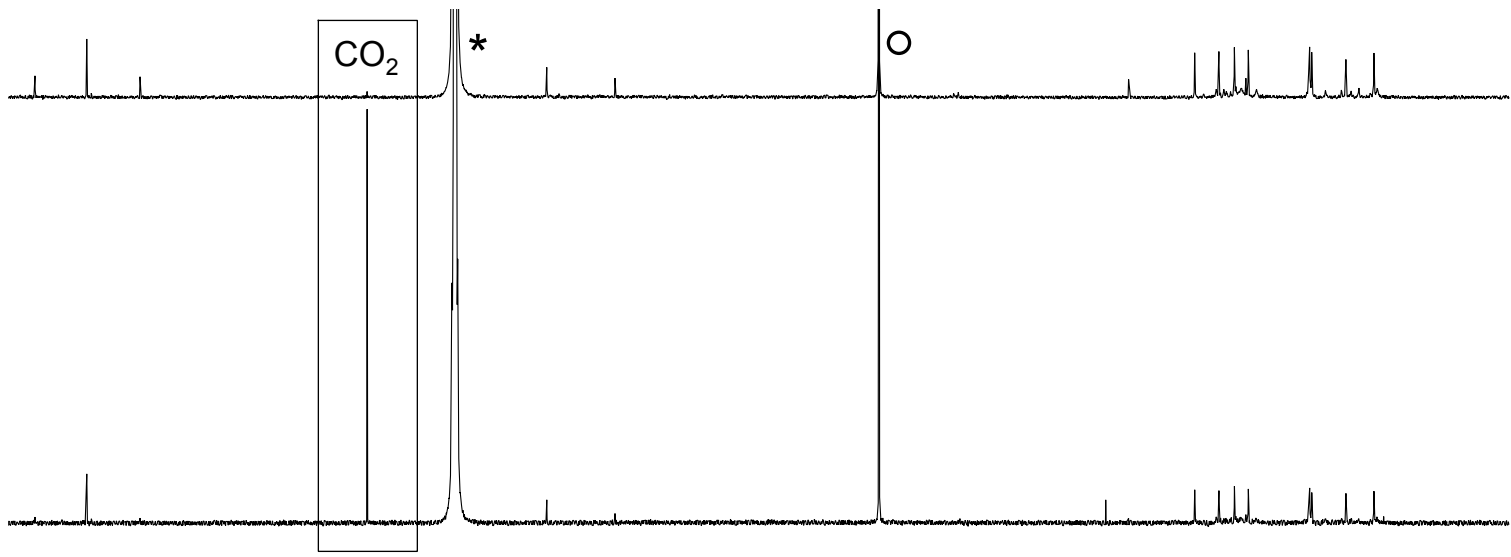

$\begin{array}{lllllllllll}150 & 140 & 130 & 120 & 110 & \begin{array}{c}100 \\ \delta / \mathrm{ppm}\end{array} & 90 & 70 & 60 & 50 & 40\end{array}$

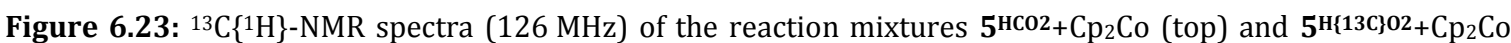
(bottom) in $\mathrm{CD}_{3} \mathrm{CN}$ at $238 \mathrm{~K}$ under inert conditions. The comparison shows that during the reaction $\mathrm{CO}_{2}$ is released.

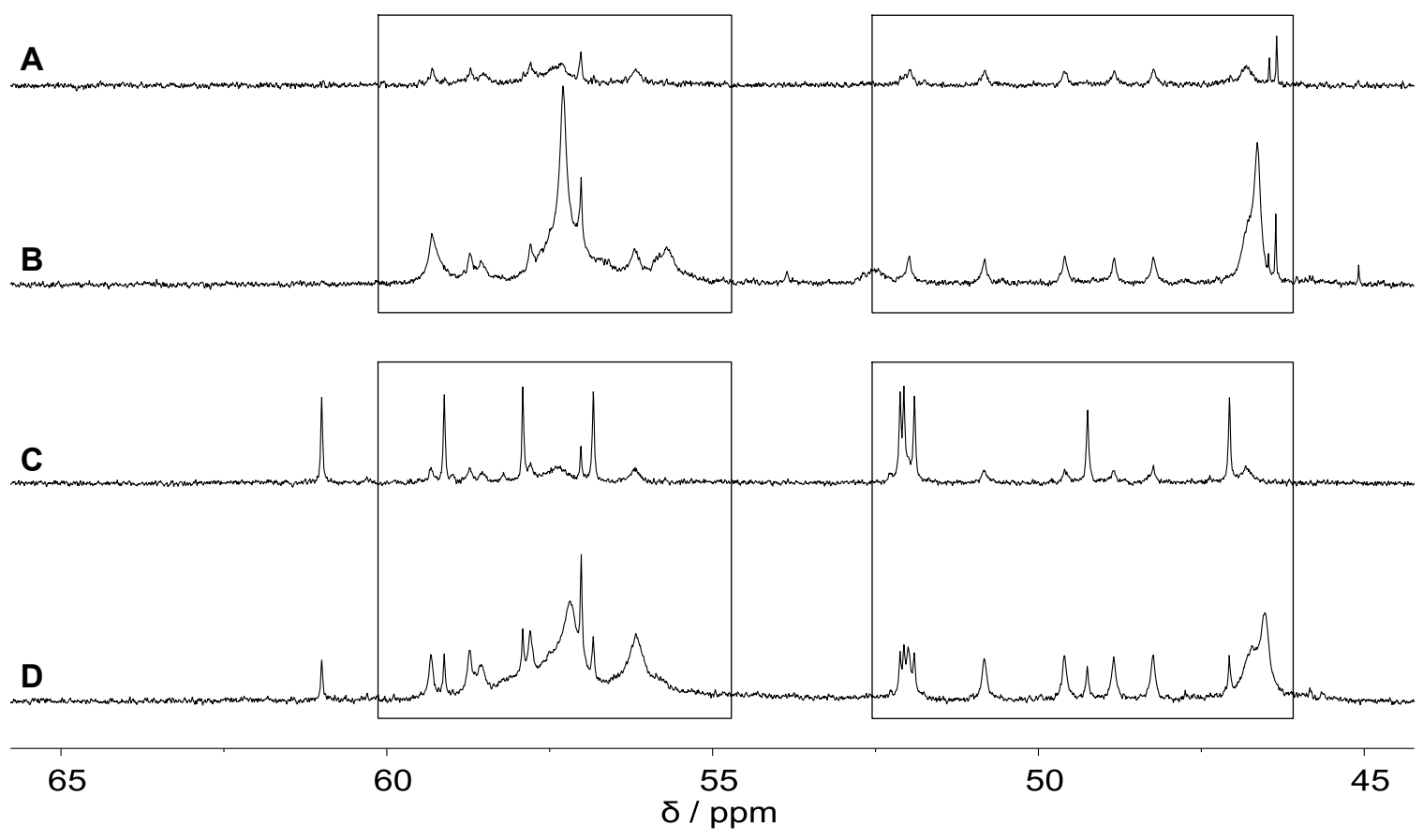

Figure 6.24: ${ }^{13} \mathrm{C}\left\{{ }^{1} \mathrm{H}\right\}-\mathrm{NMR}$ spectra $(126 \mathrm{MHz})$ in $\mathrm{CD}_{3} \mathrm{CN}$ at $238 \mathrm{~K}$ under inert conditions. A/B: Residue after crystallization of $\mathbf{9 H}^{\mathbf{H}}$ before (A) and after (B) addition of $\mathrm{HL}^{2 \mathrm{a}}$. C/D: Reaction mixture of $\mathbf{5}^{\mathbf{H C O}}+\mathrm{Cp}_{2}$ Co before (C) and after (D) addition of $\mathrm{HL}^{2 \mathrm{a}}$. The comparison shows that ligand is released during the reaction (rectangles). 
The reactivity observed upon reduction of $5^{\mathrm{HCO}}$ with cobaltocene is comparable to the decomposition of formic acid (FA) to $\mathrm{H}_{2}$ and $\mathrm{CO}_{2}$, which has received substantial attention with the intention of using FA as a hydrogen carrier/storage material.[419-423] Based on computational investigations, the decarboxylation of $\mathrm{FA}$, as well as $\mathrm{CO}_{2}$ hydrogenation, is assumed to proceed via one of two transition states (TS) as shown in Scheme 6.9. Whereas in one case, hydride abstraction occurs on one metal site with the other serving as an anchor, the reactivity is promoted by both metal ions in the $\mu$-H bridging TS. ${ }^{[424-432]}$ In each case, a rearrangement of the HCOO- moiety takes place.

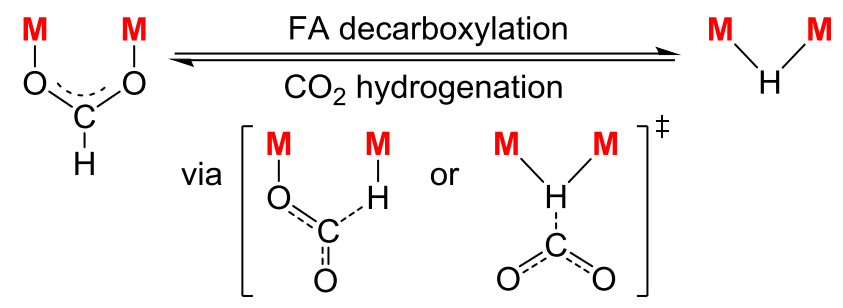

Scheme 6.9: Representation of formic acid (FA) decarboxylation and $\mathrm{CO}_{2}$ hydrogenation with possible transition states.

Previously, copper(II) formate clusters $\left[\mathrm{Cu}^{\mathrm{II}} \mathrm{n}\left(\mathrm{O}_{2} \mathrm{CH}\right)_{2 \mathrm{n}+1}\right]^{-}$were shown to decompose in the gas phase via decarboxylation associated with proton coupled electron transfer giving rise to metal reduction and $\mathrm{FA}$ formation or hydrogen radical dissociation; the resulting copper(I) formate clusters $\left[\mathrm{Cu}_{\mathrm{m}}^{\mathrm{I}}\left(\mathrm{O}_{2} \mathrm{CH}\right)_{\mathrm{m}+1}\right]^{-}(\mathrm{m}=1,2)$ subsequently decompose by decarboxylation, leading to copper(I) hydrides. ${ }^{[433]}$ Herein, the putative intermediate $\left[\left(\mathrm{L}^{2 \mathrm{a}}\right) \mathrm{Cu}_{2}{ }_{2}\left(\mathrm{O}_{2} \mathrm{CH}\right)\right]$, generated after twofold reduction of $\mathbf{5}^{\mathbf{H C O}}$, was found to spontaneously decarboxylate in solution. Since dicopper(I) complexes of bis(tetradentate) platforms akin to $\left(\mathrm{L}^{2 \mathrm{a}}\right)^{-}$have been shown to exist as species [ $\left.\mathrm{L}^{\prime} \mathrm{Cu}_{2}\right]^{+}$lacking an exogenous ligand within the bimetallic pocket (see Scheme 6.1), ${ }^{[188,380]}$ it is reasonable to assume that formate dissociation from $\left[\left(\mathrm{L}^{2 \mathrm{a}}\right) \mathrm{Cu}_{2}\left(\mathrm{O}_{2} \mathrm{CH}\right)\right]$ goes hand in hand with hydride transfer and $\mathrm{CO}_{2}$ elimination, likely via transition states such as the ones shown in Scheme 6.9. An alternative scenario could involve formate decarboxylation and hydride formation in a mixed valent $\left[\left(\mathrm{L}^{2 a}\right) \mathrm{Cu}^{\mathrm{I} C \mathrm{Cu}^{\mathrm{II}}}\left(\mathrm{O}_{2} \mathrm{CH}\right)\right]$ species, followed by further reduction. Details of the subsequent rearrangement to the hexacopper product $9^{\mathrm{H}}$ remain unclear.

Clearly, $\mathbf{9}^{\mathbf{H}}$ does not react with $\mathrm{CO}_{2}$ in solution from decomposition of $\mathbf{5}^{\mathbf{H C O}}$, but also no reaction occurs when $9^{\mathrm{H}}$ is exposed to one atmosphere of $\mathrm{CO}_{2}$. Furthermore, addition of 2 eq FA to $9^{\mathrm{H}}$ yields only minor traces of $\mathrm{H}_{2}$ after heating to $65^{\circ} \mathrm{C}$ for 3.5 hours (appendix, Figure A.96), also after addition of excess $\mathrm{H}_{2} \mathrm{O}(>100 \mathrm{eq})$. In fact, $\mathbf{9}^{\mathbf{H}}$ remains stable for over one week in the presence of water. To test whether $9^{\mathbf{H}}$ can be used for catalytic formate dehydrogenation under more extreme conditions, an excess of FA (15-26 eq) was added to $9^{\mathbf{H}}$ both in the presence and absence of NEt 3 (0.4 eq vs. FA, for details see experimental section). The solution was heated to $65{ }^{\circ} \mathrm{C}$ for several hours and the reaction progress monitored by NMR spectroscopy. The product mixture was additionally analyzed by ESI mass spectrometry after the experiment. As observed in the ${ }^{1} \mathrm{H}$ - and ${ }^{13} \mathrm{C}-\mathrm{NMR}$ spectra both $\mathrm{H}_{2}$ and $\mathrm{CO}_{2}$ are formed, however only in small amounts (appendix, Figures A.97 and A.98). Additionally, both NMR and ESI-MS indicate that the hexanuclear copper core of $2^{\mathbf{H}}$ is not retained under these conditions but dinuclear species such as $\left[\left(\mathrm{L}^{2 \mathrm{a}}\right) \mathrm{Cu}_{2}\left(\mathrm{O}_{2} \mathrm{CH}\right)_{2}\right]^{+}$and $\left[\left(\mathrm{L}^{2 \mathrm{a}}\right) \mathrm{Cu}_{2}\left(\mathrm{O}_{2} \mathrm{CH}\right)\right]^{2+}$ (cation of $\mathbf{5}^{\mathrm{HCO}}$ ) are the dominant ones according to ESI-MS (appendix, Figures A.49 and A.50).

\subsection{Reduction of $6^{\mathrm{HCO} 2}$ to a Dicopper(I) Complex}

In order to clarify if the appearance of a hexanuclear hydride complex is unique for the reduction of the $\mathrm{Me}_{2}$-tacn compound $\mathbf{5}^{\mathbf{H C O}}$, the equivalent reaction was conducted for the isopropyl analogue $6^{\mathbf{H C O}}$. Indeed, upon addition of either decamethylcobaltocene (2.0 eq) or cobaltocene (2.3 eq) to an acetonitrile solution of $\mathbf{6}^{\mathbf{H C O}}$, no peak corresponding to hydride moieties was observed in the high- 
field region of the ${ }^{1} \mathrm{H}-\mathrm{NMR}$ spectrum (Figure 6.25). Instead, a spectrum identical to the one of the known dicopper(I) compound $\mathbf{6}^{\mathrm{I} / \mathrm{I}}$ was obtained. ${ }^{[434]}$ Apart from the complex signals, a peak at $8.71 \mathrm{ppm}\left({ }^{13} \mathrm{C}\right.$-NMR: $\left.166.5 \mathrm{ppm}\right)$ could be assigned to free formate, which is released upon reduction of $6^{\mathrm{HCO}}$ to $\mathbf{6}^{\mathrm{I} / \mathrm{I}}$ (Scheme 6.10).

In line with these observations, it seems that both the formate molecule in the bimetallic cleft, as well as the methyl residues at the tacn moiety are required for the occurrence of the oligonuclear copper(I) hydride complex $9^{\mathrm{H}}$. The bulkier isopropyl residues in $6^{\mathrm{HCO2}}$ likely prevent the presence of two $\left(\mathrm{L}^{2 \mathrm{~b}}\right)^{-}$ligands in sufficiently close proximity, thus impeding the formation of an $\left[\left(\mathrm{L}^{2 \mathrm{~b}}\right)_{2} \mathrm{Cu}_{6}\right]$ species.

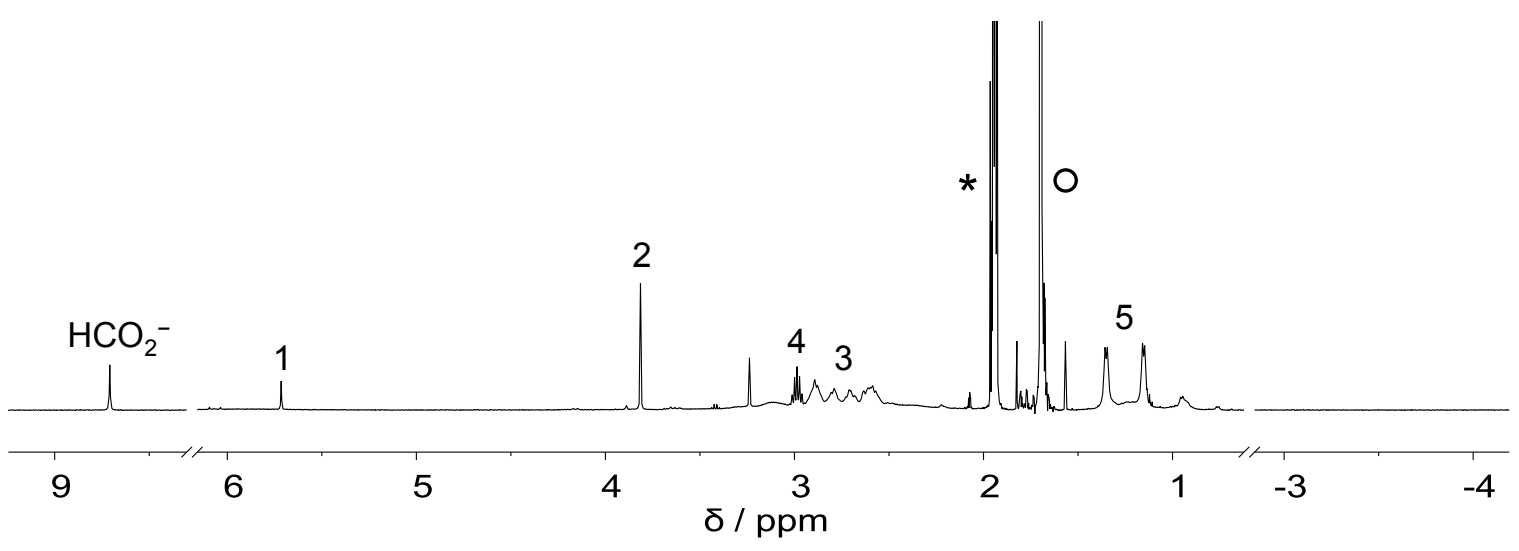

Figure 6.25: ${ }^{1} \mathrm{H}-\mathrm{NMR}$ spectrum $(500 \mathrm{MHz})$ of $6^{1 / 1} \mathrm{in} \mathrm{CD}_{3} \mathrm{CN}$ at room temperature under inert conditions. All signals are assigned according to the labeling scheme in Scheme 6.10 and in comparison to the known spectrum. ${ }^{[434]}$ The asterisk denotes the residual solvent peak, the circle points out $\left[\left(\mathrm{Cp}^{*}\right)_{2} \mathrm{Co}\right]^{+}$.

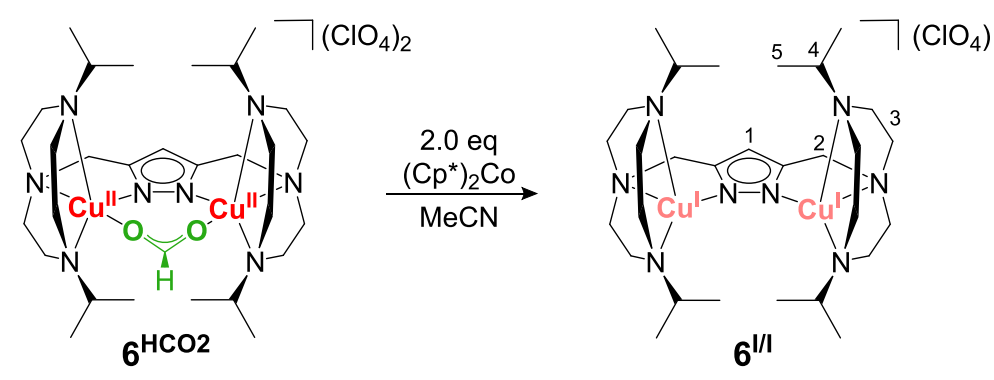

Scheme 6.10: Twofold reduction of $6^{\mathrm{HCO}}$ to the $\mathrm{Cu}^{\mathrm{I} C \mathrm{Cu}^{\mathrm{I}}}$ species $6^{\mathrm{I} / \mathrm{I}}$.

\subsection{Summary and Conclusion ${ }^{\mathrm{xxv}}$}

This chapter entails novel findings in redox transformations among the family of dicopper complexes bearing pyrazolate/tacn hybrid ligand systems. Interestingly, reduction of the dicopper(II) complexes $5^{\mathrm{OAc}}, \mathbf{5}^{\mathrm{HCO}}, \mathbf{6}^{\mathbf{O A c}}$ and $\mathbf{6}^{\mathrm{HCO}}$ results in the formation of very different products. Whereas the addition of cobaltocene to $\mathbf{5}^{\mathbf{O A c}}, \mathbf{6}^{\mathbf{O A c}}$ and $\mathbf{6}^{\mathrm{HCO2}}$ yields the expected dicopper(I) compounds, the reduction of the $\mu_{1,3}$-formate complex $\mathbf{5}^{\mathbf{H C O} 2}$ triggers rapid elimination of $\mathrm{CO}_{2}$ to give an oligonuclear copper(I) hydride cluster $\left(9^{\mathrm{H}}\right)$. Isotopic labeling experiments confirmed that the hydride ligands as well as the $\mathrm{CO}_{2}$ originate from the $\mu_{1,3}$-formate. This transformation is accompanied by major reshuffling of metal ions and ligands, as the starting complex with an $\left(\mathrm{L}^{2 \mathrm{a}}\right)^{-}: \mathrm{Cu}$ ratio of 1:2 yields a

xxxv Parts of this section were adapted from Jana Lücken, Thomas Auth, Sara Ida Mozzi, Franc Meyer, "Hexanuclear Copper(I) Hydride from the Reduction-Induced Decarboxylation of a Dicopper(II) Formate", Inorg. Chem. 2020, DOI: 10.1021/acs.inorgchem.0c02126. 
product with an $\left(\mathrm{L}^{2 a}\right)^{-}: \mathrm{Cu}$ ratio of 2:6 besides some free ligand, yet the reaction is rather clean according to NMR spectroscopy, and the full reaction equation could be determined. At this point, it is not clear whether formate decarboxylation and hydride abstraction occurs via a transient $\left[\left(\mathrm{L}^{2 a}\right) \mathrm{Cu}_{2}{ }_{2}\left(\mu-\mathrm{O}_{2} \mathrm{CH}\right)\right]$ species or a mixed-valent $\left[\left(\mathrm{L}^{2 \mathrm{a}}\right) \mathrm{Cu}^{\mathrm{I}} \mathrm{Cu}^{\mathrm{II}}\left(\mu-\mathrm{O}_{2} \mathrm{CH}\right)\right]$ species followed by further reduction.

$9^{\mathrm{H}}$ contains two $\mathrm{Cu}_{3}\left(\mu_{3}-\mathrm{H}\right)$ subunits spanned by two pyrazolate bridges of the compartmental ligand scaffold ( $\left.\mathrm{L}^{2 \mathrm{a}}\right)^{-}$. It exhibits typical hydride reactivity as demonstrated by reaction with phenylacetylene, giving the hexacopper(I) complex $\mathbf{9}^{\mathbf{C} 8 \mathrm{H} 5}$ in which the core of two pyrazolate-bridged $\mathrm{Cu}_{3}$ subunits is retained, but the latter are now capped by phenylacetylide ligands. DFT calculations revealed a rich structural landscape for both $\mathbf{9}^{\mathbf{H}}$ and $\mathbf{9}^{\mathbf{c} 8 \mathrm{H} 5}$ since both hexametallic complexes can exist as different isomers that are quite close in energy, and dynamic behavior in solution was evidenced by VT-NMR spectroscopy. However, $9^{\mathrm{H}}$ does not react with $\mathrm{CO}_{2}$ present in solution or with one atmosphere of added $\mathrm{CO}_{2}$, indicating that its thermodynamic hydride donor ability is attenuated, which is likely due to the dicationic nature of the complex ion $\left[\left(\mathrm{L}^{2 \mathrm{a}}\right)_{2} \mathrm{Cu}_{6}(\mathrm{H})_{2}\right]^{2+}$.

In combination with the recent report of an $\left[\left(\mathrm{L}^{\mathrm{XXVIII}}\right)_{2} \mathrm{Cu}_{5}(\mathrm{H})_{2}\right]^{3+}$ complex ( $\mathrm{L}^{\mathrm{XXVII}}$ is a compartmental naphthyridine ligand) that mediates catalytic FA dehydrogenation, ${ }^{[389]}$ the present findings support the view that (i) dinucleating polydentate $\mathrm{N}$-donor scaffolds can serve as suitable platforms for stabilizing a variety of novel multicopper hydride clusters, and (ii) that such cationic $\mathrm{N}$-ligated copper(I) systems may exhibit a favorably low hydricity for promoting formate decarboxylation. Most copper hydrides have been reported so far to react in the reverse direction, viz. as strong hydride donors towards $\mathrm{CO}_{2}$, and are neutral phosphine- or NHC-ligated species,[391,435-437] obtained using silane or borane reagents. ${ }^{[390,391]}$ This work now offers a convenient entry into N-donor based copper(I) hydride chemistry from easily accessible and stable dicopper(II) formate complexes.

Even though addition of cobaltocene to $\mathbf{5}^{\mathbf{0 A c}}$ and $\mathbf{6}^{\mathbf{0 A c}}$ ultimately yields the same product, viz. a dicopper(I) complex, significant differences are also observed here. Whereas the twofold reduction of $5^{\text {OAc }}$ proceeds via the mixed-valent intermediate $5^{1 / I I}, 6^{\mathbf{A c}}$ is directly reduced to $6^{\mathbf{1} / \mathbf{I}}$. The appearance of the $\mathrm{Cu}^{\mathrm{I}} \mathrm{Cu}^{\mathrm{II}}$ species $\mathbf{5}^{\mathrm{I} / \mathrm{II}}$ was observed both via UV/vis and EPR spectroscopy. Furthermore, timeresolved $\mathrm{UV} /$ vis spectra collected with a stopped-flow setup at $-40^{\circ} \mathrm{C}$, revealed the formation of the superoxo complex $\mathbf{5}^{\mathbf{S}}$ as primary $\mathrm{Cu}_{2} \mathrm{O}_{2}$ adduct upon addition of dry dioxygen to $\mathbf{5}^{\mathbf{I} / I I}$. This was not observed when $\mathbf{5}^{\mathbf{I} / \mathbf{I}}$ or an acetonitrile solution of $\mathbf{6}^{\mathbf{O A c}}$ and $1.2 \mathrm{eq} \mathrm{Cp}_{2} \mathrm{Co}$ (conditions under which a mixed-valent species could be formed) were exposed to an atmosphere of dry $\mathrm{O}_{2}$. Instead, the typical spectroscopic features of the respective peroxo complexes were formed.

The generation of a mixed-valent $\mathrm{Cu}^{\mathrm{I}} \mathrm{Cu}^{\mathrm{II}}$ complex is extraordinary for dicopper compounds based on pyrazolate/tacn hybrid ligands. This unique observation likely arises from the $\mu_{1,3}$-acetate moiety in the starting material $\mathbf{5}^{\mathbf{O A c}}$, which presumably changes its coordination mode from a bridging to a chelating fashion upon single reduction of $\mathbf{5}^{\mathbf{O A c}}$, thus stabilizing the $\mathrm{Cu}^{\mathrm{III}}$ site in $\mathbf{5}^{\mathbf{1} / \mathrm{II}}$, which requires additional ligation to the $\left\{\mathrm{N}_{4}\right\}$ donor set from the dinucleating ligand.

The superoxo complex $\mathbf{5}^{\mathrm{S}}$ is short-lived and rapidly converts to $\mathbf{5}^{\mathrm{P}}$. This conversion can take place via two possible routes: either a second $\mathbf{5}^{\mathbf{s}}$ compound or an unreacted mixed-valent species $\mathbf{5}^{\mathbf{I} / \mathbf{I I}}$ acts as reducing agent. Based on various observations, it seems likely that the former takes place in the presence of large amounts of dioxygen and the latter occurs at low $\mathrm{O}_{2}$ concentrations. In both cases, a dicopper(II) complex is formed as side-product ( $5^{\mathbf{O A c}}$ balances the equation). The short lifetime and, concomitantly, presumable high reactivity of the oxygen species bearing the ( $\left.\mathrm{L}^{2 \mathrm{a}}\right)^{-}$ligand make them promising candidates for oxidation/oxygenation reactions. The dioxygen activated species of $\mathbf{5}$ thus join the ranks of copper-oxygen complexes based on pyrazolate/tacn hybrid ligands. The reactivity of these complexes seems to depend on the accessibility of the dioxygen derived ligand in the bimetallic cleft, which is influenced by the linker $\left(\mathrm{CH}_{2}\right.$ vs. $\left.\mathrm{CH}_{2} \mathrm{CH}_{2}\right)$ between the pyrazolate and tacn sidearms as well as the periphery (Me vs. $i \mathrm{Pr}$ ) of the tacn moieties. Accordingly, this is a great example of how targeted minor changes in the ligand scaffold can elicit different reactivities, which has also been observed for the respective diiron complexes.[206] 


\section{Summary and Perspective}

In this work, a range of transition metal complexes of ruthenium, copper and cobalt were synthesized, thoroughly characterized and investigated in regard to the small molecule activation and conversion of both $\mathrm{H}_{2} \mathrm{O}$ and $\mathrm{O}_{2}$.

In the first part, an in-depth analysis of two highly water-soluble diruthenium WOCs is presented, both complexes bearing the pyrazolate/carboxylate hybrid ligand $\mathrm{H}_{3} \mathrm{~L}^{1}$ (Scheme 7.1), which was previously shown to produce a catalyst with an impressive catalytic efficiency but poor water solubility in combination with axial pyridine ligands $\left(\mathbf{X}^{\mathbf{H 2 O}}\right)$. Solubility in aqueous media could be achieved by replacing the pyridine moieties of the parent complex by pyridine-3-sulfonate and initially yielded $3^{\text {dmso }}$ with an S,O-bridging DMSO molecule in the in,in-position, which could be replaced by two $\mathrm{H}_{2} \mathrm{O}$ ligands under basic conditions $\left(3^{\mathbf{H 2 O}}\right.$ ). Both compounds were successfully employed in chemical (ceric ammonium nitrate, CAN, as oxidant), electrochemical and photo-driven water oxidation (sodium persulfate as sacrificial electron acceptor and a $\left[\mathrm{Ru}(\mathrm{bpy})_{3}\right]^{2+}$-derivative as photosensitizer). The electrocatalytic proficiency of $3^{\mathbf{H} 20}$ was further investigated by foot-of-the-wave analysis. The initial turnover frequencies obtained for $3^{\mathrm{dmso}}$ and $\mathbf{3}^{\mathrm{H} 20}$ during chemical water oxidation compare favorably to the related pyrazolate-based catalysts $\mathbf{V}^{\mathbf{H} 20}$ and $\mathbf{V I}^{\mathbf{H 2 O}}$, which lack the peripheral carboxylates, but are nearly an order of magnitude lower than for the parent complex $\mathbf{X}^{\mathbf{H 2 0}}$. Furthermore, catalysis of $3^{\text {dmso }}$ is decelerated by nitrate and Ce ${ }^{\text {III }}$ released during CAN-driven water oxidation.

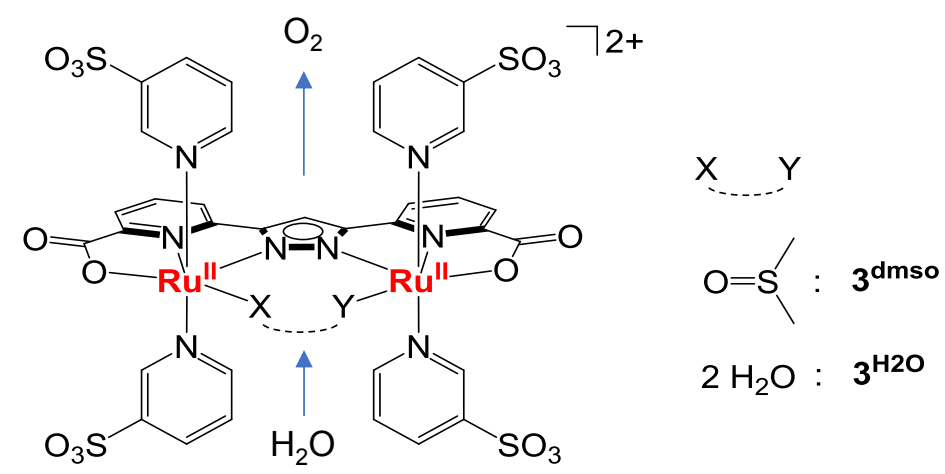

Scheme 7.1: Representation of the two diruthenium WOCs based on the pyrazolate/carboxylate hybrid ligand $\mathrm{H}_{3} \mathrm{~L}^{1}$ investigated in this work.

Mechanistic insight could be gained for $3^{\text {dmso }}$ and $3^{\text {н20 }}$ on the basis of UV/visspectroelectrochemical (SEC) experiments and the construction of a Pourbaix diagram, respectively. In the latter case, the Pourbaix diagram provides information on the proton-coupled electron transfer steps upon oxidation of $3^{\mathbf{H} 20}$, eventually yielding high-valent ruthenium-oxo units. Here, a $\left[\mathrm{Ru}^{\mathrm{IV}}=\mathrm{O} \mathrm{O}=\mathrm{Ru}^{\mathrm{V}}\right]^{2-}$ species is suggested to be reactive towards $\mathrm{O}-\mathrm{O}$ bond formation. In the case of $\mathbf{3}^{\mathrm{dmso}}$, the UV/vis-SEC analysis surprisingly indicates that the DMSO moiety remains in the bimetallic pocket 
during both electrochemical and chemical water oxidation at least for a few turnovers. As an aquo ligand is required for catalysis, this opens up three possible scenarios: (i) partial decoordination of the DMSO ligand, (ii) replacement of one carboxylate moiety with an aquo group, and (iii) expansion of the coordination environment at one ruthenium center from six to seven. In all three cases, the catalytically active species of $\mathbf{3}^{\mathrm{dms}}$ only possesses one aquo ligand, which, along with the determined first-order dependence of the catalysis on complex concentration, implies that $0-0$ bond formation proceeds via a water nucleophilic attack mechanism. Altogether, the insights gained for $\mathbf{3}^{\text {dmso }}$ emphasize that a participation of DMSO ligands during water oxidation catalysis by ruthenium-based WOCs should be taken into account in future studies. Prospective work in the context of $\mathbf{3}$ could focus on its immobilization on conducting solid support by introducing anchoring groups at the axial pyridine moieties or the ligand backbone.

With the objective of designing new water oxidation catalysts based on first-row transition metals and inspired by preceding investigations in the Meyer group, a series of dinuclear copper and cobalt complexes bearing the pyrazolate/tacn hybrid ligand scaffolds $\mathrm{HL}^{2 \mathrm{a}}(\mathrm{R}=\mathrm{Me})$ and $\mathrm{HL}^{2 \mathrm{~b}}(\mathrm{R}=i \mathrm{Pr})$ was synthesized (Scheme 7.2). Of these, the formate-bridged dicopper compound $\mathbf{5}^{\mathrm{HCO}}$ and the MeCNcoordinated dicobalt complex $7^{\mathrm{MeCN}}$ were investigated in regard to their ability to electrocatalytically oxidize water to dioxygen. In both cases, a fast degradation of the complex was observed, leading to the formation of a reactive deposit on the electrode surface, which was responsible for the catalysis (proved by rinse tests). For $\mathbf{5} \mathbf{H C O 2}$, the deposited material was identified via X-ray photoelectron spectroscopy as a copper oxide or hydroxide species (Scheme 7.2). The multiple methylene groups in $\left(\mathrm{L}^{2 \mathrm{a}}\right)^{-}$are most likely the weak spots, and are presumably oxidized under the harsh reaction conditions. Accordingly, future base-metal complexes intended for water oxidation should exclude such labile groups in ligand scaffolds.

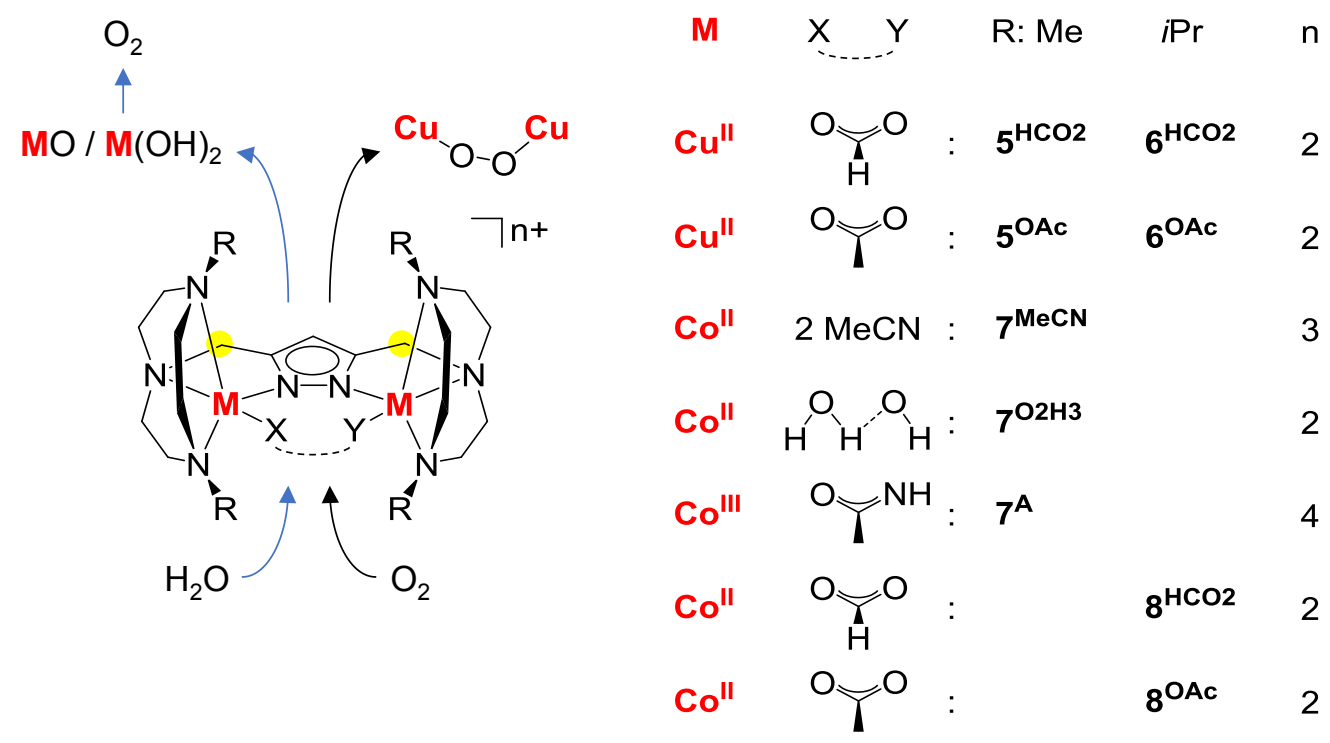

Scheme 7.2: Overview of the dinuclear copper and cobalt complexes bearing the pyrazolate/tacn hybrid ligands $\mathrm{HL}^{2 \mathrm{a}}$ and $\mathrm{HL}^{2 \mathrm{~b}}$ synthesized within this thesis.

Nevertheless, the dinuclear first-row transition metal compounds feature interesting properties. For example, SMM behavior, which is extremely rare for homodinuclear cobalt(II) species, was observed for the dicobalt complexes $\mathbf{8}^{\mathrm{HCO}}$ and $\mathbf{8}^{\mathrm{OAc}}$. Furthermore, the highly preorganized systems for coordination of two metal ions in close proximity could be a valuable platform for other types of catalysis beyond water oxidation, e.g. $\mathrm{CO}_{2}$ or $\mathrm{O}_{2}$ reduction, where cooperativity between two metal ions is desired. Indeed, dicopper(I) complexes based on pyrazolate/tacn hybrid ligands (methylene or ethylene linker, highlighted in yellow in Scheme 7.2; $\mathrm{R}=i \mathrm{Pr}$ ) have already been established for dioxygen activation in the Meyer group, and the reactivity of the respective $\mathrm{Cu}_{2} \mathrm{O}_{2}$ adducts was 
suggested to depend on the accessibility of the dioxygen derived ligand for substrates. Herein, it could be shown that replacing the isopropyl residues at the triazacyclononane macrocycles (methylene linker) with less bulky methyl groups, affords highly unstable, and thus presumably highly reactive, copper-oxygen species $\left(\mathbf{5}^{\mathbf{P}}\right.$ and $\mathbf{5}^{\mathbf{S}}$, see below), supporting this notion. Respective oxidation/oxygenation studies with organic substrates should be pursued in the future.

As the dicopper(I) complex $\mathbf{5}^{\mathbf{I} / \mathbf{I}}$ was synthetically not accessible, a different route, viz. reduction of dicopper(II) species to form $\mathbf{5}^{\mathbf{1} / \mathbf{I}}$ in situ, had to be chosen to obtain $\mathrm{Cu}_{2} \mathrm{O}_{2}$ adducts. Interestingly, stepwise reduction of $\mathbf{5}^{\mathbf{O A c}}$ gives rise to a mixed-valent $\mathrm{Cu}^{\mathrm{I}} \mathrm{Cu}^{\mathrm{II}}$ intermediate $\left(\mathbf{5}^{\mathrm{I} / \mathrm{II}}\right.$, identified by $\mathrm{UV} / \mathrm{vis}$ and EPR spectroscopy), which is unprecedented for this ligand platform. It seems likely that reorganization of the acetate ligand from a bridging to a chelating fashion stabilizes the $\mathrm{Cu}^{\mathrm{II}}$ site, enabling the formation of this unique complex. Further addition of $\mathrm{Cp}_{2} \mathrm{Co}$ then yields $\mathbf{5}^{\mathbf{1} / \mathbf{I}}$. The primary dioxygen adduct of $5^{\mathrm{I} / \mathrm{I}}$ is a $\mu$-1,2-peroxo species $\left(5^{\mathrm{P}}\right)$, whereas the addition of dry $\mathrm{O}_{2}$ to $5^{\mathrm{I} / \mathrm{II}}$ initially yields a superoxo complex $\left(\mathbf{5}^{\mathrm{S}}\right)$, as shown by their characteristic UV/vis features. The latter is so shortlived that it was only observed with a low-temperature stopped-flow UV/vis instrument and rapidly converts to $\mathbf{5}^{\mathrm{P}}$ either being reduced by a second $\mathrm{Cu}^{\mathrm{I}} \mathrm{Cu}^{\mathrm{II}}$ species or another superoxo complex. The low stability of the copper-oxygen complexes of $\mathbf{5}$ prevented more thorough investigations.

Intriguing results were also obtained for the reduction of $\mathbf{5}^{\mathbf{H C O}}$. Instead of the expected dicopper(I) compound, an oligonuclear copper(I) hydride cluster $\left(9^{\mathrm{H}}\right)$ is formed after $\mathrm{CO}_{2}$ release and major reshuffling of ligands and metal ions (Scheme 7.3). By means of labeling studies, the origin of both the hydrides and $\mathrm{CO}_{2}$ could be traced back to the formate molecule in the in,in-position of $5^{\mathbf{H C O}}$, resembling the dehydrogenation of formic acid. Typical hydride reactivity of $\mathbf{9}^{\mathrm{H}}$ was demonstrated by addition of phenylacetylene, leading to a replacement of the hydride ligands with phenylacetylide moieties while retaining the $\left[\left(\mathrm{L}^{2 \mathrm{a}}\right)_{2} \mathrm{Cu}_{6}\right]$ core $\left(\mathbf{9}^{\mathrm{C} 8 \mathrm{H}}\right)$. For both $\mathbf{9}^{\mathrm{H}}$ and $\mathbf{9}^{\mathbf{C} 8 \mathrm{H} 5}$, a rich structural landscape was evidenced by DFT calculations (four isomers each that are quite close in energy), which is consistent with the dynamic behavior monitored by variable temperature NMR spectroscopy. Overall, it has been shown that easily accessible and stable dicopper(II) formate compounds can serve as an entry point into the chemistry of copper(I) hydride complexes bearing $\mathrm{N}$-donor ligands.

The observations upon reduction of both $\mathbf{5}^{\mathbf{0 A c}}$ and $\mathbf{5}^{\mathrm{HCO}}$ were found to be unique for the complexes based on $\left(\mathrm{L}^{2 \mathrm{a}}\right)^{-}(\mathrm{R}=\mathrm{Me})$ as confirmed by control experiments with $\mathbf{6}^{\mathrm{OAc}}$ and $\mathbf{6}^{\mathrm{HCO}}$ bearing $\left(\mathrm{L}^{2 \mathrm{~b}}\right)^{-}$ $(\mathrm{R}=i \mathrm{Pr})$, suggesting that steric hindrance is too high with isopropyl periphery at the tacn moieties to accommodate the respective coordination motifs.

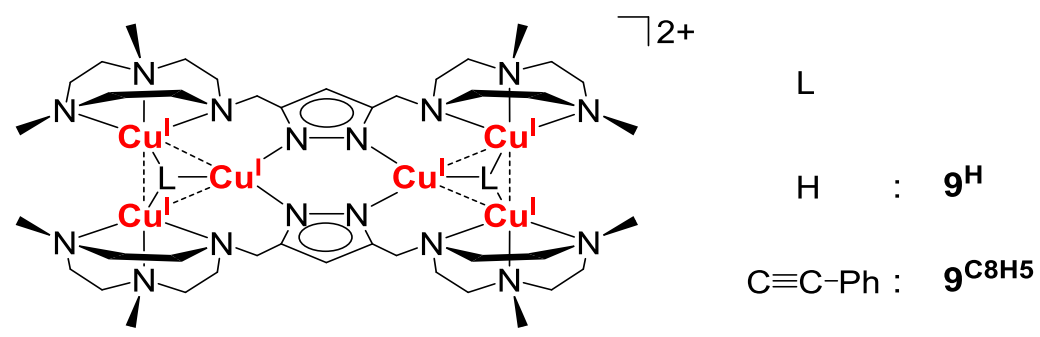

Scheme 7.3: Representation of the hexacopper(I) complexes obtained within the scope of this work. 


\section{8}

Experimental Section

\subsection{Materials and Methods}

\subsubsection{Materials}

$\mathrm{RuCl}_{2}$ (dmso) ${ }_{4}^{[438,439]}, \mathrm{H}_{3} \mathrm{~L}^{1[204,232,233]}$ and $\mathrm{HL}^{2 \mathrm{a} / \mathrm{b}[275-279]}$ were prepared according to literature procedures.

High purity deionized water was obtained by using an Aquatron A4000D water still (Bibby Scientific). Trifluoromethanesulfonic acid (99+\%), triflic acid or HOTf, was purchased from STREAM/CYMIT (packed in ampoules) and ceric ammonium nitrate (CAN) for catalysis experiments was used from ABCR (99\% ACS grade). All other reagents were purchased from Aldrich Chemical Co., ABCR, Fluka, Merck, Macherey-Nagel and ACROS Organics, and used without further purification. The utilized solvents were Chromasolve HPLC or puriss p.a. grade. Selected solvents were dried according to common procedures, distilled under a nitrogen atmosphere and degassed prior to use.

The synthesis of oxidation- and hydrolysis-sensitive substances was performed under an atmosphere of dry nitrogen by using standard Schlenk techniques or in a glovebox. Therefore, the utilized nitrogen gas was previously dried over phosphorous pentoxide on inert base material (SICAPENT® Merck) and removed from all oxygen traces by means of a copper catalyst. All glassware was dried at $120^{\circ} \mathrm{C}$ overnight before use.

\subsubsection{NMR Spectroscopy}

Nuclear magnetic resonance (NMR) spectra were recorded on Bruker Avance III 300, Bruker Avance III HD 400 and Bruker Avance III HD 500 instruments. The chemical shifts $\delta$ are given against tetramethylsilane. The residual proton signal of the respective commercially available deuterated solvent was applied as an internal standard ( $\mathrm{D}_{2} \mathrm{O} 4.790$ (1), DMF- $d_{7} 2.750$ (5), MeOD- $d_{4} 3.310$ (5), $\mathrm{CD}_{3} \mathrm{CN} 1.940$ (5)). The following abbreviations were used for the multiplicities: $\mathrm{s}=$ singlet, $\mathrm{d}=\mathrm{doublet}$, $\mathrm{t}=$ triplet, $\mathrm{m}=$ multiplet. $J$ denotes the coupling constant.

\subsubsection{Elemental Analysis}

Elemental analyses were conducted in the Analytical Laboratory of the Institute of Inorganic Chemistry at the University of Göttingen via an Elementar 4.1 Vario EL 3 instrument.

\subsubsection{IR Spectroscopy}

Infrared (IR) spectra were recorded on Bruker Alpha Platinum-ATR (solids) and Thermo Science Nicolet iZ10 (KBr pellets) instruments and evaluated with the software OPUS 7.8 and OMNIC 9.2, respectively. The positions of the bands are given in wavenumbers $\left(\mathrm{cm}^{-1}\right)$. For the band intensities, the following abbreviations were applied: $\mathrm{s}=$ strong, $\mathrm{m}=$ medium, $\mathrm{w}=$ weak.

\subsubsection{UV/vis Spectroscopy}

UV/vis-spectra of solutions were recorded on Varian CARY 5000 and Agilent Cary 60 instruments. Quartz cuvettes of 0.1 or $1.0 \mathrm{~cm}$ thickness were applied. Shoulders are denoted by sh. 


\subsubsection{EPR Spectroscopy}

Electron paramagnetic resonance (EPR) spectra were measured using a Bruker E500 ELEXSYS X-band spectrometer equipped with a standard cavity (ER4102ST, $9.45 \mathrm{GHz}$ ). By means of an Oxford instrument Helium flow cryostat (ESP910) and an Oxford temperature controller (ITC-4) the sample temperature was maintained constant. The microwave frequency was measured with the built-in frequency counter and the magnetic field was calibrated by using an NMR field probe (Bruker ER035M). The EPR spectra were simulated with the software easy spin or the SX Module from esim.SL (author: E. B., program available via mail to ebill@gwdg.de). To obtain an average from anisotropic $g$ values the following formula was applied:

$$
g_{\text {average }}=\sqrt{\frac{g_{1}^{2}+g_{2}^{2}+g_{3}^{2}}{3}}
$$

\subsubsection{ESI Mass Spectrometry}

Electrospray ionization (ESI) mass spectra were recorded with a Bruker HCTUltra (Ion Trap). M indicates the complex part of the molecule.

\subsubsection{Luminescence Measurements}

Steady-state luminescence experiments were performed using a Fluorolog-3 instrument from Horiba Jobin-Yvon with a Xenon short arc lamp (Ushio Inc.) equipped with a TBX picosecond photon detection module (Horiba Jobin-Yvon). Time-resolved measurements were conducted using a NanoLED-340 pulsed light source (Horiba Jobin-Yvon) and a TCSPC (time correlated single photon counting) module (Fluorohub FL-1061PC) as a support for the TBX picosecond photon detector. All measurements were conducted at room temperature in dry and degassed MeCN. Luminescence decays were fitted with Origin Pro 8.5 from OriginLab according to the equation:

$$
\mathrm{y}=\mathrm{y}_{0}+\mathrm{A} \cdot \exp \left(-\frac{\left(\mathrm{x}-\mathrm{x}_{0}\right)}{\tau}\right)
$$

\subsubsection{X-ray Photoelectron Spectroscopy}

XPS experiments were conducted at the Catalan Institute of Nanoscience and Nanotechnology in Barcelona, Spain, at the Institute for Solar Fuels of the Helmholtz Zentrum Berlin or at the Physics Institute of the Georg-August-University Göttingen. The measurements were performed at room temperature with a SPECS PHOIBOS 100 or 150 hemispherical XPS analyzer (SPECS GmbH, Berlin, Germany) or an Axis Supra photoelectron spectrometer (Kratos Analytical, UK) in a base pressure of $5 \cdot 10^{-10}$ mbar with a monochromatic X-ray source (SPECS FOCUS 500 monochromator, Al Ka radiation, $1486.74 \mathrm{eV}$ ). Survey and fine spectra were collected at a normal angle from the surface. The operating voltage was $15 \mathrm{keV}$. The pass energy was set to $30 \mathrm{eV}$ and $10 \mathrm{eV}$ for survey and fine spectra, respectively, with step sizes of $0.5 \mathrm{eV}$ and 0.1 or $0.05 \mathrm{eV}$, respectively. The powder samples were either deposited on carbon tape to cover a minimum of $0.5 \times 3.5 \mathrm{~cm}$ area or drop-casted on glassy carbon or gold surfaces. For the electrochemically deposited samples, a glassy carbon disc of approximately $1 \times 1 \mathrm{~cm}$ was nearly fully covered, leaving a small area free where the crocodile clamp was attached for the electrochemical measurement.

\subsubsection{Magnetic Measurements}

Temperature-dependent magnetic susceptibilities were measured using a SQUID magnetometer (Quantum Design MPMS-XL-5) equipped with a $5 \mathrm{~T}$ magnet. For these measurements, the freshly ground crystalline sample was contained in a gelatin capsule, in case of cobalt complexes fixed with polyfluorinated oil, and placed in a non-magnetic sample holder. Each raw data file for the magnetic moment was corrected for the diamagnetic contribution of the gelatin capsule according to $M^{\text {dia }}$ (capsule $)=\chi_{\mathrm{g}} \cdot m \cdot H$, where $H$ is the external magnetic field, $m$ is the mass and $\chi_{\mathrm{g}}$ is the experimentally obtained gram susceptibility of the gelatin capsule. The molar susceptibility data was corrected according to $\chi_{m}(\mathrm{dia})=-1 / 2 \cdot M \cdot 10^{6} \mathrm{~cm}^{3} \mathrm{~mol}^{-1}$, where $M$ is the molar mass. ${ }^{[440]}$ Magnetic properties were simulated with the $j u l X$ program ${ }^{[41,442]}$ using a fitting procedure to the spin 
Hamiltonian under consideration of isotropic exchange interaction, Zeeman and zero-field splitting (for cobalt complexes):

$$
\widehat{H}=-2 J \hat{S}_{1} \cdot \hat{S}_{2}+g \mu_{\mathrm{B}} \sum_{\mathrm{i}} \vec{B} \cdot \overrightarrow{S_{l}}+D \sum_{\mathrm{i}}\left[\hat{S}_{\mathrm{zi}}^{2}-\frac{1}{3} S_{i}\left(S_{i}+1\right)\right]
$$

Where $J$ is the coupling constant, $S$ the spin, $B$ the magnetic field, $D$ the axial zero-field splitting parameter, and $\mu_{\mathrm{B}}$ the Bohr magneton. AC susceptility data was fitted using the Debye model. [355] To obtain an average from anisotropic $g$ values the following formula was applied:

$$
g_{\text {average }}=\sqrt{\frac{g_{1}^{2}+g_{2}^{2}+g_{3}^{2}}{3}}
$$

\subsubsection{Electrochemistry}

Cyclic voltammetry and square wave voltammetry measurements were performed using a potentiostat Metrohm Autolab PGSTAT101 or a Gamry Instruments Interface 1000 or 1010E. A three-electrode arrangement was applied with either a glassy carbon (GC) or boron doped diamond (BDD) disk working electrode ( $3 \mathrm{~mm}$ diameter) using a Pt disk electrode ( $2 \mathrm{~mm}$ diameter) as an auxiliary. As a reference electrode, a saturated calomel electrode (SCE) or mercury sulfate electrode (MSE) were used in aqueous media. In organic solvents, a silver wire in a sample holder filled with electrolyte solution was used as the reference with ferrocene as an internal standard. The potentials were either referenced against the normal hydrogen electrode (NHE, SCE $=+241 \mathrm{mV} v s$. NHE, MSE $=+658 \mathrm{mV} v s$. NHE) or relative to the ferrocenium/ferrocene $\left(\mathrm{Fc}^{+/ 0}\right)$ redox couple. Conducting salts were used as indicated. Working disk electrodes were polished with $0.05 \mu \mathrm{m}$ alumina paste and sonicated, and all electrodes were washed with high purity deionized water and carefully dried with a paper towel before use. The electrochemical measurements were evaluated either with the software NovaSoft 1.10 or Gamry EChem Analyst.

\subsubsection{UV/vis-Spectroelectrochemistry}

For the UV/vis-spectroelectrochemical (SEC) measurements a potentiostat Metrohm Autolab PGSTAT101 or a Gamry Instruments Interface 1000 was applied. The UV/Vis spectra were recorded by an Avantes AvaSpec-ULS2048L-StarLine spectrometer using an Avantes AvaLight-DH-S-BAL Balanced Power as the light source or a BWTek Exemplar LS spectrometer with a deuterium/tungsten BWTek light source. The measurements were conducted in a quartz cuvette $(\mathrm{d}=1 \mathrm{~mm})$ at room temperature with a platinum mesh working electrode and a Pt wire as an auxiliary. As a reference electrode, an SCE or MSE were used in aqueous media (0.1 M HOTf). In organic solvents, a silver wire in a sample holder filled with electrolyte solution was used as the reference. The electrochemical and spectroscopic data was analyzed with NovaSoft 1.10 or Gamry EChem Analyst and AvaSoft 7.7 or BWSpec, respectively.

\subsubsection{Electrochemical Water Oxidation}

The electrochemical catalysis experiments were conducted in a two-compartment electrochemical cell separated by a frit and sealed by septa; one side holding the working electrode, and the other the counter and reference electrodes. For these measurements, a BDD plate was applied as working electrode, a Pt mesh as auxiliary and an MSE as reference electrode. Both compartments were filled with complex solution to prevent dilution in case solution passes through the frit. The evolution of gas was thereby monitored by a gas phase oxygen sensor ( $O x-N$ sensor and Oxy-meter, Unisense).

A typical experiment was performed in the following way: the complex solution $(0.5 \mathrm{mM}, 25 \mathrm{~mL}$, phosphate buffer) was added to the cell and degassed by an $\mathrm{N}_{2}$ flow. Once a stable baseline was reached, gas flow was stopped, and the desired potential was applied for one hour (controlled potential electrolysis, CPE). Dioxygen detection was continued post-CPE until all formed bubbles were burst. After the first electrolysis, the BDD plate was carefully rinsed with buffer solution and a second CPE was run under identical conditions, however in pure electrolyte. This was used as a so-called rinse test 
to check if a surface deposit is formed during the electrochemical experiment, which might be responsible for the catalysis.

\subsubsection{Chemical Water Oxidation}

The chemical catalysis experiments were performed at $25^{\circ} \mathrm{C}$ in a home-made glass cell $(V=16.5 \mathrm{~mL})$ which was sealed by a septum. The evolution of gas was thereby simultaneously monitored by two methods: on-line manometry with a differential pressure manometer (Testo 521-1) with respect to a reference cell of approximately equal size and a gas phase oxygen sensor (Ox-N sensor and Oxy-meter, Unisense or OXF900PT sensor and FireSting02, pyroscience) which was pierced through the septum. Prior to use, the OX-N oxygen sensor was calibrated by adding known amounts of oxygen to the cell containing $2 \mathrm{~mL}$ degassed aqueous HOTf ( $\mathrm{c}=0.1 \mathrm{M}, \mathrm{pH}$ ); the OXF900PT sensor was calibrated with a two-point calibration in air and an $\mathrm{N}_{2}$-atmosphere.

A typical experiment was performed in the following way: the tempered glass cell containing the complex $(\mathrm{n}=2.0 \mu \mathrm{mol})$ was degassed and $1.85 \mathrm{~mL}$ of degassed aqueous HOTf $(\mathrm{c}=0.1 \mathrm{M}, \mathrm{pH} 1)$ was added. After pressure equilibration between the reference (also containing $1.85 \mathrm{~mL}$ of degassed aqueous HOTf ( $\mathrm{c}=0.1 \mathrm{M}, \mathrm{pH} 1)$ ) and the measurement cell, $150 \mu \mathrm{L}$ of degassed aqueous HOTf ( $\mathrm{c}=0.1 \mathrm{M}, \mathrm{pH} 1$ ) were added to the reference cell and $150 \mu \mathrm{L}$ of a degassed solution of CAN in aqueous HOTf $(\mathrm{c}=0.1 \mathrm{M}, \mathrm{pH} 1)$ were added to the measurement cell (final concentration of the complex $\left.\mathrm{c}=1.0 \times 10^{-3} \mathrm{M}\right)$.

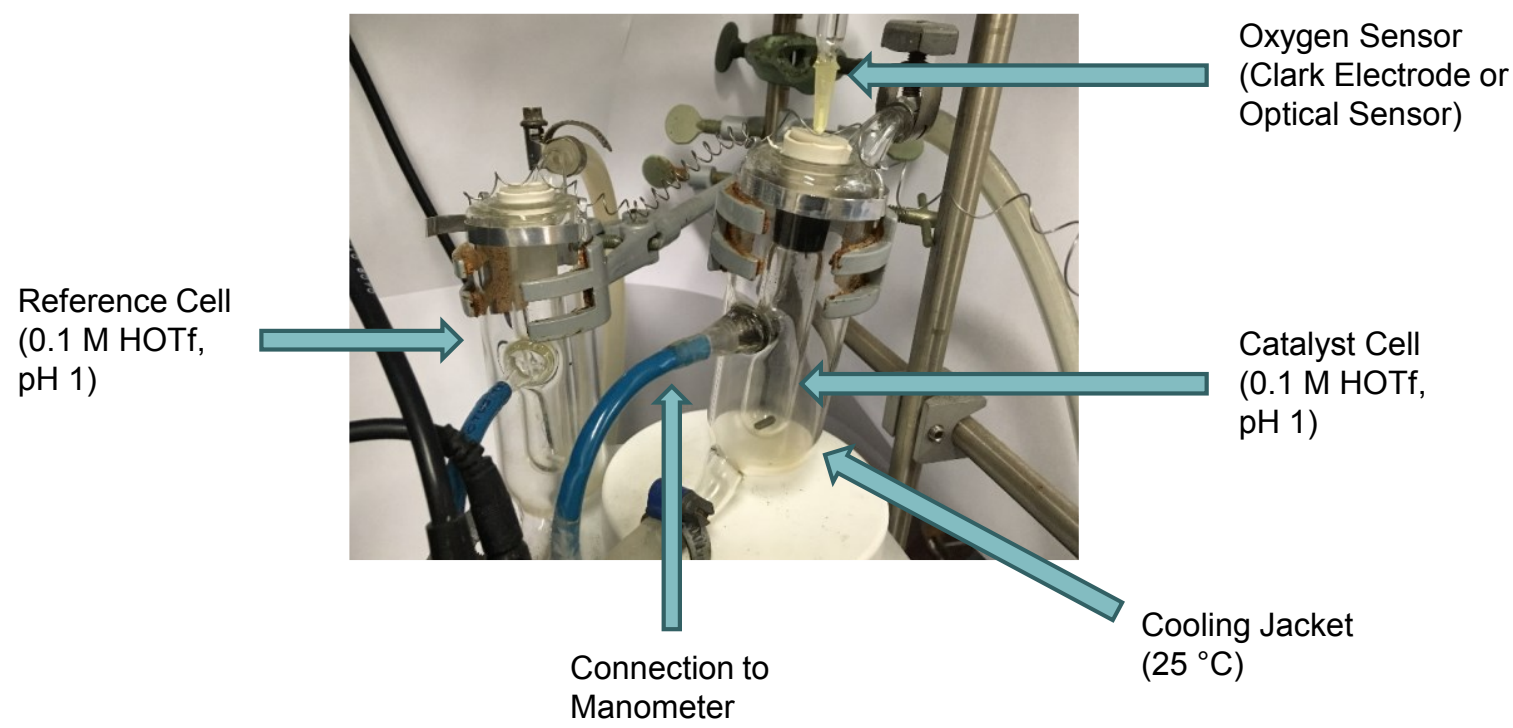

\subsubsection{Photoinduced Water Oxidation}

The photoinduced catalysis experiments were performed at $25^{\circ} \mathrm{C}$ in a specific dark chamber (DW2/2) from Hansatech Instruments with an integrated Clark-type electrode to measure the produced oxygen in the liquid phase (no headspace to guarantee that all oxygen stays in solution). Prior to use, the oxygen sensor was calibrated with the fully air- and nitrogen-saturated solution. Illumination was provided by a $150 \mathrm{~W}$ xenon Arc Lamp (LS-150, ABET technology), equipped with a $400 \mathrm{~nm}$ cutoff filter and calibrated to 1 sun $\left(100 \mathrm{~mW} \cdot \mathrm{cm}^{-2}\right)$ by using a calibrated silicon photodiode.

A typical experiment was performed in the following way: RuP1 $\left(2 \cdot 10^{-4} \mathrm{M}\right)$ as photosensitizer and $\mathrm{Na}_{2} \mathrm{~S}_{2} \mathrm{O}_{8}\left(10^{-2} \mathrm{M}\right)$ as sacrificial electron acceptor were dissolved in $\mathrm{H}_{2} \mathrm{O}$ and introduced into the dark chamber (total volume $=2 \mathrm{~mL}$ ). The catalyst $\left(2 \cdot 10^{-6} \mathrm{M}\right.$ ) was added and the solution was stirred and degassed. Finally, the dark chamber was closed by a screw cap with a septum and once a stable baseline was reached, the solution was irradiated by opening the windows of the chamber.

\subsubsection{X-ray Crystallography}

X-ray data was collected on a STOE IPDS II diffractometer with an area detector (monochromated Mo$\mathrm{K} \alpha, \lambda=0.71073 \AA$ ) by use of $\omega$ scans at $133 \mathrm{~K}$ or a Bruker D8-Quest with an I $\mu \mathrm{S}$ Micro Focus Source 
(monochromated Mo-K $\alpha, \lambda=0.71073 \AA$ ) at $100 \mathrm{~K}$. The structures were solved with SHELXT and refined on $\mathrm{F}^{2}$ using all reflections with SHELXL-2014/17/18.[443,444] Non-hydrogen atoms were refined anisotropically. Most hydrogen atoms were placed in calculated positions and assigned to an isotropic displacement parameter of $1.2 / 1.5 U_{\text {eq }}(\mathrm{C})$. Face-indexed absorption corrections were performed numerically with the program X-RED (X-RED; STOE \& CIE GmbH, Darmstadt, Germany, 2002). Crystal data and details of the data collections, as well as selected bond lengths and angles are given in the appendix.

Powder samples were measured with a Bruker Advance D8 powder diffractometer with a Dectris Eiger2 R $500 \mathrm{~K}$ detector (monochromated $\mathrm{Cu}-\mathrm{K} \alpha, \lambda=1.5406 \AA$ ) at $298.15 \mathrm{~K}$. The X-ray data was analyzed using the Bruker.Eva program and compared to the Crystallography Open Database.

\subsubsection{DFT Calculations}

Density functional theory (DFT) calculations were performed with the Gaussian 09 program. ${ }^{[445]}$ The low-resolution XRD structure of the cationic core of $\mathbf{9}^{\mathbf{H}}$ (with manually added hydride ligands, see below) and the XRD structure of the cationic core of $\mathbf{9}$ (8) 5 were used as starting points for geometry optimizations using the PBE0 hybrid functional[446] together with the atom-pairwise D3 dispersion correction featuring Becke-Johnson (BJ) damping[447,448] (Gaussian 09 settings: SCF=Tight, Int=Fine, Opt=Tight). Within these calculations, def2-SVP basis sets were applied for the main group elements $^{[449]}$ and the ECP10MWB effective core potential in combination with the corresponding valence basis set for copper.[450]

The two manually added hydride ligands of the input structure for $9^{\mathbf{H}}$ were placed in such a way that the two outer copper centers of both copper triangles were coordinated by a $\mu_{2}$ bridging hydride lying in the plane of the respective $\mathrm{Cu}_{3}$ unit. The subsequent geometry optimization resulted in a $C_{\mathrm{i}^{-}}$ symmetric structure where both copper triangles are coordinated by $\mu_{3}$ bridging hydrides, which are trans to each other with respect to the pyrazolate plane. This structure is denoted as cis- $\mathbf{-} \mathbf{9}^{\text {trans }-\mathbf{H}}$, where the prefix indicates the arrangement of the tacn moieties of the ligand relative to the pyrazolate plane. Furthermore, a $C_{\mathrm{s}}$-symmetric structure of $\mathbf{9 H}^{\mathbf{H}}$ featuring cis-standing hydrides (cis-9cis-H) could be obtained by means of a PBE0-D3BJ/def2-SVP/ECP10MWB geometry optimization starting from a manually modified variant of the calculated $C_{\mathrm{i}}$-symmetric structure. In the same manner, the $C_{2}$ symmetric structures trans $\mathbf{-} \mathbf{9}^{\text {trans-H }}$ and trans $\mathbf{-} \mathbf{- 9}{ }^{\text {cis-H }}$ were identified. The DFT geometry optimization for 9c8H5 yielded a $C_{1}$-symmetric structure similar to the input XRD geometry, where the two

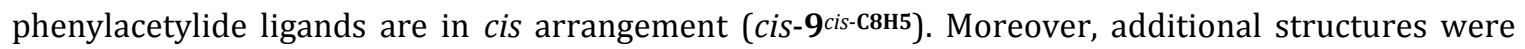
identified for 9, viz. cis-9 $\mathbf{9 t r a n s}^{-\mathbf{C 8 H 5}}\left(C_{\mathrm{i}}\right)$, trans-9 ${ }^{\text {cis-C8H5 }}\left(C_{2}\right)$ and trans-9trans-C8H5 $\left(C_{2}\right)$, by manually altering the calculated cis-9cis-c8H5 geometry and re-optimizing the resulting structures with the PBE0D3BJ/def2-SVP/ECP10MWB method.

All optimized structures were confirmed to be energy minima by the corresponding PBE0-D3BJ/def2SVP/ECP10MWB harmonic vibrational frequency calculations, since the latter yielded only positive frequencies (harmonic frequencies for the deuteride congeners cis-9trans-D and cis-9cis-D were also determined). Zero-point vibrational energies (ZPVEs) were calculated from the harmonic frequencies and thermochemical corrections were computed for $T=298.15 \mathrm{~K}$ and $p=1.01325$ bar on the basis of the optimized geometries and the harmonic frequencies using the ideal gas approximation together with the quasi-RRHO approach of Grimme. [451] In addition, PBE0-D3BJ/def2-SVP/ECP10MWB single point energy (SPE) calculations including the SMD model [452] for acetonitrile were carried out for the optimized structures and solvation energies $\left(\Delta G_{\text {solv }}\right)$ for the species of interest were determined as the difference between the PBE0-D3BJ/def2-SVP/ECP10MWB(SMD) and PBE0-D3BJ/def2SVP/ECP10MWB SPEs. The enthalpy at $0 \mathrm{~K}\left(H_{0}\right)$, the Gibbs energy at $298 \mathrm{~K}$ in the gas phase $\left(G_{298}\right)$, and the Gibbs energy at $298 \mathrm{~K}$ in acetonitrile $\left(G_{298}\right)$ for each structure were calculated by adding the ZPVE to the PBE0-D3BJ/def2-SVP/ECP10MWB electronic energy ( $E_{\text {elec }}$ ), the thermochemical correction to $H_{0}$ and $\Delta G_{\text {solv }}$ to $G_{298}$, respectively. 


\subsubsection{Formic Acid Dehydrogenation Catalysis Tests}

$9^{\mathrm{H}}$ was either crystallized before the experiment $\left(\mathbf{9 H}^{\mathrm{H}} /\left[\mathrm{Cp}_{2} \mathrm{Co}\right]\left(\mathrm{ClO}_{4}\right)\right.$ mixture $)$ or formed in situ by addition of $\mathrm{Cp}_{2} \mathrm{Co}(2.2 \mathrm{eq})$ to $\mathbf{5}^{\mathbf{H C O} 2}$. To a sample of $\mathbf{9}^{\mathbf{H}}$ and $\left[\mathrm{Cp}_{2} \mathrm{Co}\right]\left(\mathrm{ClO}_{4}\right)$ in $\mathrm{CD}_{3} \mathrm{CN}$, formic acid (FA, 1526 eq) was added and ${ }^{1} \mathrm{H} /{ }^{13} \mathrm{C}$-NMR spectra of the solution were recorded. Subsequently, the reaction mixture was heated for several hours at $65{ }^{\circ} \mathrm{C}$ and the reaction progress monitored by NMR spectroscopy. The solution was heated further as long as there were still changes in the NMR spectra. Afterwards, the solution was analyzed using ESI mass spectrometry. These experiments were conducted both in the absence and presence of $\mathrm{NEt}_{3}$ as an additive ( 0.4 eq vs. FA).

The spectra in the appendix (ESI-MS: Figures A.49 and A.50, NMR: Figures B.42 and B.43) are shown exemplarily for an experiment in the presence of $\mathrm{NEt}_{3}$ and starting from crystalline material $\left(9 \mathrm{H} /\left[\mathrm{Cp}_{2} \mathrm{Co}\right]\left(\mathrm{ClO}_{4}\right)\right.$ mixture). The NMR spectra reveal that small amounts of both $\mathrm{H}_{2}$ and $\mathrm{CO}_{2}$ are formed, however the hexacopper core of $\mathbf{9}^{\mathbf{H}}$ is not retained. All NMR spectra were measured at $238 \mathrm{~K}$. 


\subsection{Complex Synthesis}

\section{$\left[\mathrm{L}^{1} \mathrm{Ru}_{2}(\mathrm{dmso})_{2}(\mathrm{Cl})\left(\mathrm{H}_{2} \mathrm{O}\right)(\mu-\mathrm{dmso})\right](1)$}

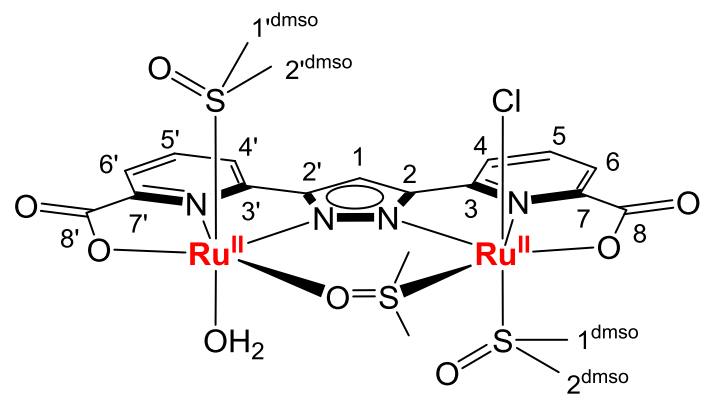

1

\section{Synthesis and Crystallization}

Under a nitrogen atmosphere, a solution of $\mathrm{H}_{3} \mathrm{~L}^{1}(400 \mathrm{mg}, 1.29 \mathrm{mmol}, 1.0 \mathrm{eq})$ and $\mathrm{NEt}_{3}(4 \mathrm{~mL}$, $28.7 \mathrm{mmol}, 22.2 \mathrm{eq})$ in a degassed mixture of EtOH $(100 \mathrm{~mL})$ and $\mathrm{H}_{2} \mathrm{O}(2 \mathrm{~mL})$ was slowly added to a refluxing solution of $\mathrm{RuCl}_{2}(\mathrm{dmso})_{4}(1.44 \mathrm{~g}, 2.97 \mathrm{mmol}, 2.3 \mathrm{eq})$ in EtOH (degassed, $25 \mathrm{~mL}$ ). After complete addition, the solution was heated to reflux overnight. The next day, the reaction mixture was cooled to room temperature and stored at $-4^{\circ} \mathrm{C}$. Once a precipitate had formed, it was removed via filtration, washed with acetone and $\mathrm{Et}_{2} \mathrm{O}$ and dried under reduced pressure. Altogether, three batches of intermediate $\mathbf{1}$ as an orange solid were obtained. Crystals of $\mathbf{1}^{\prime}$, in which the water ligand is replaced by $\mathrm{MeOH}$, were obtained by diffusion of $\mathrm{Et}_{2} \mathrm{O}$ into a solution of $\mathbf{1}$ in $\mathrm{MeOH}$.

Yield $512 \mathrm{mg}, 0.601 \mathrm{mmol}, 47 \%$

Molecular Weight for $1 \cdot 3 \mathrm{H}_{2} \mathrm{O} \quad 851.28 \mathrm{~g} / \mathrm{mol}$

Elemental Analysis for $\mathbf{1} \cdot 3 \mathrm{H}_{2} \mathrm{O}\left(\mathrm{C}_{21} \mathrm{H}_{27} \mathrm{ClN}_{4} \mathrm{O}_{8} \mathrm{Ru}_{2} \mathrm{~S}_{3}\right)$

$\begin{array}{ll}\text { Calc. / \% } & \text { C: } 29.63 \mathrm{H}: 3.91 \mathrm{~N}: 6.58 \mathrm{~S}: 11.30 \\ \text { Found / \% } & \text { C: } 29.42 \mathrm{H}: 3.96 \mathrm{~N}: 6.43 \mathrm{~S}: 10.63\end{array}$

${ }^{1} \mathbf{H}-\mathbf{N M R}\left(500 \mathrm{MHz}, \mathrm{DMF}-d_{7}\right) \delta(\mathrm{ppm})=8.25\left(\mathrm{dd}, J=8.0,1.9 \mathrm{~Hz}, 1 \mathrm{H}, 4^{\prime}-\mathrm{H}\right), 8.23\left(\mathrm{t}, J=8.0 \mathrm{~Hz}, 1 \mathrm{H}, 5^{\prime}-\mathrm{H}\right)$, $8.08(\mathrm{dd}, J=7.9,1.1 \mathrm{~Hz}, 2 \mathrm{H}, 4-\mathrm{H}), 7.98(\mathrm{~s}, 2 \mathrm{H}, 1-\mathrm{H}), 7.96(\mathrm{t}, J=7.9 \mathrm{~Hz}, 1 \mathrm{H}, 5-\mathrm{H}), 7.86(\mathrm{dd}, J=8.0,1.9 \mathrm{~Hz}$, $2 \mathrm{H}, 6$ '- $\mathrm{H}$ ), 7.74 (dd, $J=7.9,1.1 \mathrm{~Hz}, 2 \mathrm{H}, 6-\mathrm{H}$ ), 3.93 (s, 3H, $\mu$-dmso), 3.93 (s, 3H, $\mu$-dmso), 2.68 (s, 3H, 2'dmso), 2.56 (s, 3H, 2-dmso), 2.43 (s, 3H, 1'-dmso), 2.01 (s, 3H, 1-dmso).*

${ }^{13} \mathbf{C}\left\{{ }^{1} \mathbf{H}\right\}$-NMR (126 MHz, DMF- $\left.d_{7}\right) \delta(\mathrm{ppm})=172.83$ (8-C), 172.14 (8'-C), 157.29 (7-C), 156.16 (3-C), 153.51 (7'-C), 152.98 (2-C), 151.78 (3'-C), 151.76 (2'-C), 139.51 (5'-C), 135.61 (5-C), 122.15 (6'-C), 121.90 (6-C), 121.12 (4'-C), 120.07 (4-C), 105.99 (1-C), 48.57 ( $\mu$-dmso), 46.94 ( $\mu$-dmso), 44.67 (2'dmso), 42.02 (1'-dmso), 41.66 (2-dmso), 41.56 (1-dmso).*

MS (ESI $(+), \mathrm{MeOH}): m / z=802.9\left[\mathrm{M}-\mathrm{H}_{2} \mathrm{O}+\mathrm{Na}\right]^{+}, 820.9[\mathrm{M}+\mathrm{Na}]^{+}, 834.9\left[\mathrm{M}-\mathrm{H}_{2} \mathrm{O}+\mathrm{MeOH}+\mathrm{Na}\right]^{+}, 852.9$ $[\mathrm{M}+\mathrm{MeOH}+\mathrm{Na}]^{+}$.

MS $(\mathrm{ESI}(-), \mathrm{MeOH}): \quad m / z=700.9[\mathrm{M}-\mathrm{H}-\mathrm{dmso}-\mathrm{Cl}+\mathrm{OH}]^{-}, 732.9\left[\mathrm{M}-\mathrm{H}_{2} \mathrm{O}-\mathrm{dmso}+\mathrm{OMe}\right]^{-}, \quad 764.9$ $\left[\mathrm{M}-\mathrm{dmsO}-\mathrm{Cl}+\mathrm{H}_{2} \mathrm{O}+2 \mathrm{OMe}\right]^{-}, 778.9[\mathrm{M}-\mathrm{H}-\mathrm{Cl}+\mathrm{OH}]^{-}, 796.9[\mathrm{M}-\mathrm{H}]^{-}, 814.9\left[\mathrm{M}-\mathrm{Cl}+\mathrm{H}_{2} \mathrm{O}+2 \mathrm{OH}\right]^{-}$.

*the distinction between prime and non-prime is not possible and is merely done for simplification 


\section{$\mathrm{Na}_{3}\left[\mathrm{~L}^{1} \mathrm{Ru}_{2}\left(\mathrm{pyCO}_{2}\right)_{4}(\mu-\mathrm{dmso})\right]\left(2^{\mathrm{dmso}}\right)$}

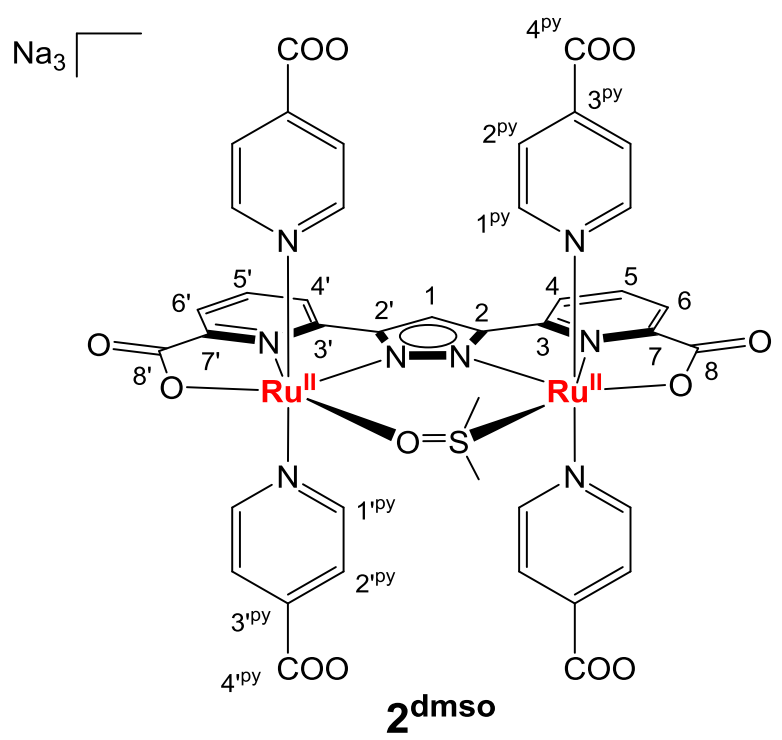

\section{Synthesis}

Intermediate 1 ( $50 \mathrm{mg}, 0.06 \mathrm{mmol}, 1.0 \mathrm{eq}$, purity $91 \%$ ), pyCO ${ }_{2} \mathrm{H}(77 \mathrm{mg}, 0.63 \mathrm{mmol}, 11.0 \mathrm{eq}$ ) and $\mathrm{NaOAc}\left(51 \mathrm{mg}, 0.62 \mathrm{mmol}, 10.9 \mathrm{eq}\right.$ ) were dissolved in $\mathrm{H}_{2} \mathrm{O}$ (bidestilled, $10 \mathrm{~mL}$ ). The solution was degassed and subsequently heated to reflux. After two days, the reaction mixture was cooled to room temperature, acetone was added until the solution turned milky and the reaction mixture was left in the freezer overnight. Subsequently, the precipitate was removed via filtration, washed with acetone and MTBE, and dried under reduced pressure. A yield was not determined.

\section{Molecular Weight $\quad 1144.90 \mathrm{~g} / \mathrm{mol}$}

\section{Empirical Formula $\mathrm{C}_{41} \mathrm{H}_{29} \mathrm{~N}_{8} \mathrm{Na}_{3} \mathrm{O}_{13} \mathrm{Ru}_{2} \mathrm{~S}$}

${ }^{1} \mathrm{H}-\mathrm{NMR}\left(500 \mathrm{MHz}, \mathrm{D}_{2} \mathrm{O}\right) \delta(\mathrm{ppm})=8.40(\mathrm{~d}, J=8.0 \mathrm{~Hz}, 1 \mathrm{H}, 4-\mathrm{H}), 8.26(\mathrm{~s}, 1 \mathrm{H}, 1-\mathrm{H}), 8.15(\mathrm{~d}, J=8.0 \mathrm{~Hz}, 1 \mathrm{H}$, $\left.4^{\prime}-\mathrm{H}\right), 8.11\left(\mathrm{~d}, J=6.7 \mathrm{~Hz}, 4 \mathrm{H}, 1^{\mathrm{py}-\mathrm{H}}\right), 7.96\left(\mathrm{t}, J=8.0 \mathrm{~Hz}, 1 \mathrm{H}, 5^{\prime}-\mathrm{H}\right), 7.85$ (t, $\left.J=8.0 \mathrm{~Hz}, 1 \mathrm{H}, 5-\mathrm{H}\right), 7.65$ (d, $\left.J=8.0 \mathrm{~Hz}, 1 \mathrm{H}, 6^{\prime}-\mathrm{H}\right), 7.59(\mathrm{~d}, J=8.0 \mathrm{~Hz}, 1 \mathrm{H}, 6-\mathrm{H}), 7.48-7.41\left(\mathrm{~m}, 8 \mathrm{H}, 1^{\prime}\right.$ py $\left.-\mathrm{H}, 2^{\mathrm{py}}-\mathrm{H}\right), 7.09(\mathrm{~d}, J=6.8 \mathrm{~Hz}, 4 \mathrm{H}$,

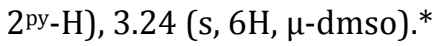

${ }^{13} \mathbf{C}\left\{{ }^{1} \mathbf{H}\right\}$-NMR $\left(126 \mathrm{MHz}, \mathrm{D}_{2} \mathrm{O}\right) \delta(\mathrm{ppm})=175.47$ (8-C), 174.77 (8'-C), 171.25 (4py-C), 170.81 (4'py-C)

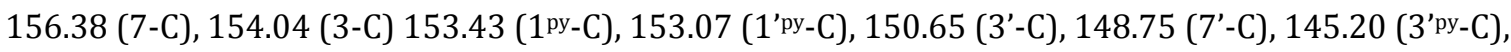
143.65 (3 ${ }^{\text {py-C) }}, 139.60$ (5'-C), 133.18 (5-C), 123.80 (2'py-C), 123.47 (2 ${ }^{\text {py-C }}$ ), 122.57 (6'-C), 122.51 (6-C), 122.47 (4-C), 122.10 (4'-C), 108.59 (1-C), 46.15 ( $\mu$-dmso).*

*the distinction between prime and non-prime is not possible and is merely done for simplification ${ }^{*}{ }^{13} \mathrm{C}\left\{{ }^{1} \mathrm{H}\right\}$-NMR: 2/2'-C as well as a peak at $172.06 \mathrm{ppm}$ could not be assigned

MS (ESI(-), MeOH): $m / z=786.8\left[\mathrm{M}-2 \mathrm{pyCO}_{2}-\mathrm{dmso}^{\mathrm{MeOH}}\right]^{-}, 864.8\left[\mathrm{M}-2 \mathrm{pyCO}_{2}+\mathrm{MeOH}\right]^{-}, 977.8$ $\left[\mathrm{M}-\mathrm{pyCO}_{2}+\mathrm{Na}\right]^{-}, 1100.8[\mathrm{M}+\mathrm{H}+\mathrm{Na}]^{-}, 1122.8[\mathrm{M}+2 \mathrm{Na}]^{-}$.

EChem $\left(\mathrm{H}_{2} \mathrm{O}, 0.1 \mathrm{M} \mathrm{NaOTf}\right): E_{1 / 2} / \mathrm{V}$ vs. NHE $=0.73\left(\mathrm{Ru}^{\mathrm{III}} \mathrm{Ru}^{\mathrm{III}} / \mathrm{Ru}^{\mathrm{II}} \mathrm{Ru}^{\mathrm{II}}\right)$. 


\section{$\mathrm{Na}_{3}\left[\mathrm{~L}^{1} \mathrm{Ru}_{2}\left(\mathrm{pySO}_{3}\right)_{4}(\mu-\mathrm{dmso})\right]\left(3^{\mathrm{dmso}}\right)$}

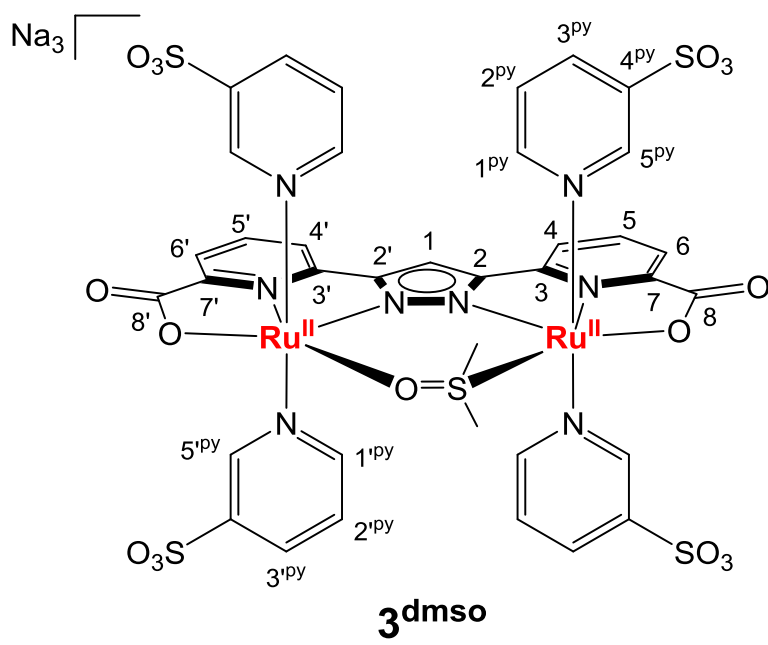

\section{Synthesis}

Intermediate 1 ( $50 \mathrm{mg}, 0.06 \mathrm{mmol}, 1.0 \mathrm{eq}), \mathrm{pySO}_{3} \mathrm{H}(100 \mathrm{mg}, 0.63 \mathrm{mmol}, 10.7 \mathrm{eq})$ and $\mathrm{NaOAc}(51 \mathrm{mg}$, $0.60 \mathrm{mmol}, 10.2 \mathrm{eq}$ ) were dissolved in $\mathrm{H}_{2} \mathrm{O}$ (bidestilled, $5 \mathrm{~mL}$ ). The solution was degassed and subsequently heated to reflux overnight. The next day, the reaction mixture was cooled to room temperature and acetone was added until the solution turned milky. The reaction mixture was left in the freezer overnight. Subsequently, the precipitate was removed via centrifugation, washed with acetone and MTBE, and dried under reduced pressure.

Yield $80 \mathrm{mg}, 0.055 \mathrm{mmol}, 94 \%$

Molecular Weight for $3^{\mathrm{dmso}} .9 \mathrm{H}_{2} \mathrm{O} \quad 1451.22 \mathrm{~g} / \mathrm{mol}$

Elemental Analysis for $3^{\text {dmso. }} 9 \mathrm{H}_{2} \mathrm{O}\left(\mathrm{C}_{37} \mathrm{H}_{47} \mathrm{~N}_{8} \mathrm{Na}_{3} \mathrm{O}_{26} \mathrm{Ru}_{2} \mathrm{~S}_{5}\right)$
Calc. / \%
C: $30.62 \mathrm{H}: 3,26 \mathrm{~N}: 7.72 \mathrm{~S}: 11.05$
Found / \%
C: $30.25 \mathrm{H}: 3.20 \mathrm{~N}: 7.30 \mathrm{~S}: 11.17$

${ }^{1} \mathbf{H}-\mathbf{N M R}\left(500 \mathrm{MHz}, \mathrm{D}_{2} \mathrm{O}\right) \delta(\mathrm{ppm})=8.46\left(\mathrm{~d}, J=7.9 \mathrm{~Hz}, 1 \mathrm{H}, 4^{\prime}-\mathrm{H}\right), 8.41\left(\mathrm{~d}, J=1.9 \mathrm{~Hz}, 2 \mathrm{H}, 5^{\prime} \mathrm{py}-\mathrm{H}\right), 8.34(\mathrm{~s}$, $1 \mathrm{H}, 1-\mathrm{H}$ ), 8.22 (d, $J=7.9 \mathrm{~Hz}, 1 \mathrm{H}, 4-\mathrm{H}), 8.12(\mathrm{~d}, J=2.0 \mathrm{~Hz}, 2 \mathrm{H}, 5 \mathrm{py}-\mathrm{H}), 8.03$ (d, $\left.J=5.7 \mathrm{~Hz}, 2 \mathrm{H}, 1^{\prime} \mathrm{py}-\mathrm{H}\right), 7.99$ $(\mathrm{t}, J=7.9 \mathrm{~Hz}, 1 \mathrm{H}, 5-\mathrm{H}), 7.93-7.85\left(\mathrm{~m}, 5 \mathrm{H}, 3^{\mathrm{py}-\mathrm{H},} 3^{\prime} \mathrm{py}-\mathrm{H}, 5^{\prime}-\mathrm{H}\right), 7.64\left(\mathrm{~d}, J=7.9 \mathrm{~Hz}, 2 \mathrm{H}, 6-\mathrm{H}, 6^{\prime}-\mathrm{H}\right), 7.25$ (dd, $J=8.0,5.7 \mathrm{~Hz}, 2 \mathrm{H}, 2^{\prime}$ py-H$), 7.14\left(\mathrm{~d}, J=5.8 \mathrm{~Hz}, 2 \mathrm{H}, 1^{\mathrm{py}}-\mathrm{H}\right), 6.91\left(\mathrm{dd}, J=8.0,5.8 \mathrm{~Hz}, 2 \mathrm{H}, 2^{\mathrm{py}}-\mathrm{H}\right), 3.33(\mathrm{~s}, 6 \mathrm{H}$, $\mu$-dmso).*

${ }^{13} \mathbf{C}\left\{{ }^{1} \mathbf{H}\right\}$-NMR (126 MHz, $\left.\mathrm{D}_{2} \mathrm{O}\right) \delta(\mathrm{ppm})=175.37$ (8'-C), 174.53 (8-C), 156.05 (7'-C), 154.99 (1'py-C),

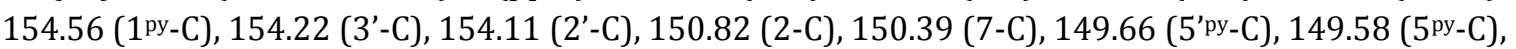

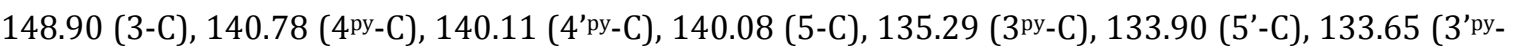
C), 125.58 (2 $2^{\text {py-C}}$ ), 125.32 (2'py-C), 122.88 (4-C), 122.82 (4'-C), 122.77 (6'-C), 122.56 (6-C), 109.18 (1C), 46.28 ( $\mu$-dmso)**

*the distinction between prime and non-prime is not possible and is merely done for simplification IR (ATR): $\tilde{v} / \mathrm{cm}^{-1}=3447$ (w), $1634(\mathrm{~m}), 1592(\mathrm{~m}), 1506(\mathrm{w}), 1464(\mathrm{w}), 1418(\mathrm{w}), 1395(\mathrm{w}), 1335$ (w), $1319(\mathrm{w}), 1204(\mathrm{~s}), 1147$ (s), $1102(\mathrm{w}), 1063(\mathrm{w}), 1026(\mathrm{~s}), 987(\mathrm{w}), 953(\mathrm{w}), 856(\mathrm{w}), 813(\mathrm{w}), 766$ (m), $698(\mathrm{~m}), 623(\mathrm{~s}), 584(\mathrm{w})$.

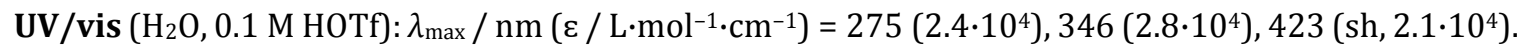
MS $\quad(\operatorname{ESI}(-), \quad \mathrm{MeOH}): \quad m / z=531.4 \quad\left[\mathrm{M}-\mathrm{pySO}_{3}\right]^{2-}, \quad 826.8 \quad\left[\mathrm{M}-2 \mathrm{pySO}_{3}-\mathrm{dmso}^{-}, \quad 858.8\right.$ $\left[\mathrm{M}-2 \mathrm{pySO}_{3}-\mathrm{dmso}+\mathrm{MeOH}^{-}, 904.8\left[\mathrm{M}-2 \mathrm{pySO}_{3}\right]^{-}, 1085.8\left[\mathrm{M}-\mathrm{pySO}_{3}+\mathrm{Na}\right]^{-}\right.$.

EChem ( $\mathrm{H}_{2} \mathrm{O}, 0.1$ M HOTf): $E_{1 / 2} / \mathrm{V} v$ s. NHE $=0.86\left(\mathrm{Ru}^{\mathrm{III}} \mathrm{Ru}^{\mathrm{III}} / \mathrm{Ru}^{\mathrm{II}} \mathrm{Ru}^{\mathrm{II}}\right)$. 


\section{$\mathrm{Na}_{3}\left[\mathrm{~L}^{1} \mathrm{Ru}_{2}\left(\mathrm{pySO}_{3}\right)_{4}\left(\mathrm{H}_{2} \mathrm{O}\right)_{2}\right]\left(3^{\mathrm{H} 20}\right)$}

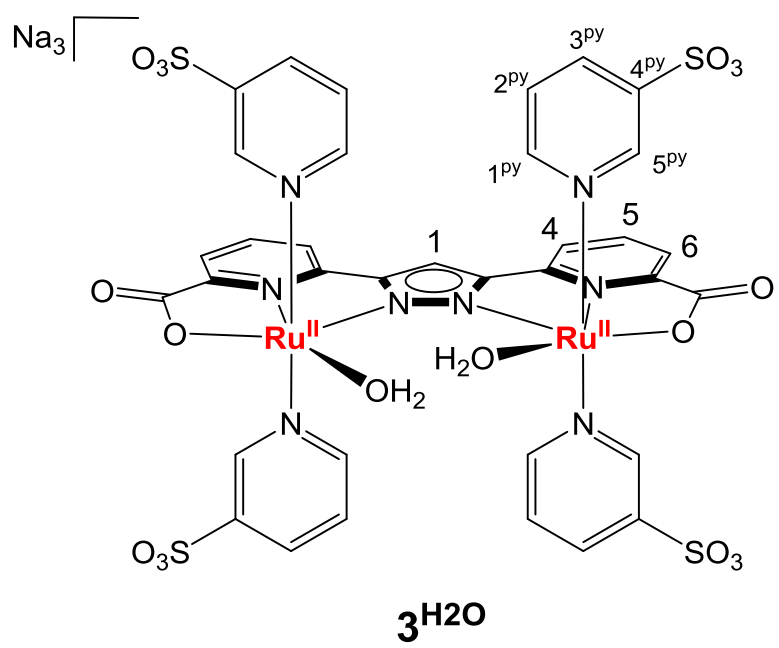

\section{Synthesis}

Complex $3^{\text {dmso }}$ (70 mg, $0.048 \mathrm{mmol}, 1.0$ eq, purity $89 \%$ ) was dissolved in $2 \mathrm{M} \mathrm{NaOH} \mathrm{(5} \mathrm{mL),} \mathrm{degassed}$ and left to stir at $50{ }^{\circ} \mathrm{C}$ for 6.5 hours. Subsequently, the solution was neutralized with aqueous $\mathrm{HBF}_{4}$ and acetone was added until a precipitate was formed. After removal of the product via filtration and washing with acetone and $\mathrm{Et}_{2} \mathrm{O}$, the partially oxidized brown solid was dried under reduced pressure.

Yield $95 \mathrm{mg}$ (crude product), $39 \mathrm{mg}$ (pure product), $0.031 \mathrm{mmol}, 65 \%$ (considering $41 \%$ purity)

\section{Molecular Weight $\quad 1246.99 \mathrm{~g} / \mathrm{mol}$}

Elemental Analysis for $3^{\mathrm{H} 20}\left(\mathrm{C}_{35} \mathrm{H}_{27} \mathrm{~N}_{8} \mathrm{Na}_{3} \mathrm{O}_{18} \mathrm{Ru}_{2} \mathrm{~S}_{4}\right)$
Calc. / \%
C: $33.71 \mathrm{H}: 2.18 \mathrm{~N}: 8.99 \mathrm{~S}: 10.28$
Found / \%
C: $13.80 \mathrm{H}: 1.44 \mathrm{~N}: 3.69 \mathrm{~S}: 4.74$ (41 \% purity; contains water and NaF)

${ }^{1} \mathbf{H}-N M R\left(500 \mathrm{MHz}, \mathrm{D}_{2} \mathrm{O} / \mathrm{NaOD}\right) \delta(\mathrm{ppm})=7.98\left(\mathrm{~d}, J=1.8 \mathrm{~Hz}, 4 \mathrm{H}, 5^{\mathrm{py}}-\mathrm{H}\right), 7.73(\mathrm{~s}, 1 \mathrm{H}, 1-\mathrm{H}), 7.70-7.63$ $\left(\mathrm{m}, 6 \mathrm{H}, 4-\mathrm{H}, 1^{\mathrm{py}}-\mathrm{H}\right), 7.60\left(\mathrm{dt}, J=8.1,1.8 \mathrm{~Hz}, 4 \mathrm{H}, 3^{\mathrm{py}}-\mathrm{H}\right), 7.35(\mathrm{~d}, J=7.7 \mathrm{~Hz}, 2 \mathrm{H}, 6-\mathrm{H}), 7.26(\mathrm{t}, J=7.7 \mathrm{~Hz}$, $2 \mathrm{H}, 5-\mathrm{H}), 6.54\left(\mathrm{dd}, J=8.1,5.7 \mathrm{~Hz}, 4 \mathrm{H}, 2^{\mathrm{py}-\mathrm{H}}\right)$.

${ }^{13} \mathbf{C}\left\{{ }^{1} \mathrm{H}\right\}$-NMR $\left(126 \mathrm{MHz}, \mathrm{D}_{2} \mathrm{O} / \mathrm{NaOD}\right) \delta(\mathrm{ppm})=176.51$ (8-C), 157.57 (3-C), 156.01 (1 $\left.{ }^{\text {py- }} \mathrm{C}\right), 153.38$ (7-

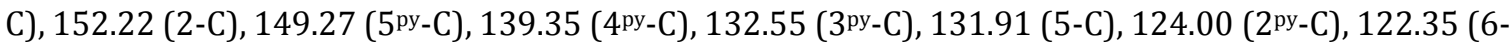
C), 120.84 (4-C), 106.59 (1-C).

UV/vis $\left(\mathrm{H}_{2} \mathrm{O}, 0.1 \mathrm{M} \mathrm{HOTf}\right): \lambda_{\max } / \mathrm{nm}\left(\varepsilon / \mathrm{L} \cdot \mathrm{mol}^{-1} \cdot \mathrm{cm}^{-1}\right)=282\left(2.3 \cdot 10^{4}\right), 352\left(\mathrm{sh}, 2.1 \cdot 10^{4}\right), 393\left(2.5 \cdot 10^{4}\right)$. IR (ATR): $\tilde{v} / \mathrm{cm}^{-1}=3435(\mathrm{w}), 1640(\mathrm{~m}), 1600(\mathrm{~m}), 1466(\mathrm{w}), 1420(\mathrm{w}), 1399(\mathrm{w}), 1329(\mathrm{w}), 1204(\mathrm{~s})$, 1149 (s), $1103(\mathrm{w}), 1064(\mathrm{w}), 1027$ (s), $736(\mathrm{~s}), 696(\mathrm{~m}), 625(\mathrm{~s}), 582(\mathrm{w}), 497(\mathrm{~m}), 474(\mathrm{~m})$.

MS (ESI(-), MeOH): $m / z=843.8\left[\mathrm{M}-2 \mathrm{pySO}_{3}-\mathrm{H}_{2} \mathrm{O}-\mathrm{H}\right]^{-}$(II/III)*, $1047.7\left[\mathrm{M}-\mathrm{pySO}_{3}-\mathrm{H}_{2} \mathrm{O}-\mathrm{H}+2 \mathrm{Na}\right]^{-}$ (II/II), $1184.0\left[\mathrm{M}-\mathrm{OH}_{2}+\mathrm{Na}\right]^{-}$(II/III).

*oxidation states of the ruthenium ions

EChem ( $\mathrm{H}_{2} \mathrm{O}, 0.1 \mathrm{M}$ HOTf): $E_{1 / 2} / \mathrm{V} v$ s. NHE $=0.69\left(\mathrm{Ru}^{\mathrm{II}} \mathrm{Ru}^{\mathrm{III}} / \mathrm{Ru}^{\mathrm{II}} \mathrm{Ru}^{\mathrm{II}}\right)$, $0.83\left(\mathrm{Ru}^{\mathrm{III}} \mathrm{Ru}^{\mathrm{III}} / \mathrm{Ru}^{\mathrm{II}} \mathrm{Ru}^{\mathrm{III}}\right)$. 


\section{$\mathrm{Na}_{5}\left[\mathrm{~L}^{1} \mathrm{Ru}_{2}\left(\mathrm{pySO}_{3}\right)_{4}\left(\mu-\mathrm{CO}_{3}\right)\right]\left(3^{\mathrm{co}}\right)$}

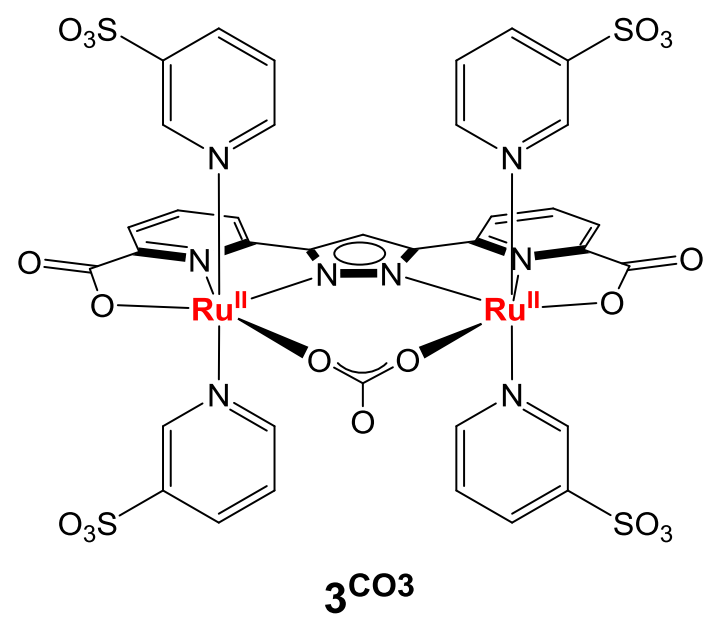

\section{Crystallization}

Crystals of $3^{\mathrm{Co3}}$ were obtained by slow diffusion of acetone into an NMR solution of $\mathbf{3}^{\mathbf{0 2} \mathrm{H}}$ in $\mathrm{NaOD} / \mathrm{D}_{2} \mathrm{O}$ under ambient conditions. Further analysis beyond X-ray diffraction was not done.

\section{$\left[\mathrm{L}^{1} \mathrm{Ru}_{2}\left(\mathrm{pySO}_{3} \mathrm{H}\right)_{3}\left(\mathrm{pySO}_{3}\right)\right]_{2}(4)$}

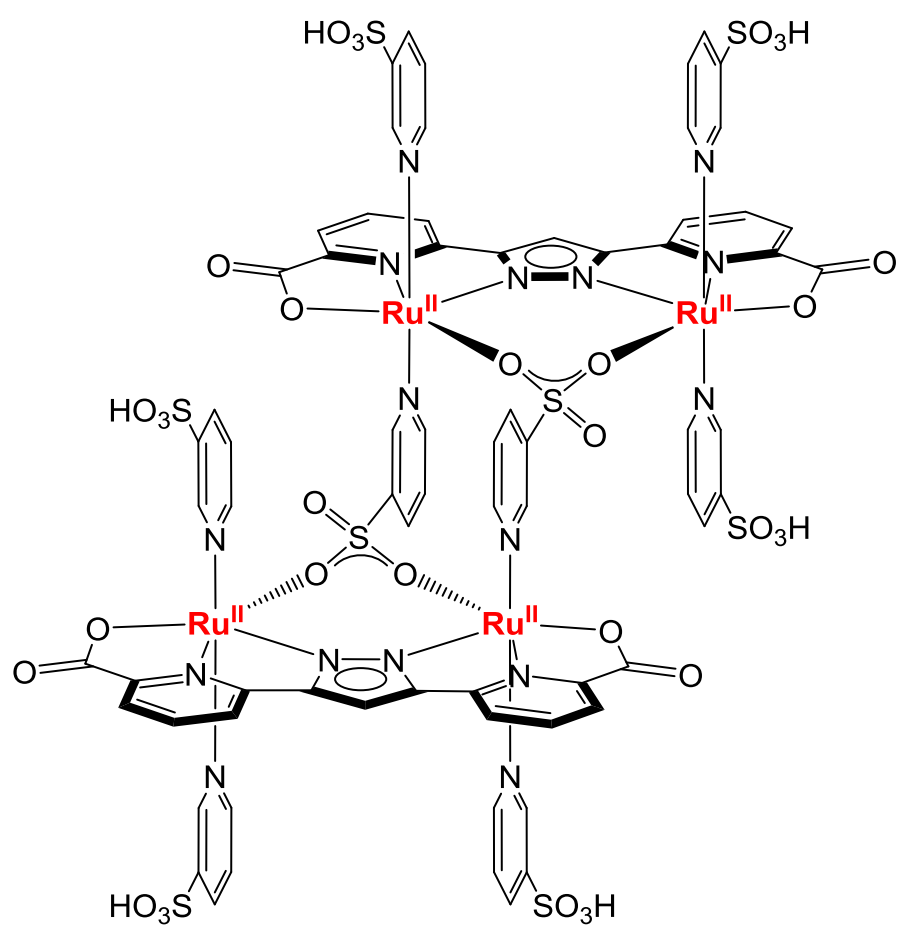

4

\section{Crystallization}

Crystals of 4 were obtained by slow diffusion of acetone into a solution of $3^{\mathbf{0 2 H}}$ in $\mathrm{H}_{2} \mathrm{O}$ under ambient conditions. Further analysis beyond X-ray diffraction was not done. 


\section{$\left[\mathrm{L}^{2 \mathrm{aa}} \mathrm{Cu}_{2}\left(\mu-\mathrm{O}_{2} \mathrm{CH}\right)\right]\left(\mathrm{ClO}_{4}\right)_{2}\left(5^{\mathrm{HCO}}\right)$}

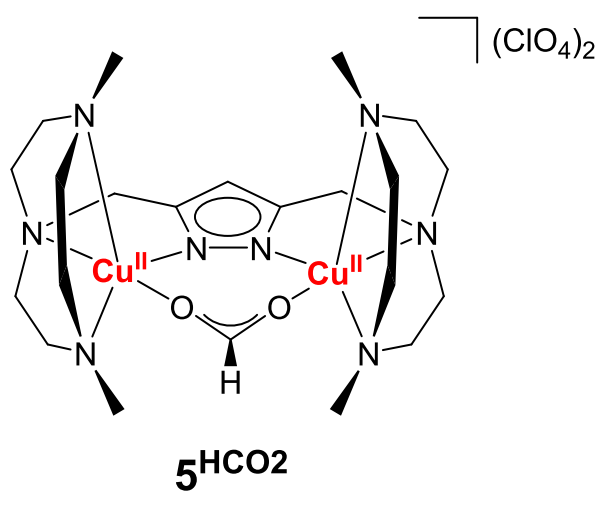

\section{Synthesis and Crystallization}

$\mathrm{HL}^{2 a}(34 \mathrm{mg}, 83.6 \mu \mathrm{mol}, 1.0 \mathrm{eq}$ ) and NEt $3(50 \mu \mathrm{L}, 358 \mu \mathrm{mol}, 4.3 \mathrm{eq})$ were dissolved in $\mathrm{MeOH}(2 \mathrm{~mL})$. Subsequently, $\mathrm{Cu}\left(\mathrm{ClO}_{4}\right)_{2} \cdot 6 \mathrm{H}_{2} \mathrm{O}(62 \mathrm{mg}, 167 \mu \mathrm{mol}, 2.0 \mathrm{eq})$ and $\mathrm{NaHCO}_{2}(58 \mathrm{mg}, 853 \mu \mathrm{mol}, 10.2 \mathrm{eq}$ ), as well as a few drops of $\mathrm{H}_{2} \mathrm{O}$ were added to ensure complete dissolution. The reaction mixture was left to stir for 1.5 hours and then left for crystallization by diffusion with $\mathrm{Et}_{2} \mathrm{O}$. The first crystallization afforded a mixture of complex and $\mathrm{NaHCO}_{2}$ crystals. The crystals were gathered and separated by addition of acetone upon which the complex crystals dissolved, and the salt crystals remained intact. Subsequent filtration, removal of the solvent and crystallization by diffusion of $\mathrm{Et}_{2} \mathrm{O}$ into a solution of $5 \mathrm{HCO2}$ in $\mathrm{MeOH} / \mathrm{H}_{2} \mathrm{O}$ yielded blue needle-shaped crystals suitable for X-ray diffraction.

Yield $38 \mathrm{mg}, 0.049 \mathrm{mmol}, 59 \%$

Molecular Weight $776.62 \mathrm{~g} / \mathrm{mol}$

Elemental Analysis for $\mathbf{5}^{\mathbf{H C O}}\left(\mathrm{C}_{22} \mathrm{H}_{42} \mathrm{Cl}_{2} \mathrm{Cu}_{2} \mathrm{~N}_{8} \mathrm{O}_{10}\right)$
Calc. / \%
C: 34.02 H: $5.45 \mathrm{~N}: 14.43$
Found / \%
C: $34.05 \mathrm{H}: 5.46 \mathrm{~N}: 14.26$

IR (ATR): $\tilde{v} / \mathrm{cm}^{-1}=2911(\mathrm{w}), 2868(\mathrm{w}), 2826(\mathrm{w}), 1570(\mathrm{~s}), 1492(\mathrm{w}), 1461(\mathrm{~m}), 1396(\mathrm{w}), 1364$ (m), 1323 (w), 1290 (w), 1230 (w), 1145 (w), 1068 (s), 1008 (s), 905 (w), 830 (m), 792 (m), 743 (m), 621 (s), $573(\mathrm{w})$.

UV/vis (EtCN): $\lambda_{\max } / \mathrm{nm}\left(\varepsilon / \mathrm{L} \cdot \mathrm{mol}^{-1} \cdot \mathrm{cm}^{-1}\right)=678$ (305).

MS (ESI $(+), \mathrm{MeOH}): m / z=287.9\left[\mathrm{M}^{2+}, 677.1\left[\mathrm{M}+\mathrm{ClO}_{4}\right]^{+}\right.$. 


\section{$\left[\mathrm{L}^{2 \mathrm{a}} \mathrm{Cu}_{2}\left(\mu-\mathrm{O}_{2} \mathrm{CD}\right)\right]\left(\mathrm{ClO}_{4}\right)_{2}\left(5^{\mathrm{DCO}}\right)$}

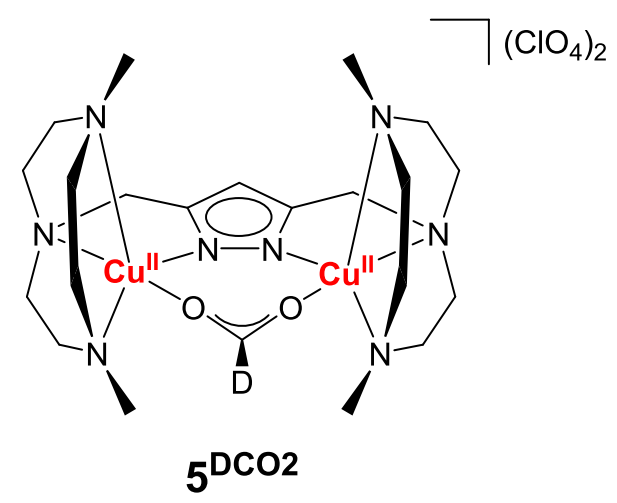

\section{Synthesis and Crystallization}

The synthesis and crystallization of $\mathbf{5}^{\mathrm{DCO} 2}$ were done identical to $\mathbf{5}^{\mathbf{H C O}}$ using $\mathrm{NaDCO}_{2}$ instead of $\mathrm{NaHCO}_{2}$ starting from $31 \mathrm{mg} \mathrm{HL}^{2 a}$.

Yield $27 \mathrm{mg}, 0.035 \mathrm{mmol}, 46 \%$

Molecular Weight $777.62 \mathrm{~g} / \mathrm{mol}$

Empirical Formula $\mathrm{C}_{22} \mathrm{H}_{41} \mathrm{DCl}_{2} \mathrm{Cu}_{2} \mathrm{~N}_{8} \mathrm{O}_{10}$

MS (ESI(+), MeOH): $m / z=288.3[\mathrm{M}]^{2+}, 678.0\left[\mathrm{M}+\mathrm{ClO}_{4}\right]^{+}$.

MS (ESI $(-), \mathrm{MeOH}): m / z=823.0\left[\mathrm{M}+\mathrm{DCO}_{2}+2 \mathrm{ClO}_{4}\right]^{-}, 875.9\left[\mathrm{M}+3 \mathrm{ClO}_{4}\right]^{-}$.

\section{$\left[\mathrm{L}^{2 \mathrm{a}} \mathrm{Cu}_{2}\left(\mu-\mathrm{O}_{2}{ }^{13} \mathrm{CH}\right)\right]\left(\mathrm{ClO}_{4}\right)_{2}\left(5^{\mathrm{H}\{13 \mathrm{C}\} 02}\right)$}

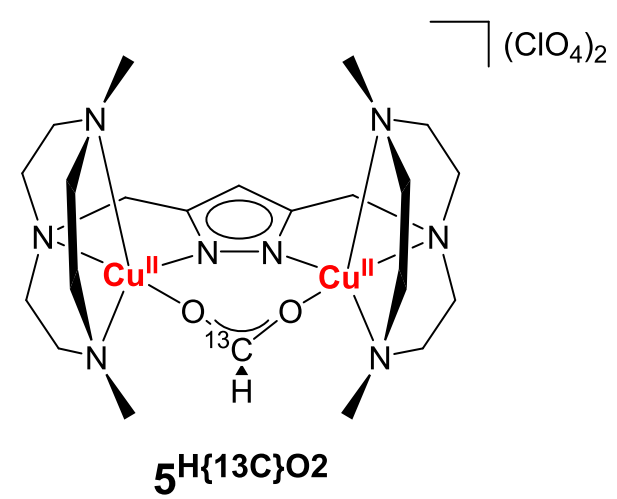

\section{Synthesis and Crystallization}

The synthesis and crystallization of $\mathbf{5}^{\mathbf{H}\{13 \mathrm{C}\} 02}$ were conducted identical to $\mathbf{5}^{\mathbf{H C O 2}}$ using $\mathrm{NaH}^{13} \mathrm{CO}_{2}$ instead of $\mathrm{NaHCO}_{2}$ starting from $31 \mathrm{mg} \mathrm{HL} 2 \mathrm{a}$.

Yield $42 \mathrm{mg}, 0.054 \mathrm{mmol}, 71 \%$

Molecular Weight $777.61 \mathrm{~g} / \mathrm{mol}$

Empirical Formula $\mathrm{C}_{21}{ }^{13} \mathrm{CH}_{42} \mathrm{Cl}_{2} \mathrm{Cu}_{2} \mathrm{~N}_{8} \mathrm{O}_{10}$

MS (ESI(+), MeOH): $m / z=288.6[\mathrm{M}]^{2+}, 678.1\left[\mathrm{M}+\mathrm{ClO}_{4}\right]^{+}$. 


\section{$\left[\mathrm{L}^{2 \mathrm{aa}} \mathrm{Cu}_{2}(\mu-\mathrm{OAc})\right]\left(\mathrm{ClO}_{4}\right)_{2}\left(5^{\mathrm{OAc}}\right)$}

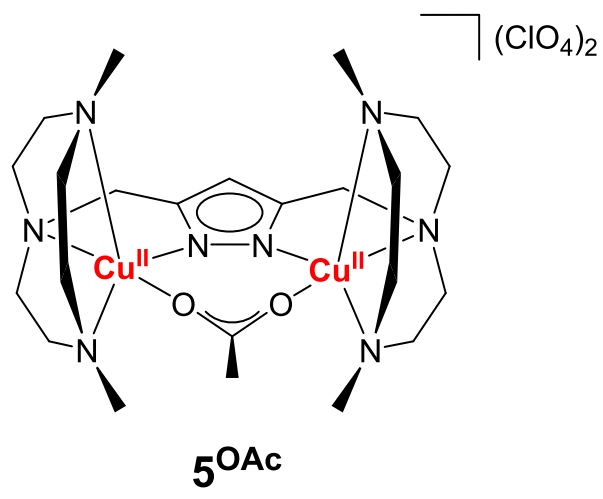

\section{Synthesis and Crystallization}

The synthesis and crystallization of $\mathbf{5}^{\mathbf{O A c}}$ were conducted identical to $\mathbf{5}^{\mathrm{HCO}}$ using $\mathrm{NaOAc}$ instead of $\mathrm{NaHCO}_{2}$ starting from $92 \mathrm{mg} \mathrm{HL}$.

Yield $140 \mathrm{mg}, 0.177 \mathrm{mmol}, 78 \%$

Molecular Weight $790.64 \mathrm{~g} / \mathrm{mol}$

Elemental Analysis for $5^{\text {oAc }}\left(\mathrm{C}_{23} \mathrm{H}_{44} \mathrm{Cl}_{2} \mathrm{Cu}_{2} \mathrm{~N}_{8} \mathrm{O}_{10}\right)$
Calc. / \%
C: 35.43 H: $5.65 \mathrm{~N}: 14.44$
Found / \%
C: $34.94 \mathrm{H}: 5.61 \mathrm{~N}: 14.17$

IR (ATR): $\tilde{v} / \mathrm{cm}^{-1}=2905(\mathrm{w}), 2868(\mathrm{w}), 2822(\mathrm{w}), 1559(\mathrm{~s}), 1491(\mathrm{w}), 1460(\mathrm{~m}), 1417(\mathrm{~m}), 1322(\mathrm{w})$, $1289(\mathrm{w}), 1230(\mathrm{w}), 1145(\mathrm{w}), 1067(\mathrm{~s}), 1008(\mathrm{~s}), 904(\mathrm{w}), 829(\mathrm{w}), 791(\mathrm{w}), 754(\mathrm{~m}), 646(\mathrm{w}), 621(\mathrm{~s})$, $573(w)$.

UV/vis (MeCN): $\lambda_{\max } / \mathrm{nm}\left(\varepsilon / \mathrm{L} \cdot \mathrm{mol}^{-1} \cdot \mathrm{cm}^{-1}\right)=684(297)$.

MS (ESI(+), MeOH): $m / z=294.9[\mathrm{M}]^{2+}, 691.1\left[\mathrm{M}+\mathrm{ClO}_{4}\right]^{+}$.

\section{$\left[\mathrm{L}^{2 \mathrm{a}} \mathrm{Cu}_{2}(\mathrm{OAc})\right]\left(\mathrm{ClO}_{4}\right)\left(5^{\mathrm{I} / \mathrm{II}}\right)$}

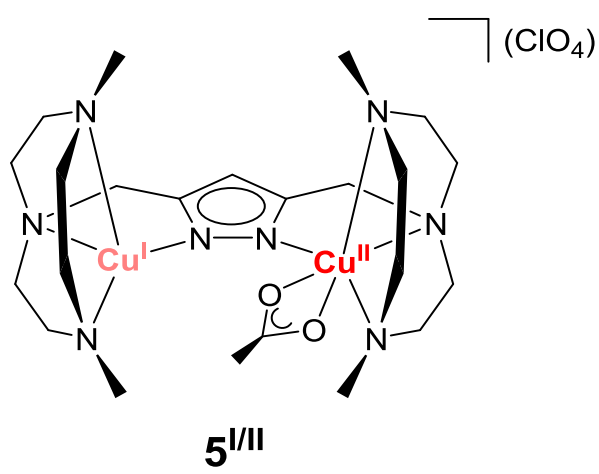

\section{Synthesis}

Under a nitrogen atmosphere, $\mathrm{Cp}_{2} \mathrm{Co}$ (1.2 eq) was added to $\mathbf{5}^{\mathbf{O A c}}$ in MeCN. $\mathbf{5}^{\mathbf{I} / \mathbf{I I}}$ was only generated in situ. A yield was not determined.

UV/vis $(\mathrm{MeCN}): \lambda_{\max } / \mathrm{nm}\left(\varepsilon / \mathrm{L} \cdot \mathrm{mol}^{-1} \cdot \mathrm{cm}^{-1}\right)=625$.

*as the exact concentration of $\mathbf{5}^{\mathbf{1} / \mathrm{II}}$ in solution is not clear, no extinction coefficients were calculated MS (ESI $(+), \operatorname{MeCN}): m / z=590.3[\mathrm{M}]^{+}$. 


\section{$\left[\mathrm{L}^{2 \mathrm{a}} \mathrm{Cu}_{2}\right]\left(\mathrm{ClO}_{4}\right)\left(5^{\mathrm{I} / 1}\right)$}

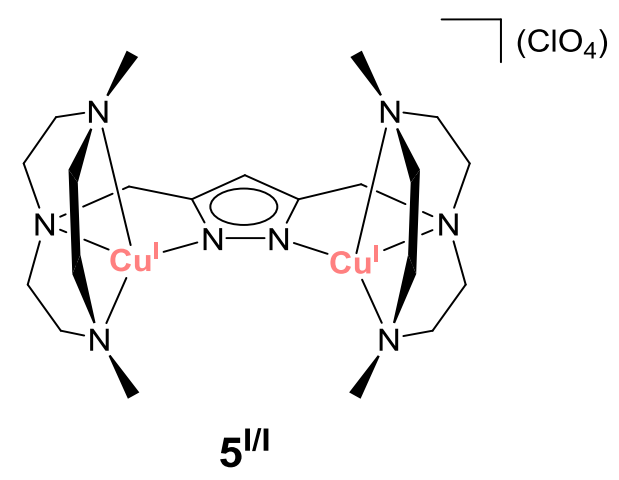

\section{Synthesis}

Under a nitrogen atmosphere, $\mathrm{Cp}_{2} \mathrm{Co}$ (2.3 eq) was added to $\mathbf{5}^{\mathbf{O A c}}$ in $\mathrm{MeCN}$. $\mathbf{5}^{\mathbf{1} / \mathbf{I}}$ was only generated in situ. A yield was not determined.

MS (ESI(+), MeCN): $m / z=531.2[\mathrm{M}]^{+}$.

\section{$\left[\mathrm{L}^{2 \mathrm{a}} \mathrm{Cu}_{2}\left(\mu-00^{--}\right)\right]\left(\mathrm{ClO}_{4}\right)\left(5^{\mathrm{s}}\right)$}

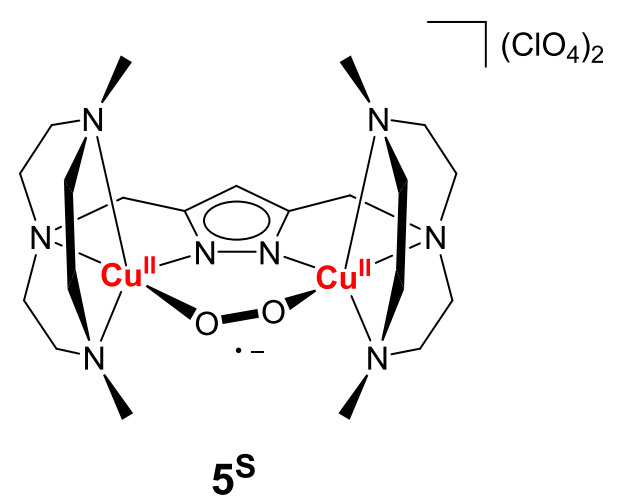

\section{Synthesis}

Under a nitrogen atmosphere, dry $\mathrm{O}_{2}$ was added to a solution of $\mathbf{5}^{\mathbf{0 A c}}$ and $\mathrm{Cp}_{2} \mathrm{Co}$ (1.2 eq) in $\mathrm{MeCN}$ at $-40{ }^{\circ} \mathrm{C} .5^{\mathrm{S}}$ is short-lived (only visible with a stopped flow setup) and rapidly converts to the peroxo complex $\mathbf{5}^{\mathbf{P}}$. A yield was not determined.

UV/vis $\left(\mathrm{MeCN}, 0^{\circ} \mathrm{C}\right): \lambda_{\max } / \mathrm{nm}=430{ }^{*}$

*as the exact concentration of $\mathbf{5}^{\mathbf{S}}$ in solution is not clear, no extinction coefficients were calculated

EChem (MeCN, $\left.0.1 \mathrm{M} \mathrm{NBu}_{4} \mathrm{PF}_{6},-45^{\circ} \mathrm{C}\right): E_{1 / 2} / \mathrm{V} v s . \mathrm{Fc}^{+/ 0}=-0.55\left(\mathrm{O}_{2}{ }^{2-} / \mathrm{O}_{2}{ }^{--}\right)$. 


\section{$\left[\mathrm{L}^{2 \mathrm{aa}} \mathrm{Cu}_{2}\left(\mu-\mathrm{OO}^{2-}\right)\right]\left(\mathrm{ClO}_{4}\right)\left(5^{\mathrm{P}}\right)$}

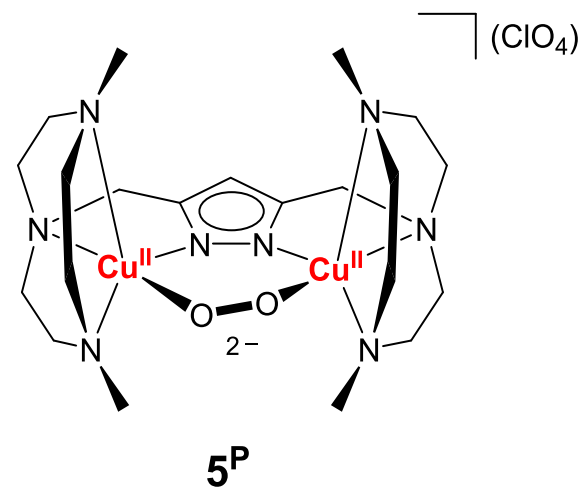

\section{Synthesis}

Under a nitrogen atmosphere, dry $\mathrm{O}_{2}$ was added to a solution of $\mathbf{5}^{\mathbf{0 A c}}$ and $\mathrm{Cp}_{2} \mathrm{Co}$ (1.2 or $2.3 \mathrm{eq}$ ) in $\mathrm{MeCN}$ at $-40^{\circ} \mathrm{C} .5^{\mathbf{p o O}}$ could not be crystallized and was only formed in situ. A yield was not determined.

UV/vis $\left(\mathrm{MeCN}, 0^{\circ} \mathrm{C}\right): \lambda_{\max } / \mathrm{nm}=510,630{ }^{*}$

*as the exact concentration of $\mathbf{5}^{\mathbf{p o o}}$ in solution is not clear, no extinction coefficients were calculated

EChem (MeCN, $\left.0.1 \mathrm{M} \mathrm{NBu}_{4} \mathrm{PF}_{6},-45^{\circ} \mathrm{C}\right): E_{1 / 2} / \mathrm{V} v s . \mathrm{Fc}^{+} / 0=-0.55\left(\mathrm{O}_{2}{ }^{2-} / \mathrm{O}_{2}{ }^{--}\right)$.

\section{$\left[\mathrm{L}^{2 \mathrm{~b}} \mathrm{Cu}_{2}\left(\mu-\mathrm{O}_{2} \mathrm{CH}\right)\right]\left(\mathrm{ClO}_{4}\right)_{2}\left(6^{\mathrm{HCO}}\right)$}

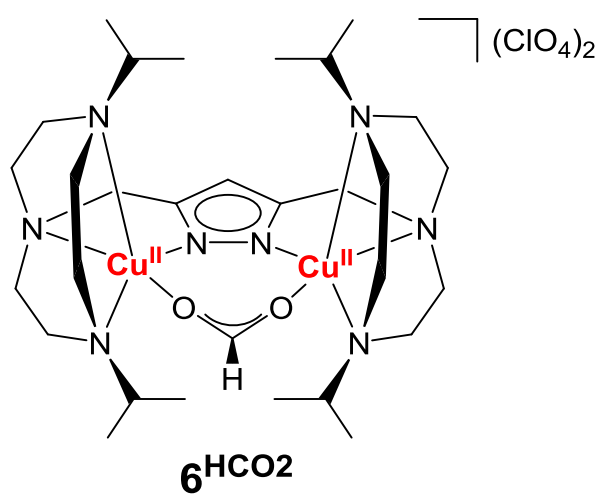

\section{Synthesis and Crystallization}

The synthesis and crystallization of $\mathbf{6}^{\mathrm{HCO}}$ were conducted identical to $\mathbf{5}^{\mathrm{HCO}}$ in a solvent mixture of $\mathrm{MeOH} / \mathrm{MeCN}$ (1/1) without adding $\mathrm{H}_{2} \mathrm{O}$ starting from $113 \mathrm{mg} \mathrm{HL}{ }^{2 b}$.

Yield $80 \mathrm{mg}, 0.090 \mathrm{mmol}, 41 \%$

Molecular Weight $888.83 \mathrm{~g} / \mathrm{mol}$

Elemental Analysis for $\mathbf{6}^{\mathbf{H C O}}\left(\mathrm{C}_{30} \mathrm{H}_{58} \mathrm{Cl}_{2} \mathrm{Cu}_{2} \mathrm{~N}_{8} \mathrm{O}_{10}\right)$
Calc. / \%
C: $40.54 \mathrm{H}: 6.58 \mathrm{~N}: 12.61$
Found / \%
C: 39.82 H: $6.37 \mathrm{~N}: 12.62$

IR (ATR): $\tilde{v} / \mathrm{cm}^{-1}=2975(\mathrm{w}), 2938(\mathrm{w}), 2870(\mathrm{w}), 1584(\mathrm{~s}), 1493(\mathrm{w}), 1454(\mathrm{w}), 1372(\mathrm{~m}), 1324(\mathrm{w})$, $1294(\mathrm{w}), 1271(\mathrm{w}), 1065(\mathrm{~s}), 996(\mathrm{~m}), 976(\mathrm{~m}), 950(\mathrm{~m}), 901(\mathrm{w}), 876(\mathrm{w}), 831(\mathrm{~m}), 792(\mathrm{~m}), 765(\mathrm{w})$, $736(\mathrm{~m}), 714(\mathrm{~m}), 664(\mathrm{w}), 621(\mathrm{~s}), 595(\mathrm{~m}), 567(\mathrm{w}), 518(\mathrm{w}), 498(\mathrm{w}), 456(\mathrm{w})$.

UV/vis (MeCN): $\lambda_{\max } / \mathrm{nm}\left(\varepsilon / \mathrm{L} \cdot \mathrm{mol}^{-1} \cdot \mathrm{cm}^{-1}\right)=727$ (397).

MS (ESI(+), MeCN): $m / z=344.2[\mathrm{M}]^{2+}, 787.3\left[\mathrm{M}+\mathrm{ClO}_{4}\right]^{+}$.

MS (ESI $(-), \operatorname{MeCN}): m / z=985.1\left[\mathrm{M}+3 \mathrm{ClO}_{4}\right]^{-}$. 


\section{$\left[\mathrm{L}^{2 \mathrm{~b}} \mathrm{Cu}_{2}(\mu-\mathrm{OAc})\right]\left(\mathrm{ClO}_{4}\right)_{2}\left(6^{\mathrm{OAc}}\right)$}

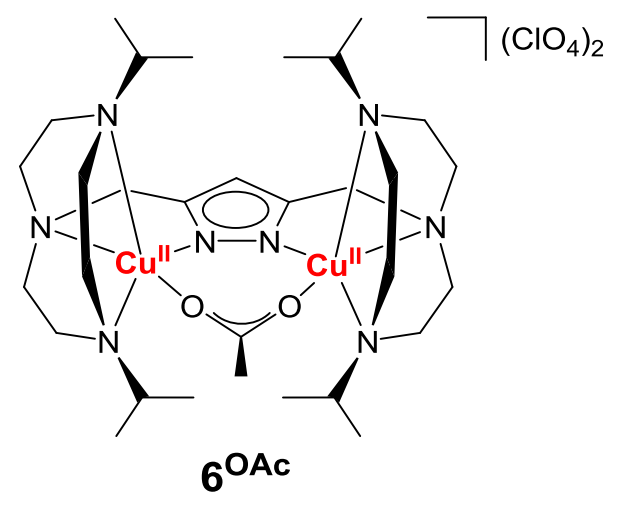

\section{Synthesis and Crystallization}

The synthesis and crystallization of $\mathbf{6}^{\mathbf{0 A c}}$ were conducted identical to $\mathbf{5}^{\mathrm{HCO}}$ using $\mathrm{NaOAc}$ instead of $\mathrm{NaHCO}_{2}$. The synthesis was done in a solvent mixture of $\mathrm{MeOH} / \mathrm{MeCN}(1 / 1)$ without adding $\mathrm{H}_{2} \mathrm{O}$ starting from $124 \mathrm{mg} \mathrm{HL}^{2 \mathrm{~b}}$.

Yield $129 \mathrm{mg}, 0.143 \mathrm{mmol}, 60 \%$

Molecular Weight $902.86 \mathrm{~g} / \mathrm{mol}$

Elemental Analysis for $6^{\mathbf{0 A c}}\left(\mathrm{C}_{31} \mathrm{H}_{60} \mathrm{Cl}_{2} \mathrm{Cu}_{2} \mathrm{~N}_{8} \mathrm{O}_{10}\right)$
Calc. / \%
C: $41.24 \mathrm{H}: 6.70 \mathrm{~N}: 12.41$
Found / \%
C: $41.01 \mathrm{H}: 6.68 \mathrm{~N}: 12.35$

IR (ATR): $\tilde{v} / \mathrm{cm}^{-1}=2972(\mathrm{w}), 2937(\mathrm{w}), 2873(\mathrm{w}), 1569(\mathrm{~s}), 1493(\mathrm{w}), 1451(\mathrm{~m}), 1426(\mathrm{~m}), 1386(\mathrm{w})$, $1350(\mathrm{w}), 1329(\mathrm{w}), 1283(\mathrm{w}), 1175(\mathrm{~m}), 1066(\mathrm{~s}), 997(\mathrm{~m}), 975(\mathrm{~m}), 949(\mathrm{~m}), 902(\mathrm{w}), 874(\mathrm{w}), 833$ (m), $794(\mathrm{~m}), 763(\mathrm{~m}), 737(\mathrm{~m}), 715(\mathrm{~m}), 665(\mathrm{w}), 621(\mathrm{~s}), 594(\mathrm{~m}), 568(\mathrm{w}), 537(\mathrm{w}), 517(\mathrm{w}), 496$ (w), $456(\mathrm{w})$.

UV/vis $(\mathrm{MeCN}): \lambda_{\max } / \mathrm{nm}\left(\varepsilon / \mathrm{L} \cdot \mathrm{mol}^{-1} \cdot \mathrm{cm}^{-1}\right)=731$ (399).

MS (ESI(+), MeCN): $m / z=351.3[\mathrm{M}]^{2+}, 801.3\left[\mathrm{M}+\mathrm{ClO}_{4}\right]^{+}$.

MS (ESI $(-), \operatorname{MeCN}): m / z=999.1\left[\mathrm{M}+3 \mathrm{ClO}_{4}\right]^{-}$. 


\section{$\left[\mathrm{L}^{2 \mathrm{a}} \mathrm{Co}_{2}(\mathrm{MeCN})_{4}\right]\left(\mathrm{ClO}_{4}\right)_{3}\left(7^{\mathrm{MeCN}}\right)$}

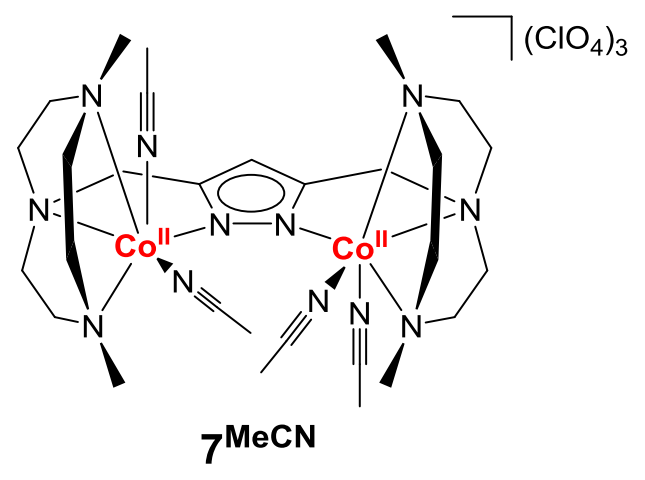

\section{Synthesis and Crystallization}

Under a nitrogen atmosphere, $\mathrm{HL}^{2 \mathrm{a}}$ (48 mg, $0.123 \mathrm{mmol}, 1.0 \mathrm{eq}$ ) was dissolved in $\mathrm{MeOH} / \mathrm{MeCN}(1 / 1$, $5 \mathrm{~mL}) . \mathrm{NEt}_{3}(0.1 \mathrm{~mL}, 0.717 \mathrm{mmol}, 5.8 \mathrm{eq})$ and subsequently $\mathrm{Co}\left(\mathrm{ClO}_{4}\right)_{2} \cdot 6 \mathrm{H}_{2} \mathrm{O}(87 \mathrm{mg}, 0.246 \mathrm{mmol}$, $2.0 \mathrm{eq}$ ) were added. After stirring for 30 minutes at room temperature, the solution was removed under reduced pressure. Crystals of $7^{\mathrm{MecN}}$ were received by diffusion of $\mathrm{Et}_{2} \mathrm{O}$ into a solution of the crude product in MeCN. Several recrystallizations under the same conditions were necessary to obtain single crystals. A yield was not determined.

Molecular Weight $986.03 \mathrm{~g} / \mathrm{mol}$

Empirical Formula $\mathrm{C}_{29} \mathrm{H}_{53} \mathrm{Cl}_{3} \mathrm{Co}_{2} \mathrm{~N}_{12} \mathrm{O}_{12}$

Elemental Analysis for $7^{\mathrm{MeCN}}+1.2 \mathrm{MeCN}\left(\mathrm{C}_{31.4} \mathrm{H}_{56.6} \mathrm{Cl}_{3} \mathrm{CO}_{2} \mathrm{~N}_{13.2} \mathrm{O}_{12}\right)$
Calc. / \%
C: $36.43 \mathrm{H}: 5.51 \mathrm{~N}: 17.86$
Found / \%
C: $36.64 \mathrm{H}: 5.60 \mathrm{~N}: 17.74$

IR (ATR): $\tilde{v} / \mathrm{cm}^{-1}=2991(\mathrm{w}), 2936(\mathrm{w}), 2873(\mathrm{w}), 2826(\mathrm{w}), 2311(\mathrm{w}), 2278(\mathrm{w}), 1490(\mathrm{w}), 1469(\mathrm{w})$, $1458(\mathrm{w}), 1375(\mathrm{w}), 1323(\mathrm{w}), 1291(\mathrm{w}), 1075(\mathrm{~s}), 1911(\mathrm{~s}), 994(\mathrm{~m}), 932(\mathrm{w}), 892(\mathrm{w}), 813(\mathrm{w}), 789$ $(w), 758(w), 621(s), 579(w), 518(w), 484(w), 439(w)$.

UV/vis $(\mathrm{MeCN}): \lambda_{\max } / \mathrm{nm}\left(\varepsilon / \mathrm{L} \cdot \mathrm{mol}^{-1} \cdot \mathrm{cm}^{-1}\right)=310\left(2.8 \cdot 10^{3}\right), 464$ (152), 543 (sh, 51).

MS (ESI $(+), \mathrm{MeCN}): m / z=310.8\left[\mathrm{M}-4 \mathrm{MeCN}+\mathrm{ClO}_{4}\right]^{2+}, 721.1\left[\mathrm{M}-4 \mathrm{MeCN}+2 \mathrm{ClO}_{4}\right]^{+}$.

MS (ESI(-), MeCN): $m / z=856.9\left[\mathrm{M}-4 \mathrm{MeCN}+\mathrm{Cl}+3 \mathrm{ClO}_{4}\right]^{-}, 918.8\left[\mathrm{M}-4 \mathrm{MeCN}+4 \mathrm{ClO}_{4}\right]^{-}$.

EChem (MeCN, $\left.0.1 \mathrm{M} \mathrm{NBu}_{4} \mathrm{PF}_{6}, 100 \mathrm{mV} / \mathrm{s}\right): E_{\mathrm{p}}{ }^{\text {ox }} / \mathrm{V}$ vs. Fc $/ \mathrm{Fc}^{+}=0.58\left(\mathrm{Co}^{\mathrm{II}} \mathrm{Co}^{\mathrm{III}} / \mathrm{Co}^{\mathrm{II}} \mathrm{Co}^{\mathrm{II}}\right)$,

$$
0.96\left(\mathrm{Co}^{\mathrm{III}} \mathrm{Co}^{\mathrm{III}} / \mathrm{Co}^{\mathrm{II}} \mathrm{Co}^{\mathrm{III}}\right) \text {. }
$$

$E_{\mathrm{p}}^{\text {red }} / \mathrm{V} v s . \mathrm{Fc} / \mathrm{Fc}^{+}=-0.08\left(\mathrm{Co}^{\mathrm{II}} \mathrm{Co}^{\mathrm{III}} / \mathrm{Co}^{\mathrm{III}} \mathrm{Co}^{\mathrm{III}}\right)$,

$-0.40\left(\mathrm{Co}^{\mathrm{II}} \mathrm{Co}^{\mathrm{II}} / \mathrm{Co}^{\mathrm{II}} \mathrm{Co}^{\mathrm{III}}\right)$. 


\section{$\left[\mathrm{L}^{2 \mathrm{a}} \mathrm{Co}_{2}\left(\mu-\mathrm{PO}_{4}\right)(\mathrm{MeCN})_{2}\right]\left(\mathrm{ClO}_{4}\right)_{2}\left(7^{\mathrm{PO} 4}\right)$}

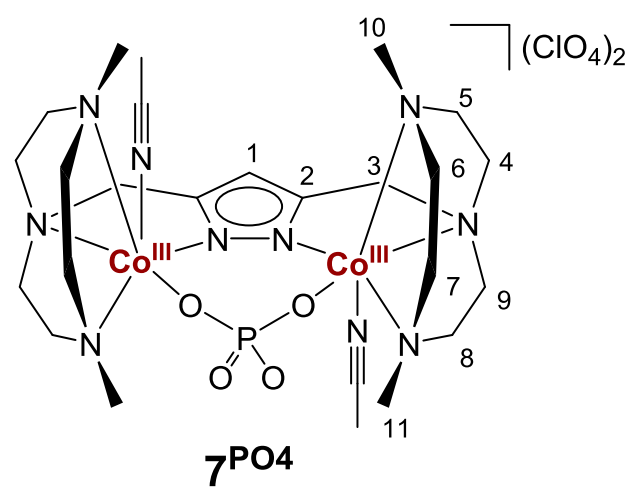

\section{Synthesis}

Dissolution of 7 MecN and $\mathrm{Na}_{2} \mathrm{HPO}_{4}$ (2 eq) in $\mathrm{D}_{2} \mathrm{O}$ led partial oxidation of the complex and exchange of the ligand in the bimetallic pocket to phosphate. The presence of axial ligands is assumed based on complexes $\mathbf{7}^{\mathrm{MeCN}}$ and $\mathbf{7}^{\mathrm{A}}$.

Molecular Weight $899.45 \mathrm{~g} / \mathrm{mol}$

Empirical Formula $\mathrm{C}_{25} \mathrm{H}_{47} \mathrm{Cl}_{2} \mathrm{Co}_{2} \mathrm{~N}_{10} \mathrm{O}_{12} \mathrm{P}$

MS $(\mathrm{ESI}(+), \mathrm{MeOH}): m / z=309.0[\mathrm{M}-2 \mathrm{MeCN}]^{2+}(\mathrm{III} / \mathrm{III}) * 309.6[\mathrm{M}-2 \mathrm{MeCN}+\mathrm{H}]^{2+}(\mathrm{II} / \mathrm{III}), 310.1$ $[\mathrm{M}-2 \mathrm{MeCN}+2 \mathrm{H}]^{2+}(\mathrm{II} / \mathrm{II}), 618.1[\mathrm{M}-2 \mathrm{MeCN}]^{+}(\mathrm{II} / \mathrm{III}), 619.2[\mathrm{M}-2 \mathrm{MeCN}+\mathrm{H}]^{+}(\mathrm{II} / \mathrm{II})$.

*oxidation states of the cobalt ions, the bridging moiety varies between $\mathrm{H}_{2} \mathrm{PO}_{4}-\mathrm{HPO}_{4}{ }^{2-}$ and $\mathrm{PO}_{4}{ }^{3-}$ 


\section{$\left[\mathrm{L}^{2 \mathrm{a}} \mathrm{Co}_{2}\left(\mu-\mathrm{CH}_{3} \mathrm{C}(\mathrm{O}) \mathrm{NH}\right)(\mathrm{MeCN})_{2}\right]\left(\mathrm{ClO}_{4}\right)_{4}\left(7^{\mathrm{A}}\right)$}

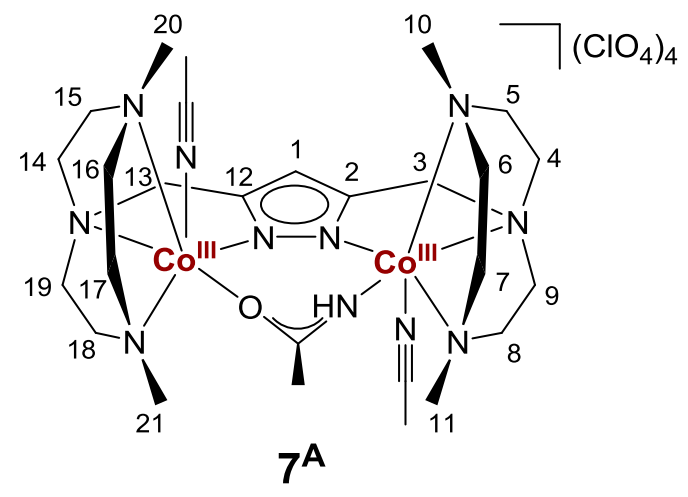

\section{Synthesis and Crystallization}

The synthesis of $7^{\mathrm{A}}$ was conducted similar to $\mathbf{7}^{\mathrm{MeCN}}$ using deaerated instead of dry solvents. During the synthetic procedure only $\mathrm{MeOH}$ was used as a solvent, $\mathrm{MeCN}$ was later used for crystallization. A polycrystalline material was obtained, which was not suitable for X-ray diffraction. The oxidation of the cobalt ions from $\mathrm{Co}^{\mathrm{II}}$ to $\mathrm{Co}^{\mathrm{III}}$ suggests that the atmosphere during synthesis or crystallization was not completely $\mathrm{O}_{2}$ free. The presence of axial ligands could not unambiguously be confirmed and are merely assumed to be $\mathrm{MeCN}$, given the tendency of cobalt(III) complexes to be octahedral. All characterization was done assuming the above shown structure.

Molecular Weight $1061.43 \mathrm{~g} / \mathrm{mol}$

Empirical Formula $\mathrm{C}_{27} \mathrm{H}_{51} \mathrm{Cl}_{4} \mathrm{Co}_{2} \mathrm{~N}_{11} \mathrm{O}_{17}$

${ }^{1}$ H-NMR $\left(500 \mathrm{MHz}, \mathrm{CD}_{3} \mathrm{CN}\right) \delta(\mathrm{ppm})=6.18(\mathrm{~s}, 1 \mathrm{H}, 1-\mathrm{H}), 4.56(\mathrm{~d}, J=16.6 \mathrm{~Hz}, 1 \mathrm{H}, 13-\mathrm{H}), 4.43(\mathrm{~d}$, $J=16.8 \mathrm{~Hz}, 1 \mathrm{H}, 3-\mathrm{H}), 4.00$ (d, $\left.J=16.6 \mathrm{~Hz}, 1 \mathrm{H}, 13^{\prime}-\mathrm{H}\right), 3.96$ (d, J = $\left.16.8 \mathrm{~Hz}, 1 \mathrm{H}, 3^{\prime}-\mathrm{H}\right), 3.84-3.75(\mathrm{~m}, 1 \mathrm{H}$, 19-H), 3.54-3.15 (m, 11H, 4-H, 4'-H, 7-H, 8-H, 9-H, 9'-H, 14-H, 14'-H, 17-H, 18-H, 19'-H), 3.06-2.91 (m, 2H, 6-H, 18'-H), 2.88-2.49 (m, 8H, 5-H, 5'-H, 6'-H, 7'-H, 8'-H, 15-H, 16-H, 17'-H), 2.78 (s, 3H, 10-H), 2.29 (s, 3H, 21-H), 2.28-2.19 (m, 2H, 15'-H, 16'-H), 2.13 (s, 3H, 20-H), 2.01 (s, 3H, 11-H), $1.74(\mathrm{~s}, 3 \mathrm{H}$, $\left.\mathrm{CH}_{3} \mathrm{C}(\mathrm{O}) \mathrm{NH}\right) *$

Pure shift ${ }^{1}$ H-NMR (500 MHz, CD 3 CN) $\delta(\mathrm{ppm})=6.18(1-\mathrm{H}), 4.55$ (13-H), $4.43(3-\mathrm{H}), 4.00\left(13^{\prime}-\mathrm{H}\right), 3.95$ (3'-H), 3.80 (19-H), 3.50 (14-H), 3.46 (4-H), 3.40 (9-H), 3.37 (19'-H), 3.34 (18-H), 3.32 (9'-H), 3.27 (14'H), $3.26\left(4^{\prime}-\mathrm{H}\right), 3.25(17-\mathrm{H}), 3.21(8-\mathrm{H}), 3.19(7-\mathrm{H}), 3.01(6-\mathrm{H}), 2.94\left(18^{\prime}-\mathrm{H}\right), 2.84(5-\mathrm{H}), 2.82(16-\mathrm{H})$, $2.79\left(8^{\prime}-\mathrm{H}\right), 2.78(10-\mathrm{H}), 2.72\left(6^{\prime}-\mathrm{H}\right), 2.66\left(7^{\prime}-\mathrm{H}\right), 2.63\left(17^{\prime}-\mathrm{H}\right), 2.57\left(5^{\prime}-\mathrm{H}\right), 2.53(15-\mathrm{H}), 2.29(21-\mathrm{H}), 2.26$ (16'-H), $2.22\left(15^{\prime}-\mathrm{H}\right), 2.13(20-\mathrm{H}), 2.00(11-\mathrm{H}), 1.74\left(\mathrm{CH}_{3} \mathrm{C}(\mathrm{O}) \mathrm{NH}\right)$. $^{*}$

${ }^{13} \mathbf{C}\left\{{ }^{1} \mathrm{H}\right\}-N M R\left(126 \mathrm{MHz}, \mathrm{CD}_{3} \mathrm{CN}\right) \delta(\mathrm{ppm})=184.64\left(\mathrm{CH}_{3} \underline{\mathrm{C}}(\mathrm{O}) \mathrm{NH}\right), 171.91(2-\mathrm{C}), 170.11(12-\mathrm{C}), 106.88$ (1-C), 64.15 (13-C), 63.84 (3-C), 63.02 (7-C), 62.64 (15-C), 61.81 (18-C), 61.55 (8-C), 61.48 (5-C), 61.22 (17-C), 60.21 (4-C), 59.77 (9-C), 59.45 (19-C), 58.00 (14-C), 57.97 (16-C), 57.41 (6-C), 51.87 (10-C), 51.29 (20-C), 49.16 (11-C), $48.53(21-\mathrm{C}), 28.61\left(\mathrm{CH}_{3} \mathrm{C}(\mathrm{O}) \mathrm{NH}\right)$ *

*the distinction between the left and right side of the complex is not possible and is merely done for simplification

*the $\mathrm{NH}$ is assumed at $3.23 \mathrm{ppm}$

*the annotation with and without prime correspond to the diastereotopic protons at the same carbon atom

UV/vis $(\mathrm{MeCN}): \lambda_{\max } / \mathrm{nm}\left(\varepsilon / \mathrm{L} \cdot \mathrm{mol}^{-1} \cdot \mathrm{cm}^{-1}\right)=306\left(4.7 \cdot 10^{3}\right), 480\left(3.6 \cdot 10^{3}\right), 686(368)$.

MS (ESI(+), MeCN): $m / z=290.3[\mathrm{M}-2 \mathrm{MeCN}]^{2+}, 680.1\left[\mathrm{M}-2 \mathrm{MeCN}+\mathrm{ClO}_{4}\right]^{+*}$.*

MS (ESI(-), MeCN): $m / z=909.9\left[\mathrm{M}-2 \mathrm{MeCN}+\mathrm{MeOH}+3 \mathrm{ClO}_{4}\right]^{-*}$.*

*all species are in the oxidation state $\mathrm{Co}^{\mathrm{II}} \mathrm{Co}^{\mathrm{II}}$

IR (KBr): $\tilde{v} / \mathrm{cm}^{-1}=3434(\mathrm{~m}), 2922$ (w), $2871(\mathrm{w}), 2360(\mathrm{w}), 2342(\mathrm{w}), 1636$ (w), $1559(\mathrm{w}), 1498$ (w), 1458 (m), 1296 (w), 1250 (w), 1095 (s), 1012 (w), 993 (w) 826 (w), 798 (w), 764 (w), 747 (w), 668 (w), $624(\mathrm{~m})$. 


\section{$\left[\mathrm{L}^{2 \mathrm{a}} \mathrm{Co}_{2}\left(\mu-\mathrm{O}_{2} \mathrm{H}_{3}\right)\right]\left(\mathrm{ClO}_{4}\right)_{2}\left(7^{\mathrm{O} 2 \mathrm{H}} 3\right)$}

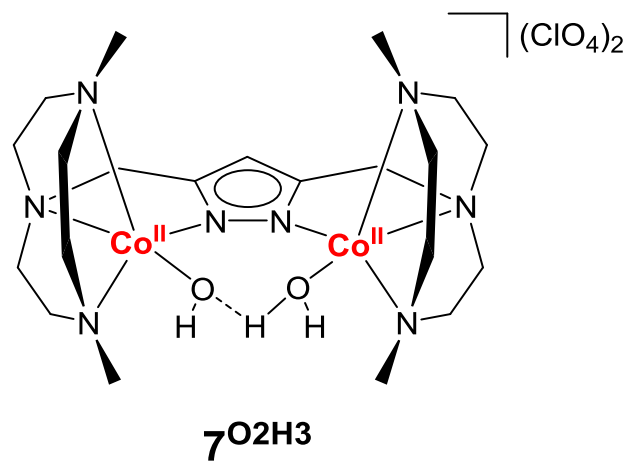

\section{Synthesis and Crystallization}

The synthesis of $\mathbf{7}^{\mathbf{0 2}} \mathbf{H} \mathbf{3}$ was conducted similar to $\mathbf{7}^{\mathrm{MecN}}$ using only $\mathrm{MeOH}$ as the solvent. After addition of $\mathrm{Co}\left(\mathrm{ClO}_{4}\right)_{2} \cdot 6 \mathrm{H}_{2} \mathrm{O}$, a pink precipitate was formed. The precipitate was washed with $\mathrm{MeOH}$ and dried under reduced pressure. Crystallization was attempted by diffusion of $\mathrm{Et}_{2} \mathrm{O}$ into a filtered solution of the crude product in acetone. Pink polycrystalline material was obtained, which was not suitable for $\mathrm{X}$-ray diffraction. The bridging ligand is presumed to be $\mathrm{O}_{2} \mathrm{H}_{3}$ based on conclusions from the mass spectrometric analysis. All characterization was done assuming the above shown structure.

Molecular Weight $839.50 \mathrm{~g} / \mathrm{mol}$

Empirical Formula $\mathrm{C}_{25} \mathrm{H}_{50} \mathrm{Cl}_{2} \mathrm{Co}_{2} \mathrm{~N}_{10} \mathrm{O}_{10}$

UV/vis (MeCN): $\lambda_{\max } / \mathrm{nm}\left(\varepsilon / \mathrm{L} \cdot \mathrm{mol}^{-1} \cdot \mathrm{cm}^{-1}\right)=452(116), 550$ (238).

MS (ESI(+), MeOH): $m / z=269.9\left[\mathrm{M}-\mathrm{H}_{2} \mathrm{O}\right]^{2+}, 639.1\left[\mathrm{M}-\mathrm{H}_{2} \mathrm{O}+\mathrm{ClO}_{4}\right]^{+}, 653.1\left[\mathrm{M}-\mathrm{O}_{2} \mathrm{H}_{3}+\mathrm{OCH}_{3}+\mathrm{ClO}_{4}\right]^{+}$.

MS $(\operatorname{ESI}(-), \mathrm{MeOH}): m / z=836.9\left[\mathrm{M}-\mathrm{H}_{2} \mathrm{O}+3 \mathrm{ClO}_{4}\right]^{-}$. 


\section{$\left[\mathrm{L}^{2 \mathrm{~b}} \mathrm{Co}_{2}\left(\mu-\mathrm{O}_{2} \mathrm{CH}\right)\right]\left(\mathrm{ClO}_{4}\right)_{2}\left(8^{\mathrm{HCO}}\right)$}

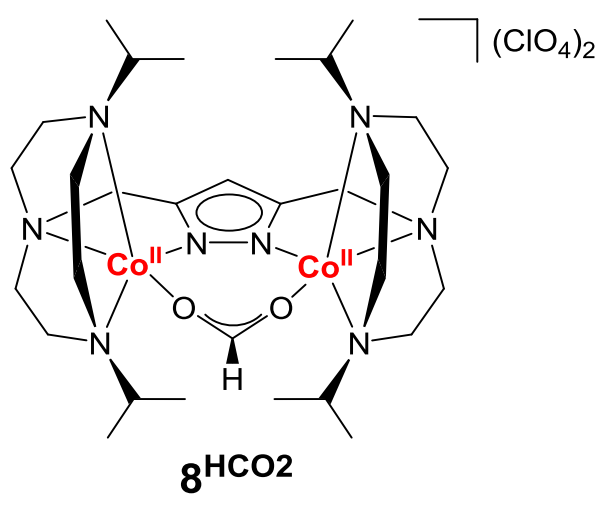

\section{Synthesis and Crystallization}

$\mathrm{HL}^{2 \mathrm{~b}}$ (95 mg, $0.183 \mathrm{mmol}, 1.0 \mathrm{eq}$ ) was dissolved in $\mathrm{MeOH}(5 \mathrm{~mL})$ and NEt 3 (100 $\left.\mu \mathrm{L}, 0.717 \mathrm{mmol}, 3.9 \mathrm{eq}\right)$ was added. Subsequent addition of $\mathrm{Co}\left(\mathrm{ClO}_{4}\right)_{2} \cdot 6 \mathrm{H}_{2} \mathrm{O}(134 \mathrm{mg}, 0.366 \mathrm{mmol}, 2.0 \mathrm{eq})$ and $\mathrm{NaHCO}_{2}(25 \mathrm{mg}$, $0.368 \mathrm{mmol}, 2.0 \mathrm{eq}$ ) in one portion yielded a pink precipitate, which was dissolved by addition of $\mathrm{MeCN}$ $(3 \mathrm{~mL})$. The reaction mixture was stirred for 1.5 hours at room temperature and then left for crystallization by diffusion of $\mathrm{Et}_{2} \mathrm{O}$. The first crystallization afforded a mixture of complex and $\mathrm{NaHCO}_{2}$ crystals. The crystals were gathered and separated by addition of MeCN upon which the complex crystals dissolved and the salt crystals remained intact. Subsequent filtration and recrystallization by diffusion with $\mathrm{Et}_{2} \mathrm{O}$ yielded pink crystals suitable for X-ray diffraction.

Yield $66 \mathrm{mg}, 0.075 \mathrm{mmol}, 41 \%$

Molecular Weight $879.61 \mathrm{~g} / \mathrm{mol}$

Empirical Formula $\mathrm{C}_{30} \mathrm{H}_{58} \mathrm{Cl}_{2} \mathrm{Co}_{2} \mathrm{~N}_{8} \mathrm{O}_{10}$

Elemental Analysis for $\mathbf{8}^{\mathrm{HCO} 2}+\mathrm{MeCN}\left(\mathrm{C}_{32} \mathrm{H}_{61} \mathrm{Cl}_{2} \mathrm{Co}_{2} \mathrm{~N}_{9} \mathrm{O}_{10}\right)$
Calc. / \%
C: $41.75 \mathrm{H}: 6.68 \mathrm{~N}: 13.69$
Found / \%
C: $41.70 \mathrm{H}: 6.71 \mathrm{~N}: 13.49$

UV/vis (MeCN): $\lambda_{\max } / \mathrm{nm}\left(\varepsilon / \mathrm{L} \cdot \mathrm{mol}^{-1} \cdot \mathrm{cm}^{-1}\right)=445$ (103), 503 (96), 523 (88), 551 (320), 722 (45).

MS $(\operatorname{ESI}(+), \operatorname{MeCN}): m / z=339.9[\mathrm{M}]^{2+}, 779.2\left[\mathrm{M}+\mathrm{ClO}_{4}\right]^{+}$.

MS (ESI(-), MeCN): $m / z=977.0\left[\mathrm{M}+3 \mathrm{ClO}_{4}\right]^{-}$.

IR (KBr): $\tilde{v} / \mathrm{cm}^{-1}=3419(\mathrm{~m}), 2969(\mathrm{w}), 2940(\mathrm{w}), 2866(\mathrm{w}), 2361(\mathrm{w}), 2341(\mathrm{w}), 1589(\mathrm{~m}), 1568(\mathrm{~m})$, 1496 (w), 1454 (w), 1375 (w), 1286 (w), 1147 (m), 1093 (s),1022 (w), 951 (w), $826(\mathrm{w}), 800$ (w), 739 $(\mathrm{w}), 720(\mathrm{w}), 623(\mathrm{~m})$.

EChem (MeCN, $\left.0.1 \mathrm{M} \mathrm{NBu}_{4} \mathrm{PF}_{6}, 100 \mathrm{mV} / \mathrm{s}\right): E_{\mathrm{p}}^{\mathrm{ox}} / \mathrm{V}$ vs. $\mathrm{Fc} / \mathrm{Fc}^{+}=0.93\left(\mathrm{Co}^{\mathrm{II}} \mathrm{Co}^{\mathrm{III}} / \mathrm{Co}^{\mathrm{II}} \mathrm{Co}^{\mathrm{II}}\right)$,

$1.18\left(\mathrm{Co}^{\mathrm{III}} \mathrm{Co}^{\mathrm{III}} / \mathrm{Co}^{\mathrm{II}} \mathrm{Co}^{\mathrm{III}}\right)$.

$$
\begin{aligned}
E_{\mathrm{p}}{ }^{\text {red }} / \mathrm{V} \text { vs. } \mathrm{Fc} / \mathrm{Fc}^{+}= & 0.18\left(\mathrm{Co}^{\mathrm{II}} \mathrm{Co}^{\mathrm{III}} / \mathrm{Co}^{\mathrm{III}} \mathrm{Co}^{\mathrm{III}}\right), \\
& -0.08\left(\mathrm{Co}^{\mathrm{II}} \mathrm{Co}^{\mathrm{II}} / \mathrm{Co}^{\mathrm{II}} \mathrm{Co}^{\mathrm{III}}\right) .
\end{aligned}
$$




\section{$\left[\mathrm{L}^{2 \mathrm{~b}} \mathrm{Co}_{2}(\mu-\mathrm{OAc})\right]\left(\mathrm{ClO}_{4}\right)_{2}\left(8^{\mathrm{OAc}}\right)$}

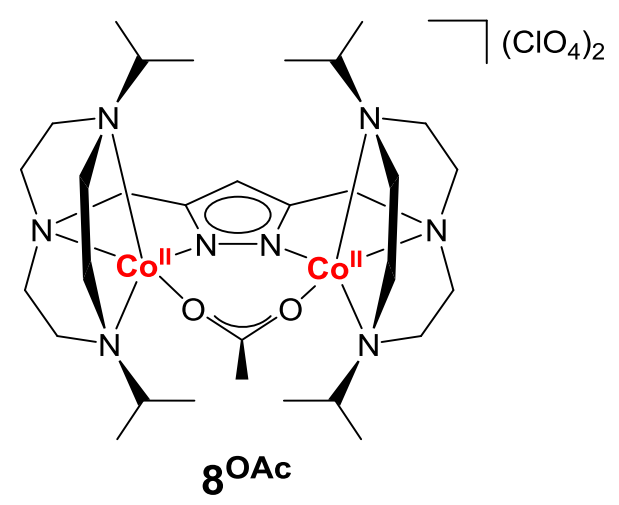

\section{Synthesis and Crystallization}

The synthesis and crystallization of $\mathbf{8}^{\mathbf{O A c}}$ were conducted identical to $\mathbf{8}^{\mathrm{HCO}}$ using $\mathrm{NaOAc}$ instead of $\mathrm{NaHCO}_{2}$ starting from $122 \mathrm{mg} \mathrm{HL}$.

Yield $79 \mathrm{mg}, 0.088 \mathrm{mmol}, 38 \%$

Molecular Weight $893.63 \mathrm{~g} / \mathrm{mol}$

Empirical Formula $\mathrm{C}_{31} \mathrm{H}_{60} \mathrm{Cl}_{2} \mathrm{Co}_{2} \mathrm{~N}_{8} \mathrm{O}_{10}$

Elemental Analysis for $8^{\text {oAc }}\left(\mathrm{C}_{33} \mathrm{H}_{63} \mathrm{Cl}_{2} \mathrm{Co}_{2} \mathrm{~N}_{9} \mathrm{O}_{10}\right)$
Calc. / \%
C: $42.41 \mathrm{H}: 6.79 \mathrm{~N}: 13.49$
Found / \%
C: $42.53 \mathrm{H}: 6.74 \mathrm{~N}: 13.51$

UV/vis $(\mathrm{MeCN}): \lambda_{\max } / \mathrm{nm}\left(\varepsilon / \mathrm{L} \cdot \mathrm{mol}^{-1} \cdot \mathrm{cm}^{-1}\right)=445$ (93), 504 (84), 524 (65), 552 (290), 729 (44).

MS (ESI(+), MeCN): $m / z=346.9[\mathrm{M}]^{2+}, 793.2\left[\mathrm{M}+\mathrm{ClO}_{4}\right]^{+}$.

MS (ESI(-), MeCN): $m / z=991.1\left[\mathrm{M}+3 \mathrm{ClO}_{4}\right]^{-}$.

IR (KBr pellet): $\tilde{v} / \mathrm{cm}^{-1}=3416(\mathrm{~m}), 2970(\mathrm{w}), 2938(\mathrm{w}), 2866(\mathrm{w}), 1638(\mathrm{w}), 1617(\mathrm{w}), 1567(\mathrm{~m})$, $1495(\mathrm{w}), 1453(\mathrm{w}), 1442(\mathrm{w}), 1327(\mathrm{w}), 1284(\mathrm{w}), 1147(\mathrm{~m}), 1092(\mathrm{~s}), 1023(\mathrm{w}), 1000(\mathrm{w}), 980(\mathrm{w})$, $951(\mathrm{w}), 826(\mathrm{w}), 799(\mathrm{w}), 765(\mathrm{w}), 741(\mathrm{w}), 721(\mathrm{w}), 624(\mathrm{~m})$.

EChem (MeCN, $\left.0.1 \mathrm{M} \mathrm{NBu}_{4} \mathrm{PF}_{6}, 100 \mathrm{mV} / \mathrm{s}\right): E_{\mathrm{p}}{ }^{\text {ox }} / \mathrm{V}$ vs. Fc $/ \mathrm{Fc}^{+}=0.87\left(\mathrm{Co}^{\mathrm{II}} \mathrm{Co}^{\mathrm{III}} / \mathrm{Co}^{\mathrm{II}} \mathrm{Co}^{\mathrm{II}}\right)$, $1.13\left(\mathrm{Co}^{\mathrm{III}} \mathrm{Co}^{\mathrm{III}} / \mathrm{Co}^{\mathrm{II}} \mathrm{Co}^{\mathrm{III}}\right)$.

$E_{\mathrm{p}}{ }^{\text {red }} / \mathrm{V} v s . \mathrm{Fc} / \mathrm{Fc}^{+}=0.15\left(\mathrm{Co}^{\mathrm{II}} \mathrm{Co}^{\mathrm{III}} / \mathrm{Co}^{\mathrm{III}} \mathrm{Co}^{\mathrm{III}}\right)$, $-0.12\left(\mathrm{Co}^{\mathrm{II}} \mathrm{Co}^{\mathrm{II}} / \mathrm{Co}^{\mathrm{II}} \mathrm{Co}^{\mathrm{III}}\right)$. 


\section{$\left[\left(\mathrm{L}^{2 \mathrm{a}}\right)_{2} \mathrm{Cu}_{6}(\mathrm{H})_{2}\right]\left(\mathrm{ClO}_{4}\right)_{2}\left({ }^{\mathrm{H}}\right)$}

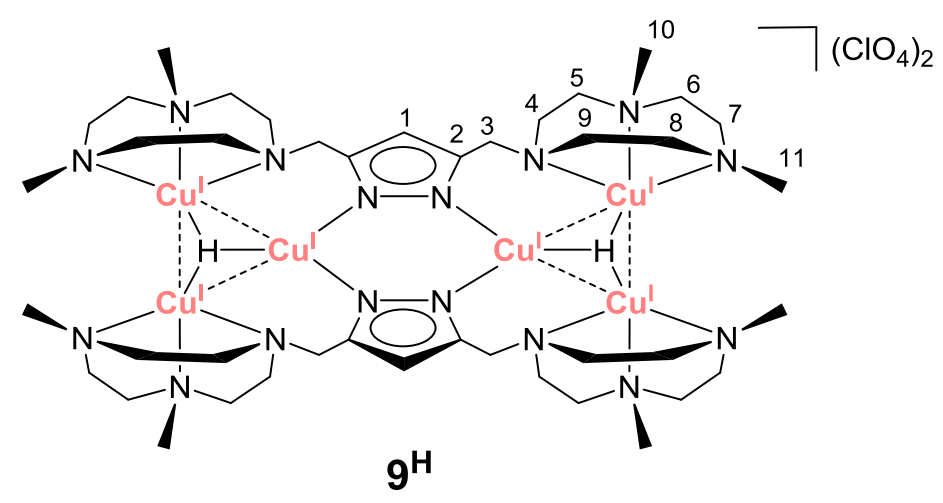

\section{Synthesis and Crystallization}

Under a nitrogen atmosphere, $\mathbf{5}^{\mathrm{HCO}}(4.97 \mathrm{mg}, 6.40 \mu \mathrm{mol}, 1.0 \mathrm{eq})$ and $\mathrm{Cp}_{2} \mathrm{Co}(2.78 \mathrm{mg}, 14.70 \mu \mathrm{mol}$, $2.3 \mathrm{eq})$ were dissolved in $\mathrm{MeCN}(700 \mu \mathrm{L})$ and left to stir for 30 minutes (yields of $60-80 \%$ were obtained for unisolated $9^{\mathrm{H}}$ by NMR spectroscopy (relative to the cobaltocenium peak) according to the reaction equation given in the Section 6.4.2). Subsequent crystallization by diffusion with $\mathrm{Et}_{2} \mathrm{O}$ yielded both transparent crystals of $\mathbf{9}^{\mathbf{H}}$ and yellow needles of $\left[\mathrm{Cp}_{2} \mathrm{Co}\right]\left(\mathrm{ClO}_{4}\right)$, which could not be separated. Consequently, all characterisation was done with a crystalline mixture of the two.

Molecular Weight $1393.41 \mathrm{~g} / \mathrm{mol}$

Empirical Formula $\mathrm{C}_{42} \mathrm{H}_{84} \mathrm{Cl}_{2} \mathrm{Cu}_{6} \mathrm{~N}_{16} \mathrm{O}_{8}$

${ }^{1}$ H-NMR $\left(500 \mathrm{MHz}, \mathrm{CD}_{3} \mathrm{CN}\right) \delta(\mathrm{ppm})=6.10(\mathrm{~s}, 2 \mathrm{H}, 1-\mathrm{H}), 4.10(\mathrm{~d}, J=11.5 \mathrm{~Hz}, 4 \mathrm{H}, 3-\mathrm{H}), 3.62(\mathrm{~d}$, $\left.J=11.5 \mathrm{~Hz}, 4 \mathrm{H}, 3^{\prime}-\mathrm{H}\right), 3.24-3.15(\mathrm{~m}, 4 \mathrm{H}, 9-\mathrm{H}), 3.10-2.96(\mathrm{~m}, 8 \mathrm{H}, 7-\mathrm{H}, 8-\mathrm{H}), 2.90-2.81$ (m, 4H, 6-H), 2.78 (s, 12H, 11-H), 2,74-2.63 (m, 4H, 4-H), 2.60-2.51 (m, 4H, 4'-H), 2.46-2.35 (m, 12H, 9'-H, 8'-H, 7'-H), $2.42(\mathrm{~s}, 12 \mathrm{H}, 10-\mathrm{H}), 2.32-2.23\left(\mathrm{~m}, 4 \mathrm{H}, 6^{\prime}-\mathrm{H}\right), 2.11-2.02(\mathrm{~m}, 4 \mathrm{H}, 5-\mathrm{H}), 1.93-1.88\left(\mathrm{~m}, 4 \mathrm{H}, 5^{\prime}-\mathrm{H}\right),-3.11$ (s, $\left.2 \mathrm{H}, \mathrm{H}^{-}\right)^{*}$

Pure shift ${ }^{1} \mathrm{H}-\mathrm{NMR}\left(500 \mathrm{MHz}, \mathrm{CD}_{3} \mathrm{CN}\right) \delta(\mathrm{ppm})=6.10(1-\mathrm{H}), 4.10(3-\mathrm{H}), 3.62\left(3^{\prime}-\mathrm{H}\right), 3.20(9-\mathrm{H}), 3.05$ (7-H), 3.02 (8-H), $2.84(6-\mathrm{H}), 2.78(11-\mathrm{H}), 2,69(4-\mathrm{H}), 2.55\left(4^{\prime}-\mathrm{H}\right), 2.43\left(9^{\prime}-\mathrm{H}\right), 2.42(10-\mathrm{H}), 2.40\left(8^{\prime}-\mathrm{H}\right)$, $2.38\left(7^{\prime}-\mathrm{H}\right), 2.28\left(6^{\prime}-\mathrm{H}\right), 2.06(5-\mathrm{H}), 1.91\left(5^{\prime}-\mathrm{H}\right){ }^{*}$

${ }^{13} \mathbf{C}\left\{{ }^{1} \mathbf{H}\right\}-N M R\left(126 \mathrm{MHz}, \mathrm{CD}_{3} \mathrm{CN}\right) \delta(\mathrm{ppm})=147.12$ (2-C), 111.36 (1-C), 61.00 (5-C), 59.12 (7-C), 57.92 (3-C), 56.84 (9-C), 52.13 (11-C), 52.07 (8-C), 51.91 (6-C), 49.25 (10-C), 47.07 (4-C).

*the annotation with and without prime correspond to the diastereotopic protons at the same carbon atom

MS (ESI(+), MeCN): $m / z=597.0[\mathrm{M}]^{2+}$. 


\section{$\left[\left(\mathrm{L}^{2 \mathrm{a}}\right)_{2} \mathrm{Cu}_{6}(\mathrm{D})_{2}\right]\left(\mathrm{ClO}_{4}\right)_{2}\left(9^{\mathrm{D}}\right)$}

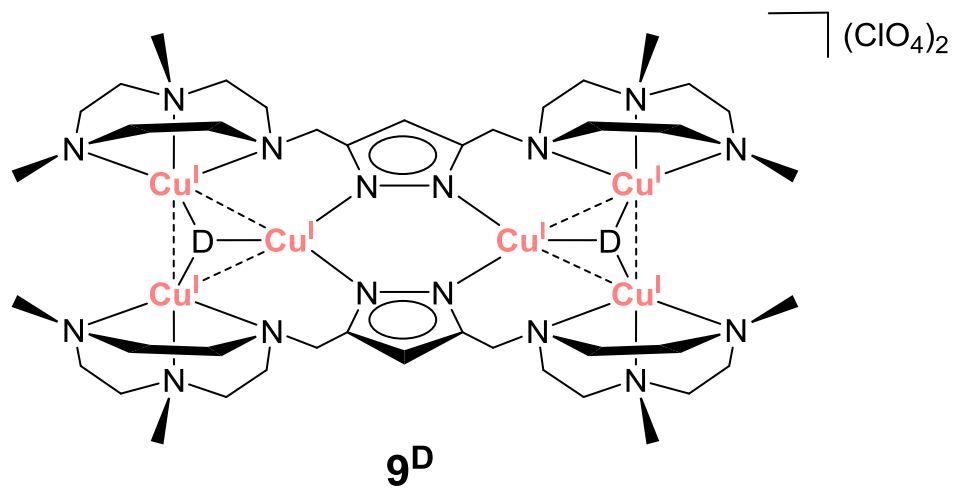

\section{Synthesis}

The synthesis of $\mathbf{9}^{\mathrm{D}}$ was conducted identical to $9^{\mathrm{H}}$ using $5^{\mathrm{DCO} 2}$ instead of $\mathbf{5}^{\mathrm{HCO}}$ as starting material. A yield was not determined.

Molecular Weight $1395.43 \mathrm{~g} / \mathrm{mol}$

Empirical Formula $\mathrm{C}_{42} \mathrm{H}_{82} \mathrm{D}_{2} \mathrm{Cl}_{2} \mathrm{Cu}_{6} \mathrm{~N}_{16} \mathrm{O}_{8}$

MS $(\operatorname{ESI}(+), \mathrm{MeCN}): m / z=598.0[\mathrm{M}]^{2+}$.

${ }^{2} \mathbf{H}-\mathrm{NMR}\left(77 \mathrm{MHz}, \mathrm{CD}_{3} \mathrm{CN}\right) \delta(\mathrm{ppm})=-3.00$ (s. 2D, $\left.\mathrm{D}^{-}\right)$. 


\section{$\left[\left(\mathrm{L}^{2 \mathrm{a}}\right)_{2} \mathrm{Cu}_{6}\left(\mathrm{C}_{8} \mathrm{H}_{5}\right)_{2}\right]\left(\mathrm{ClO}_{4}\right)_{2}\left(9^{\mathrm{C} 8 \mathrm{H} 5}\right)$}

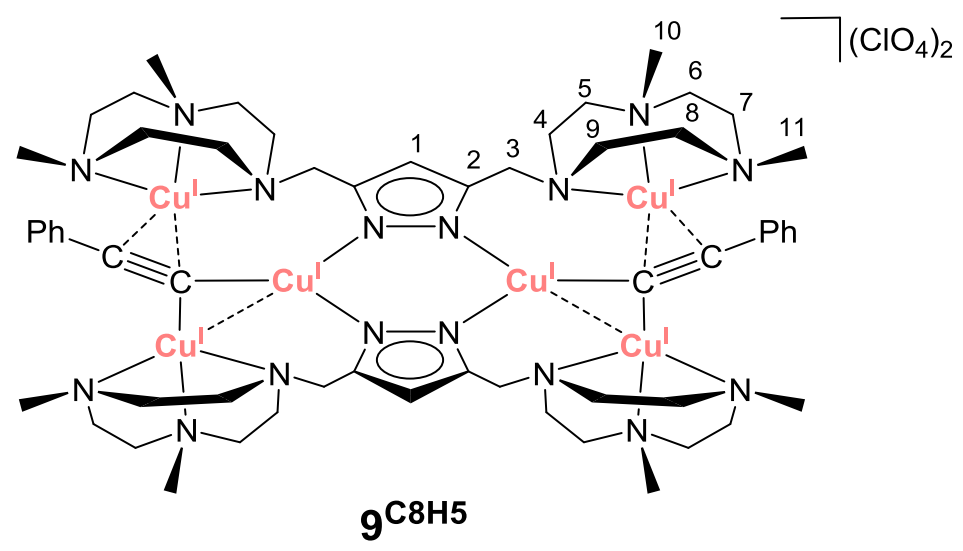

\section{Synthesis and Crystallization}

To a solution of $9 \mathrm{H}$ ( $3.73 \mathrm{mg}, 2.68 \mu \mathrm{mol}, 1.0 \mathrm{eq})$ in $\mathrm{CD}_{3} \mathrm{CN}(700 \mu \mathrm{L})$, phenyl acetylene (5.35 $\left.\mu \mathrm{mol}, 2.0 \mathrm{eq}\right)$ was added. Crystals of $\mathbf{9}^{\mathbf{c}} \mathbf{H}^{5}$ suitable for X-ray diffraction were obtained by diffusion of $\mathrm{Et}_{2} \mathrm{O}$ into the NMR sample solution. A yield was not determined.

Molecular Weight $1593.65 \mathrm{~g} / \mathrm{mol}$

Empirical Formula $\mathrm{C}_{58} \mathrm{H}_{92} \mathrm{Cl}_{2} \mathrm{Cu}_{6} \mathrm{~N}_{16} \mathrm{O}_{8}$

1H-NMR $\left(500 \mathrm{MHz}, \mathrm{CD}_{3} \mathrm{CN}\right) \delta(\mathrm{ppm})=7.42-7.13(\mathrm{~m}, 10 \mathrm{H}, \mathrm{Ph}), 6.11(\mathrm{~s}, 2 \mathrm{H}, 1-\mathrm{H}), 6.08(\mathrm{~s}, 2 \mathrm{H}, 1-\mathrm{H}), 4.13$ (d, $J=11.3 \mathrm{~Hz}, 4 \mathrm{H}, 3-\mathrm{H}), 3.51\left(\mathrm{~d}, J=11.3 \mathrm{~Hz}, 4 \mathrm{H}, 3^{\prime}-\mathrm{H}\right), 3.17-3.07(\mathrm{~m}, 4 \mathrm{H}, 9-\mathrm{H}), 3.05-2.95(\mathrm{~m}, 4 \mathrm{H}, 8-\mathrm{H})$, 2.89-2.62 (m, 16H, 6-H, 4-H, 7-H, 4'-H), 2.60 (s, 12H, 11-H), 2.56 (s, 12H, 11-H), 2.51-2.39 (m, 12H, 8'H, 9'-H, 7'-H), 2.33-2.28 (m, 4H, 6'-H), 2.34 (s, 12H, 10-H), 2.27 (s, 12H, 10-H), 2.05-1.99 (m, 4H, 5-H), 1.89-1.80 (m, 4H, 5'-H), 1.66-1.58 (m, 4H, 5'-H).*

Pure shift ${ }^{1} \mathbf{H}-\mathbf{N M R}\left(500 \mathrm{MHz}, \mathrm{CD}_{3} \mathrm{CN}\right) \delta(\mathrm{ppm})=7.41(\mathrm{Ph}), 7.37(\mathrm{Ph}), 7.26(\mathrm{Ph}), 7.19(\mathrm{Ph}), 7.18(\mathrm{Ph})$, $7.13(\mathrm{Ph}), 6.11(1-\mathrm{H}), 6.08(1-\mathrm{H}), 4.13(3-\mathrm{H}), 3.51\left(3^{\prime}-\mathrm{H}\right), 3.12(9-\mathrm{H}), 3.00(8-\mathrm{H}), 2.83(6-\mathrm{H}), 2.74(4-\mathrm{H})$, $2.73(7-\mathrm{H}), 2.65\left(4^{\prime}-\mathrm{H}\right), 2.60(11-\mathrm{H}), 2.56(11-\mathrm{H}), 2.47\left(8^{\prime}-\mathrm{H}\right), 2.43\left(9^{\prime}-\mathrm{H}\right), 2.42\left(7^{\prime}-\mathrm{H}\right), 2.34(10-\mathrm{H}), 2.31$ (6'-H), $2.27(10-\mathrm{H}), 2.01(5-\mathrm{H}), 1.84\left(5^{\prime}-\mathrm{H}\right), 1.61\left(5^{\prime}-\mathrm{H}\right)$ * $^{*}$

${ }^{13} \mathbf{C}\left\{{ }^{1} \mathbf{H}\right\}$-NMR (126 MHz, CD $\left.{ }_{3} \mathrm{CN}\right) \delta(\mathrm{ppm})=146.39$ (2-C), 129.55 (2C, Ph-C), 129.40 (2C, Ph-C), 129.35 (2C, Ph-C), 129.23 (2C, Ph-C), 126.57 (Ph-C), 126.46 (Ph-C), 121.95 (Ph-Cq), 120.07 (Ph-Cq), 110.14 (1C), 109.82 (1-C), 61.77 (5-C), 61.05 (5-C), 57.46 (7-C), 57.41 (9-C), 56.53 (3-C), 52.66 (6-C), 51.16 (8C), 49.94 (10-C), 49.86 (10-C), 48.09 (11-C), 46.92 (4-C).*

*the signals in grey are those of a second isomer

*the annotation with and without prime correspond to the diastereotopic protons at the same carbon atom

*not all signals of the phenyl acetylene could be distinguished; there are peaks at 129.93 and $129.66 \mathrm{ppm}$ in the ${ }^{13} \mathrm{C}\left\{{ }^{1} \mathrm{H}\right\}$-NMR spectrum which could not be assigned

MS (ESI(+), MeCN): $m / z=697.1[\mathrm{M}]^{2+}, 798.1\left[\mathrm{M}-\mathrm{L}^{2 \mathrm{a}}-3 \mathrm{Cu}\right]^{+}$.

IR (ATR): $v\left(\mathrm{~cm}^{-1}\right)=1852(\mathrm{C} \equiv \mathrm{C})$. 


\section{Appendix}




\section{Mass Spectrometry}

\section{$\left[\mathrm{L}^{1} \mathrm{Ru}_{2}(\mathrm{dmso})_{2}(\mathrm{Cl})\left(\mathrm{H}_{2} \mathrm{O}\right)(\mu-\mathrm{dmso})\right](1)$}

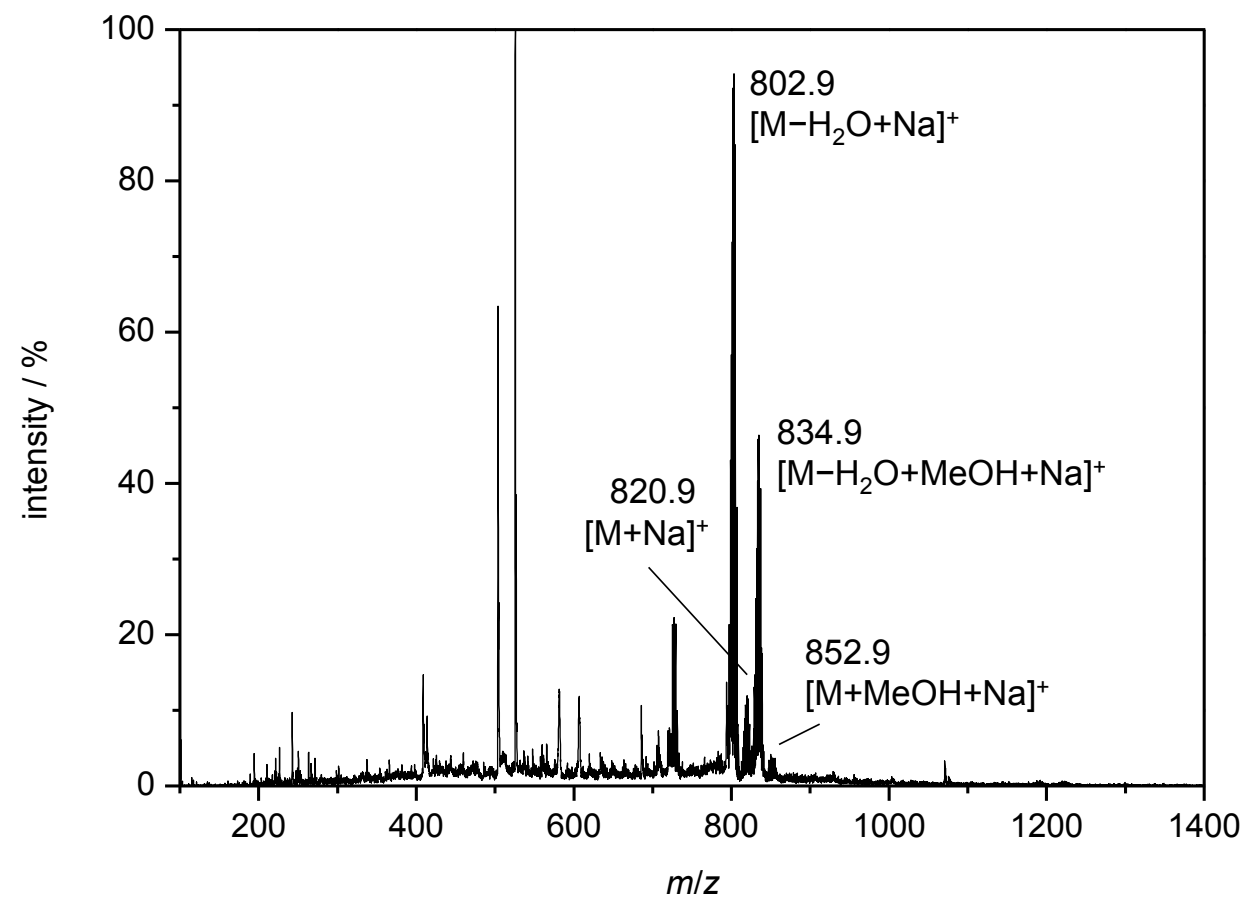

Figure A.1: ESI(+)-MS of 1 in $\mathrm{MeOH}$.

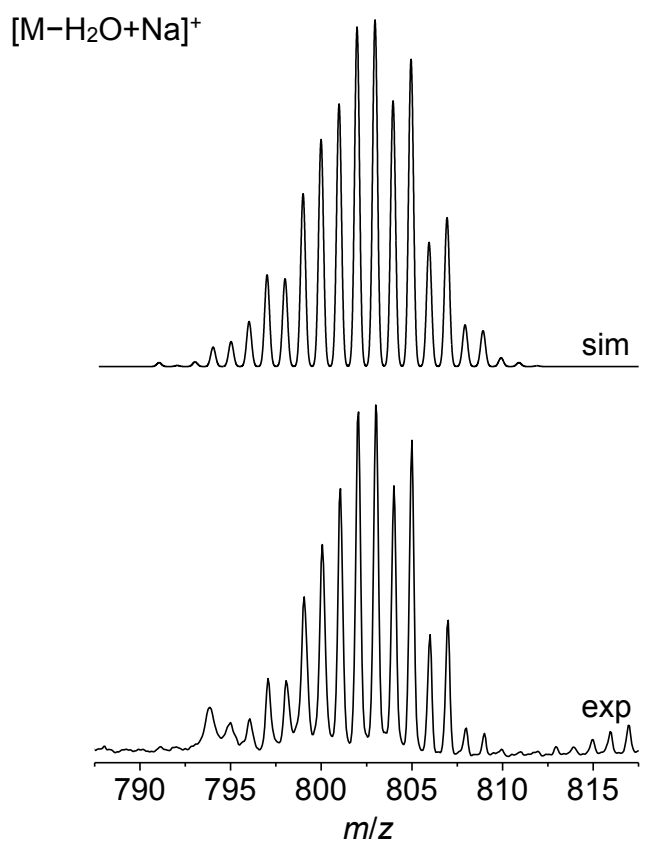

Figure A.2: $\mathrm{ESI}(+)-\mathrm{MS}$ of 1 in $\mathrm{MeOH}$. Isotopic patterns of the simulated (top) and experimental (bottom) signal corresponding to $\left[\mathrm{M}-\mathrm{H}_{2} \mathrm{O}+\mathrm{Na}\right]^{+}(m / z=802.9)$. 


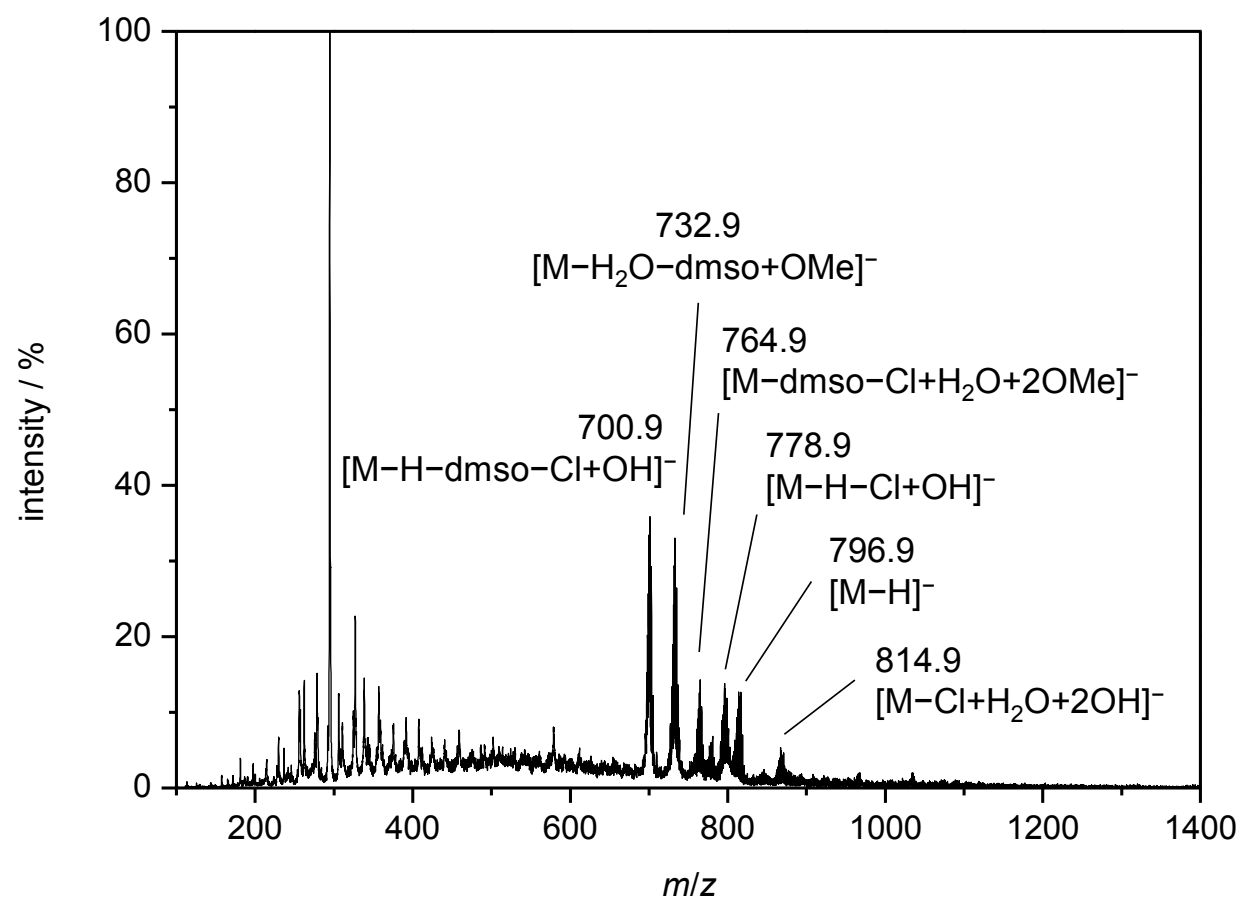

Figure A.3: ESI(-)-MS of 1 in $\mathrm{MeOH}$.

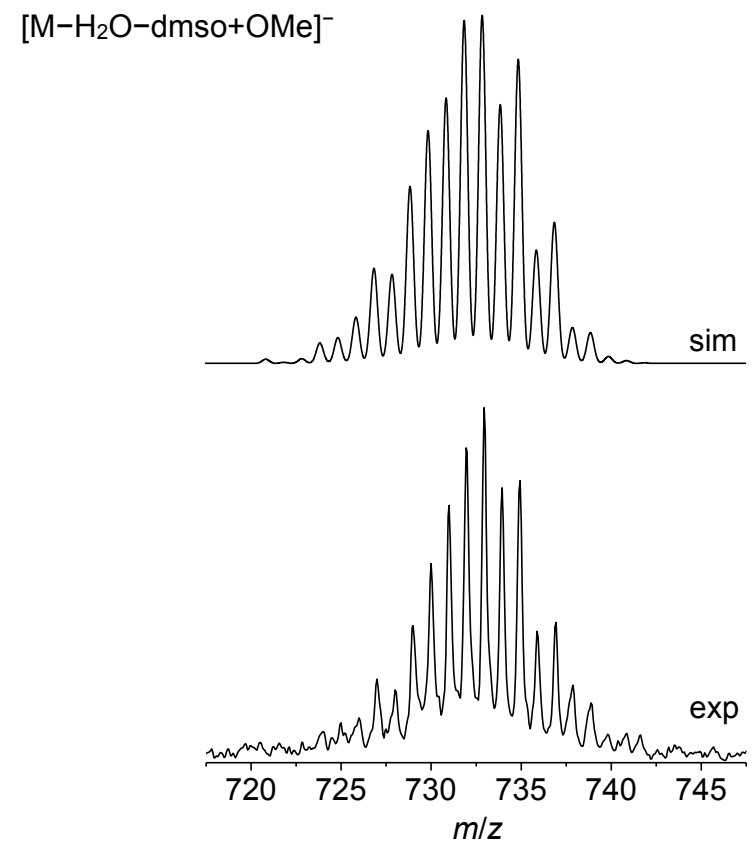

Figure A.4: ESI(-)-MS of 1 in $\mathrm{MeOH}$. Isotopic patterns of the simulated (top) and experimental (bottom) signal corresponding to $\left[\mathrm{M}-\mathrm{H}_{2} \mathrm{O}-\mathrm{dmso}+\mathrm{OMe}\right]^{-}(\mathrm{m} / \mathrm{z}=732.9)$. 


\section{$\mathrm{Na}_{3}\left[\mathrm{~L}^{1} \mathrm{Ru}_{2}\left(\mathrm{pyCO}_{2}\right)_{4}(\mu-\mathrm{dmso})\right]\left(2^{\mathrm{dmso}}\right)$}

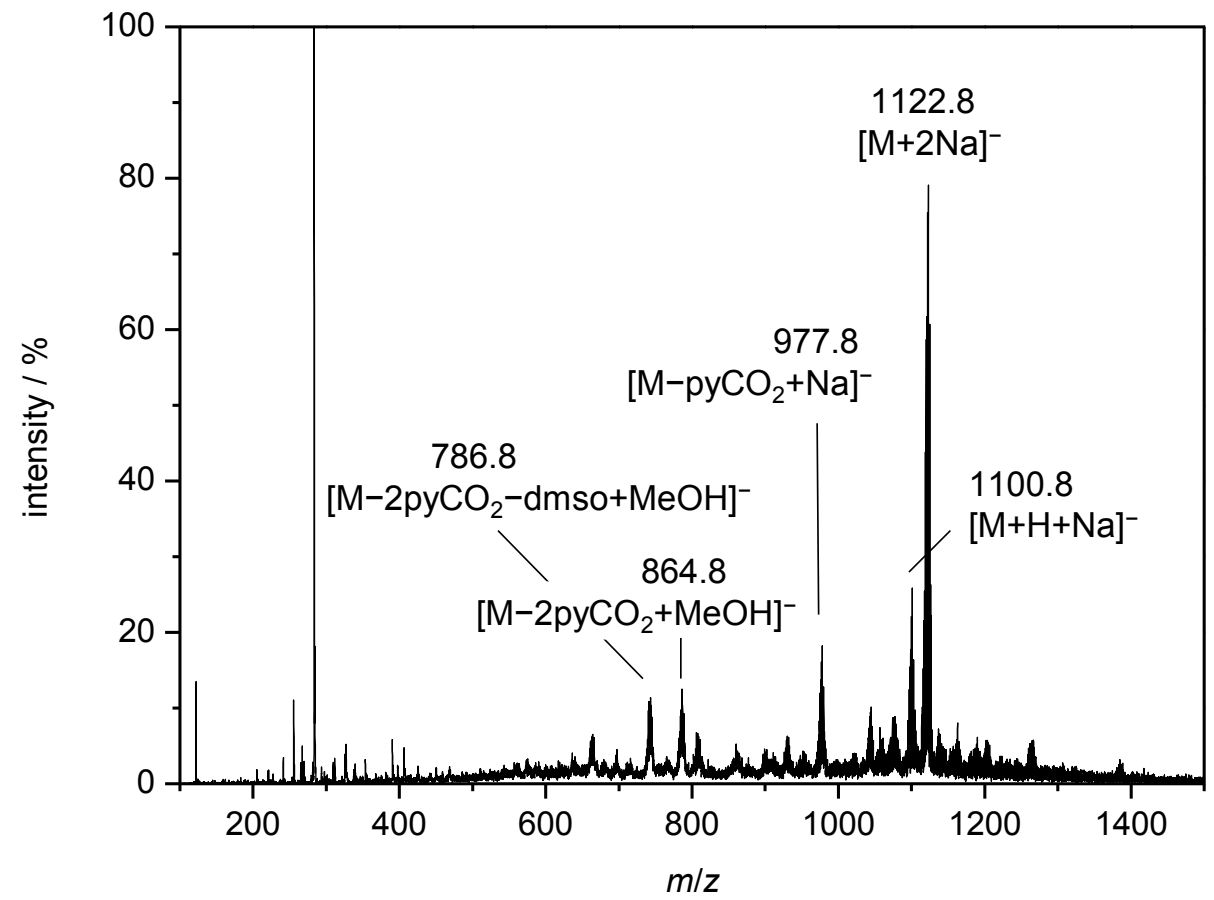

Figure A.5: ESI(-)-MS of $\mathbf{2}^{\text {dmso }}$ in $\mathrm{MeOH}$.
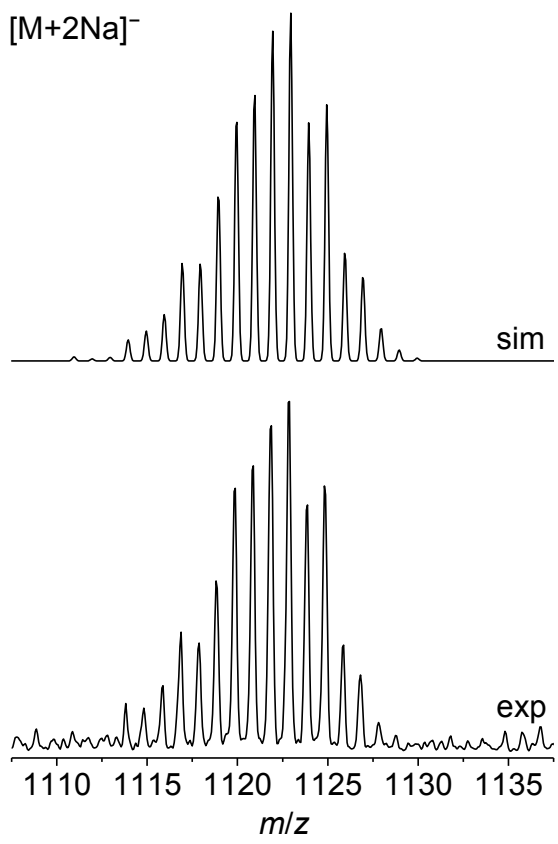

Figure A.6: $\mathrm{ESI}(-)-\mathrm{MS}$ of $\mathbf{2}^{\mathrm{dmso}}$ in $\mathrm{MeOH}$. Isotopic patterns of the simulated (top) and experimental (bottom) signal corresponding to $[\mathrm{M}+2 \mathrm{Na}]^{-}(m / z=1122.8)$. 


\section{$\mathrm{Na}_{3}\left[\mathrm{~L}^{1} \mathrm{Ru}_{2}\left(\mathrm{pySO}_{3}\right)_{4}(\mu-\mathrm{dmso})\right]\left(3^{\mathrm{dmso}}\right)$}

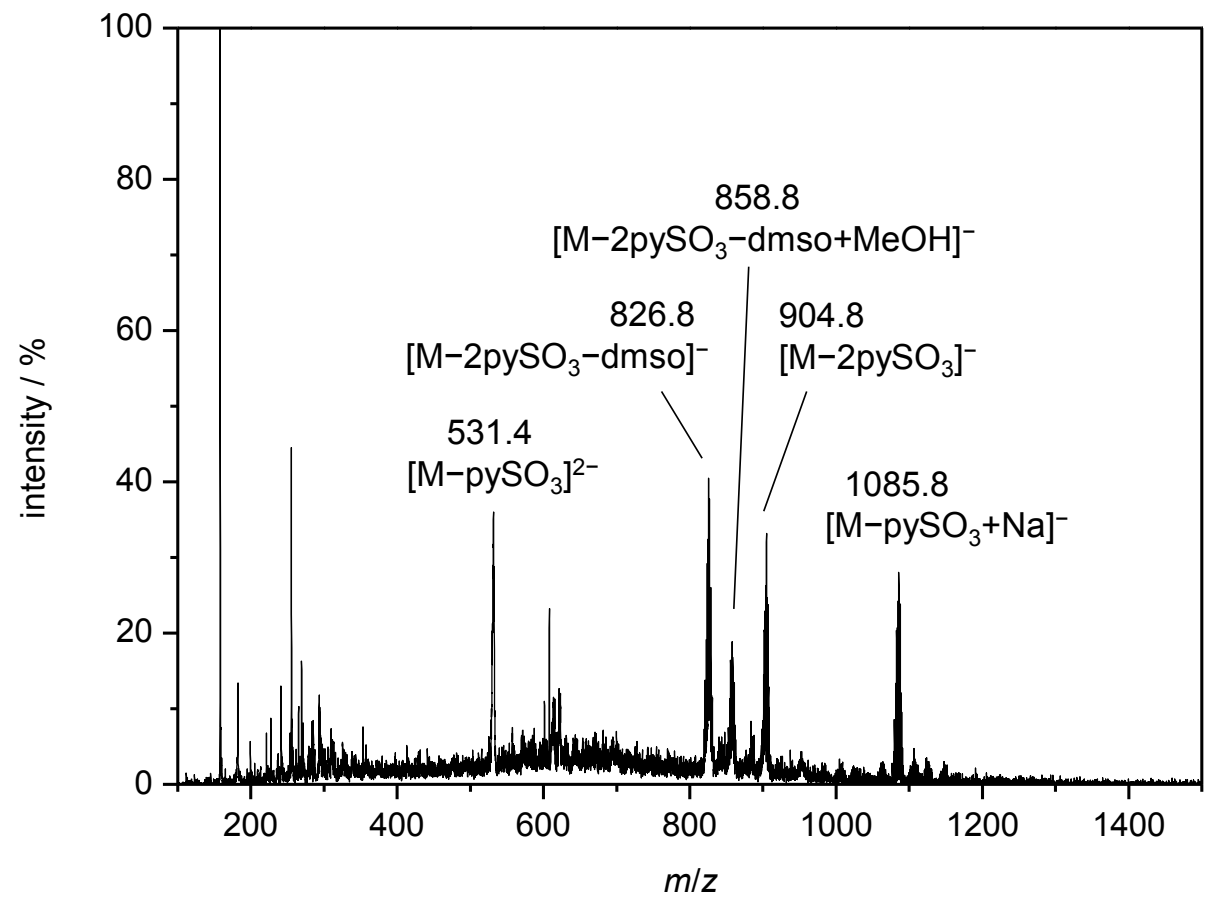

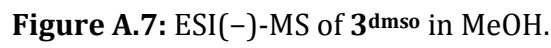
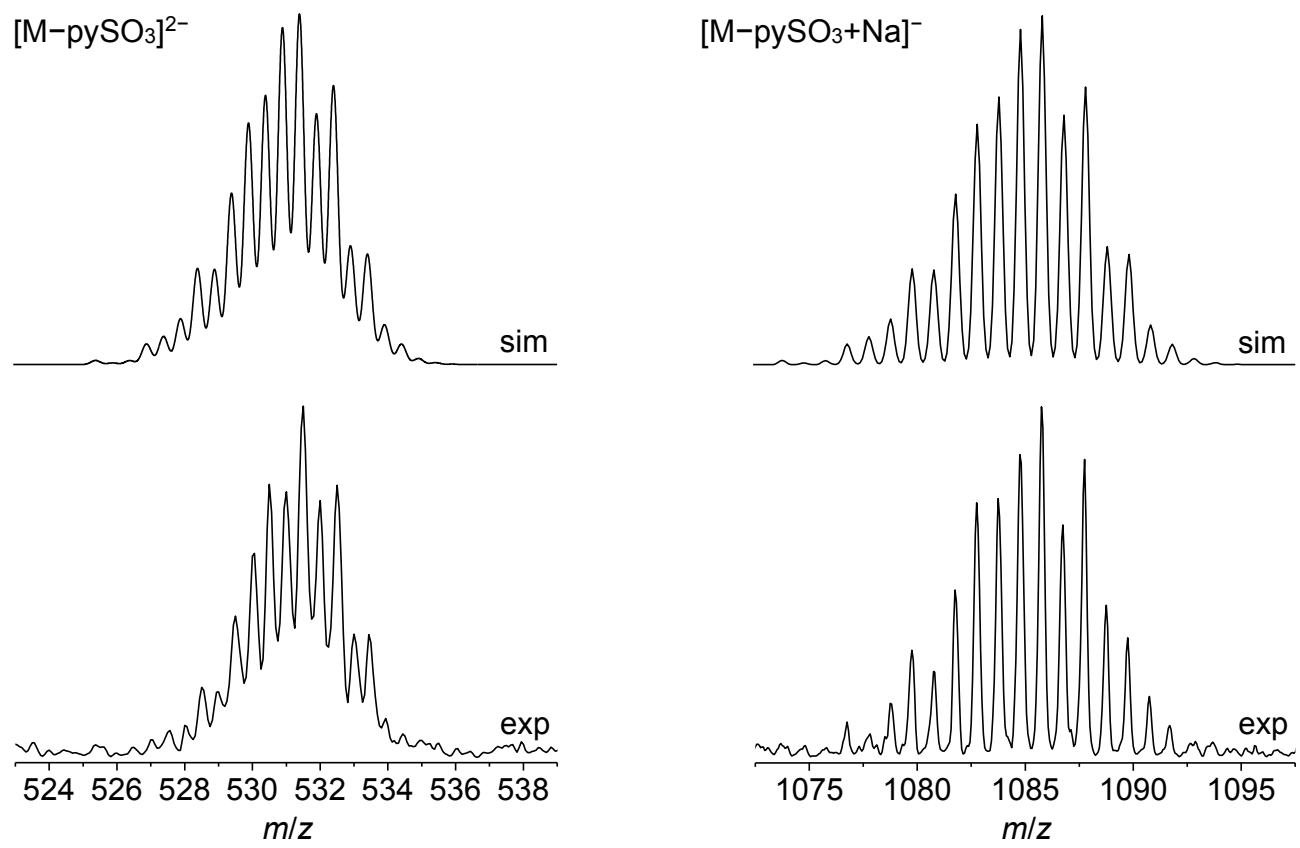

Figure A.8: $\mathrm{ESI}(-)$-MS of $3^{\mathrm{dmso}}$ in $\mathrm{MeOH}$. Isotopic patterns of the simulated (top) and experimental (bottom) signals corresponding to $\left[\mathrm{M}-\mathrm{pySO}_{3}\right]^{--}(\mathrm{m} / \mathrm{z}=531.4$, left $)$ and $\left[\mathrm{M}-\mathrm{pySO}_{3}+\mathrm{Na}\right]^{-}(\mathrm{m} / \mathrm{z}=1085.8$, left $)$. 


\section{$\mathrm{Na}_{2}\left[\mathrm{~L}^{1} \mathrm{Ru}_{2}\left(\mathrm{pySO}_{3}\right)_{4}\left(\mathrm{H}_{2} \mathrm{O}\right)_{2}\right]\left(3^{\mathrm{H} 20}\right)$}

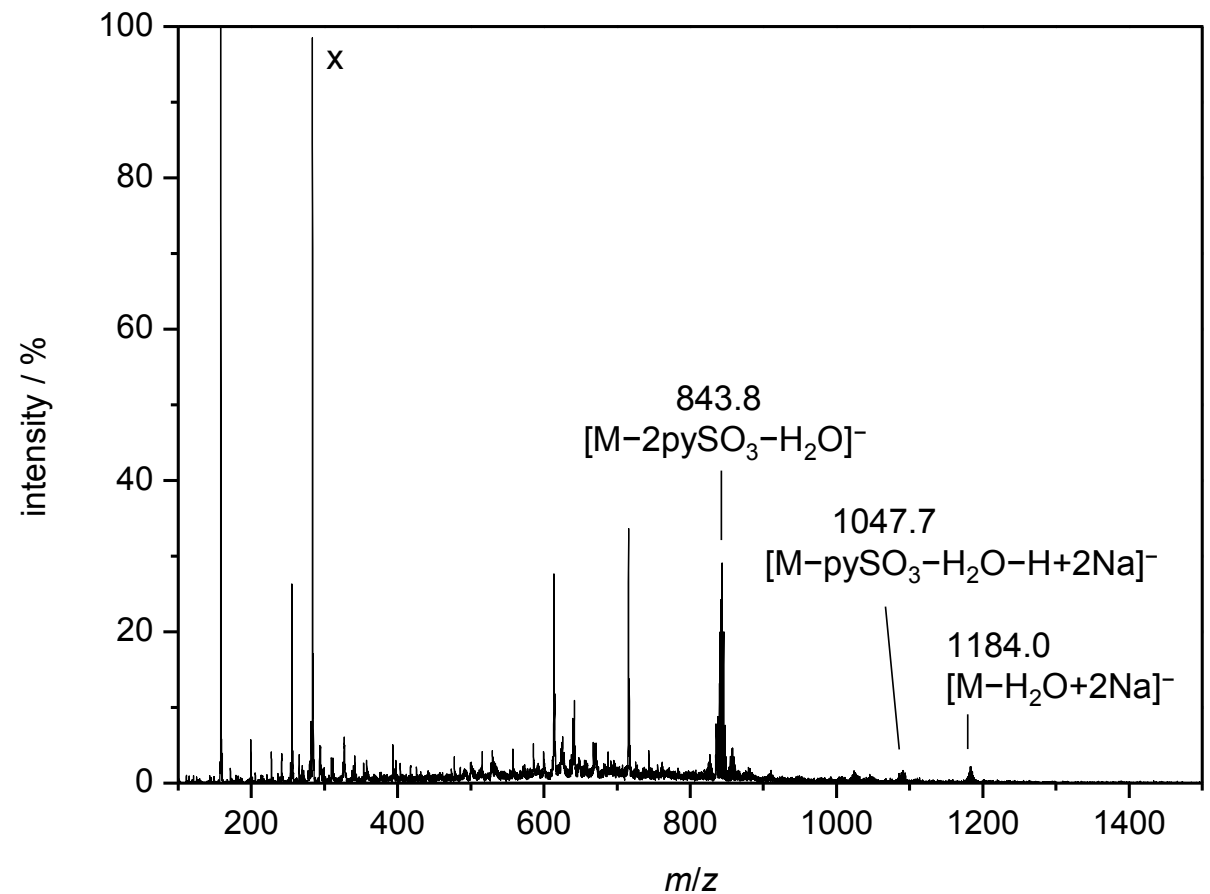

Figure A.9: $\mathrm{ESI}(-)-\mathrm{MS}$ of $3^{\mathrm{H} 20}$ in $\mathrm{MeOH}$. The x marks an impurity from the mass spectrometer.
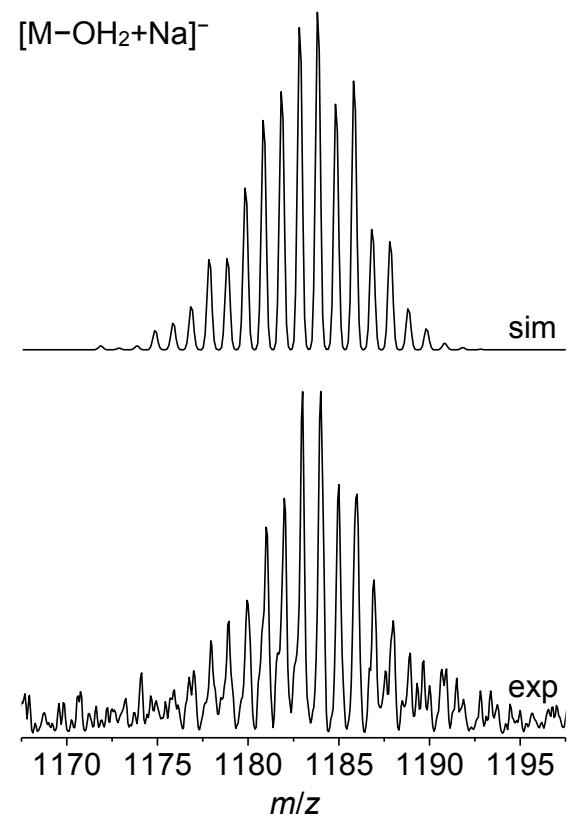

Figure A.10: $\mathrm{ESI}(-)-\mathrm{MS}$ of $\mathbf{3}^{\mathrm{H} 20}$ in $\mathrm{MeOH}$. Isotopic patterns of the simulated (top) and experimental (bottom) signal corresponding to $\left[\mathrm{M}-\mathrm{OH}_{2}+\mathrm{Na}\right]^{-}(\mathrm{m} / \mathrm{z}=1184.0$, oxidation states of ruthenium: II/III). 


\section{$\left[\mathrm{L}^{2 \mathrm{a}} \mathrm{Cu}_{2}\left(\mu-\mathrm{HCO}_{2}\right)\right]\left(\mathrm{ClO}_{4}\right)_{2}\left(5^{\mathrm{HCO}}\right)$}

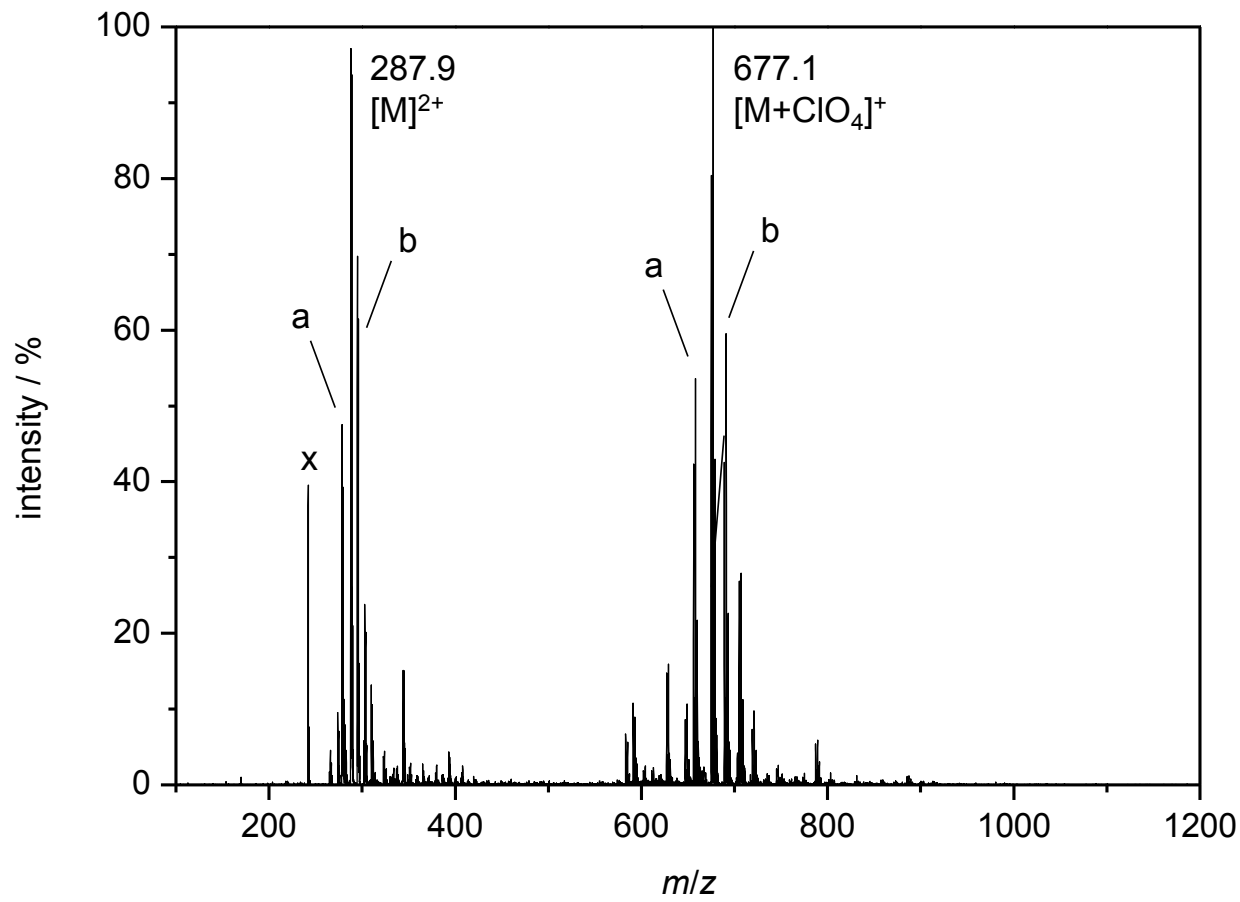

Figure A.11: $\mathrm{ESI}(+)-\mathrm{MS}$ of $\mathbf{5}^{\mathrm{HCO} 2}$ in $\mathrm{MeOH}$. The peaks denoted a and $\mathrm{b}$ correspond to the equivalent species as the main peaks bearing $\mathrm{CN}^{-}$or $\mathrm{AcO}^{-}$in the bimetallic pocket, respectively. The $\mathrm{x}$ marks an impurity from the mass spectrometer.
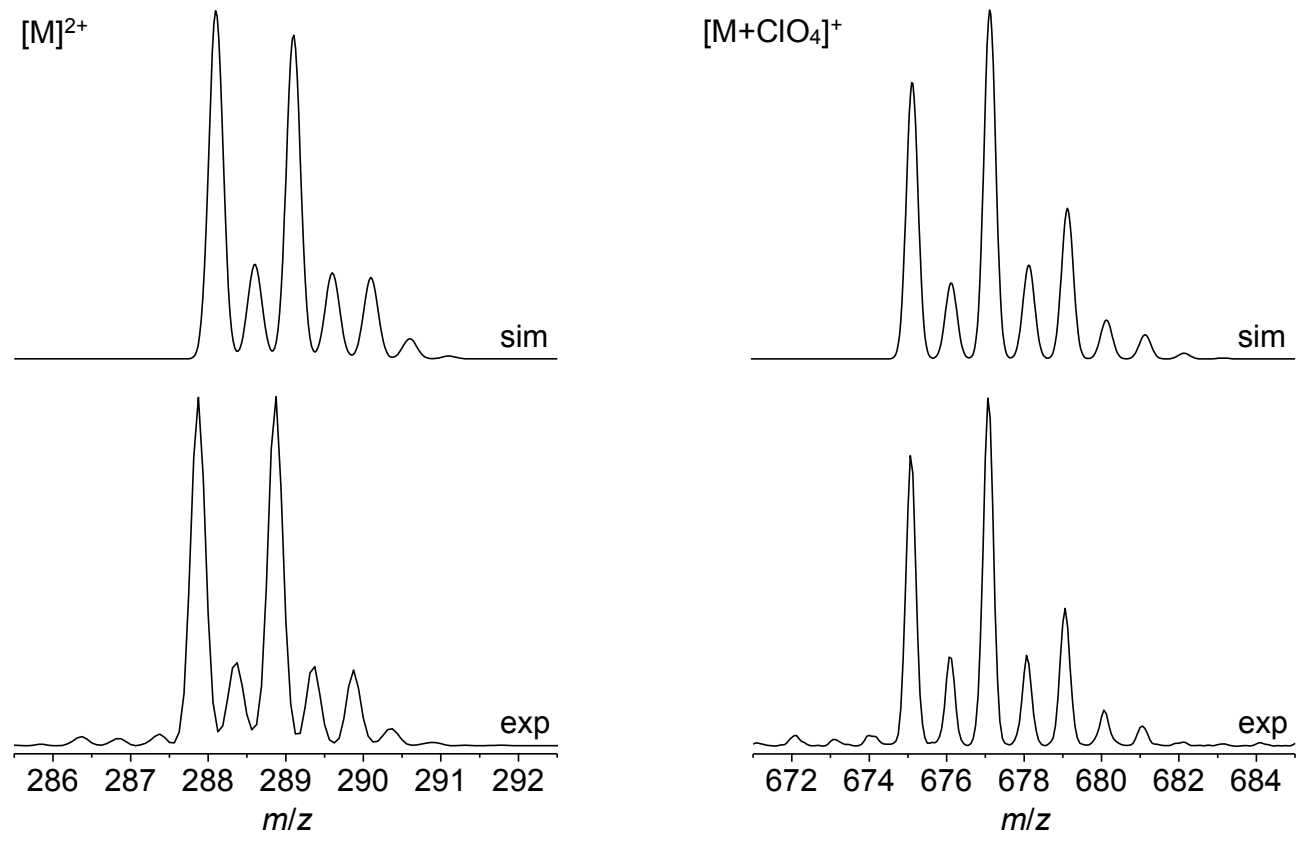

Figure A.12: $\mathrm{ESI}(+)-\mathrm{MS}$ of $\mathbf{5}^{\mathrm{HCO}}$ in MeOH. Isotopic patterns of the simulated (top) and experimental (bottom) signals corresponding to $[\mathrm{M}]^{2+}(\mathrm{m} / \mathrm{z}=287.9$, left $)$ and $\left[\mathrm{M}+\mathrm{ClO}_{4}\right]^{+}(\mathrm{m} / \mathrm{z}=677.1$, right $)$. 


\section{$\left[\mathrm{L}^{2 \mathrm{aaC}} \mathrm{Cu}_{2}\left(\mu-\mathrm{DCO}_{2}\right)\right]\left(\mathrm{ClO}_{4}\right)_{2}\left(5^{\mathrm{DCO}}\right)$}

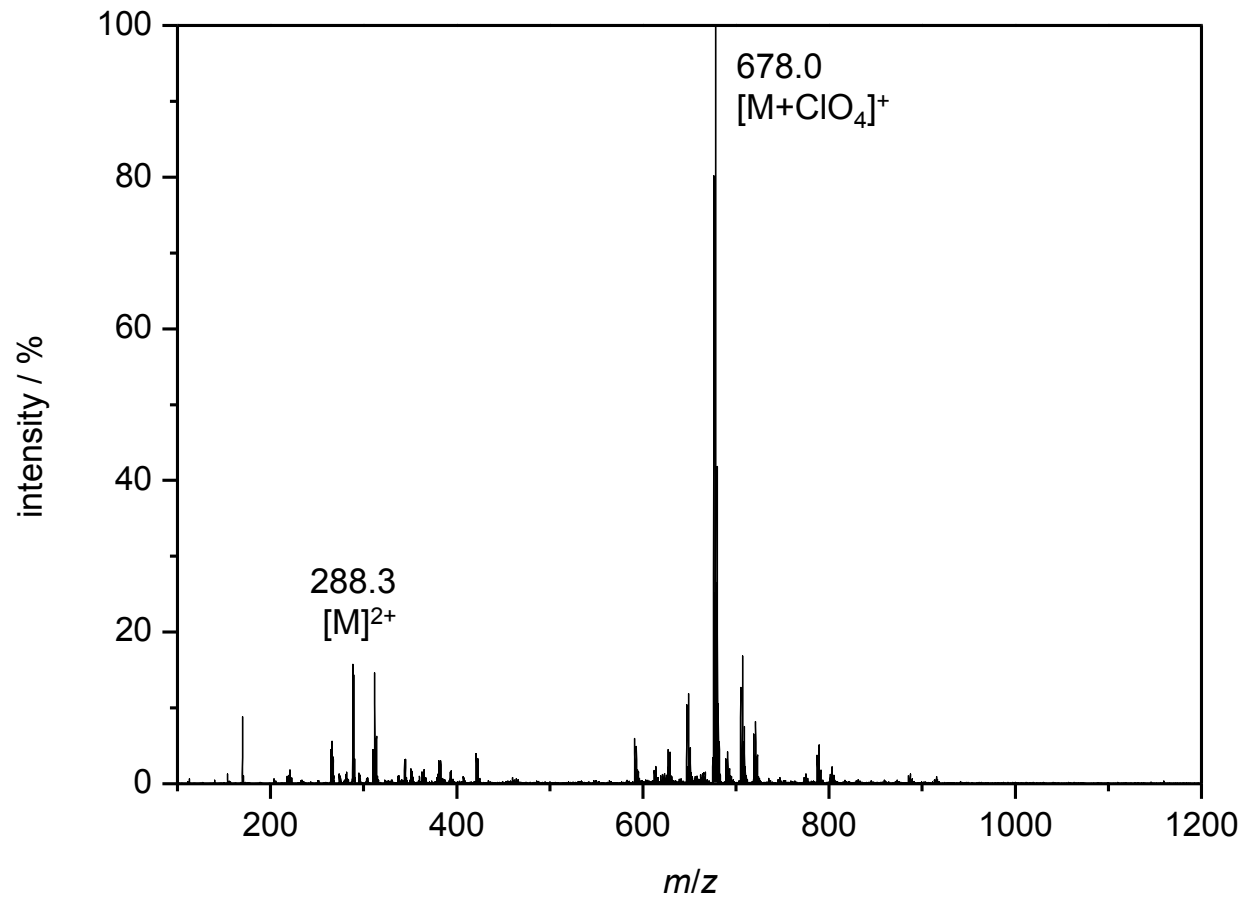

Figure A.13: ESI(+)-MS of 5 $5^{\mathrm{DCO}}$ in $\mathrm{MeOH}$.
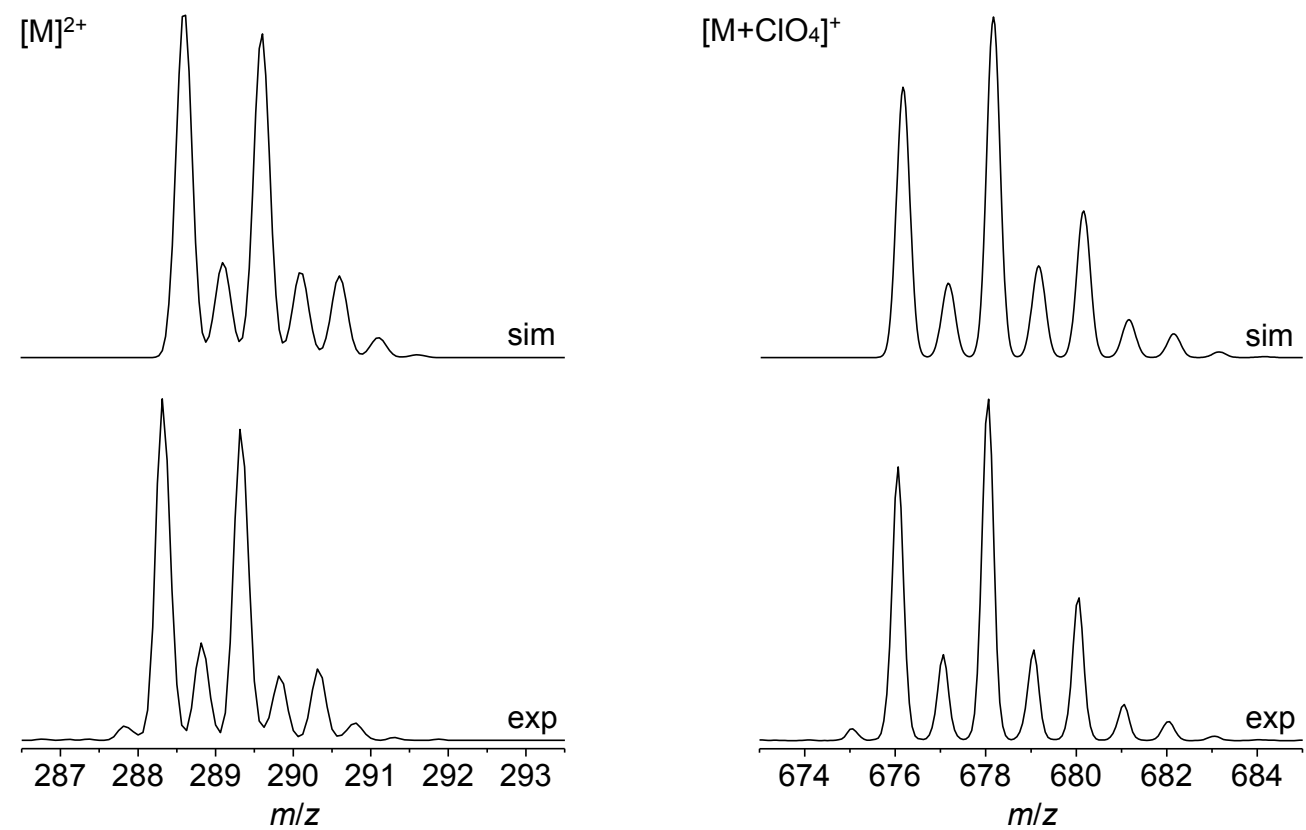

Figure A.14: $\mathrm{ESI}(+)-\mathrm{MS}$ of $\mathbf{5}^{\mathbf{D C O} 2}$ in $\mathrm{MeOH}$. Isotopic patterns of the simulated (top) and experimental (bottom) signals corresponding to $[\mathrm{M}]^{2+}(\mathrm{m} / \mathrm{z}=288.3$, left $)$ and $\left[\mathrm{M}+\mathrm{ClO}_{4}\right]^{+}(\mathrm{m} / \mathrm{z}=678.0$, right $)$. 


\section{$\left[\mathrm{L}^{2 \mathrm{a}} \mathrm{Cu}_{2}\left(\mu-\mathrm{H}^{13} \mathrm{CO}_{2}\right)\right]\left(\mathrm{ClO}_{4}\right)_{2}\left(5^{\mathrm{H}\{13 \mathrm{C}\} 02}\right)$}

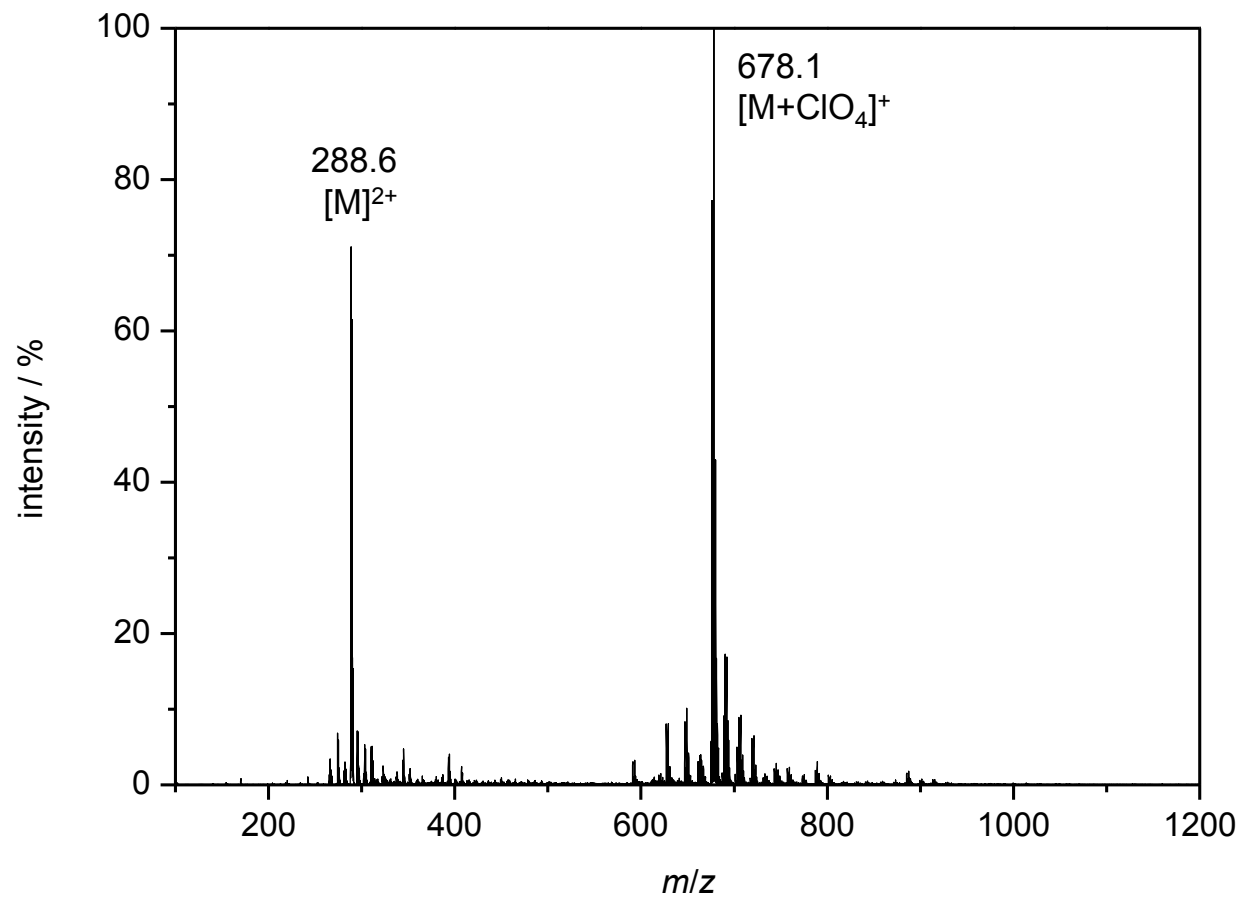

Figure A.15: $\operatorname{ESI}(+)-M S$ of $\mathbf{5}^{\mathbf{H}\{13 C\} 02}$ in $\mathrm{MeOH}$.
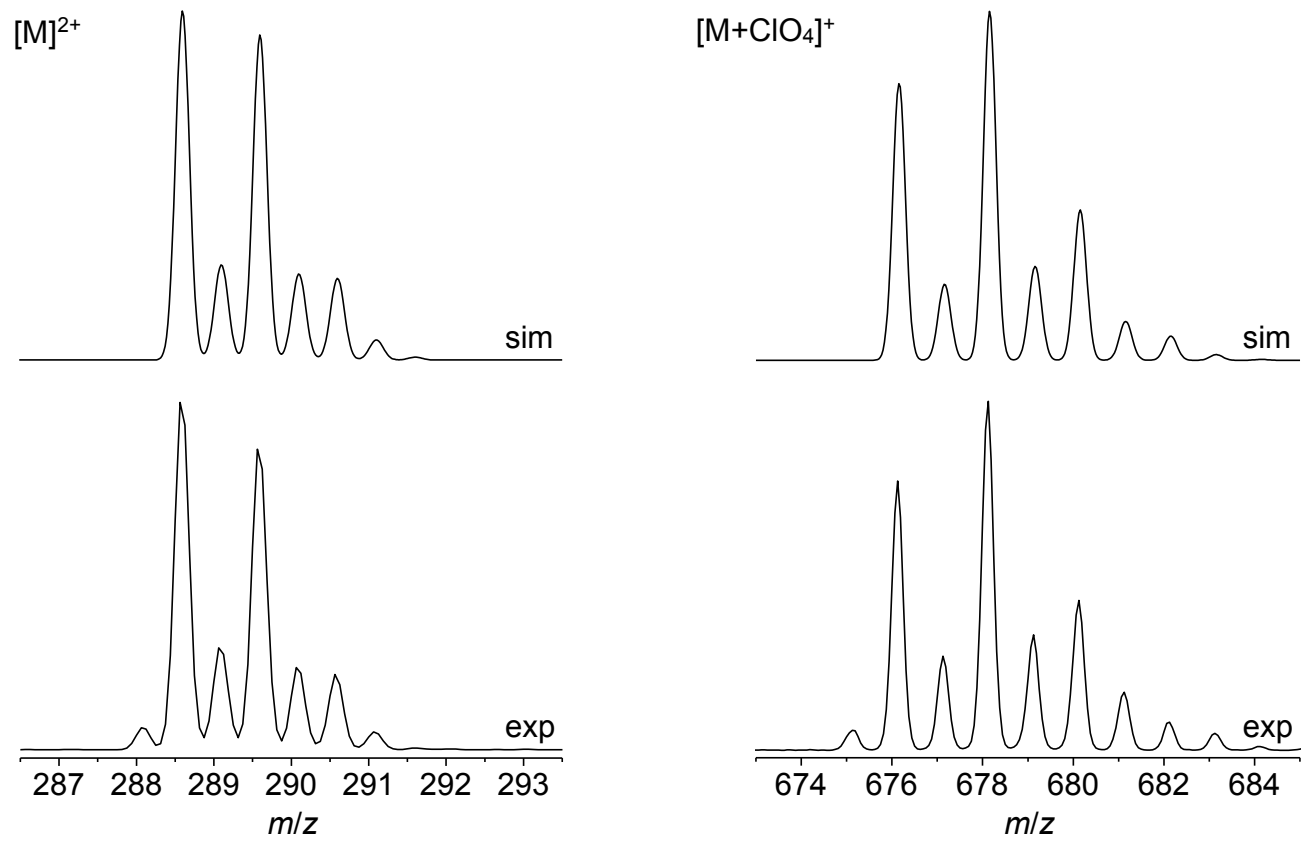

Figure A.16: $\mathrm{ESI}(+)-\mathrm{MS}$ of $\mathbf{5}^{\mathbf{H}\{13 \mathrm{C}\} \mathbf{0} \mathbf{2}}$ in $\mathrm{MeOH}$. The isotopic patterns of the simulated (top) and experimental (bottom) signals corresponding to $[\mathrm{M}]^{2+}\left(\mathrm{m} / \mathrm{z}=288.6\right.$, left) and $\left[\mathrm{M}+\mathrm{ClO}_{4}\right]^{+}(\mathrm{m} / \mathrm{z}=678.1$, right) are depicted. 


\section{$\left[\mathrm{L}^{2 \mathrm{a}} \mathrm{Cu}_{2}(\mu-\mathrm{OAc})\right]\left(\mathrm{ClO}_{4}\right)_{2}\left(5^{\mathrm{OAc}}\right)$}

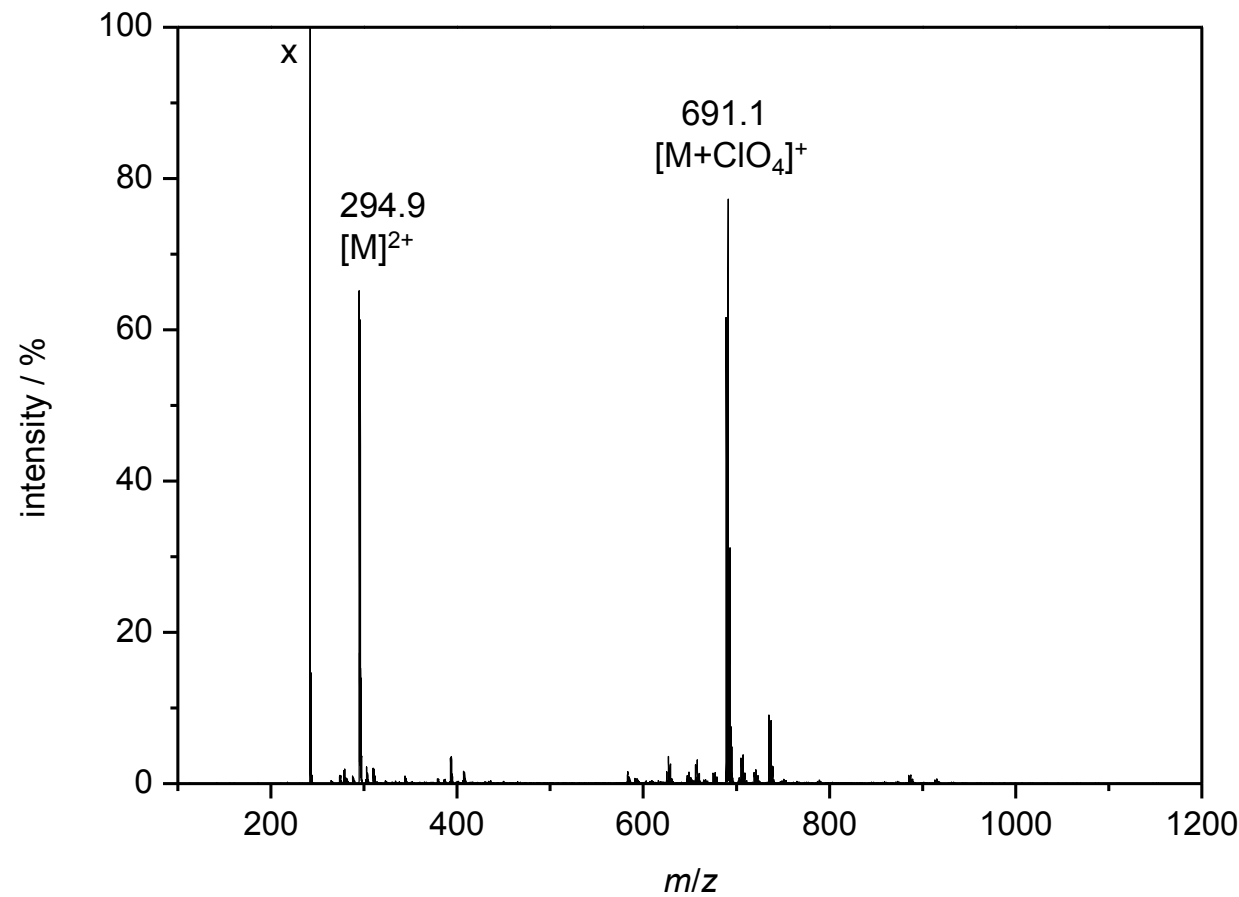

Figure A.17: ESI(+)-MS of 50Ac in MeOH. The x marks an impurity from the mass spectrometer.
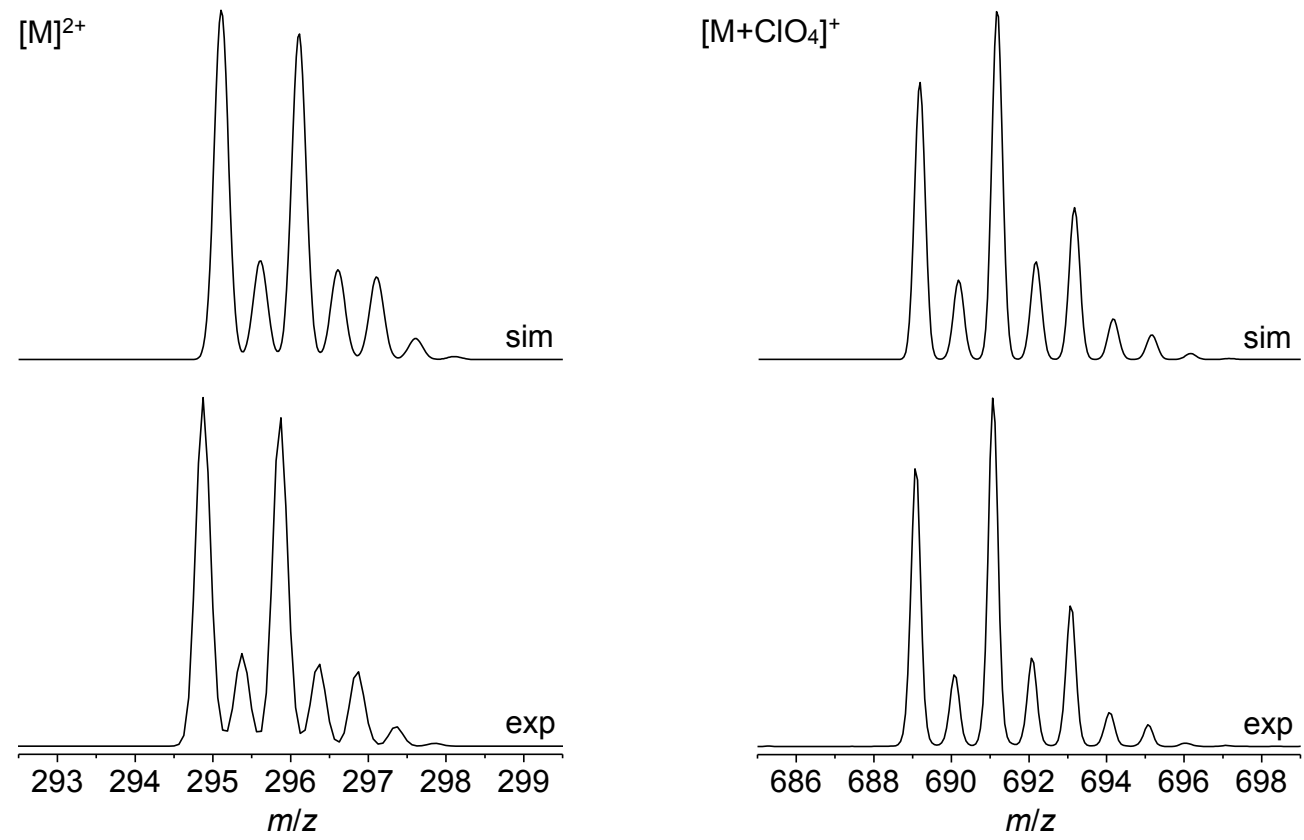

Figure A.18: $\mathrm{ESI}(+)-\mathrm{MS}$ of 50Ac in $\mathrm{MeOH}$. Isotopic patterns of the simulated (top) and experimental (bottom) signals corresponding to $[\mathrm{M}]^{2+}(\mathrm{m} / \mathrm{z}=294.9$, left $)$ and $\left[\mathrm{M}+\mathrm{ClO}_{4}\right]^{+}(\mathrm{m} / \mathrm{z}=691.1$, right $)$. 
Decomposition product of $\left[\mathrm{L}^{2 \mathrm{a}} \mathrm{Cu}_{2}\left(\mu-00^{2-}\right)\right]\left(\mathrm{ClO}_{4}\right)\left(5^{\mathrm{P}}\right)$

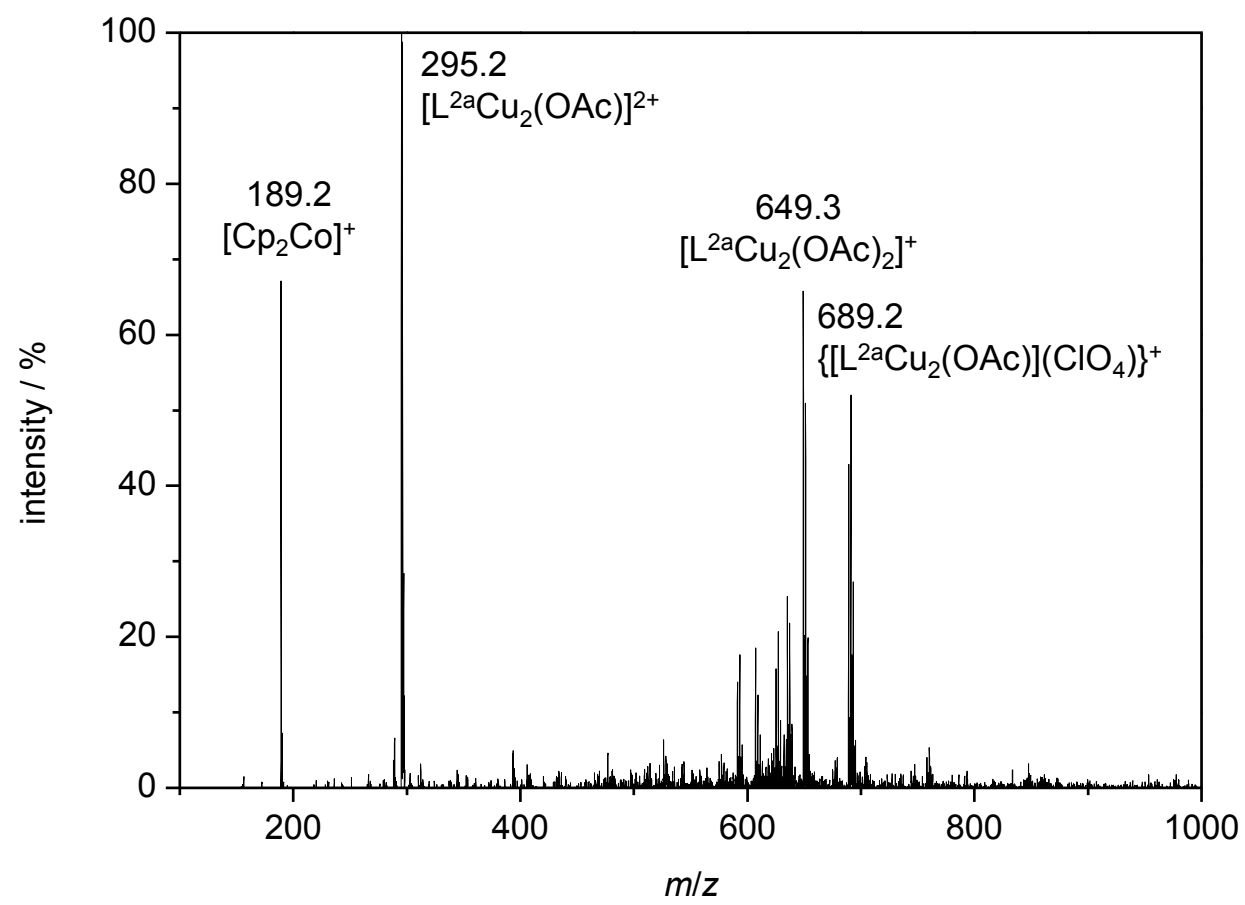

Figure A.19: ESI(+)-MS of the solution after decay of $\mathbf{5}^{\mathbf{P}}$ in MeCN. The decomposition product seems to be a dicopper(II) species with at least one acetate moiety. 


\section{$\left[\mathrm{L}^{2 \mathrm{~b}} \mathrm{Cu}_{2}\left(\mu-\mathrm{HCO}_{2}\right)\right]\left(\mathrm{ClO}_{4}\right)_{2}\left(6^{\mathrm{HCO}}\right)$}

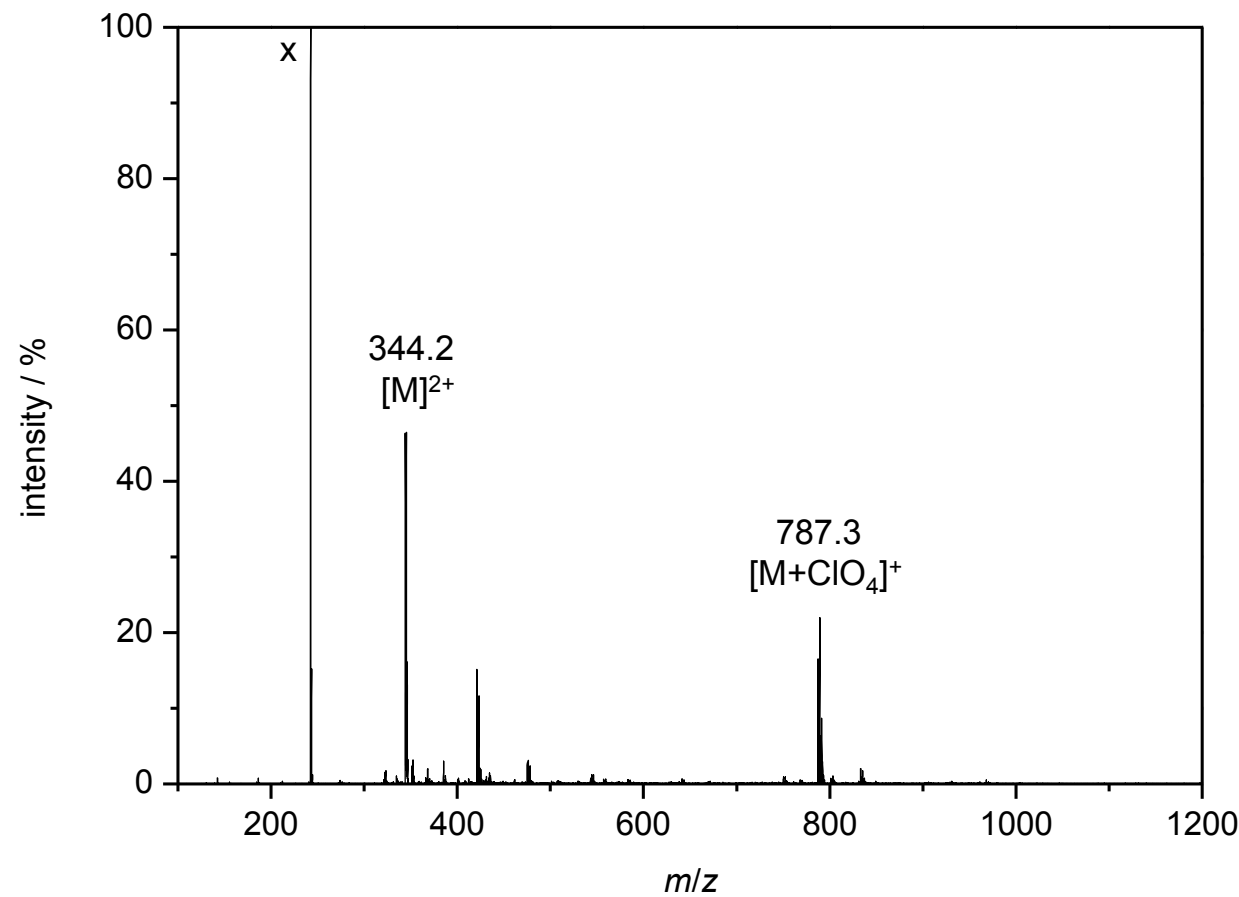

Figure A.20: ESI(+)-MS of $\mathbf{6}^{\mathrm{HCO}}$ in MeCN. The x marks an impurity from the mass spectrometer.
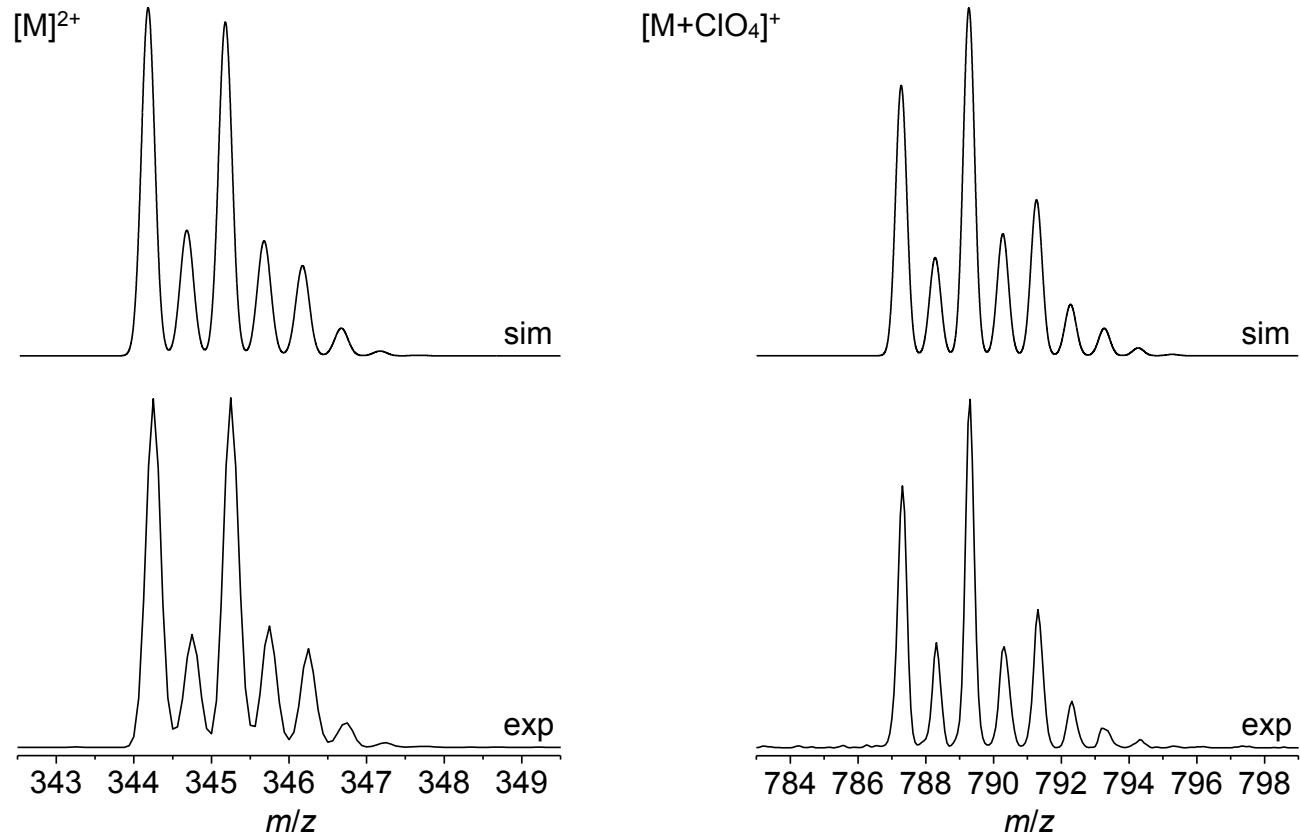

Figure A.21: ESI(+)-MS of $6^{\mathrm{HCO}}$ in MeCN. Isotopic patterns of the simulated (top) and experimental (bottom) signals corresponding to $[\mathrm{M}]^{2+}(\mathrm{m} / z=344.2$, left $)$ and $\left[\mathrm{M}+\mathrm{ClO}_{4}\right]^{+}(\mathrm{m} / \mathrm{z}=789.3$, right $)$. 


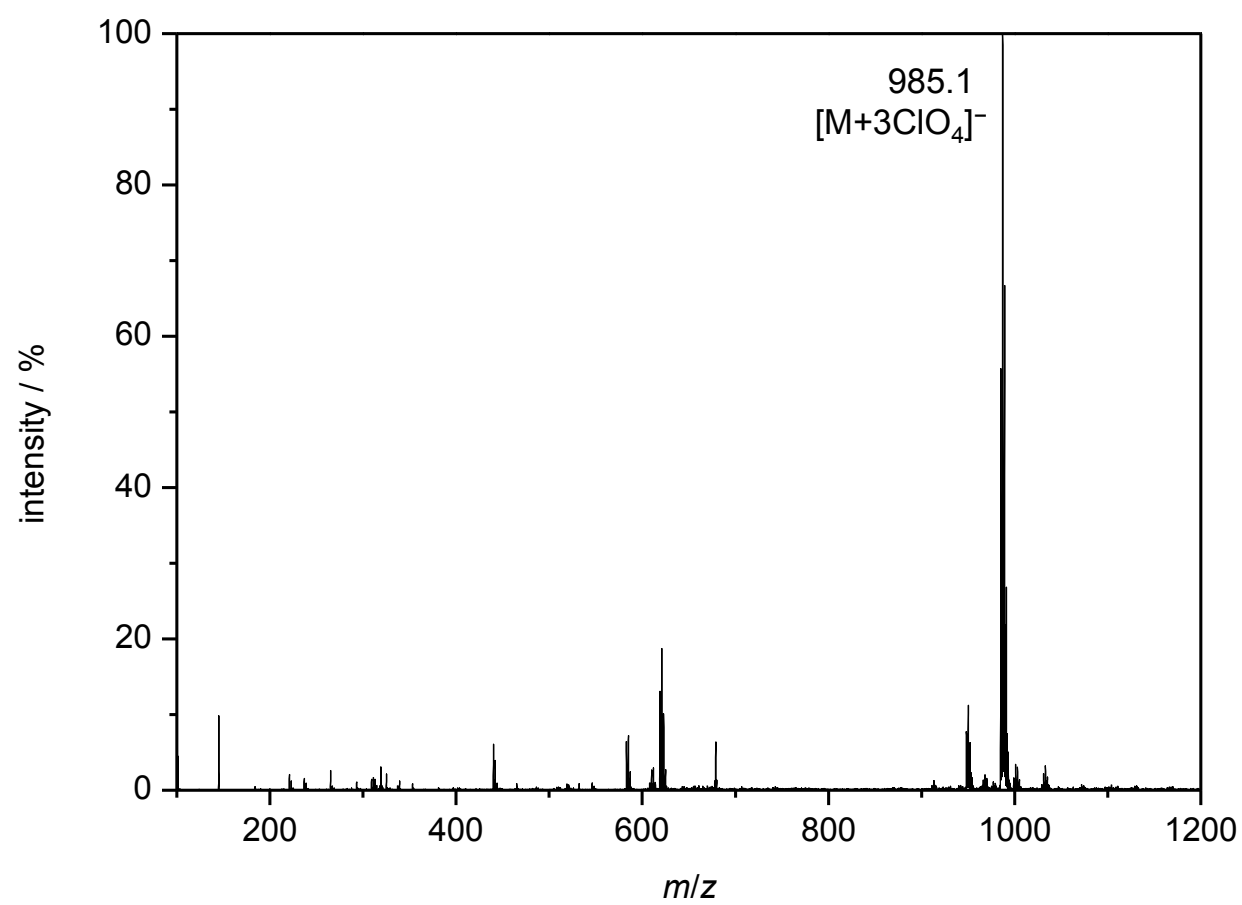

Figure A.22: $\operatorname{ESI}(-)-\mathrm{MS}$ of $6^{\mathrm{HCO}}$ in MeCN.

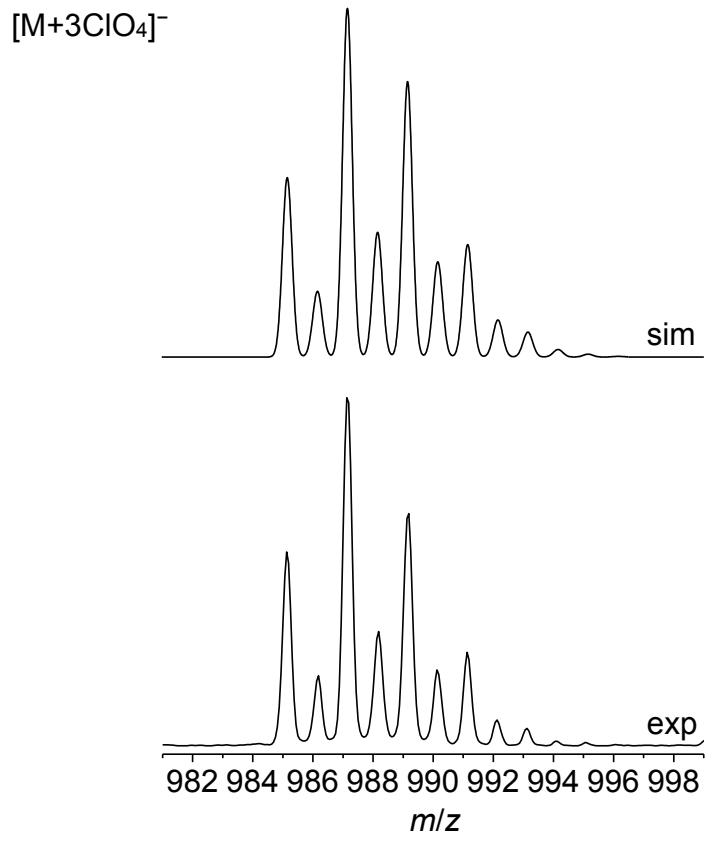

Figure A.23: ESI(-)-MS of $\mathbf{6}^{\mathrm{HCO}}$ in MeCN. Isotopic patterns of the simulated (top) and experimental (bottom) signal corresponding to $\left[\mathrm{M}+3 \mathrm{ClO}_{4}\right]^{-}(\mathrm{m} / \mathrm{z}=987.1)$. 


\section{$\left[\mathrm{L}^{2 \mathrm{~b}} \mathrm{Cu}_{2}(\mu-\mathrm{OAc})\right]\left(\mathrm{ClO}_{4}\right)_{2}\left(6^{\mathrm{OAc}}\right)$}

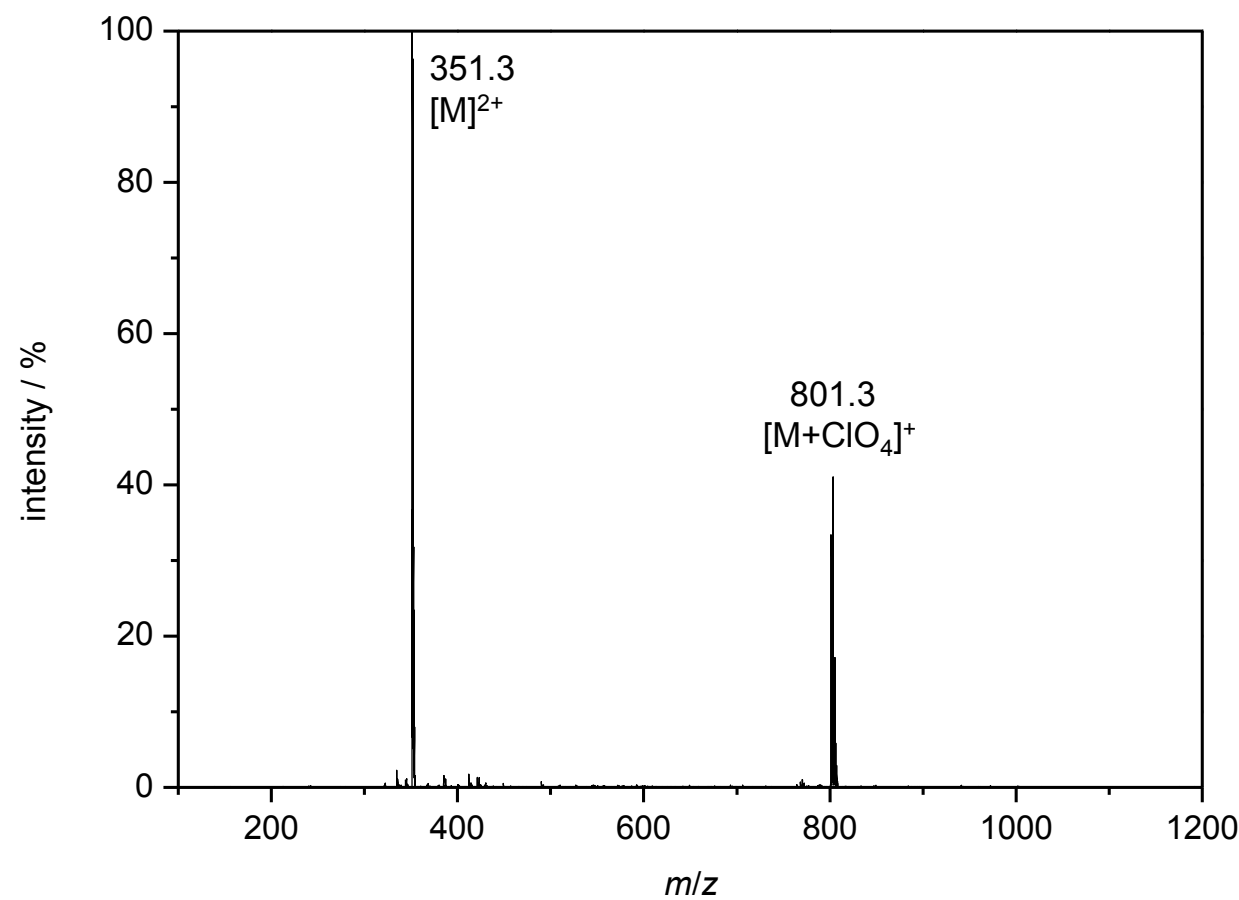

Figure A.24: $\mathrm{ESI}(+)-\mathrm{MS}$ of $6^{\mathbf{0 A c}}$ in MeCN.
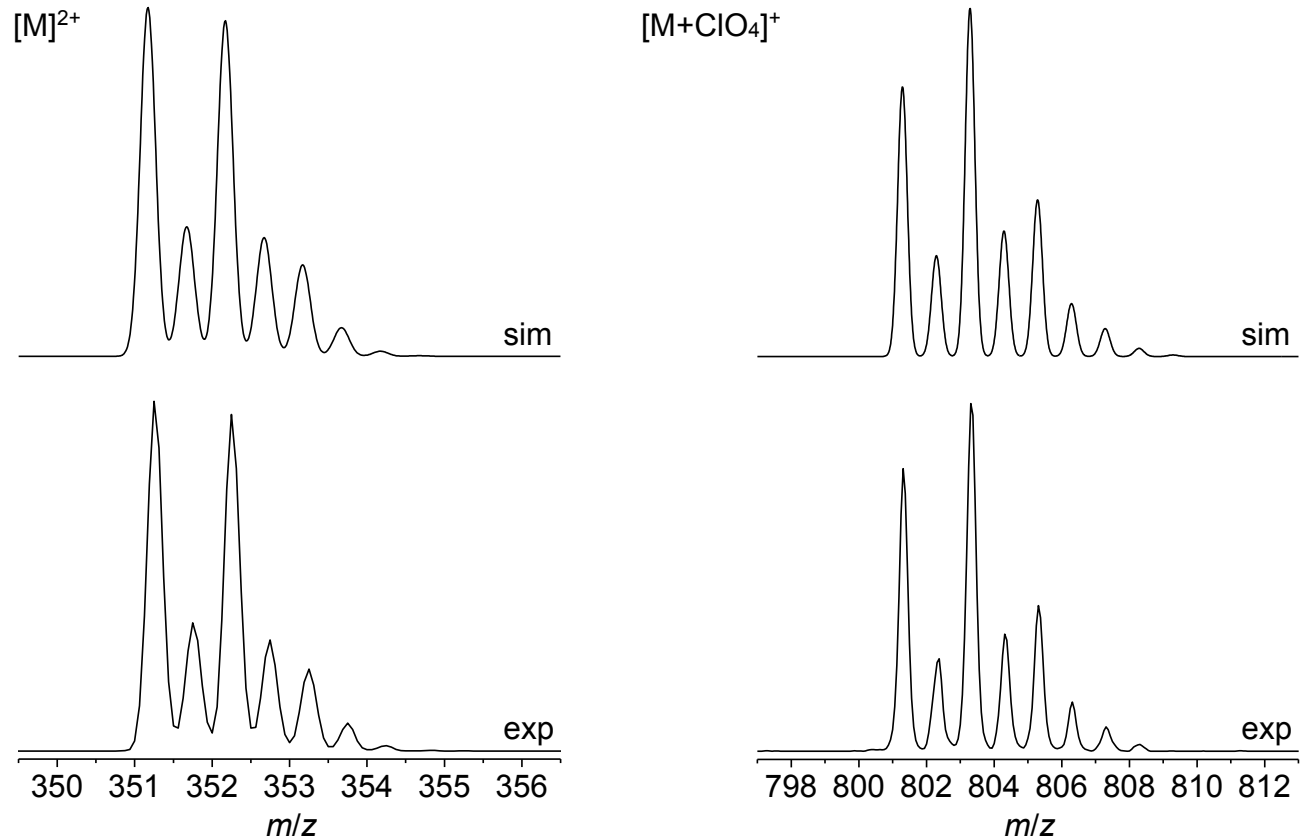

Figure A.25: $\mathrm{ESI}(+)-\mathrm{MS}$ of $\mathbf{6}^{\mathrm{OAc}}$ in MeCN. Isotopic patterns of the simulated (top) and experimental (bottom) signals corresponding to $[\mathrm{M}]^{2+}(\mathrm{m} / z=351.3$, left $)$ and $\left[\mathrm{M}+\mathrm{ClO}_{4}\right]^{+}(\mathrm{m} / z=803.3$, right $)$. 


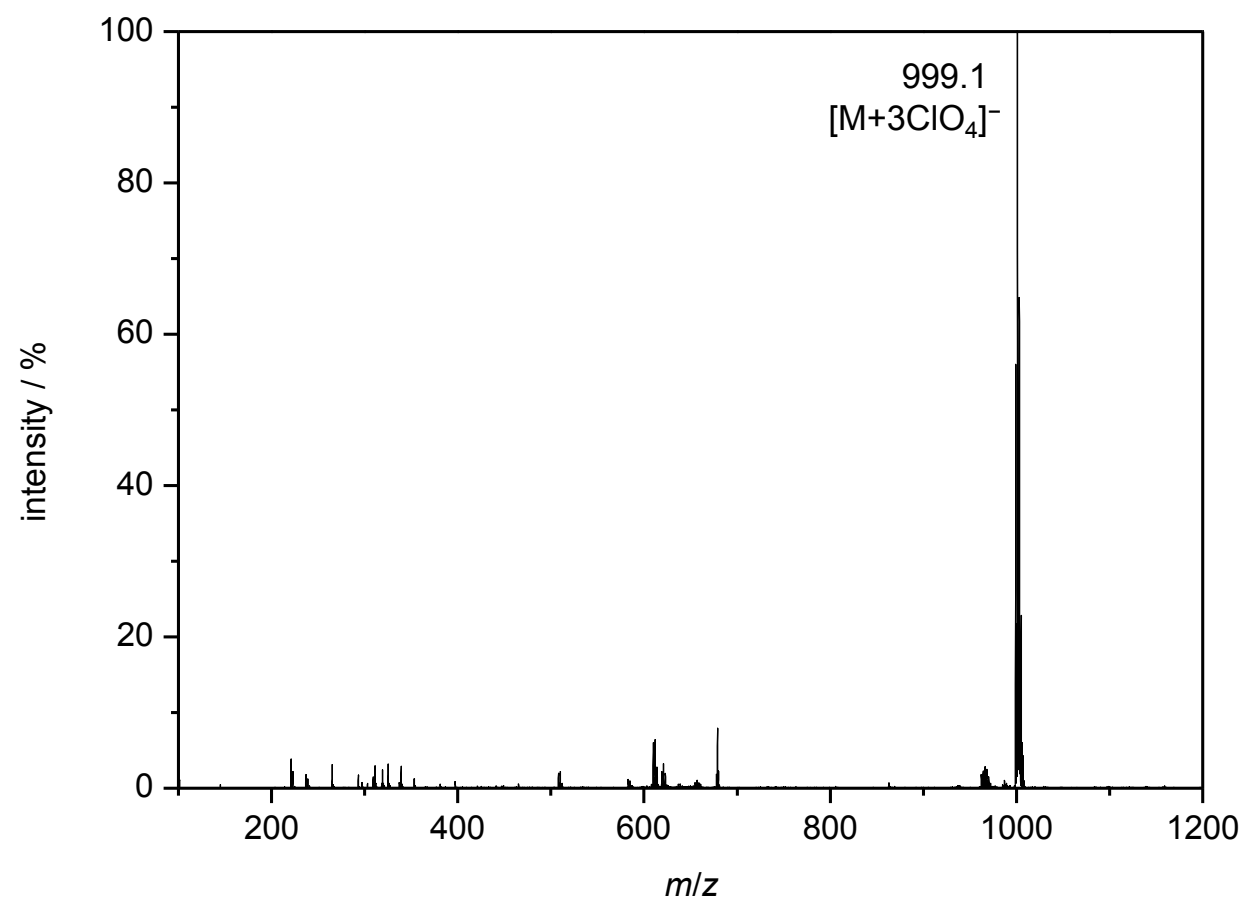

Figure A.26: $\operatorname{ESI}(-)-M S$ of $6^{\circ \mathrm{Ac}}$ in MeCN.

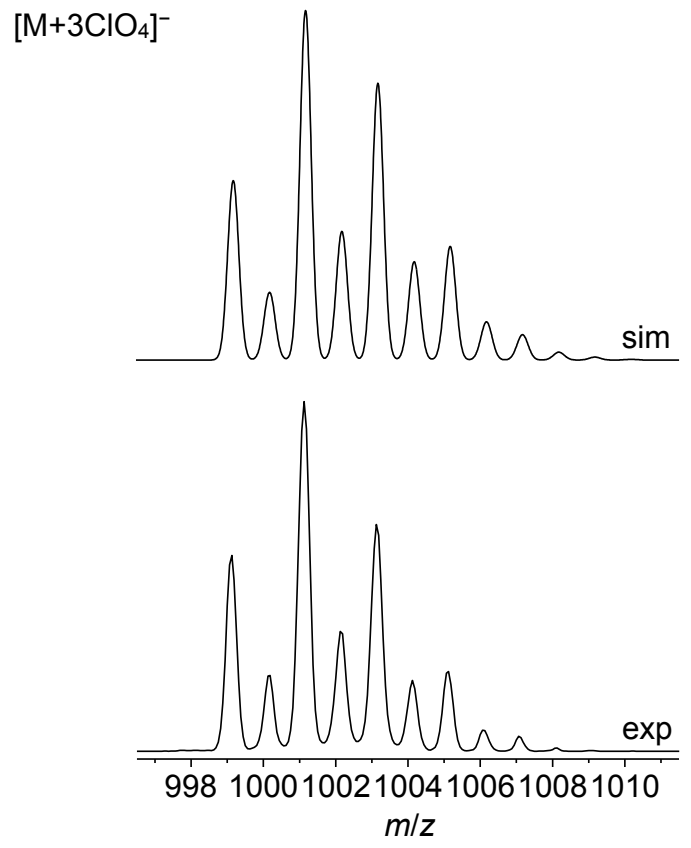

Figure A.27: ESI(-)-MS of $\mathbf{6}^{\mathbf{O A c}}$ in MeCN. Isotopic patterns of the simulated (top) and experimental (bottom) signal corresponding to $\left[\mathrm{M}+3 \mathrm{ClO}_{4}\right]^{-}(\mathrm{m} / \mathrm{z}=1001.1)$. 


\section{$\left[\mathrm{L}^{2 \mathrm{a}} \mathrm{Co}_{2}(\mathrm{MeCN})_{4}\right]\left(\mathrm{ClO}_{4}\right)_{3}\left(7^{\mathrm{MeCN}}\right)$}

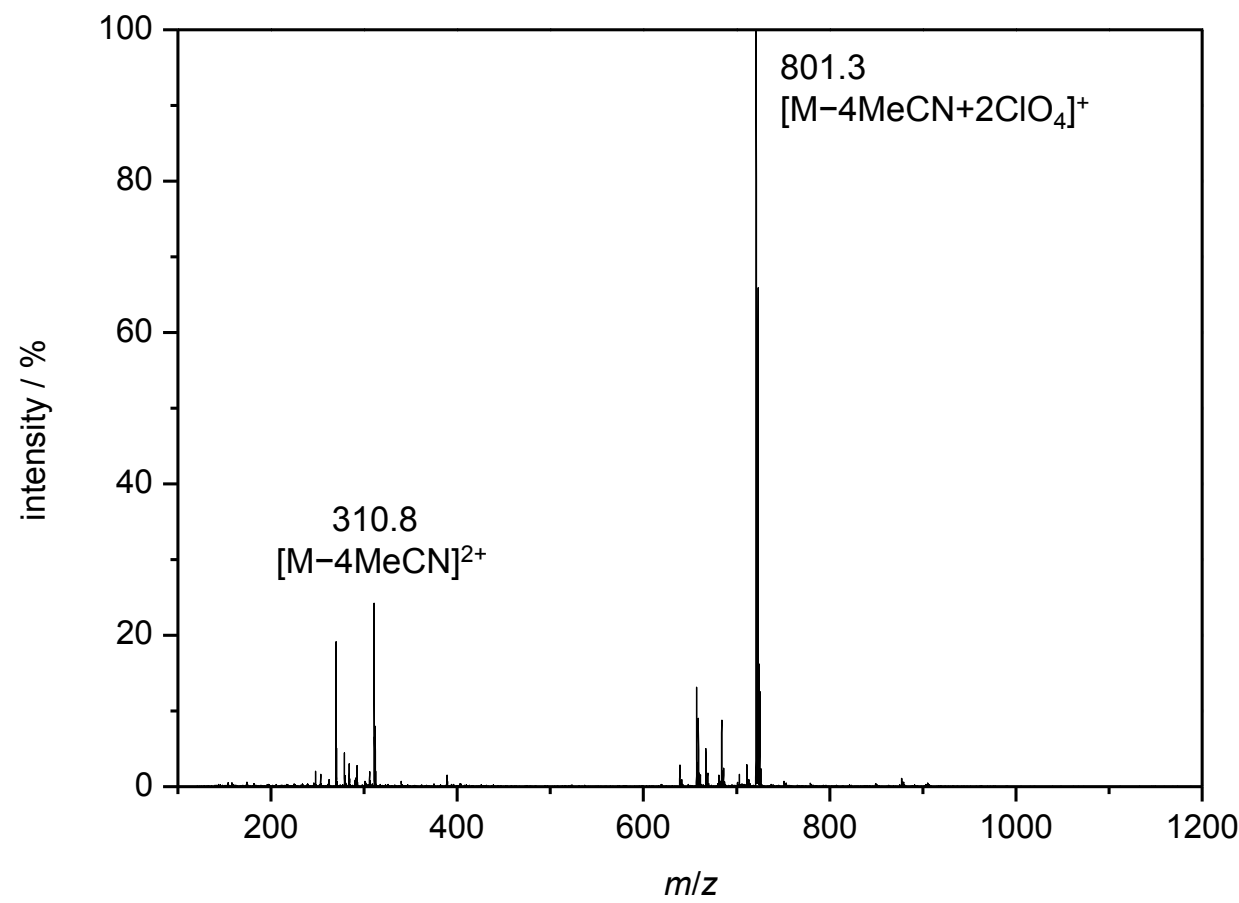

Figure A.28: $\mathrm{ESI}(+)-\mathrm{MS}$ of $\mathbf{7 M e C N}^{\mathrm{Mn}} \mathrm{MeCN}$ under inert conditions.
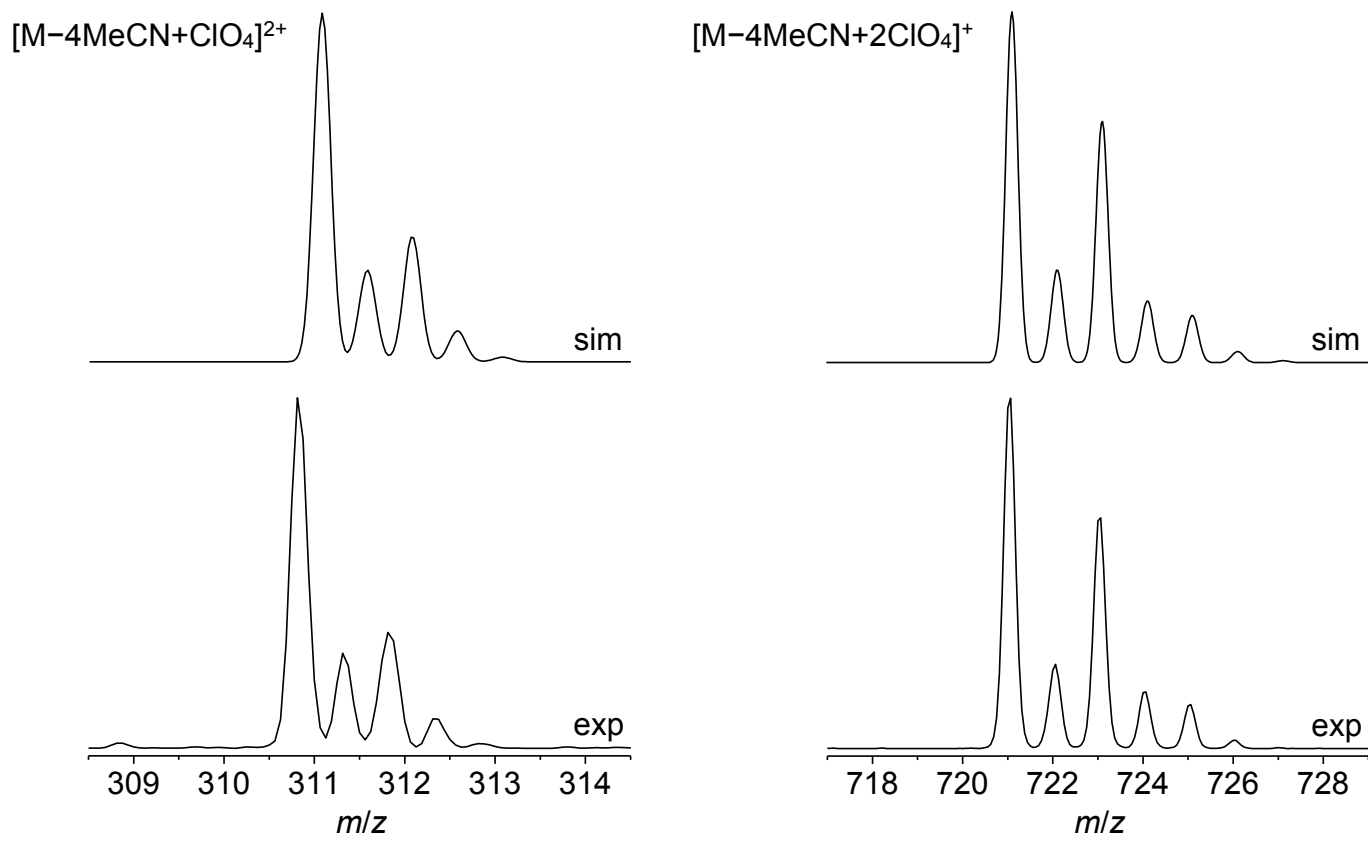

Figure A.29: $\mathrm{ESI}(+)-\mathrm{MS}$ of $\mathbf{7}^{\mathrm{MeCN}}$ in MeCN under inert conditions. Isotopic patterns of the simulated (top) and experimental (bottom) signals corresponding to $\left[\mathrm{M}-4 \mathrm{MeCN}+\mathrm{ClO}_{4}\right]^{2+}\left(\mathrm{m} / \mathrm{z}=310.8\right.$, left) and $[\mathrm{M}-4 \mathrm{MeCN}+2 \mathrm{ClO} 4]^{+}$ $(m / z=721.1$, right $)$. 


\section{$\left[\mathrm{L}^{2 \mathrm{a}} \mathrm{Co}_{2}\left(\mu-\mathrm{CH}_{3} \mathrm{C}(\mathrm{O}) \mathrm{NH}\right)(\mathrm{MeCN})_{2}\right]\left(\mathrm{ClO}_{4}\right)_{4}\left(7^{\mathrm{A}}\right)$}

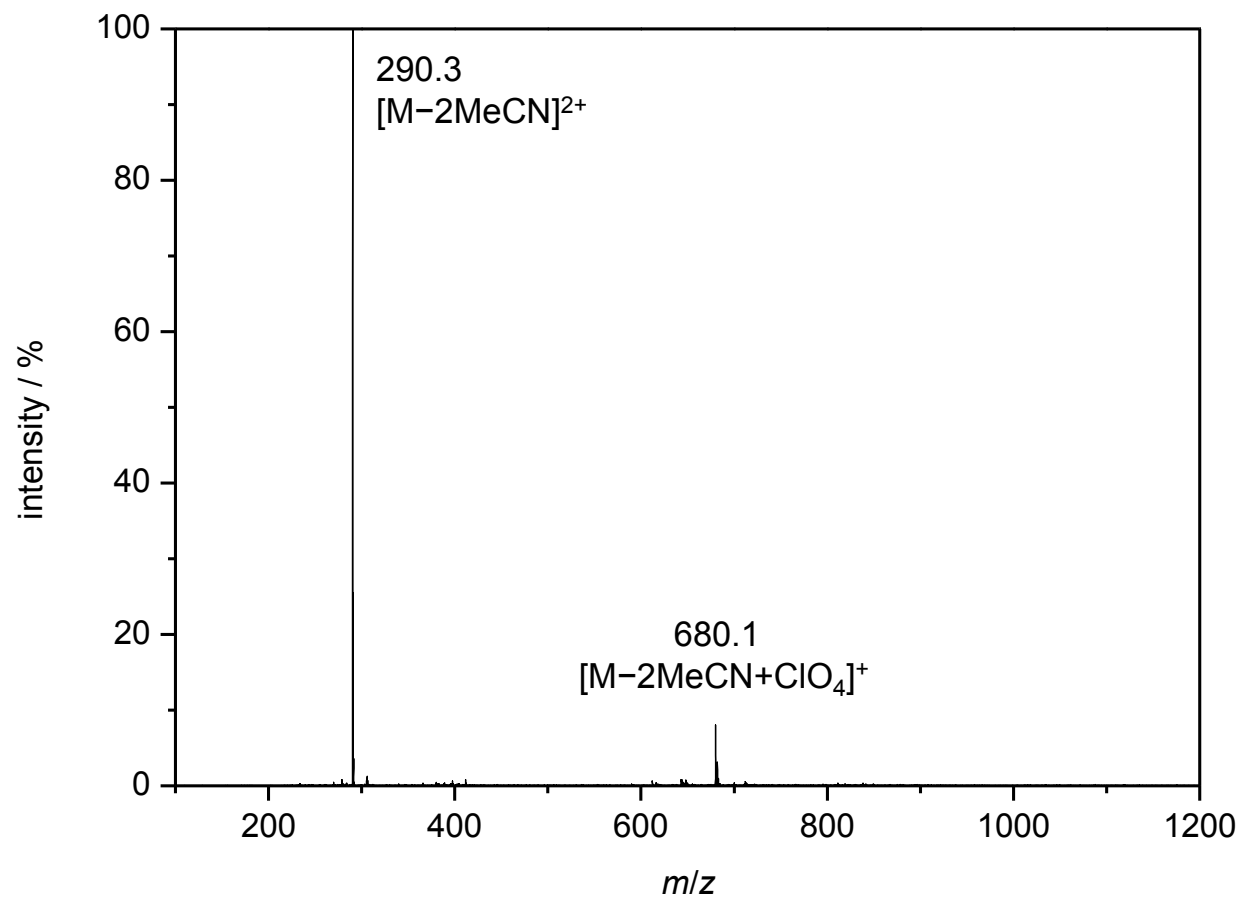

Figure A.30: $\operatorname{ESI}(+)-M S$ of $\mathbf{7}^{\mathbf{A}}$ in MeCN under inert conditions.
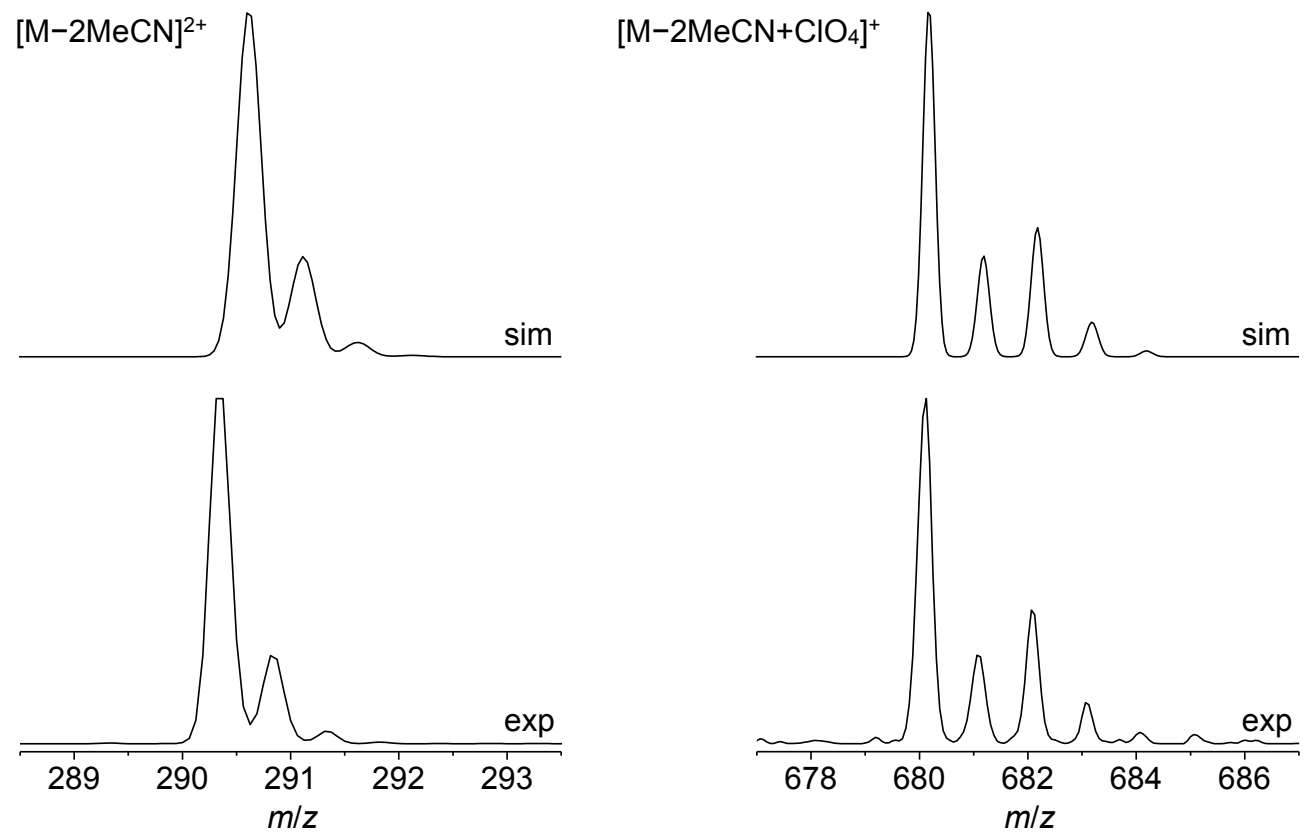

Figure A.31: $\operatorname{ESI}(+)-M S$ of $7^{\mathrm{A}}$ in $\mathrm{MeCN}$ under inert conditions. Isotopic patterns of the simulated (top) and experimental (bottom) signals corresponding to $[\mathrm{M}-2 \mathrm{MeCN}]^{2+}(\mathrm{m} / z=290.3$, oxidation states of Co: II/II, left) and $\left[\mathrm{M}-2 \mathrm{MeCN}+\mathrm{ClO}_{4}\right]^{+}(\mathrm{m} / \mathrm{z}=680.1, \mathrm{II} / \mathrm{II}$, right $)$. 
$\left[\mathrm{L}^{2 \mathrm{aa}} \mathrm{Co}_{2}\left(\mu-\mathrm{O}_{2} \mathrm{H}_{3}\right)\right]\left(\mathrm{ClO}_{4}\right)_{2}\left(7^{\mathrm{O} 2 \mathrm{H}}\right)$

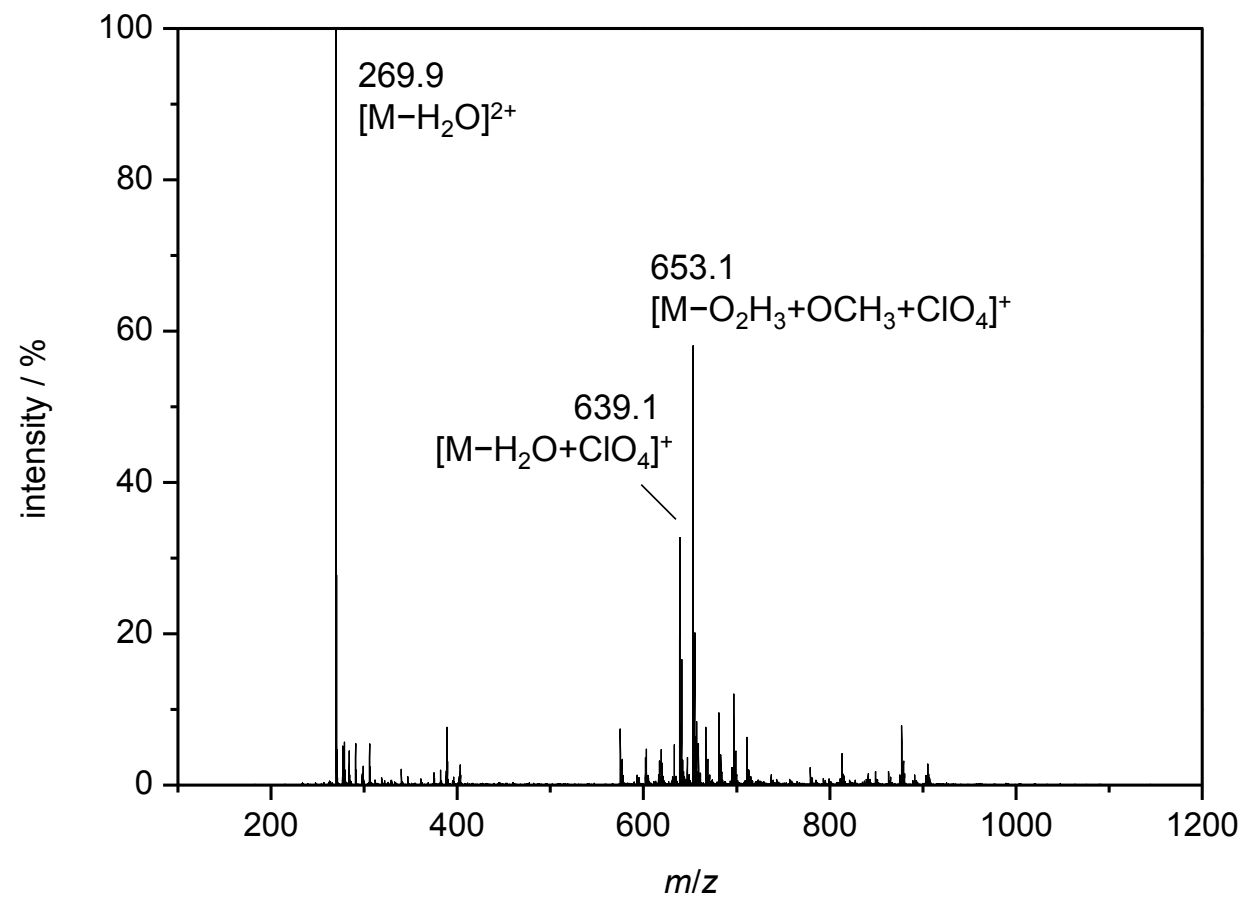

Figure A.32: ESI(+)-MS of $\mathbf{7 0 2}^{\mathbf{0} 3}$ in $\mathrm{MeOH}$ under inert conditions.
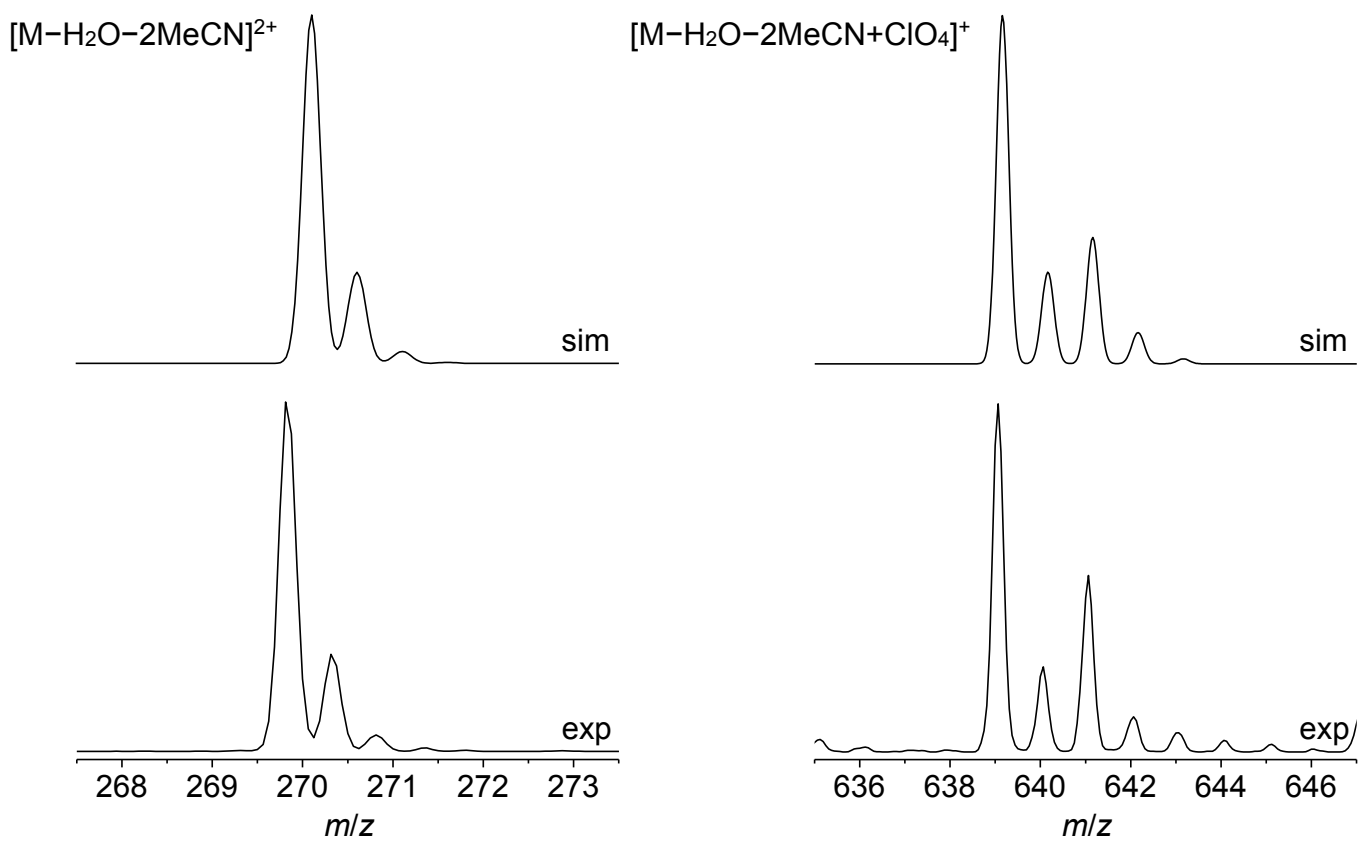

Figure A.33: $\mathrm{ESI}(+)-\mathrm{MS}$ of $\mathbf{7 0 2}^{\mathbf{0 2}} \mathbf{3}$ in $\mathrm{MeOH}$ under inert conditions. Isotopic patterns of the simulated (top) and experimental (bottom) signals corresponding to $\left[\mathrm{M}-\mathrm{H}_{2} \mathrm{O}-2 \mathrm{MeCN}\right]^{2+} \quad(\mathrm{m} / \mathrm{z}=269.9$, left) and

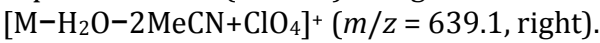




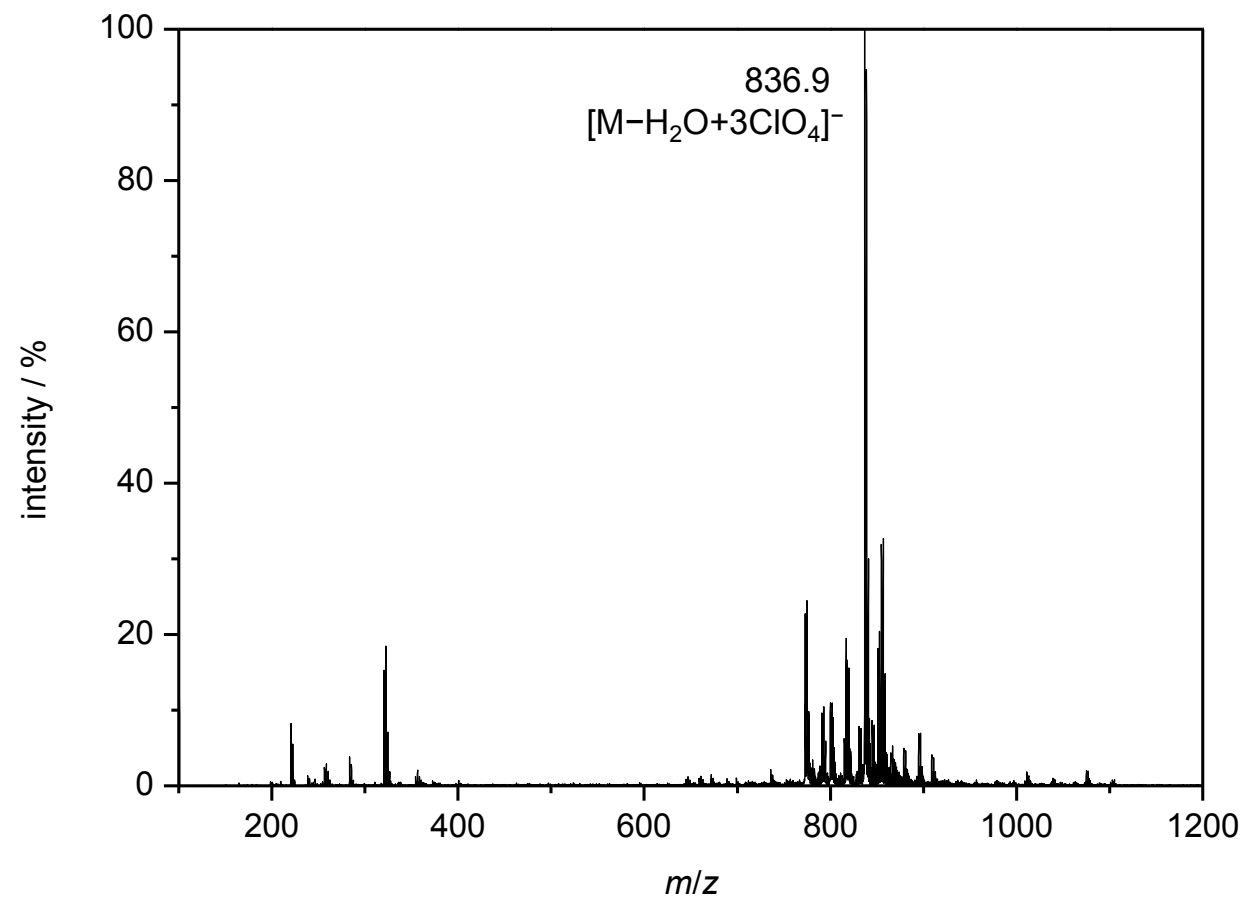

Figure A.34: $\mathrm{ESI}(-)-\mathrm{MS}$ of $\mathbf{7 0 2} \mathbf{H} 3$ in $\mathrm{MeOH}$ under inert conditions.

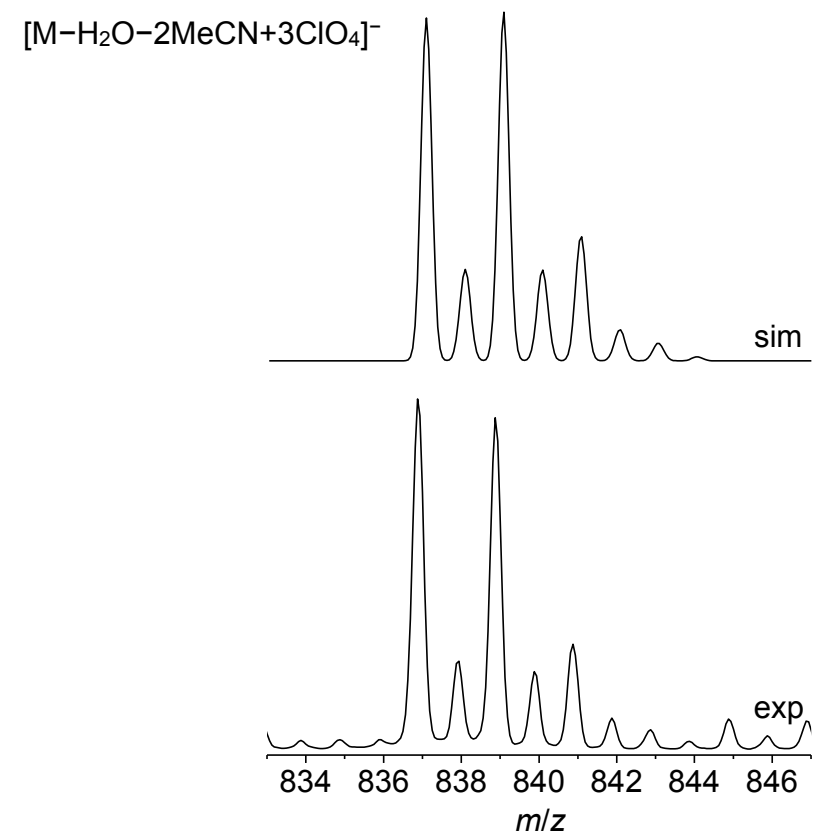

Figure A.35: ESI(-)-MS of $\mathbf{7 0 2}^{\mathbf{0}} \mathbf{H} 3$ in $\mathrm{MeOH}$ under inert conditions. Isotopic patterns of the simulated (top) and experimental (bottom) signal corresponding to $\left[\mathrm{M}-\mathrm{H}_{2} \mathrm{O}-2 \mathrm{MeCN}+3 \mathrm{ClO}_{4}\right]^{-}(\mathrm{m} / \mathrm{z}=838.9)$. 


\section{$\left[\mathrm{L}^{2 \mathrm{a}} \mathrm{Co}_{2}\left(\mu-\mathrm{PO}_{4}\right)(\mathrm{MeCN})_{2}\right]\left(\mathrm{ClO}_{4}\right)_{2}\left(7^{\mathrm{PO} 4}\right)$}

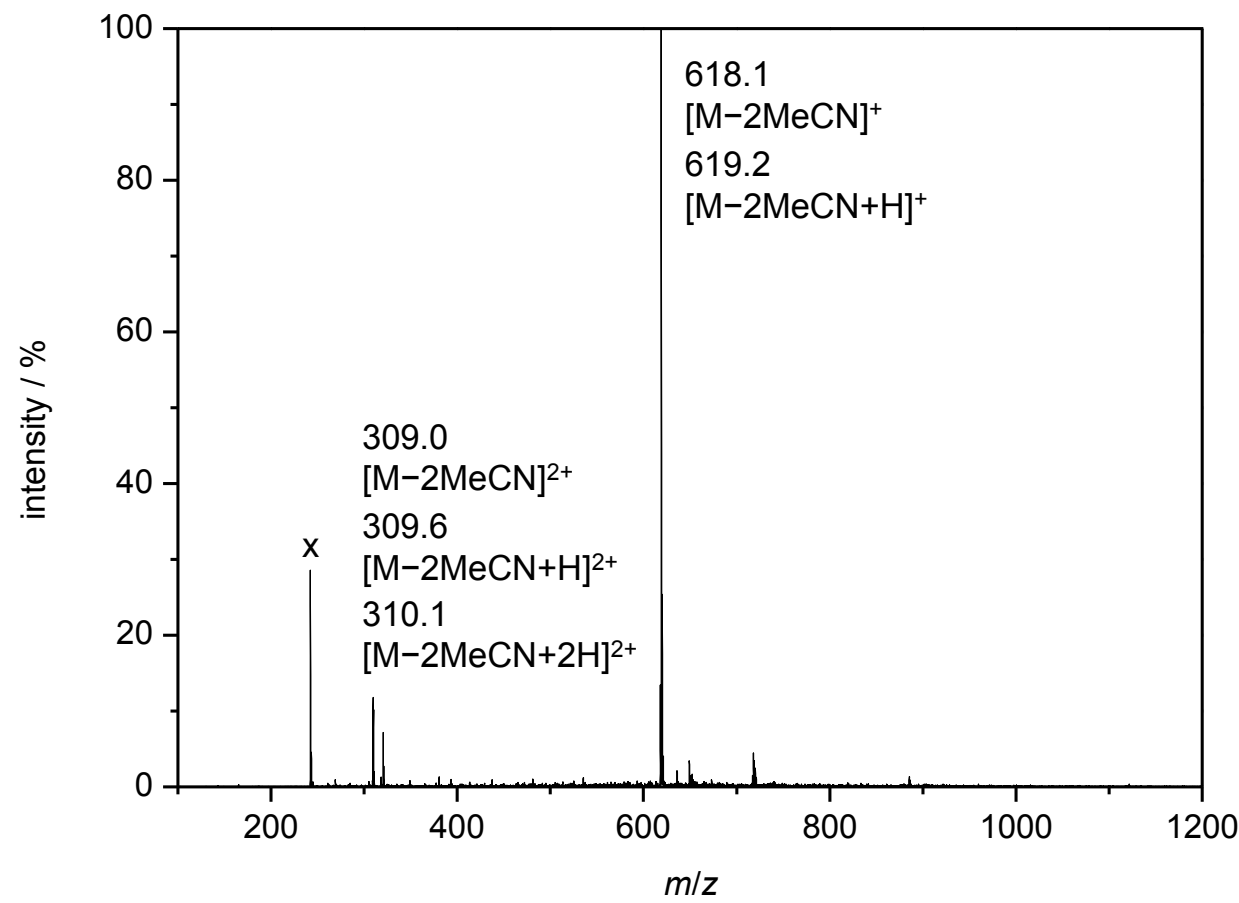

Figure A.36: ESI(+)-MS of 7P04 in MeOH. The x marks an impurity from the mass spectrometer.
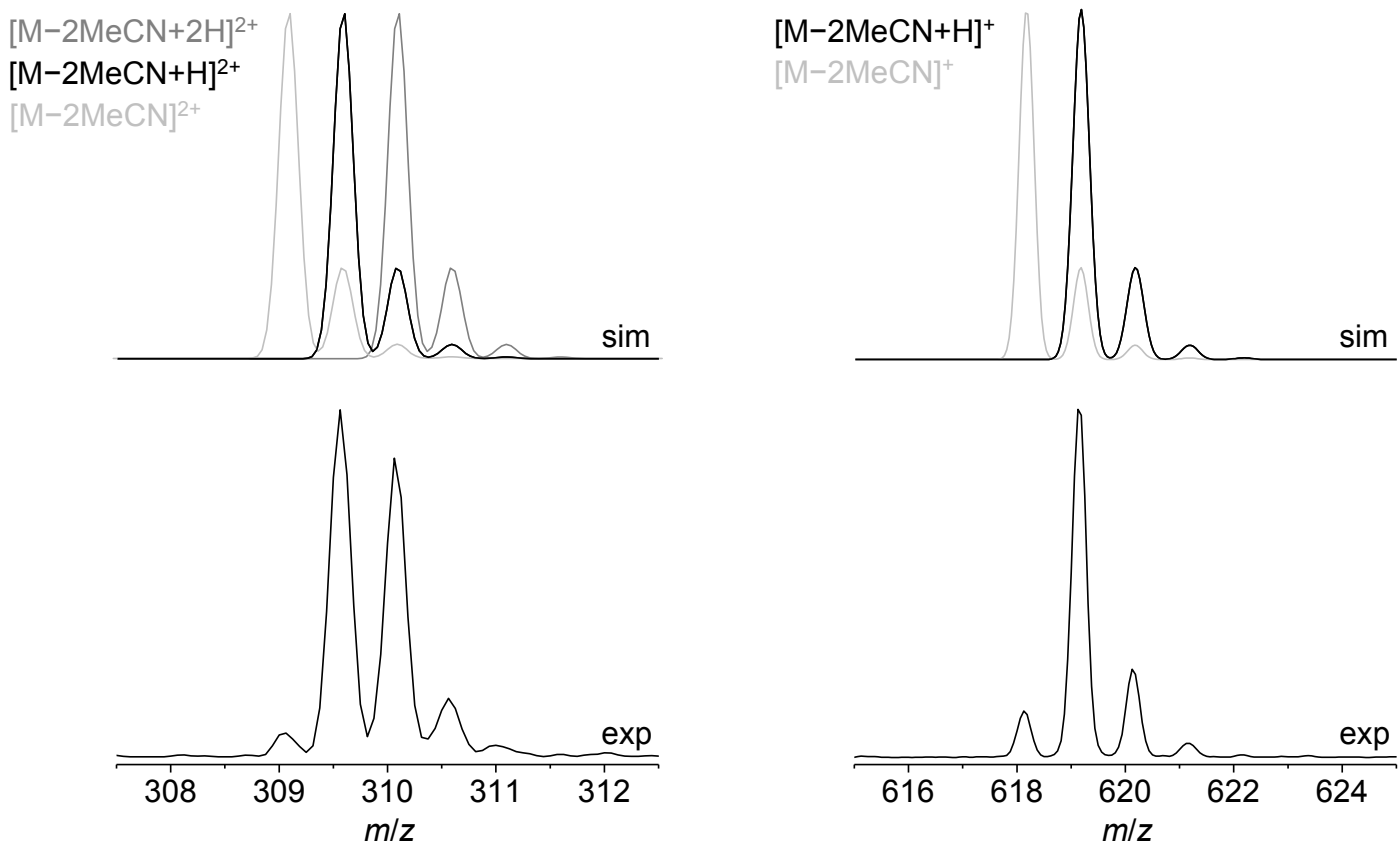

Figure A.37: ESI(+)-MS of 7P04 in MeOH. The isotopic patterns of the experimental (bottom) signals, as well as simulations (top) corresponding to $[\mathrm{M}-2 \mathrm{MeCN}]^{2+}(m / z=309.0$, oxidation states of Co: III/III, left, light grey), $[\mathrm{M}-2 \mathrm{MeCN}+\mathrm{H}]^{2+}(\mathrm{m} / \mathrm{z}=309.6$, II/III, left, black $)$ and $[\mathrm{M}-2 \mathrm{MeCN}+2 \mathrm{H}]^{2+}(\mathrm{m} / \mathrm{z}=310.1, \mathrm{II} / \mathrm{II}$, left grey $)$, as well as $[\mathrm{M}-2 \mathrm{MeCN}]^{+}(\mathrm{m} / \mathrm{z}=618.1$, II/III, right, light grey $)$ and $[\mathrm{M}-2 \mathrm{MeCN}]^{+}(\mathrm{m} / \mathrm{z}=619.2, \mathrm{II} / \mathrm{II}$, right, black $)$ are depicted. Please note, the bridging moiety varies between $\mathrm{H}_{2} \mathrm{PO}_{4}{ }^{-}, \mathrm{HPO}_{4}{ }^{2-}$ and $\mathrm{PO}_{4}{ }^{3-}$. 


\section{$\left[\mathrm{L}^{2 \mathrm{~b}} \mathrm{Co}_{2}\left(\mu-\mathrm{HCO}_{2}\right)\right]\left(\mathrm{ClO}_{4}\right)_{2}\left(8^{\mathrm{HCO}}\right)$}

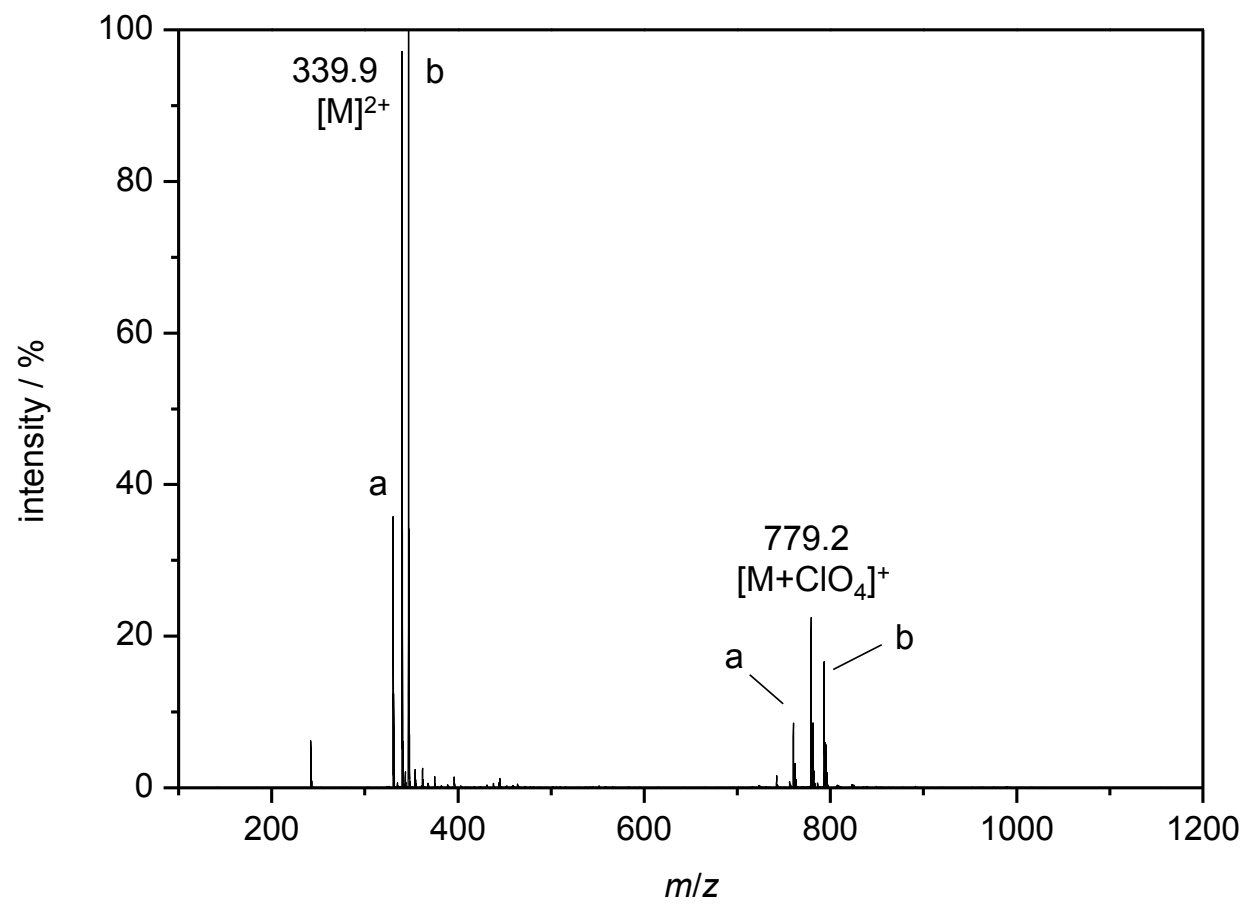

Figure A.38: ESI(+)-MS of $\mathbf{8}^{\mathrm{HCO2}}$ in MeCN. The peaks denoted a and b correspond to the equivalent species as the main peaks bearing $\mathrm{CN}^{-}$or $\mathrm{AcO}^{-}$in the bimetallic pocket, respectively.
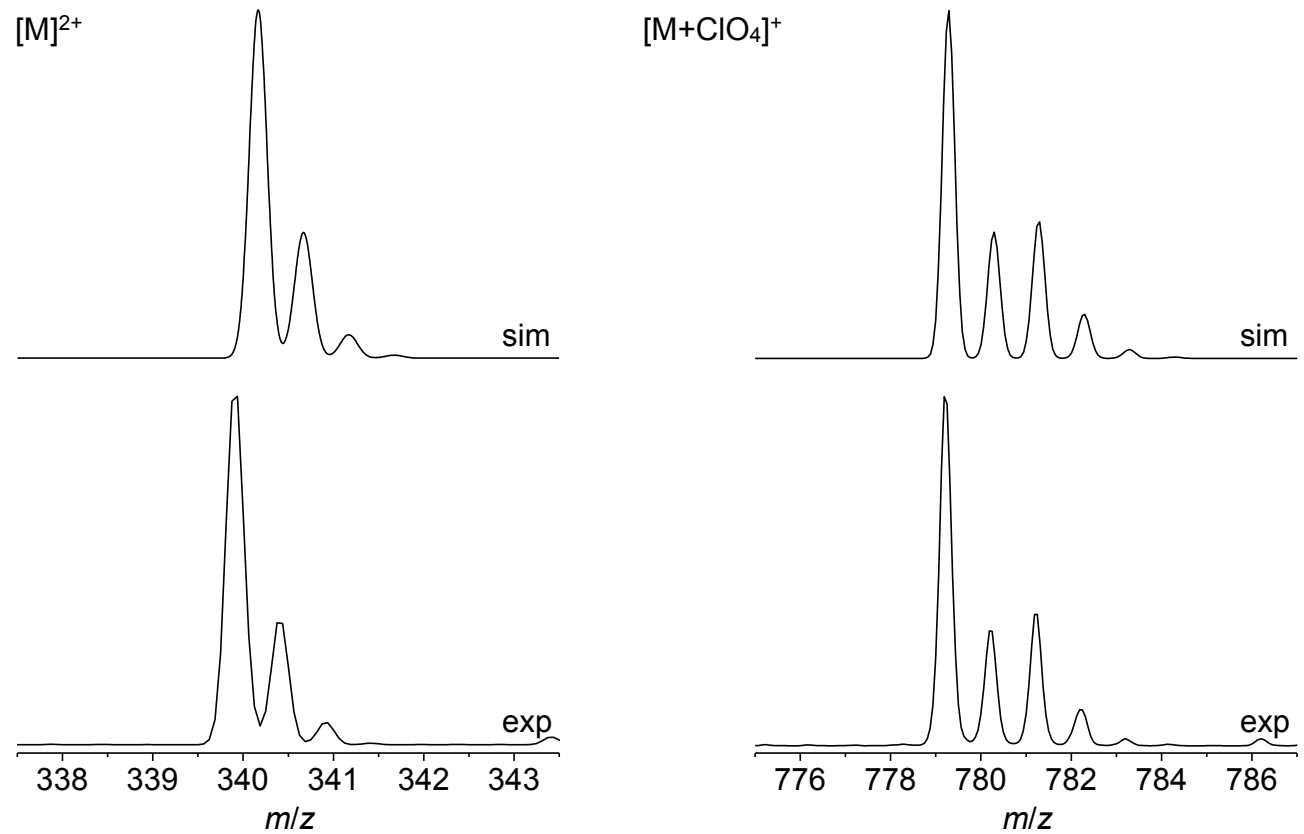

Figure A.39: ESI(+)-MS of $\mathbf{8}^{\mathrm{HCO}}$ in MeCN. Isotopic patterns of the simulated (top) and experimental (bottom) signals corresponding to $[\mathrm{M}]^{2+}(\mathrm{m} / \mathrm{z}=339.9$, left $)$ and $\left[\mathrm{M}+\mathrm{ClO}_{4}\right]^{+}(\mathrm{m} / \mathrm{z}=779.2$, right $)$. 


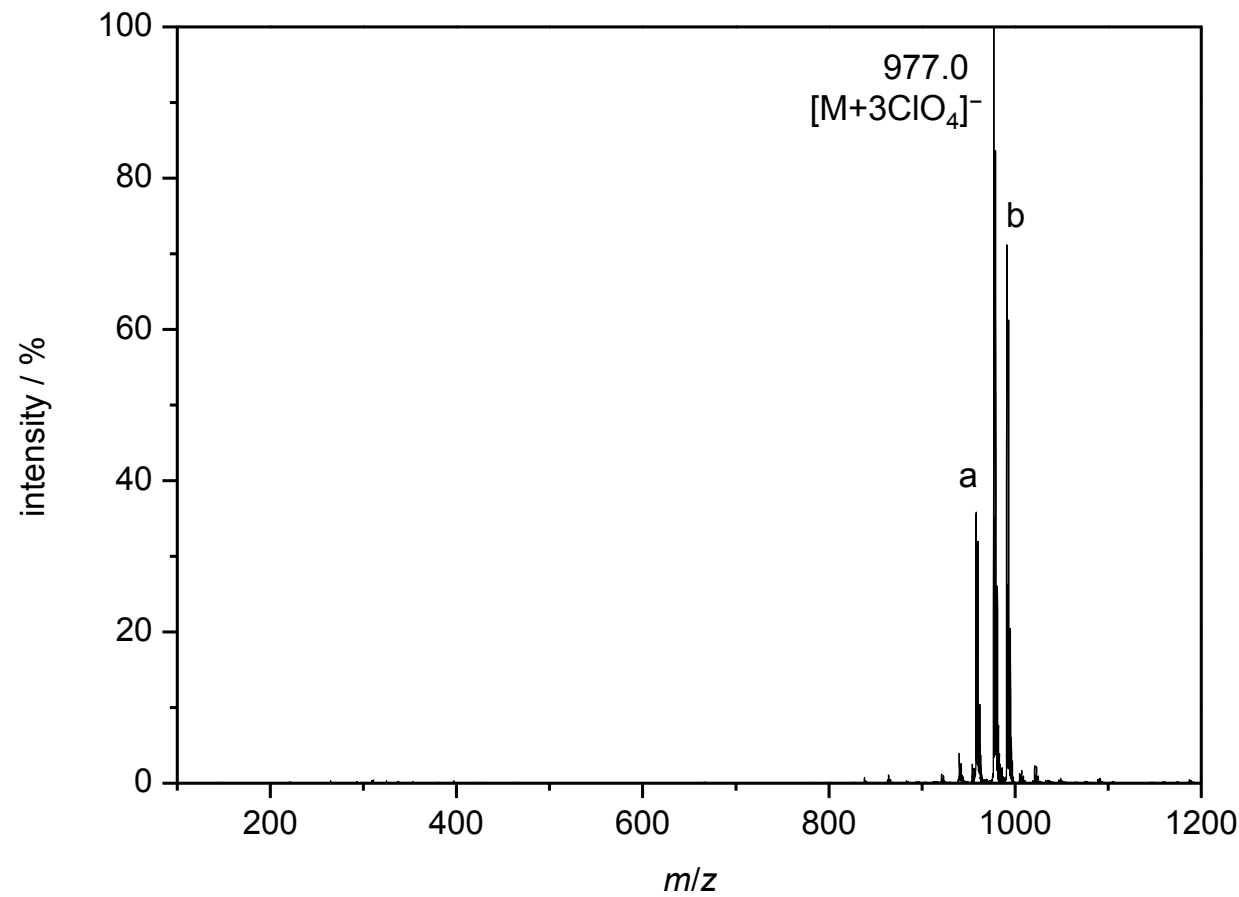

Figure A.40: $\operatorname{ESI}(-)$-MS of $\mathbf{8}^{\mathrm{HCO}}$ in MeCN. The peaks denoted a and b correspond to the equivalent species as the main peak bearing $\mathrm{CN}^{-}$or $\mathrm{AcO}^{-}$in the bimetallic pocket, respectively.

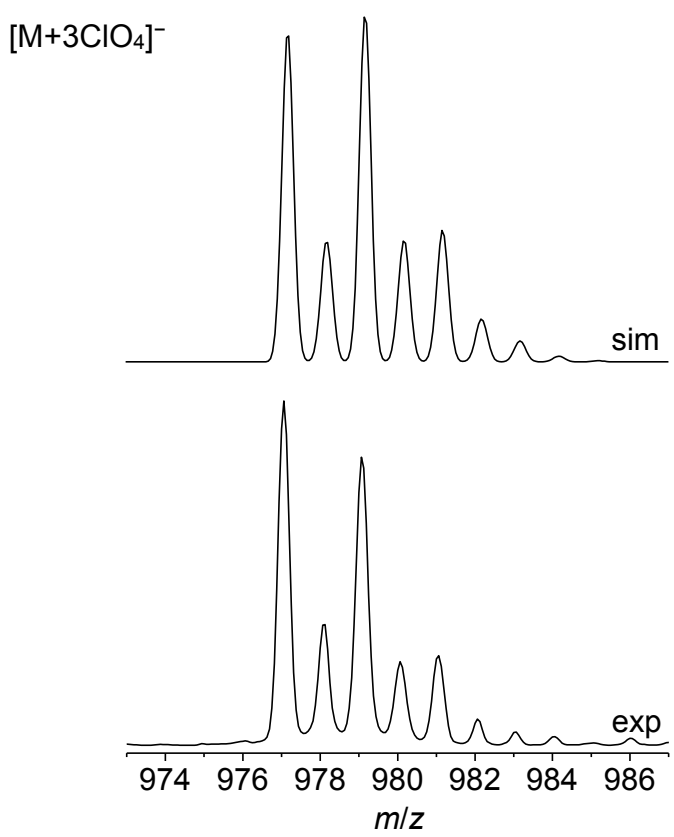

Figure A.41: ESI(-)-MS of $\mathbf{8}^{\mathbf{H C O}}$ in MeCN. Isotopic patterns of the simulated (top) and experimental (bottom) signal corresponding to $\left[\mathrm{M}+3 \mathrm{ClO}_{4}\right]^{-}(m / z=979.0)$. 


\section{$\left[\mathrm{L}^{2 \mathrm{~b}} \mathrm{Co}_{2}(\mu-\mathrm{OAc})\right]\left(\mathrm{ClO}_{4}\right)_{2}\left(8^{\mathrm{OAc}}\right)$}

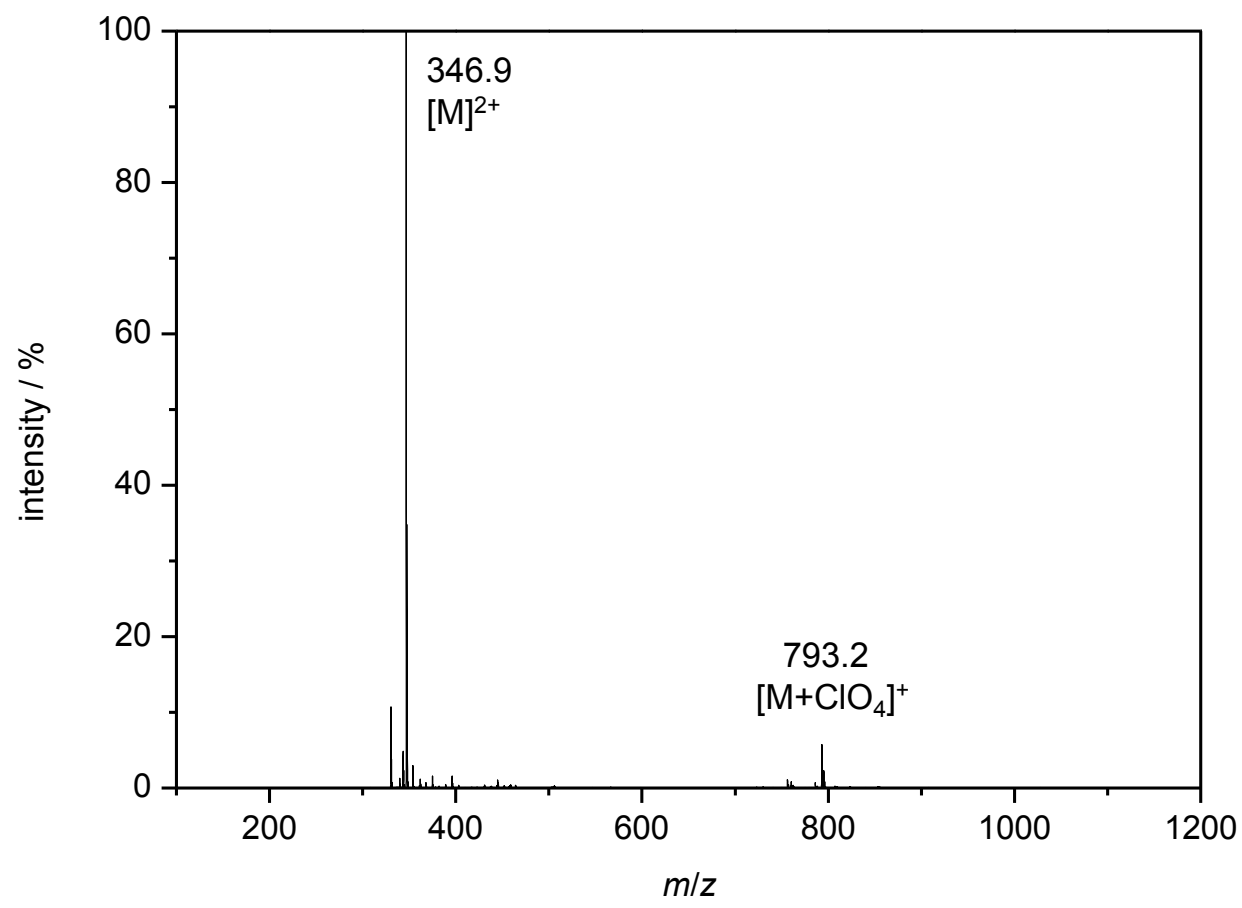

Figure A.42: $\mathrm{ESI}(+)-\mathrm{MS}$ of $\mathbf{8}^{\mathrm{OAc}}$ in MeCN.
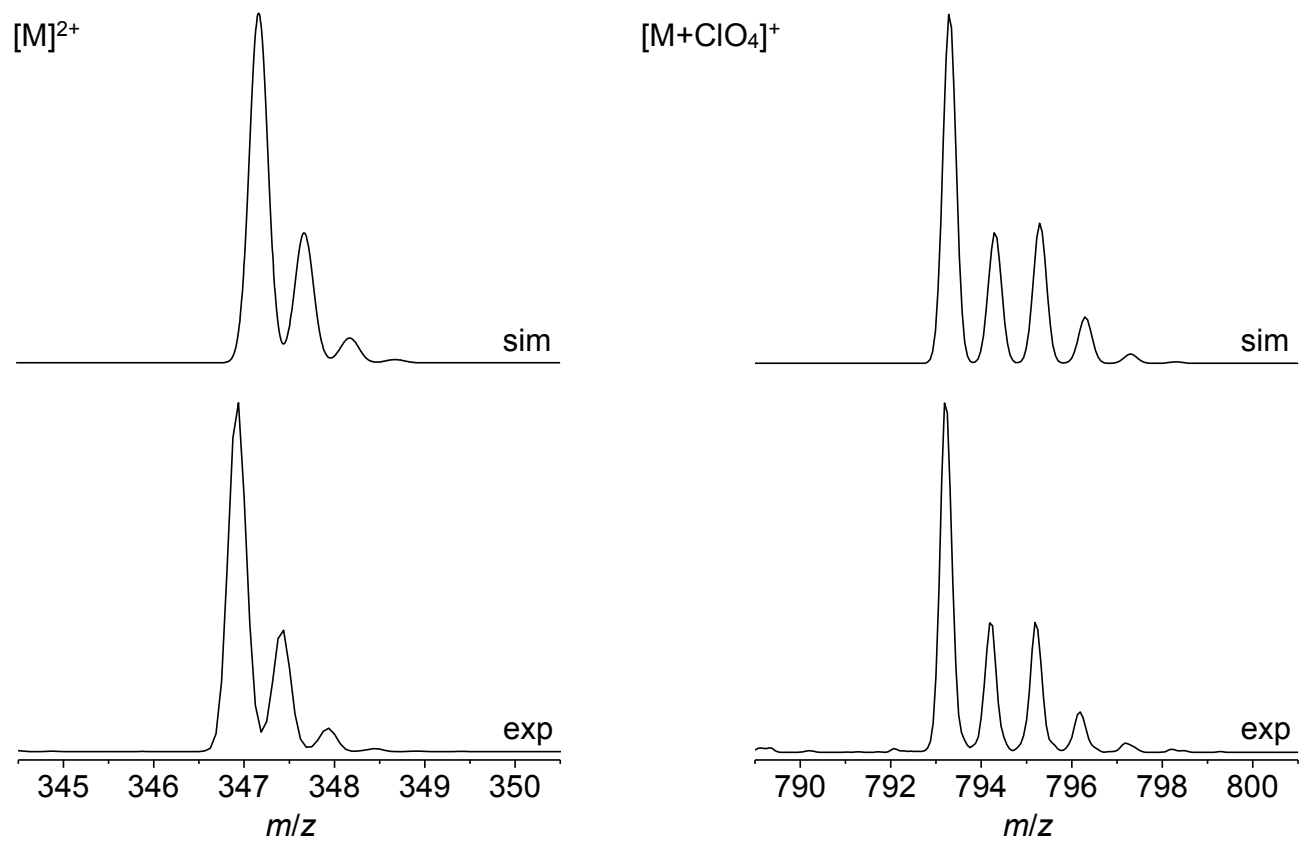

Figure A.43: ESI(+)-MS of $8^{\text {OAc }}$ in MeCN. Isotopic patterns of the simulated (top) and experimental (bottom) signals corresponding to $[\mathrm{M}]^{2+}(m / z=346.9$, left $)$ and $\left[\mathrm{M}+\mathrm{ClO}_{4}\right]^{+}(m / z=793.2$, right $)$. 


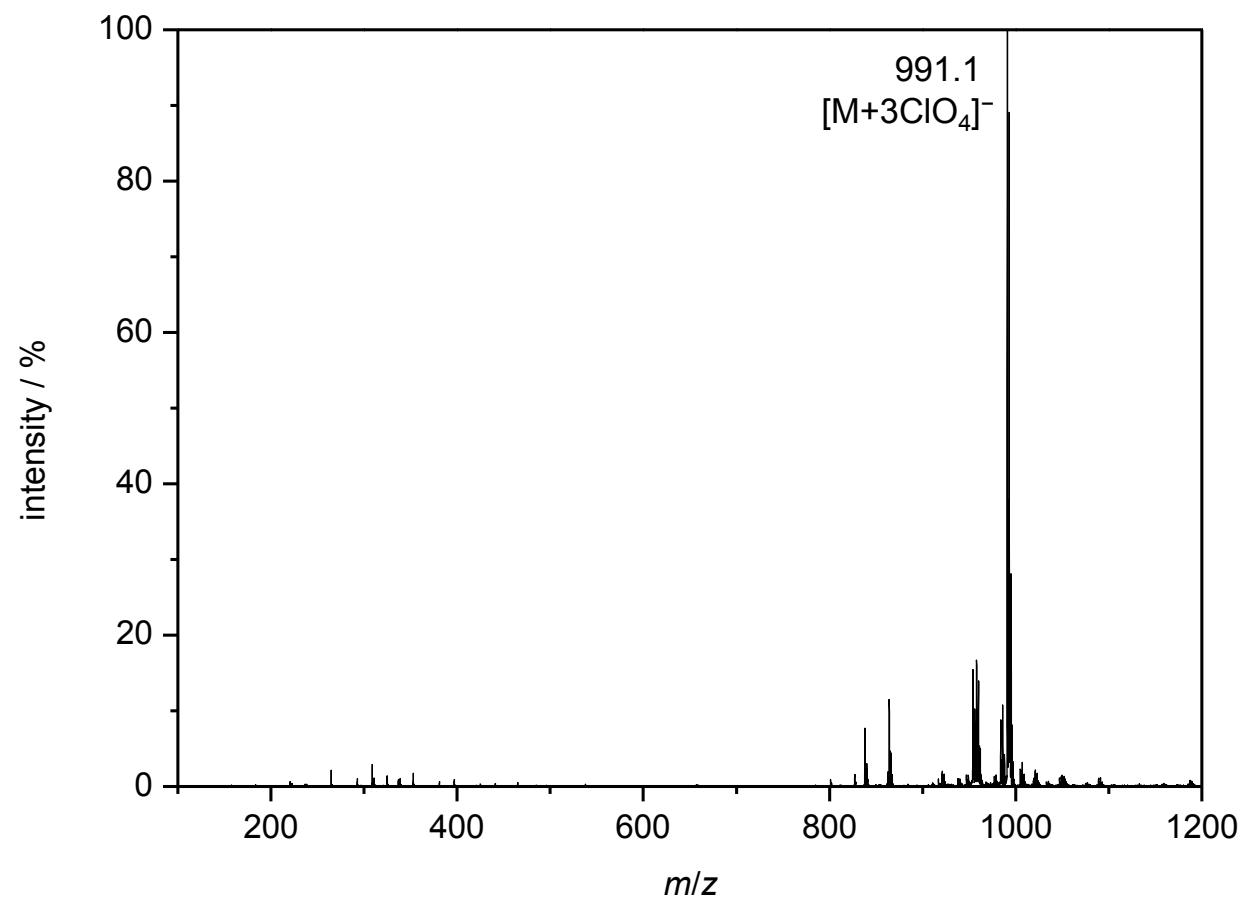

Figure A.44: $\operatorname{ESI}(-)-\mathrm{MS}$ of $8^{\mathrm{OAc}}$ in MeCN.

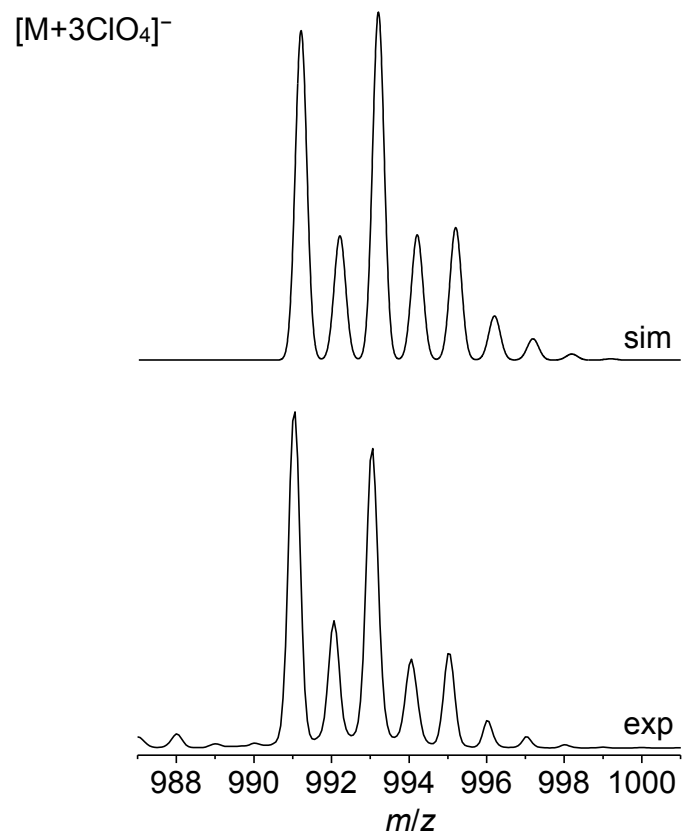

Figure A.45: ESI(-)-MS of $8^{\circ \mathrm{Ac}}$ in MeCN. Isotopic patterns of the simulated (top) and experimental (bottom) signal corresponding to $\left[\mathrm{M}+3 \mathrm{ClO}_{4}\right]^{-}(\mathrm{m} / z=993.1)$. 


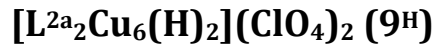

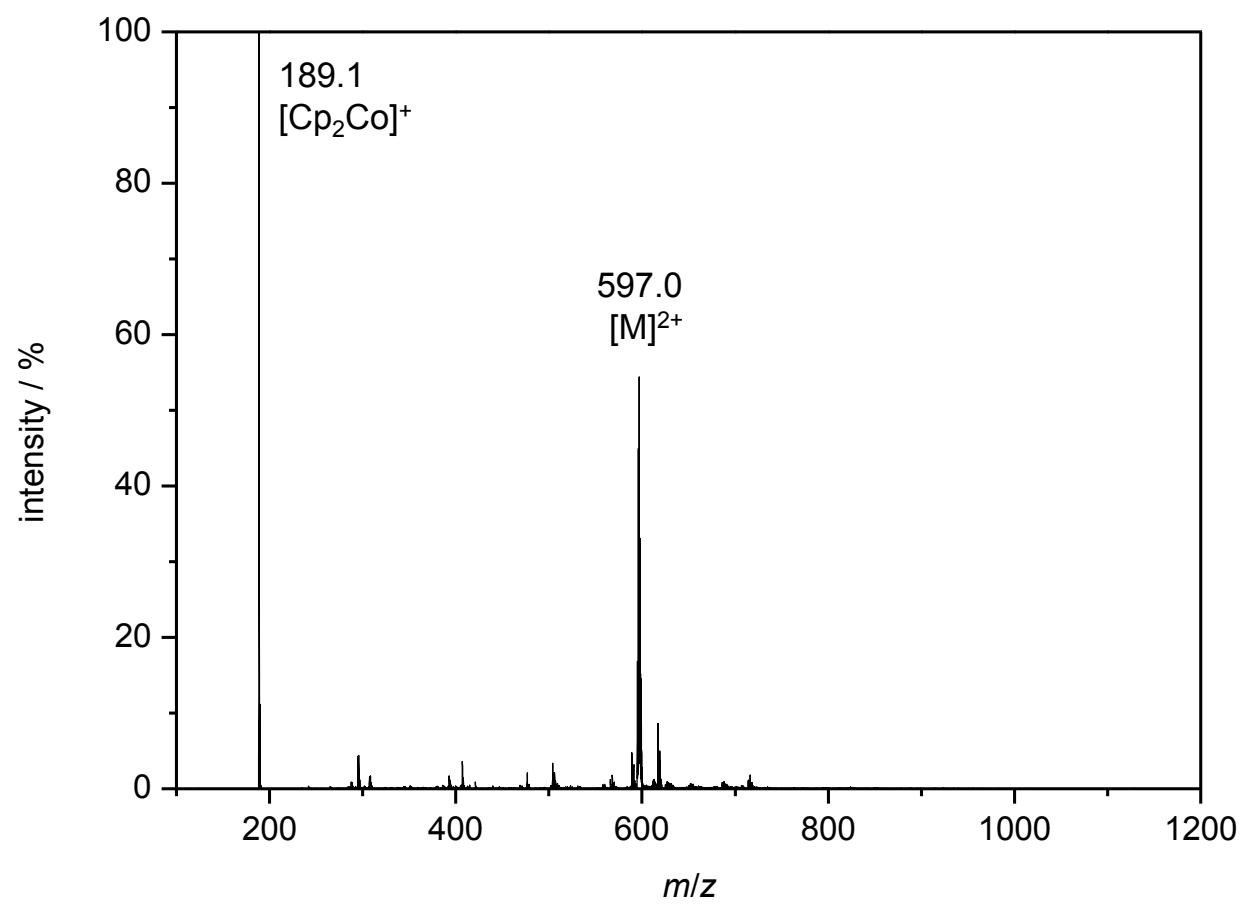

Figure A.46: $\mathrm{ESI}(+)-\mathrm{MS}$ of $\mathbf{9 H}$ in MeCN under inert conditions.
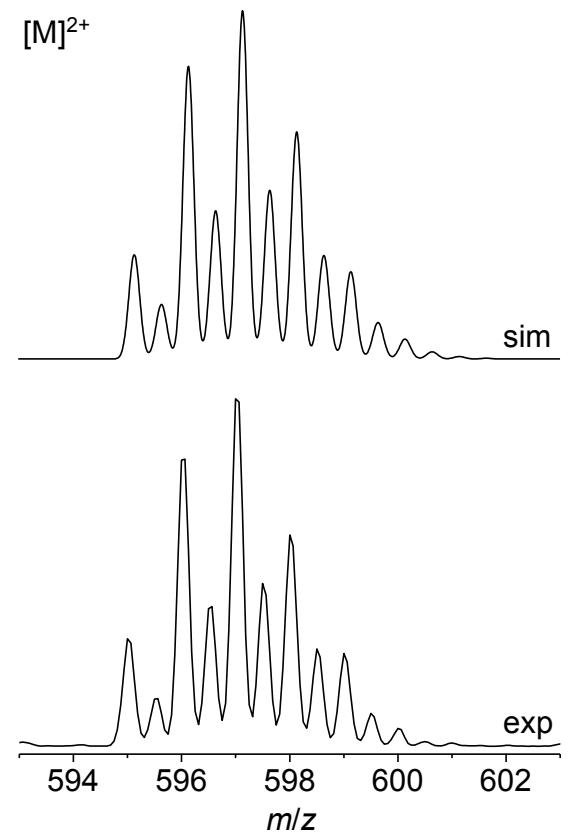

Figure A.47: ESI(+)-MS of $\mathbf{9 H}^{\mathbf{H}}$ in MeCN under inert conditions. Isotopic patterns of the simulated (top) and experimental (bottom) signal corresponding to $[\mathrm{M}]^{2+}(\mathrm{m} / \mathrm{z}=597.0)$. 


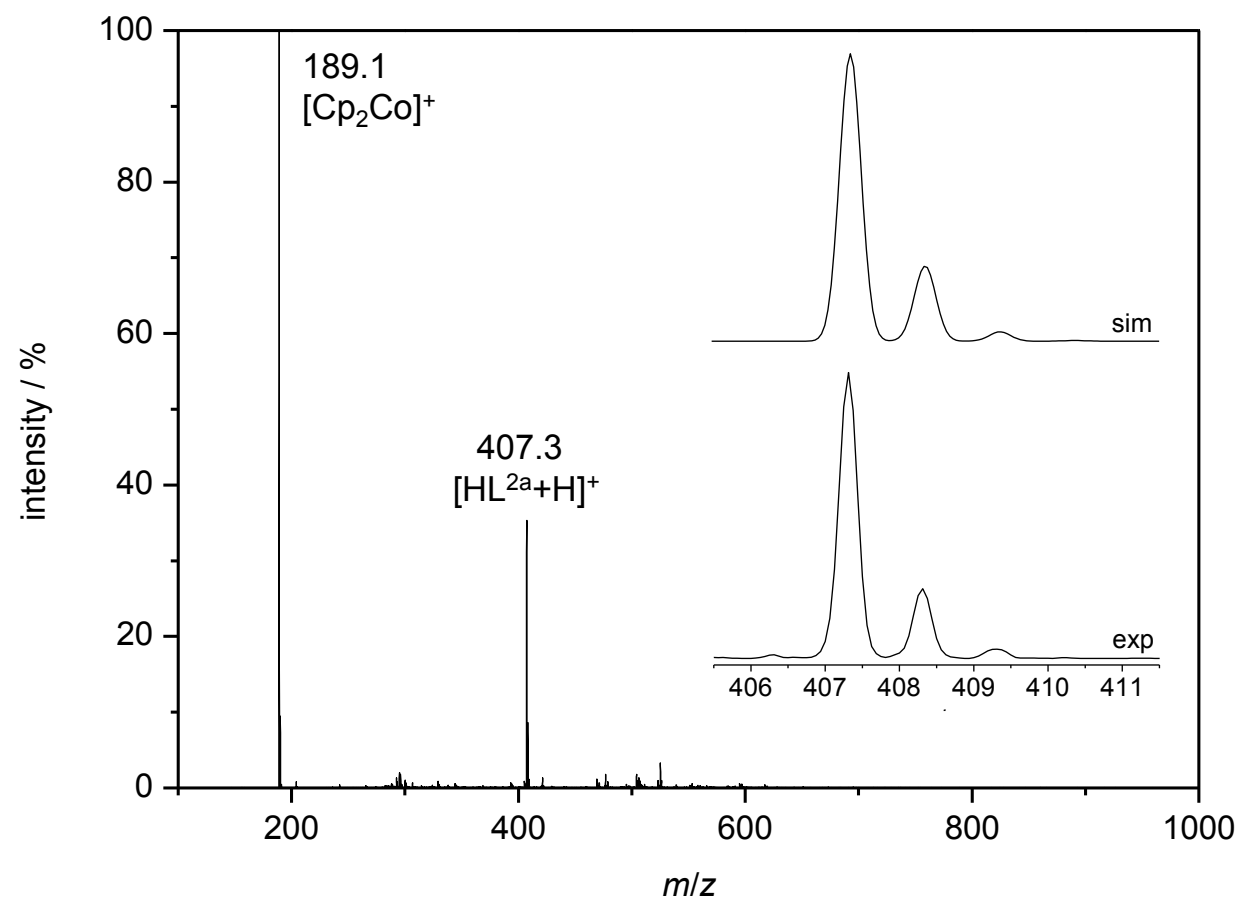

Figure A.48: ESI(+)-MS of the residue after crystallization of $\mathbf{9 H}$ in MeCN. The inset depicts the simulated (top) and experimental (bottom) signal corresponding to $\left[\mathrm{HL}^{2 \mathrm{a}}+\mathrm{H}\right]^{+}(m / z=407.3)$. The mass spectrum shows that free ligand is released during the synthesis.

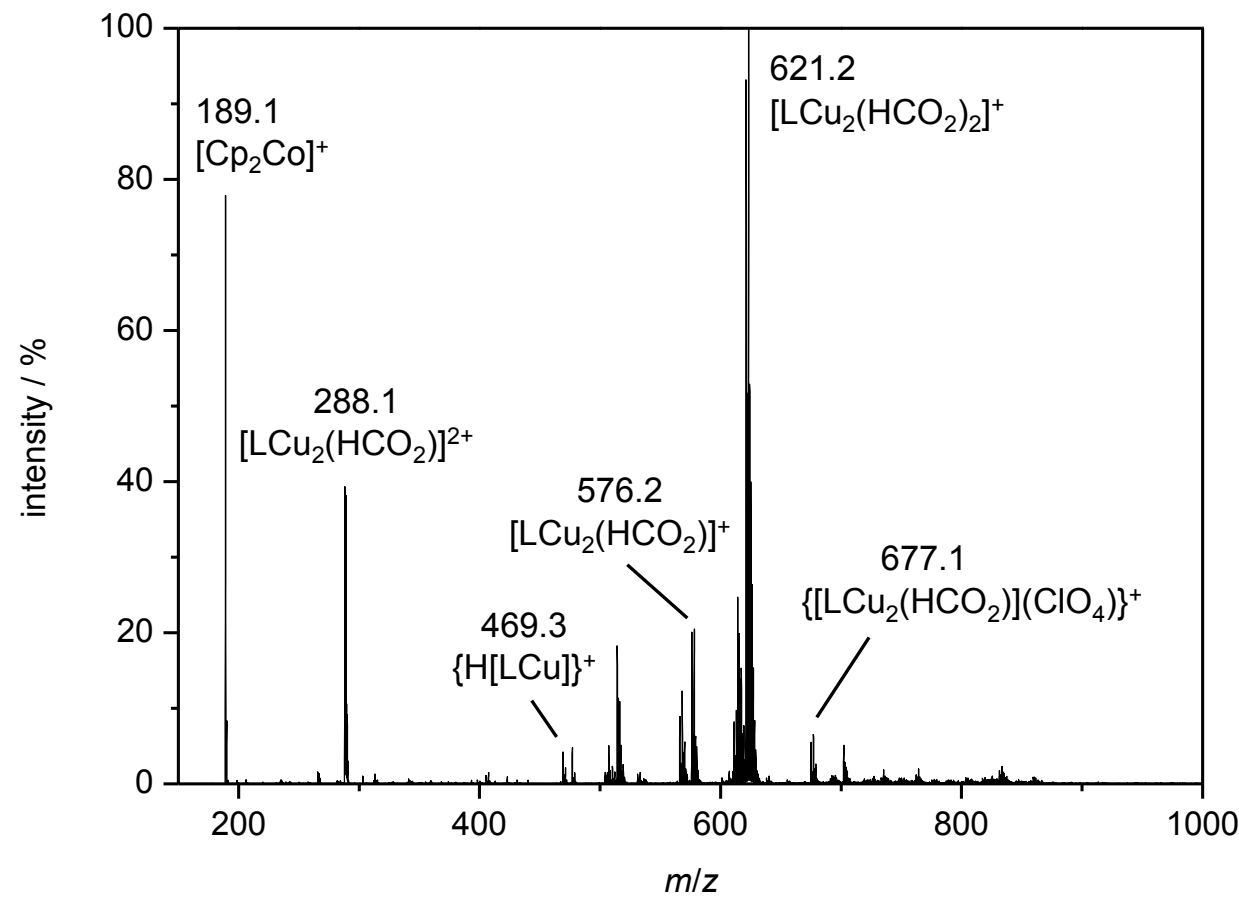

Figure A.49: ESI(+)-MS in of $\mathbf{9 H}^{\mathrm{H}}$ in the presence of formic acid (26 eq) and $\mathrm{NEt}_{3}$ (10 eq) after heating for 4 hours at $65{ }^{\circ} \mathrm{C}$ in $\mathrm{MeCN}$ under inert conditions. The mass spectrum shows various dinuclear and also mononuclear copper species ( $\mathrm{Cu}$ in both oxidation states $+\mathrm{I}$ and $+\mathrm{II})$. 


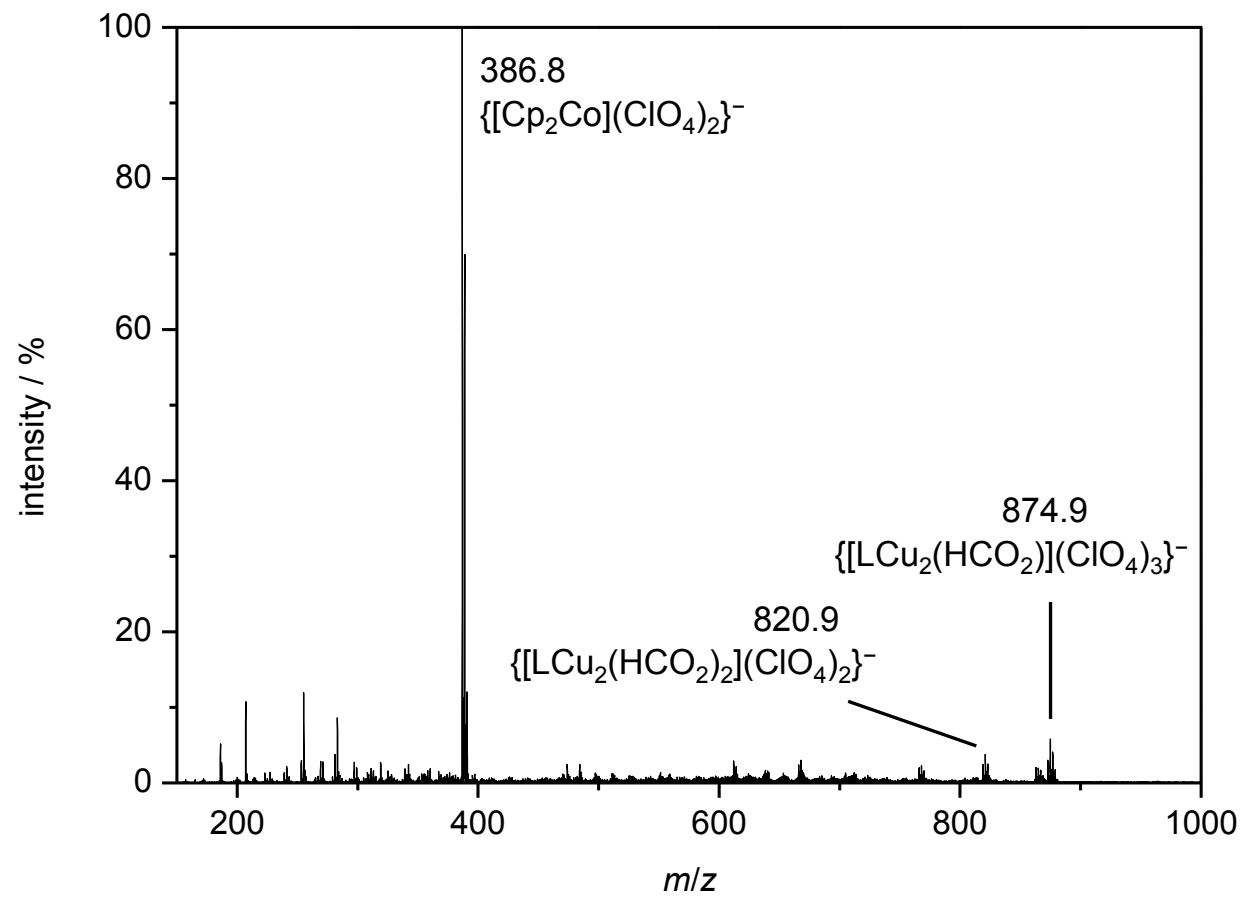

FigureA.50: ESI(-)-MS in of $9^{\mathrm{H}}$ in the presence of formic acid (26 eq) and NEt3 $(10 \mathrm{eq})$ after heating for 4 hours at $65^{\circ} \mathrm{C}$ in $\mathrm{MeCN}$ under inert conditions. Next to $\left\{\left[\mathrm{Cp}_{2} \mathrm{Co}\right]\left(\mathrm{ClO}_{4}\right)_{2}\right\}^{-}$, the mass spectrum shows dinuclear copper species ( $\mathrm{Cu}$ in the oxidation state $+\mathrm{II}$ ). 
$\left[\mathrm{L}^{2 \mathrm{a}_{2}} \mathrm{Cu}_{6}(\mathrm{D})_{2}\right]\left(\mathrm{ClO}_{4}\right)_{2}\left(9^{\mathrm{D}}\right)$

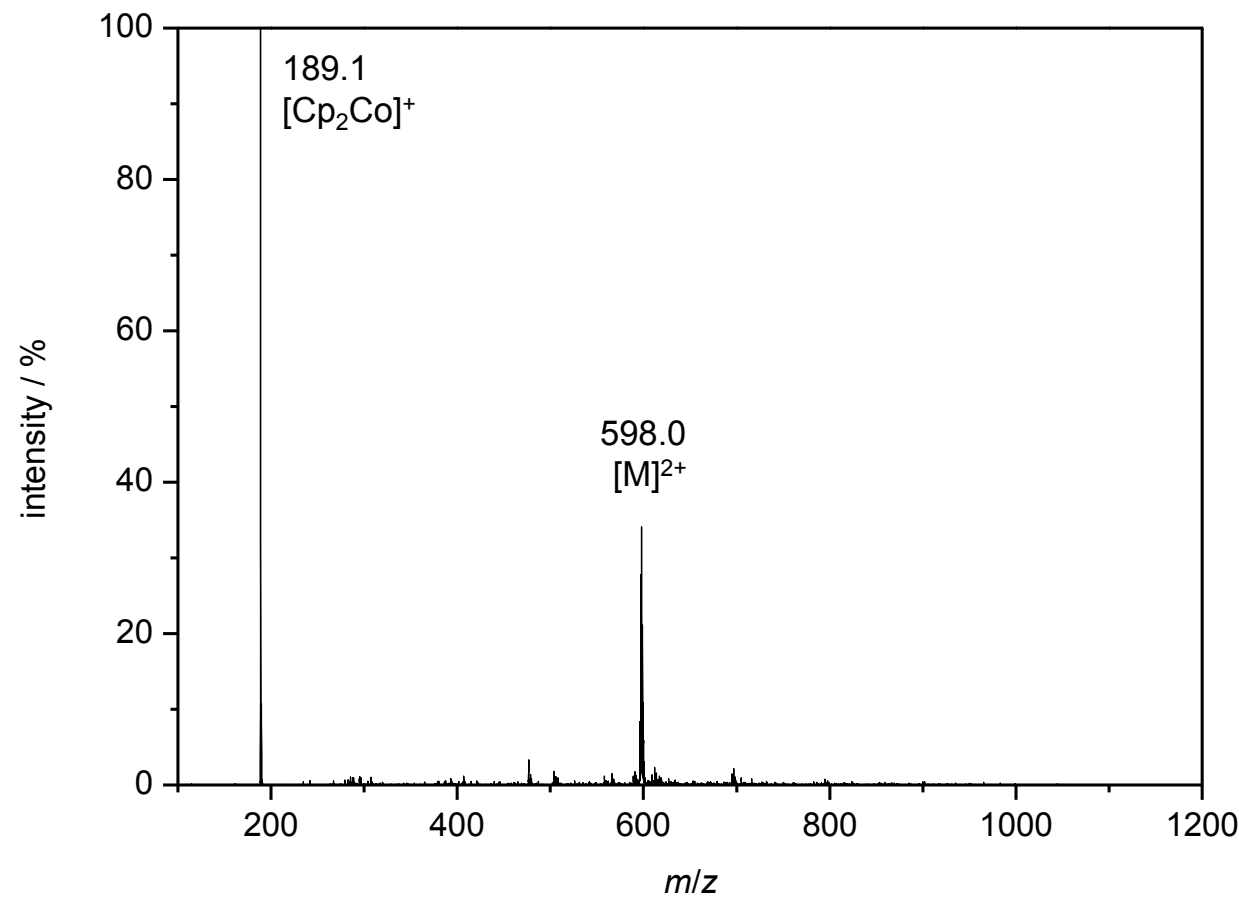

Figure A.51: $\mathrm{ESI}(+)-\mathrm{MS}$ of 9D in MeCN under inert conditions.

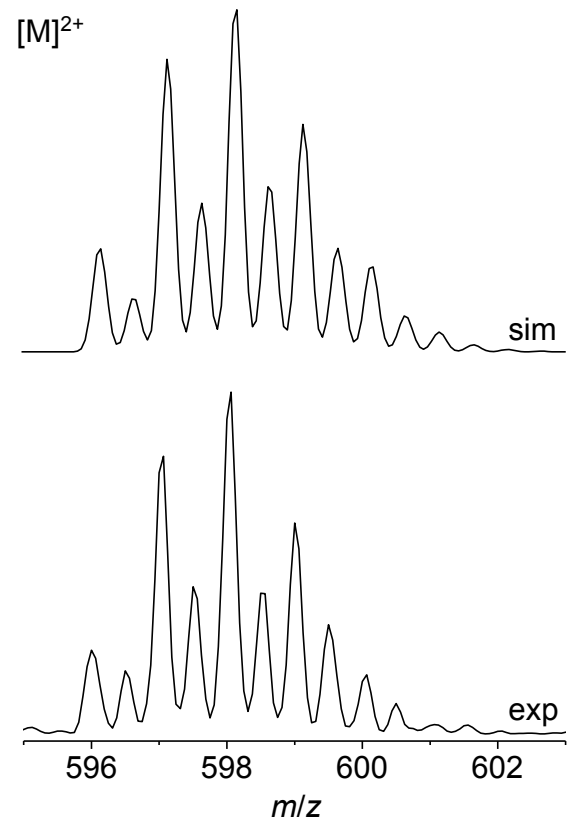

Figure A.52: ESI(+)-MS of 9D in MeCN under inert conditions. Isotopic patterns of the simulated (top) and experimental (bottom) signal corresponding to $[\mathrm{M}]^{2+}(\mathrm{m} / \mathrm{z}=598.0)$. 


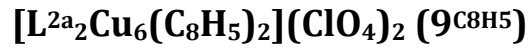

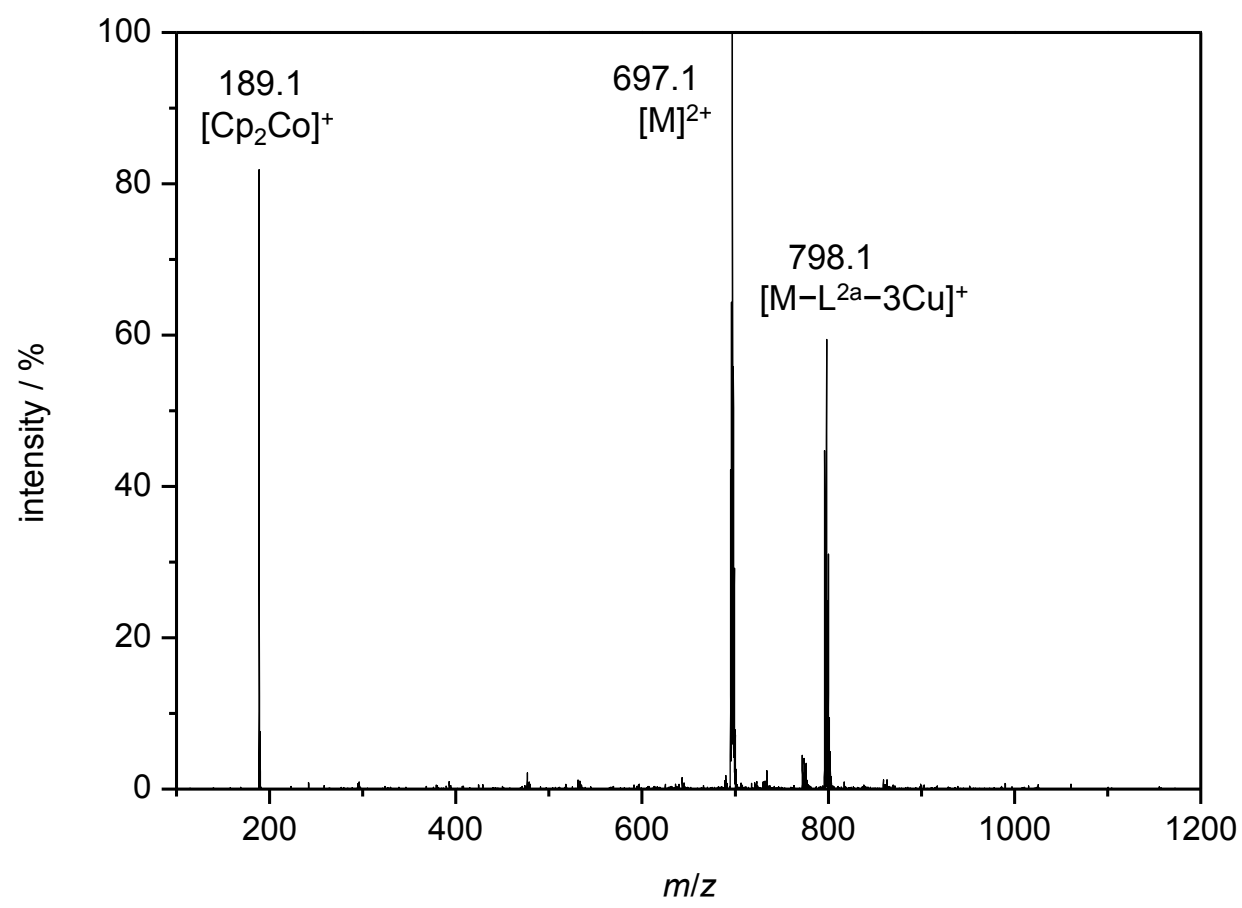

Figure A.53: ESI(+)-MS of $\mathbf{9}$ (8)5 in MeCN under inert conditions.
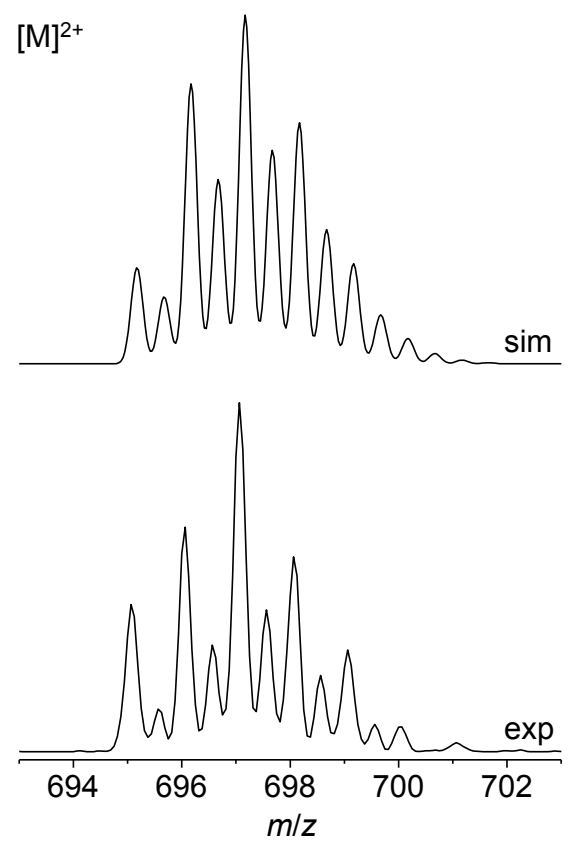

Figure A.54: ESI(+)-MS of 9C8H5 in MeCN under inert conditions. Isotopic patterns of the simulated (top) and experimental (bottom) signal corresponding to $[\mathrm{M}]^{2+}(\mathrm{m} / \mathrm{z}=697.1)$. 


\section{NMR Spectroscopy}

\section{$\left[\mathrm{L}^{1} \mathrm{Ru}_{2}(\mathrm{dmso})_{2}(\mathrm{Cl})\left(\mathrm{H}_{2} \mathrm{O}\right)(\mu-\mathrm{dmso})\right](1)$}

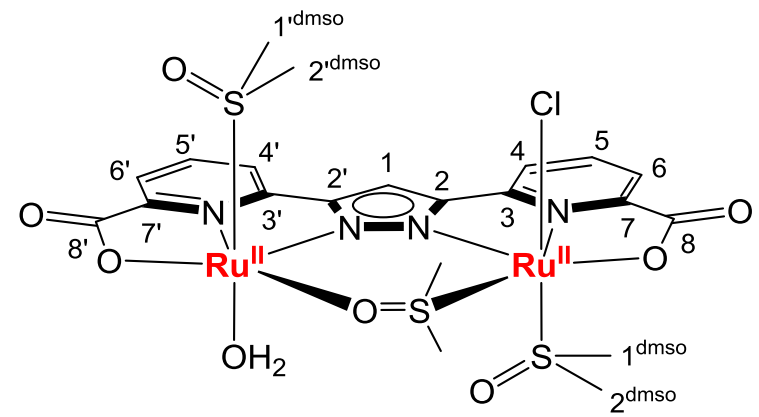

1

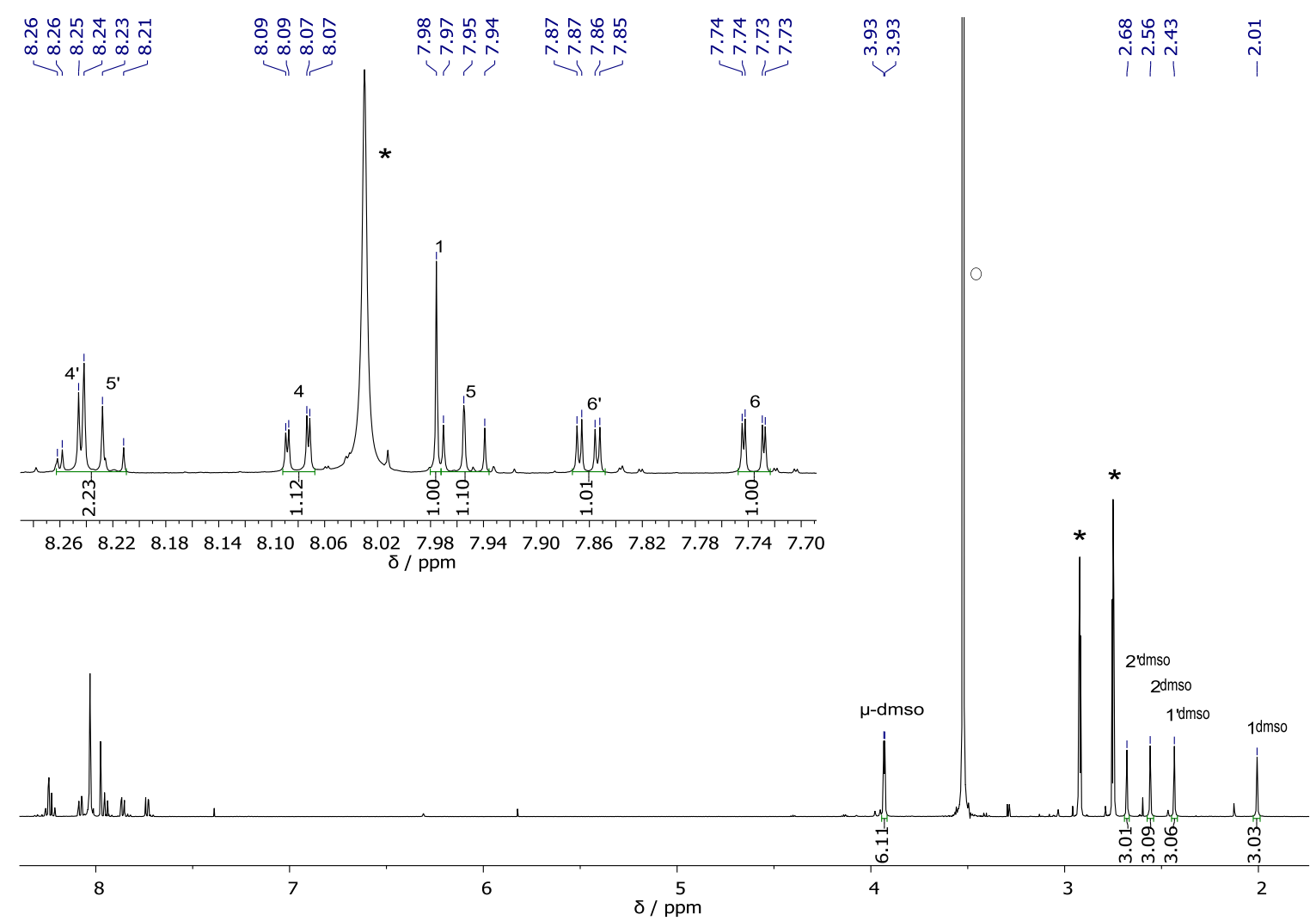

Figure A.55: ${ }^{1} \mathrm{H}-\mathrm{NMR}$ spectrum $(500 \mathrm{MHz})$ of 1 in DMF- $d_{7}$. The inset depicts an expansion of the aromatic region (7.7-8.3 ppm). The peaks are assigned according to the labeling scheme above. The asterisks denote the residual solvent peaks and the circle points out $\mathrm{H}_{2} \mathrm{O}$. Please note, the distinction between prime and non-prime is not possible and is merely done for simplification. 


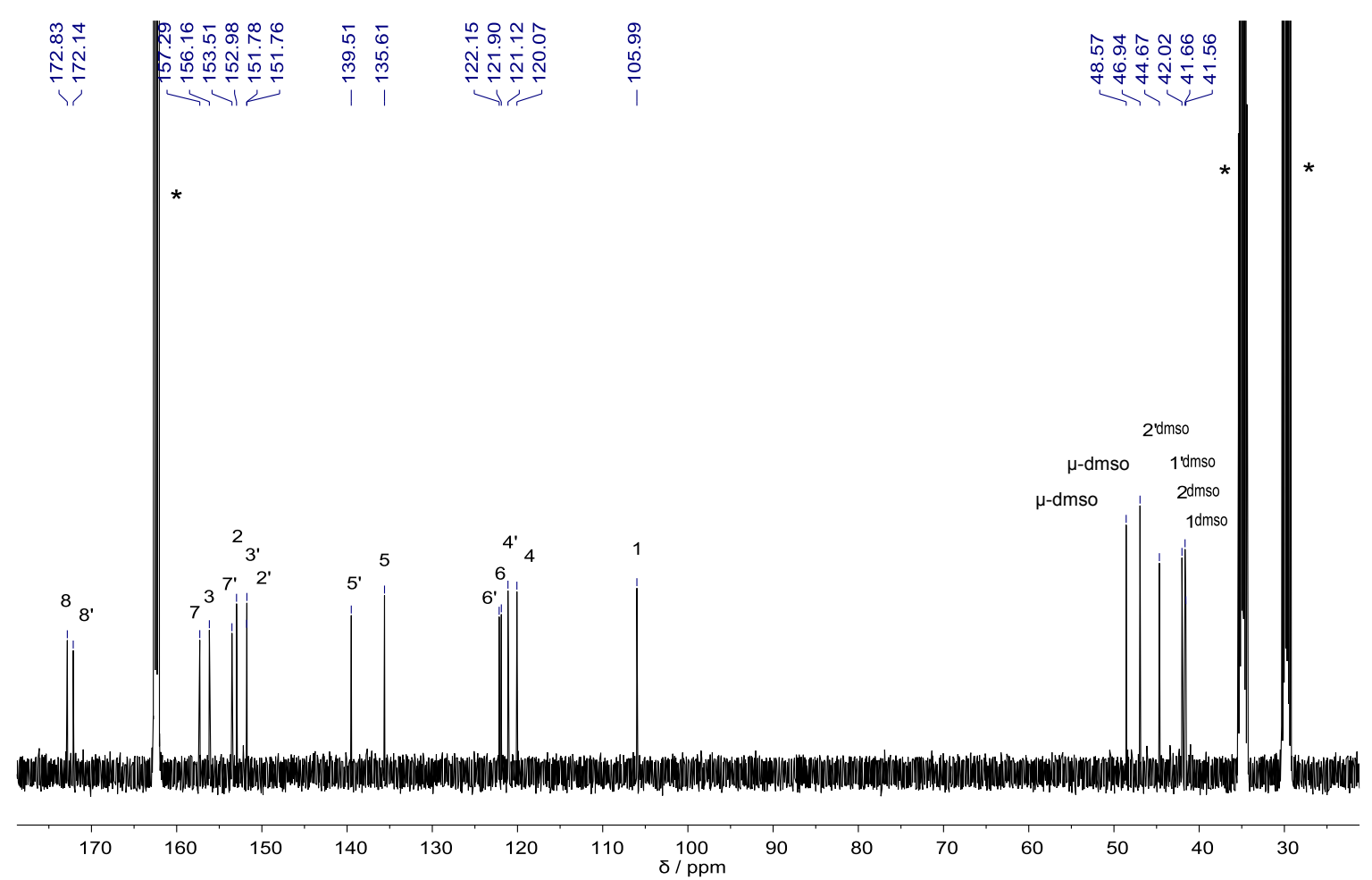

Figure A.56: ${ }^{13} \mathrm{C}\left\{{ }^{1} \mathrm{H}\right\}-\mathrm{NMR}$ spectrum $(126 \mathrm{MHz})$ of $\mathbf{1}$ in DMF- $d_{7}$. The peaks are assigned according to the labeling scheme above. The asterisks denote the residual solvent peaks. Please note, the distinction between prime and non-prime is not possible and is merely done for simplification. 

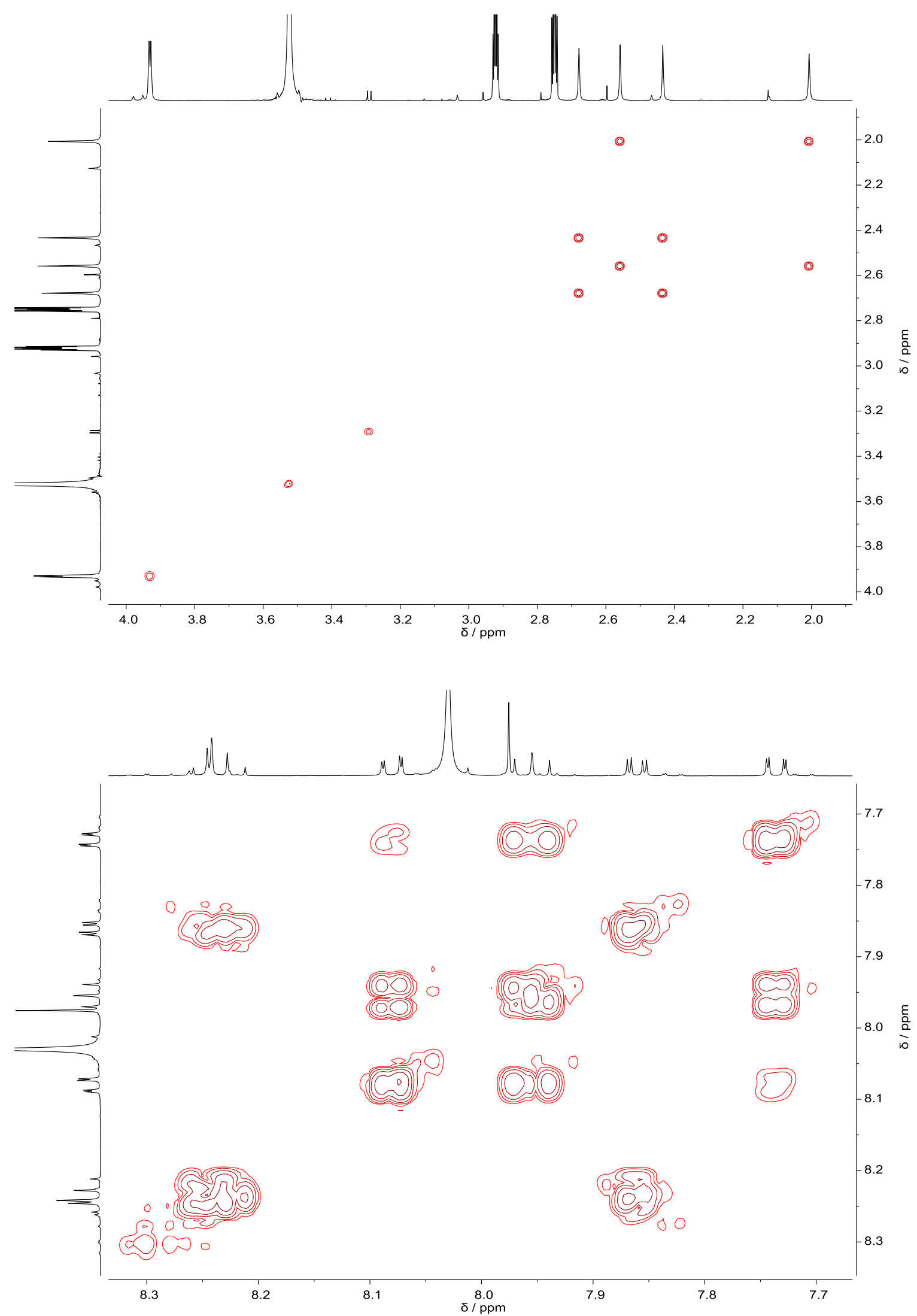

Figure A.57: ${ }^{1} \mathrm{H}-1 \mathrm{H}-\mathrm{COSY}$ spectrum $(500 \mathrm{MHz})$ of 1 in DMF- $d 7$. Top: aliphatic region (1.9-4.0 ppm), bottom: aromatic region (7.7-8.3 ppm). 

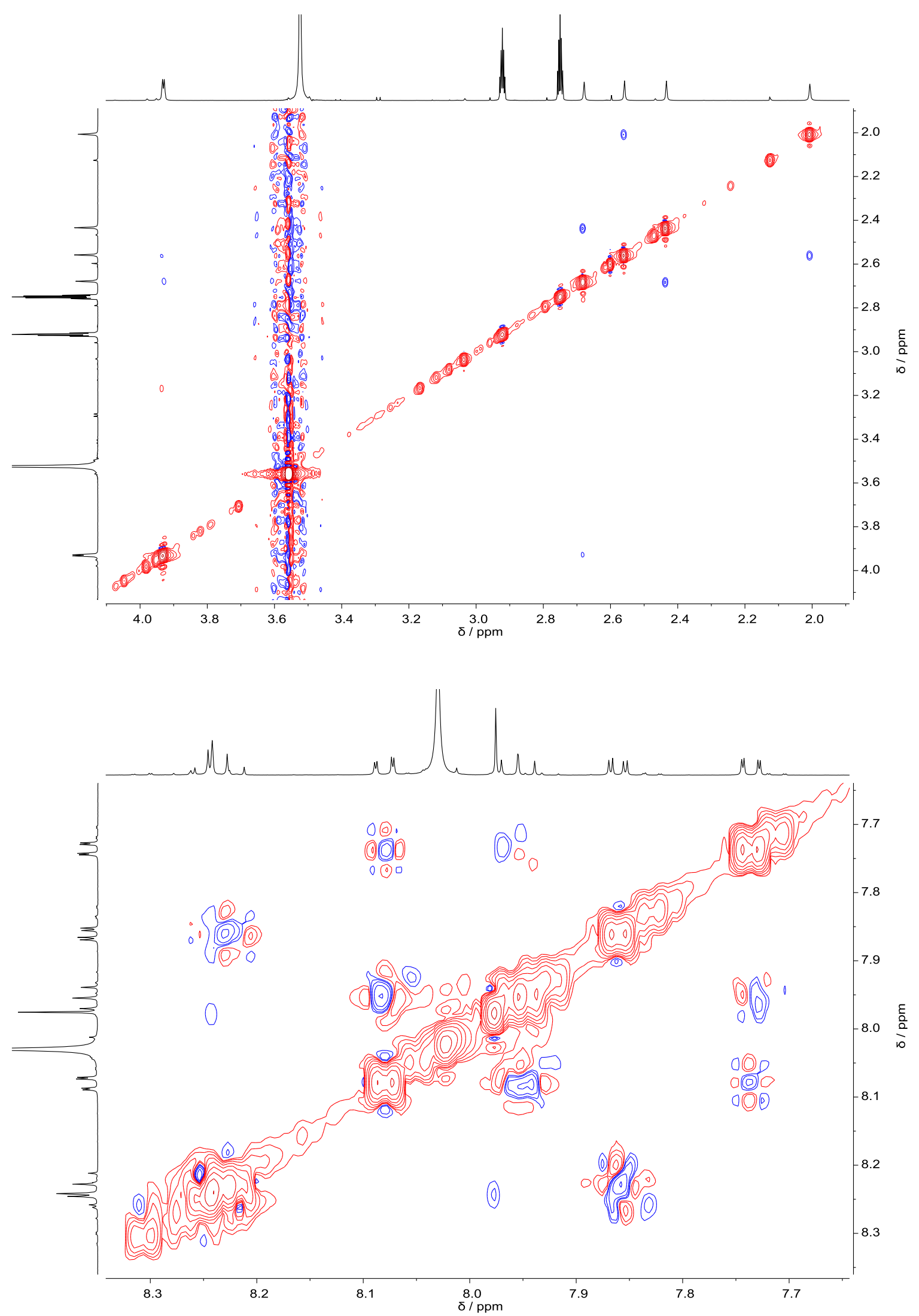

Figure A.58: ${ }^{1} \mathrm{H}-{ }^{1} \mathrm{H}-\mathrm{NOESY}$ spectrum $(500 \mathrm{MHz})$ of 1 in DMF-d7. Top: aliphatic region (1.9-4.1 ppm), bottom: aromatic region (7.7-8.3 ppm). 

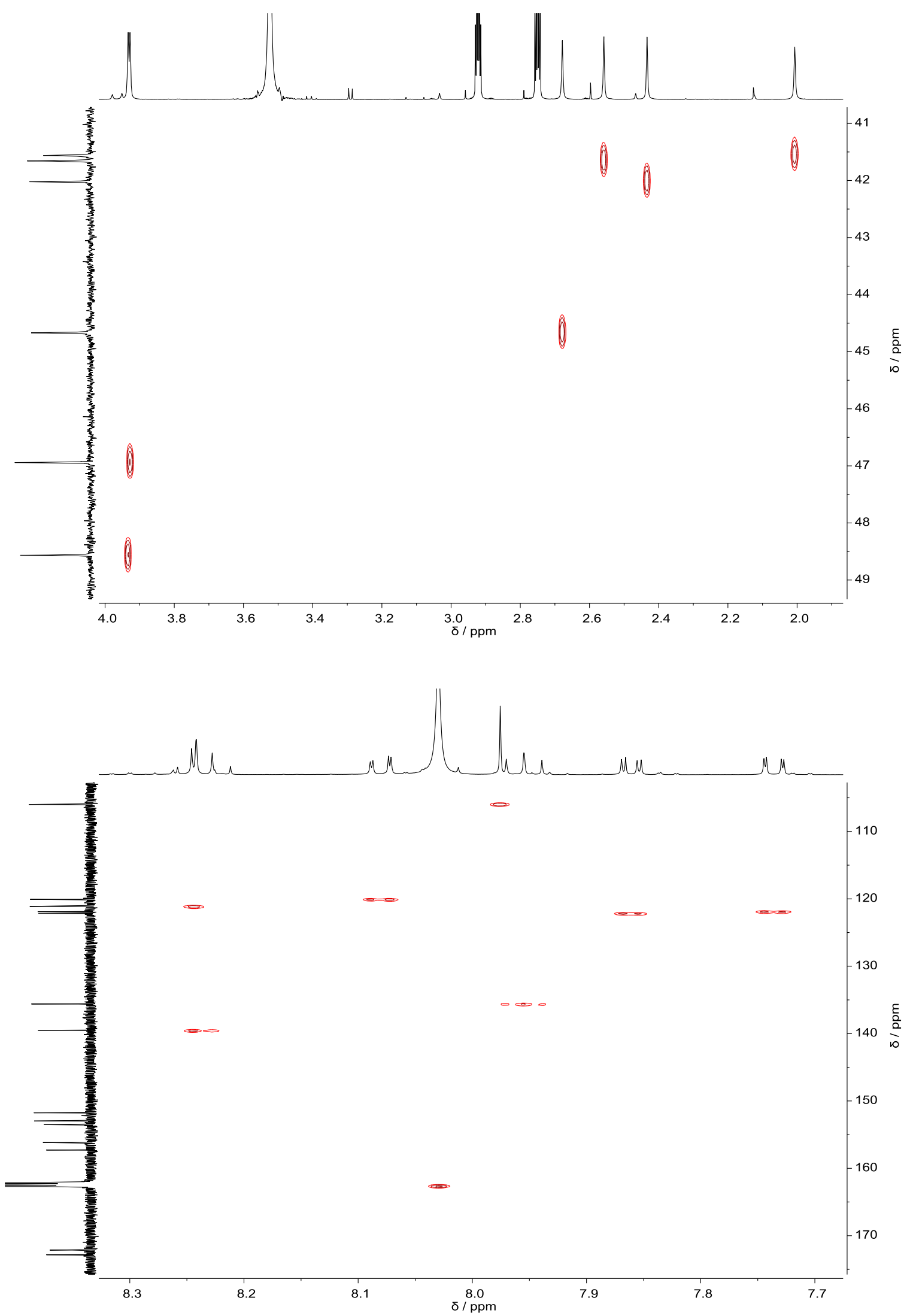

Figure A.59: ${ }^{1} \mathrm{H}-{ }^{13} \mathrm{C}\left\{{ }^{1} \mathrm{H}\right\}$-HSQC spectrum of 1 in DMF- $d 7$. Top: aliphatic region $(1.9-4.0 \mathrm{ppm})$, bottom: aromatic region (7.7-8.3 ppm). 

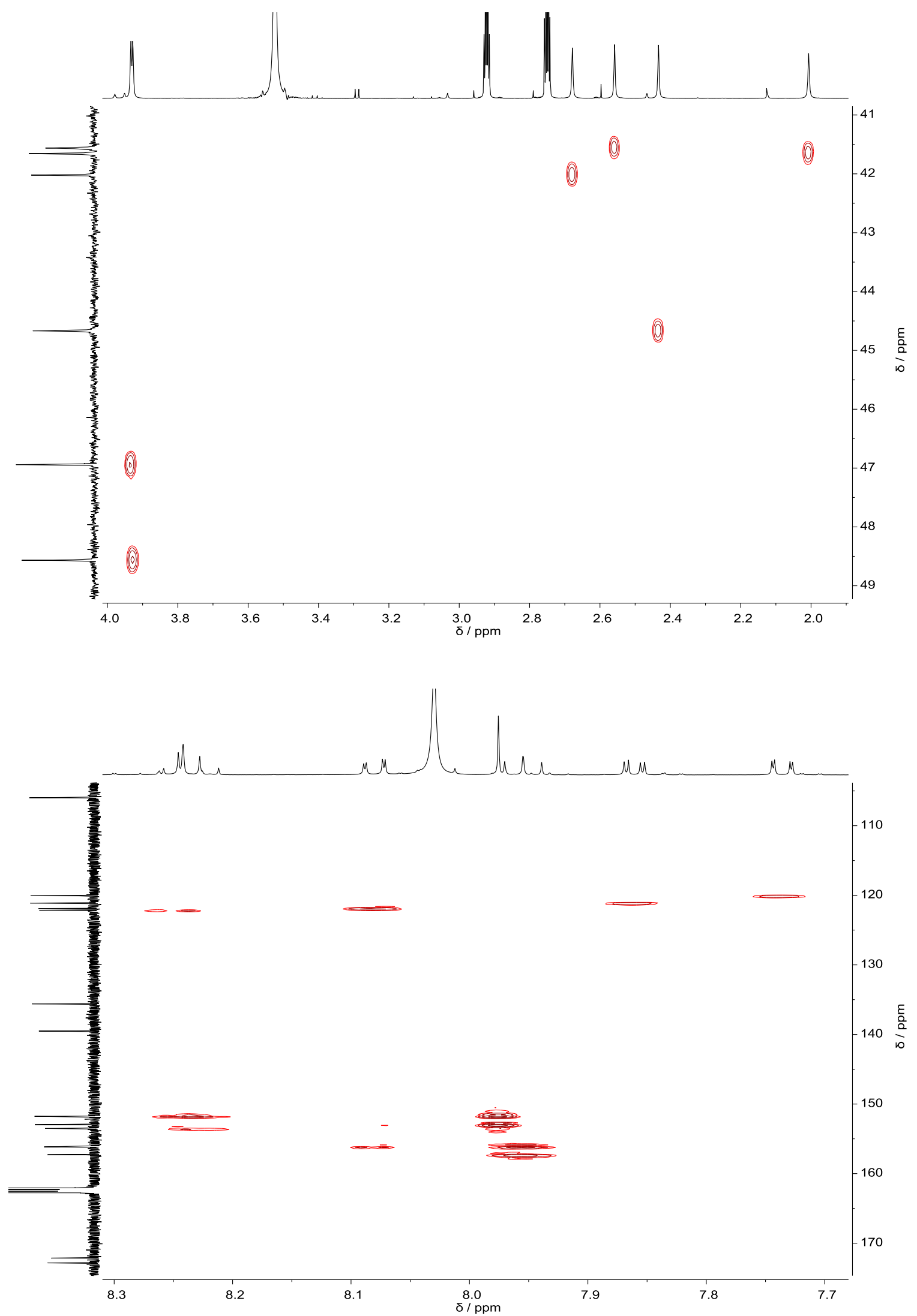

Figure A.60: ${ }^{1} \mathrm{H}-13 \mathrm{C}\left\{{ }^{1} \mathrm{H}\right\}-\mathrm{HMBC}$ spectrum of 1 in DMF- $d 7$. Top: aliphatic region (1.9-4.0 ppm), bottom: aromatic region $(7.7-8.3 \mathrm{ppm})$. 


\section{$\mathrm{Na}_{3}\left[\mathrm{~L}^{1} \mathrm{Ru}_{2}\left(\mathrm{pyCO}_{2}\right)_{4}(\mu-\mathrm{dmso})\right]\left(2^{\mathrm{dmso}}\right)$}
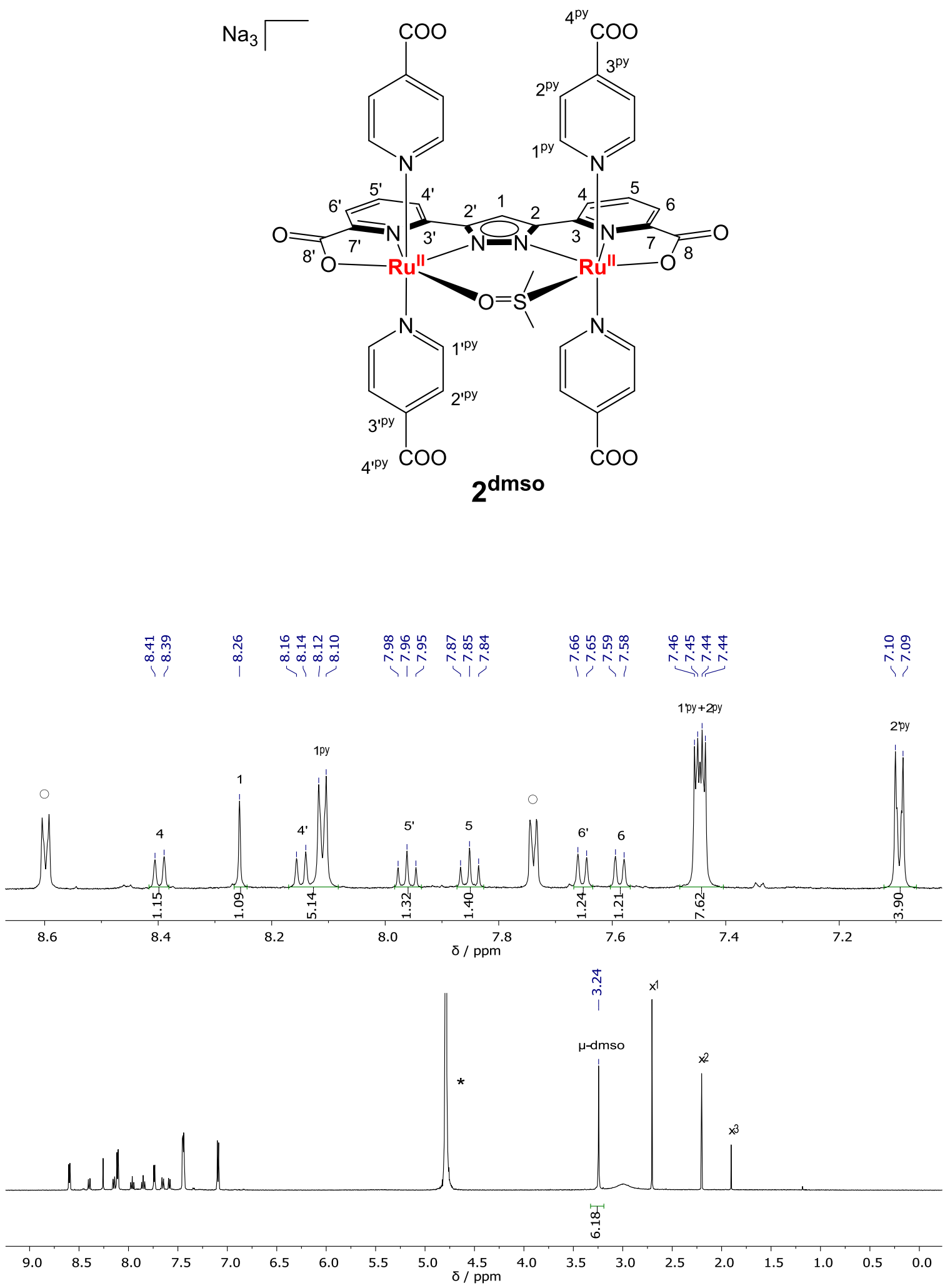

Figure A.61: ${ }^{1} \mathrm{H}-\mathrm{NMR}$ spectrum $(500 \mathrm{MHz})$ of $\mathbf{2}^{\mathrm{dmso}}$ in $\mathrm{D}_{2} \mathrm{O}$. The inset depicts an expansion of the aromatic region (7.1-8.6 ppm). The peaks are assigned according to the labeling scheme above. The asterisk denotes the residual solvent peak and the circles point out an additional pyCO $_{2}-$ molecule. $\mathrm{x}^{1}, \mathrm{x}^{2}$ and $\mathrm{x}^{3}$ mark free DMSO, acetone and acetate, respectively. Please note, the distinction between prime and non-prime for the $\left(\mathrm{L}^{1}\right)^{3-}$ ligand is not possible and is merely done for simplification. 


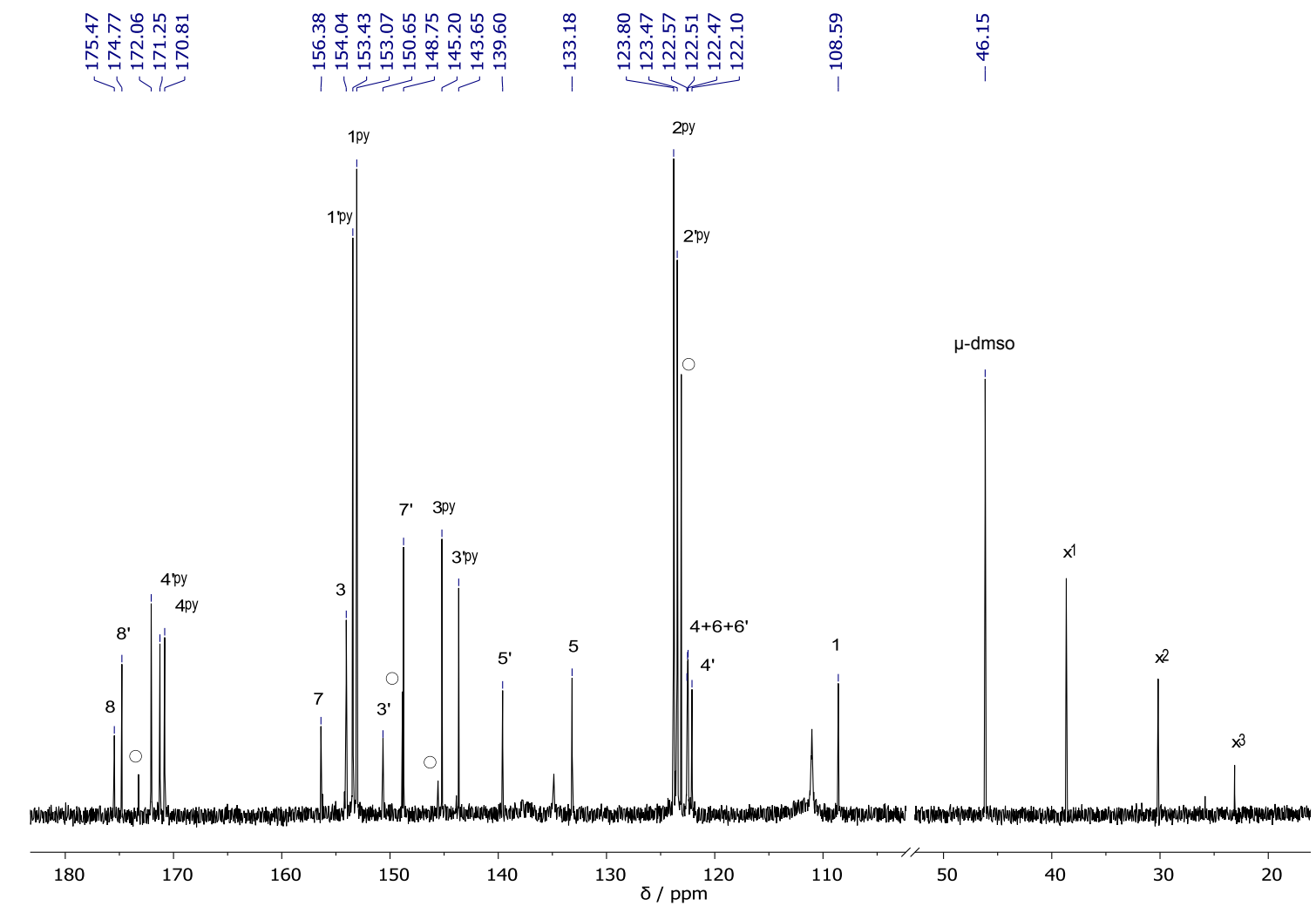

Figure A.62: ${ }^{13} \mathrm{C}\left\{{ }^{1} \mathrm{H}\right\}$-NMR spectrum $(126 \mathrm{MHz})$ of $\mathbf{2}^{\mathbf{d m s o}}$ in $\mathrm{D}_{2} \mathrm{O}$. The peaks are assigned according to the labeling scheme above. The circles point out an additional $\mathrm{pyCO}_{2}-$ molecule. $\mathrm{x}^{1}, \mathrm{x}^{2}$ and $\mathrm{x}^{3}$ mark free DMSO, acetone and acetate, respectively. The peak at $172.06 \mathrm{ppm}$ could not be assigned. Please note, the distinction between prime and non-prime for the $\left(\mathrm{L}^{1}\right)^{3-}$ ligand is not possible and is merely done for simplification.

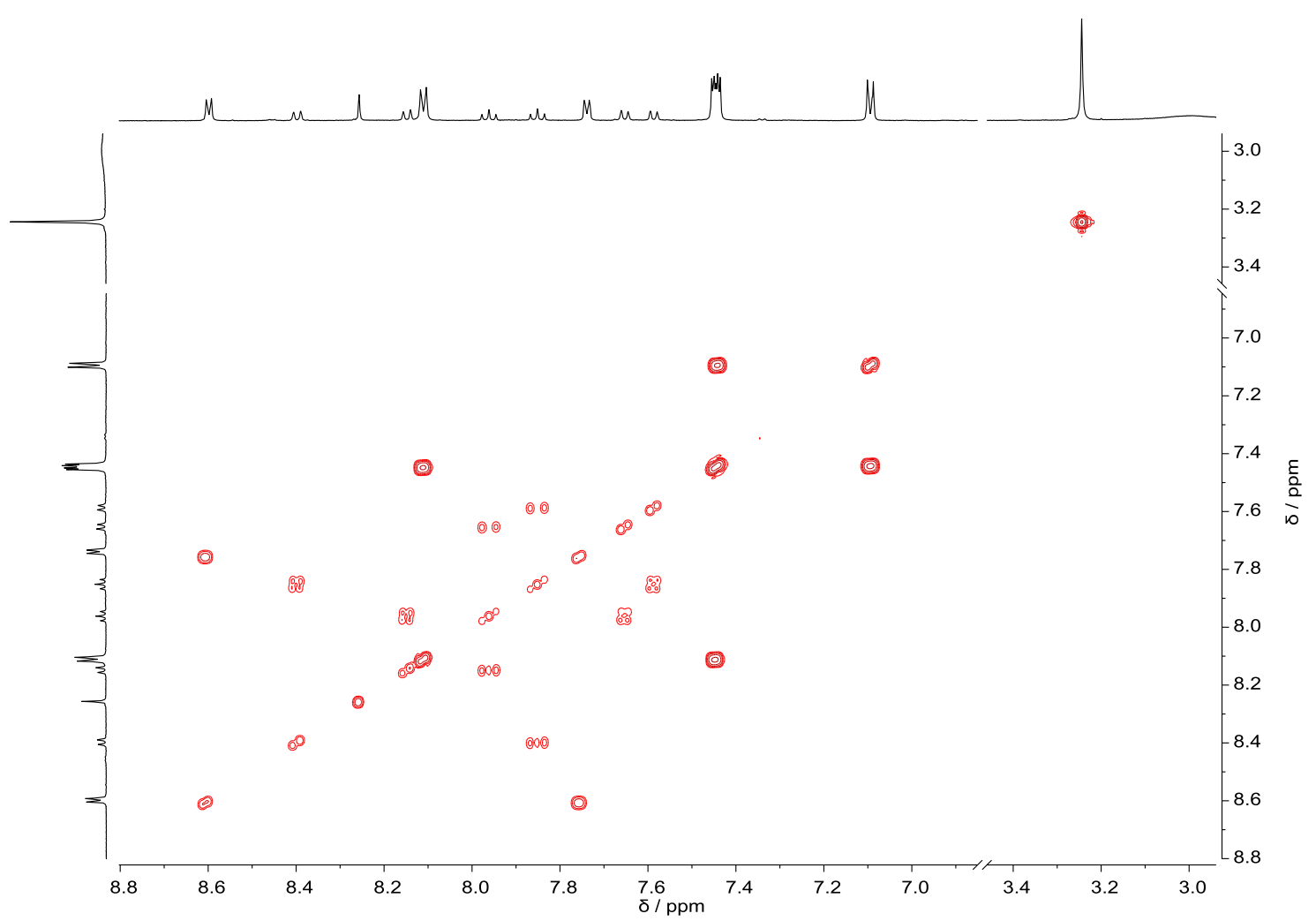

Figure A.63: ${ }^{1} \mathrm{H}-1 \mathrm{H}-\mathrm{COSY}$ spectrum $(500 \mathrm{MHz})$ of $\mathbf{2}^{\text {dmso }}$ in $\mathrm{D}_{2} \mathrm{O}$. Depicted is the full region with complex signals (3.0-8.8 ppm). 


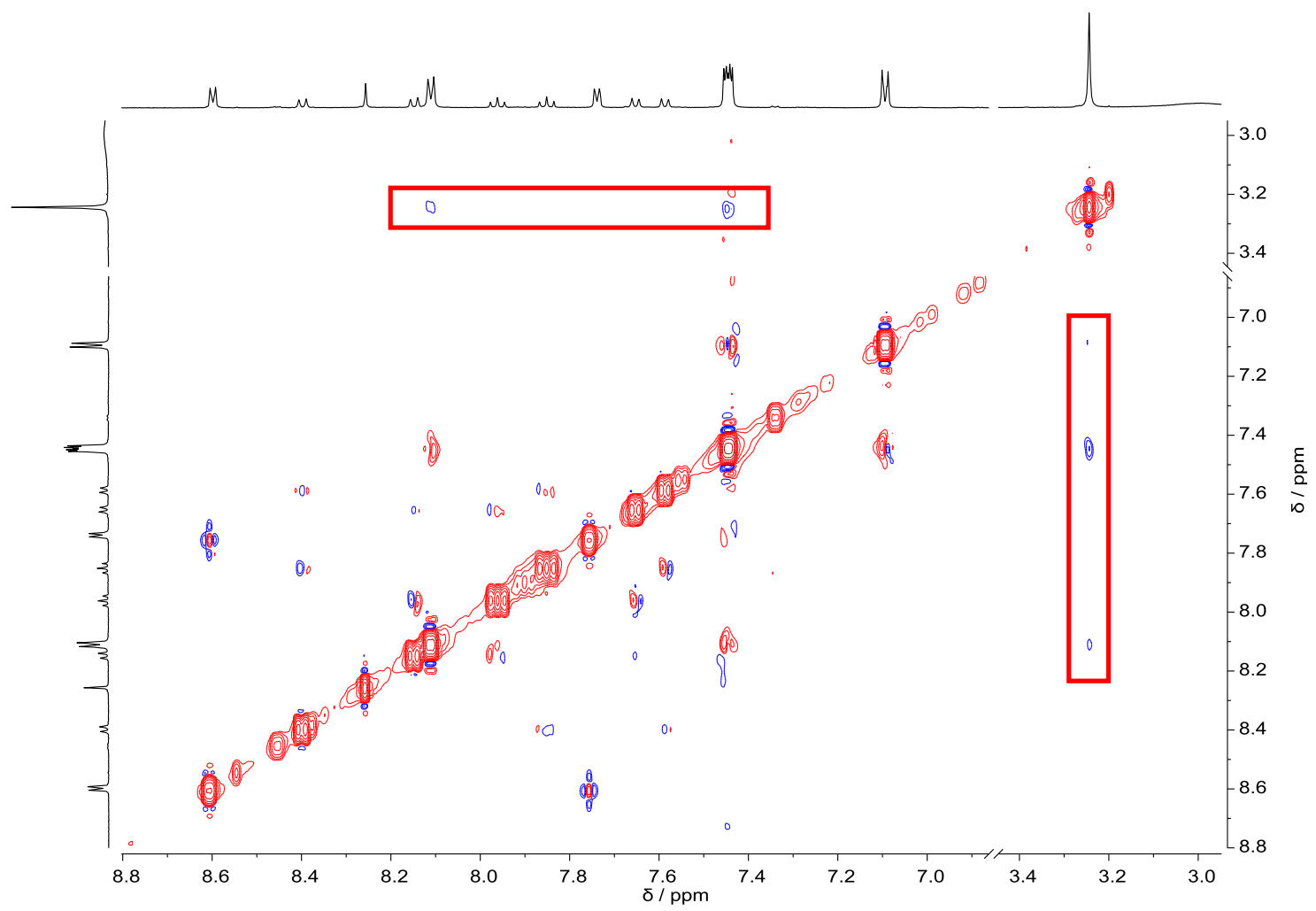

Figure A.64: ${ }^{1} \mathrm{H}-1 \mathrm{H}-\mathrm{NOESY}$ spectrum $(500 \mathrm{MHz})$ of $\mathbf{2}^{\text {dmso }}$ in $\mathrm{D}_{2} \mathrm{O}$. Depicted is the full region with complex signals (3.0-8.8 ppm). The red rectangles show the correlation of the bridging DMSO with $\mathrm{pyCO}_{2}{ }^{-}$.

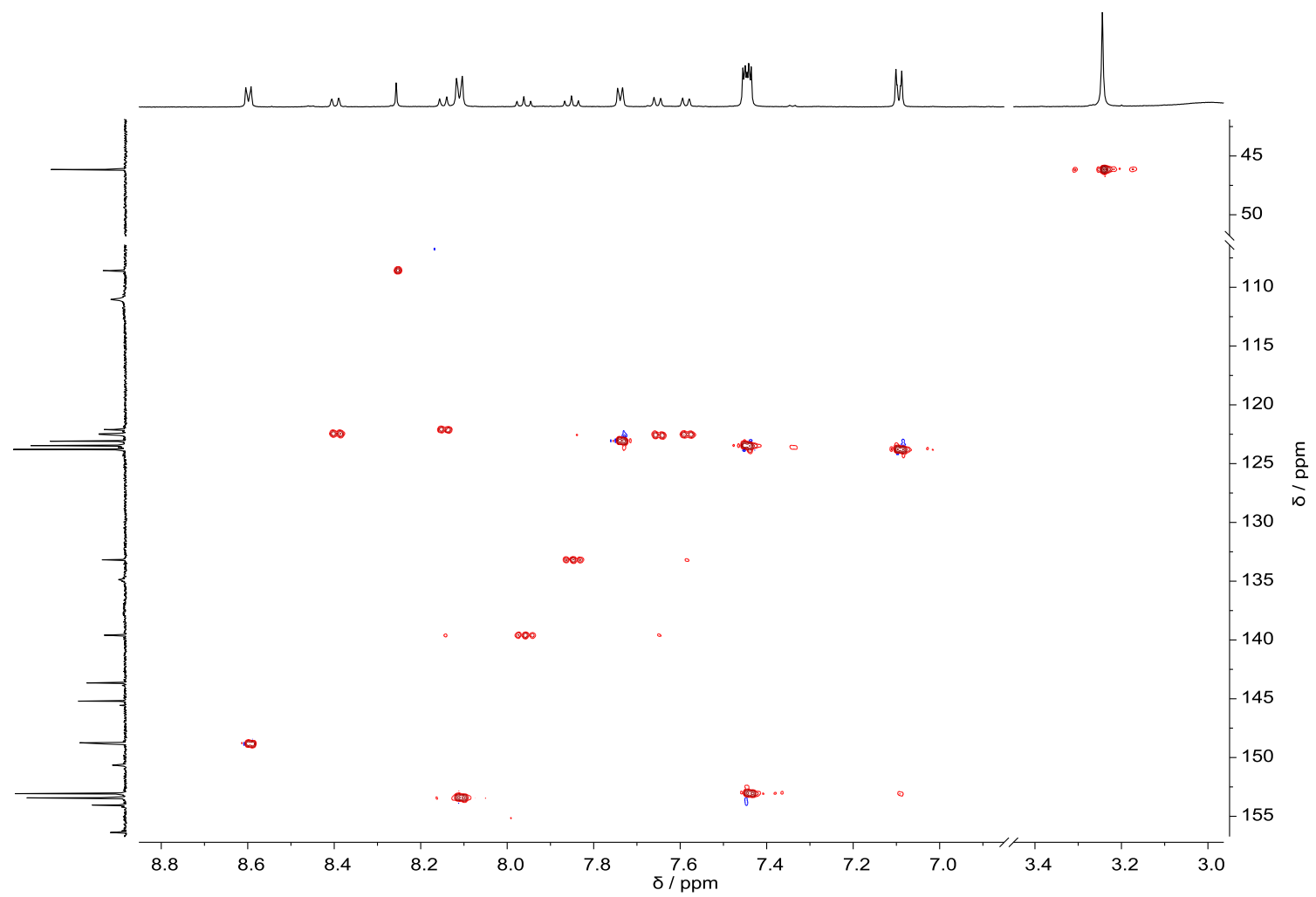

Figure A.65: ${ }^{1} \mathrm{H}^{-13} \mathrm{C}\left\{{ }^{1} \mathrm{H}\right\}-\mathrm{HSQC}$ spectrum of $\mathbf{2}^{\text {dmso }}$ in $\mathrm{D}_{2} \mathrm{O}$. Depicted is the full region with complex signals $(3.0-$ $8.8 \mathrm{ppm}$ ). 


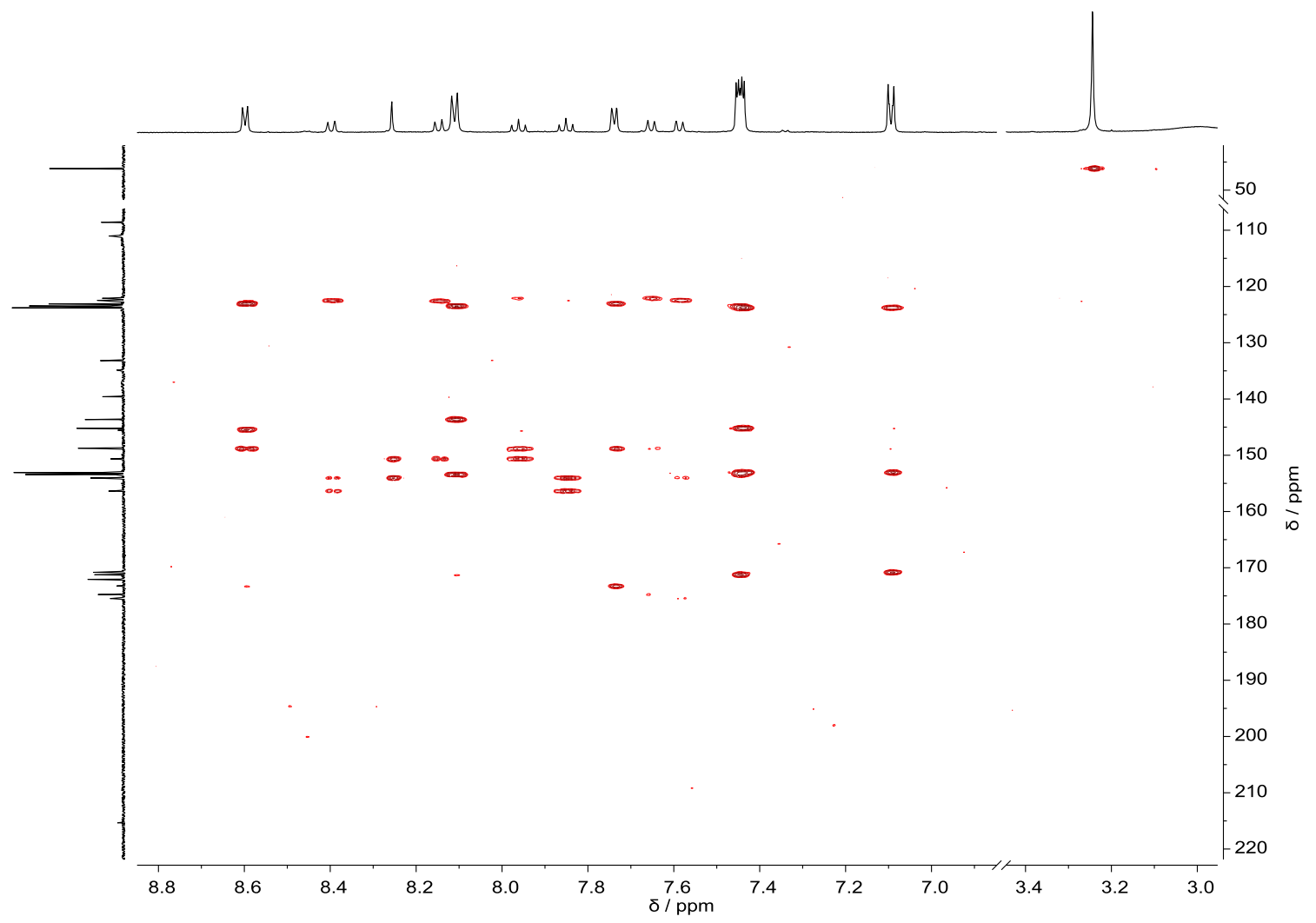

Figure A.66: ${ }^{1} \mathrm{H}-{ }^{13} \mathrm{C}\left\{{ }^{1} \mathrm{H}\right\}-\mathrm{HMBC}$ spectrum of $\mathbf{2}^{\text {dmso }}$ in $\mathrm{D}_{2} \mathrm{O}$. Depicted is the full region with complex signals (3.0$8.8 \mathrm{ppm})$.

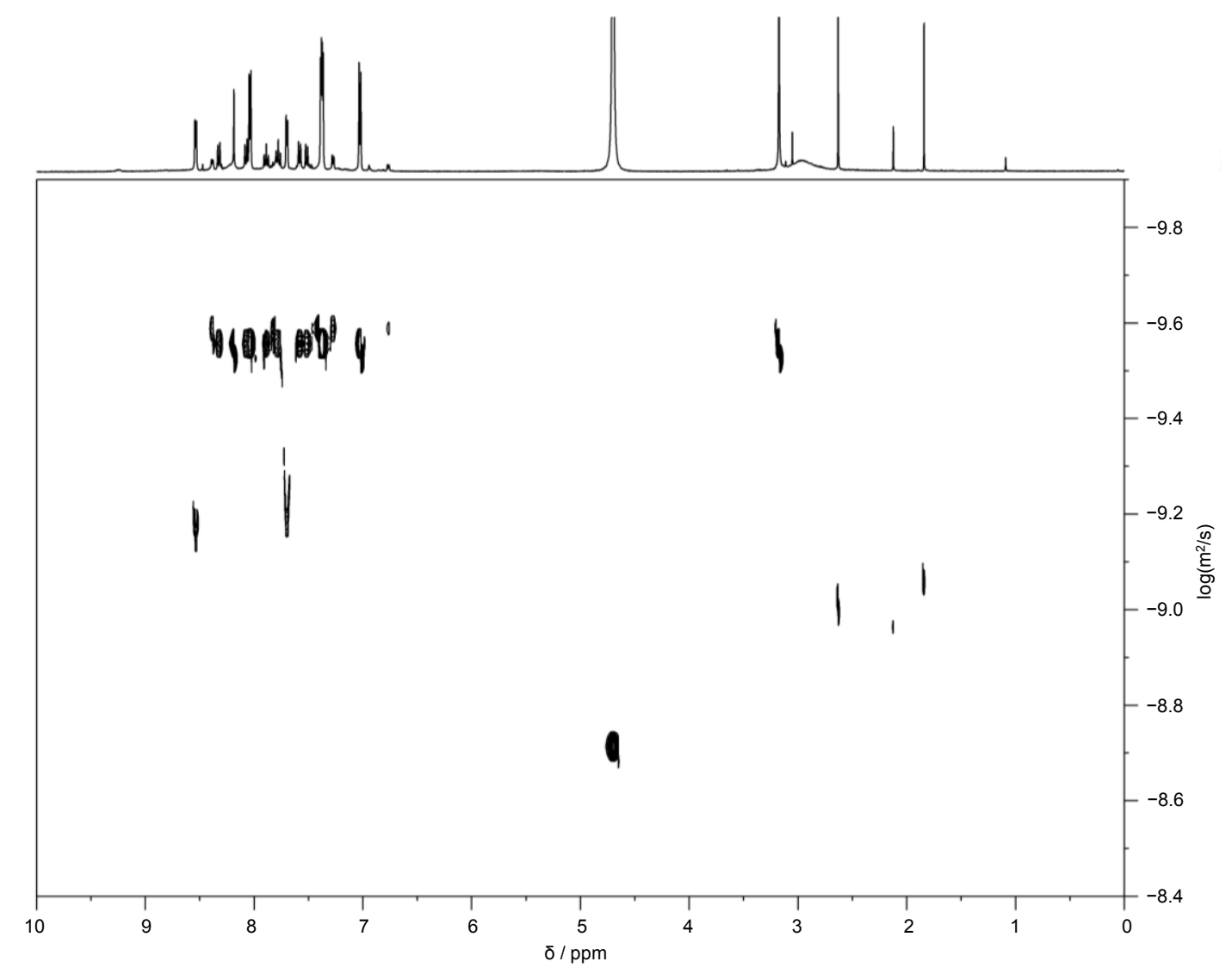

Figure A.67: DOSY spectrum of $2^{\text {dmso }}$ in $\mathrm{D}_{2} \mathrm{O}$. 


\section{$\mathrm{Na}_{3}\left[\mathrm{~L}^{1} \mathrm{Ru}_{2}\left(\mathrm{pySO}_{3}\right)_{4}(\mu-\mathrm{dmso})\right]\left(3^{\mathrm{dmso}}\right)$}
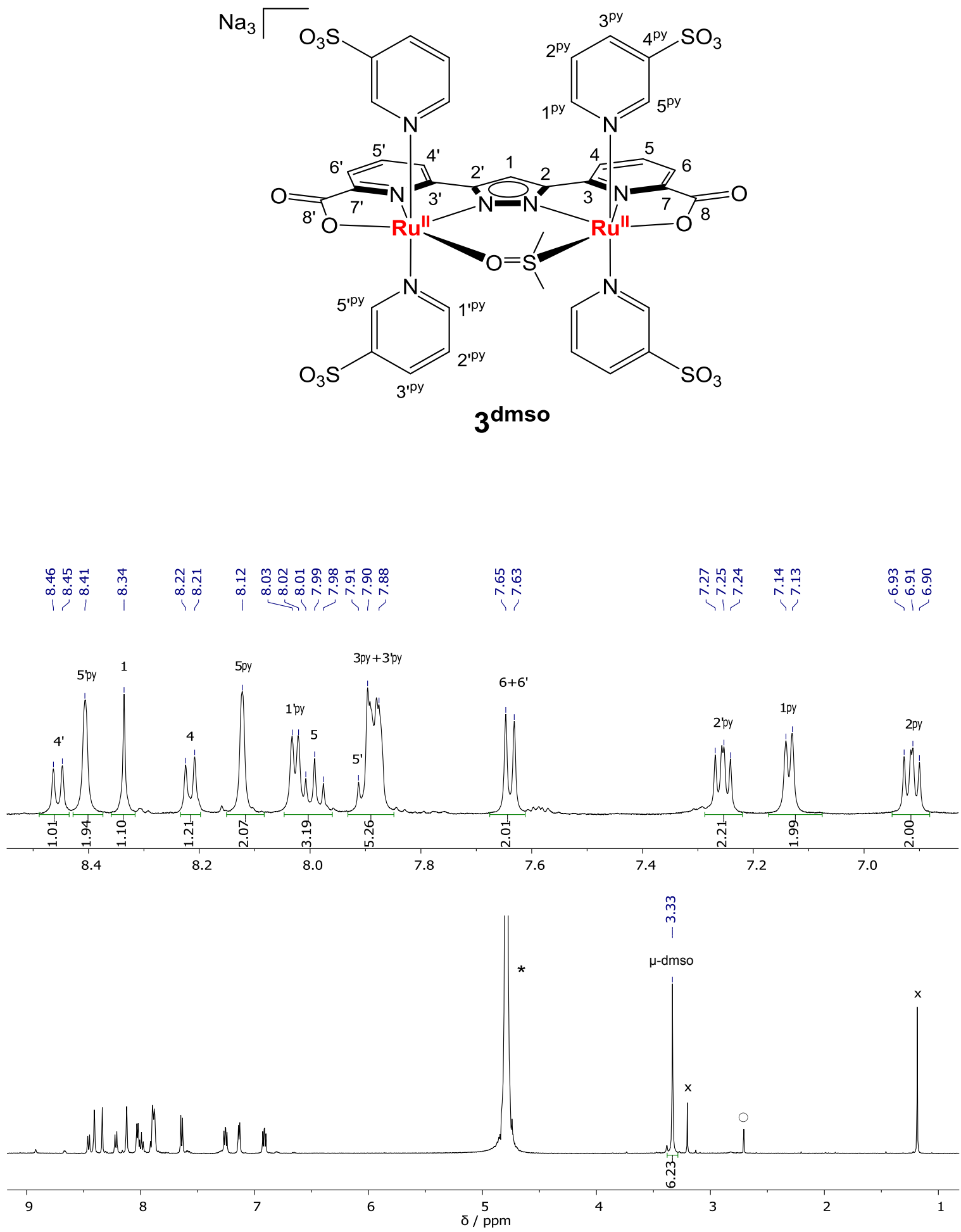

Figure A.68: ${ }^{1} \mathrm{H}-\mathrm{NMR}$ spectrum $(500 \mathrm{MHz})$ of $\mathbf{3}^{\mathrm{dmso}}$ in $\mathrm{D}_{2} \mathrm{O}$. The inset depicts an expansion of the aromatic region (6.9-8.5 p pm). The peaks are assigned according to the labeling scheme above. The asterisk denotes the residual solvent peak, the circle points out free DMSO and the x mark MTBE. Please note, the distinction between prime and non-prime for the $\left(\mathrm{L}^{1}\right)^{3-}$ ligand is not possible and is merely done for simplification. 


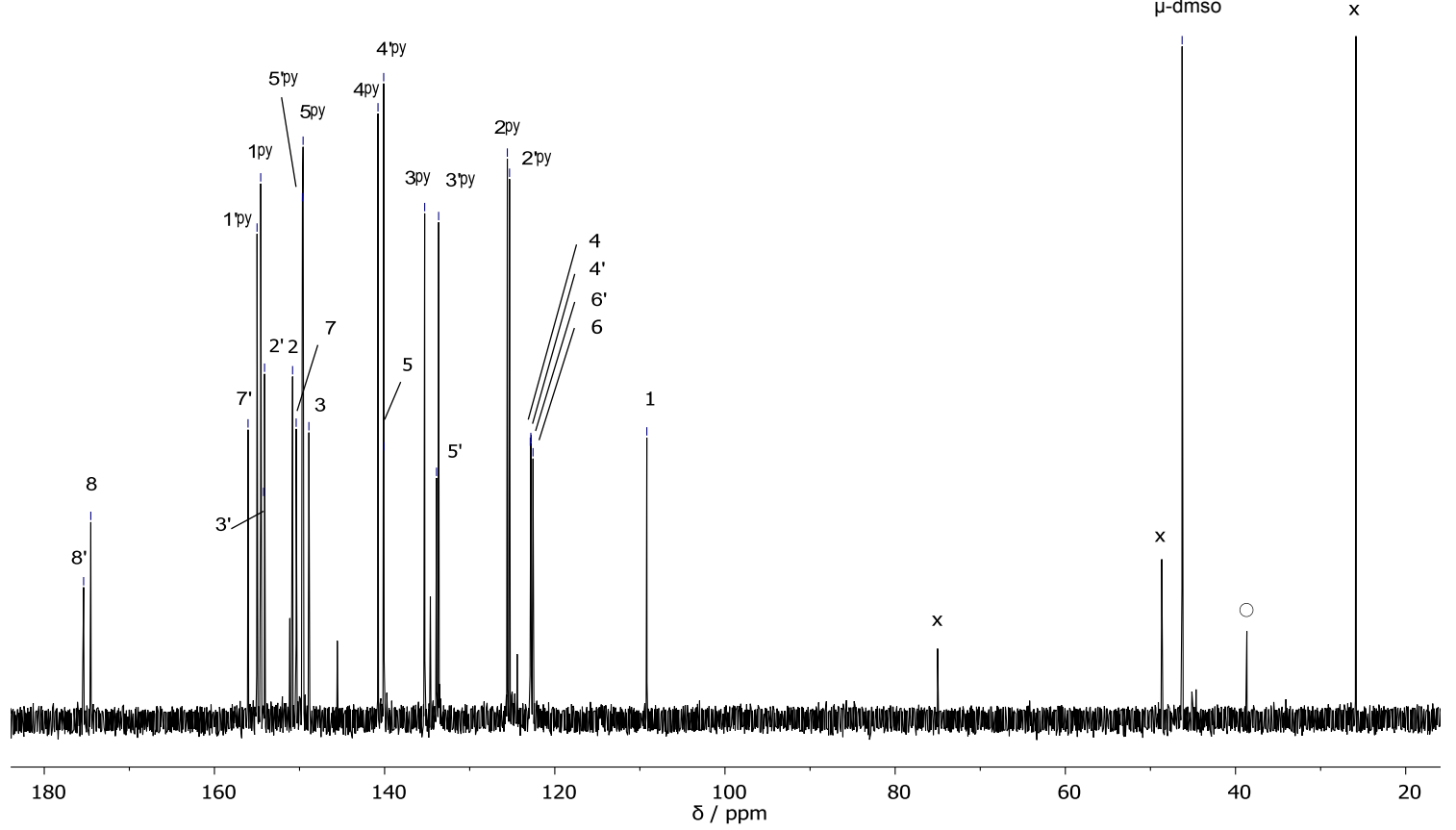

Figure A.69: ${ }^{13} \mathrm{C}\left\{{ }^{1} \mathrm{H}\right\}$-NMR spectrum $(126 \mathrm{MHz})$ of $\mathbf{3}^{\mathrm{dmso}}$ in $\mathrm{D}_{2} \mathrm{O}$. The peaks are assigned according to the labeling scheme above. The circle points out free DMSO and the $\mathrm{x}$ mark MTBE. Please note, the distinction between prime and non-prime for the $\left(\mathrm{L}^{1}\right)^{3-}$ ligand is not possible and is merely done for simplification.

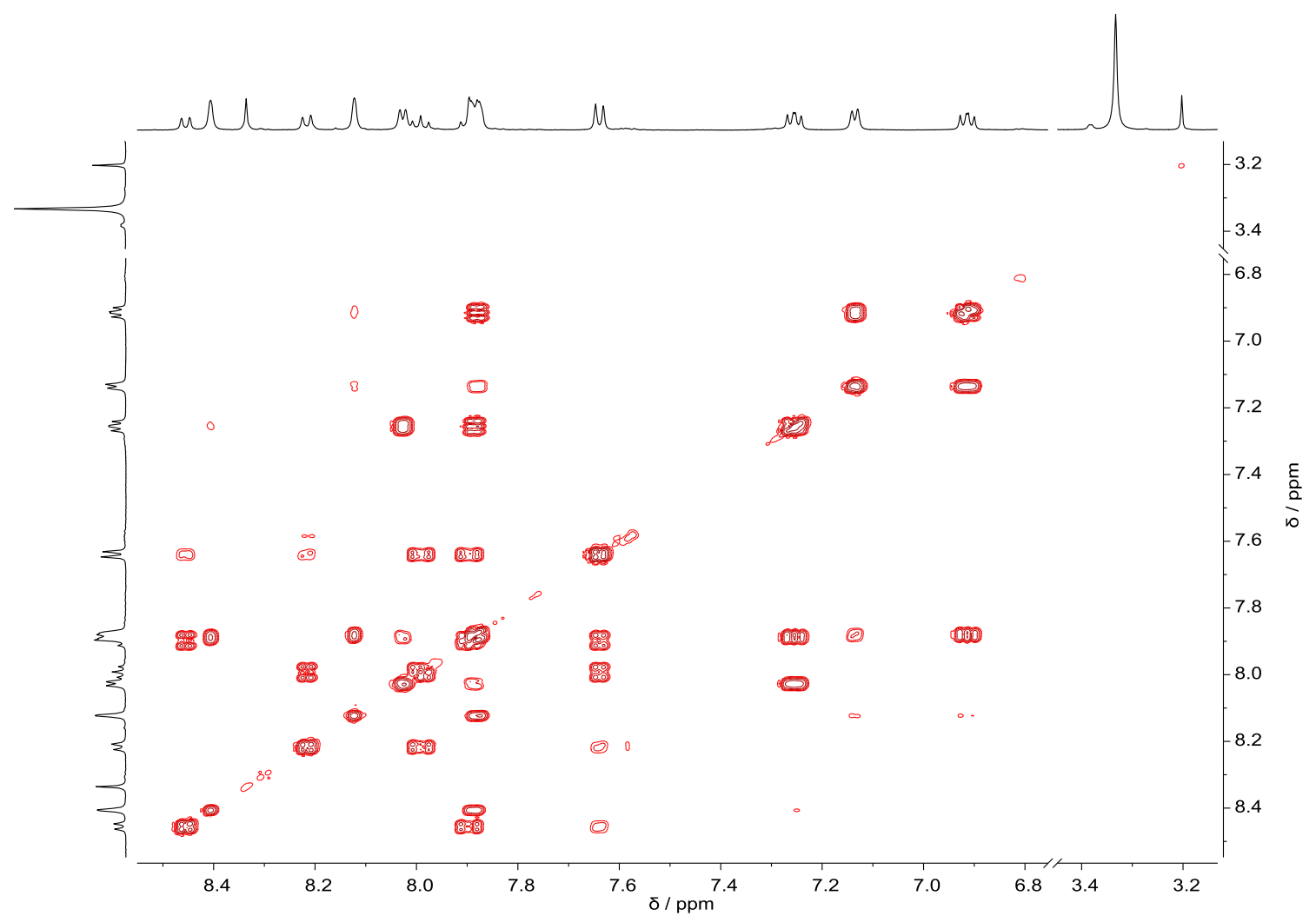

Figure A.70: ${ }^{1} \mathrm{H}-1 \mathrm{H}-\mathrm{COSY}$ spectrum $(500 \mathrm{MHz})$ of $3^{\mathrm{dmso}}$ in $\mathrm{D}_{2} \mathrm{O}$. Depicted is the full region with complex signals (3.2-8.5 ppm). 


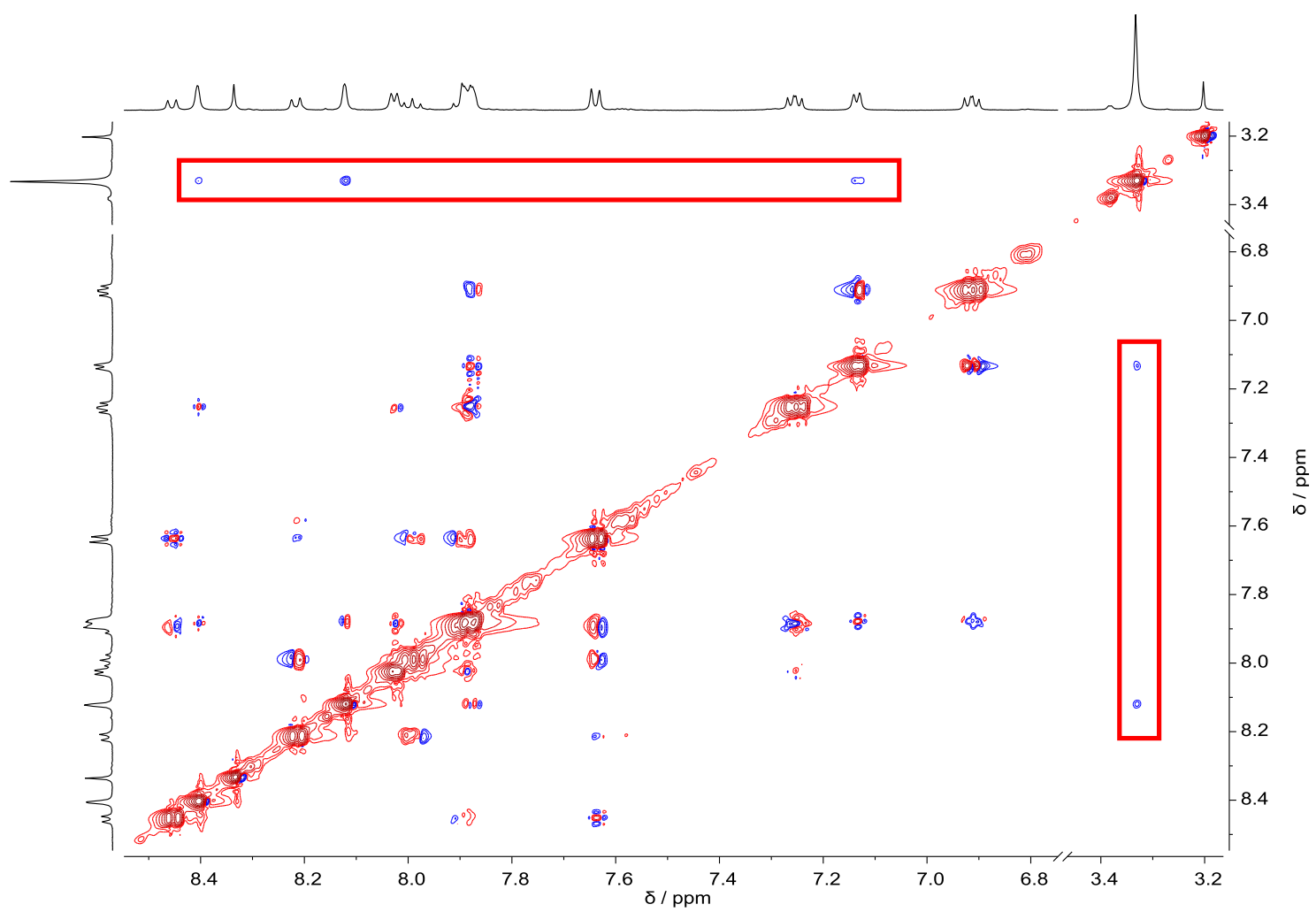

Figure A.71: ${ }^{1} \mathrm{H}-1 \mathrm{H}-\mathrm{NOESY}$ spectrum $(500 \mathrm{MHz})$ of $\mathbf{3}^{\mathrm{dmso}}$ in $\mathrm{D}_{2} \mathrm{O}$. Depicted is the full region with complex signals (3.2-8.5 ppm). The red rectangles show the correlation of the bridging DMSO with $\mathrm{pySO}_{3}{ }^{-}$.

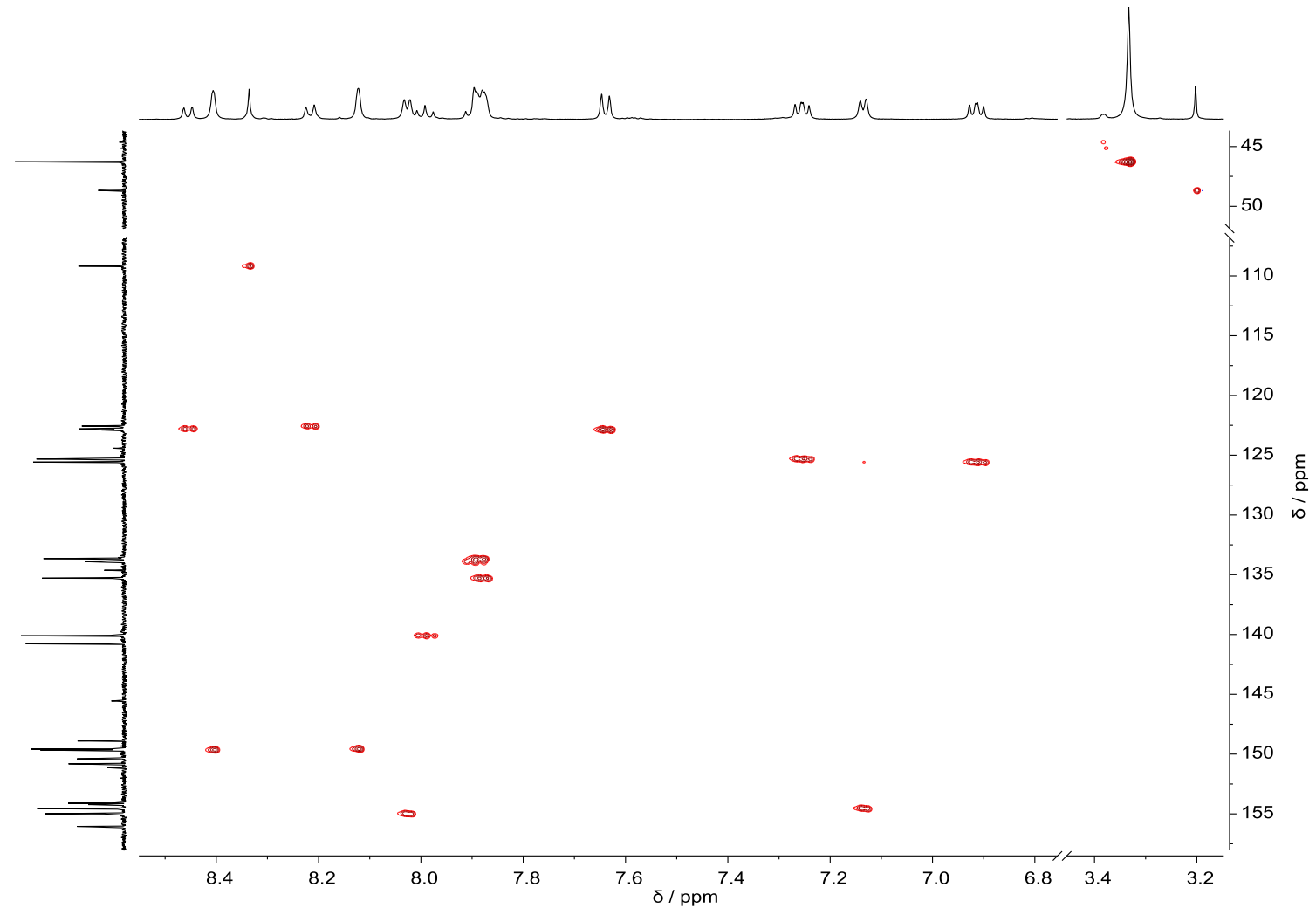

Figure A.72: ${ }^{1} \mathrm{H}-{ }^{13} \mathrm{C}\left\{{ }^{1} \mathrm{H}\right\}-\mathrm{HSQC}$ spectrum of $3^{\text {dmso }}$ in $\mathrm{D}_{2} \mathrm{O}$. Depicted is the full region with complex signals (3.2$8.5 \mathrm{ppm})$. 


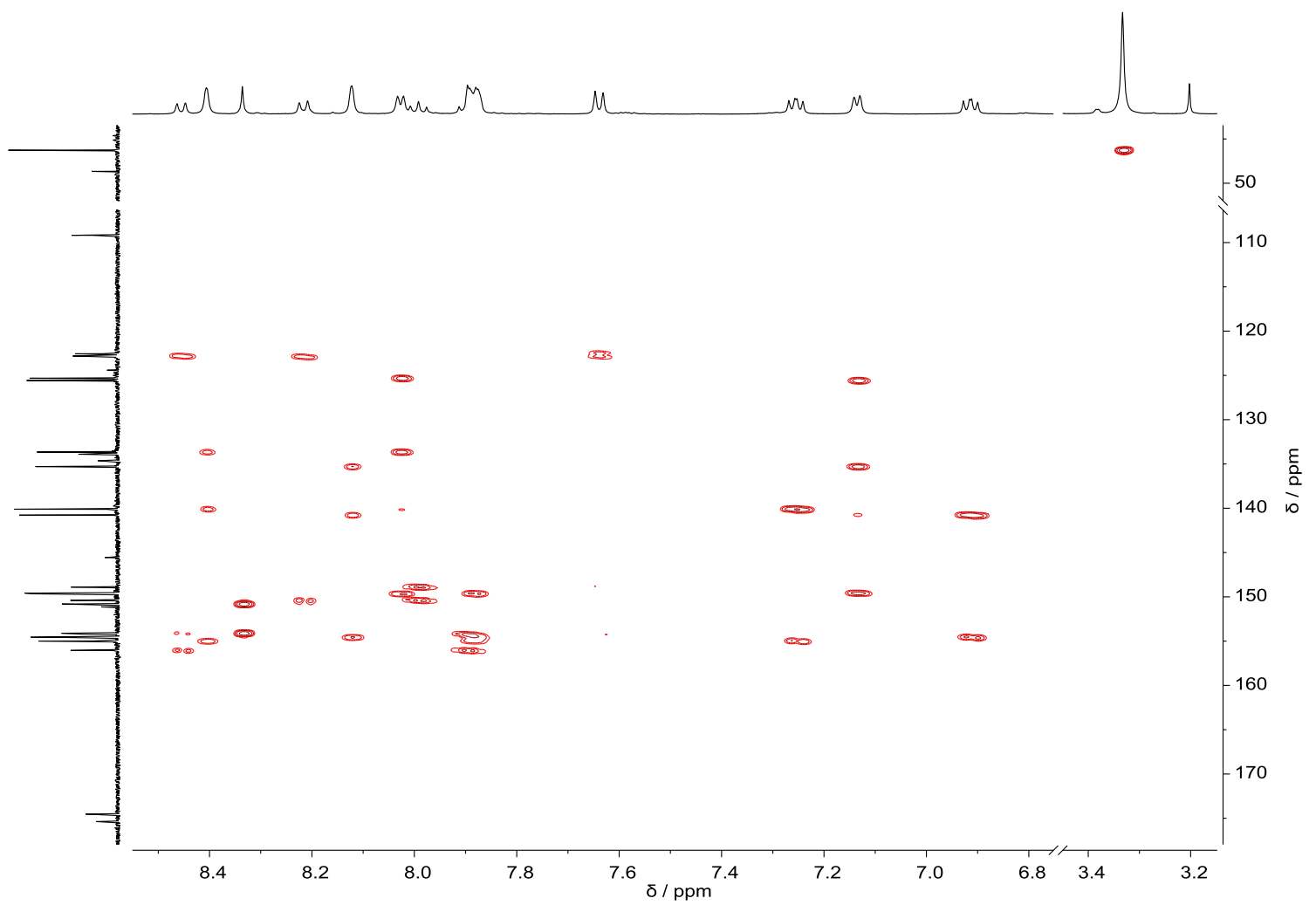

Figure A.73: ${ }^{1} \mathrm{H}^{-13} \mathrm{C}\left\{{ }^{1} \mathrm{H}\right\}-\mathrm{HMBC}$ spectrum of $\mathbf{3}^{\mathrm{dmso}}$ in $\mathrm{D}_{2} \mathrm{O}$. Depicted is the full region with complex signals $(3.2-$ $8.5 \mathrm{ppm}$ ). 


\section{$\mathrm{Na}_{4}\left[\mathrm{~L}^{1} \mathrm{Ru}_{2}\left(\mathrm{pySO}_{3}\right)_{4}\left(\mu-\mathrm{O}_{2} \mathrm{H}_{3}\right)\right]\left(3^{\mathrm{o} 2 \mathrm{H}}\right)$}
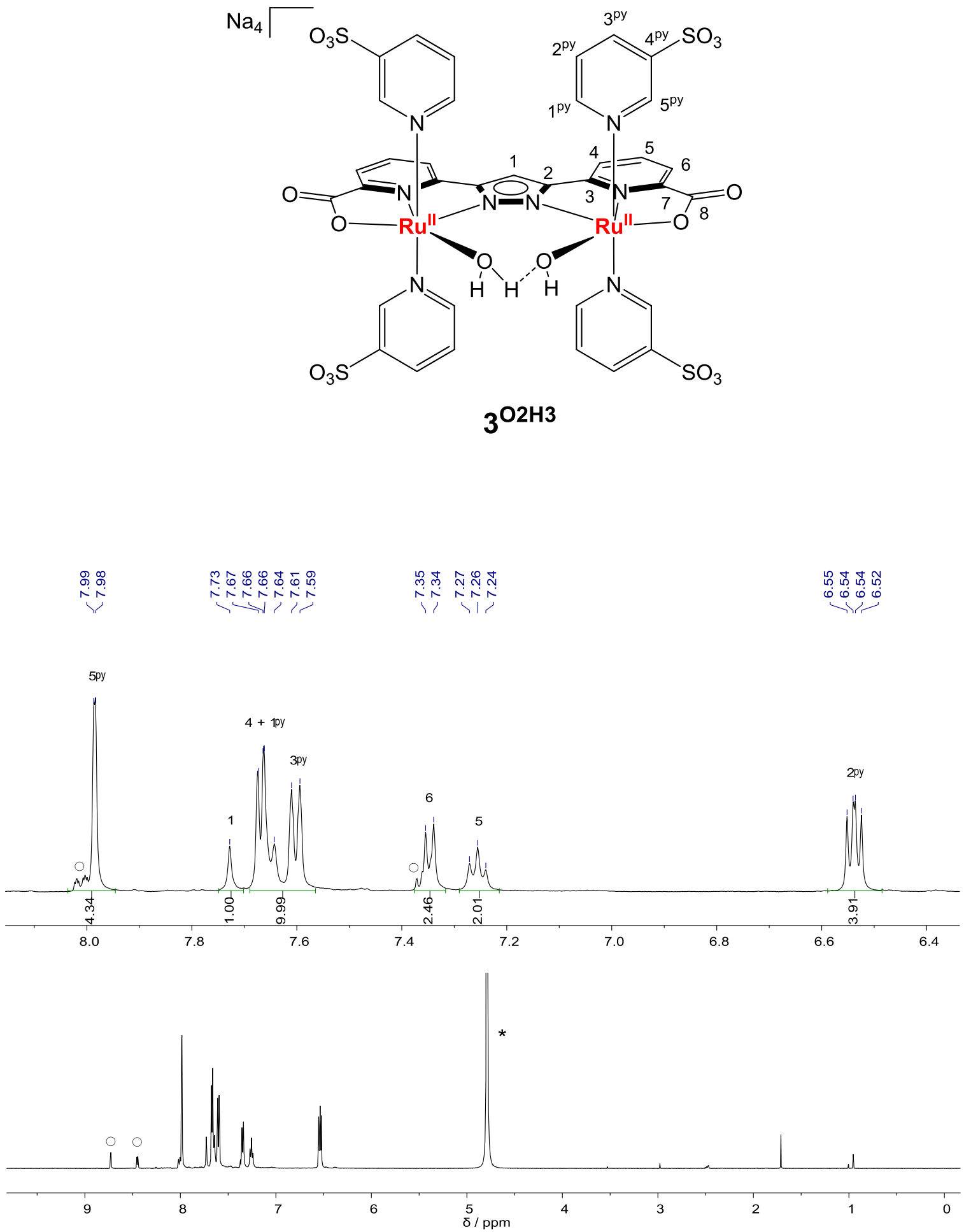

Figure A.74: ${ }^{1} \mathrm{H}-\mathrm{NMR}$ spectrum $(500 \mathrm{MHz})$ of $\mathbf{3}^{\mathbf{0 2}} \mathrm{H} 3$ in $\mathrm{D}_{2} \mathrm{O} / \mathrm{NaOD}$. The inset depicts an expansion of the aromatic region (6.4-8.2 ppm). The peaks are assigned according to the labeling scheme above. The asterisk denotes the residual solvent peak and the circles point out free $\mathrm{pySO}_{3}{ }^{-}$. 


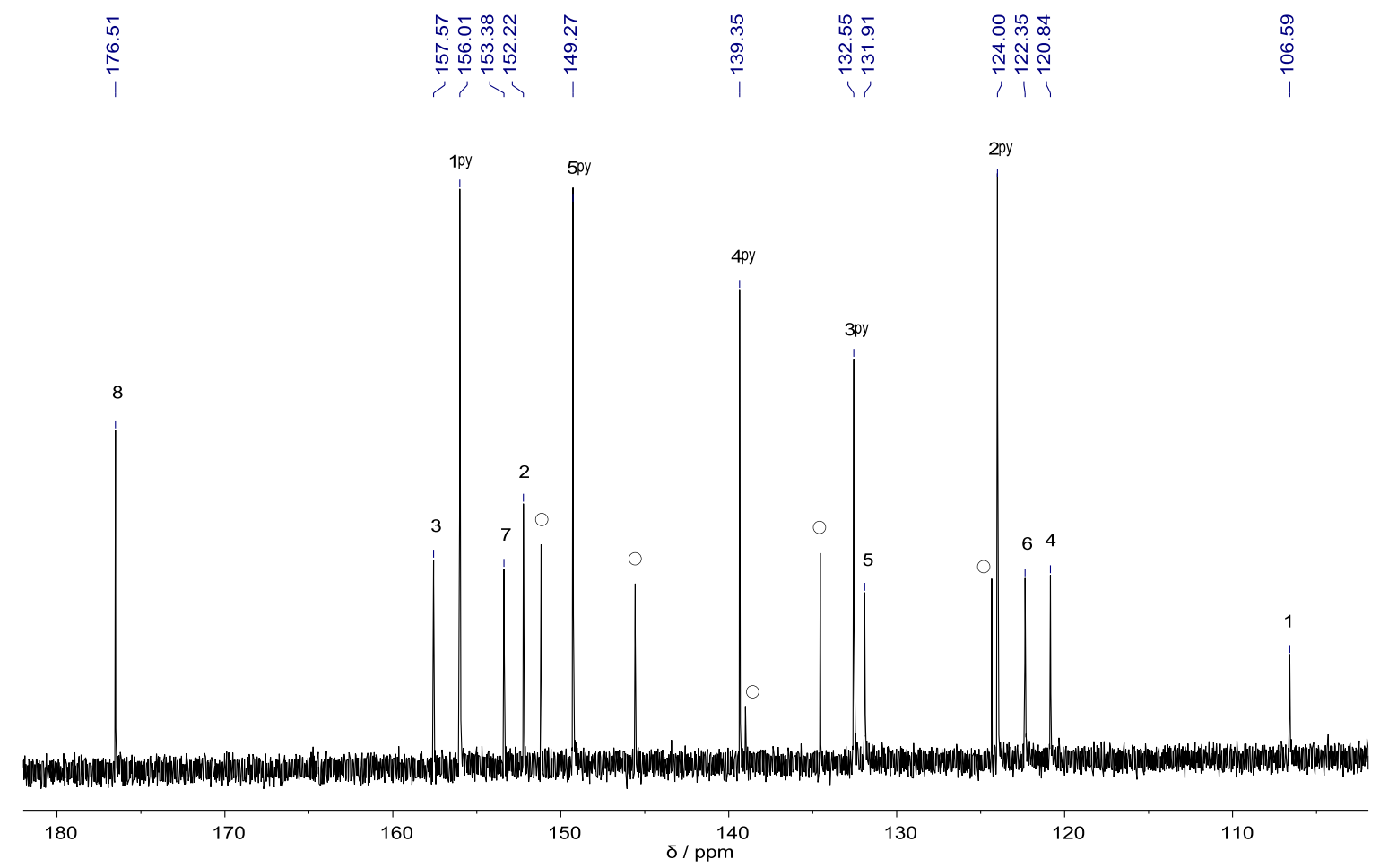

Figure A.75: ${ }^{13} \mathrm{C}\left\{{ }^{1} \mathrm{H}\right\}-\mathrm{NMR}$ spectrum $(126 \mathrm{MHz})$ of $\mathbf{3}^{\mathbf{0 2}} \mathrm{H} \mathbf{3}$ in $\mathrm{D}_{2} \mathrm{O} / \mathrm{NaOD}$. The peaks are assigned according to the labeling scheme above. The circles denote free $\mathrm{pySO}_{3}{ }^{-}$.

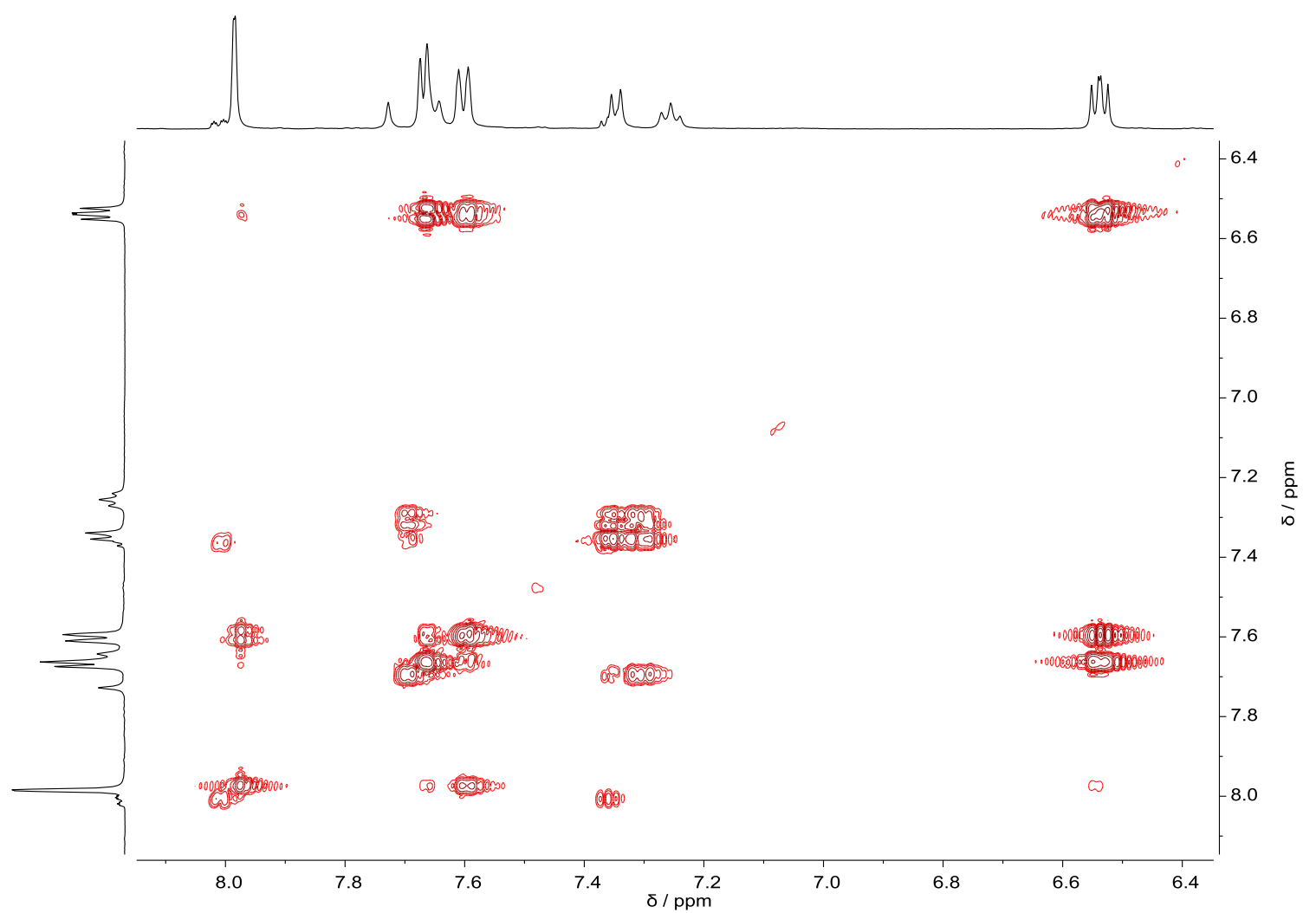

Figure A.76: ${ }^{1} \mathrm{H}-1 \mathrm{H}-\mathrm{COSY}$ spectrum $(500 \mathrm{MHz})$ of $\mathbf{3}^{\mathbf{2 0 2 H} 3}$ in $\mathrm{D}_{2} \mathrm{O} / \mathrm{NaOD}$. Depicted is the full region with complex signals (6.4-8.1 ppm). 


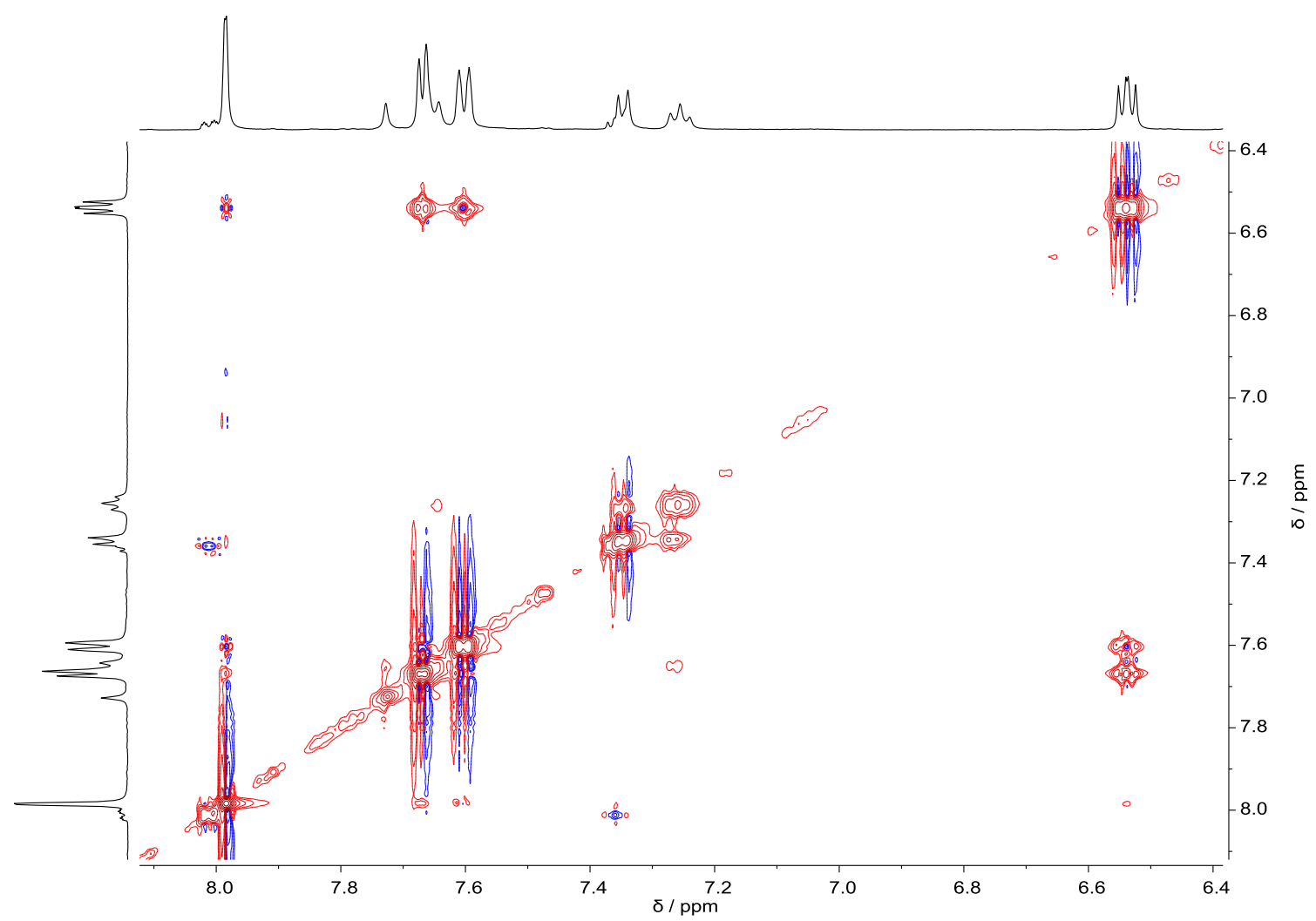

Figure A.77: ${ }^{1} \mathrm{H}-1 \mathrm{H}-\mathrm{NOESY}$ spectrum $(500 \mathrm{MHz})$ of $\mathbf{3}^{\mathbf{2} 2 \mathrm{H} 3}$ in $\mathrm{D}_{2} \mathrm{O} / \mathrm{NaOD}$. Depicted is the full region with complex signals (6.4-8.1 ppm).

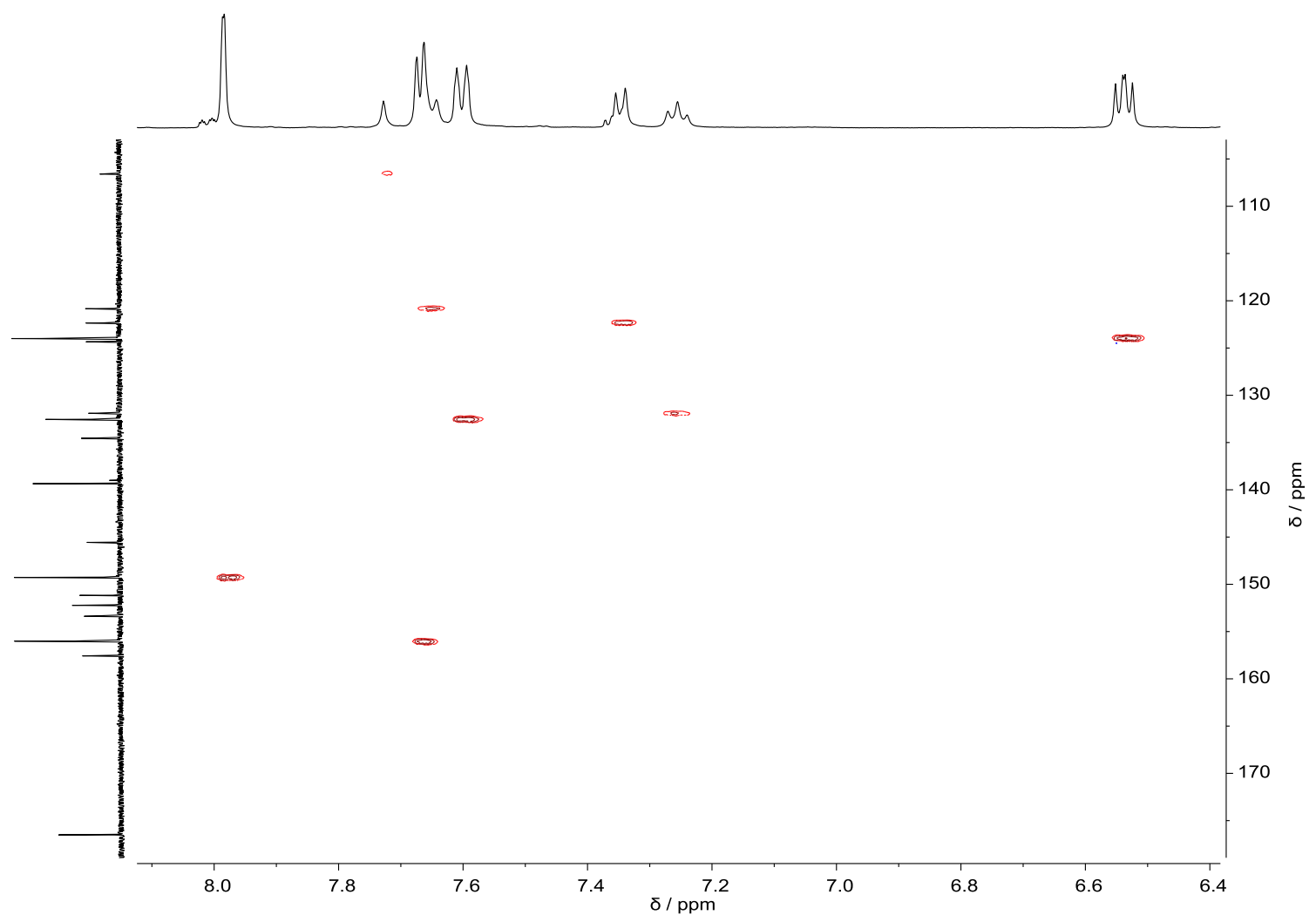

Figure A.78: ${ }^{1} \mathrm{H}-{ }^{13} \mathrm{C}\left\{{ }^{1} \mathrm{H}\right\}-\mathrm{HSQC}$ spectrum of $\mathbf{3}^{\mathbf{0 2}} \mathrm{H}$ in $\mathrm{D}_{2} \mathrm{O} / \mathrm{NaOD}$. Depicted is the full region with complex signals (6.4-8.1 ppm). 


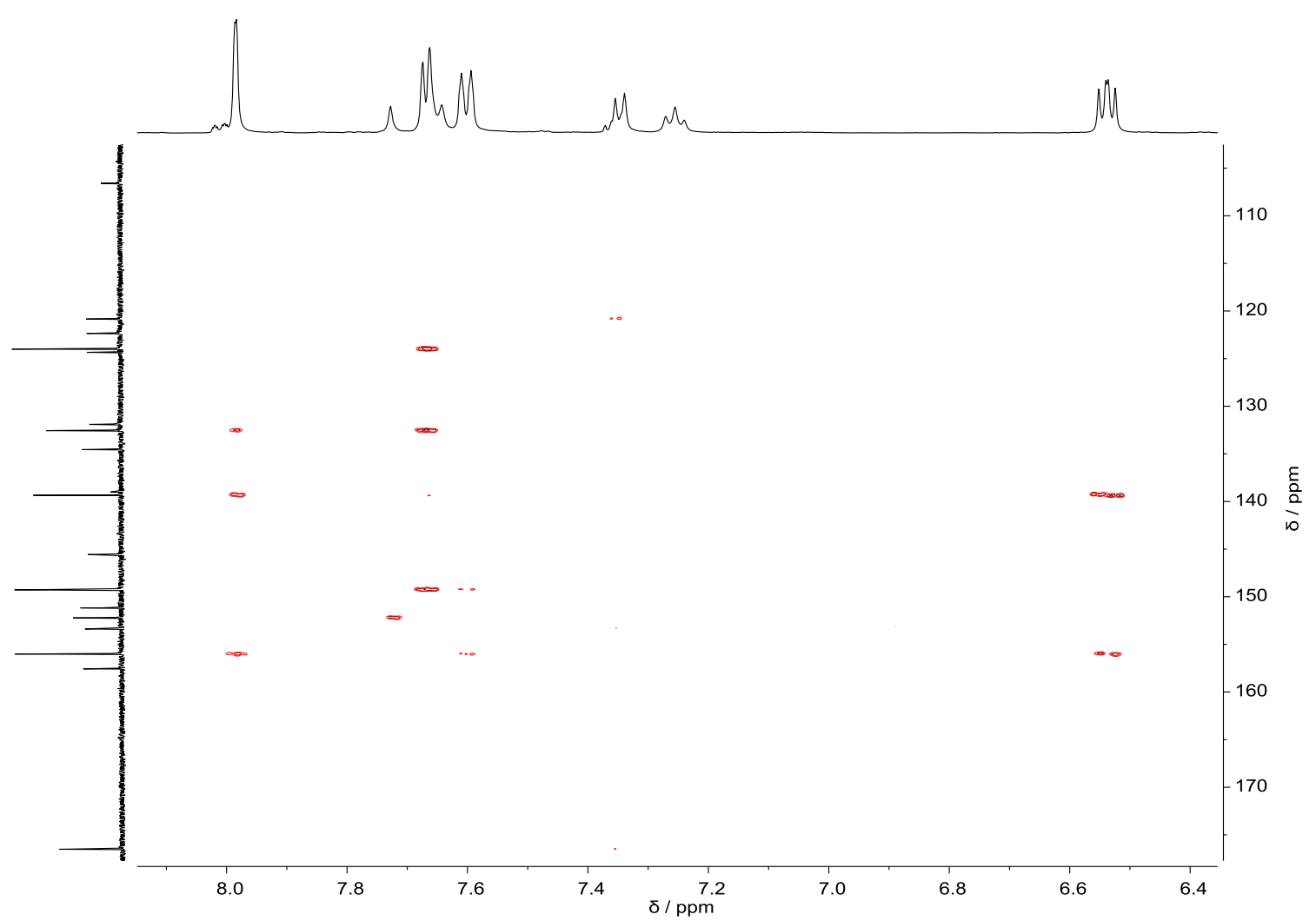

Figure A.79: ${ }^{1} \mathrm{H}^{-13} \mathrm{C}\left\{{ }^{1} \mathrm{H}\right\}-\mathrm{HMBC}$ spectrum of $\mathbf{3}^{\mathbf{0 2}} \mathrm{H}^{3}$ in $\mathrm{D}_{2} \mathrm{O} / \mathrm{NaOD}$. Depicted is the full region with complex signals (6.4-8.1 ppm). 


\section{$\left.\mathrm{L}^{2 \mathrm{a}} \mathrm{Co}_{2}\left(\mu-\mathrm{CH}_{3} \mathrm{C}(\mathrm{O}) \mathrm{NH}\right)(\mathrm{MeCN})_{2}\right]\left(\mathrm{ClO}_{4}\right)_{4}\left(7^{\mathrm{A}}\right)$}

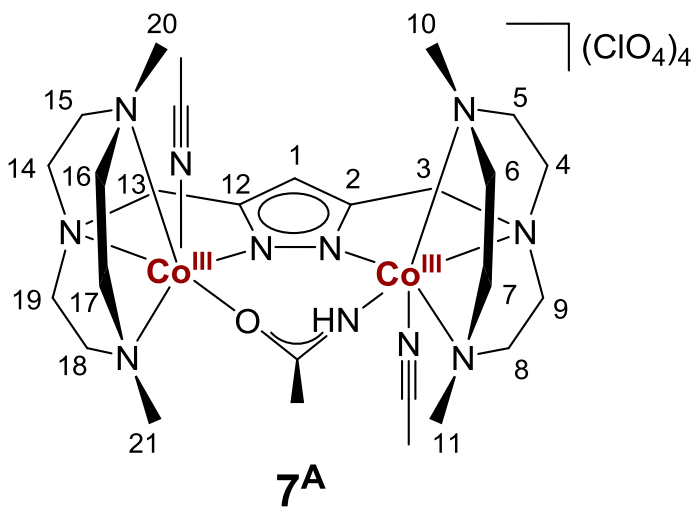

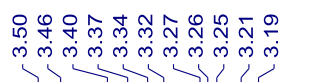

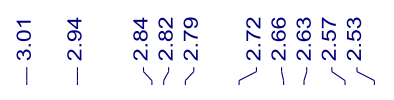

$\begin{array}{lll}\stackrel{\infty}{N} & \stackrel{\infty}{N} & \stackrel{\infty}{N} \\ \text { N } & \text { I } & 1\end{array}$

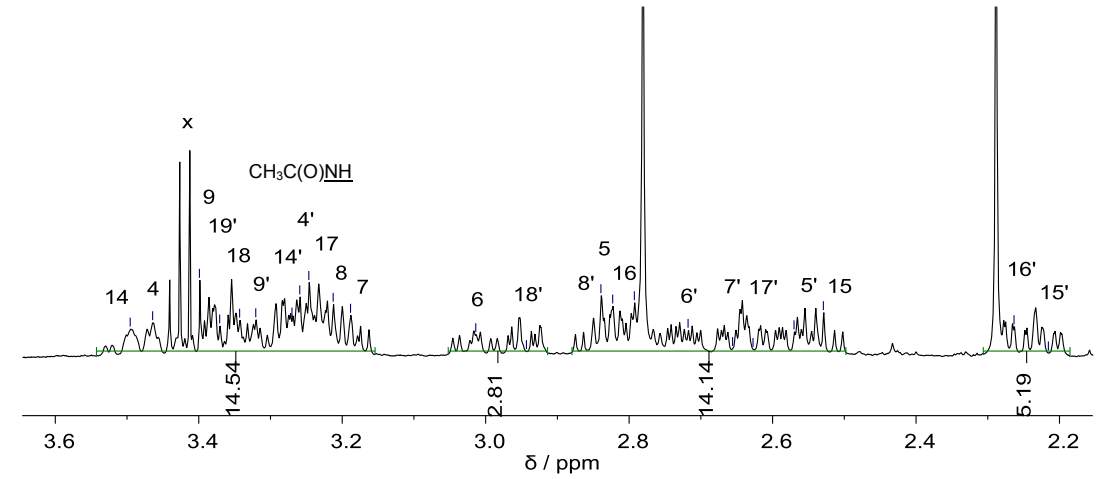

$\stackrel{\infty}{\circ}$

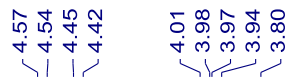

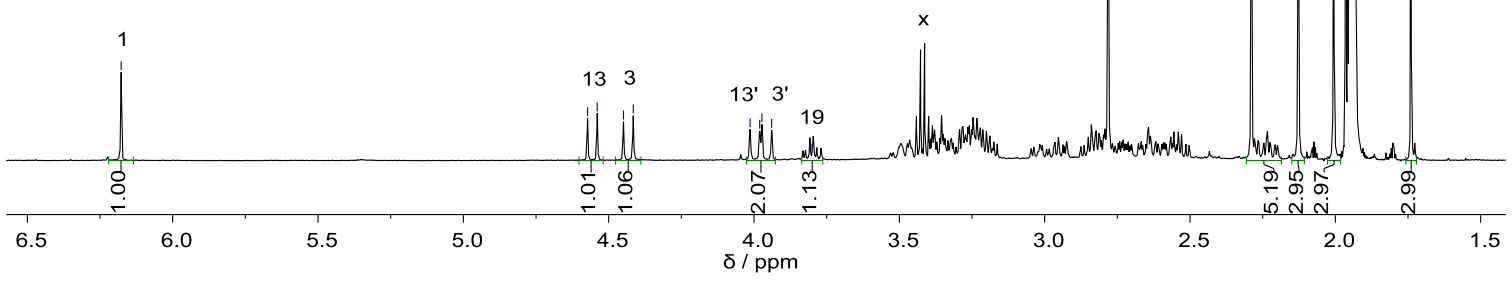

Figure A.80: ${ }^{1} \mathrm{H}-\mathrm{NMR}$ spectrum $(500 \mathrm{MHz})$ of $\mathbf{7}^{\mathrm{A}}$ in $\mathrm{CD}_{3} \mathrm{CN}$ under inert conditions. The inset depicts an expansion of the region from 2.2-3.6 ppm. The peaks are assigned according to the labeling scheme above. The asterisk denotes the residual solvent peak and $\mathrm{x}$ points out $\mathrm{Et}_{2} \mathrm{O}$. Please note, the distinction between prime and non-prime, as well as left and right side of the complex is not possible and is merely done for simplification. 

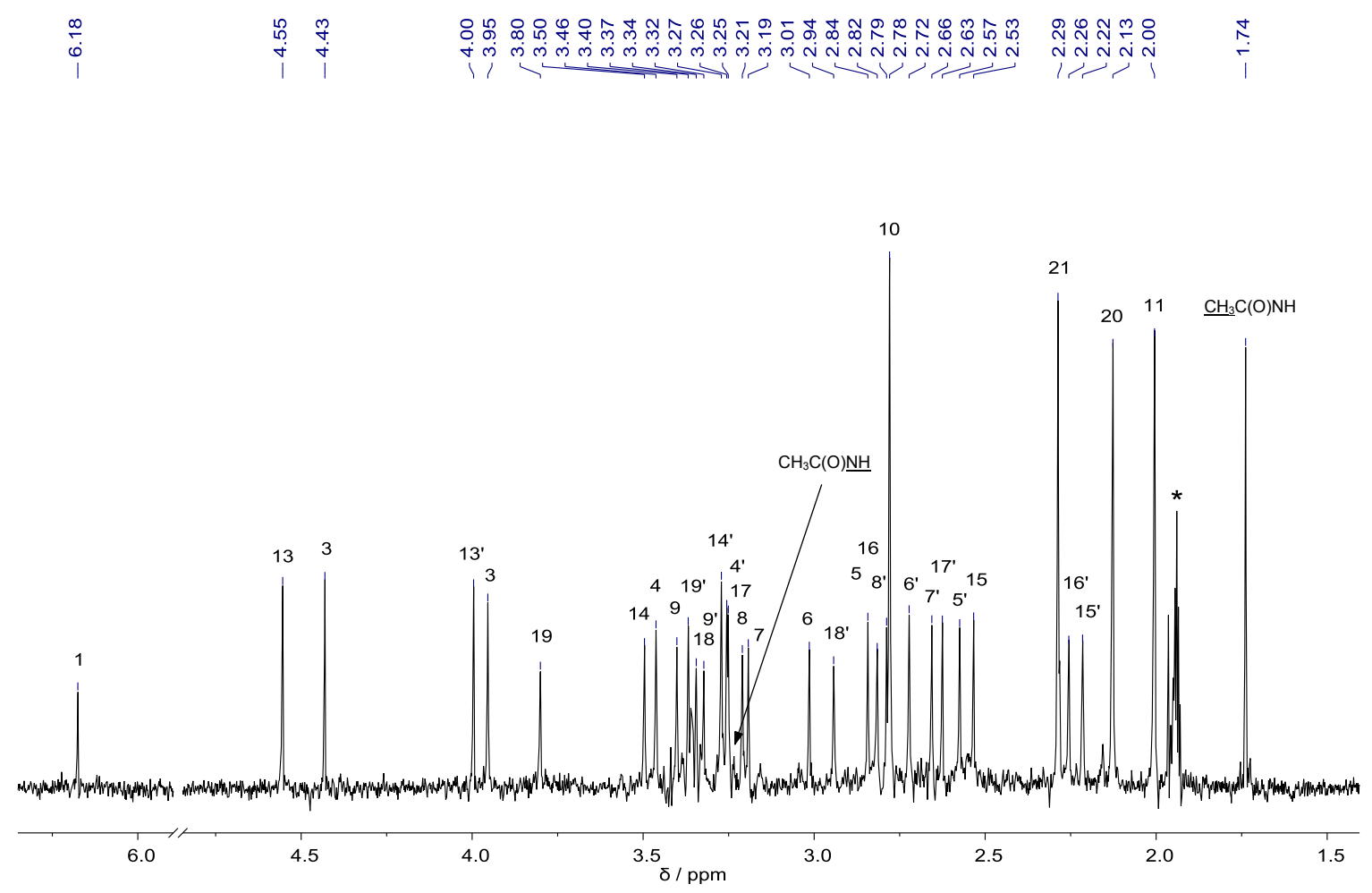

Figure A.81: Pure shift ${ }^{1} \mathrm{H}-\mathrm{NMR}$ spectrum (500 MHz) of $\mathbf{7}^{\mathbf{A}}$ in $\mathrm{CD}_{3} \mathrm{CN}$ under inert conditions. The peaks are assigned according to the labeling scheme above. The asterisk denotes the residual solvent peak. The arrow indicates where the $\mathrm{NH}$ of the acetamide is expected. Please note, the distinction between prime and non-prime is not possible and is merely done for simplification.

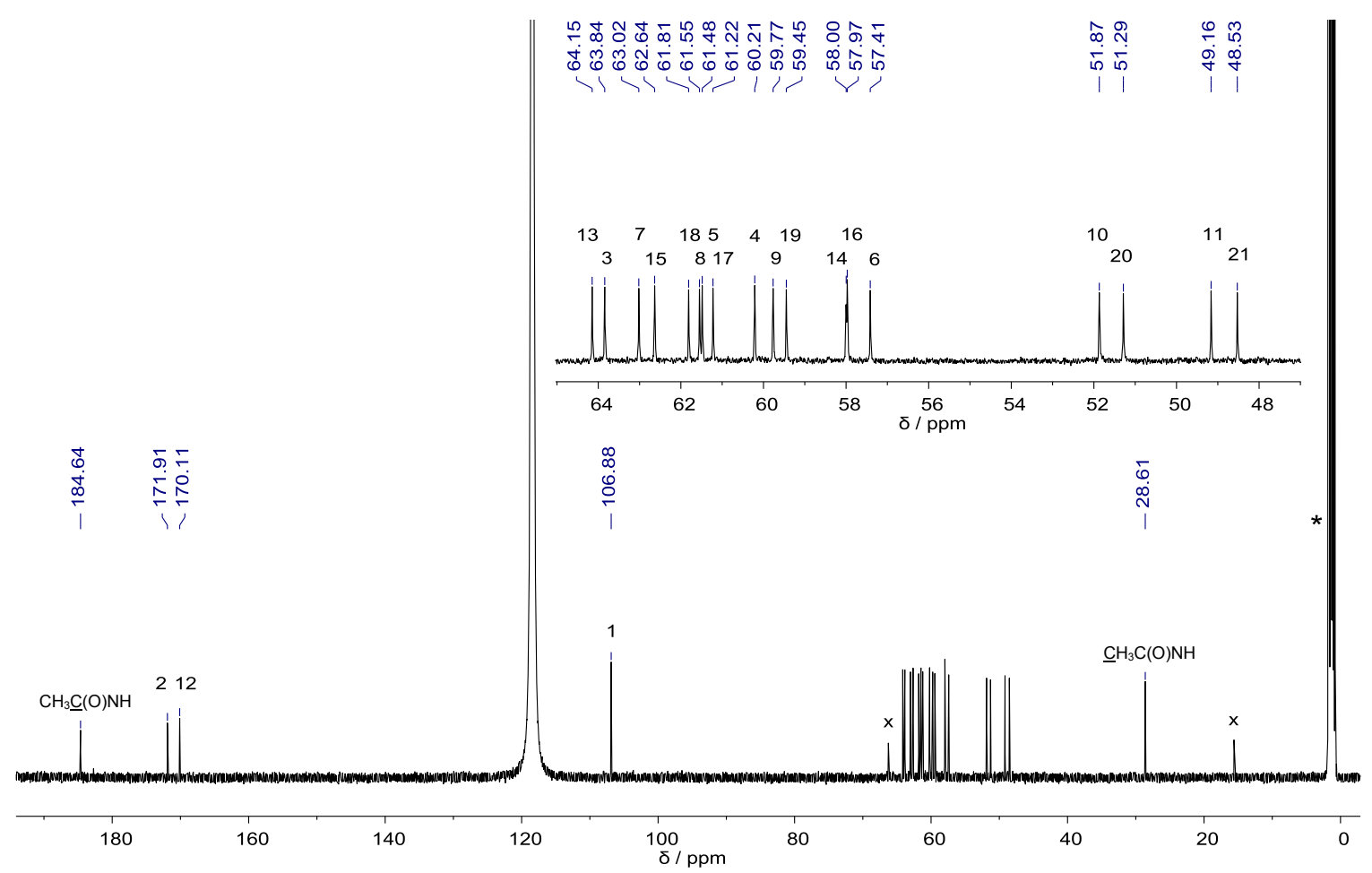

Figure A.82: ${ }^{13} \mathrm{C}\left\{{ }^{1} \mathrm{H}\right\}$-NMR spectrum $(126 \mathrm{MHz})$ of $\mathbf{7}^{\mathrm{A}}$ in $\mathrm{CD}_{3} \mathrm{CN}$ under inert conditions. The inset depicts an expansion of the region from 48-64 ppm. The peaks are assigned according to the labeling scheme above. The asterisk denotes the residual solvent peak and $\mathrm{x}$ points out $\mathrm{Et}_{2} \mathrm{O}$. 

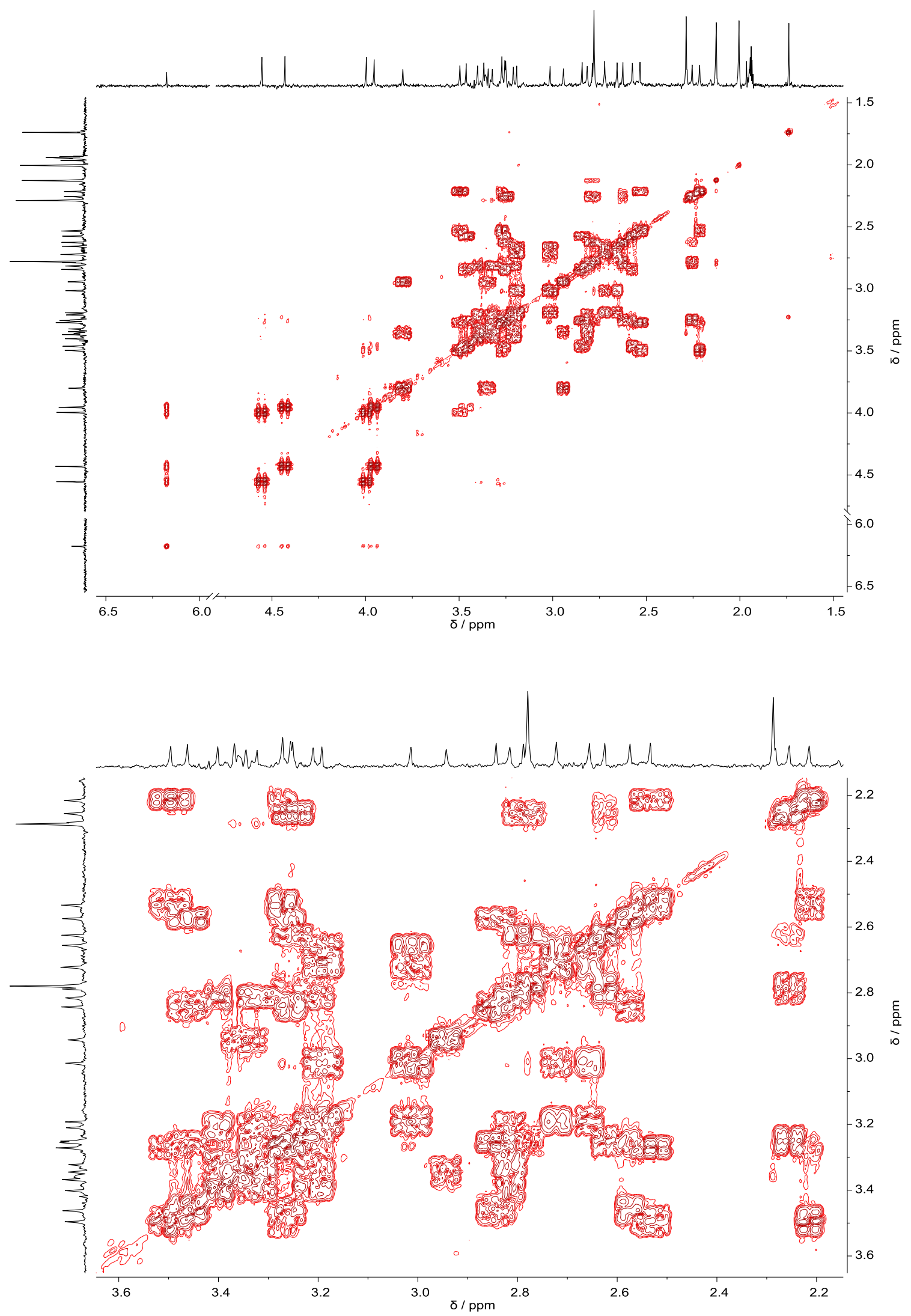

Figure A.83: ${ }^{1} \mathrm{H}-1 \mathrm{H}-\mathrm{COSY}$ spectrum of $7^{\mathrm{A}}$ in $\mathrm{CD}_{3} \mathrm{CN}$ under inert conditions. Top: complete region with complex signals (1.5-6.3 ppm), bottom: enlargement of area from 2.2-3.6 ppm. Please note, the pure shift ${ }^{1} \mathrm{H}-\mathrm{NMR}$ is shown in both traces. 

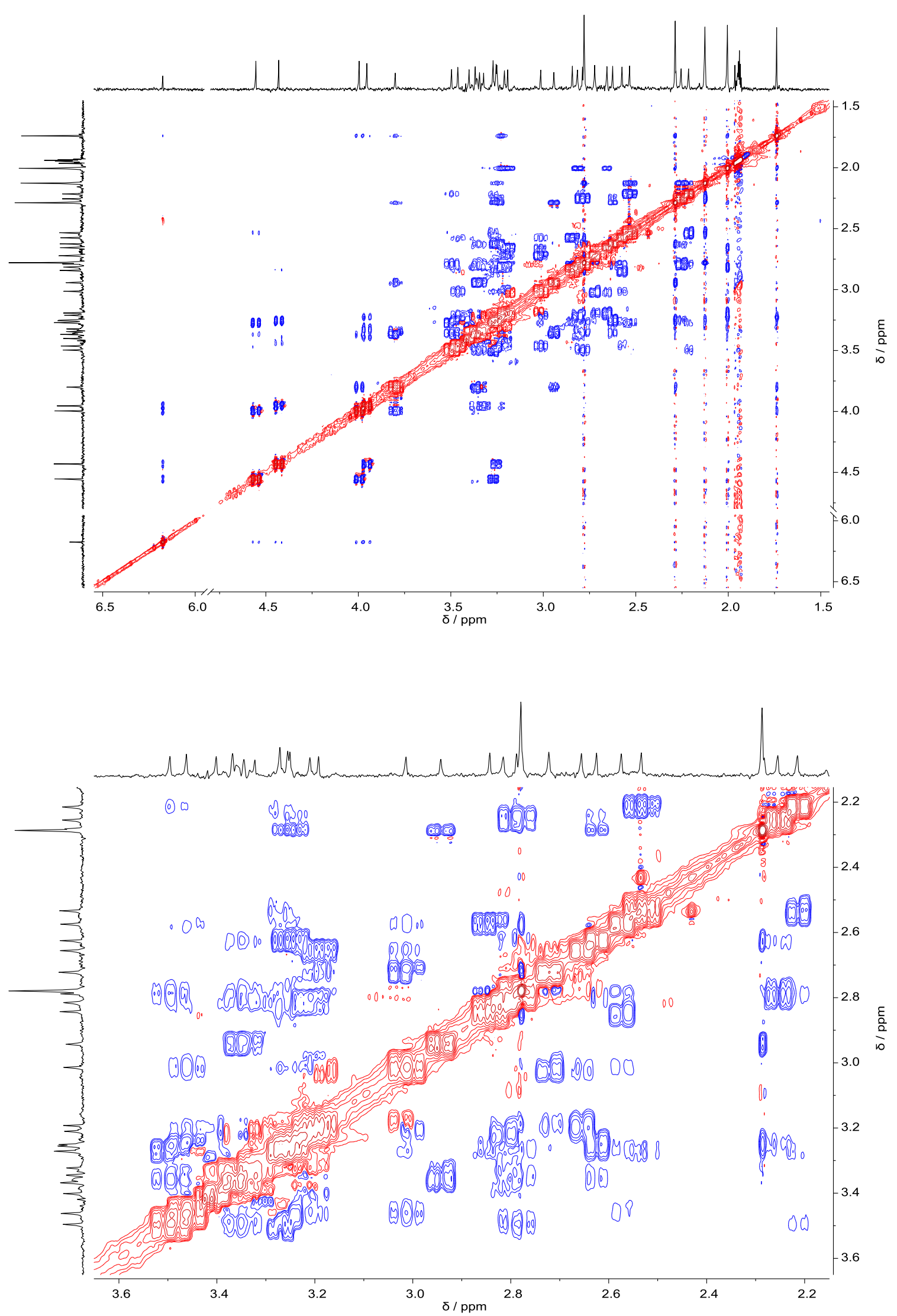

Figure A.84: ${ }^{1} \mathrm{H}-1 \mathrm{H}-\mathrm{NOESY}$ spectrum of $7^{\mathrm{A}}$ in $\mathrm{CD}_{3} \mathrm{CN}$ under inert conditions. Top: complete region with complex signals (1.5-6.3 ppm), bottom: enlargement of area from 2.2-3.6 ppm. Please note, the pure shift ${ }^{1} \mathrm{H}-\mathrm{NMR}$ is shown in both traces. 


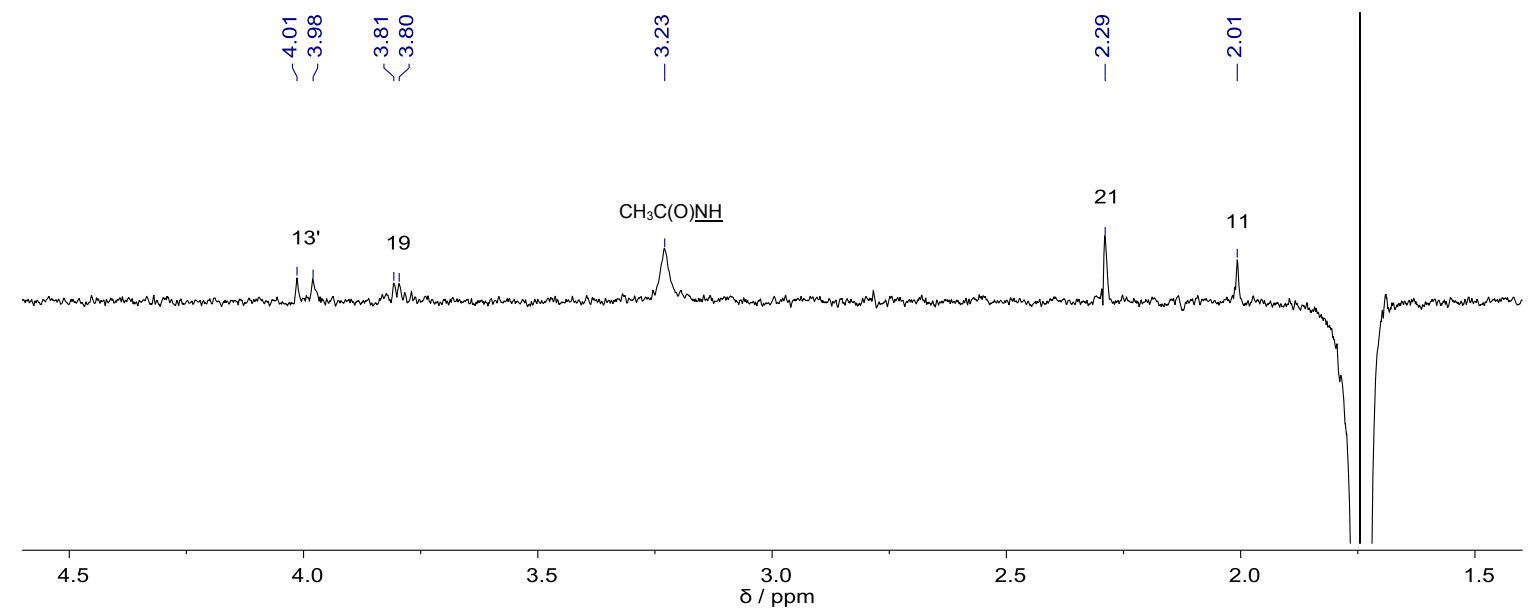

Figure A.85: ${ }^{1} \mathrm{H}-1 \mathrm{D}-\mathrm{NOESY}$ spectrum $(500 \mathrm{MHz})$ of $7^{\mathrm{A}}$ in $\mathrm{CD}_{3} \mathrm{CN}$ under inert conditions after selective excitation of the resonance at $1.74 \mathrm{ppm}$ corresponding to the methyl group of the acetamide moiety. The spectrum clearly shows a correlation to a peak at $3.23 \mathrm{ppm}$, which cannot be observed in the pure shift ${ }^{1} \mathrm{H}-\mathrm{NMR}$ spectrum and is expected to correspond to the $\mathrm{NH}$ proton of the acetamide.

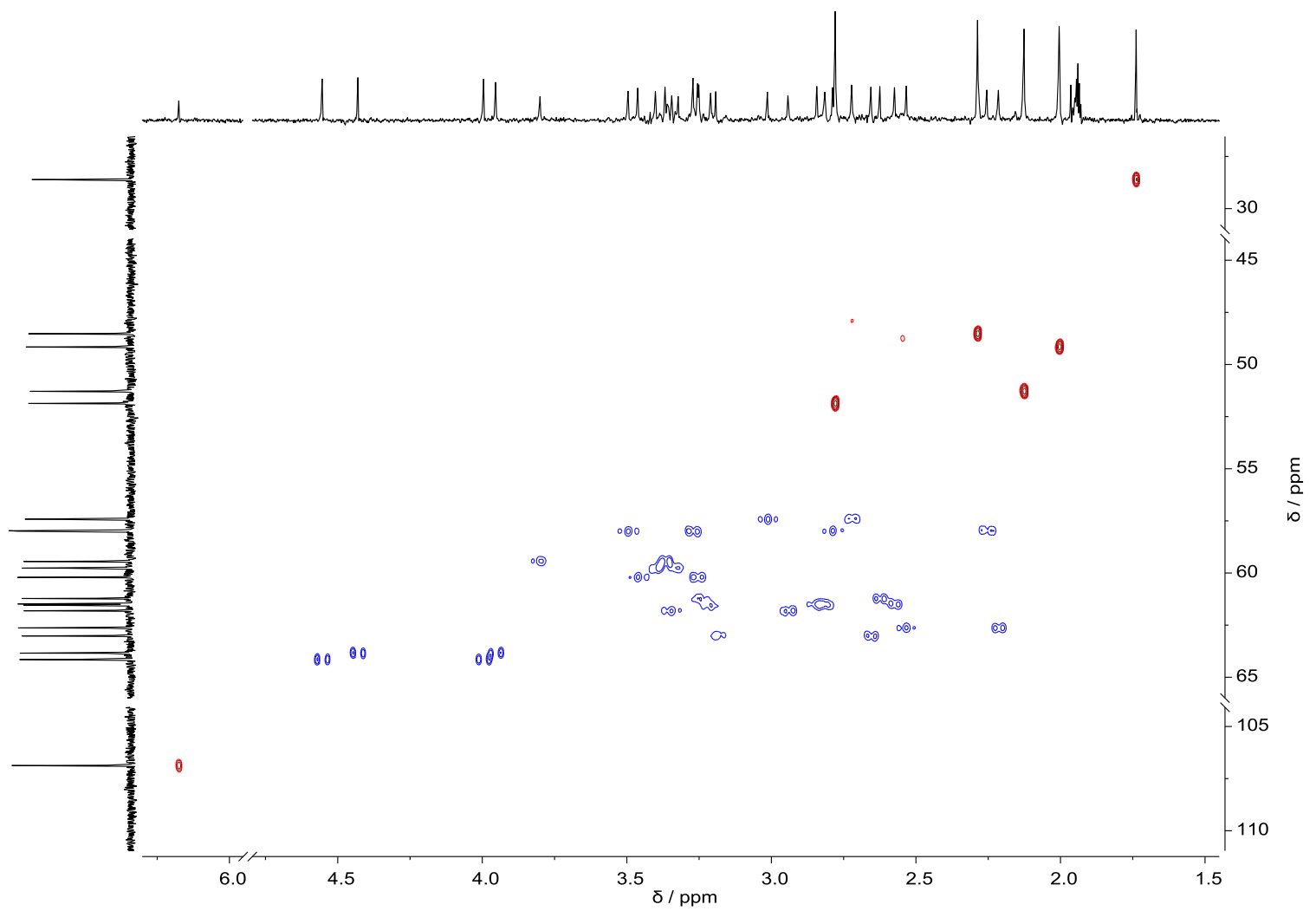

Figure A.86: ${ }^{1} \mathrm{H}-{ }^{13} \mathrm{C}\left\{{ }^{1} \mathrm{H}\right\}-\mathrm{HSQC}$ spectrum of $7^{\mathrm{A}}$ in $\mathrm{CD}_{3} \mathrm{CN}$ under inert conditions. Depicted is the full region with complex signals (1.5-6.3 ppm). Please note, the pure shift ${ }^{1} \mathrm{H}-\mathrm{NMR}$ is shown in the horizontal trace. 

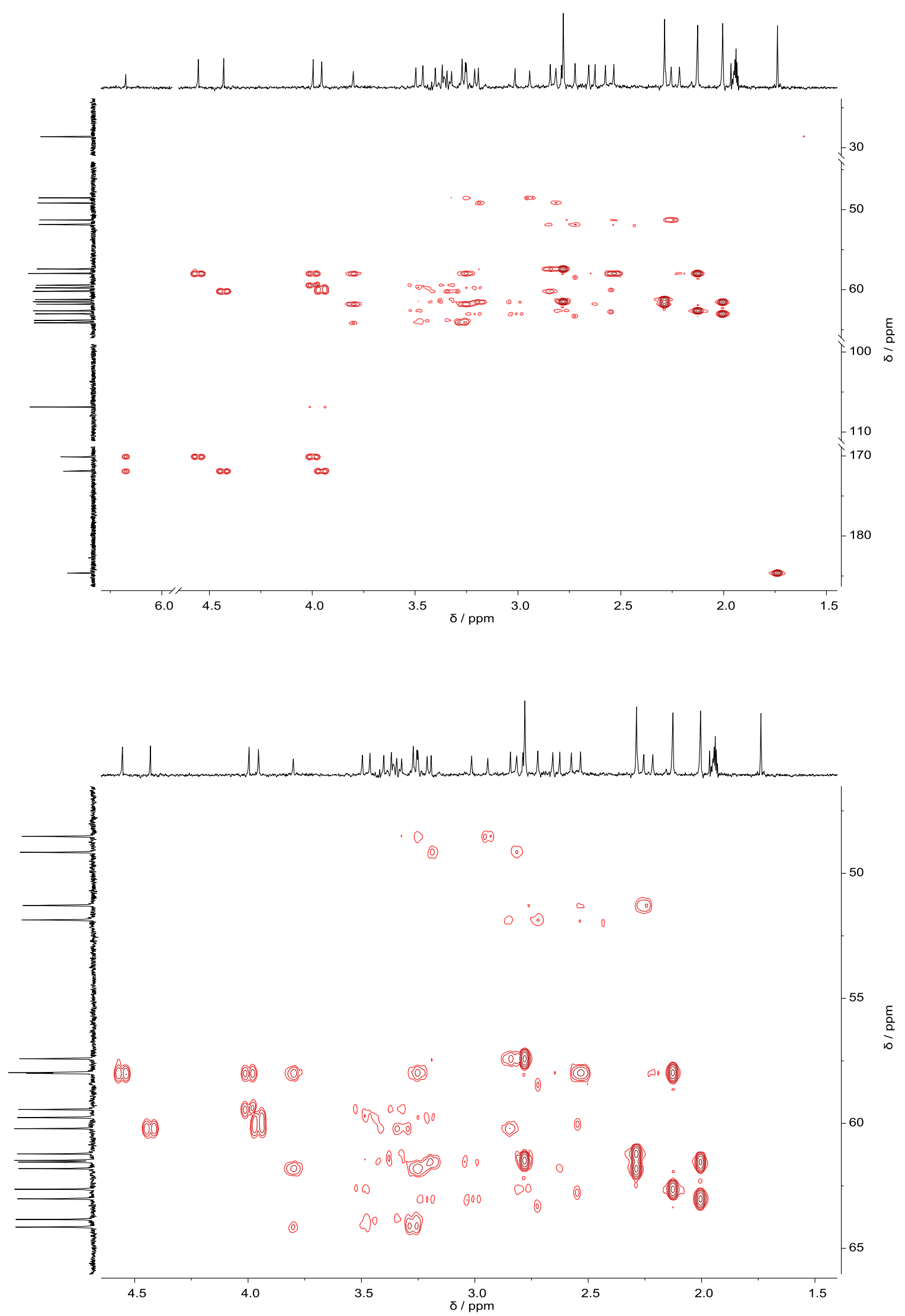

Figure A.87: ${ }^{1} \mathrm{H}-{ }^{13} \mathrm{C}\left\{{ }^{1} \mathrm{H}\right\}-\mathrm{HMBC}$ spectrum of $\mathbf{7}^{\mathbf{A}}$ in $\mathrm{CD}_{3} \mathrm{CN}$ under inert conditions. Top: complete region with complex signals (1.5-6.3 ppm), bottom: enlargement of area from 1.5-4.5 ppm. Please note, the pure shift ${ }^{1} \mathrm{H}-\mathrm{NMR}$ is shown in the horizontal trace. 


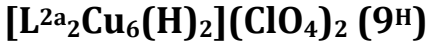
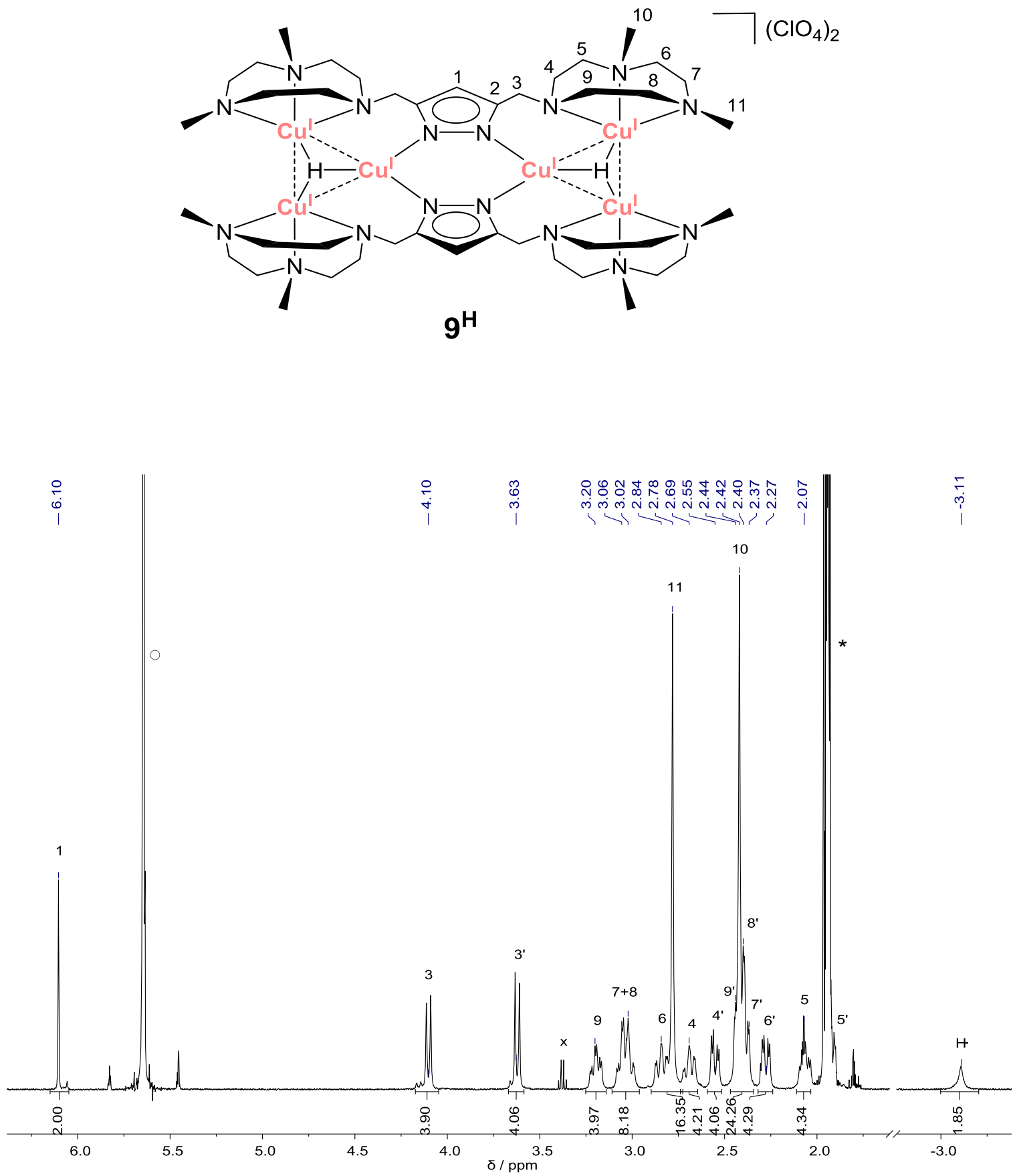

Figure A.88: ${ }^{1} \mathrm{H}-\mathrm{NMR}$ spectrum $(500 \mathrm{MHz})$ of $\mathbf{9 H}^{\mathbf{H}}$ in $\mathrm{CD}_{3} \mathrm{CN}$ at $238 \mathrm{~K}$ under inert conditions. The peaks are assigned according to the labeling scheme above. The asterisk denotes the residual solvent peak, the $\mathrm{x}$ points out $\mathrm{Et}_{2} \mathrm{O}$ and the circle marks $\left[\mathrm{Cp}_{2} \mathrm{Co}\right]^{+}$. Please note, the distinction between prime and non-prime is not possible and is merely done for simplification. 


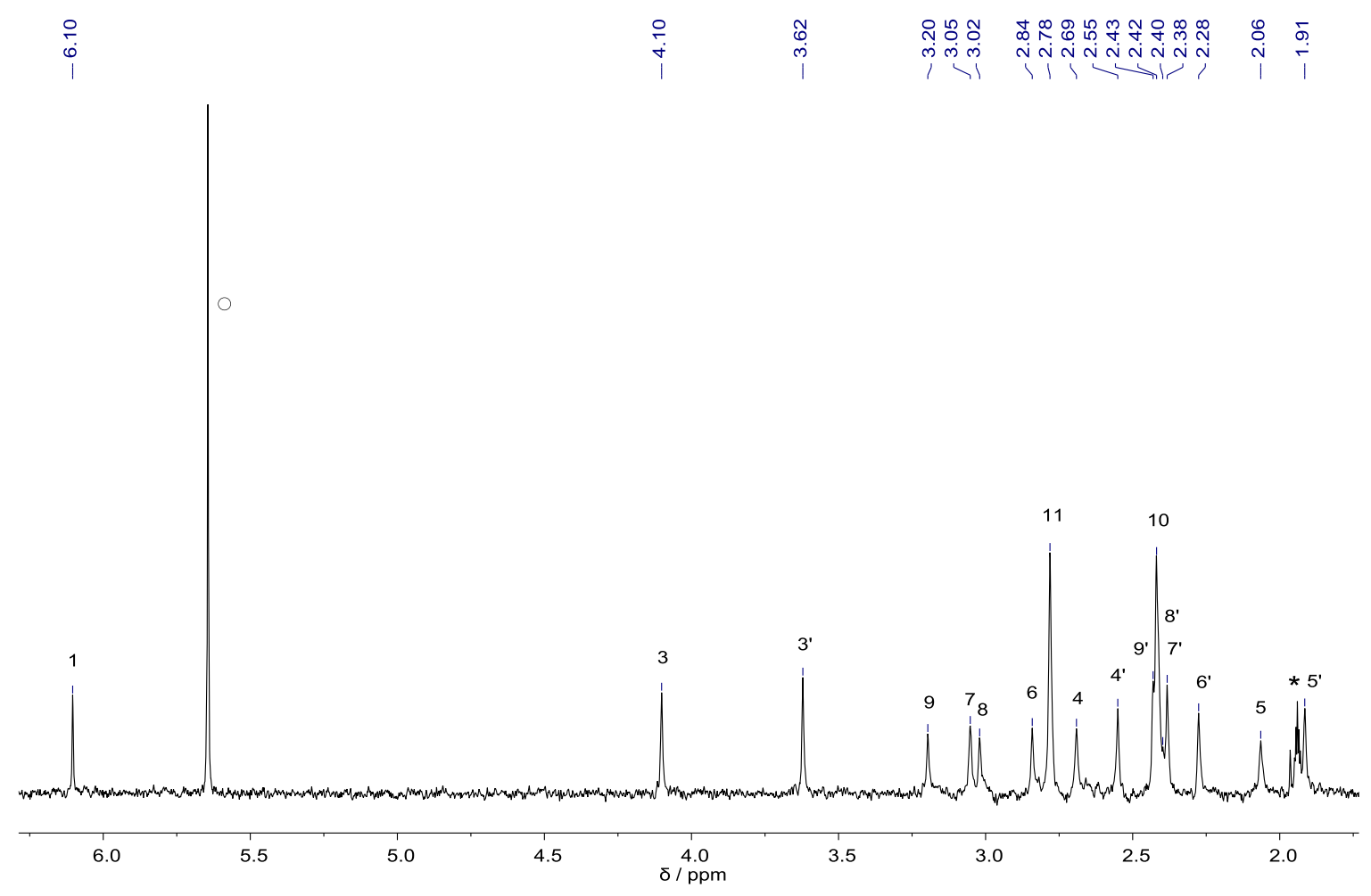

Figure A.89: Pure shift ${ }^{1} \mathrm{H}-\mathrm{NMR}$ spectrum (500 MHz) of $\mathbf{9 H}$ in $\mathrm{CD}_{3} \mathrm{CN}$ at $238 \mathrm{~K}$ under inert conditions. The peaks are assigned according to the labeling scheme above. The asterisk denotes the residual solvent peak and the circle points out $\left[\mathrm{Cp}_{2} \mathrm{Co}\right]^{+}$. Please note, the distinction between prime and non-prime is not possible and is merely done for simplification.

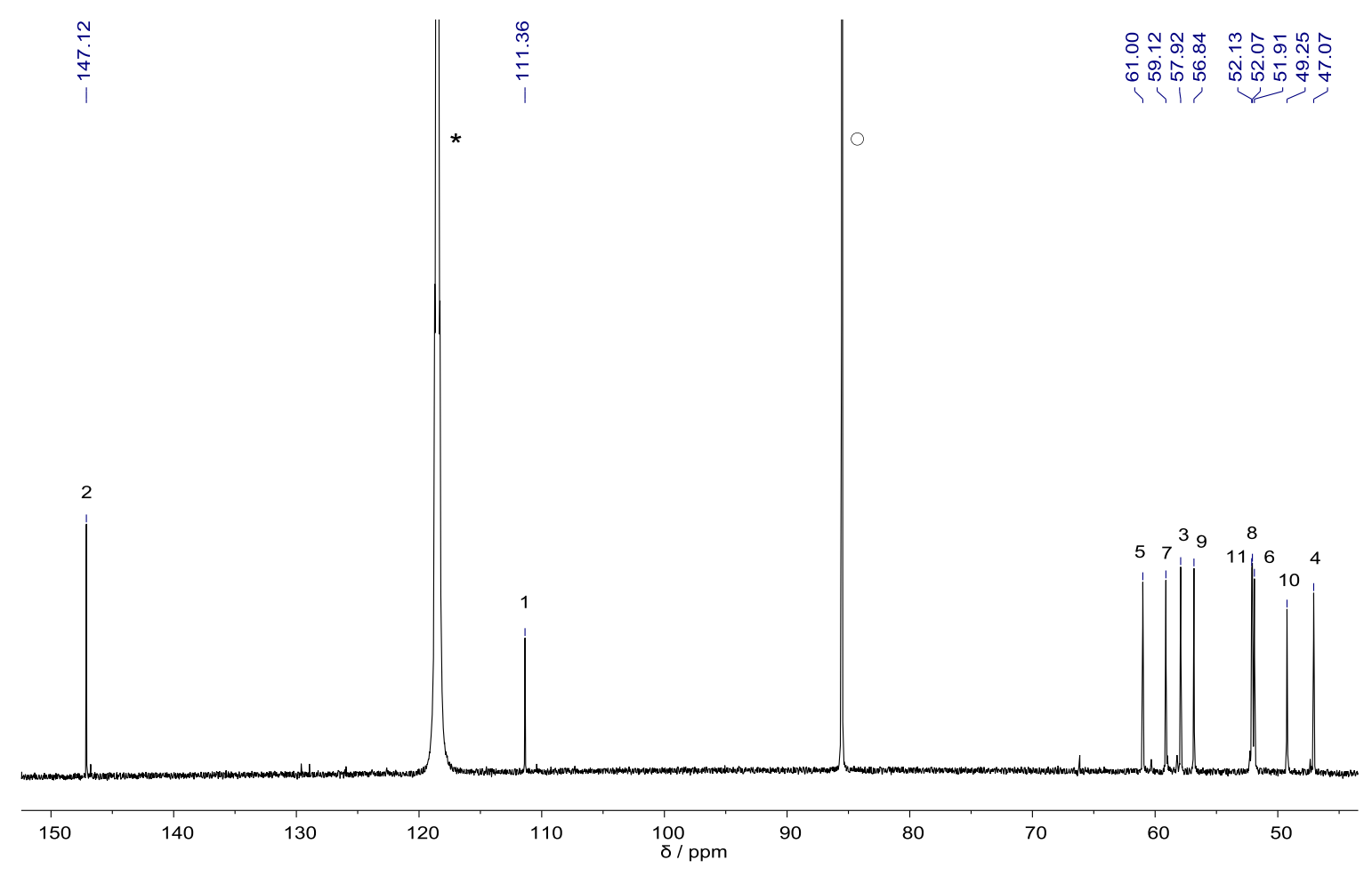

Figure A.90: ${ }^{13} \mathrm{C}\left\{{ }^{1} \mathrm{H}\right\}$-NMR spectrum $(126 \mathrm{MHz})$ of $\mathbf{9} \mathbf{H}$ in $\mathrm{CD}_{3} \mathrm{CN}$ at $238 \mathrm{~K}$ under inert conditions. The peaks are assigned according to the labeling scheme above. The asterisk denotes the residual solvent peak and the circle points out $\left[\mathrm{Cp}_{2} \mathrm{Co}\right]^{+}$. 


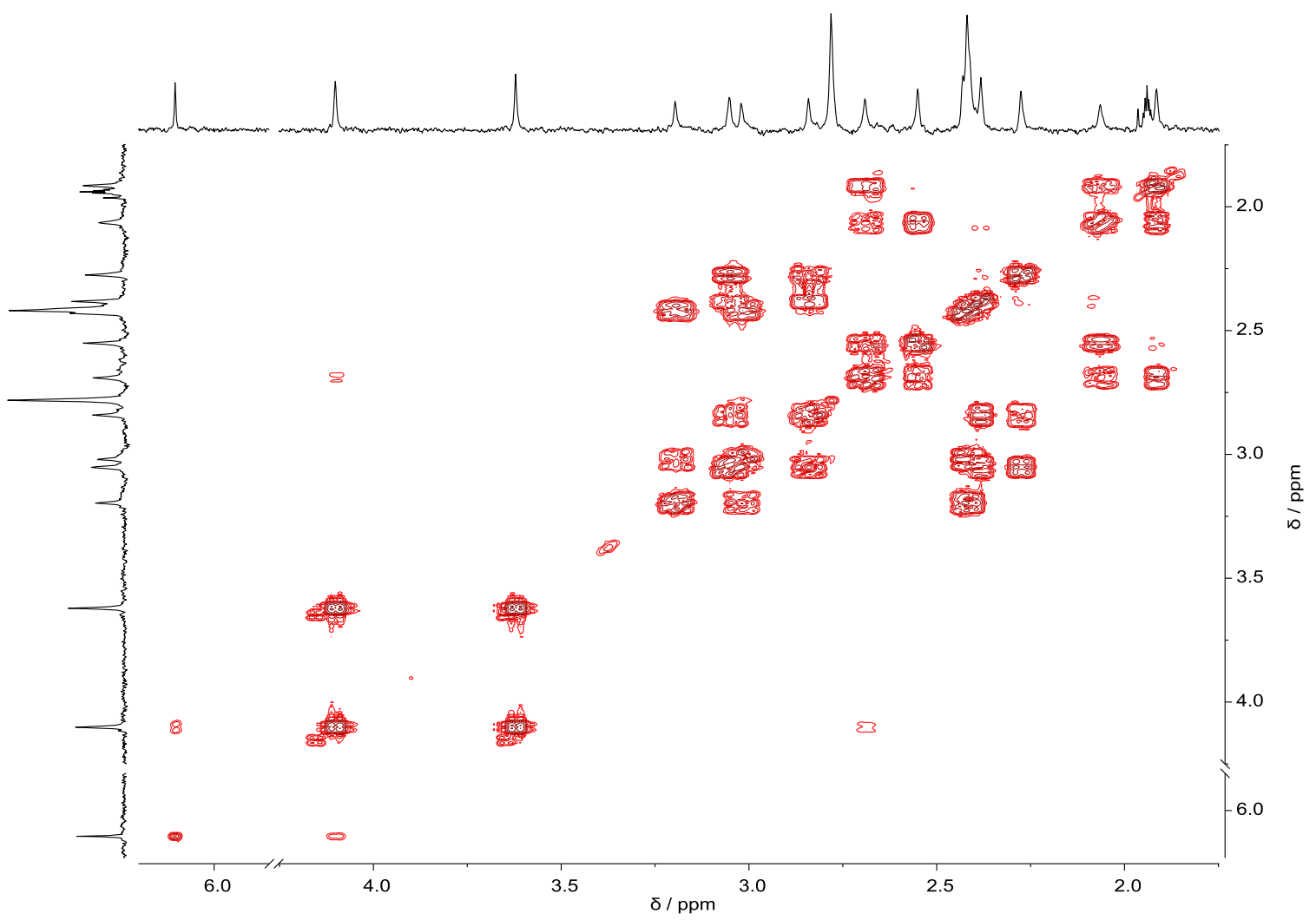

Figure A.91: ${ }^{1} \mathrm{H}-{ }^{-1} \mathrm{H}-\mathrm{COSY}$ spectrum $(500 \mathrm{MHz})$ of $\mathbf{9}^{\mathbf{H}}$ in $\mathrm{CD}_{3} \mathrm{CN}$ at $238 \mathrm{~K}$ under inert conditions. Depicted is the region from 1.8-6.2 ppm (hydrides excluded). Please note, the pure shift ${ }^{1} \mathrm{H}-\mathrm{NMR}$ is shown in both traces.

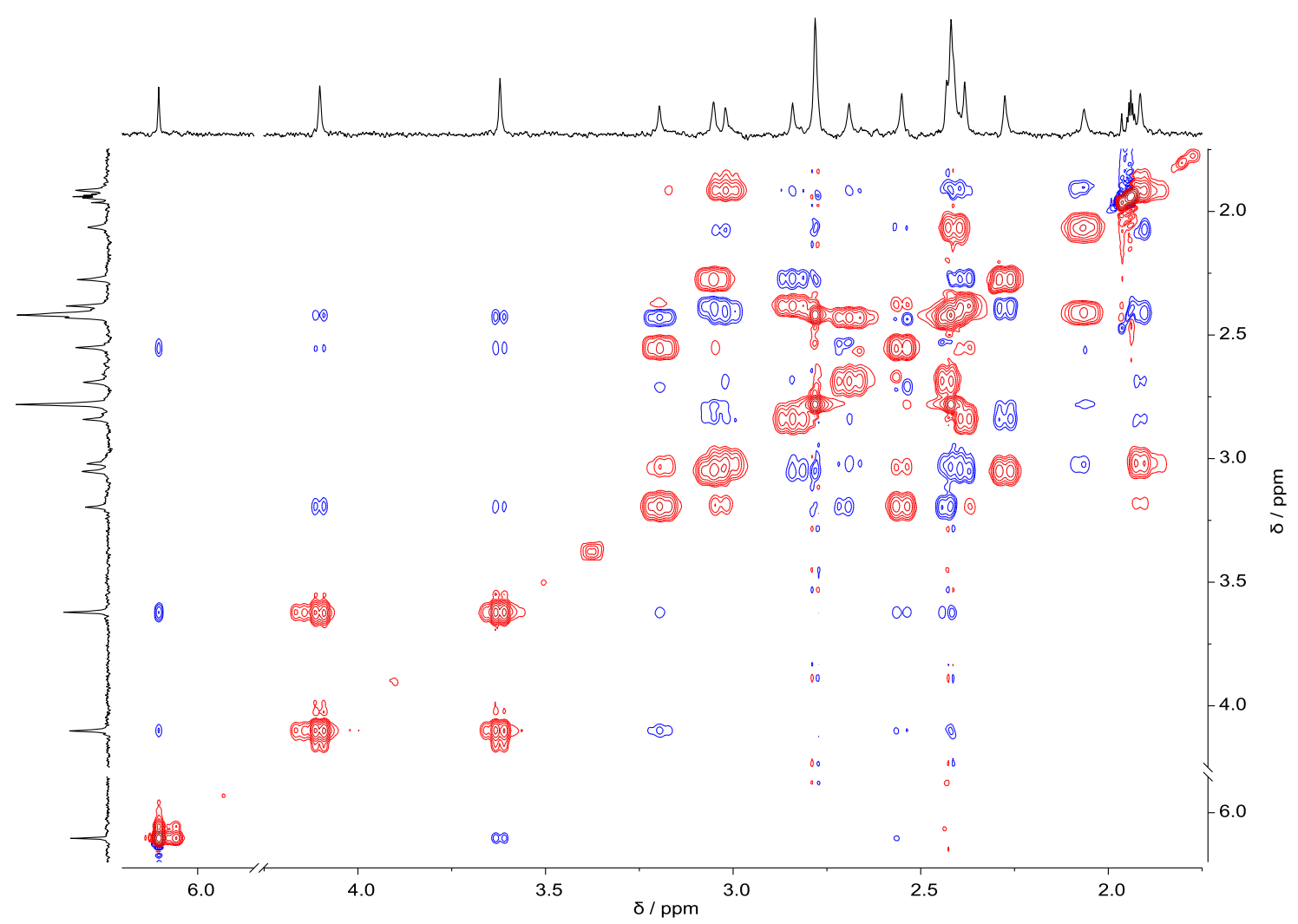

Figure A.92: ${ }^{1} \mathrm{H}-{ }^{-1} \mathrm{H}-\mathrm{NOESY}$ spectrum $(500 \mathrm{MHz})$ of $\mathbf{9}^{\mathbf{H}}$ in $\mathrm{CD}_{3} \mathrm{CN}$ at $238 \mathrm{~K}$ under inert conditions. Depicted is the region from 1.8-6.2 ppm (hydrides excluded). Please note, the pure shift ${ }^{1} \mathrm{H}-\mathrm{NMR}$ is shown in both traces. 


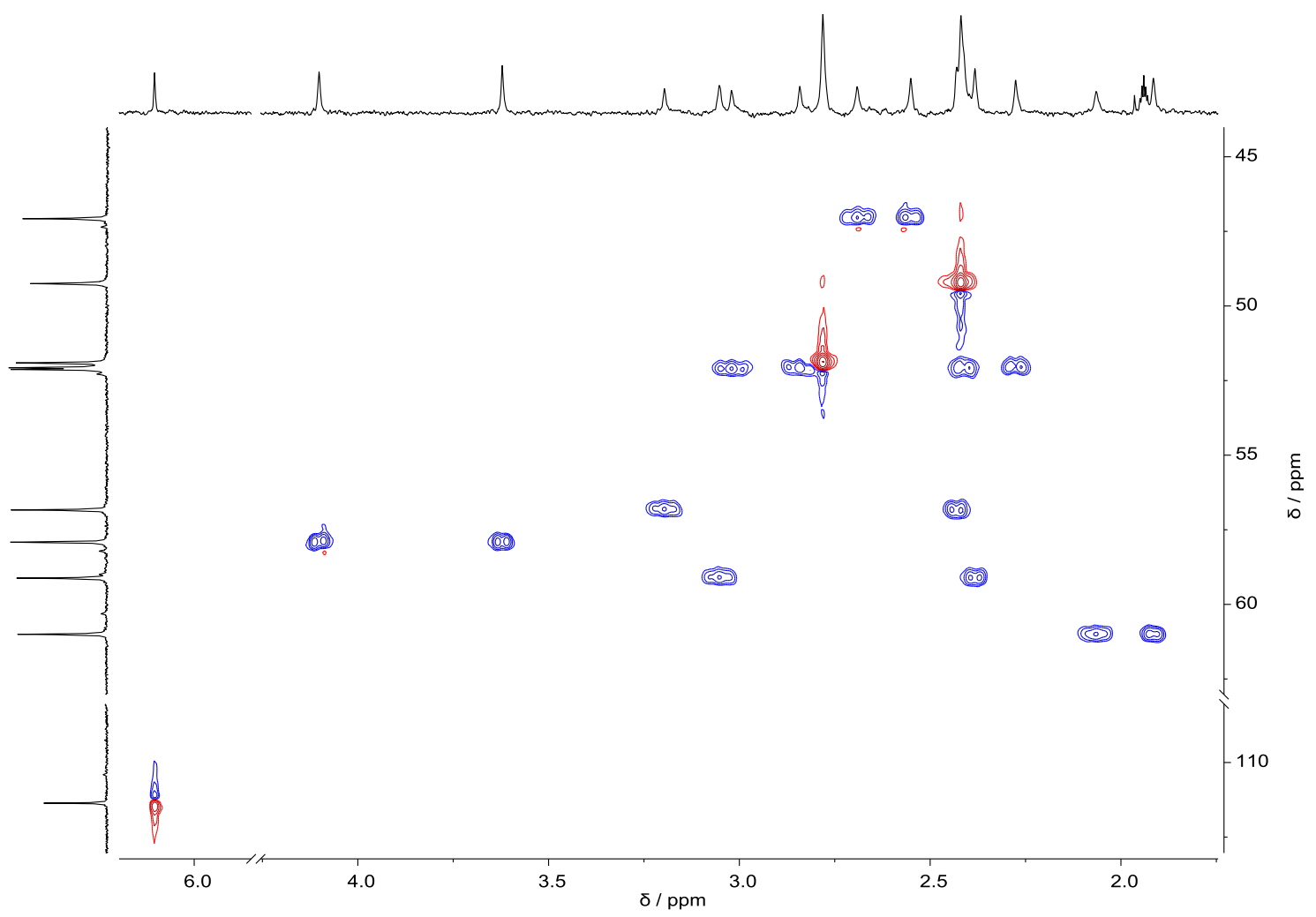

Figure A.93: ${ }^{1} \mathrm{H}-{ }^{13} \mathrm{C}\left\{{ }^{1} \mathrm{H}\right\}-\mathrm{HSQC}$ spectrum of $9^{\mathbf{H}}$ in $\mathrm{CD}_{3} \mathrm{CN}$ at $238 \mathrm{~K}$ under inert conditions. Depicted is the region from 1.8-6.2 ppm (hydrides excluded). Please note, the pure shift ${ }^{1} \mathrm{H}-\mathrm{NMR}$ is shown in the horizontal trace.

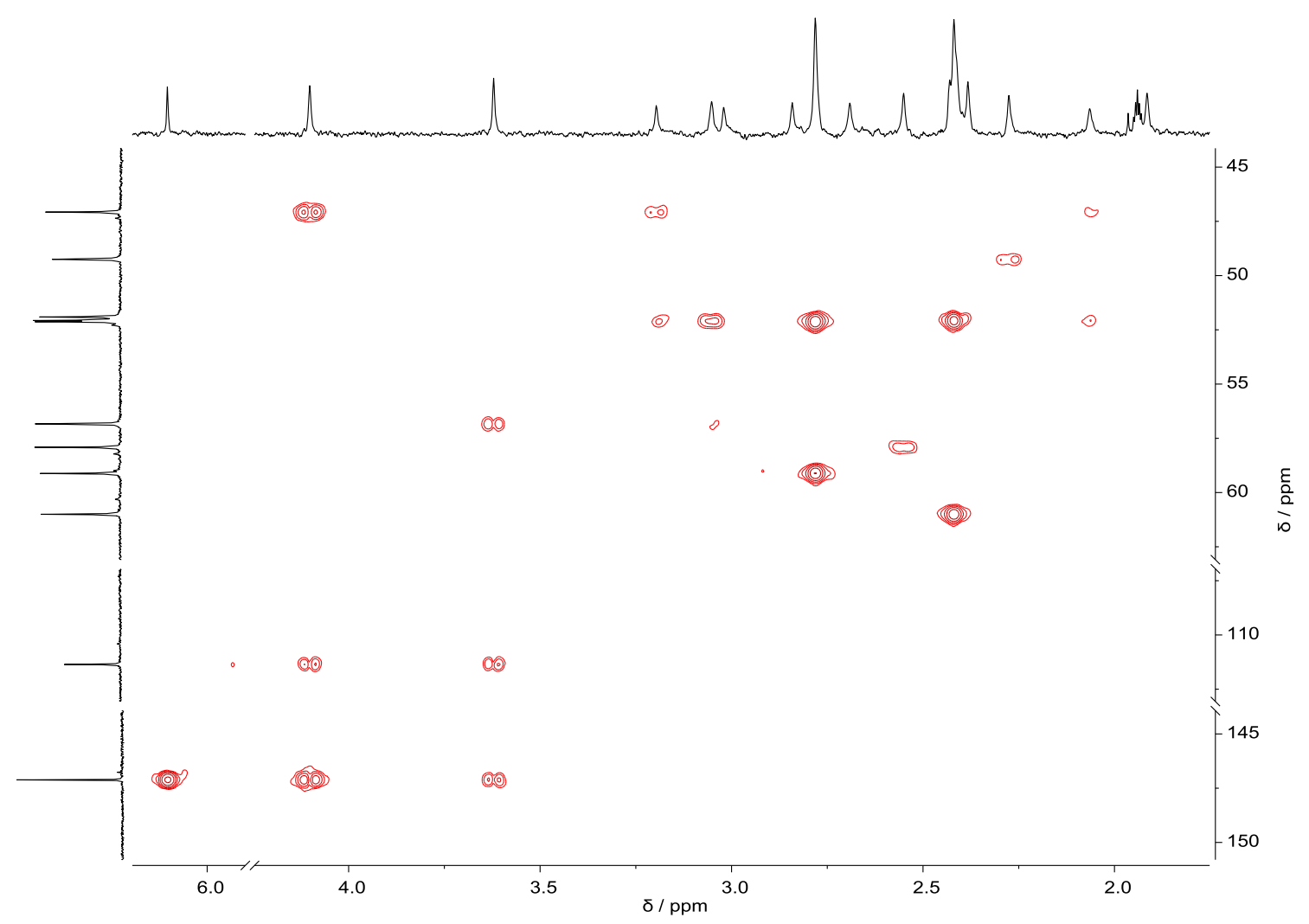

Figure A.94: ${ }^{1} \mathrm{H}^{-13} \mathrm{C}\left\{{ }^{1} \mathrm{H}\right\}-\mathrm{HMBC}$ spectrum of $\mathbf{9}^{\mathbf{H}}$ in $\mathrm{CD}_{3} \mathrm{CN}$ at $238 \mathrm{~K}$ under inert conditions. Depicted is the region from 1.8-6.2 ppm (hydrides excluded). Please note, the pure shift ${ }^{1} \mathrm{H}-\mathrm{NMR}$ is shown in the horizontal trace. 


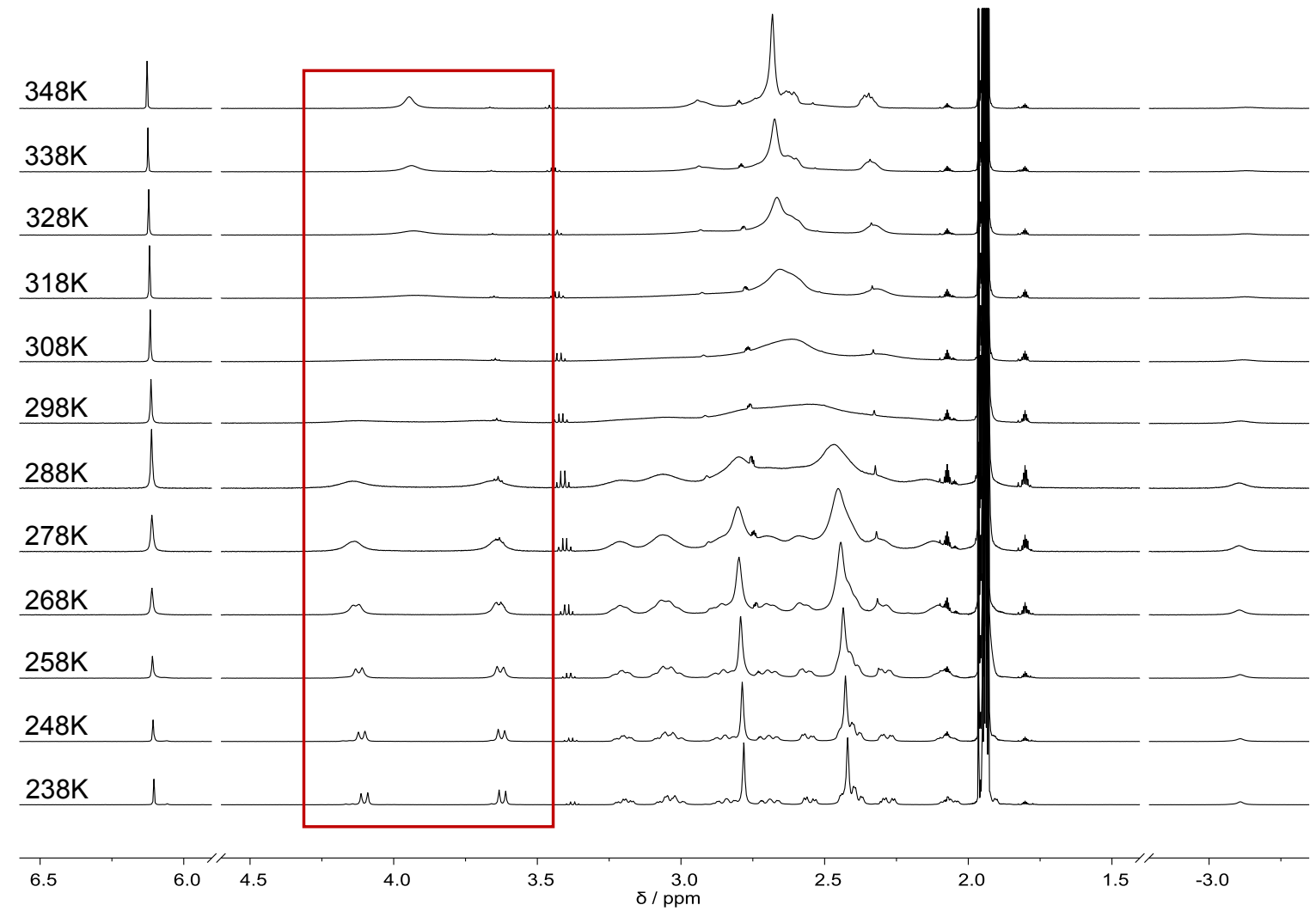

Figure A.95: Variable temperature ${ }^{1} \mathrm{H}-\mathrm{NMR}$ spectra $(500 \mathrm{MHz})$ of $\mathbf{9}^{\mathrm{H}}$ in $\mathrm{CD}_{3} \mathrm{CN}(238-348 \mathrm{~K})$ under inert conditions showing the dynamic behavior in solution. 


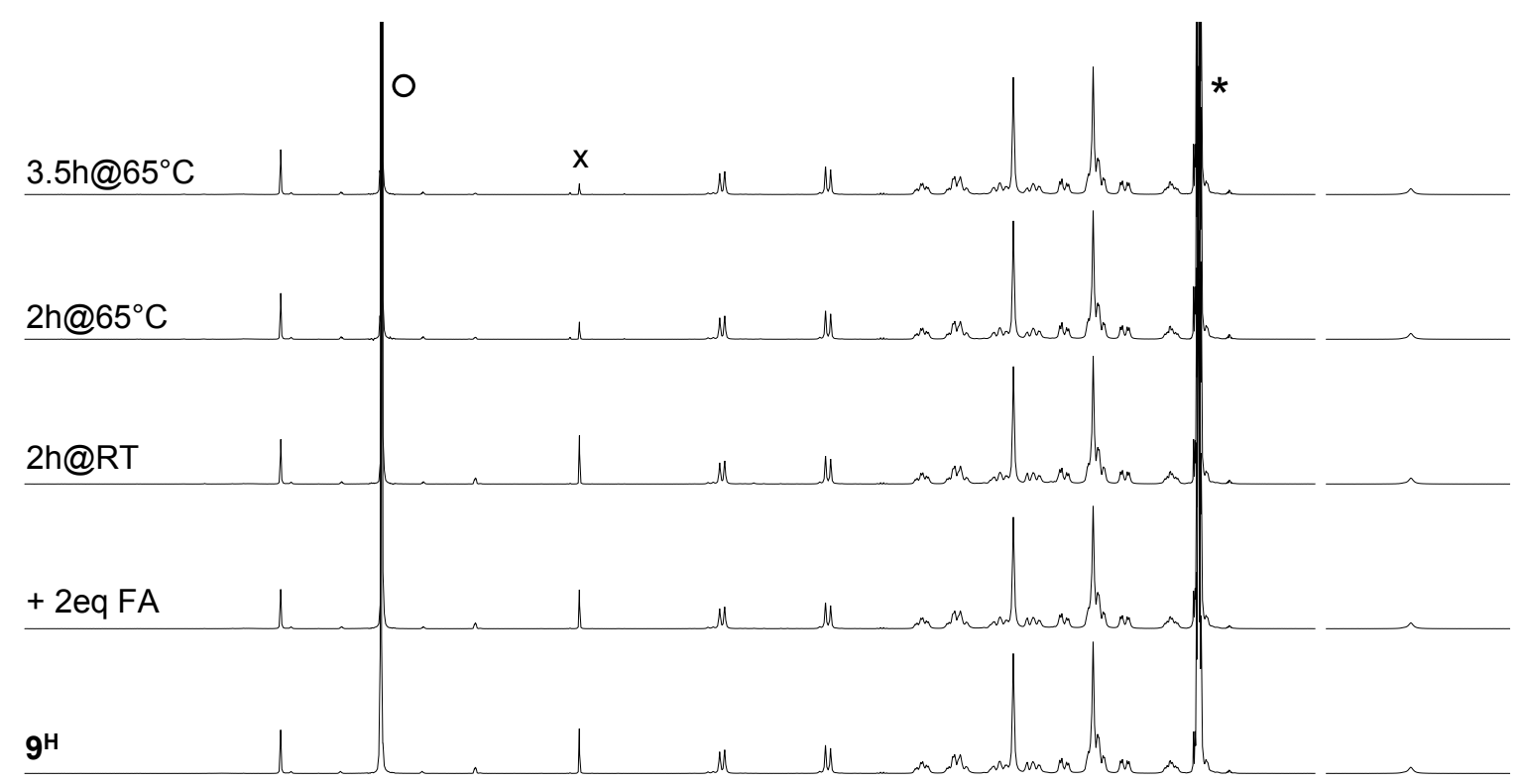

\begin{tabular}{lllllll}
\hline 7 & 6 & 5 & 4 & 3 & 2 & -3
\end{tabular}

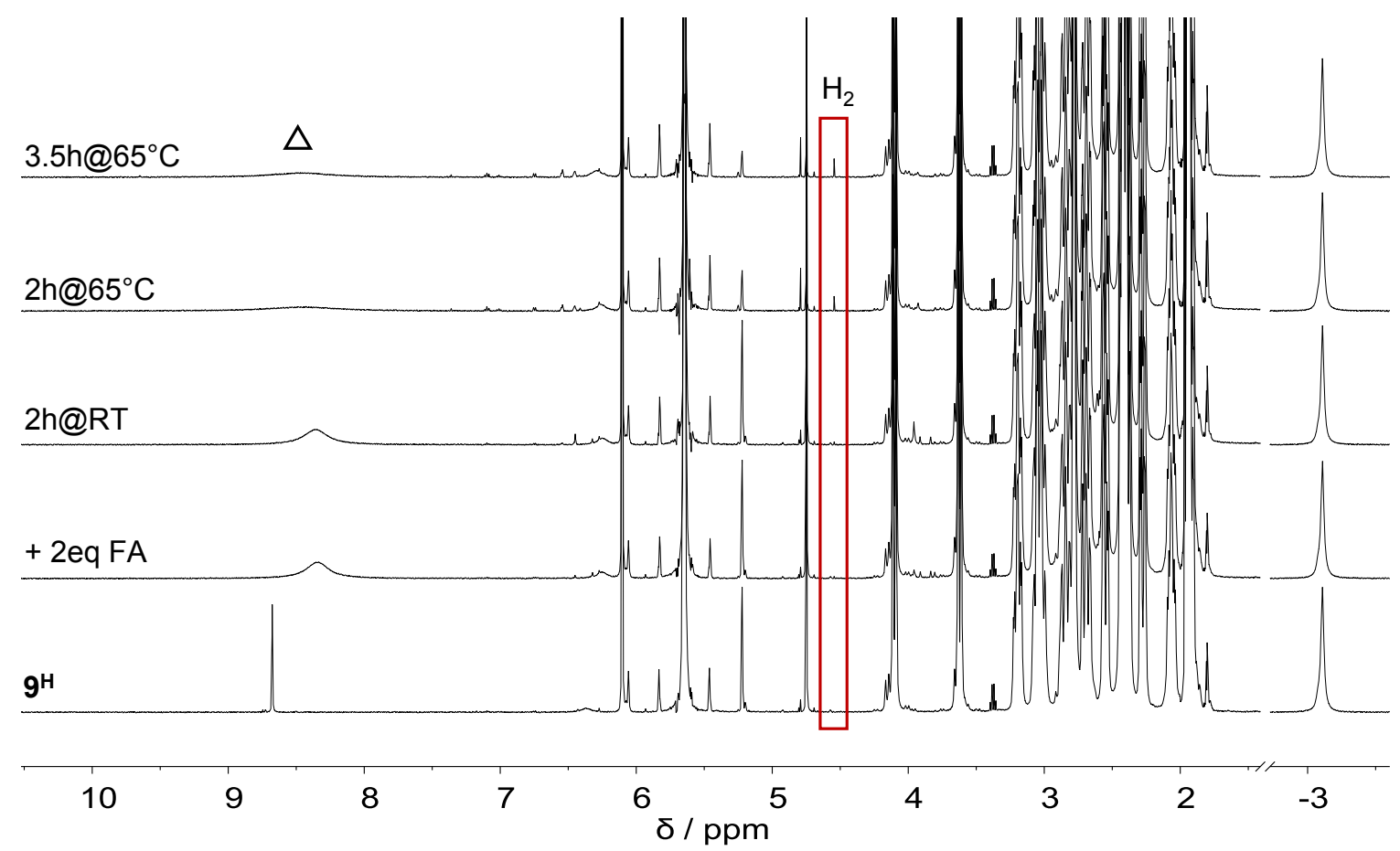

Figure A.96: ${ }^{1} \mathrm{H}-\mathrm{NMR}$ spectra $(500 \mathrm{MHz})$ at $238 \mathrm{~K}$ of $\mathbf{9 H}^{\mathrm{H}}$ before and after addition of formic acid (2 eq) and subsequent stirring for 2 hours at room temperature (RT), as well as heating for 2 and 3.5 hours at $65^{\circ} \mathrm{C}$ under inert conditions (top: (-3)-7 ppm, bottom: (-3)-10.5 ppm including the formic acid signal). The asterisk denotes the residual solvent peak and the $\mathrm{x}$ points out an impurity. The circle and triangle mark $\left[\mathrm{Cp}_{2} \mathrm{Co}\right]^{+}$and $\mathrm{HCOOH}$, respectively. 

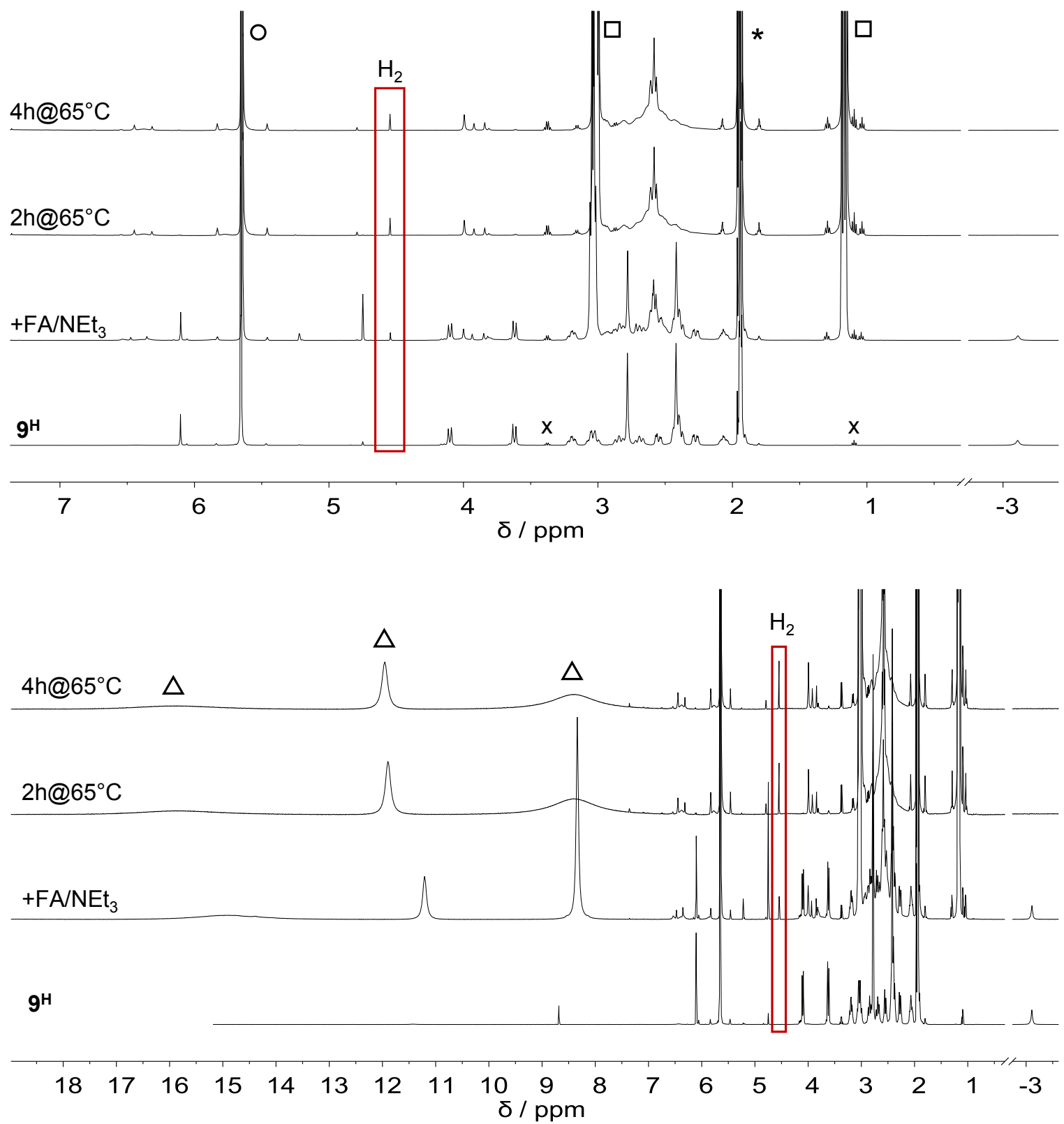

Figure A.97: ${ }^{1} \mathrm{H}-\mathrm{NMR}$ spectra (500 MHz) at $238 \mathrm{~K} \mathrm{of} \mathbf{9}^{\mathrm{H}}$ before and after addition of formic acid (26 eq) and $\mathrm{NEt}_{3}$ (10 eq) and subsequent heating for 2 and 4 hours at $65^{\circ} \mathrm{C}$ under inert conditions (top: (-3)-7 ppm, bottom: (-3)$18 \mathrm{ppm}$ including the formic acid signals). The asterisk denotes the residual solvent peak and the x point out $\mathrm{Et}_{2} \mathrm{O}$. The circle, squares and triangles mark $\left[\mathrm{Cp}_{2} \mathrm{Co}^{+}, \mathrm{NEt}_{3}\right.$ and $\mathrm{HCOOH}$, respectively. 


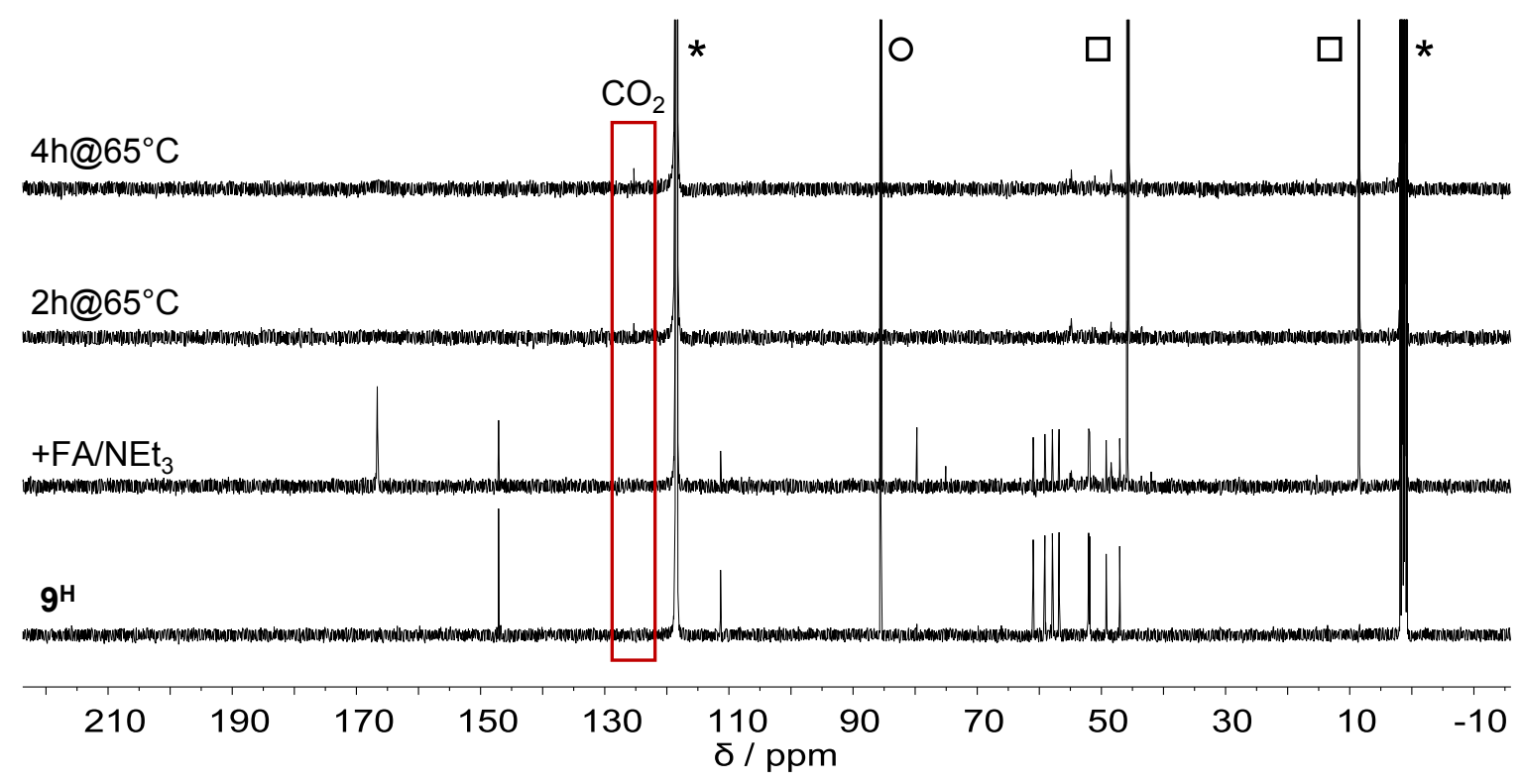

Figure A.98: ${ }^{13} \mathrm{C}$-NMR spectra (126 MHz) at $238 \mathrm{~K}$ of $\mathbf{9 H}^{\mathbf{H}}$ before and after addition of formic acid (26 eq) and $\mathrm{NEt}_{3}$ (10 eq) and subsequent heating for 2 and 4 hours at $65^{\circ} \mathrm{C}$ under inert conditions. The asterisks denote the residual solvent peak, and the circle and squares mark $\left[\mathrm{Cp}_{2} \mathrm{Co}\right]^{+}$and $\mathrm{NEt}_{3}$, respectively. 


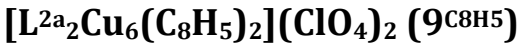
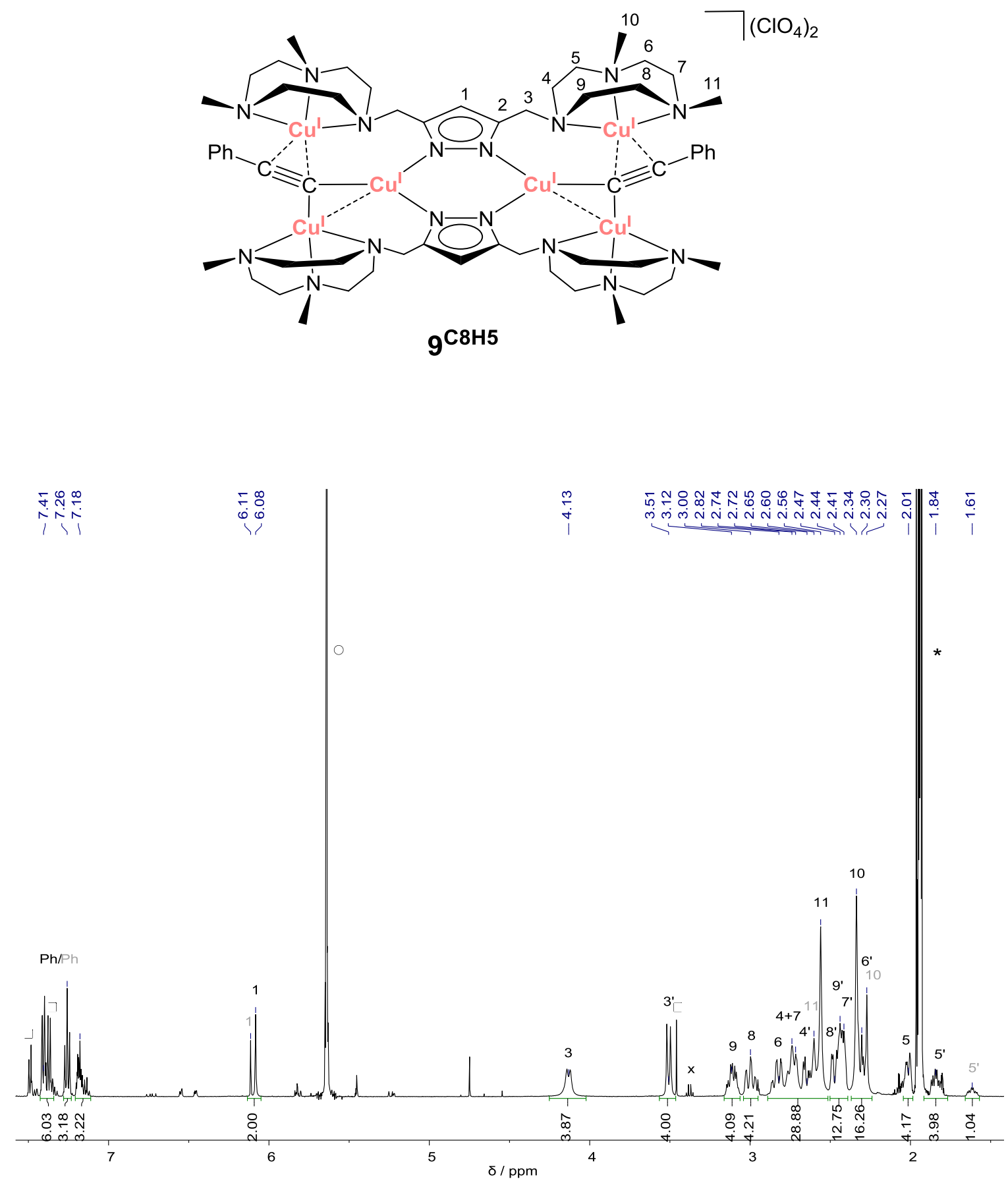

Figure A.99: ${ }^{1} \mathrm{H}-\mathrm{NMR}$ spectrum (500 MHz) of $\mathbf{9} 8 \mathbf{3} \mathbf{5}$ in $\mathrm{CD}_{3} \mathrm{CN}$ at $238 \mathrm{~K}$ under inert conditions. The peaks are assigned according to the labeling scheme above. The signals labeled in grey are those of a second isomer (ratio $3: 1$ ). The asterisk denotes the residual solvent peak and the $x$ points out $\mathrm{Et}_{2} \mathrm{O}$. The circle and squares mark $\left[\mathrm{Cp}_{2} \mathrm{Co}^{+}\right.$ and free phenyl acetylene, respectively. Please note, the distinction between prime and non-prime is not possible and is merely done for simplification. 

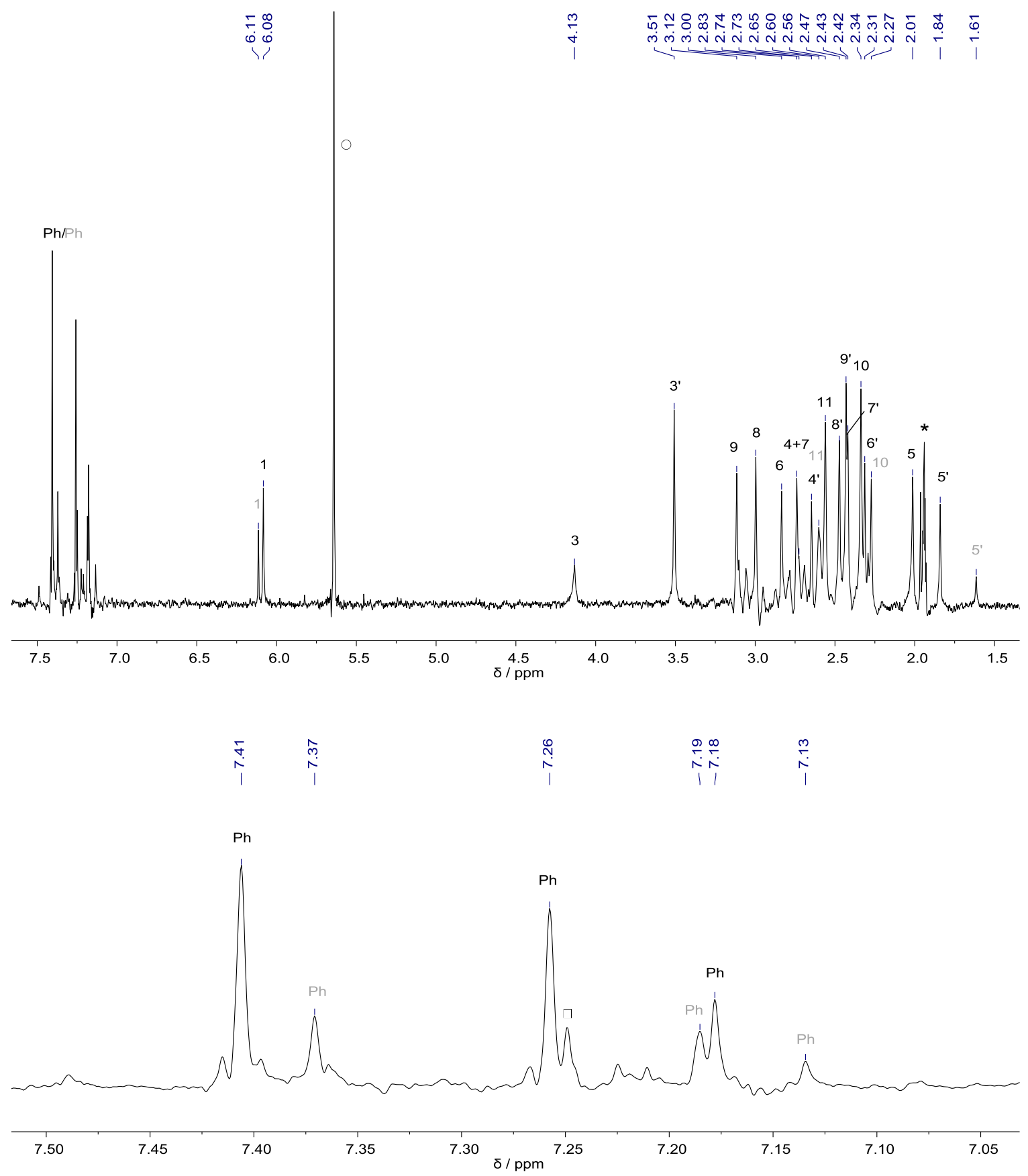

Figure A.100: Pure shift ${ }^{1} \mathrm{H}-\mathrm{NMR}$ spectrum $(500 \mathrm{MHz})$ of $\mathbf{9}$ (c8H5 in $\mathrm{CD}_{3} \mathrm{CN}$ at $238 \mathrm{~K}$ under inert conditions. Top: complete area with complex signals (1.5-7.5 ppm), bottom: enlargement of the area from 7.05-7.50 ppm. The peaks are assigned according to the labeling scheme above. The signals labeled in grey are those of a second isomer (ratio 3:1). The asterisk denotes the residual solvent peak, the circle points out [ $\left.\mathrm{Cp}_{2} \mathrm{Co}\right]^{+}$and the square marks free phenyl acetylene. Please note, the distinction between prime and non-prime is not possible and is merely done for simplification. 


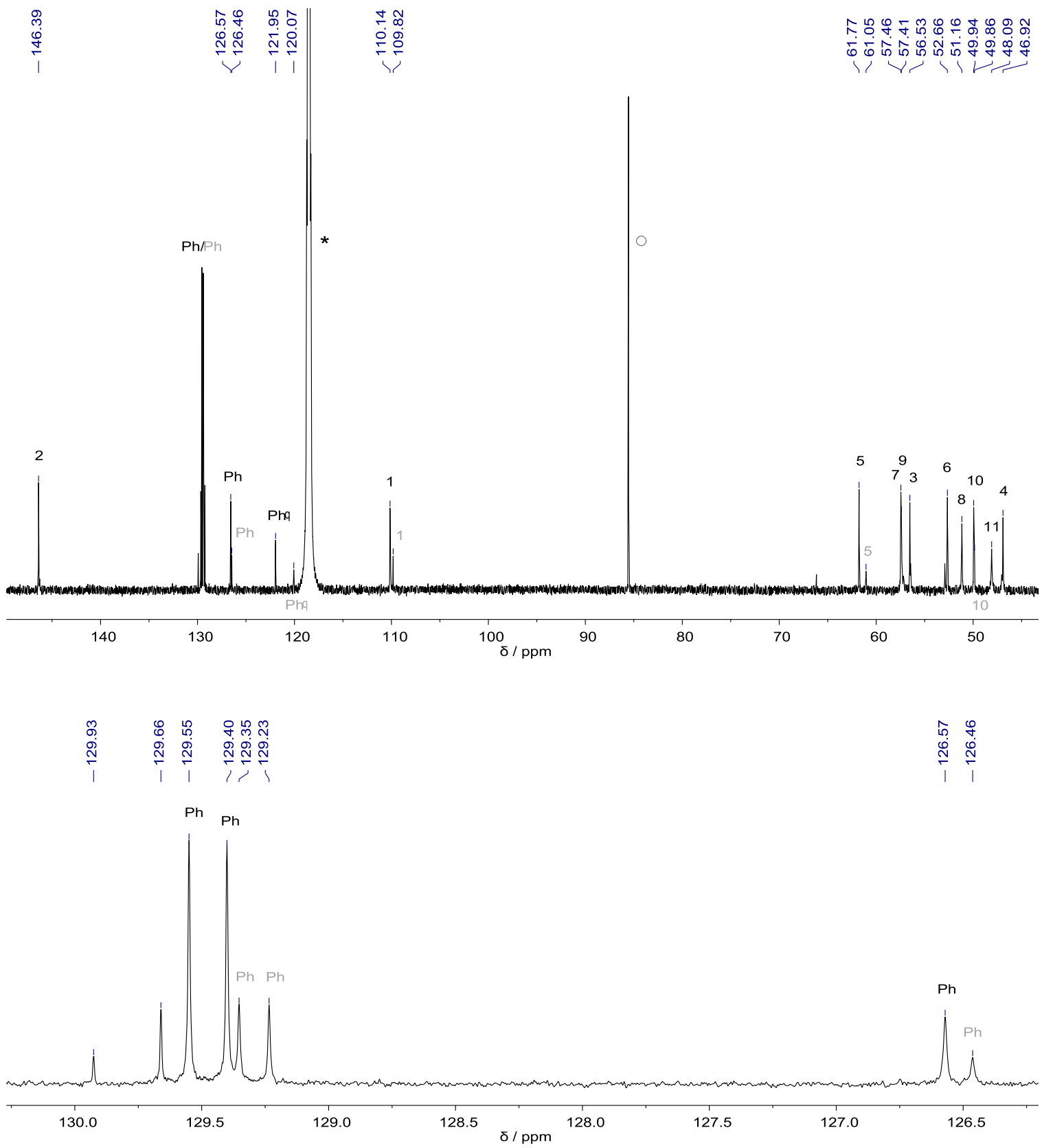

Figure A.101: ${ }^{13} \mathrm{C}\left\{{ }^{1} \mathrm{H}\right\}$-NMR spectrum (126 MHz) of $\mathbf{9} 8 \mathbf{8} \mathbf{H} 5$ in $\mathrm{CD}_{3} \mathrm{CN}$ at $238 \mathrm{~K}$ under inert conditions. Top: complete region with complex signals (45-150 ppm), bottom: enlargement of the area from 126-131 ppm. The peaks are assigned according to the labeling scheme above. The signals labeled in grey are those of a second isomer (ratio $3: 1$ ). The asterisk denotes the residual solvent peak and the circle points out $\left[\mathrm{Cp}_{2} \mathrm{Co}\right]^{+}$. Please note, not all signals of the phenyl acetylene could be distinguished and the peaks at 129.66 and 129.93 ppm could not be assigned. 


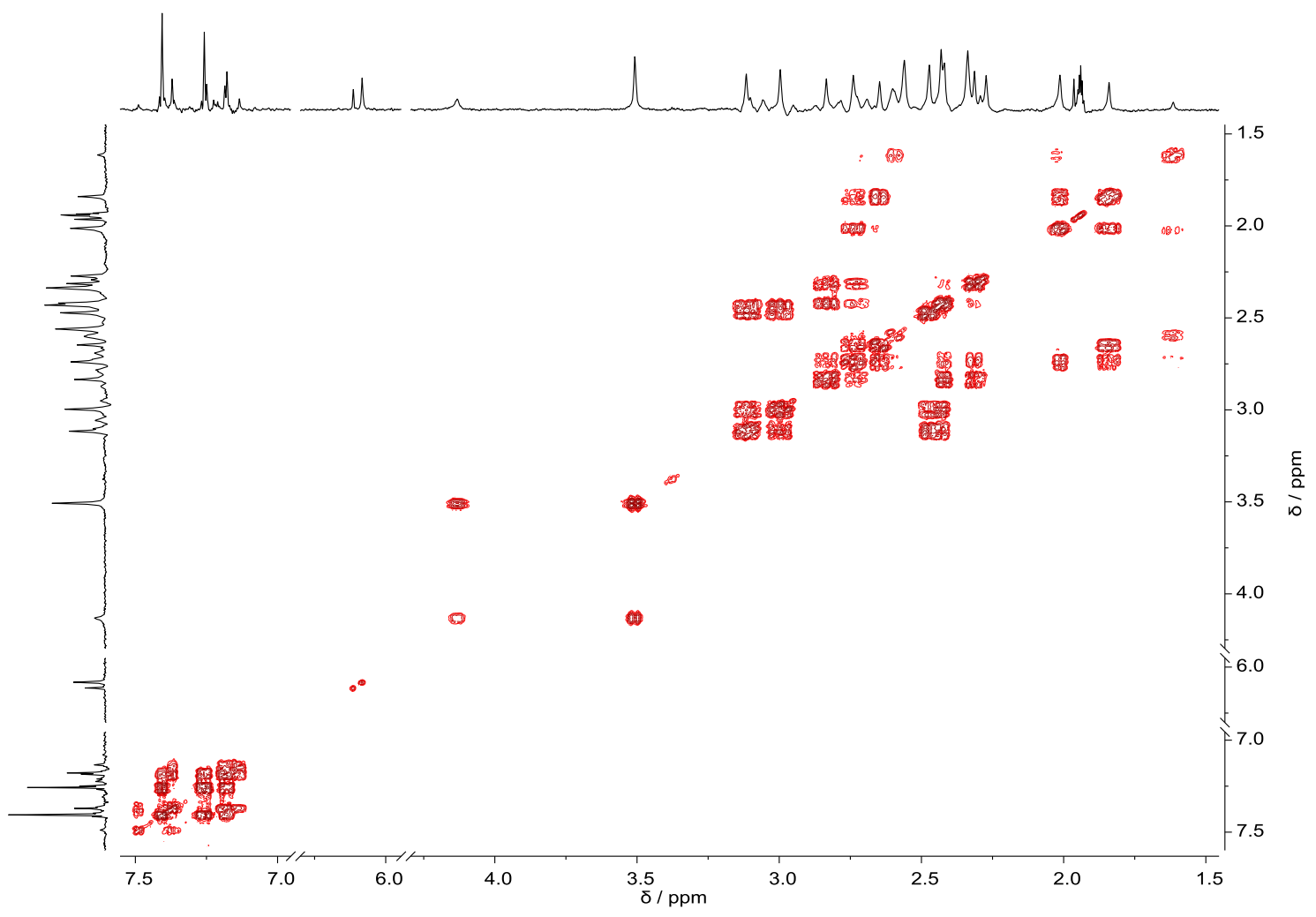

Figure A.102: ${ }^{1} \mathrm{H}-1 \mathrm{H}-\mathrm{COSY}$ spectrum $(500 \mathrm{MHz})$ of $\mathbf{9}^{\mathbf{c} 8 \mathrm{H} 5}$ in $\mathrm{CD}_{3} \mathrm{CN}$ at $238 \mathrm{~K}$ under inert conditions. Depicted is the full region with complex signals (1.5-7.5 ppm). Please note, the pure shift ${ }^{1} \mathrm{H}-\mathrm{NMR}$ is shown in both traces.

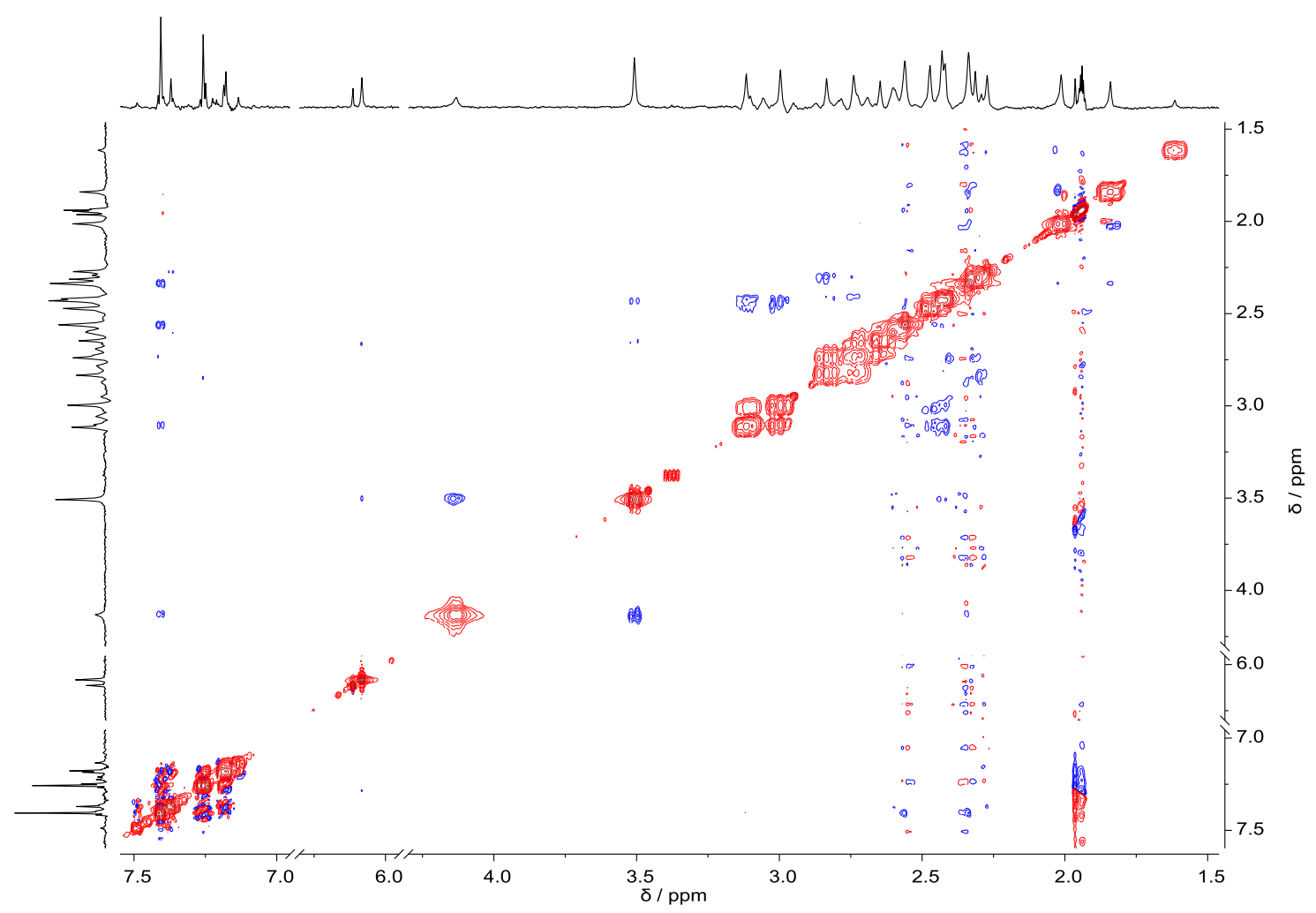

Figure A.103: ${ }^{1} \mathrm{H}^{1} \mathrm{H}-\mathrm{NOESY}$ spectrum $(500 \mathrm{MHz})$ of $\mathbf{9}^{\mathbf{C} 8 \mathrm{H} 5}$ in $\mathrm{CD}_{3} \mathrm{CN}$ at $238 \mathrm{~K}$ under inert conditions. Depicted is the full region with complex signals (1.5-7.5 ppm). Please note, the pure shift ${ }^{1} \mathrm{H}-\mathrm{NMR}$ is shown in both traces. 

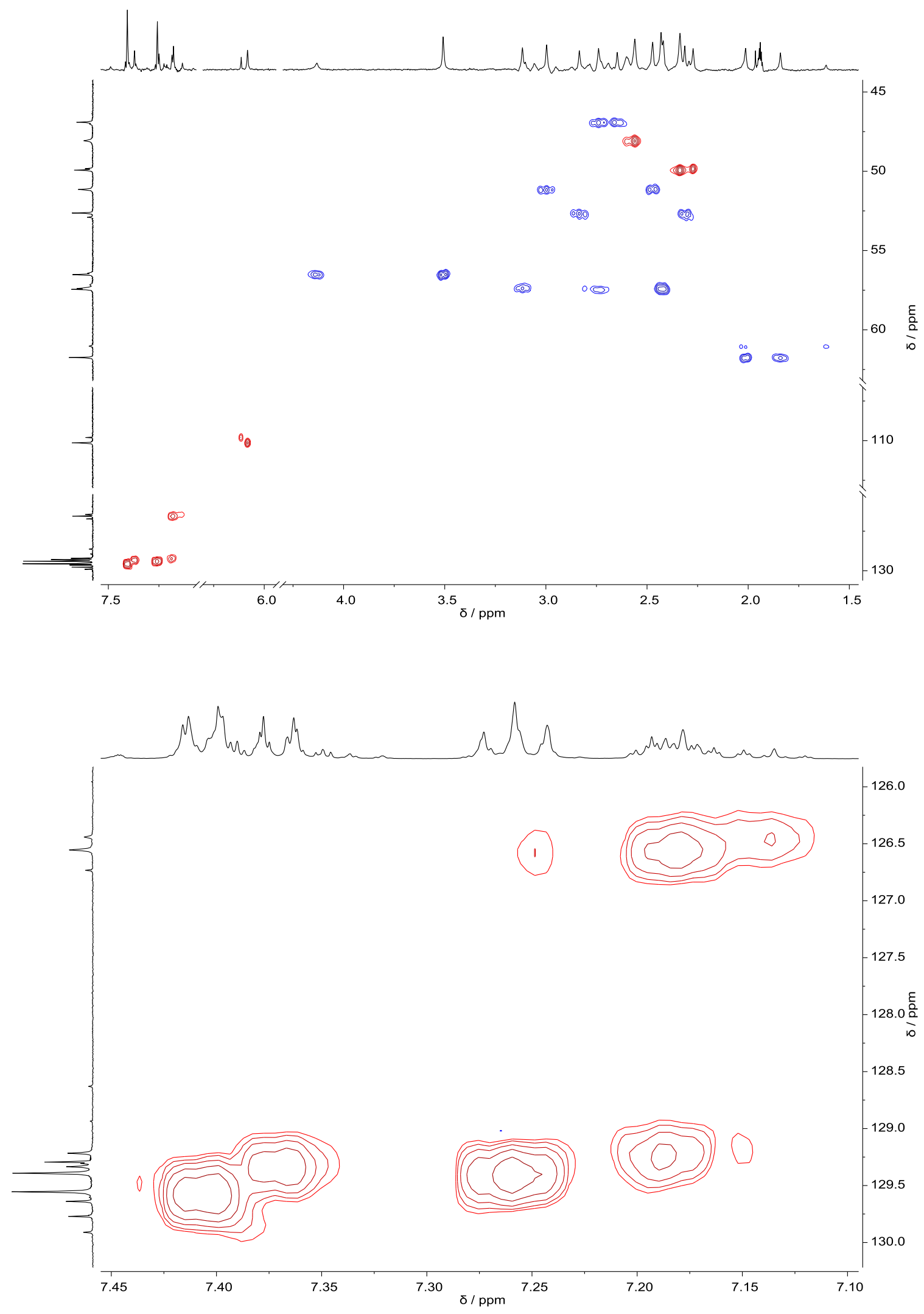

Figure A.104: ${ }^{1} \mathrm{H}-{ }^{13} \mathrm{C}\left\{{ }^{1} \mathrm{H}\right\}-\mathrm{HSQC}$ spectrum of ${ }^{9} \mathrm{C} 8 \mathrm{H} 5 \mathrm{in} \mathrm{CD}_{3} \mathrm{CN}$ at $238 \mathrm{~K}$ under inert conditions. Top: complete region with complex signals (1.5-7.5 ppm), bottom: enlargement of the area from 7.10-7.45 ppm. Please note, in the top spectrum the pure shift ${ }^{1} \mathrm{H}-\mathrm{NMR}$ is shown in the horizontal trace. 

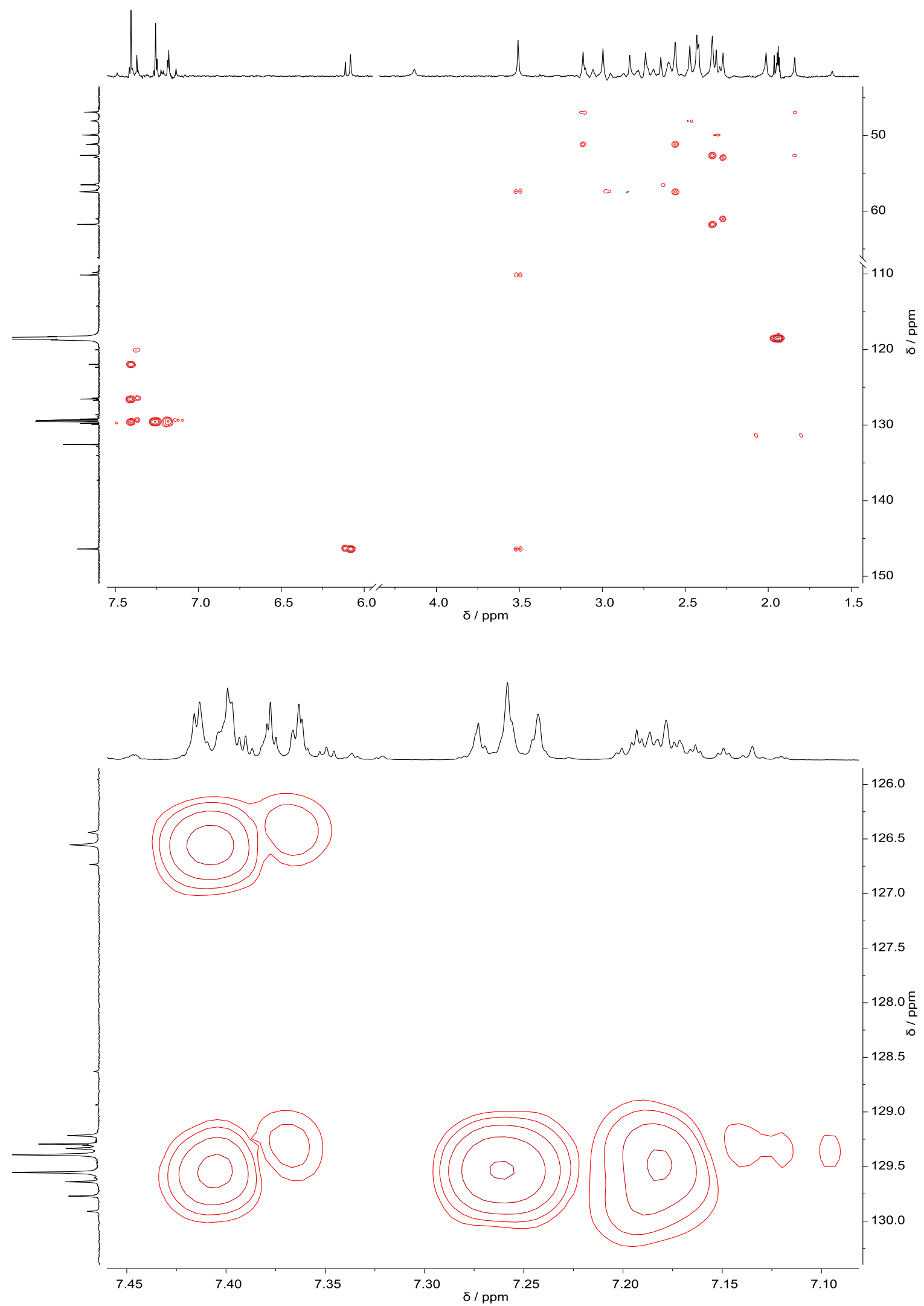

Figure A.105: ${ }^{1} \mathrm{H}-13 \mathrm{C}\left\{{ }^{1} \mathrm{H}\right\}$ - $\mathrm{HMBC}$ spectrum of $\mathbf{9}^{\mathrm{C} 8 \mathrm{H} 5}$ in $\mathrm{CD}_{3} \mathrm{CN}$ at $238 \mathrm{~K}$ under inert conditions. Top: complete region with complex signals (1.5-7.5 ppm), bottom: enlargement of the area from 7.10-7.45 ppm. Please note, in the top spectrum the pure shift ${ }^{1} \mathrm{H}-\mathrm{NMR}$ is shown in the horizontal trace. 


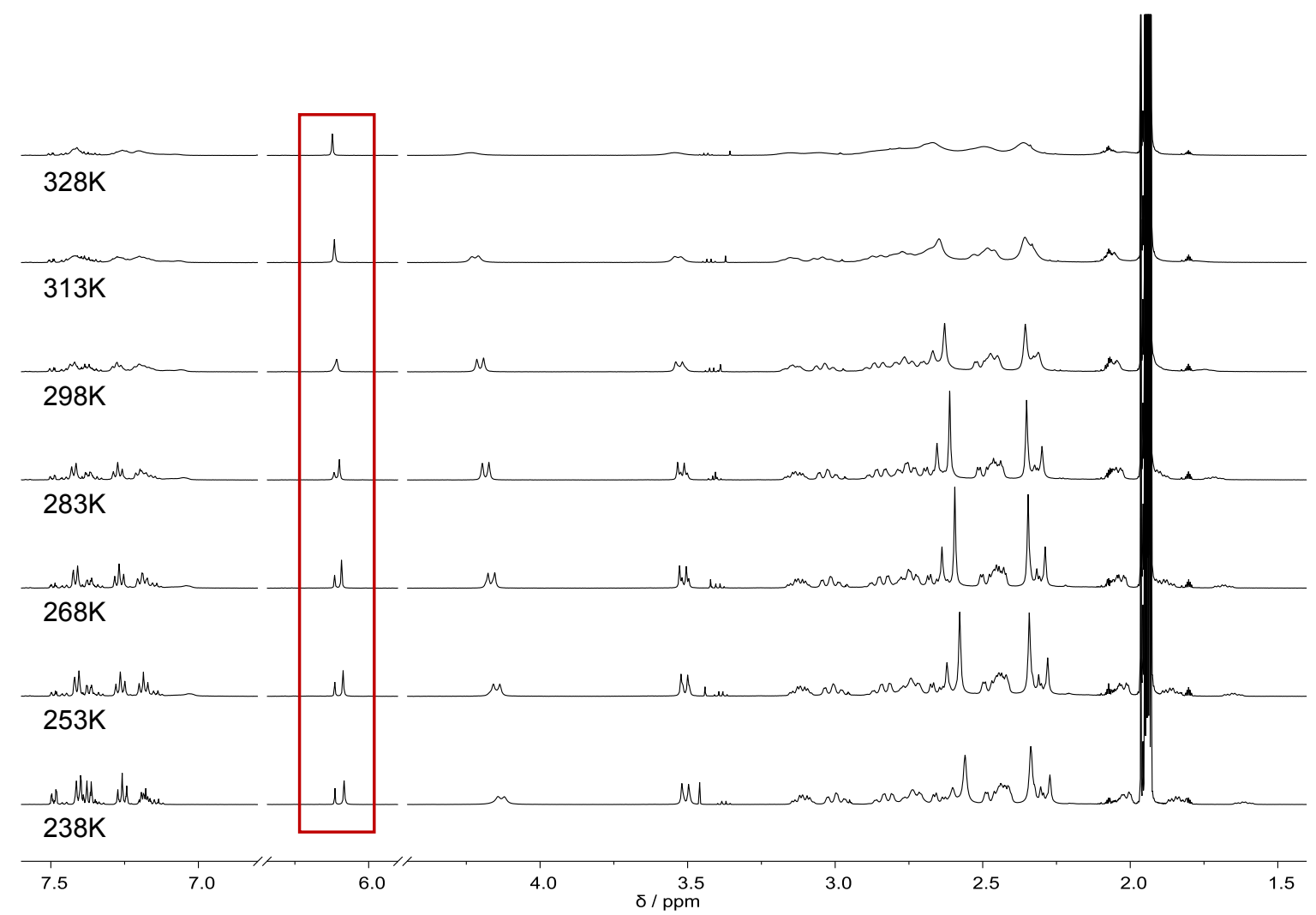

Figure A.106: Variable temperature ${ }^{1} \mathrm{H}-\mathrm{NMR}$ spectra $(500 \mathrm{MHz})$ of $\mathbf{9}^{\mathrm{C} 8 \mathrm{H} 5}$ in $\mathrm{CD}_{3} \mathrm{CN}(238-328 \mathrm{~K})$ under inert conditions showing the dynamic behavior in solution. 

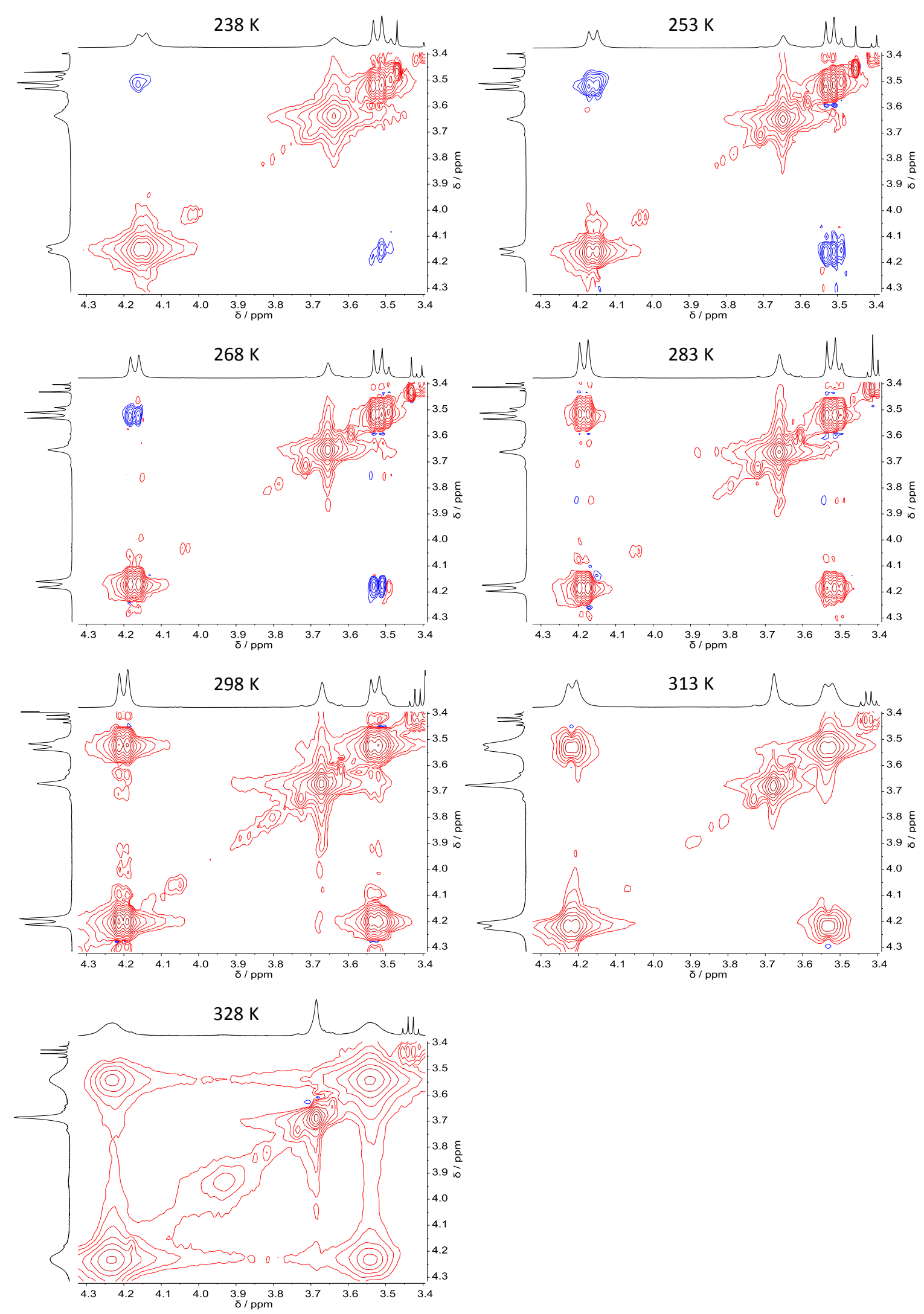

Figure A.107: Variable temperature ${ }^{1} \mathrm{H}-1 \mathrm{H}-\mathrm{NOESY}$ spectra $(500 \mathrm{MHz})$ of $\mathbf{9} \mathbf{c} \mathbf{8} \mathbf{5}$ in $\mathrm{CD}_{3} \mathrm{CN}$ under inert conditions (238-328 K). Depicted is region showing the diastereotopic $\mathrm{H}$ atoms of the methylene linker 3/3' (3.4-4.3 ppm). Until $268 \mathrm{~K}$, the cross peaks are dominated by the nuclear Overhauser effect (NOE), from $283 \mathrm{~K}$ onwards the exchange effect outweighs the NOE. 


\section{X-ray Crystallography}

\section{$\left[\mathrm{L}^{1} \mathrm{Ru}_{2}(\mathrm{dmso})_{2}(\mathrm{Cl})\left(\mathrm{H}_{2} \mathrm{O}\right)(\mu-\mathrm{dmso})\right](1)$}

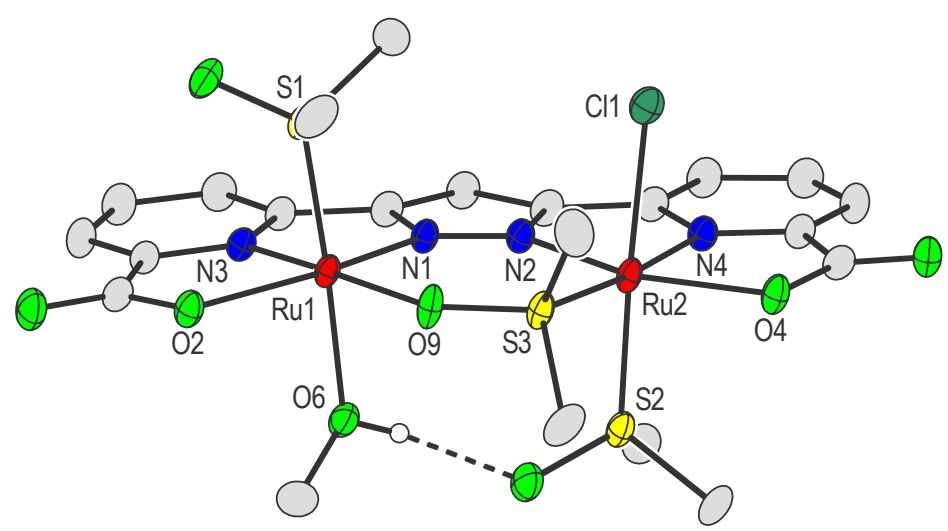

Figure A.108: Molecular structure of 1' drawn as thermal ellipsoids at the $30 \%$ probability level (ruthenium: red, nitrogen: blue, oxygen: light green, sulfur: yellow, chloride: dark green, carbon: grey, hydrogen: white). All hydrogen atoms (except those involved in intramolecular hydrogen bonding) and additional solvent molecules are omitted for clarity reasons. Please note, during crystallization the $\mathrm{H}_{2} \mathrm{O}$ molecule in $\mathbf{1}$ was exchanged for $\mathrm{MeOH}$ in $\mathbf{1}^{\prime}$.

Table A.1: Crystal data and refinement details for $\mathbf{1}$.

\begin{tabular}{|c|c|}
\hline empirical formula & $\mathrm{C}_{26} \mathrm{H}_{45} \mathrm{ClN}_{4} \mathrm{O}_{12} \mathrm{Ru}_{2} \mathrm{~S}_{3}$ \\
\hline formula weight & 939.43 \\
\hline$T / \mathrm{K}$ & $133(2)$ \\
\hline crystal size $/ \mathrm{mm}^{3}$ & $0.460 \times 0.360 \times 0.220$ \\
\hline crystal system & monoclinic \\
\hline space group & $\mathrm{Cc}$ \\
\hline$a / \AA$ & $9.4156(4)$ \\
\hline$b / \AA$ & $20.0294(6)$ \\
\hline$c / \AA$ & $19.4324(8)$ \\
\hline$\alpha /{ }^{\circ}$ & 90 \\
\hline$\beta /{ }^{\circ}$ & $92.842(3)$ \\
\hline$\gamma /{ }^{\circ}$ & 90 \\
\hline$V / \AA^{3}$ & $3660.2(2)$ \\
\hline$Z$ & 4 \\
\hline$\rho / \mathrm{g} \cdot \mathrm{cm}^{-3}$ & 1.705 \\
\hline$\mu / \mathrm{mm}^{-1}$ & 1.131 \\
\hline$F(000)$ & 1912 \\
\hline$T_{\min } / T_{\max }$ & $0.6096 / 0.8335$ \\
\hline \multirow[t]{2}{*}{$\Theta$-range $/^{\circ}$} & $2.034-26.738$ \\
\hline & $-11 \leq h \leq 11$ \\
\hline \multirow[t]{2}{*}{$h k l$-range } & $-25 \leq k \leq 24$ \\
\hline & $-24 \leq l \leq 24$ \\
\hline measured reflections & 24625 \\
\hline unique reflections $\left[R_{\text {int }}\right]$ & $7726[0.0264]$ \\
\hline
\end{tabular}


data / restraints / parameters

goodness-of-fit $\left(F^{2}\right)$

$R 1, w R 2(I>2 \sigma(I))$

$R 1, w R 2$ (all data)

residual electron density / $\mathrm{e} \cdot \AA^{3}$
$7726 / 9 / 467$

1.047

$0.0180,0.0440$

$0.0189,0.0442$

$-0.347 / 0.586$

Table A.2: Selected distances, bond lengths, torsion angles and Continuous Symmetry Measures (CSM) for 1'.

\begin{tabular}{ll}
\hline \hline atoms & distance / $\AA$ \\
\hline $\mathrm{Ru}(1) \cdots \mathrm{Ru}(2)$ & $4.1522(6)$ \\
\hline $\mathrm{Ru}(1)-\mathrm{N}(3)$ & bond length / $\AA$ \\
$\mathrm{Ru}(1)-\mathrm{N}(1)$ & $1.953(3)$ \\
$\mathrm{Ru}(1)-\mathrm{O}(2)$ & $2.003(3)$ \\
$\mathrm{Ru}(1)-\mathrm{O}(6)$ & $2.141(2)$ \\
$\mathrm{Ru}(1)-\mathrm{O}(9)$ & $2.153(2)$ \\
$\mathrm{Ru}(1)-\mathrm{S}(1)$ & $2.185(2)$ \\
$\mathrm{Ru}(2)-\mathrm{N}(4)$ & $2.1924(9)$ \\
$\mathrm{Ru}(2)-\mathrm{N}(2)$ & $2.002(3)$ \\
$\mathrm{Ru}(2)-\mathrm{O}(4)$ & $2.021(3)$ \\
$\mathrm{Ru}(2)-\mathrm{S}(2)$ & $2.123(2)$ \\
$\mathrm{Ru}(2)-\mathrm{S}(3)$ & $2.2275(10)$ \\
$\mathrm{Ru}(2)-\mathrm{Cl}(1)$ & $2.2827(8)$ \\
& $2.4362(9)$ \\
\hline $\mathrm{Ru}(1)-\mathrm{N}(1)-\mathrm{N}(2)-\mathrm{Ru}(2)$ & torsion angle ${ }^{\circ}$ \\
$\mathrm{Ru}(1)-\mathrm{O}(9)-\mathrm{S}(3)-\mathrm{Ru}(2)$ & $2.462(498)$ \\
\hline & $-0.583(243)$ \\
\hline $\mathrm{Ru}(1)$ & CSM $\boldsymbol{S ( O _ { h } ) ^ { \mathrm { a } }}$ \\
$\mathrm{Ru}(2)$ & 1.32 \\
\hline a Continuous Symmetry Measures for octahedral geometry. CSM $S(X)$ is a dimensionless variable and \\
illustrates the deviation of the coordination environment to an ideal polyhedron (not regarding the \\
type of ligands, but merely the shape). A value of $0-100$ is assigned to the MLx-system; the smaller the \\
value, the closer the structure is to the ideal polyhedron. \\
\hline
\end{tabular}




\section{$\left[\mathrm{L}^{1} \mathrm{Ru}_{2}\left(\mathrm{pySO}_{3}\right)_{4}\left(\mu-\mathrm{CO}_{3}\right)\right]\left(3^{\mathrm{CO}}\right)$}

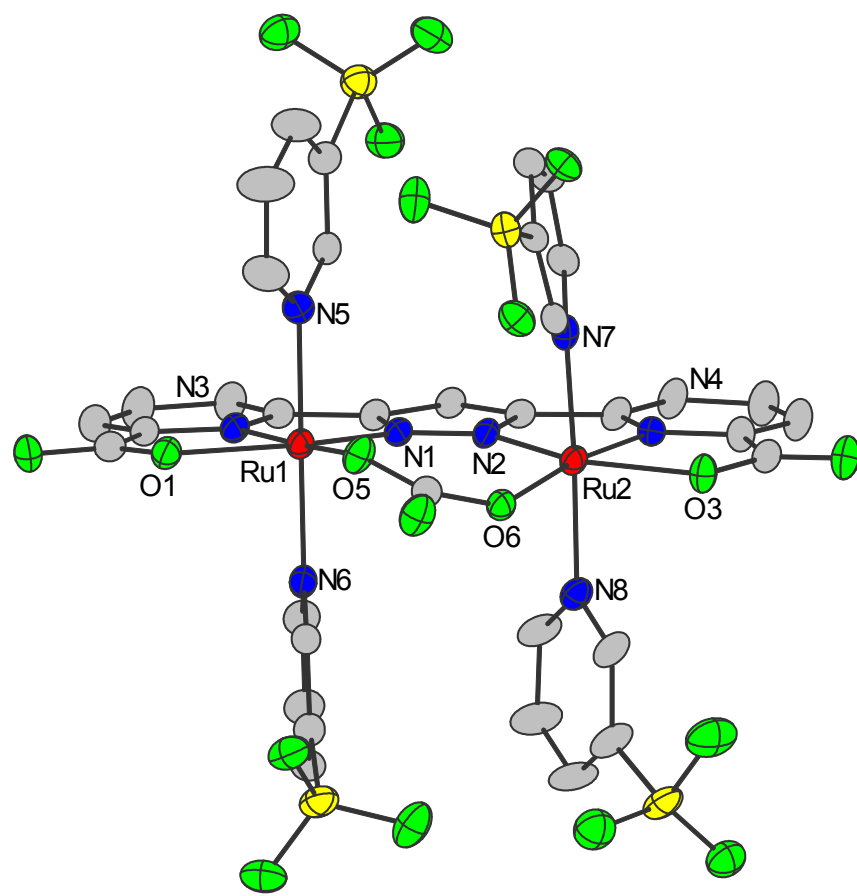

Figure A.109: Molecular structure of the anionic core of $3^{\mathrm{co3}}$ drawn as thermal ellipsoids at the $30 \%$ probability level (ruthenium: red, nitrogen: blue, oxygen: green, sulfur: yellow, carbon: grey). All hydrogen atoms, solvent molecules and counter ions are omitted for clarity reasons.

Table A.3: Crystal data and refinement details for $3^{\mathrm{co3}}$.

\begin{tabular}{ll}
\hline \hline empirical formula & $\mathrm{C}_{36} \mathrm{H}_{47.17} \mathrm{~N}_{8} \mathrm{Na}_{5} \mathrm{O}_{31.08} \mathrm{Ru}_{2} \mathrm{~S}_{4}$ \\
formula weight & 1534.64 \\
$T / \mathrm{K}$ & $133(2)$ \\
crystal size / $\mathrm{mm}^{3}$ & $0.300 \times 0.200 \times 0.200$ \\
crystal system & triclinic \\
space group & $\mathrm{P}-1$ \\
$a / \AA$ & $10.804(2)$ \\
$b / \AA$ & $12.519(3)$ \\
$c / \AA$ & $21.040(4)$ \\
$\alpha /{ }^{\circ}$ & $91.35(3)$ \\
$\beta /{ }^{\circ}$ & $90.33(3)$ \\
$\gamma /{ }^{\circ}$ & $92.54(3)$ \\
$V / \AA^{3}$ & $2842.1(10)$ \\
$Z$ & 2 \\
$\rho / \mathrm{g} \cdot \mathrm{cm}^{-3}$ & 1.793 \\
$\mu / \mathrm{mm}^{-1}$ & 0.816 \\
$F(000)$ & 1550 \\
$T_{\min } / T_{\max }$ & $0.70 / 0.74$ \\
$\Theta-r$ range $/{ }^{\circ}$ & $1.629-25.674$ \\
$h k l-$ range & $-13 \leq h \leq 11$ \\
&
\end{tabular}




\begin{tabular}{ll} 
& $-15 \leq k \leq 15$ \\
& $-25 \leq l \leq 25$ \\
measured reflections & 27113 \\
unique reflections [Rint] & $10689[0.0999]$ \\
data / restraints / parameters & $10689 / 613 / 1158$ \\
goodness-of-fit $\left(F^{2}\right)$ & 0.936 \\
$R 1, w R 2(I>2 \sigma(I))$ & $0.0597,0.1366$ \\
$R 1, w R 2$ (all data) & $0.0979,0.1535$ \\
residual electron density / $\cdot \cdot \AA^{3}$ & $-1.534 / 0.808$ \\
\hline \hline
\end{tabular}

Table A.4: Selected distances, bond lengths, torsion angles and Continuous Symmetry Measures (CSM) for $3^{\text {co3. }}$

\begin{tabular}{|c|c|}
\hline atoms & distance / Å \\
\hline \multirow[t]{2}{*}{$\operatorname{Ru}(1) \cdots \operatorname{Ru}(2)$} & $4.1349(14)$ \\
\hline & bond length / Å \\
\hline $\mathrm{Ru}(1)-\mathrm{N}(3)$ & $1.930(5)$ \\
\hline $\mathrm{Ru}(1)-\mathrm{N}(1)$ & $1.974(5)$ \\
\hline $\mathrm{Ru}(1)-\mathrm{N}(5)$ & $2.082(6)$ \\
\hline $\mathrm{Ru}(1)-\mathrm{N}(6)$ & $2.093(6)$ \\
\hline $\mathrm{Ru}(1)-\mathrm{O}(5)$ & $2.128(4)$ \\
\hline $\mathrm{Ru}(1)-\mathrm{O}(1)$ & $2.156(4)$ \\
\hline $\mathrm{Ru}(2)-\mathrm{N}(4)$ & $1.942(5)$ \\
\hline $\mathrm{Ru}(2)-\mathrm{N}(2)$ & $1.994(5)$ \\
\hline $\mathrm{Ru}(2)-\mathrm{N}(7)$ & $2.059(6)$ \\
\hline $\mathrm{Ru}(2)-\mathrm{N}(8)$ & $2.076(6)$ \\
\hline $\mathrm{Ru}(2)-\mathrm{O}(6)$ & $2.123(4)$ \\
\hline \multirow[t]{2}{*}{$\mathrm{Ru}(2)-\mathrm{O}(3)$} & $2.177(4)$ \\
\hline & torsion angle $/^{\circ}$ \\
\hline $\mathrm{Ru}(1)-\mathrm{N}(1)-\mathrm{N}(2)-\mathrm{Ru}(2)$ & $7.7(9)$ \\
\hline \multirow[t]{2}{*}{$\mathrm{Ru}(1)-0(5) \cdots O(6)-\mathrm{Ru}(2)$} & $35.004(301)$ \\
\hline & $\operatorname{CSM} S\left(O_{h}\right)^{\mathrm{a}}$ \\
\hline $\mathrm{Ru}(1)$ & 1.23 \\
\hline $\mathrm{Ru}(2)$ & 1.26 \\
\hline
\end{tabular}

a Continuous Symmetry Measures for octahedral geometry 


\section{$\left[\mathrm{L}^{1} \mathrm{Ru}_{2}\left(\mathrm{pySO}_{3}\right)_{4}\right]_{2}(4)$}

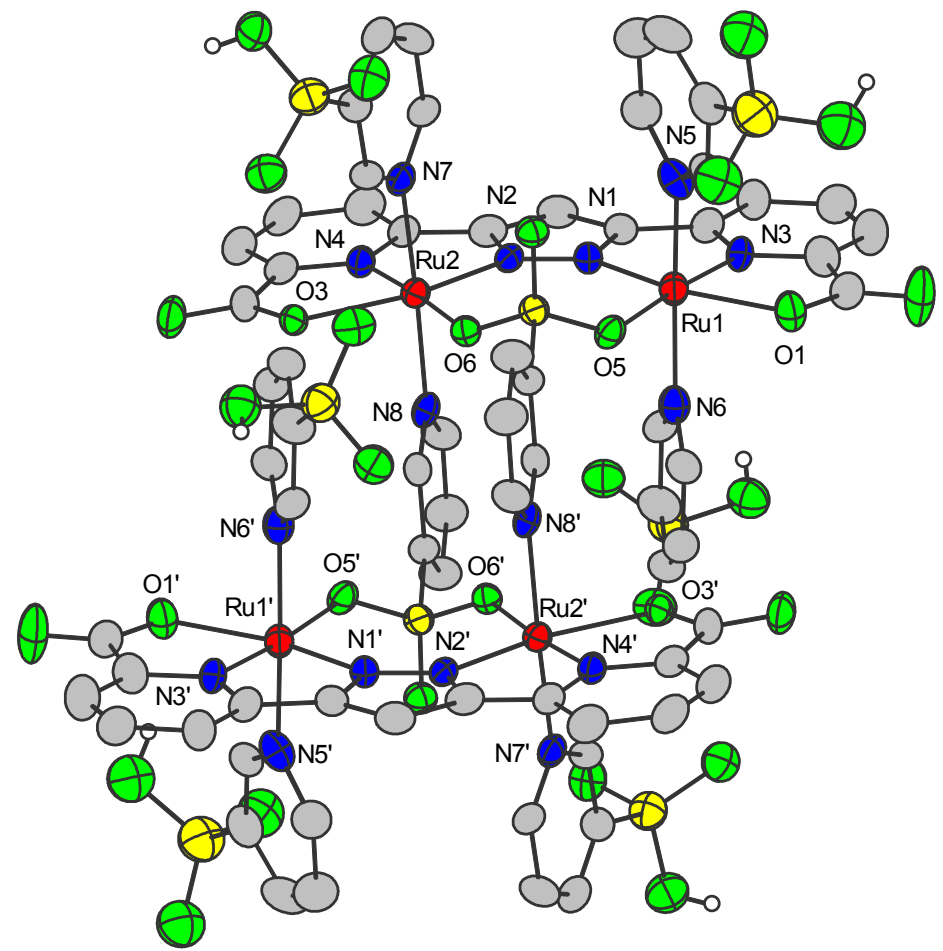

Figure A.110: Structure of the anionic core of 4 drawn as thermal ellipsoids at the $20 \%$ probability level (ruthenium: red, nitrogen: blue, oxygen: green, sulfur: yellow, carbon: grey). All hydrogen atoms (except those of the $\mathrm{SO}_{3} \mathrm{H}$ groups) and solvent molecules are omitted for clarity reasons.

Table A.5: Crystal data and refinement details for 4.

\begin{tabular}{ll}
\hline \hline empirical formula & $\mathrm{C}_{35} \mathrm{H}_{26} \mathrm{~N}_{8} \mathrm{O}_{16} \mathrm{Ru}_{2} \mathrm{~S}_{4}$ \\
formula weight & 1145.02 \\
$T / \mathrm{K}$ & $133(2)$ \\
crystal size / $\mathrm{mm}^{3}$ & $0.190 \times 0.180 \times 0.130$ \\
crystal system & monoclinic \\
space group & $\mathrm{C} 2 / \mathrm{c}$ \\
$a / \AA$ & $27.330(6)$ \\
$b / \AA$ & $14.462(3)$ \\
$c / \AA$ & $32.536(7)$ \\
$\alpha /{ }^{\circ}$ & 90 \\
$\beta /{ }^{\circ}$ & $102.42(3)$ \\
$\gamma /{ }^{\circ}$ & 90 \\
$V / \AA \AA^{3}$ & $12559(5)$ \\
$Z$ & 8 \\
$\rho / \mathrm{g} \cdot \mathrm{cm}^{-3}$ & 1.211 \\
$\mu / \mathrm{mm}^{-1}$ & 0.669 \\
$F(000)$ & 4576 \\
$T_{\min } / T_{\max }$ & $0.70 / 0.74$
\end{tabular}




\begin{tabular}{ll}
$\Theta$-range $/^{\circ}$ & $1.282-25.715$ \\
$h k l$-range & $-33 \leq h \leq 33$ \\
& $-17 \leq k \leq 16$ \\
measured reflections & $-39 \leq l \leq 39$ \\
unique reflections $[R$ int] & 57303 \\
data / restraints / parameters & $11892[0.1828]$ \\
goodness-of-fit $\left(F^{2}\right)$ & $11892 / 516 / 703$ \\
$R 1, w R 2(I>2 \sigma(I))$ & 0.841 \\
$R 1, w R 2$ (all data) & $0.0774,0.1518$ \\
residual electron density $/ \mathrm{e} \cdot \AA^{3}$ & $0.1754,0.1854$ \\
\hline
\end{tabular}

Table A.6: Selected distances, bond lengths, torsion angles and Continuous Symmetry Measures (CSM) for 4.

\begin{tabular}{ll}
\hline \hline atoms & distance / \\
\hline $\mathrm{Ru}(1) \cdots \mathrm{Ru}(2)$ & $4.3364(14)$ \\
\hline $\mathrm{Ru}(1)-\mathrm{N}(3)$ & bond length / $\AA$ \\
$\mathrm{Ru}(1)-\mathrm{N}(1)$ & $1.929(8)$ \\
$\mathrm{Ru}(1)-\mathrm{N}(5)$ & $2.011(8)$ \\
$\mathrm{Ru}(1)-\mathrm{N}(6)$ & $2.029(10)$ \\
$\mathrm{Ru}(1)-\mathrm{O}(1)$ & $2.038(10)$ \\
$\mathrm{Ru}(1)-\mathrm{O}(5)$ & $2.147(7)$ \\
$\mathrm{Ru}(2)-\mathrm{N}(4)$ & $2.162(6)$ \\
$\mathrm{Ru}(2)-\mathrm{N}(2)$ & $1.937(8)$ \\
$\mathrm{Ru}(2)-\mathrm{N}(8)$ & $2.019(7)$ \\
$\mathrm{Ru}(2)-\mathrm{N}(7)$ & $2.066(8)$ \\
$\mathrm{Ru}(2)-\mathrm{O}(3)$ & $2.092(9)$ \\
$\mathrm{Ru}(2)-\mathrm{O}(6)$ & $2.140(6)$ \\
\hline $\mathrm{Ru}(1)-\mathrm{N}(1)-\mathrm{N}(2)-\mathrm{Ru}(2)$ & $2.157(6)$ \\
$\mathrm{Ru}(1)-\mathrm{O}(5) \cdots \mathrm{O}(6)-\mathrm{Ru}(2)$ & torsion angle $/^{\circ}$ \\
\hline $\mathrm{Ru}(1)$ & $-0.434(1458)$ \\
$\mathrm{Ru}(2)$ & $2.539(437)$ \\
\hline \hline
\end{tabular}

a Continuous Symmetry Measures for octahedral geometry 


\section{$\left[\mathrm{L}^{2 \mathrm{aaCu}}\left(\mu-\mathrm{HCO}_{2}\right)\right]\left(\mathrm{ClO}_{4}\right)_{2}\left(5^{\mathrm{HCO}}\right)$}

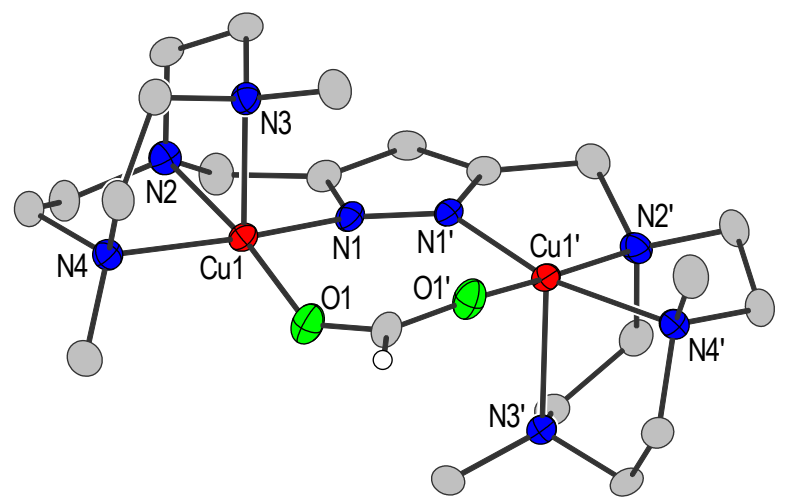

Figure A.111: Molecular structure of the cationic core of $\mathbf{5}^{\mathbf{H C O}}$ drawn as thermal ellipsoids at the $30 \%$ probability level (copper: red, nitrogen: blue, oxygen: green, carbon: grey, hydrogen: white). All hydrogen atoms (except that of $\left.\mathrm{HCO}_{2}^{-}\right)$and counter ions are omitted for clarity reasons.

Table A.7: Crystal data and refinement details for $\mathbf{5}^{\mathrm{HCO2}}$.

\begin{tabular}{|c|c|}
\hline empirical formula & $\mathrm{C}_{22} \mathrm{H}_{42} \mathrm{Cl}_{2} \mathrm{Cu}_{2} \mathrm{~N}_{8} \mathrm{O}_{10}$ \\
\hline formula weight & 776.61 \\
\hline$T / \mathrm{K}$ & $133(2)$ \\
\hline crystal size / mm³ & $0.493 \times 0.155 \times 0.125$ \\
\hline crystal system & tetragonal \\
\hline space group & I4 1 cd \\
\hline$a / \AA$ & $15.5604(3)$ \\
\hline$b / \AA$ & $15.5604(3)$ \\
\hline$c / \AA$ & $25.3987(7)$ \\
\hline$\alpha /{ }^{\circ}$ & 90 \\
\hline$\beta /{ }^{\circ}$ & 90 \\
\hline$\gamma /{ }^{\circ}$ & 90 \\
\hline$V / \AA^{3}$ & $6149.7(3)$ \\
\hline$Z$ & 8 \\
\hline$\rho / \mathrm{g} \cdot \mathrm{cm}^{-3}$ & 1.678 \\
\hline$\mu / \mathrm{mm}^{-1}$ & 1.623 \\
\hline$F(000)$ & 3216 \\
\hline$T_{\min } / T_{\max }$ & $0.6186 / 0.8334$ \\
\hline \multirow[t]{3}{*}{$\Theta$-range $/{ }^{\circ}$} & $2.449-25.660$ \\
\hline & $-18 \leq h \leq 18$ \\
\hline & $-18 \leq k \leq 18$ \\
\hline$h k l$-range & $-30 \leq l \leq 30$ \\
\hline measured reflections & 16951 \\
\hline unique reflections $\left[R_{\text {int }}\right]$ & $2882[0.0269]$ \\
\hline data / restraints / parameters & 2882 / 11 / 198 \\
\hline goodness-of-fit $\left(F^{2}\right)$ & 1.063 \\
\hline$R 1, w R 2(I>2 \sigma(I))$ & $0.0451,0.1199$ \\
\hline
\end{tabular}


$R 1, w R 2$ (all data)

residual electron density $/ \mathrm{e} \cdot \AA^{3}$
$0.0472,0.1222$

$-0.450 / 0.762$

Table A.8: Selected distances, bond lengths, torsion angles and Continuous Symmetry Measures (CSM) for $\mathbf{5}^{\text {HCO2. }}$

\begin{tabular}{|c|c|}
\hline atoms & distance / Å \\
\hline \multirow[t]{2}{*}{$\mathrm{Cu}(1) \cdots \mathrm{Cu}\left(1^{\prime}\right)$} & $4.1498(9)$ \\
\hline & bond length / Å \\
\hline $\mathrm{Cu}(1)-\mathrm{N}(1)$ & $1.948(6)$ \\
\hline $\mathrm{Cu}(1)-\mathrm{O}(1)$ & $1.991(6)$ \\
\hline $\mathrm{Cu}(1)-\mathrm{N}(2)$ & $2.072(6)$ \\
\hline $\mathrm{Cu}(1)-\mathrm{N}(3)$ & $2.205(5)$ \\
\hline \multirow[t]{2}{*}{$\mathrm{Cu}(1)-\mathrm{N}(4)$} & $2.059(6)$ \\
\hline & torsion angle $/^{\circ}$ \\
\hline $\mathrm{Cu}(1)-\mathrm{N}(1)-\mathrm{N}\left(1^{\prime}\right)-\mathrm{Cu}\left(1^{\prime}\right)$ & $33.129(994)$ \\
\hline \multirow[t]{2}{*}{$\mathrm{Cu}(1)-\mathrm{O}(1) \cdots \mathrm{O}\left(1^{\prime}\right)-\mathrm{Cu}\left(1^{\prime}\right)$} & $-41.801(446)$ \\
\hline & $\operatorname{CSM} S\left(C_{4 v}\right)^{\mathrm{a}}$ \\
\hline \multirow[t]{2}{*}{$\mathrm{Cu}(1)$} & 0.88 \\
\hline & $\operatorname{CSM} S\left(D_{3 h}\right)^{\mathrm{b}}$ \\
\hline $\mathrm{Cu}(1)$ & 6.42 \\
\hline
\end{tabular}

a Continuous Symmetry Measures for square pyramidal geometry

b Continuous Symmetry Measures for trigonal bipyramidal geometry 


\section{$\left[\mathrm{L}^{2 \mathrm{aaC}} \mathrm{Cu}_{2}(\mu-\mathrm{OAc})\right]\left(\mathrm{ClO}_{4}\right)_{2}\left(5^{\mathrm{OAc}}\right)$}

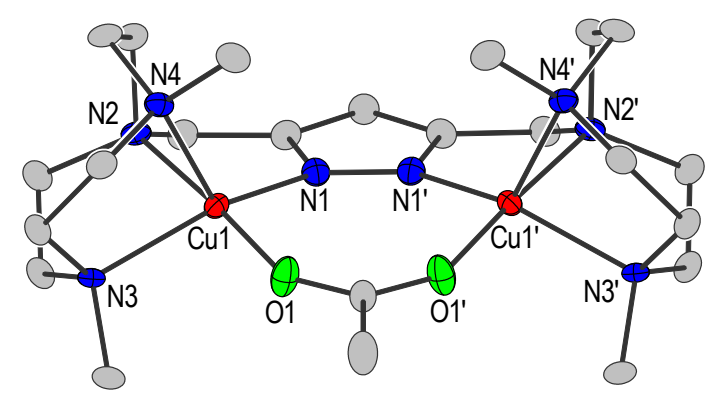

Figure A.112: Molecular structure of the cationic core of $\mathbf{5}^{\mathbf{0 A c}}$ drawn as thermal ellipsoids at the $30 \%$ probability level (copper: red, nitrogen: blue, oxygen: green, carbon: grey). All hydrogen atoms, solvent molecules and counter ions are omitted for clarity reasons.

Table A.9: Crystal data and refinement details for $5^{\mathbf{O A c} \text {. }}$

\begin{tabular}{|c|c|}
\hline empirical formula & $\mathrm{C}_{25} \mathrm{H}_{47} \mathrm{Cl}_{2} \mathrm{Cu}_{2} \mathrm{~N}_{9} \mathrm{O}_{10}$ \\
\hline formula weight & 831.69 \\
\hline$T / \mathrm{K}$ & $133(2)$ \\
\hline crystal size $/ \mathrm{mm}^{3}$ & $0.426 \times 0.184 \times 0.102$ \\
\hline crystal system & orthorhombic \\
\hline space group & Pmn21 \\
\hline$a / \AA$ & $23.6975(11)$ \\
\hline$b / \AA$ & $8.8056(3)$ \\
\hline$c / \AA$ & $8.4154(3)$ \\
\hline$\alpha /{ }^{\circ}$ & 90 \\
\hline$\beta /{ }^{\circ}$ & 90 \\
\hline$\gamma /{ }^{\circ}$ & 90 \\
\hline$V / \AA^{3}$ & $1756.05(12)$ \\
\hline$Z$ & 2 \\
\hline$\rho / \mathrm{g} \cdot \mathrm{cm}^{-3}$ & 1.573 \\
\hline$\mu / \mathrm{mm}^{-1}$ & 1.428 \\
\hline$F(000)$ & 864 \\
\hline$T_{\min } / T_{\max }$ & $0.4401 / 0.7068$ \\
\hline \multirow[t]{2}{*}{$\Theta$-range $/^{\circ}$} & $1.719-25.612$ \\
\hline & $-28 \leq h \leq 28$ \\
\hline$h k l$-range & $\begin{array}{l}-10 \leq k \leq 10 \\
-10 \leq l \leq 9\end{array}$ \\
\hline measured reflections & 15466 \\
\hline unique reflections $\left[R_{\mathrm{int}}\right]$ & $3205[0.0273]$ \\
\hline data / restraints / parameters & 3205 / 1 / 231 \\
\hline goodness-of-fit $\left(F^{2}\right)$ & 1.187 \\
\hline$R 1, w R 2(I>2 \sigma(I))$ & $0.0579,0.1459$ \\
\hline$R 1, w R 2$ (all data) & $0.0589,0.1464$ \\
\hline residual electron density / $e \cdot \AA^{3}$ & $-1.281 / 2.032$ \\
\hline
\end{tabular}


Table A.10: Selected distances, bond lengths, torsion angles and Continuous Symmetry Measures (CSM) for 50Ac.

\begin{tabular}{ll}
\hline \hline atoms & distance / \\
\hline $\mathrm{Cu}(1) \cdots \mathrm{Cu}\left(1^{\prime}\right)$ & $4.1712(14)$ \\
\hline $\mathrm{Cu}(1)-\mathrm{O}(1)$ & bond length / \\
$\mathrm{Cu}(1)-\mathrm{N}(1)$ & $1.908(8)$ \\
$\mathrm{Cu}(1)-\mathrm{N}(2)$ & $1.975(9)$ \\
$\mathrm{Cu}(1)-\mathrm{N}(3)$ & $2.057(8)$ \\
$\mathrm{Cu}(1)-\mathrm{N}(4)$ & $2.091(9)$ \\
\hline $\mathrm{Cu}(1)-\mathrm{N}(1)-\mathrm{N}\left(1^{\prime}\right)-\mathrm{Cu}\left(1^{\prime}\right)$ & $2.203(9)$ \\
$\left.\mathrm{Cu}(1)-\mathrm{O}(1) \cdots \mathrm{O}^{\prime}\right)-\mathrm{Cu}\left(1^{\prime}\right)$ & torsion angle / \\
\hline $\mathrm{Cu}(1)$ & $0.000(1647)$ \\
\hline $\mathrm{Cu}(1)$ & $0.000(755)$ \\
\hline \hline
\end{tabular}

a Continuous Symmetry Measures for square pyramidal geometry

b Continuous Symmetry Measures for trigonal bipyramidal geometry 


\section{$\left[\mathrm{L}^{2 \mathrm{~b}} \mathrm{Cu}_{2}\left(\mu-\mathrm{HCO}_{2}\right)\right]\left(\mathrm{ClO}_{4}\right)_{2}\left(6^{\mathrm{HCO}}\right)$}

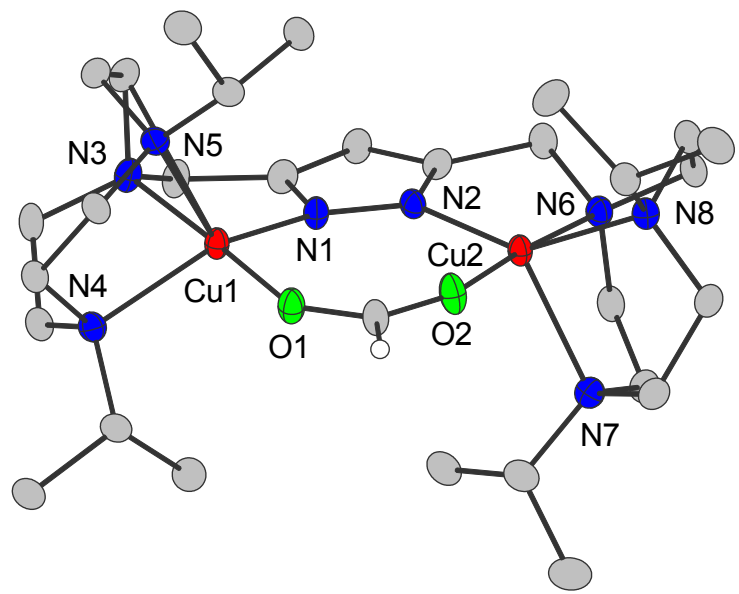

Figure A.113: Molecular structure of the cationic core of $\mathbf{6}^{\mathbf{H C O}}$ drawn as thermal ellipsoids at the 30\% probability level (copper: red, nitrogen: blue, oxygen: green, carbon: grey, hydrogen: white). All hydrogen atoms (except that of $\mathrm{HCO}_{2}^{-}$), counter ions and solvent molecules are omitted for clarity reasons.

Table A.11: Crystal data and refinement details for $\mathbf{6}^{\mathrm{HCO}}$.

\begin{tabular}{ll}
\hline \hline empirical formul & $\mathrm{C}_{32} \mathrm{H}_{61} \mathrm{Cl}_{2} \mathrm{Cu}_{2} \mathrm{~N}_{9} \mathrm{O}_{10}$ \\
formula weight & 929.87 \\
$T / \mathrm{K}$ & $133(2)$ \\
crystal size / mm & \\
crystal system & $0.440 \times 0.240 \times 0.220$ \\
space group & triclinic \\
$a / \AA$ & $\mathrm{P} 1$ \\
$b / \AA$ & $9.6063(4)$ \\
$c / \AA$ & $9.7548(4)$ \\
$\alpha /{ }^{\circ}$ & $13.0112(5)$ \\
$\beta /{ }^{\circ}$ & $94.203(3)$ \\
$\gamma /{ }^{\circ}$ & $110.773(3)$ \\
$V / \AA^{3}$ & $111.539(3)$ \\
$Z$ & $1030.94(8)$ \\
$\rho /$ g.cm-3 & 1 \\
$\mu / \mathrm{mm}^{-1}$ & 1.498 \\
$F(000)$ & 1.224 \\
$T_{\text {min }} / T_{\text {max }}$ & 488 \\
$\Theta$-range / & $0.2461 / 0.4297$ \\
& $1.722-27.144$ \\
$h k l$-range & $-12 \leq h \leq 12$ \\
measured reflections & $-12 \leq k \leq 12$ \\
unique reflections $[R$ int $]$ & $-16 \leq l \leq 16$ \\
data / restraints / parameters & 40366 \\
\end{tabular}


goodness-of-fit $\left(F^{2}\right)$

$R 1, w R 2(I>2 \sigma(I))$

$R 1, w R 2$ (all data)

residual electron density / e. $\AA^{3}$
1.084

$0.0773,0.2211$

$0.0842,0.2289$

$-0.484 / 1.126$

Table A.12: Selected distances, bond lengths, torsion angles and Continuous Symmetry Measures (CSM) for $6^{\mathrm{HCO}}$.

\begin{tabular}{|c|c|}
\hline atoms & distance / $\AA$ \\
\hline \multirow[t]{2}{*}{$\mathrm{Cu}(1) \cdots \mathrm{Cu}\left(1^{\prime}\right)$} & $4.2257(15)$ \\
\hline & bond length / Å \\
\hline $\mathrm{Cu}(1)-\mathrm{O}(1)$ & $1.953(9)$ \\
\hline $\mathrm{Cu}(1)-\mathrm{N}(1)$ & $1.964(10)$ \\
\hline $\mathrm{Cu}(1)-\mathrm{N}(3)$ & $2.059(9)$ \\
\hline $\mathrm{Cu}(1)-\mathrm{N}(4)$ & $2.118(9)$ \\
\hline $\mathrm{Cu}(1)-\mathrm{N}(5)$ & $2.210(9)$ \\
\hline $\mathrm{Cu}(2)-\mathrm{O}(2)$ & $1.947(9)$ \\
\hline $\mathrm{Cu}(2)-\mathrm{N}(2)$ & $1.954(10)$ \\
\hline $\mathrm{Cu}(2)-\mathrm{N}(6)$ & $2.053(10)$ \\
\hline $\mathrm{Cu}(2)-\mathrm{N}(8)$ & $2.117(9)$ \\
\hline \multirow[t]{2}{*}{$\mathrm{Cu}(2)-\mathrm{N}(7)$} & $2.214(9)$ \\
\hline & torsion angle $/^{\circ}$ \\
\hline $\mathrm{Cu}(1)-\mathrm{N}(1)-\mathrm{N}(2)-\mathrm{Cu}(2)$ & $22.779(1963)$ \\
\hline \multirow[t]{2}{*}{$\mathrm{Cu}(1)-\mathrm{O}(1) \cdots \mathrm{O}(2)-\mathrm{Cu}(2)$} & $-14.255(913)$ \\
\hline & $\operatorname{CSM} S\left(C_{4 v}\right)^{a}$ \\
\hline $\mathrm{Cu}(1)$ & 4.10 \\
\hline \multirow[t]{2}{*}{$\mathrm{Cu}(2)$} & 3.82 \\
\hline & $\operatorname{CSM} S\left(D_{3 h}\right)^{\mathrm{b}}$ \\
\hline $\mathrm{Cu}(1)$ & 2.93 \\
\hline $\mathrm{Cu}(2)$ & 3.03 \\
\hline
\end{tabular}

a Continuous Symmetry Measures for square pyramidal geometry

${ }^{b}$ Continuous Symmetry Measures for trigonal bipyramidal geometry 


\section{$\left[\mathrm{L}^{2 \mathrm{~b}} \mathrm{Cu}_{2}(\mu-\mathrm{OAc})\right]\left(\mathrm{ClO}_{4}\right)_{2}\left(6^{\mathrm{OAc}}\right)$}

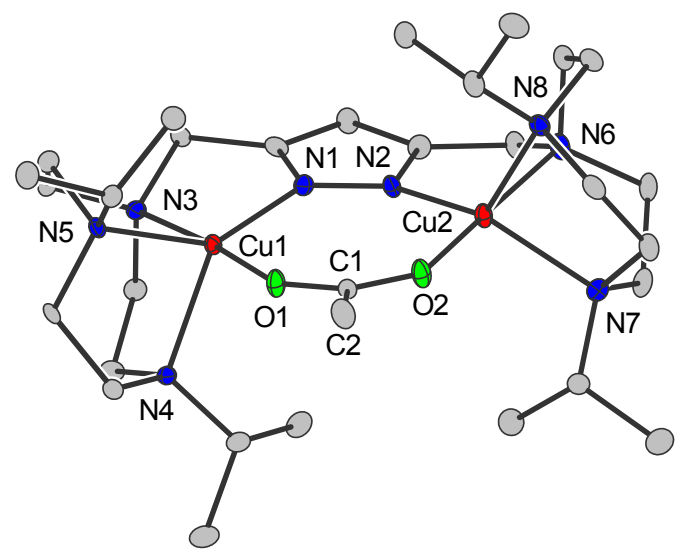

Figure A.114: Molecular structure of the cationic core of $6^{0 A c}$ drawn as thermal ellipsoids at the $30 \%$ probability level (copper: red, nitrogen: blue, oxygen: green, carbon: grey). All hydrogen atoms, solvent molecules and counter ions are omitted for clarity reasons.

Table A.13: Crystal data and refinement details for $6^{\mathbf{0 A c}}$.

\begin{tabular}{|c|c|}
\hline empirical formula & $\mathrm{C}_{32} \mathrm{H}_{64} \mathrm{Cl}_{2} \mathrm{Cu}_{2} \mathrm{~N}_{8} \mathrm{O}_{11}$ \\
\hline formula weight & 934.89 \\
\hline$T / \mathrm{K}$ & $100(2)$ \\
\hline crystal size $/ \mathrm{mm}^{3}$ & $0.353 \times 0.245 \times 0.119$ \\
\hline crystal system & monoclinic \\
\hline space group & $\mathrm{P} 21$ \\
\hline$a / \AA$ & $9.2245(3)$ \\
\hline$b / \AA$ & $47.6567(18)$ \\
\hline$c / \AA$ & $10.0655(4)$ \\
\hline$\alpha /^{\circ}$ & 90 \\
\hline$\beta /{ }^{\circ}$ & $112.7130(10)$ \\
\hline$\gamma /{ }^{\circ}$ & 90 \\
\hline$V / \AA^{3}$ & $4081.7(3)$ \\
\hline$Z$ & 4 \\
\hline$\rho / \mathrm{g} \cdot \mathrm{cm}^{-3}$ & 1.521 \\
\hline$\mu / \mathrm{mm}^{-1}$ & 1.239 \\
\hline$F(000)$ & 1968 \\
\hline$T_{\min } / T_{\max }$ & $0.74 / 0.87$ \\
\hline \multirow[t]{2}{*}{$\Theta$-range $/^{\circ}$} & $2.193-27.894$ \\
\hline & $-12 \leq h \leq 12$ \\
\hline \multirow[t]{2}{*}{ hkl-range } & $-62 \leq k \leq 62$ \\
\hline & $-11 \leq l \leq 13$ \\
\hline measured reflections & 84547 \\
\hline unique reflections [ $R$ int] & $19447[0.0264]$ \\
\hline data / restraints / parameters & 19447 / 24 / 1028 \\
\hline goodness-of-fit $\left(F^{2}\right)$ & 1.187 \\
\hline
\end{tabular}


$R 1, w R 2(I>2 \sigma(I))$

$0.0405,0.0921$

$R 1, w R 2$ (all data)

$0.0417,0.0926$

residual electron density / $\mathrm{e} \cdot \AA^{3}$

$-0.760 / 0.583$

Table A.14: Selected distances, bond lengths, torsion angles and Continuous Symmetry Measures (CSM) for $\mathbf{6}^{\mathbf{0 A c} \text {. }}$

\begin{tabular}{|c|c|}
\hline atoms & distance / Å \\
\hline \multirow[t]{2}{*}{$\mathrm{Cu}(1) \cdots \mathrm{Cu}(2)$} & $4.1850(14)$ \\
\hline & bond length / Å \\
\hline $\mathrm{Cu}(1)-\mathrm{O}(1)$ & $1.950(4)$ \\
\hline $\mathrm{Cu}(1)-\mathrm{N}(1)$ & $1.953(4)$ \\
\hline $\mathrm{Cu}(1)-\mathrm{N}(3)$ & $2.064(4)$ \\
\hline $\mathrm{Cu}(1)-\mathrm{N}(5)$ & $2.112(4)$ \\
\hline $\mathrm{Cu}(1)-\mathrm{N}(4)$ & $2.172(4)$ \\
\hline $\mathrm{Cu}(2)-\mathrm{O}(2)$ & $1.945(4)$ \\
\hline $\mathrm{Cu}(2)-\mathrm{N}(2)$ & $1.962(4)$ \\
\hline $\mathrm{Cu}(2)-\mathrm{N}(6)$ & $2.053(4)$ \\
\hline $\mathrm{Cu}(2)-\mathrm{N}(7)$ & $2.111(4)$ \\
\hline \multirow[t]{2}{*}{$\mathrm{Cu}(2)-\mathrm{N}(8)$} & $2.225(4)$ \\
\hline & torsion angle $/{ }^{\circ}$ \\
\hline $\mathrm{Cu}(1)-\mathrm{N}(1)-\mathrm{N}(2)-\mathrm{Cu}(2)$ & $-23.920(795)$ \\
\hline \multirow[t]{2}{*}{$\mathrm{Cu}(1)-\mathrm{O}(1) \cdots \mathrm{O}(2)-\mathrm{Cu}(2)$} & $5.710(368)$ \\
\hline & $\operatorname{CSM} S\left(C_{4 v}\right)^{\mathrm{a}}$ \\
\hline $\mathrm{Cu}(1)$ & 3.76 \\
\hline \multirow[t]{2}{*}{$\mathrm{Cu}(2)$} & 4.47 \\
\hline & $\operatorname{CSM} S\left(D_{3 h}\right)^{\mathrm{b}}$ \\
\hline $\mathrm{Cu}(1)$ & 2.74 \\
\hline $\mathrm{Cu}(2)$ & 2.82 \\
\hline
\end{tabular}

a Continuous Symmetry Measures for square pyramidal geometry

b Continuous Symmetry Measures for trigonal bipyramidal geometry 


\section{$\left[\mathrm{L}^{2 \mathrm{aaCo}}(\mathrm{MeCN})_{4}\right]\left(\mathrm{ClO}_{4}\right)_{3}\left(7^{\mathrm{MeCN}}\right)$}

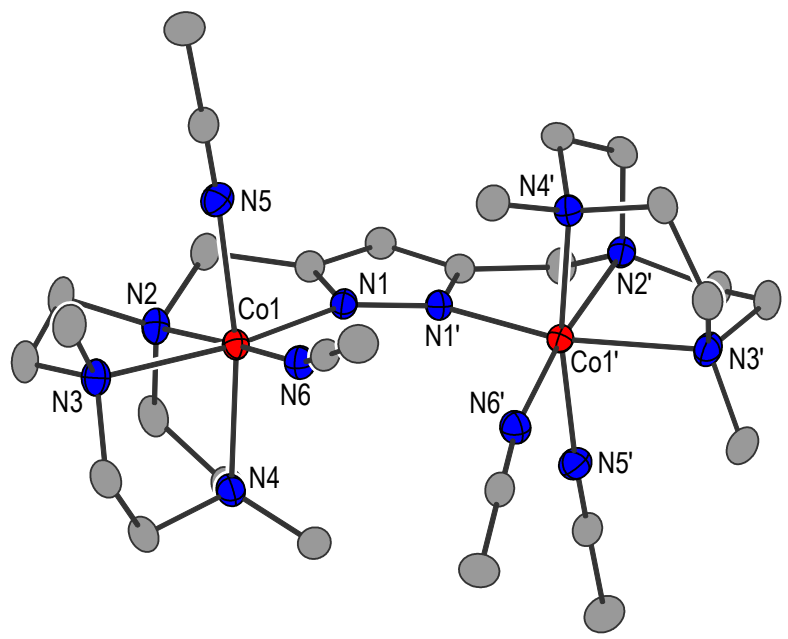

Figure A.115: Molecular structure of the cationic core of $7^{\mathrm{MeCN}}$ drawn as thermal ellipsoids at the $30 \%$ probability level (cobalt: red, nitrogen: blue, carbon: grey). All hydrogen atoms, solvent molecules and counter ions are omitted for clarity reasons.

Table A.15: Crystal data and refinement details for 7MeCN.

\begin{tabular}{|c|c|}
\hline empirical formula & $\mathrm{C}_{33} \mathrm{H}_{60.61} \mathrm{Cl}_{3} \mathrm{Co}_{2} \mathrm{~N}_{13.20} \mathrm{O}_{12.40}$ \\
\hline formula weight & 1064.93 \\
\hline$T / \mathrm{K}$ & $133(2)$ \\
\hline crystal size / mm ${ }^{3}$ & $0.500 \times 0.250 \times 0.180$ \\
\hline crystal system & orthorhombic \\
\hline space group & $\mathrm{P} 2{ }_{1} 2{ }_{1} 2$ \\
\hline$a / \AA$ & $18.5599(5)$ \\
\hline$b / \AA$ & $20.5556(4)$ \\
\hline$c / \AA$ & $12.4717(2)$ \\
\hline$\alpha /{ }^{\circ}$ & 90 \\
\hline$\beta /{ }^{\circ}$ & 90 \\
\hline$\gamma /{ }^{\circ}$ & 90 \\
\hline$V / \AA^{3}$ & $4758.08(18)$ \\
\hline$Z$ & 4 \\
\hline$\rho / \mathrm{g} \cdot \mathrm{cm}^{-3}$ & 1.487 \\
\hline$\mu / \mathrm{mm}^{-1}$ & 0.936 \\
\hline$F(000)$ & 2221 \\
\hline$T_{\min } / T_{\max }$ & $0.7381 / 0.8933$ \\
\hline \multirow[t]{2}{*}{$\Theta$-range $/^{\circ}$} & $1.478-25.681$ \\
\hline & $-22 \leq h \leq 22$ \\
\hline \multirow[t]{2}{*}{$h k l$-range } & $-24 \leq k \leq 25$ \\
\hline & $-13 \leq l \leq 15$ \\
\hline measured reflections & 61703 \\
\hline unique reflections $\left[R_{\mathrm{int}}\right]$ & $8988[0.0425]$ \\
\hline
\end{tabular}



data / restraints / parameters
8988 / 18 / 682
goodness-of-fit $\left(F^{2}\right)$
1.019
$R 1, w R 2(I>2 \sigma(I))$
$0.0310,0.0722$
$R 1, w R 2$ (all data)
$0.0400,0.0758$
residual electron density / $\cdot \cdot \AA^{3}$
$-0.339 / 0.553$

Table A.16: Selected distances, bond lengths, torsion angles and Continuous Symmetry Measures (CSM) for 7MeCN.

\begin{tabular}{|c|c|}
\hline atoms & distance / ̊̊ \\
\hline \multirow[t]{2}{*}{$\operatorname{Co}(1) \cdots \operatorname{Co}\left(1^{\prime}\right)$} & $4.6553(6)$ \\
\hline & bond length / Å \\
\hline $\operatorname{Co}(1)-N(6)$ & $2.086(3)$ \\
\hline $\operatorname{Co}(1)-N(1)$ & $2.092(3)$ \\
\hline $\operatorname{Co}(1)-N(2)$ & $2.147(3)$ \\
\hline $\operatorname{Co}(1)-N(4)$ & $2.163(3)$ \\
\hline $\operatorname{Co}(1)-N(5)$ & $2.163(3)$ \\
\hline \multirow[t]{2}{*}{$\operatorname{Co}(1)-N(3)$} & $2.199(3)$ \\
\hline & torsion angle $/{ }^{\circ}$ \\
\hline \multirow[t]{2}{*}{$\operatorname{Co}(1)-\mathrm{N}(1)-\mathrm{N}\left(1^{\prime}\right)-\mathrm{Co}\left(1^{\prime}\right)$} & $-7.233(577)$ \\
\hline & $\operatorname{CSM} S\left(O_{h}\right)^{\mathrm{a}}$ \\
\hline $\operatorname{Co}(1)$ & 0.78 \\
\hline
\end{tabular}

${ }^{\mathrm{a} C o n t i n u o u s ~ S y m m e t r y ~ M e a s u r e s ~ f o r ~ o c t a h e d r a l ~ g e o m e t r y ~}$ 


\section{$\left[\mathrm{L}^{2 \mathrm{~b}} \mathrm{Co}_{2}\left(\mu-\mathrm{HCO}_{2}\right)\right]\left(\mathrm{ClO}_{4}\right)_{2}\left(8^{\mathrm{HCO}}\right)$}

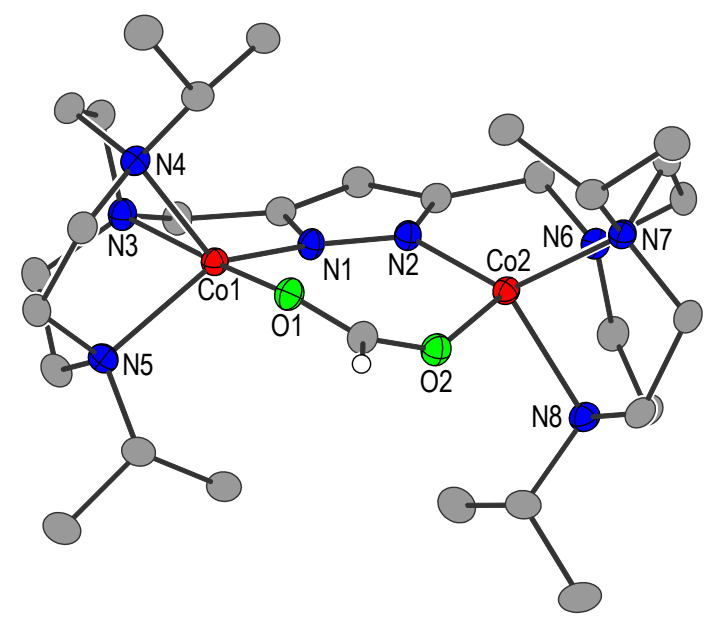

Figure A.116: Molecular structure of the cationic core of $\mathbf{8}^{\mathrm{HCO}}$ drawn as thermal ellipsoids at the 30\% probability level (cobalt: red, nitrogen: blue, oxygen: green, carbon: grey, hydrogen: white). All hydrogen atoms (except that of $\mathrm{HCO}_{2}^{-}$), solvent molecules and counter ions are omitted for clarity reasons.

Table A.17: Crystal data and refinement details for $\mathbf{8}^{\mathrm{HCO}}$.

\begin{tabular}{ll}
\hline \hline empirical formula & $\mathrm{C}_{38} \mathrm{H}_{70} \mathrm{Cl}_{2} \mathrm{C}_{2} \mathrm{~N}_{12} \mathrm{O}_{10}$ \\
formula weight & 1043.82 \\
$T / \mathrm{K}$ & $133(2)$ \\
crystal size / $\mathrm{mm}^{3}$ & $0.431 \times 0.284 \times 0.256$ \\
crystal system & monoclinic \\
space group & $\mathrm{C} 2 / \mathrm{c}$ \\
$a / \AA$ & $36.2714(13)$ \\
$b / \AA$ & $10.6705(3)$ \\
$c / \AA$ & $26.3292(9)$ \\
$\alpha /{ }^{\circ}$ & 90 \\
$\beta /{ }^{\circ}$ & $102.206(3)$ \\
$\gamma /{ }^{\circ}$ & 90 \\
$V / \AA^{3}$ & $9959.9(6)$ \\
$Z$ & 8 \\
$\rho /$ g.cm-3 & 1.392 \\
$\mu / \mathrm{mm}^{-1}$ & 0.837 \\
$F(000)$ & 4400 \\
$T_{\text {min }} / T_{\text {max }}$ & $0.4560 / 0.7523$ \\
$\Theta$-range / & $1.748-26.005$ \\
& $-43 \leq h \leq 44$ \\
$h k l$-range & $-13 \leq k \leq 12$ \\
measured reflections & $-31 \leq l \leq 31$ \\
unique reflections $\left[R_{\text {int }}\right]$ & 37771 \\
data / restraints / parameters & $9374[0.0396]$ \\
\end{tabular}


goodness-of-fit $\left(F^{2}\right)$

$R 1, w R 2(I>2 \sigma(I))$

$R 1, w R 2$ (all data)

residual electron density / $\mathrm{e} \cdot \AA^{3}$
1.057

$0.0435,0.1129$

$0.0561,0.1253$

-0.433 / 1.357

Table A.18: Selected distances, bond lengths, torsion angles and Continuous Symmetry Measures (CSM) for $\mathbf{8}^{\mathrm{HCO} 2}$.

\begin{tabular}{|c|c|}
\hline atoms & distance / ^̊ \\
\hline \multirow[t]{2}{*}{$\operatorname{Co}(1) \cdots \operatorname{Co}(2)$} & $4.1324(10)$ \\
\hline & bond length / Å \\
\hline $\operatorname{Co}(1)-N(1)$ & $1.993(2)$ \\
\hline $\operatorname{Co}(1)-O(1)$ & $2.034(2)$ \\
\hline $\operatorname{Co}(1)-N(5)$ & $2.094(2)$ \\
\hline $\operatorname{Co}(1)-N(4)$ & $2.097(2)$ \\
\hline $\operatorname{Co}(1)-N(3)$ & $2.221(2)$ \\
\hline $\operatorname{Co}(2)-N(2)$ & $1.992(2)$ \\
\hline $\operatorname{Co}(2)-\mathrm{O}(2)$ & $2.021(2)$ \\
\hline $\operatorname{Co}(2)-N(8)$ & $2.108(2)$ \\
\hline $\operatorname{Co}(2)-N(7)$ & $2.111(2)$ \\
\hline \multirow[t]{2}{*}{$\operatorname{Co}(2)-N(6)$} & $2.218(2)$ \\
\hline & torsion angle $/^{\circ}$ \\
\hline $\operatorname{Co}(1)-\mathrm{N}(1)-\mathrm{N}(2)-\operatorname{Co}(2)$ & $32.777(350)$ \\
\hline \multirow[t]{2}{*}{$\mathrm{Co}(1)-\mathrm{O}(1) \cdots \mathrm{O}(2)-\mathrm{Co}(2)$} & $36.165(157)$ \\
\hline & $\operatorname{CSM} S\left(C_{4 v}\right)^{\mathrm{a}}$ \\
\hline $\operatorname{Co}(1)$ & 5.97 \\
\hline \multirow[t]{2}{*}{$\operatorname{Co}(2)$} & 5.70 \\
\hline & $\operatorname{CSM} S\left(D_{3 h}\right)^{\mathrm{b}}$ \\
\hline $\operatorname{Co}(1)$ & 2.45 \\
\hline $\operatorname{Co}(2)$ & 2.50 \\
\hline
\end{tabular}

a Continuous Symmetry Measures for square pyramidal geometry

b Continuous Symmetry Measures for trigonal bipyramidal geometry 


\section{$\left[\mathrm{L}^{2 \mathrm{~b}} \mathrm{Co}_{2}(\mu-\mathrm{OAc})\right]\left(\mathrm{ClO}_{4}\right)_{2}\left(8^{\mathrm{OAc}}\right)$}

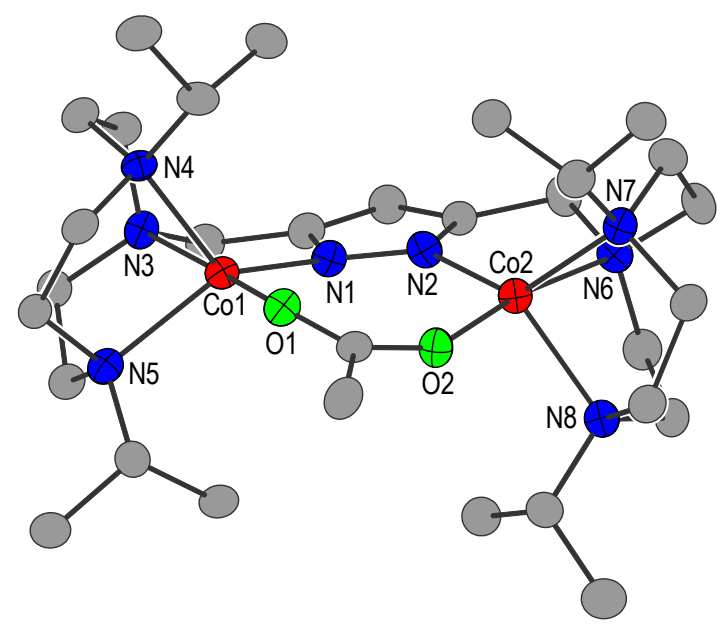

Figure A.117: Molecular structure of the cationic core of $\mathbf{8}^{\text {OAc }}$ drawn as thermal ellipsoids at the 30\% probability level (cobalt: red, nitrogen: blue, oxygen: green, carbon: grey). All hydrogen atoms, solvent molecules and counter ions are omitted for clarity reasons.

Table A.19: Crystal data and refinement details for $\mathbf{8}^{\text {oAc. }}$

\begin{tabular}{|c|c|}
\hline empirical formula & $\mathrm{C}_{32} \mathrm{H}_{64} \mathrm{Cl}_{2} \mathrm{Co}_{2} \mathrm{~N}_{8} \mathrm{O}_{11}$ \\
\hline formula weight & 925.67 \\
\hline$T / \mathrm{K}$ & $133(2)$ \\
\hline crystal size / $\mathrm{mm}^{3}$ & $0.495 \times 0.258 \times 0.149$ \\
\hline crystal system & monoclinic \\
\hline space group & $\mathrm{P} 2{ }_{1}$ \\
\hline$a / \AA$ & $9.1964(3)$ \\
\hline$b / \AA$ & $47.6751(10)$ \\
\hline$c / \AA$ & $10.1840(3)$ \\
\hline$\alpha /{ }^{\circ}$ & 90 \\
\hline$\beta /{ }^{\circ}$ & $112.964(2)$ \\
\hline$\gamma /{ }^{\circ}$ & 90 \\
\hline$V / \AA^{3}$ & $4111.2(2)$ \\
\hline$Z$ & 4 \\
\hline$\rho / \mathrm{g} \cdot \mathrm{cm}^{-3}$ & 1.496 \\
\hline$\mu / \mathrm{mm}^{-1}$ & 1.002 \\
\hline$F(000)$ & 1952 \\
\hline$T_{\min } / T_{\max }$ & $0.3133 / 0.8300$ \\
\hline \multirow[t]{2}{*}{$\Theta$-range $/^{\circ}$} & $1.709-25.608$ \\
\hline & $-11 \leq h \leq 9$ \\
\hline \multirow[t]{2}{*}{$h k l$-range } & $-57 \leq k \leq 57$ \\
\hline & $-12 \leq l \leq 12$ \\
\hline measured reflections & 34007 \\
\hline unique reflections $\left[R_{\mathrm{int}}\right]$ & $15088[0.0646]$ \\
\hline data / restraints / parameters & $15088 / 11 / 1029$ \\
\hline
\end{tabular}


goodness-of-fit $\left(F^{2}\right)$

$R 1, w R 2(I>2 \sigma(I))$

$R 1, w R 2$ (all data)

residual electron density / $\mathrm{e} \cdot \AA^{3}$
1.029

$0.0578,0.1509$

$0.0683,0.1648$

$-0.568 / 0.688$

Table A.20: Selected distances, bond lengths, torsion angles and Continuous Symmetry Measures (CSM) for 80Ac.

\begin{tabular}{|c|c|}
\hline atoms & distance / Å \\
\hline \multirow[t]{2}{*}{$\operatorname{Co}(1) \cdots \operatorname{Co}(2)$} & $4.1437(14)$ \\
\hline & bond length / Å \\
\hline $\mathrm{Co}(1)-\mathrm{O}(1)$ & $2.006(6)$ \\
\hline $\operatorname{Co}(1)-\mathrm{N}(1)$ & $2.009(7)$ \\
\hline $\operatorname{Co}(1)-\mathrm{N}(4)$ & $2.086(7)$ \\
\hline $\operatorname{Co}(1)-N(5)$ & $2.108(8)$ \\
\hline $\operatorname{Co}(1)-N(3)$ & $2.232(7)$ \\
\hline $\mathrm{Co}(2)-\mathrm{O}(2)$ & $2.004(6)$ \\
\hline $\operatorname{Co}(2)-\mathrm{N}(2)$ & $2.014(7)$ \\
\hline $\operatorname{Co}(2)-\mathrm{N}(8)$ & $2.111(7)$ \\
\hline $\operatorname{Co}(2)-\mathrm{N}(7)$ & $2.121(8)$ \\
\hline \multirow[t]{2}{*}{$\operatorname{Co}(2)-N(6)$} & $2.219(7)$ \\
\hline & torsion angle $/^{\circ}$ \\
\hline $\operatorname{Co}(1)-\mathrm{N}(1)-\mathrm{N}(2)-\mathrm{Co}(2)$ & $28.660(1208)$ \\
\hline \multirow[t]{2}{*}{$\mathrm{Co}(1)-\mathrm{O}(1) \cdots \mathrm{O}(2)-\mathrm{Co}(2)$} & $25.152(525)$ \\
\hline & $\operatorname{CSM} S\left(C_{4 v}\right)^{\mathrm{a}}$ \\
\hline $\operatorname{Co}(1)$ & 5.28 \\
\hline \multirow[t]{2}{*}{$\mathrm{Co}(2)$} & 6.10 \\
\hline & $\operatorname{CSM} S\left(D_{3 h}\right)^{\mathrm{b}}$ \\
\hline $\operatorname{Co}(1)$ & 2.41 \\
\hline $\mathrm{Co}(2)$ & 2.61 \\
\hline
\end{tabular}

a Continuous Symmetry Measures for square pyramidal geometry

${ }^{b}$ Continuous Symmetry Measures for trigonal bipyramidal geometry 


\section{$\left[\left(\mathrm{L}^{2 \mathrm{a}}\right)_{2} \mathrm{Cu}_{6}\left(\mathrm{C}_{8} \mathrm{H}_{5}\right)_{2}\right]\left(\mathrm{ClO}_{4}\right)_{2}\left(9^{\mathrm{C} 8 \mathrm{H} 5}\right)$}

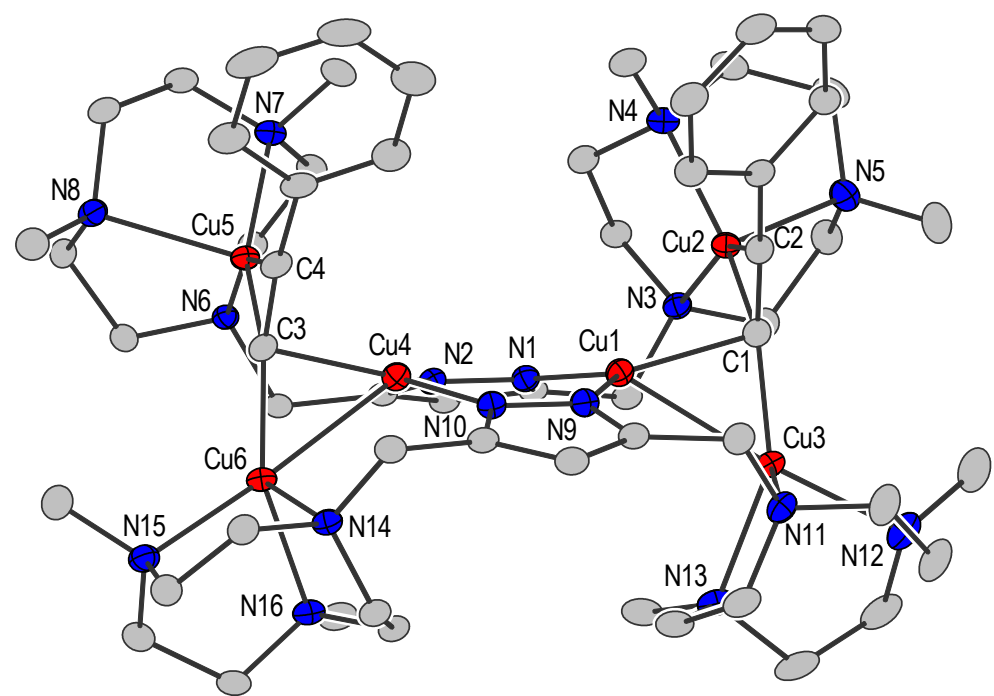

Figure A.118: Molecular structure of the cationic core of $\mathbf{9} \mathbf{6} \mathbf{H 5}$ drawn as thermal ellipsoids at the 30\% probability level (copper: red, nitrogen: blue, carbon: grey). All hydrogen atoms, solvent molecules and counter ions are omitted for clarity reasons.

Table A.21: Crystal data and refinement details for 9c8H5.

\begin{tabular}{ll}
\hline \hline empirical formula & $\mathrm{C}_{60} \mathrm{H}_{95} \mathrm{Cl}_{2} \mathrm{Cu}_{6} \mathrm{~N}_{17} \mathrm{O}_{8}$ \\
formula weight & 1634.66 \\
$T / \mathrm{K}$ & $133(2)$ \\
crystal size / $\mathrm{mm}^{3}$ & $0.490 \times 0.218 \times 0.109$ \\
crystal system & triclinic \\
space group & $\mathrm{P}-1$ \\
$a / \AA$ & $15.4370(5)$ \\
$b / \AA$ & $15.6281(5)$ \\
$c / \AA$ & $16.0641(5)$ \\
$\alpha /{ }^{\circ}$ & $96.553(3)$ \\
$\beta /{ }^{\circ}$ & $116.853(2)$ \\
$\gamma /{ }^{\circ}$ & $95.477(3)$ \\
$V / \AA^{3}$ & $3387.4(2)$ \\
$Z$ & 2 \\
$\rho /$ g.cm-3 & 1.603 \\
$\mu / \mathrm{mm}^{-1}$ & 1.994 \\
$F(000)$ & 1692 \\
$T_{\text {min }} / T_{\text {max }}$ & $0.3815 / 0.6327$ \\
$\Theta$-range / & $1.330-26.827$ \\
& $-19 \leq h \leq 19$ \\
$h k l$-range & $-19 \leq k \leq 17$ \\
measured reflections & $-20 \leq l \leq 20$ \\
unique reflections $[R$ int $]$ & 40537 \\
data / restraints / parameters & $14348[0.0333]$ \\
\end{tabular}


goodness-of-fit $\left(F^{2}\right)$

$R 1, w R 2(I>2 \sigma(I))$

$R 1, w R 2$ (all data)

residual electron density / e $\cdot \AA^{3}$
1.020

$0.0311,0.0709$

$0.0495,0.0771$

$-0.335 / 0.509$

Table A.22: Selected distances, bond lengths and torsion angles for 9c8H5.

\begin{tabular}{|c|c|}
\hline atoms & distance / $\AA$ \\
\hline $\mathrm{Cu}(1) \cdots \mathrm{Cu}(2)$ & $2,9484(4)$ \\
\hline $\mathrm{Cu}(1) \cdots \mathrm{Cu}(3)$ & $2.5062(4)$ \\
\hline $\mathrm{Cu}(2) \cdots \mathrm{Cu}(3)$ & $3.5898(5)$ \\
\hline $\mathrm{Cu}(4) \cdots \mathrm{Cu}(5)$ & $2.8849(4)$ \\
\hline $\mathrm{Cu}(4) \cdots \mathrm{Cu}(6)$ & $2.5512(4)$ \\
\hline \multirow[t]{2}{*}{$\mathrm{Cu}(5) \cdots \mathrm{Cu}(6)$} & $3.6119(5)$ \\
\hline & bond length / Å \\
\hline $\mathrm{Cu}(1)-\mathrm{N}(1)$ & $1.9427(19)$ \\
\hline $\mathrm{Cu}(1)-\mathrm{N}(9)$ & $1.976(2)$ \\
\hline $\mathrm{Cu}(1)-\mathrm{C}(1)$ & $2.045(3)$ \\
\hline $\mathrm{Cu}(2)-\mathrm{N}(3)$ & 2.1131(19) \\
\hline $\mathrm{Cu}(2)-\mathrm{N}(4)$ & $2.102(2)$ \\
\hline $\mathrm{Cu}(2)-\mathrm{N}(5)$ & $2.280(2)$ \\
\hline $\mathrm{Cu}(2)-\mathrm{C}(1)$ & $2.028(2)$ \\
\hline $\mathrm{Cu}(2)-\mathrm{C}(2)$ & $2.000(2)$ \\
\hline $\mathrm{Cu}(3)-\mathrm{N}(11)$ & $2.224(2)$ \\
\hline $\mathrm{Cu}(3)-\mathrm{N}(12)$ & $2.138(2)$ \\
\hline $\mathrm{Cu}(3)-\mathrm{N}(13)$ & $2.117(2)$ \\
\hline $\mathrm{Cu}(3)-\mathrm{C}(1)$ & $1.890(2)$ \\
\hline $\mathrm{Cu}(4)-\mathrm{N}(2)$ & $1.951(2)$ \\
\hline $\mathrm{Cu}(4)-\mathrm{N}(10)$ & $1.979(2)$ \\
\hline $\mathrm{Cu}(4)-\mathrm{C}(3)$ & $2.055(2)$ \\
\hline $\mathrm{Cu}(5)-\mathrm{N}(6)$ & $2.1087(19)$ \\
\hline $\mathrm{Cu}(5)-\mathrm{N}(7)$ & $2.096(2)$ \\
\hline $\mathrm{Cu}(5)-\mathrm{N}(8)$ & $2.281(2)$ \\
\hline $\mathrm{Cu}(5)-\mathrm{C}(3)$ & $2.051(2)$ \\
\hline $\mathrm{Cu}(5)-\mathrm{C}(4)$ & $2.006(2)$ \\
\hline $\mathrm{Cu}(6)-\mathrm{N}(14)$ & $2.226(2)$ \\
\hline $\mathrm{Cu}(6)-\mathrm{N}(15)$ & $2.158(2)$ \\
\hline $\mathrm{Cu}(6)-\mathrm{N}(16)$ & $2.107(2)$ \\
\hline $\mathrm{Cu}(6)-\mathrm{C}(3)$ & $1.886(2)$ \\
\hline$C(1)-C(2)$ & $1.254(3)$ \\
\hline \multirow[t]{2}{*}{$C(3)-C(4)$} & $1.255(3)$ \\
\hline & torsion angle $/{ }^{\circ}$ \\
\hline $\mathrm{Cu}(1)-\mathrm{N}(1)-\mathrm{N}(2)-\mathrm{Cu}(4)$ & $1.004(247)$ \\
\hline $\mathrm{Cu}(1)-\mathrm{N}(9)-\mathrm{N}(10)-\mathrm{Cu}(4)$ & $-1.002(249)$ \\
\hline
\end{tabular}




\section{Electrochemistry}

Table A.23: Redox potentials of $3^{\mathbf{H} 20}$ at various $\mathrm{pH}$ values used for the formation of the Pourbaix diagram. The potentials were distinguished by cyclic voltammetry and differential pulse voltammetry.

\begin{tabular}{|c|c|c|c|c|c|}
\hline \multirow[t]{2}{*}{$\mathrm{pH}$} & \multicolumn{5}{|c|}{$E_{1 / 2} / \mathrm{V} v s . \mathrm{NHE}$} \\
\hline & II,III / II,II & III,III / II,III & III,IV / III,III & IV,IV / III,IV & IV,V / IV,IV \\
\hline 1.31 & 0.699 & 0.832 & - & & a \\
\hline 1.45 & 0.696 & 0.835 & - & & a \\
\hline 1.64 & 0.687 & 0.842 & 1.250 & & a \\
\hline 1.73 & 0.688 & 0.839 & 1.250 & & a \\
\hline 1.82 & 0.682 & 0.839 & 1.229 & & a \\
\hline 1.92 & 0.681 & 0.841 & 1.222 & & a \\
\hline 2.03 & 0.678 & 0.842 & 1.207 & & a \\
\hline 2.14 & 0.673 & 0.843 & 1.217 & & a \\
\hline 2.25 & 0.667 & 0.847 & 1.205 & & a \\
\hline 2.42 & 0.659 & 0.847 & 1.190 & & a \\
\hline 2.58 & 0.650 & 0.848 & 1.183 & - & 1.428 \\
\hline 2.86 & 0.634 & 0.850 & 1.171 & - & 1.413 \\
\hline 3.11 & 0.619 & 0.849 & 1.149 & - & 1.420 \\
\hline 3.31 & 0.609 & 0.850 & 1.137 & - & 1.418 \\
\hline 3.56 & 0.593 & 0.850 & 1.115 & - & 1.420 \\
\hline 3.73 & 0.581 & 0.850 & 1.098 & - & 1.415 \\
\hline 3.89 & 0.576 & 0.849 & 1.093 & - & 1.415 \\
\hline 4.04 & 0.572 & 0.851 & 1.095 & - & 1.420 \\
\hline 4.19 & 0.566 & 0.851 & 1.085 & - & 1.418 \\
\hline 4.33 & 0.554 & 0.849 & 1.069 & 1.243 & 1.411 \\
\hline 4.67 & 0.527 & 0.843 & 1.023 & 1.212 & 1.437 \\
\hline 4.81 & 0.510 & 0.840 & 0.997 & 1.198 & - \\
\hline 4.98 & 0.499 & 0.840 & 0.987 & 1.195 & - \\
\hline 5.14 & 0.493 & 0.837 & 0.977 & 1.183 & - \\
\hline 5.33 & 0.470 & 0.831 & 0.961 & 1.181 & - \\
\hline 5.63 & 0.461 & 0.822 & 0.946 & 1.172 & - \\
\hline 5.84 & 0.452 & 0.819 & 0.940 & 1.170 & - \\
\hline 5.99 & 0.423 & 0.808 & 0.916 & 1.155 & - \\
\hline 6.16 & 0.433 & 0.800 & 0.920 & - & - \\
\hline 6.37 & 0.426 & 0.789 & 0.903 & - & - \\
\hline 6.52 & 0.417 & 0.779 & 0.887 & - & - \\
\hline 6.85 & 0.391 & 0.754 & 0.890 & - & - \\
\hline 7.10 & 0.370 & 0.736 & 0.871 & - & - \\
\hline 7.64 & 0.351 & 0.709 & 0.881 & - & - \\
\hline 8.14 & 0.327 & 0.652 & 0.881 & - & - \\
\hline
\end{tabular}

atwo-electron process: IV,V / III,IV 


\section{Bibliography}

[1] International Energy Agency, World Energy Outlook 2019, Paris, 2019.

[2] N. S. Lewis, D. G. Nocera, Proc. Natl. Acad. Sci. 2006, 103, 15729-15735.

[3] Intergovernmental Panel on Climate Change, Climate Change 2013: The Physical Science Basis. Contribution of Working Group I to the Fifth Assessment Report of the Intergovernmental Panel on Climate Change, Cambridge University Press, Cambridge, UK and New York, NY, USA, 2013.

[4] International Energy Agency, International Renewable Energy Agency, Perspectives for the Energy Transition - Investment Needs for a Low-Carbon Energy System, 2017.

[5] International Renewable Energy Agency, Global Energy Transformation: A Roadmap to 2050, Abu Dhabi, 2018.

[6] AG Energiebilanzen e.V., Energieverbrauch 2019, Berlin, Bergheim, 2020.

[7] J. Barber, A. V. Ruban, P. J. Nixon, Oxygen Production and Reduction in Artificial and Natural Systems, World Scientific, 2019.

[8] H. Dau, P. Kurz, M.-D. Weitze, Künstliche Photosynthese: Besser als die Natur?, Springer-Verlag, Berlin Heidelberg, 2019.

[9] Bundesministerium für Wirtschaft und Energie, Energieeffizienz in Zahlen 2019 - Entwicklungen und Trends in Deutschland 2019, Berlin, 2019.

[10] A. Llobet, F. Meyer, Angew. Chem. Int. Ed. 2011, 50, A30-A33.

[11] H. Inoue, T. Shimada, Y. Kou, Y. Nabetani, D. Masui, S. Takagi, H. Tachibana, ChemSusChem 2011, 4, 173-179.

[12] W. Zhang, W. Lai, R. Cao, Chem. Rev. 2017, 117, 3717-3797.

[13] C. Gimbert-Suriñach, D. Moonshiram, L. Francàs, N. Planas, V. Bernales, F. Bozoglian, A. Guda, L. Mognon, I. López, M. A. Hoque, L. Gagliardi, C. J. Cramer, A. Llobet, J. Am. Chem. Soc. 2016, 138, 15291-15294.

[14] M. P. Johnson, Essays Biochem. 2016, 60, 255-273.

[15] W. Lubitz, E. J. Reijerse, J. Messinger, Energy Environ. Sci. 2008, 1, 15.

[16] L. Stryer, Biochemistry, W. H. Freeman and Company, New York, 1988.

[17] J. Yano, V. Yachandra, Chem. Rev. 2014, 114, 4175-4205.

[18] J. E. Penner-Hahn, C. F. Yocum, Science 2005, 310, 982-983.

[19] J. Barber, Chem Soc Rev 2009, 38, 185-196.

[20] R. D. Britt, P. H. Oyala, Science 2014, 345, 736-736.

[21] W. Lubitz, M. Chrysina, N. Cox, Photosynth. Res. 2019, 142, 105-125.

[22] W. Kaim, B. Schwederski, Bioanorganische Chemie, Vieweg+Teubner Verlag, Wiesbaden, 2005.

[23] Y. Umena, K. Kawakami, J.-R. Shen, N. Kamiya, Nature 2011, 473, 55-60.

[24] B. Kok, B. Forbush, M. McGloin, Photochem. Photobiol. 1970, 11, 457-475.

[25] C. Cady, R. Crabtree, G. Brudvig, Coord. Chem. Rev. 2008, 252, 444-455.

[26] H. Dau, C. Limberg, T. Reier, M. Risch, S. Roggan, P. Strasser, ChemCatChem 2010, 2, 724-761.

[27] N. Cox, M. Retegan, F. Neese, D. A. Pantazis, A. Boussac, W. Lubitz, Science 2014, 345, 804-808.

[28] J. Barber, Nat. Plants 2017, 3, 1-5.

[29] S. Styring, Faraday Discuss 2012, 155, 357-376.

[30] D. Gust, T. A. Moore, A. L. Moore, Acc. Chem. Res. 2009, 42, 1890-1898.

[31] S. Berardi, S. Drouet, L. Francàs, C. Gimbert-Suriñach, M. Guttentag, C. Richmond, T. Stoll, A. Llobet, Chem. Soc. Rev. 2014, 43, 7501-7519.

[32] J. Seo, H. Nishiyama, T. Yamada, K. Domen, Angew. Chem. Int. Ed. 2018, 57, 8396-8415.

[33] M. Wang, Y. Yang, J. Shen, J. Jiang, L. Sun, Sustain. Energy Fuels 2017, 1, 1641-1663.

[34] D. L. Ashford, M. K. Gish, A. K. Vannucci, M. K. Brennaman, J. L. Templeton, J. M. Papanikolas, T. J. Meyer, Chem. Rev. 2015, 115, 13006-13049.

[35] X. Ding, L. Zhang, Y. Wang, A. Liu, Y. Gao, Coord. Chem. Rev. 2018, 357, 130-143.

[36] E. A. Gibson, Chem. Soc. Rev. 2017, 46, 6194-6209.

[37] B. O'Regan, M. Grätzel, Nature 1991, 353, 737-740.

[38] A. Hagfeldt, G. Boschloo, L. Sun, L. Kloo, H. Pettersson, Chem. Rev. 2010, 110, 6595-6663.

[39] B. Zhang, L. Sun, Chem. Soc. Rev. 2019, 48, 2216-2264.

[40] K. J. Young, L. A. Martini, R. L. Milot, R. C. Snoeberger, V. S. Batista, C. A. Schmuttenmaer, R. H. Crabtree, G. W. Brudvig, Coord. Chem. Rev. 2012, 256, 2503-2520.

[41] C. C. L. McCrory, S. Jung, I. M. Ferrer, S. M. Chatman, J. C. Peters, T. F. Jaramillo, J. Am. Chem. Soc. 2015, 137, 4347-4357.

[42] J. J. Concepcion, J. W. Jurss, M. K. Brennaman, P. G. Hoertz, A. O. T. Patrocinio, N. Y. Murakami Iha, J. L. Templeton, T. J. Meyer, Acc. Chem. Res. 2009, 42, 1954-1965. 
[43] K. S. Joya, Y. F. Joya, K. Ocakoglu, R. van de Krol, Angew. Chem. Int. Ed. 2013, 52, 10426-10437.

[44] Z. Yu, F. Li, L. Sun, Energy Environ. Sci. 2015, 8, 760-775.

[45] E. D. Cline, S. E. Adamson, S. Bernhard, Inorg. Chem. 2008, 47, 10378-10388.

[46] S. Fukuzumi, T. Kobayashi, T. Suenobu, Angew. Chem. Int. Ed. 2011, 50, 728-731.

[47] T. Stoll, M. Gennari, I. Serrano, J. Fortage, J. Chauvin, F. Odobel, M. Rebarz, O. Poizat, M. Sliwa, A. Deronzier, M.-N. Collomb, Chem. Eur. J. 2013, 19, 782-792.

[48] K. Sakai, H. Ozawa, Coord. Chem. Rev. 2007, 251, 2753-2766.

[49] M. Wang, L. Chen, L. Sun, Energy Environ. Sci. 2012, 5, 6763-6778.

[50] V. S. Thoi, Y. Sun, J. R. Long, C. J. Chang, Chem. Soc. Rev. 2013, 42, 2388-2400.

[51] J. R. McKone, S. C. Marinescu, B. S. Brunschwig, J. R. Winkler, H. B. Gray, Chem. Sci. 2014, 5, 865878.

[52] M. R. DuBois, D. L. DuBois, Chem. Soc. Rev. 2008, 38, 62-72.

[53] M. Rakowski Dubois, D. L. Dubois, Acc. Chem. Res. 2009, 42, 1974-1982.

[54] M. L. Helm, M. P. Stewart, R. M. Bullock, M. R. DuBois, D. L. DuBois, Science 2011, 333, 863-866.

[55] U. J. Kilgore, J. A. S. Roberts, D. H. Pool, A. M. Appel, M. P. Stewart, M. R. DuBois, W. G. Dougherty, W. S. Kassel, R. M. Bullock, D. L. DuBois, J. Am. Chem. Soc. 2011, 133, 5861-5872.

[56] P. Garrido-Barros, C. Gimbert-Suriñach, R. Matheu, X. Sala, A. Llobet, Chem. Soc. Rev. 2017, 46, 6088-6098.

[57] L. Tong, R. P. Thummel, Chem. Sci. 2016, 7, 6591-6603.

[58] M. D. Kärkäs, E. V. Johnston, O. Verho, B. Åkermark, Acc. Chem. Res. 2014, 47, 100-111.

[59] M. D. Kärkäs, O. Verho, E. V. Johnston, B. Åkermark, Chem. Rev. 2014, 114, 11863-12001.

[60] J. D. Blakemore, R. H. Crabtree, G. W. Brudvig, Chem. Rev. 2015, 115, 12974-13005.

[61] D. J. Wasylenko, R. D. Palmer, C. P. Berlinguette, Chem. Commun. 2013, 49, 218-227.

[62] D. G. H. Hetterscheid, J. N. H. Reek, Angew. Chem. Int. Ed. 2012, 51, 9740-9747.

[63] N. Wang, H. Zheng, W. Zhang, R. Cao, Chin. J. Catal. 2018, 39, 228-244.

[64] R. Matheu, P. Garrido-Barros, M. Gil-Sepulcre, M. Z. Ertem, X. Sala, C. Gimbert-Suriñach, A. Llobet, Nat. Rev. Chem. 2019, 3, 331-341.

[65] A. Sartorel, M. Carraro, F. M. Toma, M. Prato, M. Bonchio, Energy Environ. Sci. 2012, 5, 55925603.

[66] J. R. Galán-Mascarós, ChemElectroChem 2015, 2, 37-50.

[67] B. M. Hunter, H. B. Gray, A. M. Müller, Chem. Rev. 2016, 116, 14120-14136.

[68] X. Liu, S. Inagaki, J. Gong, Angew. Chem. Int. Ed. 2016, 55, 14924-14950.

[69] P. Garrido-Barros, R. Matheu, C. Gimbert-Suriñach, A. Llobet, Curr. Opin. Electrochem. 2019, 15, 140-147.

[70] Z. N. Zahran, Y. Tsubonouchi, E. A. Mohamed, M. Yagi, ChemSusChem 2019, 12, 1775-1793.

[71] X. Sala, I. Romero, M. Rodríguez, L. Escriche, A. Llobet, Angew. Chem. Int. Ed. 2009, 48, $2842-$ 2852.

[72] A. Shatskiy, M. D. Kärkäs, B. Åkermark, Eur. J. Inorg. Chem. 2019, 2019, 2020-2024.

[73] B. Zhang, L. Sun, J. Am. Chem. Soc. 2019, 141, 5565-5580.

[74] D. W. Shaffer, Y. Xie, J. J. Concepcion, Chem. Soc. Rev. 2017, 46, 6170-6193.

[75] E. Y. Tsui, J. S. Kanady, T. Agapie, Inorg. Chem. 2013, 52, 13833-13848.

[76] A. Singh, L. Spiccia, Coord. Chem. Rev. 2013, 257, 2607-2622.

[77] A. R. Parent, K. Sakai, ChemSusChem 2014, 7, 2070-2080.

[78] M. D. Kärkäs, B. Åkermark, Dalton Trans. 2016, 45, 14421-14461.

[79] J.-W. Wang, D.-C. Zhong, T.-B. Lu, Coord. Chem. Rev. 2018, 377, 225-236.

[80] J. Lin, Q. Han, Y. Ding, Chem. Rec. 2018, 18, 1531-1547.

[81] M. Mahdi Najafpour, H. Feizi, Dalton Trans. 2018, 47, 6519-6527.

[82] J.-W. Wang, P. Sahoo, T.-B. Lu, ACS Catal. 2016, 6, 5062-5068.

[83] M. Mahdi Najafpour, H. Feizi, Catal. Sci. Technol. 2018, 8, 1840-1848.

[84] R. Safdari, M. R. Mohammadi, M. Hołyńska, P. Chernev, H. Dau, M. M. Najafpour, Dalton Trans. 2018, 47, 16668-16673.

[85] P. Garrido-Barros, S. Grau, S. Drouet, J. Benet-Buchholz, C. Gimbert-Suriñach, A. Llobet, ACS Catal. 2019, 9, 3936-3945.

[86] S. Fukuzumi, D. Hong, Eur. J. Inorg. Chem. 2014, 2014, 645-659.

[87] A. Singh, S. L. Y. Chang, R. K. Hocking, U. Bach, L. Spiccia, Catal. Sci. Technol. 2013, 3, 1725-1732.

[88] A. Singh, S. L. Y. Chang, R. K. Hocking, U. Bach, L. Spiccia, Energy Environ. Sci. 2013, 6, 579-586.

[89] R. K. Hocking, R. Brimblecombe, L.-Y. Chang, A. Singh, M. H. Cheah, C. Glover, W. H. Casey, L. Spiccia, Nat. Chem. 2011, 3, 461-466.

[90] I. Rapaport, L. Helm, A. E. Merbach, P. Bernhard, A. Ludi, Inorg. Chem. 1988, 27, 873-879. 
[91] L. Helm, A. E. Merbach, Chem. Rev. 2005, 105, 1923-1960.

[92] A. Draksharapu, Q. Li, H. Logtenberg, T. A. van den Berg, A. Meetsma, J. S. Killeen, B. L. Feringa, R. Hage, G. Roelfes, W. R. Browne, Inorg. Chem. 2012, 51, 900-913.

[93] D. Hong, S. Mandal, Y. Yamada, Y.-M. Lee, W. Nam, A. Llobet, S. Fukuzumi, Inorg. Chem. 2013, 52, 9522-9531.

[94] D. Wang, G. Ghirlanda, J. P. Allen, J. Am. Chem. Soc. 2014, 136, 10198-10201.

[95] B. A. Moyer, T. J. Meyer, J. Am. Chem. Soc. 1978, 100, 3601-3603.

[96] R. A. Binstead, T. J. Meyer, J. Am. Chem. Soc. 1987, 109, 3287-3297.

[97] D. R. Weinberg, C. J. Gagliardi, J. F. Hull, C. F. Murphy, C. A. Kent, B. C. Westlake, A. Paul, D. H. Ess, D. G. McCafferty, T. J. Meyer, Chem. Rev. 2012, 112, 4016-4093.

[98] T. R. Weaver, T. J. Meyer, S. A. Adeyemi, G. M. Brown, R. P. Eckberg, W. E. Hatfield, E. C. Johnson, R. W. Murray, D. Untereker, J. Am. Chem. Soc. 1975, 97, 3039-3048.

[99] X. Sala, S. Maji, R. Bofill, J. García-Antón, L. Escriche, A. Llobet, Acc. Chem. Res. 2014, 47, 504-516.

[100] P. Garrido-Barros, I. Funes-Ardoiz, S. Drouet, J. Benet-Buchholz, F. Maseras, A. Llobet, J. Am. Chem. Soc. 2015, 137, 6758-6761.

[101] I. Funes-Ardoiz, P. Garrido-Barros, A. Llobet, F. Maseras, ACS Catal. 2017, 1712-1719.

[102] S. Romain, L. Vigara, A. Llobet, Acc. Chem. Res. 2009, 42, 1944-1953.

[103] S. Romain, F. Bozoglian, X. Sala, A. Llobet, J. Am. Chem. Soc. 2009, 131, 2768-2769.

[104] L. Duan, F. Bozoglian, S. Mandal, B. Stewart, T. Privalov, A. Llobet, L. Sun, Nat. Chem. 2012, 4, 418-423.

[105] J. Nyhlén, L. Duan, B. Åkermark, L. Sun, T. Privalov, Angew. Chem. Int. Ed. 2010, 49, 1773-1777.

[106] E. A. Lewis, W. B. Tolman, Chem. Rev. 2004, 104, 1047-1076.

[107] S. J. Koepke, K. M. Light, P. E. VanNatta, K. M. Wiley, M. T. Kieber-Emmons, J. Am. Chem. Soc. 2017, $139,8586-8600$.

[108] S. Neudeck, S. Maji, I. López, S. Meyer, F. Meyer, A. Llobet, J. Am. Chem. Soc. 2014, 136, 24-27.

[109] D. J. Wasylenko, C. Ganesamoorthy, M. A. Henderson, B. D. Koivisto, H. D. Osthoff, C. P. Berlinguette, J. Am. Chem. Soc. 2010, 132, 16094-16106.

[110] S. Maji, L. Vigara, F. Cottone, F. Bozoglian, J. Benet-Buchholz, A. Llobet, Angew. Chem. Int. Ed. 2012, 51, 5967-5970.

[111] L. Vigara, M. Z. Ertem, N. Planas, F. Bozoglian, N. Leidel, H. Dau, M. Haumann, L. Gagliardi, C. J. Cramer, A. Llobet, Chem. Sci. 2012, 3, 2576.

[112] A. R. Parent, R. H. Crabtree, G. W. Brudvig, Chem. Soc. Rev. 2013, 42, 2247-2252.

[113] M. Yoshida, S. Masaoka, J. Abe, K. Sakai, Chem. - Asian J. 2010, 5, 2369-2378.

[114] A. Kimoto, K. Yamauchi, M. Yoshida, S. Masaoka, K. Sakai, Chem. Commun. 2012, 48, $239-241$.

[115] M. Yoshida, M. Kondo, S. Torii, K. Sakai, S. Masaoka, Angew. Chem. Int. Ed. 2015, 54, 7981-7984.

[116] A. Kundu, S. K. Dey, S. Dey, A. Anoop, S. Mandal, Inorg. Chem. 2020, 59, 1461-1470.

[117] J. Limburg, J. S. Vrettos, L. M. Liable-Sands, A. L. Rheingold, R. H. Crabtree, G. W. Brudvig, Science 1999, 283, 1524-1527.

[118] A. R. Parent, T. P. Brewster, W. De Wolf, R. H. Crabtree, G. W. Brudvig, Inorg. Chem. 2012, 51, 6147-6152.

[119] D. G. H. Hetterscheid, J. N. H. Reek, Eur. J. Inorg. Chem. 2014, 2014, 742-749.

[120] J. Limburg, R. H. Crabtree*, G. W. Brudvig*, Inorganica Chim. Acta 2000, 297, 301-306.

[121] A. Ikeda-Ohno, S. Tsushima, C. Hennig, T. Yaita, G. Bernhard, Dalton Trans. 2012, 41, 7190-7192.

[122] K. J. Lee, N. Elgrishi, B. Kandemir, J. L. Dempsey, Nat. Rev. Chem. 2017, 1, 0039.

[123] C. Costentin, S. Drouet, M. Robert, J.-M. Savéant, J. Am. Chem. Soc. 2012, 134, 11235-11242.

[124] V. C.-C. Wang, B. A. Johnson, ACS Catal. 2019, 7109-7123.

[125] R. Matheu, S. Neudeck, F. Meyer, X. Sala, A. Llobet, ChemSusChem 2016, 9, 3361-3369.

[126] H. S. White, W. G. Becker, A. J. Bard, J. Phys. Chem. 1984, 88, 1840-1846.

[127] K. Henbest, P. Douglas, M. S. Garley, A. Mills, J. Photochem. Photobiol. Chem. 1994, 80, $299-305$.

[128] B. C. Gau, H. Chen, Y. Zhang, M. L. Gross, Anal. Chem. 2010, 82, 7821-7827.

[129] L. Duan, Y. Xu, P. Zhang, M. Wang, L. Sun, Inorg. Chem. 2010, 49, 209-215.

[130] S. W. Gersten, G. J. Samuels, T. J. Meyer, J. Am. Chem. Soc. 1982, 104, 4029-4030.

[131] J. A. Gilbert, D. S. Eggleston, W. R. Murphy, D. A. Geselowitz, S. W. Gersten, D. J. Hodgson, T. J. Meyer, J. Am. Chem. Soc. 1985, 107, 3855-3864.

[132] J. P. Collin, J. P. Sauvage, Inorg. Chem. 1986, 25, 135-141.

[133] K. Nagoshi, S. Yamashita, M. Yagi, M. Kaneko, J. Mol. Catal. Chem. 1999, 144, 71-76.

[134] F. Liu, J. J. Concepcion, J. W. Jurss, T. Cardolaccia, J. L. Templeton, T. J. Meyer, Inorg. Chem. 2008, 47, 1727-1752.

[135] C. W. Chronister, R. A. Binstead, J. Ni, T. J. Meyer, Inorg. Chem. 1997, 36, 3814-3815. 
[136] J. L. Cape, J. K. Hurst, J. Am. Chem. Soc. 2008, 130, 827-829.

[137] B. Limburg, E. Bouwman, S. Bonnet, Coord. Chem. Rev. 2012, 256, 1451-1467.

[138] P. K. Ghosh, B. S. Brunschwig, M. Chou, C. Creutz, N. Sutin, J. Am. Chem. Soc. 1984, 106, 47724783.

[139] I. Romero, M. Rodríguez, C. Sens, J. Mola, M. Rao Kollipara, L. Francàs, E. Mas-Marza, L. Escriche, A. Llobet, Inorg. Chem. 2008, 47, 1824-1834.

[140] E. L. Lebeau, S. A. Adeyemi, T. J. Meyer, Inorg. Chem. 1998, 37, 6476-6484.

[141] R. A. Binstead, C. W. Chronister, J. Ni, C. M. Hartshorn, T. J. Meyer, J. Am. Chem. Soc. 2000, 122, 8464-8473.

[142] F. P. Rotzinger, S. Munavalli, P. Comte, J. K. Hurst, M. Graetzel, F. J. Pern, A. J. Frank, J. Am. Chem. Soc. 1987, 109, 6619-6626.

[143] M. K. Nazeeruddin, F. P. Rotzinger, P. Comte, M. Grätzel, J. Chem. Soc. Chem. Commun. 1988, 872 874.

[144] S. Trasatti, Electrochimica Acta 1984, 29, 1503-1512.

[145] E. I. Solomon, P. Chen, M. Metz, S.-K. Lee, A. E. Palmer, Angew. Chem. Int. Ed. 2001, 40, 45704590.

[146] L. Que, W. B. Tolman, Nature 2008, 455, 333-340.

[147] E. I. Solomon, D. E. Heppner, E. M. Johnston, J. W. Ginsbach, J. Cirera, M. Qayyum, M. T. KieberEmmons, C. H. Kjaergaard, R. G. Hadt, L. Tian, Chem. Rev. 2014, 114, 3659-3853.

[148] E. I. Solomon, F. Tuczek, D. E. Root, C. A. Brown, Chem. Rev. 1994, 94, 827-856.

[149] I. A. Koval, P. Gamez, C. Belle, K. Selmeczi, J. Reedijk, Chem. Soc. Rev. 2006, 35, 814-840.

[150] W. P. J. Gaykema, A. Volbeda, W. G. J. Hol, J. Mol. Biol. 1986, 187, 255-275.

[151] A. Volbeda, W. G. J. Hol, J. Mol. Biol. 1989, 209, 249-279.

[152] K. A. Magnus, H. Ton-That, J. E. Carpenter, Chem. Rev. 1994, 94, 727-735.

[153] C. Eicken, B. Krebs, J. C. Sacchettini, Curr. Opin. Struct. Biol. 1999, 9, 677-683.

[154] H. Decker, T. Schweikardt, F. Tuczek, Angew. Chem. Int. Ed. 2006, 45, 4546-4550.

[155] E. I. Solomon, U. M. Sundaram, T. E. Machonkin, Chem. Rev. 1996, 96, 2563-2606.

[156] E. I. Solomon, R. Sarangi, J. S. Woertink, A. J. Augustine, J. Yoon, S. Ghosh, Acc. Chem. Res. 2007, 40, 581-591.

[157] E. I. Solomon, J. W. Ginsbach, D. E. Heppner, M. T. Kieber-Emmons, C. H. Kjaergaard, P. J. Smeets, L. Tian, J. S. Woertink, Faraday Discuss. 2011, 148, 11-39.

[158] M. Metz, E. I. Solomon, J. Am. Chem. Soc. 2001, 123, 4938-4950.

[159] D. E. Wilcox, A. G. Porras, Y. T. Hwang, K. Lerch, M. E. Winkler, E. I. Solomon, J. Am. Chem. Soc. 1985, 107, 4015-4027.

[160] Y. Matoba, T. Kumagai, A. Yamamoto, H. Yoshitsu, M. Sugiyama, J. Biol. Chem. 2006, 281, 89818990.

[161] M. Rolff, J. Schottenheim, H. Decker, F. Tuczek, Chem. Soc. Rev. 2011, 40, 4077-4098.

[162] H. S. Mason, W. L. Fowlks, E. Peterson, J. Am. Chem. Soc. 1955, 77, 2914-2915.

[163] P. E. M. Siegbahn, J. Biol. Inorg. Chem. 2003, 8, 567-576.

[164] W. B. Tolman, Acc. Chem. Res. 1997, 30, 227-237.

[165] M. J. Baldwin, D. E. Root, J. E. Pate, K. Fujisawa, N. Kitajima, E. I. Solomon, J. Am. Chem. Soc. 1992, 114, 10421-10431.

[166] S. Schindler, Eur. J. Inorg. Chem. 2000, 2000, 2311-2326.

[167] L. M. Mirica, X. Ottenwaelder, T. D. P. Stack, Chem. Rev. 2004, 104, 1013-1046.

[168] C. E. Elwell, N. L. Gagnon, B. D. Neisen, D. Dhar, A. D. Spaeth, G. M. Yee, W. B. Tolman, Chem. Rev. 2017, 117, 2059-2107.

[169] R. R. Jacobson, Zoltan. Tyeklar, Amjad. Farooq, K. D. Karlin, Shuncheng. Liu, Jon. Zubieta, J. Am. Chem. Soc. 1988, 110, 3690-3692.

[170] K. E. Dalle, T. Gruene, S. Dechert, S. Demeshko, F. Meyer, J. Am. Chem. Soc. 2014, 136, 7428-7434.

[171] N. Kitajima, K. Fujisawa, Y. Morooka, K. Toriumi, J. Am. Chem. Soc. 1989, 111, 8975-8976.

[172] J. A. Halfen, S. Mahapatra, E. C. Wilkinson, S. Kaderli, V. G. Young, L. Que, A. D. Zuberbühler, W. B. Tolman, Science 1996, 271, 1397-1400.

[173] S. Mahapatra, J. A. Halfen, E. C. Wilkinson, G. Pan, X. Wang, V. G. Young, C. J. Cramer, Que Lawrence, W. B. Tolman, J. Am. Chem. Soc. 1996, 118, 11555-11574.

[174] L. Q. Hatcher, M. A. Vance, A. A. Narducci Sarjeant, E. I. Solomon, K. D. Karlin, Inorg. Chem. 2006, 45, 3004-3013.

[175] S. Kim, J. W. Ginsbach, A. I. Billah, M. A. Siegler, C. D. Moore, E. I. Solomon, K. D. Karlin, J. Am. Chem. Soc. 2014, 136, 8063-8071. 
[176] H. R. Lucas, L. Li, A. A. N. Sarjeant, M. A. Vance, E. I. Solomon, K. D. Karlin, J. Am. Chem. Soc. 2009, $131,3230-3245$.

[177] D. Maiti, J. S. Woertink, A. A. Narducci Sarjeant, E. I. Solomon, K. D. Karlin, Inorg. Chem. 2008, 47, 3787-3800.

[178] M. T. Kieber-Emmons, J. W. Ginsbach, P. K. Wick, H. R. Lucas, M. E. Helton, B. Lucchese, M. Suzuki, A. D. Zuberbühler, K. D. Karlin, E. I. Solomon, Angew. Chem. Int. Ed. 2014, 53, 4935-4939.

[179] C. Würtele, O. Sander, V. Lutz, T. Waitz, F. Tuczek, S. Schindler, J. Am. Chem. Soc. 2009, 131, 75447545.

[180] T. Hoppe, S. Schaub, J. Becker, C. Würtele, S. Schindler, Angew. Chem. Int. Ed. 2013, 52, 870-873.

[181] K. D. Karlin, N. Wei, B. Jung, S. Kaderli, A. D. Zuberbuehler, J. Am. Chem. Soc. 1991, 113, 58685870.

[182] K. D. Karlin, N. Wei, B. Jung, S. Kaderli, P. Niklaus, A. D. Zuberbuehler, J. Am. Chem. Soc. 1993, 115, 9506-9514.

[183] K. Fujisawa, M. Tanaka, Y. Moro-oka, N. Kitajima, J. Am. Chem. Soc. 1994, 116, 12079-12080.

[184] P. Chen, D. E. Root, C. Campochiaro, K. Fujisawa, E. I. Solomon, J. Am. Chem. Soc. 2003, 125, 466474.

[185] M. Schatz, V. Raab, S. P. Foxon, G. Brehm, S. Schneider, M. Reiher, M. C. Holthausen, J. Sundermeyer, S. Schindler, Angew. Chem. Int. Ed. 2004, 43, 4360-4363.

[186] C. Würtele, E. Gaoutchenova, K. Harms, M. C. Holthausen, J. Sundermeyer, S. Schindler, Angew. Chem. Int. Ed. 2006, 45, 3867-3869.

[187] N. Kindermann, C.-J. Günes, S. Dechert, F. Meyer, J. Am. Chem. Soc. 2017, 139, 9831-9834.

[188] A. Brinkmeier, Bimetallic Copper Complexes for Bioinspired Dioxygen Activation and Catalytic Water Oxidation, Georg-August Universität, 2018.

[189] M. Mahroof-Tahir, K. D. Karlin, J. Am. Chem. Soc. 1992, 114, 7599-7601.

[190] R. Cao, C. Saracini, J. W. Ginsbach, M. T. Kieber-Emmons, M. A. Siegler, E. I. Solomon, S. Fukuzumi, K. D. Karlin, J. Am. Chem. Soc. 2016, 138, 7055-7066.

[191] P. Chen, K. Fujisawa, E. I. Solomon, J. Am. Chem. Soc. 2000, 122, 10177-10193.

[192] M. Kodera, T. Kita, I. Miura, N. Nakayama, T. Kawata, K. Kano, S. Hirota, J. Am. Chem. Soc. 2001, $123,7715-7716$.

[193] T. Kamachi, Y.-M. Lee, T. Nishimi, J. Cho, K. Yoshizawa, W. Nam, J. Phys. Chem. A 2008, 112, 13102-13108.

[194] S. Kim, C. Saracini, M. A. Siegler, N. Drichko, K. D. Karlin, Inorg. Chem. 2012, 51, 12603-12605.

[195] D. Maiti, A. A. Narducci Sarjeant, K. D. Karlin, J. Am. Chem. Soc. 2007, 129, 6720-6721.

[196] D. Maiti, A. A. Narducci Sarjeant, K. D. Karlin, Inorg. Chem. 2008, 47, 8736-8747.

[197] A. Wada, M. Harata, K. Hasegawa, K. Jitsukawa, H. Masuda, M. Mukai, T. Kitagawa, H. Einaga, Angew. Chem. Int. Ed. 1998, 37, 798-799.

[198] S. Yamaguchi, A. Wada, S. Nagatomo, T. Kitagawa, K. Jitsukawa, H. Masuda, Chem. Lett. 2004, 33, 1556-1557.

[199] N. Kindermann, S. Dechert, S. Demeshko, F. Meyer, J. Am. Chem. Soc. 2015, 137, 8002-8005.

[200] K. Itoh, H. Hayashi, H. Furutachi, T. Matsumoto, S. Nagatomo, T. Tosha, S. Terada, S. Fujinami, M. Suzuki, T. Kitagawa, J. Am. Chem. Soc. 2005, 127, 5212-5223.

[201] S. Neudeck, S. Maji, I. López, S. Dechert, J. Benet-Buchholz, A. Llobet, F. Meyer, Inorg. Chem. 2016, $55,2508-2521$.

[202] J. Odrobina, J. Scholz, A. Pannwitz, L. Francàs, S. Dechert, A. Llobet, C. Jooss, F. Meyer, ACS Catal. 2017, 7, 2116-2125.

[203] J. Odrobina, J. Scholz, M. Risch, S. Dechert, C. Jooss, F. Meyer, ACS Catal. 2017, 7, 6235-6244.

[204] A. C. Sander, S. Maji, L. Francàs, T. Böhnisch, S. Dechert, A. Llobet, F. Meyer, ChemSusChem 2015, 8, 1697-1702.

[205] K. E. Dalle, F. Meyer, Eur. J. Inorg. Chem. 2015, 2015, 3391-3405.

[206] N. Kindermann, Diiron and Dicopper Complexes with Pyrazolate/Tacn Hybrid Ligands for Small Molecule Activation, Georg-August Universität, 2015.

[207] K. E. Dalle, Bioinspired Activation of Oxygen with Pyrazole-Supported Dinuclear Copper Complexes, Georg-August Universität, 2016.

[208] R. Zong, R. P. Thummel, J. Am. Chem. Soc. 2005, 127, 12802-12803.

[209] H.-W. Tseng, R. Zong, J. T. Muckerman, R. Thummel, Inorg. Chem. 2008, 47, 11763-11773.

[210] D. E. Polyansky, J. T. Muckerman, J. Rochford, R. Zong, R. P. Thummel, E. Fujita, J. Am. Chem. Soc. 2011, 133, 14649-14665.

[211] L. Duan, A. Fischer, Y. Xu, L. Sun, J. Am. Chem. Soc. 2009, 131, 10397-10399. 
[212] G. C. Dismukes, R. Brimblecombe, G. A. N. Felton, R. S. Pryadun, J. E. Sheats, L. Spiccia, G. F. Swiegers, Acc. Chem. Res. 2009, 42, 1935-1943.

[213] L. Duan, C. M. Araujo, M. S. G. Ahlquist, L. Sun, Proc. Natl. Acad. Sci. 2012, 109, 15584-15588.

[214] L. Duan, L. Wang, A. K. Inge, A. Fischer, X. Zou, L. Sun, Inorg. Chem. 2013, 52, 7844-7852.

[215] L. Wang, L. Duan, Y. Wang, M. S. G. Ahlquist, L. Sun, Chem Commun 2014, 50, 12947-12950.

[216] B. Zhang, L. Sun, J. Am. Chem. Soc. 2019, 141, 5565-5580.

[217] R. Matheu, A. Ghaderian, L. Francàs, P. Chernev, M. Z. Ertem, J. Benet-Buchholz, V. S. Batista, M. Haumann, C. Gimbert-Suriñach, X. Sala, A. Llobet, Chem. Eur. J. 2018, 24, 12838-12847.

[218] R. Matheu, M. Z. Ertem, C. Gimbert-Suriñach, X. Sala, A. Llobet, Chem. Rev. 2019, 119, 3453-3471.

[219] R. Matheu, M. Z. Ertem, J. Benet-Buchholz, E. Coronado, V. S. Batista, X. Sala, A. Llobet, J. Am. Chem. Soc. 2015, 137, 10786-10795.

[220] J. Creus, R. Matheu, I. Peñafiel, D. Moonshiram, P. Blondeau, J. Benet-Buchholz, J. García-Antón, X. Sala, C. Godard, A. Llobet, Angew. Chem. Int. Ed. 2016, 55, 15382-15386.

[221] R. Matheu, I. A. Moreno-Hernandez, X. Sala, H. B. Gray, B. S. Brunschwig, A. Llobet, N. S. Lewis, J. Am. Chem. Soc. 2017, 139, 11345-11348.

[222] S. Grau, S. Berardi, A. Moya, R. Matheu, V. Cristino, J. J. Vilatela, C. A. Bignozzi, S. Caramori, C. Gimbert-Suriñach, A. Llobet, Sustain. Energy Fuels 2018, 2, 1979-1985.

[223] R. Matheu, L. Francàs, P. Chernev, M. Z. Ertem, V. Batista, M. Haumann, X. Sala, A. Llobet, ACS Catal. 2015, 5, 3422-3429.

[224] N. Vereshchuk, R. Matheu, J. Benet-Buchholz, M. Pipelier, J. Lebreton, D. Dubreuil, A. Tessier, C. Gimbert-Suriñach, M. Z. Ertem, A. Llobet, J. Am. Chem. Soc. 2020, 142, 5068-5077.

[225] C. Sens, I. Romero, M. Rodríguez, A. Llobet, T. Parella, J. Benet-Buchholz, J. Am. Chem. Soc. 2004, 126, 7798-7799.

[226] F. Bozoglian, S. Romain, M. Z. Ertem, T. K. Todorova, C. Sens, J. Mola, M. Rodríguez, I. Romero, J. Benet-Buchholz, X. Fontrodona, C. J. Cramer, L. Gagliardi, A. Llobet, J. Am. Chem. Soc. 2009, 131, 15176-15187.

[227] Y. Xu, A. Fischer, L. Duan, L. Tong, E. Gabrielsson, B. Åkermark, L. Sun, Angew. Chem. Int. Ed. 2010, 49, 8934-8937.

[228] A. Shatskiy, R. Lomoth, A. F. Abdel-Magied, W. Rabten, T. M. Laine, H. Chen, J. Sun, P. G. Andersson, M. D. Kärkäs, E. V. Johnston, B. Åkermark, Dalton Trans. 2016, 45, 19024-19033.

[229] T. M. Laine, M. D. Kärkäs, R.-Z. Liao, T. Åkermark, B.-L. Lee, E. A. Karlsson, P. E. M. Siegbahn, B. Åkermark, Chem Commun 2015, 51, 1862-1865.

[230] T. M. Laine, M. D. Kärkäs, R.-Z. Liao, P. E. M. Siegbahn, B. Åkermark, Chem. Eur. J. 2015, 21, 1003910048.

[231] S. Berardi, L. Francàs, S. Neudeck, S. Maji, J. Benet-Buchholz, F. Meyer, A. Llobet, ChemSusChem 2015, 8, 3688-3696.

[232] W. Zhang, J. Liu, H. Zhu, W. Gao, L. Sun, Synth. Commun. 2007, 37, 3393-3402.

[233] A. C. Sander, Studies of New Pyrazolate-Based Binuclear Ruthenium Complexes for Catalytic Water Oxidation, Georg-August-Universität, 2015.

[234] B. Schneider, S. Demeshko, S. Dechert, F. Meyer, Angew. Chem. Int. Ed. 2010, 49, 9274-9277.

[235] B. Schneider, S. Demeshko, S. Dechert, F. Meyer, Inorg. Chem. 2012, 51, 4912-4914.

[236] B. Schneider, S. Demeshko, S. Neudeck, S. Dechert, F. Meyer, Inorg. Chem. 2013, 52, 1323013237.

[237] M. Steinert, B. Schneider, S. Dechert, S. Demeshko, F. Meyer, Angew. Chem. Int. Ed. 2014, 53, 6135-6139.

[238] M. Steinert, B. Schneider, S. Dechert, S. Demeshko, F. Meyer, Inorg. Chem. 2016, 55, 2363-2373.

[239] J. Tong, S. Demeshko, M. John, S. Dechert, F. Meyer, Inorg. Chem. 2016, 55, 4362-4372.

[240] J. Tong, S. Demeshko, S. Dechert, F. Meyer, Eur. J. Inorg. Chem. 2017, 2017, 4333-4343.

[241] J. W. L. Wong, S. Demeshko, S. Dechert, F. Meyer, Inorg. Chem. 2019, 58, 13337-13345.

[242] H. Zabrodsky, S. Peleg, D. Avnir, J. Am. Chem. Soc. 1992, 114, 7843-7851.

[243] H. Zabrodsky, S. Peleg, D. Avnir, J. Am. Chem. Soc. 1993, 115, 8278-8289.

[244] Avnir, David, "Continuous Symmetry Measures | Shape | Simple mode," can be found under http://www.csm.huji.ac.il/new/?cmd=shape.26., n.d.

[245] S. Alvarez, D. Avnir, M. Llunell, M. Pinsky, New J. Chem. 2002, 26, 996-1009.

[246] V. A. Russell, M. D. Ward, J. Mater. Chem. 1997, 7, 1123-1133.

[247] J. L. Atwood, L. J. Barbour, S. Dalgarno, C. L. Raston, H. R. Webb, J. Chem. Soc. Dalton Trans. 2002, 4351-4356.

[248] S. Baskar Raj, V. Sethuraman, S. Francis, M. Hemamalini, P. T. Muthiah, G. Bocelli, A. Cantoni, U. Rychlewska, B. Warzajtis, CrystEngComm 2003, 5, 70-76. 
[249] Y. Liu, C. Hu, A. Comotti, M. D. Ward, Science 2011, 333, 436-440.

[250] P. Zanello, C. Nervi, F. F. de Biani, Inorganic Electrochemistry. Theory, Practice and Application., Royal Society Of Chemistry, Cambridge, 2011.

[251] E. S. Rountree, B. D. McCarthy, T. T. Eisenhart, J. L. Dempsey, Inorg. Chem. 2014, 53, 9983-10002.

[252] A. E. Clark, J. K. Hurst, in Prog. Inorg. Chem., John Wiley \& Sons, Ltd, 2011, pp. 1-54.

[253] X. Sala, L. Escriche, A. Llobet, in Mol. Sol. Fuels, 2011, pp. 273-287.

[254] D. J. Wasylenko, C. Ganesamoorthy, M. A. Henderson, C. P. Berlinguette, Inorg. Chem. 2011, 50, 3662-3672.

[255] L. Wang, L. Duan, B. Stewart, M. Pu, J. Liu, T. Privalov, L. Sun, J. Am. Chem. Soc. 2012, 134, 1886818880.

[256] A. Shatskiy, A. A. Bardin, M. Oschmann, R. Matheu, J. Benet-Buchholz, L. Eriksson, M. D. Kärkäs, E. V. Johnston, C. Gimbert-Suriñach, A. Llobet, B. Åkermark, ChemSusChem 2019, 12, 2251-2262.

[257] R. Matheu, J. Benet-Buchholz, X. Sala, A. Llobet, Inorg. Chem. 2018, 57, 1757-1765.

[258] T. Fan, L. Duan, P. Huang, H. Chen, Q. Daniel, M. S. G. Ahlquist, L. Sun, ACS Catal. 2017, 7, 29562966.

[259] U. S. Akhtar, E. L. Tae, Y. S. Chun, I. C. Hwang, K. B. Yoon, ACS Catal. 2016, 6, 8361-8369.

[260] P. K. Ghosh, B. S. Brunschwig, M. Chou, C. Creutz, N. Sutin, J. Am. Chem. Soc. 1984, 106, 47724783.

[261] A. Juris, V. Balzani, F. Barigelletti, S. Campagna, P. Belser, A. von Zelewsky, Coord. Chem. Rev. 1988, 84, 85-277.

[262] A. Lewandowska-Andralojc, D. E. Polyansky, R. Zong, R. P. Thummel, E. Fujita, Phys. Chem. Chem. Phys. 2013, 15, 14058-14068.

[263] W. Su, H. A. Younus, S. Chaemchuen, C. Chen, F. Verpoort, ChemCatChem 2017, 9, 2565-2573.

[264] D. Lukács, Ł. Szyrwiel, J. S. Pap, Catalysts 2019, 9, 83.

[265] S. M. Barnett, K. I. Goldberg, J. M. Mayer, Nat. Chem. 2012, 4, 498-502.

[266] M.-T. Zhang, Z. Chen, P. Kang, T. J. Meyer, J. Am. Chem. Soc. 2013, 135, 2048-2051.

[267] J. S. Pap, Ł. Szyrwiel, D. Srankó, Z. Kerner, B. Setner, Z. Szewczuk, W. Malinka, Chem. Commun. 2015, 51, 6322-6324.

[268] Ł. Szyrwiel, D. Lukács, D. F. Srankó, Z. Kerner, A. Kotynia, J. Brasuń, B. Setner, Z. Szewczuk, K. Malec, J. S. Pap, RSC Adv. 2017, 7, 24657-24666.

[269] T. Zhang, C. Wang, S. Liu, J.-L. Wang, W. Lin, J. Am. Chem. Soc. 2014, 136, 273-281.

[270] D. L. Gerlach, S. Bhagan, A. A. Cruce, D. B. Burks, I. Nieto, H. T. Truong, S. P. Kelley, C. J. HerbstGervasoni, K. L. Jernigan, M. K. Bowman, S. Pan, M. Zeller, E. T. Papish, Inorg. Chem. 2014, 53, 12689-12698.

[271] A. de Aguirre, P. Garrido-Barros, I. Funes-Ardoiz, F. Maseras, Eur. J. Inorg. Chem. 2019, 2019, 2109-2114.

[272] P. Garrido-Barros, C. Gimbert-Suriñach, D. Moonshiram, A. Picón, P. Monge, V. S. Batista, A. Llobet, J. Am. Chem. Soc. 2017, 139, 12907-12910.

[273] X.-J. Su, M. Gao, L. Jiao, R.-Z. Liao, P. E. M. Siegbahn, J.-P. Cheng, M.-T. Zhang, Angew. Chem. Int. Ed. 2015, 54, 4909-4914.

[274] X.-J. Su, C. Zheng, Q.-Q. Hu, H.-Y. Du, R.-Z. Liao, M.-T. Zhang, Dalton Trans. 2018, 47, 8670-8675.

[275] N. Bridonneau, L.-M. Chamoreau, G. Gontard, J.-L. Cantin, J. von Bardeleben, V. Marvaud, Dalton Trans. 2016, 45, 9412-9418.

[276] D. A. Valyaev, S. Clair, L. Patrone, M. Abel, L. Porte, O. Chuzel, J.-L. Parrain, Chem. Sci. 2013, 4, 2815-2821.

[277] J. A. Halfen, W. B. Tolman, K. Weighardt, in Inorg. Synth. (Ed.: Marcetta.Y. Darensbourg), John Wiley \& Sons, Ltd, Hoboken, NJ, USA, 1998, pp. 75-81.

[278] C. Acerete, J. M. Bueno, L. Campayo, P. Navarro, M. I. Rodriguez-Franco, A. Samat, Tetrahedron 1994, 50, 4765-4774.

[279] S. Buchler, F. Meyer, E. Kaifer, H. Pritzkow, Inorganica Chim. Acta 2002, 337, 371-386.

[280] F. Meyer, S. Beyreuther, K. Heinze, L. Zsolnai, Chem. Ber. 1997, 130, 605-613.

[281] J. Ackermann, S. Buchler, F. Meyer, Comptes Rendus Chim. 2007, 10, 421-432.

[282] B. Burger, Structure, Dynamics and Bioinspired Reactivity of Novel Pyrazolate-Based Diiron Complexes, Georg-August Universität, 2012.

[283] N. Kindermann, A. Schober, S. Demeshko, N. Lehnert, F. Meyer, Inorg. Chem. 2016, 55, 1153811550.

[284] B. Bauer-Siebenlist, F. Meyer, E. Farkas, D. Vidovic, J. A. Cuesta-Seijo, R. Herbst-Irmer, H. Pritzkow, Inorg. Chem. 2004, 43, 4189-4202.

[285] S. Nestke, E. Ronge, I. Siewert, Dalton Trans. 2018, 47, 10737-10741. 
[286] M. Gross, J. Jordan, Pure Appl. Chem. 1984, 56, 1095-1129.

[287] Z. Chen, T. J. Meyer, Angew. Chem. Int. Ed. 2013, 52, 700-703.

[288] V. Artero, M. Fontecave, Chem. Soc. Rev. 2013, 42, 2338-2356.

[289] X. Wu, F. Li, B. Zhang, L. Sun, J. Photochem. Photobiol. C Photochem. Rev. 2015, 25, 71-89.

[290] N. S. McIntyre, M. G. Cook, Anal. Chem. 1975, 47, 2208-2213.

[291] R. P. Vasquez, Surf. Sci. Spectra 1998, 5, 262-266.

[292] R. P. Vasquez, Surf. Sci. Spectra 1998, 5, 267-272.

[293] M. C. Biesinger, Surf. Interface Anal. 2017, 49, 1325-1334.

[294] L. Francàs, X. Sala, J. Benet-Buchholz, L. Escriche, A. Llobet, ChemSusChem 2009, 2, 321-329.

[295] B. Radaram, J. A. Ivie, W. M. Singh, R. M. Grudzien, J. H. Reibenspies, C. E. Webster, X. Zhao, Inorg. Chem. 2011, 50, 10564-10571.

[296] A. C. Sander, A. Schober, S. Dechert, F. Meyer, Eur. J. Inorg. Chem. 2015, 2015, 4348-4353.

[297] M. Anbar, I. Pecht, J. Am. Chem. Soc. 1967, 89, 2553-2556.

[298] G. L. Elizarova, L. G. Matvienko, N. V. Lozhkina, V. N. Parmon, K. I. Zamaraev, React. Kinet. Catal. Lett. 1981, 16, 191-194.

[299] A. Harriman, G. Porter, P. Walters, J. Chem. Soc. Faraday Trans. 2 1981, 77, 2373-2383.

[300] B. S. Brunschwig, M. H. Chou, C. Creutz, P. Ghosh, N. Sutin, J. Am. Chem. Soc. 1983, 105, 48324833.

[301] G. L. Elizarova, L. G. Matvienko, N. V. Lozhkina, V. N. Parmon, React. Kinet. Catal. Lett. 1984, 26, 67-72.

[302] M. W. Kanan, D. G. Nocera, Science 2008, 321, 1072-1075.

[303] J. G. McAlpin, Y. Surendranath, M. Dincă, T. A. Stich, S. A. Stoian, W. H. Casey, D. G. Nocera, R. D. Britt, J. Am. Chem. Soc. 2010, 132, 6882-6883.

[304] M. Risch, K. Klingan, F. Ringleb, P. Chernev, I. Zaharieva, A. Fischer, H. Dau, ChemSusChem 2012, $5,542-549$.

[305] K. Dimitrou, K. Folting, W. E. Streib, G. Christou, J. Am. Chem. Soc. 1993, 115, 6432-6433.

[306] N. S. McCool, D. M. Robinson, J. E. Sheats, G. C. Dismukes, J. Am. Chem. Soc. 2011, 133, 1144611449.

[307] G. L. Ganga, F. Puntoriero, S. Campagna, I. Bazzan, S. Berardi, M. Bonchio, A. Sartorel, M. Natali, F. Scandola, Faraday Discuss. 2012, 155, 177-190.

[308] S. Berardi, G. La Ganga, M. Natali, I. Bazzan, F. Puntoriero, A. Sartorel, F. Scandola, S. Campagna, M. Bonchio, J. Am. Chem. Soc. 2012, 134, 11104-11107.

[309] M. D. Symes, D. A. Lutterman, T. S. Teets, B. L. Anderson, J. J. Breen, D. G. Nocera, ChemSusChem 2013, 6, 65-69.

[310] X. Zhou, F. Li, H. Li, B. Zhang, F. Yu, L. Sun, ChemSusChem 2014, 7, 2453-2456.

[311] B. Zhang, F. Li, F. Yu, X. Wang, X. Zhou, H. Li, Y. Jiang, L. Sun, ACS Catal. 2014, 4, 804-809.

[312] Y. Wang, F. Li, H. Li, L. Bai, L. Sun, Chem. Commun. 2016, 52, 3050-3053.

[313] S. Ye, C. Ding, R. Chen, F. Fan, P. Fu, H. Yin, X. Wang, Z. Wang, P. Du, C. Li, J. Am. Chem. Soc. 2018, 140, 3250-3256.

[314] Y. Wang, F. Li, X. Zhou, F. Yu, J. Du, L. Bai, L. Sun, Angew. Chem. Int. Ed. 2017, 56, 6911-6915.

[315] F. Evangelisti, R. Moré, F. Hodel, S. Luber, G. R. Patzke, J. Am. Chem. Soc. 2015, 137, 11076-11084.

[316] F. Song, R. Moré, M. Schilling, G. Smolentsev, N. Azzaroli, T. Fox, S. Luber, G. R. Patzke, J. Am. Chem. Soc. 2017, 139, 14198-14208.

[317] Q. Yin, J. M. Tan, C. Besson, Y. V. Geletii, D. G. Musaev, A. E. Kuznetsov, Z. Luo, K. I. Hardcastle, C. L. Hill, Science 2010, 328, 342-345.

[318] Z. Huang, Z. Luo, Y. V. Geletii, J. W. Vickers, Q. Yin, D. Wu, Y. Hou, Y. Ding, J. Song, D. G. Musaev, C. L. Hill, T. Lian, J. Am. Chem. Soc. 2011, 133, 2068-2071.

[319] S. Goberna-Ferrón, L. Vigara, J. Soriano-López, J. R. Galán-Mascarós, Inorg. Chem. 2012, 51, 11707-11715.

[320] H. Lv, Y. V. Geletii, C. Zhao, J. W. Vickers, G. Zhu, Z. Luo, J. Song, T. Lian, D. G. Musaev, C. L. Hill, Chem. Soc. Rev. 2012, 41, 7572-7589.

[321] S. Tanaka, M. Annaka, K. Sakai, Chem. Commun. 2012, 48, 1653-1655.

[322] G. Zhu, Y. V. Geletii, P. Kögerler, H. Schilder, J. Song, S. Lense, C. Zhao, K. I. Hardcastle, D. G. Musaev, C. L. Hill, Dalton Trans. 2012, 41, 2084-2090.

[323] H. Lv, J. A. Rudd, P. F. Zhuk, J. Young Lee, E. C. Constable, C. E. Housecroft, C. L. Hill, D. G. Musaev, Y. V. Geletii, RSC Adv. 2013, 3, 20647-20654.

[324] F. Song, Y. Ding, B. Ma, C. Wang, Q. Wang, X. Du, S. Fu, J. Song, Energy Environ. Sci. 2013, 6, 1170 1184.

[325] F. Evangelisti, P.-E. Car, O. Blacque, G. R. Patzke, Catal. Sci. Technol. 2013, 3, 3117-3129. 
[326] S.-S. Wang, G.-Y. Yang, Chem. Rev. 2015, 115, 4893-4962.

[327] M. Blasco-Ahicart, J. Soriano-López, J. J. Carbó, J. M. Poblet, J. R. Galan-Mascaros, Nat. Chem. 2018, 10, 24-30.

[328] D. J. Wasylenko, C. Ganesamoorthy, J. Borau-Garcia, C. P. Berlinguette, Chem. Commun. 2011, 47, 4249-4251.

[329] D. J. Wasylenko, R. D. Palmer, E. Schott, C. P. Berlinguette, Chem. Commun. 2012, 48, 2107-2109.

[330] D. W. Crandell, S. Ghosh, C. P. Berlinguette, M.-H. Baik, ChemSusChem 2015, 8, 844-852.

[331] D. K. Dogutan, R. McGuire, D. G. Nocera, J. Am. Chem. Soc. 2011, 133, 9178-9180.

[332] R. McGuire Jr., D. K. Dogutan, T. S. Teets, J. Suntivich, Y. Shao-Horn, D. G. Nocera, Chem. Sci. 2010, 1, 411-414.

[333] M. Z. Ertem, C. J. Cramer, Dalton Trans. 2012, 41, 12213-12219.

[334] W. Lai, R. Cao, G. Dong, S. Shaik, J. Yao, H. Chen, J. Phys. Chem. Lett. 2012, 3, 2315-2319.

[335] C.-Y. Yeh, C. J. Chang, D. G. Nocera, J. Am. Chem. Soc. 2001, 123, 1513-1514.

[336] D. Wang, J. T. Groves, Proc. Natl. Acad. Sci. 2013, 110, 15579-15584.

[337] T. Nakazono, A. R. Parent, K. Sakai, Chem. Commun. 2013, 49, 6325-6327.

[338] M. L. Rigsby, S. Mandal, W. Nam, L. C. Spencer, A. Llobet, S. S. Stahl, Chem. Sci. 2012, 3, 30583062.

[339] S. Fukuzumi, S. Mandal, K. Mase, K. Ohkubo, H. Park, J. Benet-Buchholz, W. Nam, A. Llobet, J. Am. Chem. Soc. 2012, 134, 9906-9909.

[340] R. H. Crabtree, Chem. Rev. 2012, 112, 1536-1554.

[341] J. J. Stracke, R. G. Finke, J. Am. Chem. Soc. 2011, 133, 14872-14875.

[342] Q. Daniel, R. B. Ambre, B. Zhang, B. Philippe, H. Chen, F. Li, K. Fan, S. Ahmadi, H. Rensmo, L. Sun, ACS Catal. 2017, 7, 1143-1149.

[343] A. M. Ullman, Y. Liu, M. Huynh, D. K. Bediako, H. Wang, B. L. Anderson, D. C. Powers, J. J. Breen, H. D. Abruña, D. G. Nocera, J. Am. Chem. Soc. 2014, 136, 17681-17688.

[344] H.-Y. Wang, E. Mijangos, S. Ott, A. Thapper, Angew. Chem. Int. Ed. 2014, 53, 14499-14502.

[345] M. Risch, V. Khare, I. Zaharieva, L. Gerencser, P. Chernev, H. Dau, J. Am. Chem. Soc. 2009, 131, 6936-6937.

[346] M. W. Kanan, J. Yano, Y. Surendranath, M. Dincă, V. K. Yachandra, D. G. Nocera, J. Am. Chem. Soc. 2010, 132, 13692-13701.

[347] D. Hong, J. Jung, J. Park, Y. Yamada, T. Suenobu, Y.-M. Lee, W. Nam, S. Fukuzumi, Energy Environ. Sci. 2012, 5, 7606-7616.

[348] S. Fu, Y. Liu, Y. Ding, X. Du, F. Song, R. Xiang, B. Ma, Chem. Commun. 2014, 50, 2167-2169.

[349] H. Chen, Z. Sun, X. Liu, A. Han, P. Du, J. Phys. Chem. C 2015, 119, 8998-9004.

[350] N. Wiberg, Lehrbuch der Anorganischen Chemie, Walter De Gruyter, Berlin, New York, 2008.

[351] O. Kahn, C. J. Martinez, Science 1998, 279, 44-48.

[352] S. A. Wolf, D. D. Awschalom, R. A. Buhrman, J. M. Daughton, S. von Molnár, M. L. Roukes, A. Y. Chtchelkanova, D. M. Treger, Science 2001, 294, 1488-1495.

[353] P. C. Bunting, M. Atanasov, E. Damgaard-Møller, M. Perfetti, I. Crassee, M. Orlita, J. Overgaard, J. van Slageren, F. Neese, J. R. Long, Science 2018, 362, eaat7319.

[354] R. L. Carlin, Magnetochemistry, Springer-Verlag, Berlin Heidelberg, 1986.

[355] D. Reta, N. F. Chilton, Phys. Chem. Chem. Phys. 2019, 21, 23567-23575.

[356] D. Gatteschi, R. Sessoli, Angew. Chem. Int. Ed. 2003, 42, 268-297.

[357] M. Murrie, Chem. Soc. Rev. 2010, 39, 1986-1995.

[358] M. Lamouchi, E. Jeanneau, G. Novitchi, D. Luneau, A. Brioude, C. Desroches, Inorg. Chem. 2014, $53,63-72$.

[359] V. Chandrasekhar, A. Dey, A. J. Mota, E. Colacio, Inorg. Chem. 2013, 52, 4554-4561.

[360] A. K. Mondal, V. S. Parmar, S. Biswas, S. Konar, Dalton Trans. 2016, 45, 4548-4557.

[361] S. Manna, A. Bhunia, S. Mistri, J. Vallejo, E. Zangrando, H. Puschmann, J. Cano, S. C. Manna, Eur. J. Inorg. Chem. 2017, 2017, 2585-2594.

[362] G. A. Craig, M. Murrie, Chem. Soc. Rev. 2015, 44, 2135-2147.

[363] J. Vallejo, I. Castro, L. Cañadillas-Delgado, C. Ruiz-Pérez, J. Ferrando-Soria, R. Ruiz-García, J. Cano, F. Lloret, M. Julve, Dalton Trans. 2010, 39, 2350-2358.

[364] R. Boča, Coord. Chem. Rev. 2004, 248, 757-815.

[365] M. Ciampolini, N. Nardi, Inorg. Chem. 1966, 5, 41-44.

[366] L. Sacconi, Pure Appl. Chem. 1968, 17, 95-127.

[367] R. Morassi, I. Bertini, L. Sacconi, Coord. Chem. Rev. 1973, 11, 343-402.

[368] I. Bertini, M. Ciampolini, D. Gatteschi, Inorg. Chem. 1973, 12, 693-696.

[369] L. K. Thompson, B. S. Ramaswamy, E. A. Seymour, Can. J. Chem. 1977, 55, 878-888. 
[370] C. Di Giovanni, C. Gimbert-Suriñach, M. Nippe, J. Benet-Buchholz, J. R. Long, X. Sala, A. Llobet, Chem. Eur. J. 2016, 22, 361-369.

[371] M. Nippe, R. S. Khnayzer, J. A. Panetier, D. Z. Zee, B. S. Olaiya, M. Head-Gordon, C. J. Chang, F. N. Castellano, J. R. Long, Chem. Sci. 2013, 4, 3934-3945.

[372] A. Coehn, M. Gläser, Z. Für Anorg. Chem. 1902, 33, 9-24.

[373] F. W. Skirrow, Z. Für Anorg. Chem. 1902, 33, 25-30.

[374] A. C. C. Tseung, S. Jasem, Electrochimica Acta 1977, 22, 31-34.

[375] C. Iwakura, A. Honji, H. Tamura, Electrochimica Acta 1981, 26, 1319-1326.

[376] R. Boggio, A. Carugati, S. Trasatti, J. Appl. Electrochem. 1987, 17, 828-840.

[377] A. Harriman, I. J. Pickering, J. M. Thomas, P. A. Christensen, J. Chem. Soc. Faraday Trans. 1 1988, 84, 2795-2806.

[378] Y. Surendranath, M. Dincă, D. G. Nocera, J. Am. Chem. Soc. 2009, 131, 2615-2620.

[379] D. G. Nocera, Acc. Chem. Res. 2012, 45, 767-776.

[380] N. Kindermann, E. Bill, S. Dechert, S. Demeshko, E. J. Reijerse, F. Meyer, Angew. Chem. Int. Ed. 2015, 54, 1738-1743.

[381] N. G. Connelly, W. E. Geiger, Chem. Rev. 1996, 96, 877-910.

[382] R. C. Stevenson, J. Magn. Reson. 1984, 57, 24-42.

[383] J. Svorec, M. Valko, J. Moncol, M. Mazúr, M. Melník, J. Telser, Transit. Met. Chem. 2009, 34, 129134.

[384] H. Khanmohammadi, M. Erfantalab, E. J. Reijerse, J. Iran. Chem. Soc. 2014, 11, 323-333.

[385] R. P. Sartoris, O. R. Nascimento, R. C. Santana, M. Perec, R. F. Baggio, R. Calvo, Dalton Trans. 2015, $44,4732-4743$.

[386] R. R. Jacobson, Zoltan. Tyeklar, Amjad. Farooq, K. D. Karlin, Shuncheng. Liu, Jon. Zubieta, J. Am. Chem. Soc. 1988, 110, 3690-3692.

[387] M. Weitzer, S. Schindler, G. Brehm, S. Schneider, E. Hörmann, B. Jung, S. Kaderli, A. D. Zuberbühler, Inorg. Chem. 2003, 42, 1800-1806.

[388] J. M. Achord, C. L. Hussey, Anal. Chem. 1980, 52, 601-602.

[389] A. N. Desnoyer, A. Nicolay, M. S. Ziegler, N. A. Torquato, T. D. Tilley, Angew. Chem. Int. Ed. 2020, $59,12769-12773$.

[390] A. J. Jordan, G. Lalic, J. P. Sadighi, Chem. Rev. 2016, 116, 8318-8372.

[391] T. Nakajima, K. Nakamae, Y. Ura, T. Tanase, Eur. J. Inorg. Chem. 2020, 2020, 2211-2226.

[392] S. A. Bezman, M. R. Churchill, J. A. Osborn, J. Wormald, J. Am. Chem. Soc. 1971, 93, 2063-2065.

[393] R. C. Stevens, M. R. McLean, R. Bau, T. F. Koetzle, J. Am. Chem. Soc. 1989, 111, 3472-3473.

[394] L. Hou, W.-J. Shi, Y.-Y. Wang, H.-H. Wang, L. Cui, P.-X. Chen, Q.-Z. Shi, Inorg. Chem. 2011, 50, $261-$ 270.

[395] J. Zheng, H. Yang, M. Xie, D. Li, Chem. Commun. 2019, 55, 7134-7146.

[396] A. Kishimura, T. Yamashita, K. Yamaguchi, T. Aida, Nat. Mater. 2005, 4, 546-549.

[397] K. Fujisawa, Y. Ishikawa, Y. Miyashita, K. Okamoto, Inorganica Chim. Acta 2010, 363, 2977-2989.

[398] A. Kishimura, T. Yamashita, T. Aida, J. Am. Chem. Soc. 2005, 127, 179-183.

[399] J.-X. Zhang, J. He, Y.-G. Yin, M.-H. Hu, D. Li, X.-C. Huang, Inorg. Chem. 2008, 47, 3471-3473.

[400] Q. Xiao, J. Zheng, M. Li, S.-Z. Zhan, J.-H. Wang, D. Li, Inorg. Chem. 2014, 53, 11604-11615.

[401] V. W.-W. Yam, V. K.-M. Au, S. Y.-L. Leung, Chem. Rev. 2015, 115, 7589-7728.

[402] F. Gong, Q. Wang, J. Chen, Z. Yang, M. Liu, S. Li, G. Yang, L. Bai, J. Liu, Y. Dong, Inorg. Chem. 2010, 49, 1658-1666.

[403] E. Cariati, E. Lucenti, C. Botta, U. Giovanella, D. Marinotto, S. Righetto, Coord. Chem. Rev. 2016, 306, 566-614.

[404] M. Veronelli, N. Kindermann, S. Dechert, S. Meyer, F. Meyer, Inorg. Chem. 2014, 53, 2333-2341.

[405] M. Enomoto, A. Kishimura, T. Aida, J. Am. Chem. Soc. 2001, 123, 5608-5609.

[406] H. V. R. Dias, H. V. K. Diyabalanage, M. G. Eldabaja, O. Elbjeirami, M. A. Rawashdeh-Omary, M. A. Omary, J. Am. Chem. Soc. 2005, 127, 7489-7501.

[407] H. V. R. Dias, H. V. K. Diyabalanage, M. A. Rawashdeh-Omary, M. A. Franzman, M. A. Omary, J. Am. Chem. Soc. 2003, 125, 12072-12073.

[408] C. E. Kivi, D. Song, Dalton Trans. 2016, 45, 17087-17090.

[409] H. Yang, J. Zheng, S.-K. Peng, X.-W. Zhu, M.-Y. Wan, W. Lu, D. Li, Chem. Commun. 2019, 55, 46354638.

[410] H. Lang, A. Jakob, B. Milde, Organometallics 2012, 31, 7661-7693.

[411] J. C. Evans, R. A. Nyquist, Spectrochim. Acta 1960, 16, 918-928.

[412] R. A. Nyquist, S. Fiedler, Vib. Spectrosc. 1994, 7, 149-162. 
[413] R. A. Nyquist, Interpreting Infrared, Raman, and Nuclear Magnetic Resonance Spectra, Academic Press, Cambridge, MA, 2001.

[414] D. L. Reger, M. F. Huff, Organometallics 1992, 11, 69-73.

[415] H. Lang, K. Köhler, G. Rheinwald, L. Zsolnai, M. Büchner, A. Driess, G. Huttner, J. Strähle, Organometallics 1999, 18, 598-605.

[416] H. Lang, M. Leschke, M. Melter, B. Walfort, K. Köhler, S. E. Schulz, T. Geßner, Z. Für Anorg. Allg. Chem. 2003, 629, 2371-2380.

[417] Y. Sevryugina, A. Yu. Rogachev, M. A. Petrukhina, Inorg. Chem. 2007, 46, 7870-7879.

[418] J. S. Thompson, A. Z. Bradley, K.-H. Park, K. D. Dobbs, W. Marshall, Organometallics 2006, 25, 2712-2714.

[419] J. Eppinger, K.-W. Huang, ACS Energy Lett. 2017, 2, 188-195.

[420] M. Grasemann, G. Laurenczy, Energy Environ. Sci. 2012, 5, 8171-8181.

[421] D. Mellmann, P. Sponholz, H. Junge, M. Beller, Chem. Soc. Rev. 2016, 45, 3954-3988.

[422] F. Valentini, V. Kozell, C. Petrucci, A. Marrocchi, Y. Gu, D. Gelman, L. Vaccaro, Energy Environ. Sci. 2019, 12, 2646-2664.

[423] A. K. Singh, S. Singh, A. Kumar, Catal. Sci. Technol. 2016, 6, 12-40.

[424] W. H. Bernskoetter, N. Hazari, Eur. J. Inorg. Chem. 2013, 2013, 4032-4041.

[425] J. E. Heimann, W. H. Bernskoetter, N. Hazari, J. Am. Chem. Soc. 2019, 141, 10520-10529.

[426] T. Nakajima, Y. Kamiryo, M. Kishimoto, K. Imai, K. Nakamae, Y. Ura, T. Tanase, J. Am. Chem. Soc. 2019, 141, 8732-8736.

[427] A. Zavras, M. Krstić, P. Dugourd, V. Bonačić-Koutecký, R. A. J. O’Hair, ChemCatChem 2017, 9, 1298-1302.

[428] M. Krstić, Q. Jin, G. N. Khairallah, R. A. J. O’Hair, V. Bonačić-Koutecký, ChemCatChem 2018, 10, 1173-1177.

[429] T. J. Schmeier, G. E. Dobereiner, R. H. Crabtree, N. Hazari, J. Am. Chem. Soc. 2011, 133, 9274-9277.

[430] A. Zavras, G. N. Khairallah, M. Krstić, M. Girod, S. Daly, R. Antoine, P. Maitre, R. J. Mulder, S.-A. Alexander, V. Bonačić-Koutecký, P. Dugourd, R. A. J. O’Hair, Nat. Commun. 2016, 7, 1-8.

[431] H.-W. Suh, T. J. Schmeier, N. Hazari, R. A. Kemp, M. K. Takase, Organometallics 2012, 31, 82258236.

[432] N. Hazari, J. E. Heimann, Inorg. Chem. 2017, 56, 13655-13678.

[433] T. F. Pascher, M. Ončák, C. van der Linde, M. K. Beyer, ChemistryOpen 2019, 8, 1453-1459.

[434] A. Brinkmeier, Bioinspired Dinuclear Copper Complexes for Dioxygen Activation, Georg-August Universität, 2014.

[435] R. S. Dhayal, W. E. van Zyl, C. W. Liu, Dalton Trans. 2019, 48, 3531-3538.

[436] C. M. Zall, J. C. Linehan, A. M. Appel, ACS Catal. 2015, 5, 5301-5305.

[437] E. A. Romero, T. Zhao, R. Nakano, X. Hu, Y. Wu, R. Jazzar, G. Bertrand, Nat. Catal. 2018, 1, 743747.

[438] I. P. Evans, A. Spencer, G. Wilkinson, J. Chem. Soc. Dalton Trans. 1973, 0, 204-209.

[439] E. Dulière, M. Devillers, J. Marchand-Brynaert, Organometallics 2003, 22, 804-811.

[440] O. Kahn, Molecular Magnetism, Wiley-VCH, New York, NY, 1993.

[441] E. Bill, JulX, Max Planck Institute For Chemical Energy Conversion, Mülheim/Ruhr, Germany, 2008.

[442] E. Bill,JulX_2S, Max Planck Institute For Chemical Energy Conversion, Mülheim/Ruhr, Germany, 2014.

[443] G. M. Sheldrick, Acta Crystallogr. Sect. Found. Adv. 2015, 71, 3-8.

[444] G. M. Sheldrick, Acta Crystallogr. Sect. C Struct. Chem. 2015, 71, 3-8.

[445] M. J. Frisch, G. W. Trucks, H. B. Schlegel, G. E. Scuseria, M. A. Robb, J. R. Cheeseman, G. Scalmani, V. Barone, B. Mennucci, G. A. Petersson, H. Nakatsuji, M. Caricato, X. Li, H. P. Hratchian, A. F. Izmaylov, J. Bloino, G. Zheng, J. L. Sonnenberg, M. Hada, M. Ehara, K. Toyota, R. Fukuda, J. Hasegawa, M. Ishida, T. Nakajima, Y. Honda, O. Kitao, H. Nakai, T. Vreven, J. A. Montgomery Jr., J. E. Peralta, F. Ogliaro, M. Bearpark, J. J. Heyd, E. Brothers, K. N. Kudin, V. N. Staroverov, T. Keith, R. Kobayashi, J. Normand, K. Raghavachari, A. Rendell, J. C. Burant, S. S. Iyengar, J. Tomasi, M. Cossi, N. Rega, J. M. Millam, M. Klene, J. E. Knox, J. B. Cross, V. Bakken, C. Adamo, J. Jaramillo, R. Gomperts, R. E. Stratmann, O. Yazyev, A. J. Austin, R. Cammi, C. Pomelli, J. W. Ochterski, R. L. Martin, K. Morokuma, V. G. Zakrzewski, G. A. Voth, P. Salvador, J. J. Dannenberg, S. Dapprich, A. D. Daniels, O. Farkas, J. B. Foresman, J. V. Ortiz, J. Cioslowski, D. J. Fox, Gaussian 09, Gaussian, Inc., Wallingford, CT, 2013.

[446] C. Adamo, V. Barone, J. Chem. Phys. 1999, 110, 6158-6170.

[447] S. Grimme, J. Antony, S. Ehrlich, H. Krieg, J. Chem. Phys. 2010, 132, 154104. 
[448] S. Grimme, S. Ehrlich, L. Goerigk, J. Comput. Chem. 2011, 32, 1456-1465.

[449] F. Weigend, R. Ahlrichs, Phys. Chem. Chem. Phys. 2005, 7, 3297.

[450] M. Dolg, U. Wedig, H. Stoll, H. Preuss, J. Chem. Phys. 1987, 86, 866-872.

[451] S. Grimme, Chem. - Eur. J. 2012, 18, 9955-9964.

[452] A. V. Marenich, C. J. Cramer, D. G. Truhlar, J. Phys. Chem. B 2009, 113, 6378-6396. 


\section{Structures of Complexes}

Ruthenium Complexes:

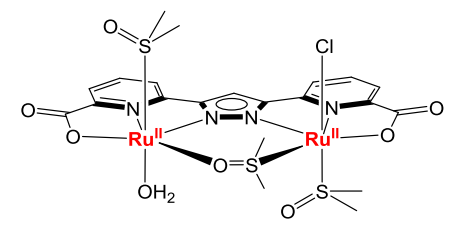

1

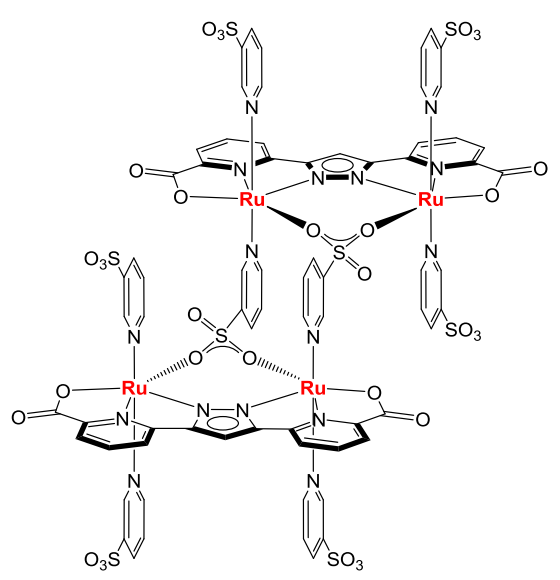

4
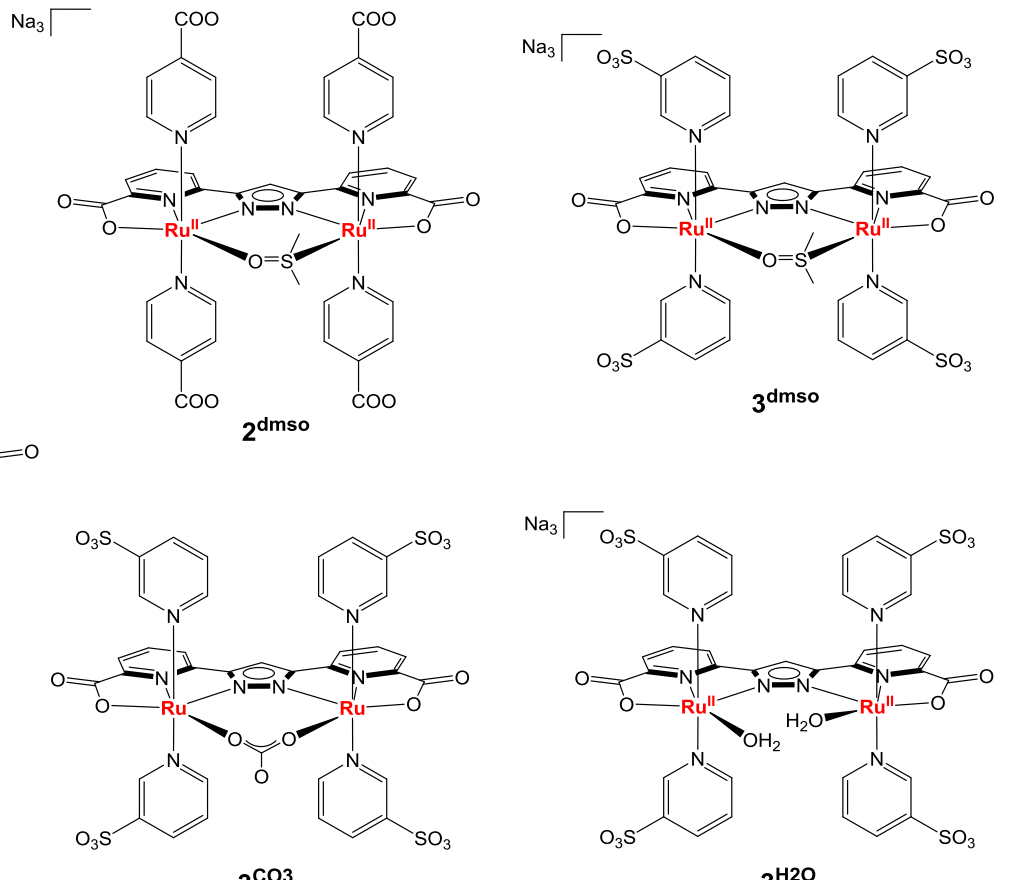

$3^{\mathrm{CO} 3}$

$3^{\mathrm{H} 2 \mathrm{O}}$

Dinuclear Copper Complexes:
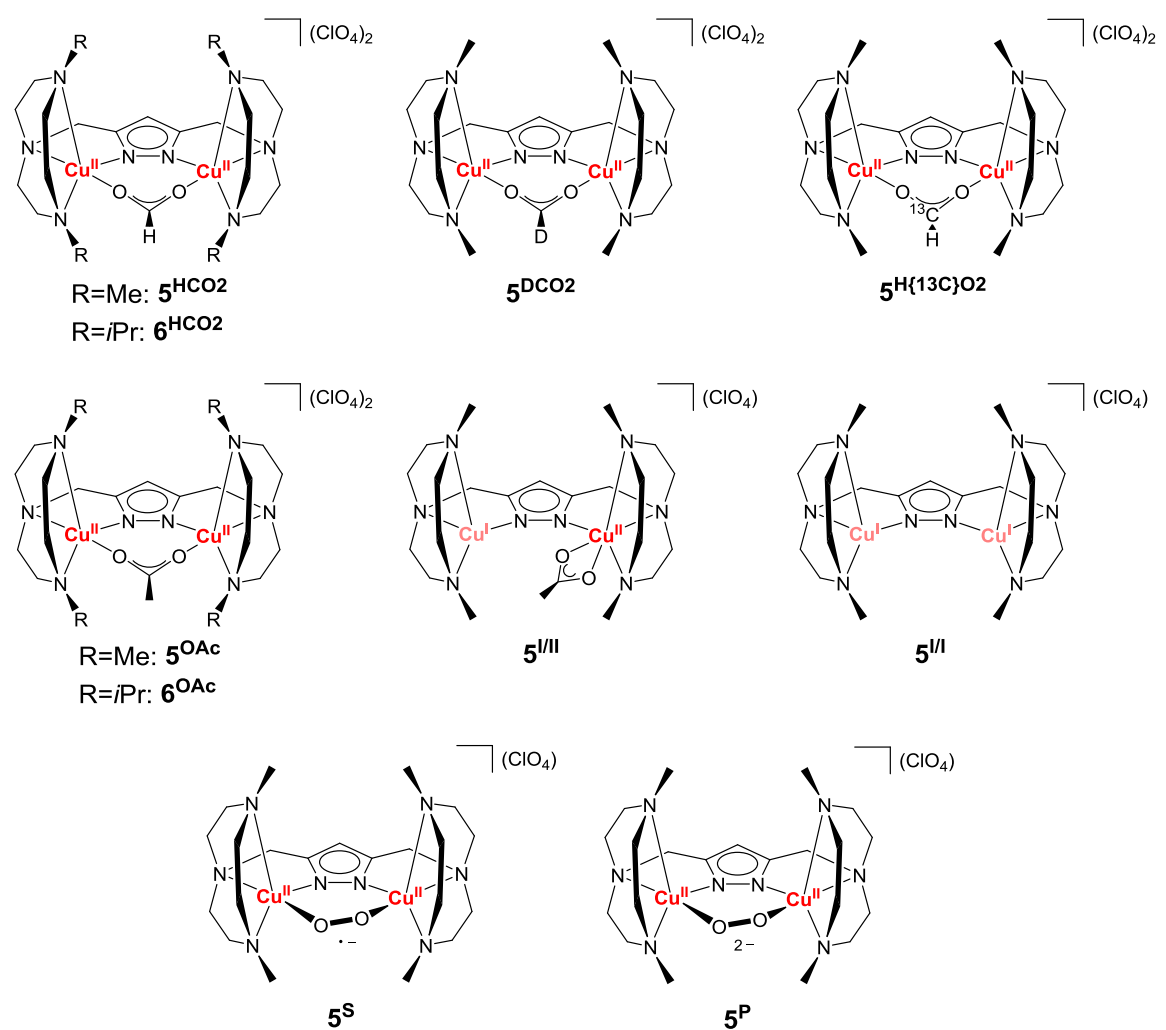
Cobalt Complexes:
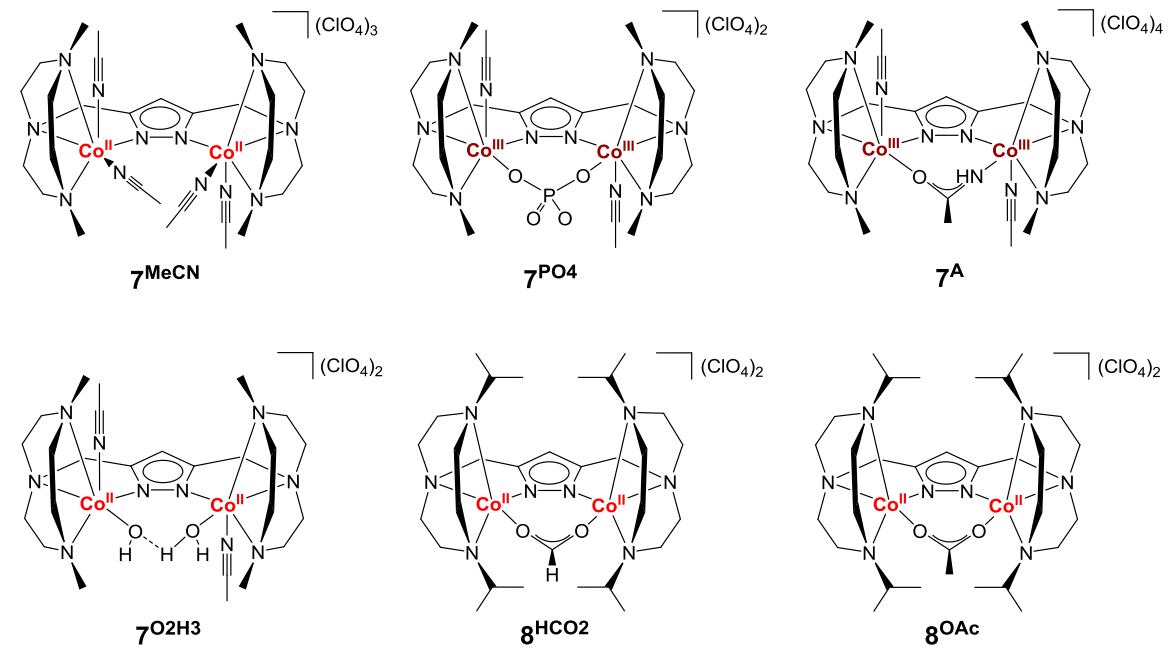

Hexanuclear Copper Complexes:

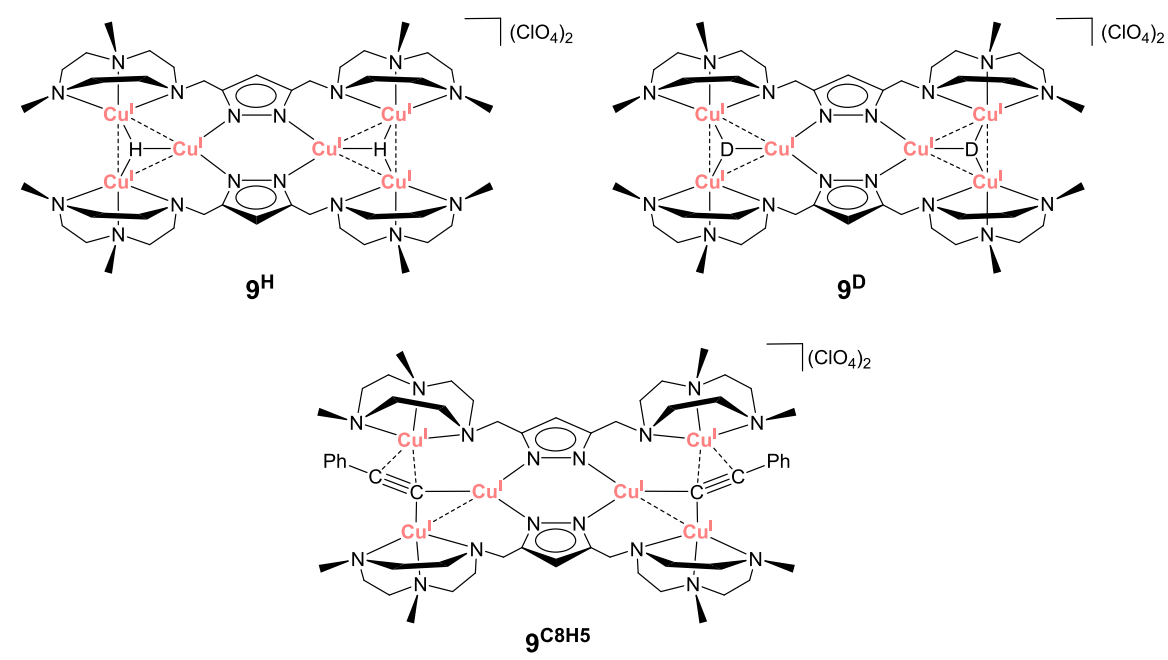




\section{Abbreviations}

\begin{tabular}{|c|c|}
\hline ADP & adenosine diphosphate \\
\hline AP & artificial photosynthesis \\
\hline APT & atom proton transfer \\
\hline ATP & adenosine triphosphate \\
\hline ATR & attenuated total reflection \\
\hline Mebbp & 3,5-bis $\{6-(2,2$ '-bipyridyl $)\}$-4-methyl-pyrazolato \\
\hline BA & butylamine \\
\hline $\mathrm{bda}^{2-}$ & 2,2'-bipyridine-6,6'-dicarboxylate \\
\hline BDD & boron doped diamond \\
\hline BDFE & bond dissociation free energy \\
\hline BJ & Becke-Johnson \\
\hline${ }^{\mathrm{H}} \mathrm{bpp}^{-}$ & 3,5-bis(2-pyridyl)pyrazolato \\
\hline bpy & 2,2'-bipyridine \\
\hline $\mathrm{Bz}$ & benzyl \\
\hline CAN & ceric ammonium nitrate \\
\hline $\mathrm{CB}$ & conduction band \\
\hline $\mathrm{CE}$ & counter electrode \\
\hline $\mathrm{CN}$ & coordination number \\
\hline COSY & correlated spectroscopy \\
\hline $\mathrm{COx}$ & catechol oxidase \\
\hline $\mathrm{Cp} / \mathrm{Cp}^{*}$ & cyclopentadienyl/pentamethylcyclopentadienyl \\
\hline CPE & controlled potential electrolysis \\
\hline CSM & Continuous Symmetry Measures \\
\hline CT & charge transfer \\
\hline $\mathrm{CV}$ & cyclic voltammogram \\
\hline cyt $b_{6} f$ & cytochrome $b_{6} f$ complex \\
\hline $\mathrm{d}$ & doublet (NMR) \\
\hline DBU & diazabicycloundecene \\
\hline DCM & dichloromethane \\
\hline DFT & density functional theory \\
\hline DLS & dynamic light scattering \\
\hline $\mathrm{DMF}$ & dimethylformamide \\
\hline DMSO & dimethylsulfoxide \\
\hline L-DOPA & 3,4-dihydroxy-L-phenylalanine \\
\hline DOSY & diffusion-ordered spectroscopy \\
\hline DPV & differential pulse voltammogram \\
\hline DSSC & dye-sensitized solar cell \\
\hline EDX & energy-dispersive X-ray spectroscopy \\
\hline e.g. & exempli gratia, for example \\
\hline EMPA & electron microprobe analysis \\
\hline EPR & electron paramagnetic resonance \\
\hline ESI & electrospray ionization \\
\hline et al. & et alii, to complete a list \\
\hline $\mathrm{Et}_{2} \mathrm{O}$ & diethylether \\
\hline EtOAc & ethyl acetate \\
\hline $\mathrm{EtOH}$ & ethanol \\
\hline FA & formic acid \\
\hline FAME & flexibility, adaptability, multidenticity, equatori \\
\hline $\mathrm{Fc}^{+/ 0}$ & ferrocenium/ferrocene \\
\hline
\end{tabular}




\begin{tabular}{|c|c|}
\hline FD & ferredoxin \\
\hline $\mathrm{FE}$ & faradaic efficiency \\
\hline $\mathrm{FeS}$ & iron-sulfur cluster \\
\hline FNR & ferredoxin-NADP ${ }^{+}$reductase \\
\hline FOWA & foot-of-the-wave analysis \\
\hline FWHM & full width half maximum \\
\hline GC & glassy carbon \\
\hline HAT & hydrogen atom transfer/abstraction \\
\hline Hc & hemocyanin \\
\hline HEC & hydrogen evolving catalyst \\
\hline His & histidine \\
\hline HLutOTf & lutidinium triflate \\
\hline HMBC & heteronuclear multiple bond correlation \\
\hline HOTf & triflic acid \\
\hline HSQC & heteronuclear single quantum coherance \\
\hline $\mathrm{H}_{3} \mathrm{tpha}^{-}$ & $2,2^{\prime}: 6^{\prime}, 2^{\prime \prime}$-terpyridine-6,6"-diphosphononic acid \\
\hline $\mathrm{I} 2 \mathrm{M}$ & interaction of two $\mathrm{M}-\mathrm{O}$ entities \\
\hline i.e. & id est, that is \\
\hline$i \operatorname{Pr}$ & isopropyl \\
\hline IR & infrared \\
\hline ISC & intersystem crossing \\
\hline isoq & isoquinoline \\
\hline LMCT & ligand-metal charge transfer \\
\hline $\mathrm{m}$ & multiplet (NMR), medium (IR) \\
\hline $\mathrm{Me}$ & methyl \\
\hline $\mathrm{MeCN}$ & acetonitrile, $\mathrm{CH}_{3} \mathrm{CN}$ \\
\hline $\mathrm{MeOH}$ & methanol \\
\hline MLCT & metal-ligand charge transfer \\
\hline MS & mass spectrometry \\
\hline MSE & mercury sulfate electrode \\
\hline MTBE & methyl tert-butyl ether \\
\hline$m / z$ & mass-to-charge ratio \\
\hline NADPH & nicotinamide adenine dinucleotide phosphate \\
\hline $\mathrm{NaOAc}$ & sodium acetate \\
\hline NaOEt & sodium ethanolate \\
\hline NaOTf & sodium triflate \\
\hline $\mathrm{NEt}_{3}$ & triethyl amine \\
\hline NHE & normal hydrogen electrode \\
\hline NMR & nuclear magnetic resonance \\
\hline NOESY & nuclear overhauser enhancement spectroscopy \\
\hline$o$ & ortho \\
\hline OCP & open circuit potential \\
\hline OEC & oxygen evolving complex \\
\hline OER & oxygen evolution reaction \\
\hline ORR & oxygen reduction reaction \\
\hline $\mathrm{P}$ & photosensitizer \\
\hline PC & plastocyanin \\
\hline PCET & proton-coupled electron transfer \\
\hline PEC & photoelectrochemical cell \\
\hline PEM & proton exchange membrane \\
\hline Pheo & pheophytin \\
\hline
\end{tabular}




\begin{tabular}{|c|c|}
\hline pic & picoline \\
\hline PS & photosystem \\
\hline PV & photovoltaics \\
\hline py & pyridine \\
\hline $\mathrm{Q} / \mathrm{H}_{2} \mathrm{Q}$ & plastoquinone/plastoquinol \\
\hline RDS & rate-determining step \\
\hline $\mathrm{RE}$ & reference electrode \\
\hline RI & redox isomerization \\
\hline ROS & reactive oxygen species \\
\hline s & singlet (NMR), strong (IR) \\
\hline$S$ & spin \\
\hline SC & semiconductor \\
\hline SCE & saturated calomel electrode \\
\hline SEA & sacrificial electron acceptor \\
\hline SEC & spectroelectrochemical \\
\hline SEM & scanning electron microscopy \\
\hline SET & single electron transfer \\
\hline SIM & single ion magnet \\
\hline SMD & solvation model based on density \\
\hline SMM & single molecule magnet \\
\hline SPE & single point energy \\
\hline SWV & square wave voltammogram \\
\hline $\mathrm{t}$ & triplet (NMR) \\
\hline $\operatorname{tacn}$ & 1,4,7-triazacyclononane \\
\hline$t \mathrm{Bu}$ & tert-butyl \\
\hline $\mathrm{tda}^{2-}$ & $2,2^{\prime}: 6^{\prime}, 2^{\prime \prime}$-terpyridine- $6,6^{\prime \prime}$-dicarboxylate \\
\hline TEM & transmission electron microscopy \\
\hline TEMPO & $(2,2,6,6$-tetramethylpiperidinyl)oxyl \\
\hline TFE & 2,2,2-trifluoroethanol \\
\hline TGG & triglycylglycine \\
\hline TIP & temperature-independent paramagnetism \\
\hline TMG & 1,1,3,3-tetramethylguanidine \\
\hline TOF & turnover frequency \\
\hline TON & turnover number \\
\hline $\mathrm{Tp}$ & tris(pyrazolyl)borate \\
\hline tpa & tris(2-pyridylmethyl)amine \\
\hline tren & tris(2-aminoethyl)amine \\
\hline trpy & $2,2^{\prime}: 6^{\prime}, 2^{\prime \prime}$-terpyridine \\
\hline TS & transition state \\
\hline Tyr & tyrosine \\
\hline $\mathrm{UV} / \mathrm{vis}$ & ultraviolet/visible \\
\hline VB & valence band \\
\hline viz. & videlicet, namely \\
\hline vs. & versus \\
\hline VT & variable temperature \\
\hline VTVH & variable temperature/variable field \\
\hline $\mathrm{w}$ & weak (IR) \\
\hline WE & working electrode \\
\hline WNA & water nucleophilic attack \\
\hline WO & water oxidation \\
\hline WOC & water oxidation catalyst \\
\hline
\end{tabular}


XPS

XRD

$\mathrm{Y}$

ZPVE
X-ray photoelectron spectroscopy

$\mathrm{X}$-ray diffraction

tyrosine

zero-point vibrational energy 


\section{Acknowledgments}

Many people have supported me throughout my PhD thesis. With these few words I would like to acknowledge their manifold contributions.

First and foremost, I want to thank Prof. Dr. Franc Meyer for the opportunity to conduct my PhD thesis in his working group. Your guidance, support and motivation were ever present over the last years, and it was a pleasure to work on this interesting and challenging topic. Furthermore, I thank Prof. Dr. Inke Siewert for being my second supervisor and for the many fruitful scientific discussions, which went far beyond the annual meetings.

Entering the world of water oxidation catalysis with ruthenium complexes went hand in hand with an intense scientific collaboration to the group of Prof. Dr. Antoni Llobet at the Institut Català d'Investigació Química (ICIQ) in Tarragona (Spain). I would like to thank Prof. Llobet for giving me the opportunity to join his group, for the many invaluable discussions on my topic (either in person or via email) and the constant new ideas and feedback. Furthermore, I owe a big thank you to Dr. Roc Matheu and Dr. Carolina Gimbert-Suriñach, who spent many hours discussing my scientific work and who supervised me in and outside the lab. Dr. Roc Matheu I would further like to thank for introducing me to all necessities within the lab and especially the photochemical water oxidation catalysis setup. My stays at the ICIQ were not only scientifically an indispensable part of my PhD, I also met a lot of great people there. Sergi and Nataliia, you two made me feel at home in Tarragona and I am very happy to say that our friendship has lasted beyond my time in Spain.

I am truly grateful for all those analytically-involved people: Dr. Sebastian Dechert for teaching me how to measure X-ray crystallography, as well as for solving all my computer problems; Dr. Jordi BenetBuchholz for calculating my ruthenium XRD structures; Dr. Serhiy Demeshko for collecting my SQUID data and providing me with all the knowledge I need on magnetic investigations in numerous sessions; Dr. A. Claudia Stückl for the EPR measurements, as well as Dr. Eckhard Bill and Dr. Yang Liu for helping me simulate the respective EPR spectra; Thomas Auth for the theoretical calculations; Dr. Fatemeh Ebrahimi, Christian Höhn at the Helmholz-Zentrum Berlin (Institute for Solar Fuels) and the Catalan Institute of Nanoscience and Nanotechnology in Barcelona for the XPS analyses; Dr. Holm Frauendorf and the mass department for mass spectrometric investigations; the analytical lab for the multiple combustion analyses; Dr. Allyssa A. Massie for the stopped-flow UV/vis measurements; Dr. Dennis Manz for equipping me with the ability to conduct NMR measurements myself; the NMR department and especially Dr. Michael John and Ralf Schöne for thoroughly increasing my knowledge on NMR spectroscopy and for the numerous high-quality NMR spectra; and finally, Dr. Jann Odrobina, Dr. Mauricio Cattaneo, Massimiliano Morganti and Dr. Shao-An Hua for supporting me in all electrochemical and spectroelectrochemical issues, and the former two for introducing me to water oxidation catalysis measurements, as well as for teaching me to use the ESI mass spectrometer. Thank you!

Most of the work in the laboratories would not be possible without many people working in the background. These include Petra Unger, Britta Müller-Wollmann, Dr. A. Claudia Stückl and Maria-José Gutiérrez (administrative issues), Matthias Hesse, Holger Tucholla and Katja Grube (chemicals), Andreas Schwarz (safety issues, orders, technical equipment), Jörg Teichgräber (orders, technical equipment), Hille, Uwe, Niklas, Sascha and Wolfram (caretakers), and all the workshops. Andreas Schwarz I would additionally like to thank for the synthesis of the ligand precursors. Furthermore, I gratefully acknowledge the Studienstiftung des deutschen Volkes for my PhD scholarship and both the Studienstiftung and the ICASEC for financing conferences and research stays abroad.

The old (Jann, Sven, Anett and Mauricio) and new (Sheida and Shao-An) "Water Oxidation Crew", the "Copper $\mathrm{O}_{2}$ Activation Crew" (Jerome and Martin), as well as Massi and Sara, I would like to thank for the helpful discussions and encouraging words when things were not going as planned. You all have 
had an open ear for me whenever I needed it and have kept me going strong. Jerome, Shao-An, Massi, Sara, Manuel, Allyssa, Carolina and Thomas I would like to thank for the conscientious proofreading of my thesis. I would further like to thank my Bachelor (Tanja) and Master students (Lukas, Saskia, Hannah and Sara) for the scientific work they did and the fun time we had. A very big thank you in this regard goes out to Sara, who worked on multiple different projects and greatly contributed to this thesis.

A special thanks goes to my old and new labmates Jann and Sheida. During the time we spent together in the lab, we have found common ground on all emerging issues, even concerning the music. You made the lab a most entertaining place to work and I feel confident to say that we make a great team.

All members of the Meyer group I would like to thank for the good working atmosphere. I had a great time in these past years, and I can say, without a doubt, that there are many good bakers and cooks among yourselves.

Outside of work, I would like to thank my friends who help me with words and deeds under all circumstances and provide me with the necessary moral support and distraction (You know who you are!). A special thanks goes to Thomas who has had to endure all my moaning and groaning and has continuously supported and encouraged me. I really appreciate you always being there for me.

Last, but certainly not least, I would like to thank my family who have supported me in all issues throughout my life. 


\title{
Curriculum Vitae
}

\author{
JANA ELENA LÜCKEN
}

\section{Personal data:}

DATE AND PLACE OF BIRTH

ADDRESS

EMAIL

NATIONALITY

\section{Education:}

UNIVERSITY

SCHOOL

\section{Work Experience:}

INDUSTRY
09 November 1989 / Oldenburg, Germany Lichtenbergstraße 1, 37075 Göttingen, Germany jana.luecken@chemie.uni-goettingen.de german

since 09/2015, PhD Thesis Georg-August University, Göttingen, Germany supervisor: Prof. Dr. Franc Meyer

10/2018 and 09/2016-12/2016, Research Stays Abroad Institute of Chemical Research of Catalonia, Tarragona, Spain supervisor: Prof. Dr. Antoni Llobet

10/2012-06/2015, Master in Chemistry (MSc, grade: 1.0) Georg-August University, Göttingen, Germany Supervisor of Master thesis: Prof. Dr. Franc Meyer

04/2014-07/2014, Research Stay Abroad University of Oxford, UK supervisor: Prof. Dr. Fraser Armstrong

10/2009-07/2012, Bachelor in Chemistry (BSc, grade: 1.1) Georg-August University, Göttingen, Germany Supervisor of Bachelor thesis: Prof. Dr. Franc Meyer

08/2008-05/2009, Stay Abroad (declared major: Chemistry) Valdosta State University, Georgia, USA

08/2002-06/2008, Abitur (grade: 1.8)

Herbartgymnasium, Oldenburg, Germany

08/2001-06/2002, School Abroad

International Secondary School Eindhoven, Netherlands

04/1994-06/2001, School Abroad

Regional International School Eindhoven, Netherlands

07/2014-10/2014, Industrial Internship

Research and Development, Altana AG in Wesel, Germany

\section{Scholarships and Awards:}

10/2019, Best Poster Award ICASEC Summer School 2019 in Göttingen, Germany

03/2019, Best Presentation Award $15^{\text {th }}$ annual Coordination Chemistry Meeting in Munich, Germany

05/2016-05/2019, PhD Scholarship Studienstiftung des deutschen Volkes 
MASTER

OTHER

\section{Scientific Contributions:}

POSTERS

TALKS

PUBLICATIONS
12/2015, Gustav-Tammann Award Georg-August-University in Göttingen, Germany For an outstanding Master's degree

10/2013-06/2014, Member

Studienstiftung des deutschen Volkes

08/2008-05/2009, Gulf South Conference Athletic Scholarship Valdosta State University (Basketball) in Georgia, USA

10/2019, ICASEC Summer School in Göttingen, Germany, "Reactivity and Mechanism of a Highly Active Molecular Diruthenium Water Oxidation Catalyst"

09/2018, $5^{\text {th }}$ Lower Saxony Catalysis Symposium in Göttingen, Germany, "Mechanistic Studies of a Highly Active Molecular Diruthenium Water Oxidation Catalyst", Göttingen, Germany

08/2017, Anglo-German Inorganic Chemistry Meeting in Göttingen, Germany, "Mechanistic Studies of a Highly Active Molecular Diruthenium Water Oxidation Catalyst", Göttingen, Germany

07/2017, International Solar Fuels Conference in San Diego, California, USA, "Mechanistic Studies of a Highly Active Molecular Diruthenium Water Oxidation Catalyst", Göttingen, Germany

03/2019, $15^{\text {th }}$ annual Coordination Chemistry Meeting in Munich, Germany, "Reactivity and Mechanism of a Highly Active Molecular Diruthenium Water Oxidation Catalyst", Göttingen, Germany

Jana Lücken,* Thomas Auth, Sara Ida Mozzi, Franc Meyer, "Hexanuclear Copper(I) Hydride from the Reduction-Induced Decarboxylation of a Dicopper(II) Formate", Inorg. Chem. 2020, 59, 14347-14354.

Jana Lücken,* Carolina Gimbert-Suriñach, Jordi Benet-Buchholz, Antoni Llobet, Franc Meyer, "Diruthenium Complexes of a Pyrazolate/Carboxylate Hybrid Ligand for Chemically, Electro- and Photo-Driven Catalytic Water Oxidation", ACS Catalysis (under revision) 